\title{
Analytical Data Report of Grab Samples Collected From BP-5 Operable Unit A Well (C5858)
}

Michael Lindberg

September 2008

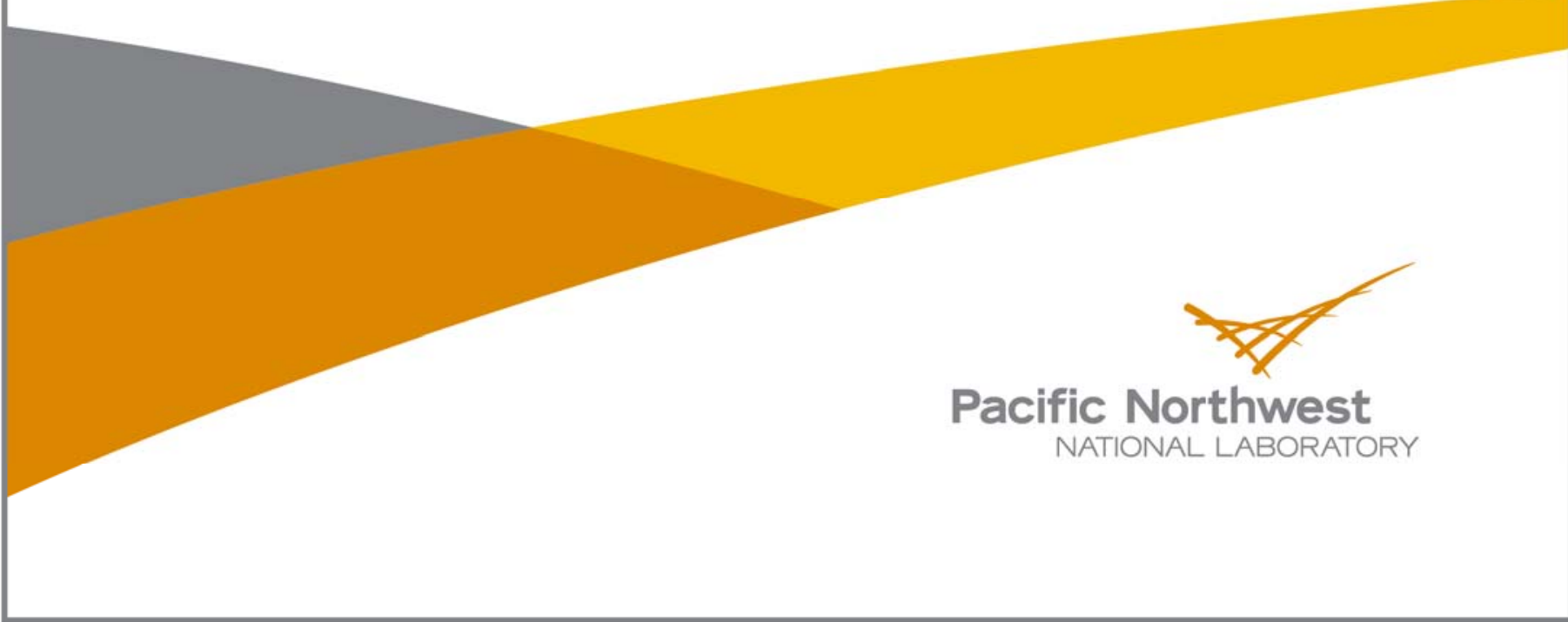




\section{To: Dana Widrig}

From: Michael J. Lindberg

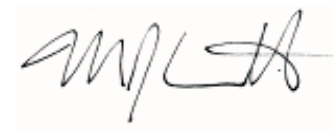

Environmental Sciences Laboratory

Energy and Environment Directorate, Pacific Northwest National Laboratory

Subject: Analytical Data Report of Grab Samples Collected From BP-5 Operable Unit A Well (5858), Sample Delivery Group ESL080002, SAF Number F08-036

This letter contains the following information for sample delivery group ESL080002

- Cover Sheet

- Narrative

- Analytical Results

- Quality Control

- Geologic Logs

- Geologic Photos

- Chain of Custodies 


\section{Introduction}

Between January 22, 2008 and February 26, 2008 soil cores and grab samples were received from BP-5 Operable Unit A Well (5858) for geochemical studies.

\section{Analytical Results/Methodology}

The analyses for this project were performed at the 325 building located in the 300 Area of the Hanford Site. The analyses were performed according to Pacific Northwest National Laboratory (PNNL) approved procedures and/or nationally recognized test procedures. The data sets include the sample identification numbers, analytical results, estimated quantification limits (EQL), and quality control data.

\section{Quality Control}

The preparatory and analytical quality control requirements, calibration requirements, acceptance criteria, and failure actions are defined in the on-line QA plan “Conducting Analytical Work in Support of Regulatory Programs” (CAW). This QA plan implements the Hanford Analytical Services Quality Assurance Requirements Documents (HASQARD) for PNNL.

\section{Definitions}

Dup Duplicate

RPD Relative Percent Difference

\section{Sample Receipt}

Samples were received with a chain of custody (COC) and were analyzed according to the sample identification numbers supplied by the client. All Samples were refrigerated upon receipt until prepared for analysis.

All samples were received with custody seals intact unless noted in the Case Narrative.

\section{Holding Times}

Holding time is defined as the time from sample preparation to the time of analyses. The prescribed holding times were met for all analytes unless noted in the Case Narrative.

\section{Analytical Results}

All reported analytical results meet the requirements of the CAW or client specified SOW unless noted in the case narrative. 


\section{Case Narrative Report}

\section{Hold time:}

No discrepancies noted.

\section{Preparation Blank (PB):}

QC Sample 8D28004-BLK1 failed criteria for Barium in ICP-OES Vadose-WE.

$\mathrm{MDL}=0.00879 \mathrm{ug} / \mathrm{g}$

$\mathrm{MRL}=0.00879 \mathrm{ug} / \mathrm{g}$

Result $=0.0368 \mathrm{ug} / \mathrm{g}$

Criterion = $1 \times$ MRL

Sample concentration was less than 5 times the blank concentration. Barium was not reported for these samples.

QC Sample 8D28004-BLK1 failed criteria for Zinc in ICP-OES Vadose-WE.

$\mathrm{MDL}=0.0927 \mathrm{ug} / \mathrm{g}$

MRL $=0.0927 \mathrm{ug} / \mathrm{g}$

Result $=0.196 \mathrm{ug} / \mathrm{g}$

Criterion = $1 \times$ MRL

The concentration of the samples were less than detectable. The blank contamination is not representative of the analytical data. There should be no impact to data as reported.

\section{Duplicate (DUP):}

Duplicate RPD for Uranium 238 (40.8\%) was above the acceptance limit (35) in 8D15001-DUP2 for ICPMS-Tc_U-WE

Potential analytical preparation error of the duplicate. Duplicate was in control for all other elements. There should be not impact to data as reported.

\section{Laboratory control samples (LCS):}

The Blank spike failed criteria for silver. It was determined that silver is not stable in the nitric acid digestion as performed. Silver is not reported for acid extracted samples.

\section{Post spike (PS) and post spike duplicate (PSD):}

Post-Spike Recovery for Calcium (464\%) was outside acceptance limits (75-125) in 8D28004-PS1 for ICP-OES Vadose-WE The native sample concentration was greater than 5 times the spike concentration. There should be not impact to data as reported.

Post-Spike Recovery for Magnesium (249\%) was outside acceptance limits (75-125) in 8D28004-PS1 for ICP-OES Vadose-WE The native sample concentration was greater than 5 times the spike concentration. There should be not impact to data as reported.

Post-Spike Recovery for Sodium (298\%) was outside acceptance limits (75-125) in 8D28004-PS1 for ICP-OES Vadose-WE The native sample concentration was greater than 5 times the spike concentration. There should be not impact to data as reported.

Post-Spike Recovery for Aluminum (227\%) was outside acceptance limits (75-125) in 8E12002-PS1 for ICP-OES Vadose-AE The native sample concentration was greater than 5 times the spike concentration. There should be not impact to data as reported.

Post-Spike Recovery for Calcium (212\%) was outside acceptance limits (75-125) in 8E12002-PS1 for ICP-OES Vadose-AE The native sample concentration was greater than 5 times the spike concentration. There should be not impact to data as reported.

Post-Spike Recovery for Iron (316\%) was outside acceptance limits (75-125) in 8E12002-PS1 for ICP-OES Vadose-AE The native sample concentration was greater than 5 times the spike concentration. There should be not impact to data as reported. 


\section{Case Narrative Report}

Post spike (PS) and post spike duplicate (PSD):

Post-Spike Recovery for Magnesium (192\%) was outside acceptance limits (75-125) in 8E12002-PS1 for ICP-OES Vadose-AE The native sample concentration was greater than 5 times the spike concentration. There should be not impact to data as reported.

Matrix spike (MS) and matrix spike duplicate (MSD):

No discrepancies noted.

Other QC Criteria:

No discrepancies noted.

\section{DISCLAIMER}

This report was prepared as an account of work sponsored by an agency of the United States Government. Neither the United States Government nor any agency thereof, nor Battelle Memorial Institute, nor any of their employees, makes any warranty, express or implied, or assumes any legal liability or responsibility for the accuracy, completeness, or usefulness of any information, apparatus, product, or process disclosed, or represents that its use would not infringe privately owned rights. Reference herein to any specific commercial product, process, or service by trade name, trademark, manufacturer, or otherwise does not necessarily constitute or imply its endorsement, recommendation, or favoring by the United States Government or any agency thereof, or Battelle Memorial Institute. The views and opinions of authors expressed herein do not necessarily state or reflect those of the United States Government or any agency thereof.

\section{SAMPLES INCLUDED IN THIS REPORT}

200 BP 5 OU, C5858 A-Well VZ

$\begin{array}{lll}\text { HEIS No. } & \text { Laboratory ID } & \text { Matrix } \\ \text { B1T170 } & 0802013-12 & \text { SOIL } \\ \text { B1T172 } & 0802013-14 & \text { SOIL } \\ \text { B1T152 } & 0802014-10 & \text { SOIL } \\ \text { B1T154 } & 0802014-12 & \text { SOIL } \\ \text { B1T156 } & 0802014-14 & \text { SOIL } \\ \text { B1T158 } & 0802014-16 & \text { SOIL } \\ \text { B1T142 } & 0802015-15 & \text { SOIL } \\ \text { B1T198 } & 0802016-26 & \text { SOIL } \\ \text { B1T1C4 } & 0802018-03 & \text { SOIL } \\ \text { B1T1H0 } & 0802022-01 & \text { SOIL } \\ \text { B1T1H2 } & 0802022-04 & \text { SOIL } \\ \text { B1T1H4 } & 0802022-07 & \text { SOIL } \\ \text { B1T1H6 } & 0802022-11 & \text { SOIL } \\ \text { B1T1D8 } & 0802024-15 & \text { SOIL } \\ \text { B1T1F0 } & 0802024-18 & \text { SOIL } \\ \text { B1T1F6 } & 0802024-27 & \text { SOIL } \\ \text { B1T1M0 } & 0802024-56 & \text { SOIL }\end{array}$

\begin{tabular}{|c|c|}
\hline $\begin{array}{l}\text { Date Collected } \\
1 / 23 / 0815: 02\end{array}$ & $\begin{array}{l}\text { Date Received } \\
1 / 24 / 0813: 10\end{array}$ \\
\hline $1 / 23 / 08 \quad 15: 26$ & $1 / 24 / 08 \quad 13: 10$ \\
\hline 1/22/08 12:56 & $1 / 23 / 08 \quad 13: 50$ \\
\hline 1/22/08 13:57 & $1 / 23 / 08 \quad 13: 50$ \\
\hline $1 / 22 / 08 \quad 14: 26$ & $1 / 23 / 08 \quad 13: 50$ \\
\hline 1/22/08 14:56 & $1 / 23 / 08 \quad 13: 50$ \\
\hline 1/21/08 15:25 & $1 / 22 / 08 \quad 13: 30$ \\
\hline 1/25/08 14:34 & $1 / 29 / 08 \quad 14: 00$ \\
\hline 1/30/08 09:05 & $1 / 31 / 08 \quad 14: 00$ \\
\hline 2/11/08 08:35 & $2 / 14 / 08 \quad 14: 00$ \\
\hline 2/11/08 10:20 & 2/14/08 14:00 \\
\hline 2/11/08 11:05 & $2 / 14 / 08 \quad 14: 00$ \\
\hline 2/11/08 15:14 & $2 / 14 / 08 \quad 14: 00$ \\
\hline 2/6/08 15:10 & 2/11/08 09:30 \\
\hline 2/7/08 09:25 & 2/11/08 09:30 \\
\hline 2/8/08 11:15 & 2/11/08 09:30 \\
\hline $2 / 22 / 08 \quad 10: 50$ & 2/26/08 13:50 \\
\hline
\end{tabular}


The following analyses were performed on the following samples included in this report:

Metals 1:1 DI Water Extract by ICPMS

Metals Acid Extract by ICPMS

Alkalinity, Titrimetic ( $\mathrm{pH} 4.5)$

Anions By Ion Chromatography

GEA No Preparation

Geological Description

Metals 1:1 Water Extract by ICPOES

Metals Acid Extract by ICPOES

Moisture Content

$\mathrm{pH}$ of Waters By Electrode

Specific Conductance

Tc_U Acid Extract by ICPMS

Tc_U 1:1 DI Water Extract by ICPMS

Tc_U UFA by ICPMS

Total Alpha Total Beta Acid Extract By LSC

\section{SAMPLES ANALYZED IN THIS REPORT}

$\begin{array}{lll}\text { HEIS No. } & \text { Laboratory ID } & \text { Matrix } \\ \text { B1T170 } & 0802013-12 & \text { SOIL } \\ \text { B1T172 } & 0802013-14 & \text { SOIL } \\ \text { B1T152 } & 0802014-10 & \text { SOIL } \\ \text { B1T154 } & 0802014-12 & \text { SOIL } \\ \text { B1T156 } & 0802014-14 & \text { SOIL } \\ \text { B1T158 } & 0802014-16 & \text { SOIL } \\ \text { B1T142 } & 0802015-15 & \text { SOIL } \\ \text { B1T198 } & 0802016-26 & \text { SOIL } \\ \text { B1T1C4 } & 0802018-03 & \text { SOIL } \\ \text { B1T1H0 } & 0802022-01 & \text { SOIL } \\ \text { B1T1H2 } & 0802022-04 & \text { SOIL } \\ \text { B1T1H4 } & 0802022-07 & \text { SOIL } \\ \text { B1T1H6 } & 0802022-11 & \text { SOIL } \\ \text { B1T1D8 } & 0802024-15 & \text { SOIL } \\ \text { B1T1F0 } & 0802024-18 & \text { SOIL } \\ \text { B1T1F6 } & 0802024-27 & \text { SOIL } \\ \text { B1T1M0 } & 0802024-56 & \text { SOIL }\end{array}$

\begin{tabular}{|c|c|}
\hline Date Collected & Date Received \\
\hline 1/23/08 15:02 & $1 / 24 / 08 \quad 13: 10$ \\
\hline 1/23/08 15:26 & $1 / 24 / 08 \quad 13: 10$ \\
\hline $1 / 22 / 08 \quad 12: 56$ & $1 / 23 / 08 \quad 13: 50$ \\
\hline 1/22/08 13:57 & $1 / 23 / 08 \quad 13: 50$ \\
\hline 1/22/08 14:26 & $1 / 23 / 08 \quad 13: 50$ \\
\hline $1 / 22 / 08 \quad 14: 56$ & $1 / 23 / 08 \quad 13: 50$ \\
\hline 1/21/08 15:25 & $1 / 22 / 08 \quad 13: 30$ \\
\hline $1 / 25 / 08 \quad 14: 34$ & $1 / 29 / 08 \quad 14: 00$ \\
\hline 1/30/08 09:05 & $1 / 31 / 08 \quad 14: 00$ \\
\hline 2/11/08 08:35 & $2 / 14 / 08 \quad 14: 00$ \\
\hline 2/11/08 10:20 & $2 / 14 / 08 \quad 14: 00$ \\
\hline 2/11/08 11:05 & $2 / 14 / 08 \quad 14: 00$ \\
\hline 2/11/08 15:14 & $2 / 14 / 08 \quad 14: 00$ \\
\hline 2/6/08 15:10 & 2/11/08 09:30 \\
\hline 2/7/08 09:25 & 2/11/08 09:30 \\
\hline 2/8/08 11:15 & 2/11/08 09:30 \\
\hline 2/22/08 10:50 & 2/26/08 13:50 \\
\hline
\end{tabular}




\section{Wet Chemistry}

\begin{tabular}{|c|c|c|c|c|c|}
\hline $\begin{array}{l}\text { Alkalinity } \\
\text { Lab ID }\end{array}$ & $\begin{array}{c}\text { CaCO3 ( } \\
\text { HEIS No. }\end{array}$ & $\begin{array}{l}\text { Method } \\
\text { Results }\end{array}$ & $\begin{array}{l}\text { OB } \\
\text { EQL }\end{array}$ & Analyzed & Batch \\
\hline 0802013-12 & B1T170 & $6.08 \mathrm{E} 1$ & $2.35 \mathrm{E} 1$ & $3 / 31 / 08$ & 8C31001 \\
\hline 0802013-14 & B1T172 & 4.73E1 & 2.32E1 & 3/31/08 & 8C31001 \\
\hline 0802014-10 & B1T152 & 4.44E1 & 2.33E1 & 3/31/08 & 8С31001 \\
\hline 0802015-15 & B1T142 & 4.28E1 & 2.36E1 & 3/31/08 & 8C31001 \\
\hline 0802016-26 & B1T198 & 3.87E1 & 2.35E1 & $3 / 31 / 08$ & 8C31001 \\
\hline 0802018-03 & B1T1C4 & $5.11 \mathrm{E} 1$ & 2.36E1 & 3/31/08 & 8C31001 \\
\hline 0802022-01 & B1T1H0 & 7.44E1 & 2.35E1 & 3/31/08 & 8C31001 \\
\hline 0802022-04 & B1T1H2 & $1.11 \mathrm{E} 2$ & 2.33E1 & 3/31/08 & 8C31001 \\
\hline 0802022-07 & B1T1H4 & $2.21 \mathrm{E} 2$ & 2.35E1 & $3 / 31 / 08$ & 8C31001 \\
\hline 0802022-11 & B1T1H6 & 1.32E2 & 2.35E1 & 3/31/08 & 8С31001 \\
\hline 0802024-15 & B1T1D8 & 3.81E1 & 2.36E1 & 3/31/08 & 8C31001 \\
\hline 0802024-18 & B1T1F0 & 7.16E1 & 2.36E1 & $3 / 31 / 08$ & 8C31001 \\
\hline 0802024-27 & B1T1F6 & $6.56 \mathrm{E} 1$ & 2.36E1 & $3 / 31 / 08$ & 8C31001 \\
\hline 0802024-56 & B1T1M0 & $5.24 \mathrm{E} 1$ & 2.38E1 & 3/31/08 & 8C31001 \\
\hline
\end{tabular}




\section{Wet Chemistry}

\begin{tabular}{llcccc}
\multicolumn{2}{l}{$\begin{array}{l}\text { Specific Conductance } \\
\text { Lab ID }\end{array}$} & HEIS No. & (mS/cm) by EPA 120.1 & Analyzed & Batch \\
\hline $0802013-12$ & B1T170 & Results & EQL & $3 / 28 / 08$ & $8 \mathrm{C} 28004$ \\
$0802013-14$ & B1T172 & $3.15 \mathrm{E}-1$ & $1.00 \mathrm{E}-2$ & $3 / 28 / 08$ & $8 \mathrm{C} 28004$ \\
$0802014-10$ & B1T152 & $1.56 \mathrm{E}-1$ & $1.00 \mathrm{E}-2$ & $3 / 28 / 08$ & $8 \mathrm{C} 28004$ \\
$0802015-15$ & B1T142 & $2.24 \mathrm{E}-1$ & $1.00 \mathrm{E}-2$ & $3 / 28 / 08$ & $8 \mathrm{C} 28004$ \\
$0802016-26$ & B1T198 & $1.37 \mathrm{E}-1$ & $1.00 \mathrm{E}-2$ & $3 / 28 / 08$ & $8 \mathrm{C} 28004$ \\
$0802018-03$ & B1T1C4 & $1.39 \mathrm{E}-1$ & $1.00 \mathrm{E}-2$ & $3 / 28 / 08$ & $8 \mathrm{C} 28004$ \\
$0802022-01$ & B1T1H0 & $1.73 \mathrm{E}-1$ & $1.00 \mathrm{E}-2$ & $3 / 28 / 08$ & $8 \mathrm{C} 28004$ \\
$0802022-04$ & B1T1H2 & $1.10 \mathrm{E} 0$ & $1.00 \mathrm{E}-2$ & $3 / 28 / 08$ & $8 \mathrm{C} 28004$ \\
$0802022-07$ & B1T1H4 & $1.47 \mathrm{E} 0$ & $1.00 \mathrm{E}-2$ & $3 / 28 / 08$ & $8 \mathrm{C} 28004$ \\
$0802022-11$ & B1T1H6 & $1.71 \mathrm{E} 0$ & $1.00 \mathrm{E}-2$ & $3 / 28 / 08$ & $8 \mathrm{C} 28004$ \\
$0802024-15$ & B1T1D8 & $1.65 \mathrm{E} 0$ & $1.00 \mathrm{E}-2$ & $3 / 28 / 08$ & $8 \mathrm{C} 28004$ \\
$0802024-18$ & B1T1F0 & $1.56 \mathrm{E}-1$ & $1.00 \mathrm{E}-2$ & $3 / 28 / 08$ & $8 \mathrm{C} 28004$ \\
$0802024-27$ & B1T1F6 & $4.60 \mathrm{E}-1$ & $1.00 \mathrm{E}-2$ & $3 / 28 / 08$ & $8 \mathrm{C} 28004$ \\
$0802024-56$ & B1T1M0 & $4.57 \mathrm{E}-1$ & $1.00 \mathrm{E}-2$ & $3 / 28 / 08$ & $8 \mathrm{C} 28004$ \\
\hline
\end{tabular}




\section{Wet Chemistry}

\begin{tabular}{|c|c|c|c|c|c|}
\hline $\begin{array}{l}\text { Moisture } \\
\text { Lab ID }\end{array}$ & $\begin{array}{l}\text { ntent (\% } \\
\text { HEIS No. }\end{array}$ & $\begin{array}{l}\text { C-001 } \\
\text { Results }\end{array}$ & EQL & Analyzed & Batch \\
\hline 0802013-12 & B1T170 & $1.78 \mathrm{E} 1$ & N/A & $3 / 17 / 08$ & $8 C 05001$ \\
\hline 0802013-14 & B1T172 & 5.85E0 & N/A & $3 / 17 / 08$ & 8C05001 \\
\hline 0802014-10 & B1T152 & $5.88 \mathrm{E} 0$ & N/A & $3 / 17 / 08$ & 8C05001 \\
\hline 0802014-12 & B1T154 & 2.34E0 & N/A & $3 / 17 / 08$ & 8C05001 \\
\hline 0802014-14 & B1T156 & $6.62 \mathrm{E} 0$ & N/A & $3 / 17 / 08$ & 8C05001 \\
\hline 0802014-16 & B1T158 & $3.16 \mathrm{E} 0$ & N/A & $3 / 17 / 08$ & 8C05001 \\
\hline 0802015-15 & B1T142 & 4.03E0 & N/A & $3 / 17 / 08$ & 8C05001 \\
\hline 0802016-26 & B1T198 & 3.64E0 & N/A & $3 / 17 / 08$ & 8C05001 \\
\hline 0802018-03 & B1T1C4 & 5.97E0 & N/A & $3 / 17 / 08$ & 8C05001 \\
\hline 0802022-01 & B1T1H0 & 1.83E1 & N/A & $3 / 17 / 08$ & 8C05001 \\
\hline 0802022-04 & B1T1H2 & $3.11 \mathrm{E} 1$ & N/A & $3 / 17 / 08$ & 8C05001 \\
\hline 0802022-07 & B1T1H4 & 2.77E1 & N/A & $3 / 17 / 08$ & 8C05001 \\
\hline 0802022-11 & B1T1H6 & 2.35E1 & N/A & $3 / 17 / 08$ & 8C05001 \\
\hline 0802024-15 & B1T1D8 & 4.79E0 & N/A & $3 / 17 / 08$ & 8C05001 \\
\hline 0802024-18 & B1T1F0 & 2.82E1 & N/A & $3 / 17 / 08$ & 8C05001 \\
\hline 0802024-27 & B1T1F6 & 1.91E1 & N/A & $3 / 17 / 08$ & 8C05001 \\
\hline 0802024-56 & B1T1M0 & 5.89E0 & N/A & $3 / 17 / 08$ & 8C05001 \\
\hline
\end{tabular}




\section{Wet Chemistry}

pH (pH Units) by AGG-pH-001

\begin{tabular}{|c|c|c|c|c|c|}
\hline Lab ID & HEIS No. & Results & EQL & Analyzed & Batch \\
\hline 0802013-12 & B1T170 & 8.05E0 & N/A & $3 / 28 / 08$ & 8C28004 \\
\hline 0802013-14 & B1T172 & 7.98E0 & N/A & 3/28/08 & 8C28004 \\
\hline 0802014-10 & B1T152 & 7.88E0 & N/A & $3 / 28 / 08$ & 8C28004 \\
\hline 0802015-15 & B1T142 & 8.08E0 & N/A & $3 / 28 / 08$ & 8C28004 \\
\hline 0802016-26 & B1T198 & 7.89E0 & N/A & $3 / 28 / 08$ & 8C28004 \\
\hline 0802018-03 & B1T1C4 & 7.89E0 & N/A & 3/28/08 & 8C28004 \\
\hline 0802022-01 & B1T1H0 & 7.82E0 & N/A & $3 / 28 / 08$ & $8 C 28004$ \\
\hline 0802022-04 & B1T1H2 & 8.03E0 & N/A & $3 / 28 / 08$ & $8 C 28004$ \\
\hline 0802022-07 & B1T1H4 & 8.32E0 & N/A & 3/28/08 & 8C28004 \\
\hline 0802022-11 & B1T1H6 & 8.04E0 & N/A & $3 / 28 / 08$ & $8 C 28004$ \\
\hline 0802024-15 & B1T1D8 & 7.85E0 & N/A & $3 / 28 / 08$ & 8C28004 \\
\hline 0802024-18 & B1T1F0 & $8.13 \mathrm{E} 0$ & N/A & $3 / 28 / 08$ & 8C28004 \\
\hline 0802024-27 & B1T1F6 & 8.18E0 & N/A & 3/28/08 & 8C28004 \\
\hline 0802024-56 & B1T1M0 & 8.07E0 & N/A & $3 / 28 / 08$ & 8C28004 \\
\hline
\end{tabular}




\section{Anions by Ion Chromatography}

\begin{tabular}{|c|c|c|c|c|c|c|c|}
\hline CAS \# & Analyte & Results & Units & EQL & Analyzed & Batch & Method \\
\hline HEIS No. & B1T170 & \multicolumn{3}{|c|}{ Lab ID: $\quad$ 0802013-12 } & & & \\
\hline $16984-48-8$ & Fluoride & $<2.00 \mathrm{E} 0$ & ug/g dry & $2.00 \mathrm{E} 0$ & 3/28/08 & 8C28005 & AGG-IC-001 \\
\hline $16887-00-6$ & Chloride & $1.32 \mathrm{E} 1$ & ug/g dry & $5.00 \mathrm{E} 0$ & 3/28/08 & 8C28005 & AGG-IC-001 \\
\hline $14797-65-0$ & Nitrite & $<1.00 \mathrm{E} 1$ & ug/g dry & $1.00 \mathrm{E} 1$ & 3/28/08 & 8C28005 & AGG-IC-001 \\
\hline 24959-67-9 & Bromide & $<1.00 \mathrm{E} 1$ & ug/g dry & $1.00 \mathrm{E} 1$ & 3/28/08 & 8C28005 & AGG-IC-001 \\
\hline 14797-55-8 & Nitrate & $<1.00 \mathrm{E} 1$ & ug/g dry & $1.00 \mathrm{E} 1$ & 3/28/08 & 8C28005 & AGG-IC-001 \\
\hline $14808-79-8$ & Sulfate & $5.55 \mathrm{E} 1$ & ug/g dry & $1.50 \mathrm{E} 1$ & 3/28/08 & 8C28005 & AGG-IC-001 \\
\hline $14265-44-2$ & Phosphate & $<1.50 \mathrm{E} 1$ & ug/g dry & $1.50 \mathrm{E} 1$ & 3/28/08 & 8C28005 & AGG-IC-001 \\
\hline HEIS No. & B1T172 & \multicolumn{3}{|c|}{ Lab ID: $\quad$ 0802013-14 } & & & \\
\hline $16984-48-8$ & Fluoride & 3.37E-1 & ug/g dry & $1.98 \mathrm{E}-1$ & 3/28/08 & 8C28005 & AGG-IC-001 \\
\hline $16887-00-6$ & Chloride & 2.67E0 & ug/g dry & 4.94E-1 & 3/28/08 & 8C28005 & AGG-IC-001 \\
\hline $14797-65-0$ & Nitrite & $<9.88 \mathrm{E}-1$ & ug/g dry & $9.88 \mathrm{E}-1$ & 3/28/08 & 8C28005 & AGG-IC-001 \\
\hline 24959-67-9 & Bromide & $<9.88 \mathrm{E}-1$ & ug/g dry & $9.88 \mathrm{E}-1$ & 3/28/08 & 8C28005 & AGG-IC-001 \\
\hline $14797-55-8$ & Nitrate & 2.75E0 & ug/g dry & $9.88 \mathrm{E}-1$ & 3/28/08 & 8C28005 & AGG-IC-001 \\
\hline 14808-79-8 & Sulfate & $1.71 \mathrm{E} 1$ & ug/g dry & $1.48 \mathrm{E} 0$ & 3/28/08 & 8C28005 & AGG-IC-001 \\
\hline $14265-44-2$ & Phosphate & $<1.48 \mathrm{E} 0$ & ug/g dry & $1.48 \mathrm{E} 0$ & 3/28/08 & 8C28005 & AGG-IC-001 \\
\hline HEIS No. & B1T152 & \multicolumn{3}{|c|}{ Lab ID: $\quad$ 0802014-10 } & & & \\
\hline $16984-48-8$ & Fluoride & $3.25 \mathrm{E}-1$ & ug/g dry & $1.98 \mathrm{E}-1$ & 3/28/08 & 8C28005 & AGG-IC-001 \\
\hline $16887-00-6$ & Chloride & 3.99E0 & ug/g dry & 4.95E-1 & 3/28/08 & 8C28005 & AGG-IC-001 \\
\hline $14797-65-0$ & Nitrite & $<9.90 \mathrm{E}-1$ & ug/g dry & $9.90 \mathrm{E}-1$ & 3/28/08 & 8C28005 & AGG-IC-001 \\
\hline 24959-67-9 & Bromide & $<9.90 \mathrm{E}-1$ & ug/g dry & $9.90 \mathrm{E}-1$ & 3/28/08 & 8C28005 & AGG-IC-001 \\
\hline $14797-55-8$ & Nitrate & 3.76E0 & ug/g dry & $9.90 \mathrm{E}-1$ & 3/28/08 & 8C28005 & AGG-IC-001 \\
\hline 14808-79-8 & Sulfate & $4.80 \mathrm{E} 1$ & ug/g dry & 1.49E0 & 3/28/08 & 8C28005 & AGG-IC-001 \\
\hline $14265-44-2$ & Phosphate & $<1.49 \mathrm{E} 0$ & ug/g dry & $1.49 \mathrm{E} 0$ & 3/28/08 & 8C28005 & AGG-IC-001 \\
\hline HEIS No. & B1T142 & \multicolumn{3}{|c|}{ Lab ID: $\quad$ 0802015-15 } & & & \\
\hline $16984-48-8$ & Fluoride & $3.62 \mathrm{E}-1$ & ug/g dry & $2.01 \mathrm{E}-1$ & 3/28/08 & 8C28005 & AGG-IC-001 \\
\hline $16887-00-6$ & Chloride & $1.95 \mathrm{E} 0$ & ug/g dry & 5.03E-1 & 3/28/08 & 8C28005 & AGG-IC-001 \\
\hline $14797-65-0$ & Nitrite & $<1.01 \mathrm{E} 0$ & ug/g dry & $1.01 \mathrm{E} 0$ & 3/28/08 & 8C28005 & AGG-IC-001 \\
\hline 24959-67-9 & Bromide & $<1.01 \mathrm{E} 0$ & ug/g dry & $1.01 \mathrm{E} 0$ & 3/28/08 & 8C28005 & AGG-IC-001 \\
\hline $14797-55-8$ & Nitrate & $1.60 \mathrm{E} 0$ & ug/g dry & $1.01 \mathrm{E} 0$ & 3/28/08 & 8C28005 & AGG-IC-001 \\
\hline $14808-79-8$ & Sulfate & $1.55 \mathrm{E} 1$ & ug/g dry & $1.51 \mathrm{E} 0$ & 3/28/08 & 8C28005 & AGG-IC-001 \\
\hline 14265-44-2 & Phosphate & $<1.51 \mathrm{E} 0$ & ug/g dry & $1.51 \mathrm{E} 0$ & 3/28/08 & 8C28005 & AGG-IC-001 \\
\hline HEIS No. & B1T198 & \multicolumn{3}{|c|}{ Lab ID: $\quad$ 0802016-26 } & & & \\
\hline $16984-48-8$ & Fluoride & $2.31 \mathrm{E}-1$ & ug/g dry & 2.00E-1 & 3/28/08 & 8C28005 & AGG-IC-001 \\
\hline $16887-00-6$ & Chloride & 4.06Е0 & ug/g dry & 4.99E-1 & 3/28/08 & 8C28005 & AGG-IC-001 \\
\hline $14797-65-0$ & Nitrite & $<9.98 \mathrm{E}-1$ & ug/g dry & $9.98 \mathrm{E}-1$ & 3/28/08 & 8C28005 & AGG-IC-001 \\
\hline 24959-67-9 & Bromide & $<9.98 \mathrm{E}-1$ & ug/g dry & $9.98 \mathrm{E}-1$ & 3/28/08 & $8 C 28005$ & AGG-IC-001 \\
\hline $14797-55-8$ & Nitrate & 3.92E0 & ug/g dry & $9.98 \mathrm{E}-1$ & 3/28/08 & 8C28005 & AGG-IC-001 \\
\hline 14808-79-8 & Sulfate & $1.67 \mathrm{E} 1$ & ug/g dry & $1.50 \mathrm{E} 0$ & 3/28/08 & 8C28005 & AGG-IC-001 \\
\hline $14265-44-2$ & Phosphate & $<1.50 \mathrm{E} 0$ & ug/g dry & $1.50 \mathrm{E} 0$ & 3/28/08 & 8C28005 & AGG-IC-001 \\
\hline HEIS No. & B1T1C4 & \multicolumn{3}{|c|}{ Lab ID: $\quad$ 0802018-03 } & & & \\
\hline $16984-48-8$ & Fluoride & $3.44 \mathrm{E}-1$ & ug/g dry & $2.01 \mathrm{E}-1$ & 3/28/08 & 8C28005 & AGG-IC-001 \\
\hline $16887-00-6$ & Chloride & 3.39E0 & ug/g dry & $5.01 \mathrm{E}-1$ & $3 / 28 / 08$ & 8C28005 & AGG-IC-001 \\
\hline $14797-65-0$ & Nitrite & $<1.00 \mathrm{E} 0$ & ug/g dry & $1.00 \mathrm{E} 0$ & 3/28/08 & 8C28005 & AGG-IC-001 \\
\hline 24959-67-9 & Bromide & $<1.00 \mathrm{E} 0$ & ug/g dry & $1.00 \mathrm{E} 0$ & 3/28/08 & 8C28005 & AGG-IC-001 \\
\hline $14797-55-8$ & Nitrate & 4.33E0 & ug/g dry & $1.00 \mathrm{E} 0$ & 3/28/08 & 8C28005 & AGG-IC-001 \\
\hline 14808-79-8 & Sulfate & 2.32E1 & ug/g dry & $1.50 \mathrm{E} 0$ & 3/28/08 & 8C28005 & AGG-IC-001 \\
\hline 14265-44-2 & Phosphate & $<1.50 \mathrm{E} 0$ & ug/g dry & $1.50 \mathrm{E} 0$ & 3/28/08 & 8C28005 & AGG-IC-001 \\
\hline HEIS No. & B1T1H0 & \multicolumn{2}{|c|}{ Lab ID: } & 2022-01 & & & \\
\hline
\end{tabular}




\section{Anions by Ion Chromatography}

\begin{tabular}{|c|c|c|c|c|c|c|c|}
\hline CAS \# & Analyte & Results & Units & EQL & Analyzed & Batch & Method \\
\hline HEIS No. & B1T1H0 & \multicolumn{3}{|c|}{ Lab ID: $\quad$ 0802022-01 } & & & \\
\hline $16984-48-8$ & Fluoride & $<2.00 \mathrm{E} 0$ & ug/g dry & $2.00 \mathrm{E} 0$ & 3/28/08 & 8C28005 & AGG-IC-001 \\
\hline 16887-00-6 & Chloride & $1.76 \mathrm{E} 1$ & ug/g dry & 4.99E0 & 3/28/08 & 8C28005 & AGG-IC-001 \\
\hline 14797-65-0 & Nitrite & $<9.99 \mathrm{E} 0$ & ug/g dry & 9.99E0 & 3/28/08 & 8C28005 & AGG-IC-001 \\
\hline 24959-67-9 & Bromide & $<9.99 \mathrm{E} 0$ & ug/g dry & 9.99E0 & 3/28/08 & 8C28005 & AGG-IC-001 \\
\hline $14797-55-8$ & Nitrate & 2.83E2 & ug/g dry & 9.99E0 & 3/28/08 & 8C28005 & AGG-IC-001 \\
\hline 14808-79-8 & Sulfate & $1.87 \mathrm{E} 2$ & ug/g dry & $1.50 \mathrm{E} 1$ & 3/28/08 & 8C28005 & AGG-IC-001 \\
\hline $14265-44-2$ & Phosphate & $<1.50 \mathrm{E} 1$ & ug/g dry & $1.50 \mathrm{E} 1$ & 3/28/08 & 8C28005 & AGG-IC-001 \\
\hline HEIS No. & B1T1H2 & \multicolumn{3}{|c|}{ Lab ID: $\quad$ 0802022-04 } & & & \\
\hline 16984-48-8 & Fluoride & $<1.98 \mathrm{E} 0$ & ug/g dry & $1.98 \mathrm{E} 0$ & 3/29/08 & 8C28005 & AGG-IC-001 \\
\hline $16887-00-6$ & Chloride & 3.27E1 & ug/g dry & 4.96E0 & 3/29/08 & 8C28005 & AGG-IC-001 \\
\hline 14797-65-0 & Nitrite & $1.00 \mathrm{E} 1$ & ug/g dry & 9.92E0 & 3/29/08 & 8C28005 & AGG-IC-001 \\
\hline 24959-67-9 & Bromide & $<9.92 \mathrm{E} 0$ & ug/g dry & 9.92E0 & 3/29/08 & 8C28005 & AGG-IC-001 \\
\hline $14797-55-8$ & Nitrate & $3.48 \mathrm{E} 2$ & ug/g dry & 9.92E0 & 3/29/08 & 8C28005 & AGG-IC-001 \\
\hline 14808-79-8 & Sulfate & 2.53E2 & ug/g dry & 1.49E1 & 3/29/08 & 8C28005 & AGG-IC-001 \\
\hline $14265-44-2$ & Phosphate & $<1.49 \mathrm{E} 1$ & ug/g dry & 1.49E1 & 3/29/08 & 8C28005 & AGG-IC-001 \\
\hline HEIS No. & B1T1H4 & \multicolumn{3}{|c|}{ Lab ID: $\quad$ 0802022-07 } & & & \\
\hline 16984-48-8 & Fluoride & $<2.00 \mathrm{E} 0$ & ug/g dry & $2.00 \mathrm{E} 0$ & 3/29/08 & 8C28005 & AGG-IC-001 \\
\hline 16887-00-6 & Chloride & 2.86E1 & ug/g dry & $5.00 \mathrm{E} 0$ & 3/29/08 & 8C28005 & AGG-IC-001 \\
\hline $14797-65-0$ & Nitrite & 4.93E1 & ug/g dry & $1.00 \mathrm{E} 1$ & 3/29/08 & 8C28005 & AGG-IC-001 \\
\hline 24959-67-9 & Bromide & $<1.00 \mathrm{E} 1$ & ug/g dry & $1.00 \mathrm{E} 1$ & 3/29/08 & 8C28005 & AGG-IC-001 \\
\hline 14797-55-8 & Nitrate & $4.58 \mathrm{E} 2$ & ug/g dry & $1.00 \mathrm{E} 1$ & 3/29/08 & 8C28005 & AGG-IC-001 \\
\hline 14808-79-8 & Sulfate & 3.63E2 & ug/g dry & 1.50E1 & 3/29/08 & 8C28005 & AGG-IC-001 \\
\hline $14265-44-2$ & Phosphate & $<1.50 \mathrm{E} 1$ & ug/g dry & $1.50 \mathrm{E} 1$ & 3/29/08 & 8C28005 & AGG-IC-001 \\
\hline HEIS No. & B1T1H6 & \multicolumn{3}{|c|}{ Lab ID: $\quad$ 0802022-11 } & & & \\
\hline 16984-48-8 & Fluoride & $<2.00 \mathrm{E} 0$ & ug/g dry & $2.00 \mathrm{E} 0$ & 3/29/08 & 8C28005 & AGG-IC-001 \\
\hline $16887-00-6$ & Chloride & $2.78 \mathrm{E} 1$ & ug/g dry & $5.00 \mathrm{E} 0$ & 3/29/08 & 8C28005 & AGG-IC-001 \\
\hline 14797-65-0 & Nitrite & $<1.00 \mathrm{E} 1$ & ug/g dry & $1.00 \mathrm{E} 1$ & 3/29/08 & 8C28005 & AGG-IC-001 \\
\hline 24959-67-9 & Bromide & $<1.00 \mathrm{E} 1$ & ug/g dry & $1.00 \mathrm{E} 1$ & 3/29/08 & 8C28005 & AGG-IC-001 \\
\hline $14797-55-8$ & Nitrate & $6.00 \mathrm{E} 2$ & ug/g dry & $1.00 \mathrm{E} 1$ & 3/29/08 & 8C28005 & AGG-IC-001 \\
\hline 14808-79-8 & Sulfate & $4.01 \mathrm{E} 2$ & ug/g dry & $1.50 \mathrm{E} 1$ & 3/29/08 & 8C28005 & AGG-IC-001 \\
\hline $14265-44-2$ & Phosphate & $<1.50 \mathrm{E} 1$ & ug/g dry & $1.50 \mathrm{E} 1$ & 3/29/08 & 8C28005 & AGG-IC-001 \\
\hline HEIS No. & B1T1D8 & \multicolumn{3}{|c|}{ Lab ID: $\quad$ 0802024-15 } & & & \\
\hline $16984-48-8$ & Fluoride & $2.70 \mathrm{E}-1$ & ug/g dry & $2.01 \mathrm{E}-1$ & 3/29/08 & 8C28005 & AGG-IC-001 \\
\hline $16887-00-6$ & Chloride & $3.58 \mathrm{E} 0$ & ug/g dry & $5.02 \mathrm{E}-1$ & 3/29/08 & 8C28005 & AGG-IC-001 \\
\hline $14797-65-0$ & Nitrite & $<1.00 \mathrm{E} 0$ & ug/g dry & $1.00 \mathrm{E} 0$ & 3/29/08 & 8C28005 & AGG-IC-001 \\
\hline 24959-67-9 & Bromide & $<1.00 \mathrm{E} 0$ & ug/g dry & $1.00 \mathrm{E} 0$ & 3/29/08 & $8 C 28005$ & AGG-IC-001 \\
\hline $14797-55-8$ & Nitrate & 6.32E0 & ug/g dry & $1.00 \mathrm{E} 0$ & 3/29/08 & 8C28005 & AGG-IC-001 \\
\hline 14808-79-8 & Sulfate & 2.13E1 & ug/g dry & $1.50 \mathrm{E} 0$ & 3/29/08 & 8C28005 & AGG-IC-001 \\
\hline $14265-44-2$ & Phosphate & $<1.50 \mathrm{E} 0$ & ug/g dry & $1.50 \mathrm{E} 0$ & 3/29/08 & 8C28005 & AGG-IC-001 \\
\hline HEIS No. & B1T1F0 & \multicolumn{3}{|c|}{ Lab ID: $\quad$ 0802024-18 } & & & \\
\hline 16984-48-8 & Fluoride & $4.11 \mathrm{E}-1$ & ug/g dry & $2.00 \mathrm{E}-1$ & 3/29/08 & 8C28005 & AGG-IC-001 \\
\hline $16887-00-6$ & Chloride & $1.69 \mathrm{E} 1$ & ug/g dry & $5.01 \mathrm{E}-1$ & 3/29/08 & 8C28005 & AGG-IC-001 \\
\hline $14797-65-0$ & Nitrite & $<1.00 \mathrm{E} 0$ & ug/g dry & $1.00 \mathrm{E} 0$ & 3/29/08 & 8C28005 & AGG-IC-001 \\
\hline 24959-67-9 & Bromide & $<1.00 \mathrm{E} 0$ & ug/g dry & $1.00 \mathrm{E} 0$ & 3/29/08 & 8C28005 & AGG-IC-001 \\
\hline $14797-55-8$ & Nitrate & $1.86 \mathrm{E} 1$ & ug/g dry & $1.00 \mathrm{E} 0$ & 3/29/08 & 8C28005 & AGG-IC-001 \\
\hline 14808-79-8 & Sulfate & 9.91E1 & ug/g dry & $1.50 \mathrm{E} 0$ & 3/29/08 & 8C28005 & AGG-IC-001 \\
\hline $14265-44-2$ & Phosphate & $<1.50 \mathrm{E} 0$ & ug/g dry & $1.50 \mathrm{E} 0$ & 3/29/08 & 8C28005 & AGG-IC-001 \\
\hline HEIS No. & B1T1F6 & \multicolumn{2}{|c|}{ Lab ID: } & 2024-27 & & & \\
\hline
\end{tabular}




\section{Anions by Ion Chromatography}

\begin{tabular}{|c|c|c|c|c|c|c|c|}
\hline CAS \# & Analyte & Results & Units & EQL & Analyzed & Batch & Method \\
\hline HEIS No. & B1T1F6 & \multicolumn{3}{|c|}{ Lab ID: $\quad$ 0802024-27 } & & & \\
\hline $16984-48-8$ & Fluoride & 4.26E-1 & ug/g dry & $2.01 \mathrm{E}-1$ & 3/29/08 & 8C28005 & AGG-IC-001 \\
\hline $16887-00-6$ & Chloride & $1.55 \mathrm{E} 1$ & ug/g dry & $5.02 \mathrm{E}-1$ & 3/29/08 & 8C28005 & AGG-IC-001 \\
\hline $14797-65-0$ & Nitrite & $<1.00 \mathrm{E} 0$ & ug/g dry & $1.00 \mathrm{E} 0$ & 3/29/08 & 8C28005 & AGG-IC-001 \\
\hline 24959-67-9 & Bromide & $<1.00 \mathrm{E} 0$ & ug/g dry & $1.00 \mathrm{E} 0$ & 3/29/08 & 8C28005 & AGG-IC-001 \\
\hline $14797-55-8$ & Nitrate & 2.66E1 & ug/g dry & $1.00 \mathrm{E} 0$ & 3/29/08 & 8C28005 & AGG-IC-001 \\
\hline 14808-79-8 & Sulfate & $1.02 \mathrm{E} 2$ & ug/g dry & $1.51 \mathrm{E} 0$ & 3/29/08 & 8C28005 & AGG-IC-001 \\
\hline $14265-44-2$ & Phosphate & $<1.51 \mathrm{E} 0$ & ug/g dry & $1.51 \mathrm{E} 0$ & 3/29/08 & 8C28005 & AGG-IC-001 \\
\hline HEIS No. & B1T1M0 & \multicolumn{3}{|c|}{ Lab ID: $\quad$ 0802024-56 } & & & \\
\hline $16984-48-8$ & Fluoride & $<2.03 \mathrm{E} 0$ & ug/g dry & 2.03E0 & 3/29/08 & 8C28005 & AGG-IC-001 \\
\hline $16887-00-6$ & Chloride & $<5.07 \mathrm{E} 0$ & ug/g dry & 5.07E0 & 3/29/08 & 8C28005 & AGG-IC-001 \\
\hline $14797-65-0$ & Nitrite & $<1.01 \mathrm{E} 1$ & ug/g dry & $1.01 \mathrm{E} 1$ & 3/29/08 & 8C28005 & AGG-IC-001 \\
\hline 24959-67-9 & Bromide & $<1.01 \mathrm{E} 1$ & ug/g dry & $1.01 \mathrm{E} 1$ & 3/29/08 & 8C28005 & AGG-IC-001 \\
\hline $14797-55-8$ & Nitrate & $<1.01 \mathrm{E} 1$ & ug/g dry & $1.01 \mathrm{E} 1$ & 3/29/08 & 8C28005 & AGG-IC-001 \\
\hline $14808-79-8$ & Sulfate & $1.78 \mathrm{E} 1$ & ug/g dry & $1.52 \mathrm{E} 1$ & 3/29/08 & 8C28005 & AGG-IC-001 \\
\hline 14265-44-2 & Phosphate & $<1.52 \mathrm{E} 1$ & ug/g dry & $1.52 \mathrm{E} 1$ & 3/29/08 & 8C28005 & AGG-IC-001 \\
\hline
\end{tabular}


Total Metals by PNNL-AGG-ICP-AES/Water Extract

\begin{tabular}{|c|c|c|c|c|c|c|c|}
\hline CAS \# & Analyte & Results & Units & EQL & Analyzed & Batch & Method \\
\hline HEIS No. & B1T170 & \multicolumn{3}{|c|}{ Lab ID: $\quad$ 0802013-12 } & & & \\
\hline 7429-90-5 & Aluminum & $3.29 \mathrm{E}-1$ & ug/g dry & $8.58 \mathrm{E}-2$ & $5 / 01 / 08$ & 8D28004 & PNNL-AGG-ICP-AES \\
\hline $7440-70-2$ & Calcium & 2.15E1 & ug/g dry & 3.87E-1 & $5 / 01 / 08$ & 8D28004 & PNNL-AGG-ICP-AES \\
\hline 7440-48-4 & Cobalt & $<9.60 \mathrm{E}-2$ & ug/g dry & $9.60 \mathrm{E}-2$ & $5 / 01 / 08$ & 8D28004 & PNNL-AGG-ICP-AES \\
\hline $7440-47-3$ & Chromium & $<3.33 \mathrm{E}-2$ & ug/g dry & 3.33E-2 & $5 / 01 / 08$ & 8D28004 & PNNL-AGG-ICP-AES \\
\hline $7440-50-8$ & Copper & $<8.04 \mathrm{E}-2$ & ug/g dry & $8.04 \mathrm{E}-2$ & $5 / 01 / 08$ & 8D28004 & PNNL-AGG-ICP-AES \\
\hline 7439-89-6 & Iron & $<1.42 \mathrm{E}-1$ & ug/g dry & $1.42 \mathrm{E}-1$ & $5 / 01 / 08$ & 8D28004 & PNNL-AGG-ICP-AES \\
\hline $7440-09-7$ & Potassium & $6.24 \mathrm{E} 0$ & ug/g dry & 2.33Е0 & $5 / 01 / 08$ & 8D28004 & PNNL-AGG-ICP-AES \\
\hline 7439-95-4 & Magnesium & 5.69E0 & ug/g dry & 8.34E-2 & $5 / 01 / 08$ & 8D28004 & PNNL-AGG-ICP-AES \\
\hline 7439-96-5 & Manganese & $<1.71 \mathrm{E}-2$ & ug/g dry & $1.71 \mathrm{E}-2$ & $5 / 01 / 08$ & 8D28004 & PNNL-AGG-ICP-AES \\
\hline $7440-02-0$ & Nickel & $<9.33 \mathrm{E}-2$ & ug/g dry & $9.33 \mathrm{E}-2$ & $5 / 01 / 08$ & 8D28004 & PNNL-AGG-ICP-AES \\
\hline $7782-49-2$ & Selenium & $<1.95 \mathrm{E} 0$ & ug/g dry & $1.95 \mathrm{E} 0$ & $5 / 01 / 08$ & 8D28004 & PNNL-AGG-ICP-AES \\
\hline $7440-62-2$ & Vanadium & $<4.53 \mathrm{E}-2$ & ug/g dry & 4.53E-2 & $5 / 01 / 08$ & 8D28004 & PNNL-AGG-ICP-AES \\
\hline $7440-66-6$ & Zinc & $<9.27 \mathrm{E}-2$ & ug/g dry & $9.27 \mathrm{E}-2$ & $5 / 01 / 08$ & 8D28004 & PNNL-AGG-ICP-AES \\
\hline 7440-23-5 & Sodium & 2.13E1 & ug/g dry & $6.69 \mathrm{E}-1$ & $5 / 01 / 08$ & 8D28004 & PNNL-AGG-ICP-AES \\
\hline HEIS No. & B1T172 & \multicolumn{3}{|c|}{ Lab ID: $\quad$ 0802013-14 } & & & \\
\hline $7429-90-5$ & Aluminum & $2.26 \mathrm{E}-1$ & ug/g dry & $8.48 \mathrm{E}-2$ & $5 / 01 / 08$ & 8D28004 & PNNL-AGG-ICP-AES \\
\hline $7440-70-2$ & Calcium & 8.96E0 & ug/g dry & 3.83E-1 & $5 / 01 / 08$ & 8D28004 & PNNL-AGG-ICP-AES \\
\hline $7440-48-4$ & Cobalt & $<9.49 \mathrm{E}-2$ & ug/g dry & $9.49 \mathrm{E}-2$ & $5 / 01 / 08$ & 8D28004 & PNNL-AGG-ICP-AES \\
\hline $7440-47-3$ & Chromium & $<3.29 \mathrm{E}-2$ & ug/g dry & 3.29E-2 & $5 / 01 / 08$ & 8D28004 & PNNL-AGG-ICP-AES \\
\hline $7440-50-8$ & Copper & $<7.95 \mathrm{E}-2$ & ug/g dry & 7.95E-2 & $5 / 01 / 08$ & 8D28004 & PNNL-AGG-ICP-AES \\
\hline 7439-89-6 & Iron & $<1.41 \mathrm{E}-1$ & ug/g dry & $1.41 \mathrm{E}-1$ & $5 / 01 / 08$ & 8D28004 & PNNL-AGG-ICP-AES \\
\hline 7440-09-7 & Potassium & 3.73E0 & ug/g dry & $2.30 \mathrm{E} 0$ & $5 / 01 / 08$ & 8D28004 & PNNL-AGG-ICP-AES \\
\hline 7439-95-4 & Magnesium & 2.33E0 & ug/g dry & $8.24 \mathrm{E}-2$ & $5 / 01 / 08$ & 8D28004 & PNNL-AGG-ICP-AES \\
\hline 7439-96-5 & Manganese & $<1.69 \mathrm{E}-2$ & ug/g dry & $1.69 \mathrm{E}-2$ & $5 / 01 / 08$ & 8D28004 & PNNL-AGG-ICP-AES \\
\hline 7440-02-0 & Nickel & $<9.22 \mathrm{E}-2$ & ug/g dry & $9.22 \mathrm{E}-2$ & $5 / 01 / 08$ & 8D28004 & PNNL-AGG-ICP-AES \\
\hline $7782-49-2$ & Selenium & $<1.93 \mathrm{E} 0$ & ug/g dry & $1.93 \mathrm{E} 0$ & $5 / 01 / 08$ & 8D28004 & PNNL-AGG-ICP-AES \\
\hline $7440-62-2$ & Vanadium & $<4.48 \mathrm{E}-2$ & ug/g dry & 4.48E-2 & $5 / 01 / 08$ & 8D28004 & PNNL-AGG-ICP-AES \\
\hline $7440-66-6$ & Zinc & $<9.16 \mathrm{E}-2$ & ug/g dry & $9.16 \mathrm{E}-2$ & $5 / 01 / 08$ & 8D28004 & PNNL-AGG-ICP-AES \\
\hline $7440-23-5$ & Sodium & $1.64 \mathrm{E} 1$ & ug/g dry & $6.61 \mathrm{E}-1$ & $5 / 01 / 08$ & 8D28004 & PNNL-AGG-ICP-AES \\
\hline HEIS No. & B1T152 & \multicolumn{3}{|c|}{ Lab ID: $\quad$ 0802014-10 } & & & \\
\hline $7429-90-5$ & Aluminum & $2.19 \mathrm{E}-1$ & ug/g dry & $8.50 \mathrm{E}-2$ & $5 / 01 / 08$ & 8D28004 & PNNL-AGG-ICP-AES \\
\hline $7440-70-2$ & Calcium & $1.59 \mathrm{E} 1$ & ug/g dry & 3.83E-1 & $5 / 01 / 08$ & 8D28004 & PNNL-AGG-ICP-AES \\
\hline $7440-48-4$ & Cobalt & $<9.51 \mathrm{E}-2$ & ug/g dry & $9.51 \mathrm{E}-2$ & $5 / 01 / 08$ & 8D28004 & PNNL-AGG-ICP-AES \\
\hline $7440-47-3$ & Chromium & $<3.30 \mathrm{E}-2$ & ug/g dry & $3.30 \mathrm{E}-2$ & $5 / 01 / 08$ & 8D28004 & PNNL-AGG-ICP-AES \\
\hline $7440-50-8$ & Copper & $<7.96 \mathrm{E}-2$ & ug/g dry & 7.96E-2 & $5 / 01 / 08$ & 8D28004 & PNNL-AGG-ICP-AES \\
\hline 7439-89-6 & Iron & $<1.41 \mathrm{E}-1$ & ug/g dry & $1.41 \mathrm{E}-1$ & $5 / 01 / 08$ & 8D28004 & PNNL-AGG-ICP-AES \\
\hline 7440-09-7 & Potassium & $1.29 \mathrm{E} 1$ & ug/g dry & 2.31E0 & $5 / 01 / 08$ & 8D28004 & PNNL-AGG-ICP-AES \\
\hline 7439-95-4 & Magnesium & 4.33E0 & ug/g dry & $8.26 \mathrm{E}-2$ & 5/01/08 & 8D28004 & PNNL-AGG-ICP-AES \\
\hline 7439-96-5 & Manganese & $<1.70 \mathrm{E}-2$ & ug/g dry & $1.70 \mathrm{E}-2$ & $5 / 01 / 08$ & 8D28004 & PNNL-AGG-ICP-AES \\
\hline $7440-02-0$ & Nickel & $<9.24 \mathrm{E}-2$ & ug/g dry & $9.24 \mathrm{E}-2$ & 5/01/08 & 8D28004 & PNNL-AGG-ICP-AES \\
\hline $7782-49-2$ & Selenium & $<1.93 \mathrm{E} 0$ & ug/g dry & $1.93 \mathrm{E} 0$ & 5/01/08 & 8D28004 & PNNL-AGG-ICP-AES \\
\hline 7440-66-6 & Zinc & $<9.18 \mathrm{E}-2$ & ug/g dry & $9.18 \mathrm{E}-2$ & $5 / 01 / 08$ & 8D28004 & PNNL-AGG-ICP-AES \\
\hline 7440-23-5 & Sodium & $1.20 \mathrm{E} 1$ & ug/g dry & $6.63 \mathrm{E}-1$ & $5 / 01 / 08$ & 8D28004 & PNNL-AGG-ICP-AES \\
\hline HEIS No. & B1T142 & \multicolumn{3}{|c|}{ Lab ID: $\quad$ 0802015-15 } & & & \\
\hline 7429-90-5 & Aluminum & $6.64 \mathrm{E}-1$ & ug/g dry & 8.63E-2 & $5 / 01 / 08$ & 8D28004 & PNNL-AGG-ICP-AES \\
\hline $7440-70-2$ & Calcium & $8.11 \mathrm{E} 0$ & ug/g dry & $3.89 \mathrm{E}-1$ & $5 / 01 / 08$ & 8D28004 & PNNL-AGG-ICP-AES \\
\hline $7440-48-4$ & Cobalt & $<9.66 \mathrm{E}-2$ & ug/g dry & $9.66 \mathrm{E}-2$ & $5 / 01 / 08$ & 8D28004 & PNNL-AGG-ICP-AES \\
\hline 7440-47-3 & Chromium & $<3.35 \mathrm{E}-2$ & ug/g dry & $3.35 \mathrm{E}-2$ & $5 / 01 / 08$ & 8D28004 & PNNL-AGG-ICP-AES \\
\hline
\end{tabular}


Total Metals by PNNL-AGG-ICP-AES/Water Extract

\begin{tabular}{|c|c|c|c|c|c|c|c|}
\hline CAS \# & Analyte & Results & Units & EQL & Analyzed & Batch & Method \\
\hline HEIS No. & B1T142 & \multicolumn{3}{|c|}{ Lab ID: $\quad$ 0802015-15 } & & & \\
\hline 7440-50-8 & Copper & $<8.09 \mathrm{E}-2$ & ug/g dry & 8.09E-2 & $5 / 01 / 08$ & 8D28004 & PNNL-AGG-ICP-AES \\
\hline 7439-89-6 & Iron & $<1.43 \mathrm{E}-1$ & ug/g dry & $1.43 \mathrm{E}-1$ & $5 / 01 / 08$ & 8D28004 & PNNL-AGG-ICP-AES \\
\hline $7440-09-7$ & Potassium & 4.45E0 & ug/g dry & $2.34 \mathrm{E} 0$ & $5 / 01 / 08$ & 8D28004 & PNNL-AGG-ICP-AES \\
\hline 7439-95-4 & Magnesium & 2.57E0 & ug/g dry & 8.39E-2 & $5 / 01 / 08$ & 8D28004 & PNNL-AGG-ICP-AES \\
\hline $7439-96-5$ & Manganese & $<1.72 \mathrm{E}-2$ & ug/g dry & $1.72 \mathrm{E}-2$ & $5 / 01 / 08$ & 8D28004 & PNNL-AGG-ICP-AES \\
\hline 7440-02-0 & Nickel & $<9.39 \mathrm{E}-2$ & ug/g dry & $9.39 \mathrm{E}-2$ & $5 / 01 / 08$ & 8D28004 & PNNL-AGG-ICP-AES \\
\hline $7782-49-2$ & Selenium & $<1.96 \mathrm{E} 0$ & ug/g dry & $1.96 \mathrm{E} 0$ & $5 / 01 / 08$ & 8D28004 & PNNL-AGG-ICP-AES \\
\hline $7440-62-2$ & Vanadium & $<4.56 \mathrm{E}-2$ & ug/g dry & 4.56E-2 & $5 / 01 / 08$ & 8D28004 & PNNL-AGG-ICP-AES \\
\hline 7440-66-6 & Zinc & $7.54 \mathrm{E}-1$ & ug/g dry & $9.32 \mathrm{E}-2$ & $5 / 01 / 08$ & 8D28004 & PNNL-AGG-ICP-AES \\
\hline $7440-23-5$ & Sodium & $1.12 \mathrm{E} 1$ & ug/g dry & 6.73E-1 & $5 / 01 / 08$ & 8D28004 & PNNL-AGG-ICP-AES \\
\hline HEIS No. & B1T198 & \multicolumn{3}{|c|}{ Lab ID: $\quad$ 0802016-26 } & & & \\
\hline $7429-90-5$ & Aluminum & $2.32 \mathrm{E}-1$ & ug/g dry & 8.57E-2 & $5 / 01 / 08$ & 8D28004 & PNNL-AGG-ICP-AES \\
\hline $7440-70-2$ & Calcium & 8.32E0 & ug/g dry & 3.86E-1 & $5 / 01 / 08$ & 8D28004 & PNNL-AGG-ICP-AES \\
\hline $7440-48-4$ & Cobalt & $<9.58 \mathrm{E}-2$ & ug/g dry & $9.58 \mathrm{E}-2$ & $5 / 01 / 08$ & 8D28004 & PNNL-AGG-ICP-AES \\
\hline $7440-47-3$ & Chromium & $<3.32 \mathrm{E}-2$ & ug/g dry & 3.32E-2 & $5 / 01 / 08$ & 8D28004 & PNNL-AGG-ICP-AES \\
\hline $7440-50-8$ & Copper & $<8.03 \mathrm{E}-2$ & ug/g dry & 8.03E-2 & $5 / 01 / 08$ & 8D28004 & PNNL-AGG-ICP-AES \\
\hline 7439-89-6 & Iron & $<1.42 \mathrm{E}-1$ & ug/g dry & $1.42 \mathrm{E}-1$ & $5 / 01 / 08$ & 8D28004 & PNNL-AGG-ICP-AES \\
\hline 7440-09-7 & Potassium & 3.67E0 & ug/g dry & $2.32 \mathrm{E} 0$ & $5 / 01 / 08$ & 8D28004 & PNNL-AGG-ICP-AES \\
\hline 7439-95-4 & Magnesium & 2.36E0 & ug/g dry & 8.33E-2 & $5 / 01 / 08$ & 8D28004 & PNNL-AGG-ICP-AES \\
\hline 7439-96-5 & Manganese & $<1.71 \mathrm{E}-2$ & ug/g dry & $1.71 \mathrm{E}-2$ & $5 / 01 / 08$ & 8D28004 & PNNL-AGG-ICP-AES \\
\hline 7440-02-0 & Nickel & $<9.32 \mathrm{E}-2$ & ug/g dry & $9.32 \mathrm{E}-2$ & $5 / 01 / 08$ & 8D28004 & PNNL-AGG-ICP-AES \\
\hline $7782-49-2$ & Selenium & $<1.95 \mathrm{E} 0$ & ug/g dry & $1.95 \mathrm{E} 0$ & $5 / 01 / 08$ & 8D28004 & PNNL-AGG-ICP-AES \\
\hline $7440-62-2$ & Vanadium & $<4.52 \mathrm{E}-2$ & ug/g dry & $4.52 \mathrm{E}-2$ & $5 / 01 / 08$ & 8D28004 & PNNL-AGG-ICP-AES \\
\hline $7440-66-6$ & Zinc & $<9.26 \mathrm{E}-2$ & ug/g dry & $9.26 \mathrm{E}-2$ & $5 / 01 / 08$ & 8D28004 & PNNL-AGG-ICP-AES \\
\hline $7440-23-5$ & Sodium & $8.94 \mathrm{E} 0$ & ug/g dry & $6.68 \mathrm{E}-1$ & $5 / 01 / 08$ & 8D28004 & PNNL-AGG-ICP-AES \\
\hline HEIS No. & B1T1C4 & \multicolumn{3}{|c|}{ Lab ID: $\quad$ 0802018-03 } & & & \\
\hline $7429-90-5$ & Aluminum & $1.98 \mathrm{E}-1$ & ug/g dry & $8.60 \mathrm{E}-2$ & $5 / 01 / 08$ & 8D28004 & PNNL-AGG-ICP-AES \\
\hline $7440-70-2$ & Calcium & $1.06 \mathrm{E} 1$ & ug/g dry & $3.88 \mathrm{E}-1$ & $5 / 01 / 08$ & 8D28004 & PNNL-AGG-ICP-AES \\
\hline $7440-48-4$ & Cobalt & $<9.63 \mathrm{E}-2$ & ug/g dry & $9.63 \mathrm{E}-2$ & $5 / 01 / 08$ & 8D28004 & PNNL-AGG-ICP-AES \\
\hline $7440-47-3$ & Chromium & $<3.34 \mathrm{E}-2$ & ug/g dry & $3.34 \mathrm{E}-2$ & $5 / 01 / 08$ & 8D28004 & PNNL-AGG-ICP-AES \\
\hline $7440-50-8$ & Copper & $<8.06 \mathrm{E}-2$ & ug/g dry & 8.06E-2 & $5 / 01 / 08$ & 8D28004 & PNNL-AGG-ICP-AES \\
\hline 7439-89-6 & Iron & $<1.43 \mathrm{E}-1$ & ug/g dry & $1.43 \mathrm{E}-1$ & $5 / 01 / 08$ & 8D28004 & PNNL-AGG-ICP-AES \\
\hline 7440-09-7 & Potassium & $4.46 \mathrm{E} 0$ & ug/g dry & $2.33 \mathrm{E} 0$ & $5 / 01 / 08$ & 8D28004 & PNNL-AGG-ICP-AES \\
\hline 7439-95-4 & Magnesium & $2.96 \mathrm{E} 0$ & ug/g dry & 8.36E-2 & $5 / 01 / 08$ & 8D28004 & PNNL-AGG-ICP-AES \\
\hline 7439-96-5 & Manganese & $<1.72 \mathrm{E}-2$ & ug/g dry & $1.72 \mathrm{E}-2$ & $5 / 01 / 08$ & 8D28004 & PNNL-AGG-ICP-AES \\
\hline $7440-02-0$ & Nickel & $<9.36 \mathrm{E}-2$ & ug/g dry & $9.36 \mathrm{E}-2$ & $5 / 01 / 08$ & 8D28004 & PNNL-AGG-ICP-AES \\
\hline $7782-49-2$ & Selenium & $<1.96 \mathrm{E} 0$ & ug/g dry & $1.96 \mathrm{E} 0$ & $5 / 01 / 08$ & 8D28004 & PNNL-AGG-ICP-AES \\
\hline $7440-62-2$ & Vanadium & $<4.54 \mathrm{E}-2$ & ug/g dry & $4.54 \mathrm{E}-2$ & $5 / 01 / 08$ & 8D28004 & PNNL-AGG-ICP-AES \\
\hline $7440-66-6$ & Zinc & $<9.30 \mathrm{E}-2$ & ug/g dry & $9.30 \mathrm{E}-2$ & $5 / 01 / 08$ & 8D28004 & PNNL-AGG-ICP-AES \\
\hline $7440-23-5$ & Sodium & $1.37 \mathrm{E} 1$ & ug/g dry & $6.71 \mathrm{E}-1$ & $5 / 01 / 08$ & 8D28004 & PNNL-AGG-ICP-AES \\
\hline HEIS No. & B1T1H0 & \multicolumn{3}{|c|}{ Lab ID: $\quad$ 0802022-01 } & & & \\
\hline 7429-90-5 & Aluminum & $<8.57 \mathrm{E}-2$ & ug/g dry & 8.57E-2 & $5 / 01 / 08$ & 8D28004 & PNNL-AGG-ICP-AES \\
\hline $7440-70-2$ & Calcium & $7.20 \mathrm{E} 1$ & ug/g dry & 3.86E-1 & $5 / 01 / 08$ & 8D28004 & PNNL-AGG-ICP-AES \\
\hline $7440-48-4$ & Cobalt & $<9.59 \mathrm{E}-2$ & ug/g dry & $9.59 \mathrm{E}-2$ & $5 / 01 / 08$ & 8D28004 & PNNL-AGG-ICP-AES \\
\hline $7440-47-3$ & Chromium & $<3.33 \mathrm{E}-2$ & ug/g dry & 3.33E-2 & $5 / 01 / 08$ & 8D28004 & PNNL-AGG-ICP-AES \\
\hline $7440-50-8$ & Copper & $<8.03 \mathrm{E}-2$ & ug/g dry & 8.03E-2 & $5 / 01 / 08$ & 8D28004 & PNNL-AGG-ICP-AES \\
\hline 7439-89-6 & Iron & $<1.42 \mathrm{E}-1$ & ug/g dry & $1.42 \mathrm{E}-1$ & $5 / 01 / 08$ & 8D28004 & PNNL-AGG-ICP-AES \\
\hline 7440-09-7 & Potassium & 1.13E1 & ug/g dry & $2.32 \mathrm{E} 0$ & $5 / 01 / 08$ & 8D28004 & PNNL-AGG-ICP-AES \\
\hline
\end{tabular}


Total Metals by PNNL-AGG-ICP-AES/Water Extract

\begin{tabular}{|c|c|c|c|c|c|c|c|}
\hline CAS \# & Analyte & Results & Units & EQL & Analyzed & Batch & Method \\
\hline HEIS No. & B1T1H0 & \multicolumn{3}{|c|}{ Lab ID: $\quad$ 0802022-01 } & & & \\
\hline 7439-95-4 & Magnesium & 2.06E1 & ug/g dry & 8.33E-2 & $5 / 01 / 08$ & 8D28004 & PNNL-AGG-ICP-AES \\
\hline 7439-96-5 & Manganese & $<1.71 \mathrm{E}-2$ & ug/g dry & $1.71 \mathrm{E}-2$ & $5 / 01 / 08$ & 8D28004 & PNNL-AGG-ICP-AES \\
\hline $7440-02-0$ & Nickel & $<9.32 \mathrm{E}-2$ & ug/g dry & $9.32 \mathrm{E}-2$ & $5 / 01 / 08$ & 8D28004 & PNNL-AGG-ICP-AES \\
\hline 7782-49-2 & Selenium & $<1.95 \mathrm{E} 0$ & ug/g dry & $1.95 \mathrm{E} 0$ & $5 / 01 / 08$ & 8D28004 & PNNL-AGG-ICP-AES \\
\hline $7440-62-2$ & Vanadium & $<4.52 \mathrm{E}-2$ & ug/g dry & $4.52 \mathrm{E}-2$ & $5 / 01 / 08$ & 8D28004 & PNNL-AGG-ICP-AES \\
\hline 7440-66-6 & Zinc & $<9.26 \mathrm{E}-2$ & ug/g dry & $9.26 \mathrm{E}-2$ & $5 / 01 / 08$ & 8D28004 & PNNL-AGG-ICP-AES \\
\hline $7440-23-5$ & Sodium & $1.00 \mathrm{E} 2$ & ug/g dry & $6.68 \mathrm{E}-1$ & $5 / 01 / 08$ & 8D28004 & PNNL-AGG-ICP-AES \\
\hline HEIS No. & B1T1H2 & \multicolumn{3}{|c|}{ Lab ID: $\quad$ 0802022-04 } & & & \\
\hline $7429-90-5$ & Aluminum & $1.01 \mathrm{E}-1$ & ug/g dry & $8.51 \mathrm{E}-2$ & $5 / 01 / 08$ & 8D28004 & PNNL-AGG-ICP-AES \\
\hline $7440-70-2$ & Calcium & $9.16 \mathrm{E} 1$ & ug/g dry & 3.84E-1 & $5 / 01 / 08$ & 8D28004 & PNNL-AGG-ICP-AES \\
\hline $7440-48-4$ & Cobalt & $<9.52 \mathrm{E}-2$ & ug/g dry & $9.52 \mathrm{E}-2$ & $5 / 01 / 08$ & 8D28004 & PNNL-AGG-ICP-AES \\
\hline $7440-47-3$ & Chromium & $<3.30 \mathrm{E}-2$ & ug/g dry & $3.30 \mathrm{E}-2$ & $5 / 01 / 08$ & 8D28004 & PNNL-AGG-ICP-AES \\
\hline $7440-50-8$ & Copper & $8.11 \mathrm{E}-2$ & ug/g dry & 7.97E-2 & $5 / 01 / 08$ & 8D28004 & PNNL-AGG-ICP-AES \\
\hline 7439-89-6 & Iron & $<1.41 \mathrm{E}-1$ & ug/g dry & $1.41 \mathrm{E}-1$ & $5 / 01 / 08$ & 8D28004 & PNNL-AGG-ICP-AES \\
\hline 7440-09-7 & Potassium & $1.45 \mathrm{E} 1$ & ug/g dry & $2.31 \mathrm{E} 0$ & $5 / 01 / 08$ & 8D28004 & PNNL-AGG-ICP-AES \\
\hline 7439-95-4 & Magnesium & 2.77E1 & ug/g dry & $8.27 \mathrm{E}-2$ & $5 / 01 / 08$ & 8D28004 & PNNL-AGG-ICP-AES \\
\hline 7439-96-5 & Manganese & $2.70 \mathrm{E}-1$ & ug/g dry & $1.70 \mathrm{E}-2$ & $5 / 01 / 08$ & 8D28004 & PNNL-AGG-ICP-AES \\
\hline 7440-02-0 & Nickel & $<9.25 \mathrm{E}-2$ & ug/g dry & $9.25 \mathrm{E}-2$ & $5 / 01 / 08$ & 8D28004 & PNNL-AGG-ICP-AES \\
\hline $7782-49-2$ & Selenium & $<1.93 \mathrm{E} 0$ & ug/g dry & 1.93E0 & $5 / 01 / 08$ & 8D28004 & PNNL-AGG-ICP-AES \\
\hline $7440-62-2$ & Vanadium & $<4.49 \mathrm{E}-2$ & ug/g dry & 4.49E-2 & $5 / 01 / 08$ & 8D28004 & PNNL-AGG-ICP-AES \\
\hline 7440-66-6 & Zinc & $<9.19 \mathrm{E}-2$ & ug/g dry & $9.19 \mathrm{E}-2$ & $5 / 01 / 08$ & 8D28004 & PNNL-AGG-ICP-AES \\
\hline $7440-23-5$ & Sodium & $1.33 \mathrm{E} 2$ & ug/g dry & 6.63E-1 & $5 / 01 / 08$ & 8D28004 & PNNL-AGG-ICP-AES \\
\hline HEIS No. & B1T1H4 & \multicolumn{3}{|c|}{ Lab ID: $\quad 0802022-07$} & & & \\
\hline $7429-90-5$ & Aluminum & $2.01 \mathrm{E}-1$ & ug/g dry & $8.58 \mathrm{E}-2$ & $5 / 01 / 08$ & 8D28004 & PNNL-AGG-ICP-AES \\
\hline $7440-70-2$ & Calcium & $1.62 \mathrm{E} 2$ & ug/g dry & 3.87E-1 & $5 / 01 / 08$ & 8D28004 & PNNL-AGG-ICP-AES \\
\hline $7440-48-4$ & Cobalt & $<9.60 \mathrm{E}-2$ & ug/g dry & $9.60 \mathrm{E}-2$ & $5 / 01 / 08$ & 8D28004 & PNNL-AGG-ICP-AES \\
\hline $7440-47-3$ & Chromium & $<3.33 \mathrm{E}-2$ & ug/g dry & 3.33E-2 & $5 / 01 / 08$ & 8D28004 & PNNL-AGG-ICP-AES \\
\hline $7440-50-8$ & Copper & $2.47 \mathrm{E}-1$ & ug/g dry & 8.04E-2 & $5 / 01 / 08$ & 8D28004 & PNNL-AGG-ICP-AES \\
\hline 7439-89-6 & Iron & $<1.42 \mathrm{E}-1$ & ug/g dry & $1.42 \mathrm{E}-1$ & $5 / 01 / 08$ & 8D28004 & PNNL-AGG-ICP-AES \\
\hline 7440-09-7 & Potassium & 1.67E1 & ug/g dry & 2.33E0 & $5 / 01 / 08$ & 8D28004 & PNNL-AGG-ICP-AES \\
\hline 7439-95-4 & Magnesium & $6.14 \mathrm{E} 1$ & ug/g dry & 8.34E-2 & $5 / 01 / 08$ & 8D28004 & PNNL-AGG-ICP-AES \\
\hline $7439-96-5$ & Manganese & $2.72 \mathrm{E}-1$ & ug/g dry & $1.71 \mathrm{E}-2$ & $5 / 01 / 08$ & 8D28004 & PNNL-AGG-ICP-AES \\
\hline 7440-02-0 & Nickel & $<9.33 E-2$ & ug/g dry & $9.33 \mathrm{E}-2$ & $5 / 01 / 08$ & 8D28004 & PNNL-AGG-ICP-AES \\
\hline $7782-49-2$ & Selenium & $<1.95 \mathrm{E} 0$ & ug/g dry & $1.95 \mathrm{E} 0$ & $5 / 01 / 08$ & 8D28004 & PNNL-AGG-ICP-AES \\
\hline $7440-62-2$ & Vanadium & $<4.53 \mathrm{E}-2$ & ug/g dry & 4.53E-2 & $5 / 01 / 08$ & 8D28004 & PNNL-AGG-ICP-AES \\
\hline $7440-66-6$ & Zinc & $<9.27 \mathrm{E}-2$ & ug/g dry & $9.27 \mathrm{E}-2$ & $5 / 01 / 08$ & 8D28004 & PNNL-AGG-ICP-AES \\
\hline $7440-23-5$ & Sodium & $1.46 \mathrm{E} 2$ & ug/g dry & $6.69 \mathrm{E}-1$ & $5 / 01 / 08$ & 8D28004 & PNNL-AGG-ICP-AES \\
\hline HEIS No. & B1T1H6 & \multicolumn{3}{|c|}{ Lab ID: $\quad$ 0802022-11 } & & & \\
\hline $7429-90-5$ & Aluminum & $1.01 \mathrm{E}-1$ & ug/g dry & $8.59 \mathrm{E}-2$ & $5 / 01 / 08$ & 8D28004 & PNNL-AGG-ICP-AES \\
\hline $7440-70-2$ & Calcium & $1.60 \mathrm{E} 2$ & ug/g dry & 3.87E-1 & $5 / 01 / 08$ & 8D28004 & PNNL-AGG-ICP-AES \\
\hline $7440-48-4$ & Cobalt & $<9.61 \mathrm{E}-2$ & ug/g dry & $9.61 \mathrm{E}-2$ & $5 / 01 / 08$ & 8D28004 & PNNL-AGG-ICP-AES \\
\hline $7440-47-3$ & Chromium & $<3.33 \mathrm{E}-2$ & ug/g dry & 3.33E-2 & $5 / 01 / 08$ & 8D28004 & PNNL-AGG-ICP-AES \\
\hline $7440-50-8$ & Copper & $1.01 \mathrm{E}-1$ & ug/g dry & 8.05E-2 & $5 / 01 / 08$ & 8D28004 & PNNL-AGG-ICP-AES \\
\hline 7439-89-6 & Iron & $<1.42 \mathrm{E}-1$ & ug/g dry & $1.42 \mathrm{E}-1$ & $5 / 01 / 08$ & 8D28004 & PNNL-AGG-ICP-AES \\
\hline 7440-09-7 & Potassium & $1.49 \mathrm{E} 1$ & ug/g dry & $2.33 \mathrm{E} 0$ & $5 / 01 / 08$ & 8D28004 & PNNL-AGG-ICP-AES \\
\hline 7439-95-4 & Magnesium & 8.69E1 & ug/g dry & 8.35E-2 & $5 / 01 / 08$ & 8D28004 & PNNL-AGG-ICP-AES \\
\hline 7439-96-5 & Manganese & 4.99E-2 & ug/g dry & $1.71 \mathrm{E}-2$ & $5 / 01 / 08$ & 8D28004 & PNNL-AGG-ICP-AES \\
\hline $7440-02-0$ & Nickel & $<9.34 \mathrm{E}-2$ & ug/g dry & $9.34 \mathrm{E}-2$ & $5 / 01 / 08$ & 8D28004 & PNNL-AGG-ICP-AES \\
\hline
\end{tabular}


Total Metals by PNNL-AGG-ICP-AES/Water Extract

\begin{tabular}{|c|c|c|c|c|c|c|c|}
\hline CAS \# & Analyte & Results & Units & EQL & Analyzed & Batch & Method \\
\hline HEIS No. & B1T1H6 & \multicolumn{3}{|c|}{ Lab ID: $\quad$ 0802022-11 } & & & \\
\hline $7782-49-2$ & Selenium & $<1.95 \mathrm{E} 0$ & ug/g dry & $1.95 \mathrm{E} 0$ & $5 / 01 / 08$ & 8D28004 & PNNL-AGG-ICP-AES \\
\hline $7440-62-2$ & Vanadium & $<4.53 \mathrm{E}-2$ & ug/g dry & 4.53E-2 & $5 / 01 / 08$ & 8D28004 & PNNL-AGG-ICP-AES \\
\hline $7440-66-6$ & Zinc & $1.11 \mathrm{E}-1$ & ug/g dry & $9.28 \mathrm{E}-2$ & $5 / 01 / 08$ & 8D28004 & PNNL-AGG-ICP-AES \\
\hline $7440-23-5$ & Sodium & 8.62E1 & ug/g dry & $6.70 \mathrm{E}-1$ & $5 / 01 / 08$ & 8D28004 & PNNL-AGG-ICP-AES \\
\hline HEIS No. & B1T1D8 & \multicolumn{3}{|c|}{ Lab ID: $\quad$ 0802024-15 } & & & \\
\hline $7429-90-5$ & Aluminum & $1.87 \mathrm{E}-1$ & ug/g dry & $8.61 \mathrm{E}-2$ & $5 / 01 / 08$ & 8D28004 & PNNL-AGG-ICP-AES \\
\hline $7440-70-2$ & Calcium & $1.00 \mathrm{E} 1$ & ug/g dry & $3.88 \mathrm{E}-1$ & $5 / 01 / 08$ & 8D28004 & PNNL-AGG-ICP-AES \\
\hline $7440-48-4$ & Cobalt & $<9.63 \mathrm{E}-2$ & ug/g dry & $9.63 \mathrm{E}-2$ & $5 / 01 / 08$ & 8D28004 & PNNL-AGG-ICP-AES \\
\hline $7440-47-3$ & Chromium & $<3.34 \mathrm{E}-2$ & ug/g dry & $3.34 \mathrm{E}-2$ & $5 / 01 / 08$ & 8D28004 & PNNL-AGG-ICP-AES \\
\hline $7440-50-8$ & Copper & $<8.07 \mathrm{E}-2$ & ug/g dry & 8.07E-2 & $5 / 01 / 08$ & 8D28004 & PNNL-AGG-ICP-AES \\
\hline 7439-89-6 & Iron & $<1.43 \mathrm{E}-1$ & ug/g dry & 1.43E-1 & $5 / 01 / 08$ & 8D28004 & PNNL-AGG-ICP-AES \\
\hline 7440-09-7 & Potassium & 3.68E0 & ug/g dry & $2.34 \mathrm{E} 0$ & $5 / 01 / 08$ & 8D28004 & PNNL-AGG-ICP-AES \\
\hline 7439-95-4 & Magnesium & $2.65 \mathrm{E} 0$ & ug/g dry & 8.37E-2 & $5 / 01 / 08$ & 8D28004 & PNNL-AGG-ICP-AES \\
\hline 7439-96-5 & Manganese & $<1.72 \mathrm{E}-2$ & ug/g dry & $1.72 \mathrm{E}-2$ & $5 / 01 / 08$ & 8D28004 & PNNL-AGG-ICP-AES \\
\hline $7440-02-0$ & Nickel & $<9.36 \mathrm{E}-2$ & ug/g dry & $9.36 \mathrm{E}-2$ & $5 / 01 / 08$ & 8D28004 & PNNL-AGG-ICP-AES \\
\hline $7782-49-2$ & Selenium & $<1.96 \mathrm{E} 0$ & ug/g dry & $1.96 \mathrm{E} 0$ & $5 / 01 / 08$ & 8D28004 & PNNL-AGG-ICP-AES \\
\hline $7440-62-2$ & Vanadium & $<4.54 \mathrm{E}-2$ & ug/g dry & $4.54 \mathrm{E}-2$ & $5 / 01 / 08$ & 8D28004 & PNNL-AGG-ICP-AES \\
\hline $7440-66-6$ & Zinc & $<9.30 \mathrm{E}-2$ & ug/g dry & $9.30 \mathrm{E}-2$ & $5 / 01 / 08$ & 8D28004 & PNNL-AGG-ICP-AES \\
\hline $7440-23-5$ & Sodium & $9.17 \mathrm{E} 0$ & ug/g dry & $6.71 \mathrm{E}-1$ & $5 / 01 / 08$ & 8D28004 & PNNL-AGG-ICP-AES \\
\hline HEIS No. & B1T1F0 & \multicolumn{3}{|c|}{ Lab ID: $\quad$ 0802024-18 } & & & \\
\hline $7429-90-5$ & Aluminum & $<8.60 \mathrm{E}-2$ & ug/g dry & $8.60 \mathrm{E}-2$ & $5 / 01 / 08$ & 8D28004 & PNNL-AGG-ICP-AES \\
\hline $7440-70-2$ & Calcium & $3.75 \mathrm{E} 1$ & ug/g dry & $3.88 \mathrm{E}-1$ & $5 / 01 / 08$ & 8D28004 & PNNL-AGG-ICP-AES \\
\hline $7440-48-4$ & Cobalt & $<9.62 \mathrm{E}-2$ & ug/g dry & $9.62 \mathrm{E}-2$ & $5 / 01 / 08$ & 8D28004 & PNNL-AGG-ICP-AES \\
\hline $7440-47-3$ & Chromium & $<3.34 \mathrm{E}-2$ & ug/g dry & 3.34E-2 & $5 / 01 / 08$ & 8D28004 & PNNL-AGG-ICP-AES \\
\hline $7440-50-8$ & Copper & $<8.06 \mathrm{E}-2$ & ug/g dry & 8.06E-2 & $5 / 01 / 08$ & 8D28004 & PNNL-AGG-ICP-AES \\
\hline 7439-89-6 & Iron & $<1.43 \mathrm{E}-1$ & ug/g dry & $1.43 \mathrm{E}-1$ & $5 / 01 / 08$ & 8D28004 & PNNL-AGG-ICP-AES \\
\hline 7440-09-7 & Potassium & $6.01 \mathrm{E} 0$ & ug/g dry & 2.33E0 & $5 / 01 / 08$ & 8D28004 & PNNL-AGG-ICP-AES \\
\hline 7439-95-4 & Magnesium & $1.04 \mathrm{E} 1$ & ug/g dry & $8.36 \mathrm{E}-2$ & $5 / 01 / 08$ & 8D28004 & PNNL-AGG-ICP-AES \\
\hline 7439-96-5 & Manganese & $<1.72 \mathrm{E}-2$ & ug/g dry & $1.72 \mathrm{E}-2$ & $5 / 01 / 08$ & 8D28004 & PNNL-AGG-ICP-AES \\
\hline $7440-02-0$ & Nickel & $<9.35 \mathrm{E}-2$ & ug/g dry & $9.35 \mathrm{E}-2$ & $5 / 01 / 08$ & 8D28004 & PNNL-AGG-ICP-AES \\
\hline $7782-49-2$ & Selenium & $<1.95 \mathrm{E} 0$ & ug/g dry & $1.95 \mathrm{E} 0$ & $5 / 01 / 08$ & 8D28004 & PNNL-AGG-ICP-AES \\
\hline $7440-62-2$ & Vanadium & $<4.54 \mathrm{E}-2$ & ug/g dry & $4.54 \mathrm{E}-2$ & $5 / 01 / 08$ & 8D28004 & PNNL-AGG-ICP-AES \\
\hline 7440-66-6 & Zinc & $<9.29 \mathrm{E}-2$ & ug/g dry & $9.29 \mathrm{E}-2$ & $5 / 01 / 08$ & 8D28004 & PNNL-AGG-ICP-AES \\
\hline $7440-23-5$ & Sodium & $2.59 \mathrm{E} 1$ & ug/g dry & $6.71 \mathrm{E}-1$ & $5 / 01 / 08$ & 8D28004 & PNNL-AGG-ICP-AES \\
\hline HEIS No. & B1T1F6 & \multicolumn{3}{|c|}{ Lab ID: $\quad 0802024-27$} & & & \\
\hline $7429-90-5$ & Aluminum & $<8.61 \mathrm{E}-2$ & ug/g dry & $8.61 \mathrm{E}-2$ & $5 / 01 / 08$ & 8D28004 & PNNL-AGG-ICP-AES \\
\hline $7440-70-2$ & Calcium & 3.08E1 & ug/g dry & 3.88E-1 & $5 / 01 / 08$ & 8D28004 & PNNL-AGG-ICP-AES \\
\hline $7440-48-4$ & Cobalt & $<9.63 \mathrm{E}-2$ & ug/g dry & $9.63 \mathrm{E}-2$ & $5 / 01 / 08$ & 8D28004 & PNNL-AGG-ICP-AES \\
\hline $7440-47-3$ & Chromium & $<3.34 \mathrm{E}-2$ & ug/g dry & 3.34E-2 & $5 / 01 / 08$ & 8D28004 & PNNL-AGG-ICP-AES \\
\hline $7440-50-8$ & Copper & $<8.07 \mathrm{E}-2$ & ug/g dry & 8.07E-2 & $5 / 01 / 08$ & 8D28004 & PNNL-AGG-ICP-AES \\
\hline 7439-89-6 & Iron & $<1.43 \mathrm{E}-1$ & ug/g dry & $1.43 \mathrm{E}-1$ & $5 / 01 / 08$ & 8D28004 & PNNL-AGG-ICP-AES \\
\hline 7440-09-7 & Potassium & $6.33 \mathrm{E} 0$ & ug/g dry & $2.34 \mathrm{E} 0$ & $5 / 01 / 08$ & 8D28004 & PNNL-AGG-ICP-AES \\
\hline 7439-95-4 & Magnesium & $1.13 \mathrm{E} 1$ & ug/g dry & 8.37E-2 & $5 / 01 / 08$ & 8D28004 & PNNL-AGG-ICP-AES \\
\hline 7439-96-5 & Manganese & $2.59 \mathrm{E}-2$ & ug/g dry & $1.72 \mathrm{E}-2$ & $5 / 01 / 08$ & 8D28004 & PNNL-AGG-ICP-AES \\
\hline 7440-02-0 & Nickel & $<9.36 \mathrm{E}-2$ & ug/g dry & $9.36 \mathrm{E}-2$ & $5 / 01 / 08$ & 8D28004 & PNNL-AGG-ICP-AES \\
\hline $7782-49-2$ & Selenium & $<1.96 \mathrm{E} 0$ & ug/g dry & $1.96 \mathrm{E} 0$ & $5 / 01 / 08$ & 8D28004 & PNNL-AGG-ICP-AES \\
\hline $7440-62-2$ & Vanadium & $<4.55 \mathrm{E}-2$ & ug/g dry & 4.55E-2 & $5 / 01 / 08$ & 8D28004 & PNNL-AGG-ICP-AES \\
\hline $7440-66-6$ & Zinc & $<9.30 \mathrm{E}-2$ & ug/g dry & $9.30 \mathrm{E}-2$ & $5 / 01 / 08$ & 8D28004 & PNNL-AGG-ICP-AES \\
\hline
\end{tabular}


Total Metals by PNNL-AGG-ICP-AES/Water Extract

\begin{tabular}{|c|c|c|c|c|c|c|c|}
\hline CAS \# & Analyte & Results & Units & EQL & Analyzed & Batch & Method \\
\hline HEIS No. & B1T1F6 & \multicolumn{3}{|c|}{ Lab ID: $\quad$ 0802024-27 } & & & \\
\hline $7440-23-5$ & Sodium & 2.80E1 & ug/g dry & 6.71E-1 & $5 / 01 / 08$ & 8D28004 & PNNL-AGG-ICP-AES \\
\hline HEIS No. & B1T1M0 & \multicolumn{3}{|c|}{ Lab ID: $\quad$ 0802024-56 } & & & \\
\hline 7429-90-5 & Aluminum & $1.94 \mathrm{E}-1$ & ug/g dry & 8.71E-2 & $5 / 01 / 08$ & 8D28004 & PNNL-AGG-ICP-AES \\
\hline $7440-70-2$ & Calcium & 9.57E0 & ug/g dry & 3.93E-1 & $5 / 01 / 08$ & 8D28004 & PNNL-AGG-ICP-AES \\
\hline $7440-48-4$ & Cobalt & $<9.74 \mathrm{E}-2$ & ug/g dry & $9.74 \mathrm{E}-2$ & $5 / 01 / 08$ & 8D28004 & PNNL-AGG-ICP-AES \\
\hline $7440-47-3$ & Chromium & $<3.38 \mathrm{E}-2$ & ug/g dry & 3.38E-2 & $5 / 01 / 08$ & 8D28004 & PNNL-AGG-ICP-AES \\
\hline $7440-50-8$ & Copper & $<8.16 \mathrm{E}-2$ & ug/g dry & $8.16 \mathrm{E}-2$ & $5 / 01 / 08$ & 8D28004 & PNNL-AGG-ICP-AES \\
\hline 7439-89-6 & Iron & $1.56 \mathrm{E}-1$ & ug/g dry & 1.44E-1 & $5 / 01 / 08$ & 8D28004 & PNNL-AGG-ICP-AES \\
\hline 7440-09-7 & Potassium & 4.15E0 & ug/g dry & 2.36E0 & $5 / 01 / 08$ & 8D28004 & PNNL-AGG-ICP-AES \\
\hline 7439-95-4 & Magnesium & 2.99E0 & ug/g dry & $8.46 \mathrm{E}-2$ & $5 / 01 / 08$ & 8D28004 & PNNL-AGG-ICP-AES \\
\hline 7439-96-5 & Manganese & $<1.74 \mathrm{E}-2$ & ug/g dry & $1.74 \mathrm{E}-2$ & $5 / 01 / 08$ & 8D28004 & PNNL-AGG-ICP-AES \\
\hline 7440-02-0 & Nickel & $<9.47 \mathrm{E}-2$ & ug/g dry & $9.47 \mathrm{E}-2$ & $5 / 01 / 08$ & 8D28004 & PNNL-AGG-ICP-AES \\
\hline 7782-49-2 & Selenium & $<1.98 \mathrm{E} 0$ & ug/g dry & $1.98 \mathrm{E} 0$ & $5 / 01 / 08$ & 8D28004 & PNNL-AGG-ICP-AES \\
\hline $7440-62-2$ & Vanadium & $<4.60 \mathrm{E}-2$ & ug/g dry & $4.60 \mathrm{E}-2$ & $5 / 01 / 08$ & 8D28004 & PNNL-AGG-ICP-AES \\
\hline $7440-66-6$ & Zinc & $<9.41 \mathrm{E}-2$ & ug/g dry & $9.41 \mathrm{E}-2$ & $5 / 01 / 08$ & 8D28004 & PNNL-AGG-ICP-AES \\
\hline $7440-23-5$ & Sodium & $1.07 \mathrm{E} 1$ & ug/g dry & 6.79E-1 & $5 / 01 / 08$ & 8D28004 & PNNL-AGG-ICP-AES \\
\hline
\end{tabular}


Total Metals by PNNL-AGG-ICP-AES/Acid Extract

\begin{tabular}{|c|c|c|c|c|c|c|c|}
\hline CAS \# & Analyte & Results & Units & EQL & Analyzed & Batch & Method \\
\hline HEIS No. & B1T170 & \multicolumn{3}{|c|}{ Lab ID: $\quad$ 0802013-12 } & & & \\
\hline $7429-90-5$ & Aluminum & $5.17 \mathrm{E} 3$ & ug/g dry & 4.13E1 & 6/13/08 & 8E12002 & PNNL-AGG-ICP-AES \\
\hline $7440-39-3$ & Barium & 5.73E1 & ug/g dry & 3.93E0 & 6/13/08 & 8E12002 & PNNL-AGG-ICP-AES \\
\hline $7440-70-2$ & Calcium & $7.00 \mathrm{E} 3$ & ug/g dry & $1.53 \mathrm{E} 2$ & 6/13/08 & 8E12002 & PNNL-AGG-ICP-AES \\
\hline $7440-48-4$ & Cobalt & $<8.57 \mathrm{E} 0$ & ug/g dry & 8.57Е0 & 6/13/08 & 8E12002 & PNNL-AGG-ICP-AES \\
\hline $7440-47-3$ & Chromium & $1.04 \mathrm{E} 1$ & ug/g dry & 3.43E0 & 6/13/08 & 8E12002 & PNNL-AGG-ICP-AES \\
\hline $7440-50-8$ & Copper & $<3.66 \mathrm{E} 1$ & ug/g dry & $3.66 \mathrm{E} 1$ & 6/13/08 & 8E12002 & PNNL-AGG-ICP-AES \\
\hline 7439-89-6 & Iron & $9.28 \mathrm{E} 3$ & ug/g dry & $1.05 \mathrm{E} 2$ & 6/13/08 & 8E12002 & PNNL-AGG-ICP-AES \\
\hline 7440-09-7 & Potassium & $1.10 \mathrm{E} 3$ & ug/g dry & $9.74 \mathrm{E} 1$ & 6/13/08 & 8E12002 & PNNL-AGG-ICP-AES \\
\hline 7439-95-4 & Magnesium & 3.90E3 & ug/g dry & $3.25 \mathrm{E} 1$ & 6/13/08 & 8E12002 & PNNL-AGG-ICP-AES \\
\hline $7439-96-5$ & Manganese & $2.44 \mathrm{E} 2$ & ug/g dry & 1.23E0 & 6/13/08 & 8E12002 & PNNL-AGG-ICP-AES \\
\hline $7440-02-0$ & Nickel & $1.27 \mathrm{E} 1$ & ug/g dry & $8.44 \mathrm{E} 0$ & 6/13/08 & 8E12002 & PNNL-AGG-ICP-AES \\
\hline $7782-49-2$ & Selenium & $<1.43 \mathrm{E} 2$ & ug/g dry & $1.43 \mathrm{E} 2$ & 6/13/08 & 8E12002 & PNNL-AGG-ICP-AES \\
\hline $7440-62-2$ & Vanadium & $1.05 \mathrm{E} 1$ & ug/g dry & 3.82E0 & 6/13/08 & 8E12002 & PNNL-AGG-ICP-AES \\
\hline $7440-66-6$ & Zinc & 2.53E1 & ug/g dry & $6.44 \mathrm{E} 0$ & 6/13/08 & 8E12002 & PNNL-AGG-ICP-AES \\
\hline $7440-23-5$ & Sodium & $<1.10 \mathrm{E} 3$ & ug/g dry & $1.10 \mathrm{E} 3$ & 6/13/08 & 8E12002 & PNNL-AGG-ICP-AES \\
\hline HEIS No. & $\begin{array}{l}\text { B1T172 } \\
\end{array}$ & \multicolumn{3}{|c|}{ Lab ID: $\quad$ 0802013-14 } & & & \\
\hline $7429-90-5$ & Aluminum & $4.94 \mathrm{E} 3$ & ug/g dry & $3.70 \mathrm{E} 1$ & 6/13/08 & 8E12002 & PNNL-AGG-ICP-AES \\
\hline $7440-39-3$ & Barium & 5.91E1 & ug/g dry & 3.53E0 & 6/13/08 & 8E12002 & PNNL-AGG-ICP-AES \\
\hline $7440-70-2$ & Calcium & 5.67E3 & ug/g dry & $1.38 \mathrm{E} 2$ & 6/13/08 & 8E12002 & PNNL-AGG-ICP-AES \\
\hline $7440-48-4$ & Cobalt & $<7.68 \mathrm{E} 0$ & ug/g dry & 7.68E0 & 6/13/08 & 8E12002 & PNNL-AGG-ICP-AES \\
\hline $7440-47-3$ & Chromium & 5.87E0 & ug/g dry & $3.08 \mathrm{E} 0$ & 6/13/08 & 8E12002 & PNNL-AGG-ICP-AES \\
\hline $7440-50-8$ & Copper & $<3.28 \mathrm{E} 1$ & ug/g dry & $3.28 \mathrm{E} 1$ & 6/13/08 & 8E12002 & PNNL-AGG-ICP-AES \\
\hline 7439-89-6 & Iron & $1.04 \mathrm{E} 4$ & ug/g dry & $9.41 \mathrm{E} 1$ & 6/13/08 & 8E12002 & PNNL-AGG-ICP-AES \\
\hline 7440-09-7 & Potassium & $1.07 \mathrm{E} 3$ & ug/g dry & 8.73E1 & 6/13/08 & 8E12002 & PNNL-AGG-ICP-AES \\
\hline $7439-95-4$ & Magnesium & 3.39E3 & ug/g dry & $2.91 \mathrm{E} 1$ & 6/13/08 & 8E12002 & PNNL-AGG-ICP-AES \\
\hline 7439-96-5 & Manganese & $2.21 \mathrm{E} 2$ & ug/g dry & $1.10 \mathrm{E} 0$ & 6/13/08 & 8E12002 & PNNL-AGG-ICP-AES \\
\hline 7440-02-0 & Nickel & 7.77E0 & ug/g dry & 7.57E0 & 6/13/08 & 8E12002 & PNNL-AGG-ICP-AES \\
\hline $7782-49-2$ & Selenium & $<1.29 \mathrm{E} 2$ & ug/g dry & $1.29 \mathrm{E} 2$ & 6/13/08 & 8E12002 & PNNL-AGG-ICP-AES \\
\hline $7440-62-2$ & Vanadium & 1.33E1 & ug/g dry & 3.43E0 & 6/13/08 & 8E12002 & PNNL-AGG-ICP-AES \\
\hline $7440-66-6$ & Zinc & 2.49E1 & ug/g dry & 5.77E0 & 6/13/08 & 8E12002 & PNNL-AGG-ICP-AES \\
\hline $7440-23-5$ & Sodium & $<9.87 \mathrm{E} 2$ & ug/g dry & $9.87 \mathrm{E} 2$ & 6/13/08 & 8E12002 & PNNL-AGG-ICP-AES \\
\hline HEIS No. & $\begin{array}{l}\text { B1T152 } \\
\end{array}$ & \multicolumn{3}{|c|}{ Lab ID: $\quad$ 0802014-10 } & & & \\
\hline $7429-90-5$ & Aluminum & 4.47E3 & ug/g dry & $3.51 \mathrm{E} 1$ & 6/13/08 & 8E12002 & PNNL-AGG-ICP-AES \\
\hline $7440-39-3$ & Barium & 3.85E1 & ug/g dry & 3.35E0 & 6/13/08 & 8E12002 & PNNL-AGG-ICP-AES \\
\hline $7440-70-2$ & Calcium & $5.70 \mathrm{E} 3$ & ug/g dry & $1.30 \mathrm{E} 2$ & 6/13/08 & 8E12002 & PNNL-AGG-ICP-AES \\
\hline $7440-48-4$ & Cobalt & $<7.29 \mathrm{E} 0$ & ug/g dry & 7.29E0 & 6/13/08 & 8E12002 & PNNL-AGG-ICP-AES \\
\hline $7440-47-3$ & Chromium & 7.12E0 & ug/g dry & $2.92 \mathrm{E} 0$ & 6/13/08 & 8E12002 & PNNL-AGG-ICP-AES \\
\hline 7439-89-6 & Iron & 8.04E3 & ug/g dry & 8.92E1 & 6/13/08 & 8E12002 & PNNL-AGG-ICP-AES \\
\hline $7440-09-7$ & Potassium & $1.07 \mathrm{E} 3$ & ug/g dry & $8.28 \mathrm{E} 1$ & 6/13/08 & 8E12002 & PNNL-AGG-ICP-AES \\
\hline 7439-95-4 & Magnesium & 3.39E3 & ug/g dry & $2.76 \mathrm{E} 1$ & 6/13/08 & 8E12002 & PNNL-AGG-ICP-AES \\
\hline 7439-96-5 & Manganese & $2.05 \mathrm{E} 2$ & ug/g dry & $1.05 \mathrm{E} 0$ & 6/13/08 & 8E12002 & PNNL-AGG-ICP-AES \\
\hline 7439-98-7 & Molybdenum & $<1.98 \mathrm{E} 1$ & ug/g dry & $1.98 \mathrm{E} 1$ & 6/13/08 & 8E12002 & PNNL-AGG-ICP-AES \\
\hline $7440-02-0$ & Nickel & $1.03 \mathrm{E} 1$ & ug/g dry & $7.18 \mathrm{E} 0$ & 6/13/08 & 8E12002 & PNNL-AGG-ICP-AES \\
\hline $7782-49-2$ & Selenium & $<1.22 \mathrm{E} 2$ & ug/g dry & $1.22 \mathrm{E} 2$ & 6/13/08 & 8E12002 & PNNL-AGG-ICP-AES \\
\hline $7440-62-2$ & Vanadium & 8.65E0 & ug/g dry & $3.25 \mathrm{E} 0$ & 6/13/08 & 8E12002 & PNNL-AGG-ICP-AES \\
\hline $7440-66-6$ & Zinc & $2.25 \mathrm{E} 1$ & ug/g dry & $5.48 \mathrm{E} 0$ & 6/13/08 & 8E12002 & PNNL-AGG-ICP-AES \\
\hline $7440-23-5$ & Sodium & $<9.36 \mathrm{E} 2$ & ug/g dry & $9.36 \mathrm{E} 2$ & 6/13/08 & 8E12002 & PNNL-AGG-ICP-AES \\
\hline HEIS No. & B1T142 & $\mathbf{L}$ & D: & 015-15 & & & \\
\hline
\end{tabular}


Total Metals by PNNL-AGG-ICP-AES/Acid Extract

\begin{tabular}{|c|c|c|c|c|c|c|c|}
\hline CAS \# & Analyte & Results & Units & EQL & Analyzed & Batch & Method \\
\hline HEIS No. & B1T142 & \multicolumn{3}{|c|}{ Lab ID: $\quad$ 0802015-15 } & & & \\
\hline $7429-90-5$ & Aluminum & 4.84E3 & ug/g dry & $3.45 \mathrm{E} 1$ & 6/13/08 & 8E12002 & PNNL-AGG-ICP-AES \\
\hline $7440-39-3$ & Barium & 4.79E1 & ug/g dry & 3.29E0 & 6/13/08 & 8E12002 & PNNL-AGG-ICP-AES \\
\hline $7440-70-2$ & Calcium & $6.72 \mathrm{E} 3$ & ug/g dry & $1.28 \mathrm{E} 2$ & 6/13/08 & 8E12002 & PNNL-AGG-ICP-AES \\
\hline 7440-43-9 & Cadmium & $<1.96 \mathrm{E} 0$ & ug/g dry & 1.96E0 & 6/13/08 & 8E12002 & PNNL-AGG-ICP-AES \\
\hline $7440-48-4$ & Cobalt & $<7.17 \mathrm{E} 0$ & ug/g dry & 7.17E0 & 6/13/08 & 8E12002 & PNNL-AGG-ICP-AES \\
\hline $7440-47-3$ & Chromium & 6.97E0 & ug/g dry & 2.87E0 & 6/13/08 & 8E12002 & PNNL-AGG-ICP-AES \\
\hline $7440-50-8$ & Copper & $<3.06 \mathrm{E} 1$ & ug/g dry & 3.06E1 & 6/13/08 & 8E12002 & PNNL-AGG-ICP-AES \\
\hline 7439-89-6 & Iron & $1.07 \mathrm{E} 4$ & ug/g dry & 8.77E1 & 6/13/08 & 8E12002 & PNNL-AGG-ICP-AES \\
\hline $7440-09-7$ & Potassium & $9.48 \mathrm{E} 2$ & ug/g dry & 8.15E1 & 6/13/08 & 8E12002 & PNNL-AGG-ICP-AES \\
\hline 7439-95-4 & Magnesium & 3.92E3 & ug/g dry & $2.72 \mathrm{E} 1$ & 6/13/08 & 8E12002 & PNNL-AGG-ICP-AES \\
\hline 7439-96-5 & Manganese & 2.23E2 & ug/g dry & 1.03E0 & 6/13/08 & 8E12002 & PNNL-AGG-ICP-AES \\
\hline $7440-02-0$ & Nickel & $1.02 \mathrm{E} 1$ & ug/g dry & 7.06E0 & 6/13/08 & 8E12002 & PNNL-AGG-ICP-AES \\
\hline $7782-49-2$ & Selenium & $<1.20 \mathrm{E} 2$ & ug/g dry & $1.20 \mathrm{E} 2$ & 6/13/08 & 8E12002 & PNNL-AGG-ICP-AES \\
\hline $7440-62-2$ & Vanadium & $1.28 \mathrm{E} 1$ & ug/g dry & $3.20 \mathrm{E} 0$ & 6/13/08 & 8E12002 & PNNL-AGG-ICP-AES \\
\hline $7440-66-6$ & Zinc & $2.41 \mathrm{E} 1$ & ug/g dry & 5.38E0 & 6/13/08 & 8E12002 & PNNL-AGG-ICP-AES \\
\hline $7440-23-5$ & Sodium & $<9.20 \mathrm{E} 2$ & ug/g dry & $9.20 \mathrm{E} 2$ & 6/13/08 & 8E12002 & PNNL-AGG-ICP-AES \\
\hline $7440-36-0$ & Antimony & $<9.61 \mathrm{E} 1$ & ug/g dry & $9.61 \mathrm{E} 1$ & 6/13/08 & 8E12002 & PNNL-AGG-ICP-AES \\
\hline HEIS No. & B1T198 & \multicolumn{3}{|c|}{ Lab ID: $\quad 0802016-26$} & & & \\
\hline $7429-90-5$ & Aluminum & $4.51 \mathrm{E} 3$ & ug/g dry & 3.67E1 & 6/13/08 & 8E12002 & PNNL-AGG-ICP-AES \\
\hline $7440-39-3$ & Barium & 4.55E1 & ug/g dry & 3.49E0 & 6/13/08 & 8E12002 & PNNL-AGG-ICP-AES \\
\hline $7440-70-2$ & Calcium & $5.62 \mathrm{E} 3$ & ug/g dry & $1.36 \mathrm{E} 2$ & 6/13/08 & 8E12002 & PNNL-AGG-ICP-AES \\
\hline $7440-48-4$ & Cobalt & $<7.60 \mathrm{E} 0$ & ug/g dry & 7.60E0 & 6/13/08 & 8E12002 & PNNL-AGG-ICP-AES \\
\hline $7440-47-3$ & Chromium & 7.61E0 & ug/g dry & $3.05 \mathrm{E} 0$ & 6/13/08 & 8E12002 & PNNL-AGG-ICP-AES \\
\hline $7440-50-8$ & Copper & $<3.25 \mathrm{E} 1$ & ug/g dry & $3.25 \mathrm{E} 1$ & 6/13/08 & 8E12002 & PNNL-AGG-ICP-AES \\
\hline 7439-89-6 & Iron & 8.89E3 & ug/g dry & $9.31 \mathrm{E} 1$ & 6/13/08 & 8E12002 & PNNL-AGG-ICP-AES \\
\hline 7440-09-7 & Potassium & 8.59E2 & ug/g dry & 8.64E1 & 6/13/08 & 8E12002 & PNNL-AGG-ICP-AES \\
\hline 7439-95-4 & Magnesium & 3.68E3 & ug/g dry & $2.88 \mathrm{E} 1$ & 6/13/08 & 8E12002 & PNNL-AGG-ICP-AES \\
\hline 7439-96-5 & Manganese & $2.08 \mathrm{E} 2$ & ug/g dry & 1.09E0 & 6/13/08 & 8E12002 & PNNL-AGG-ICP-AES \\
\hline $7440-02-0$ & Nickel & $1.17 \mathrm{E} 1$ & ug/g dry & 7.49E0 & 6/13/08 & 8E12002 & PNNL-AGG-ICP-AES \\
\hline $7782-49-2$ & Selenium & $<1.27 \mathrm{E} 2$ & ug/g dry & $1.27 \mathrm{E} 2$ & 6/13/08 & 8E12002 & PNNL-AGG-ICP-AES \\
\hline $7440-62-2$ & Vanadium & 9.97E0 & ug/g dry & 3.39E0 & 6/13/08 & 8E12002 & PNNL-AGG-ICP-AES \\
\hline $7440-66-6$ & Zinc & 2.15E1 & ug/g dry & 5.71E0 & 6/13/08 & 8E12002 & PNNL-AGG-ICP-AES \\
\hline $7440-23-5$ & Sodium & $<9.76 \mathrm{E} 2$ & ug/g dry & $9.76 \mathrm{E} 2$ & 6/13/08 & 8E12002 & PNNL-AGG-ICP-AES \\
\hline HEIS No. & B1T1C4 & \multicolumn{3}{|c|}{ Lab ID: $\quad$ 0802018-03 } & & & \\
\hline $7429-90-5$ & Aluminum & 4.95E3 & ug/g dry & 3.52E1 & 6/13/08 & 8E12002 & PNNL-AGG-ICP-AES \\
\hline $7440-39-3$ & Barium & 7.40E1 & ug/g dry & 3.36E0 & 6/13/08 & 8E12002 & PNNL-AGG-ICP-AES \\
\hline $7440-70-2$ & Calcium & $7.58 \mathrm{E} 3$ & ug/g dry & $1.31 \mathrm{E} 2$ & 6/13/08 & 8E12002 & PNNL-AGG-ICP-AES \\
\hline $7440-43-9$ & Cadmium & $<2.00 \mathrm{E} 0$ & ug/g dry & $2.00 \mathrm{E} 0$ & 6/13/08 & 8E12002 & PNNL-AGG-ICP-AES \\
\hline $7440-48-4$ & Cobalt & $<7.31 \mathrm{E} 0$ & ug/g dry & 7.31E0 & 6/13/08 & 8E12002 & PNNL-AGG-ICP-AES \\
\hline $7440-47-3$ & Chromium & $3.45 \mathrm{E} 0$ & ug/g dry & 2.93E0 & 6/13/08 & 8E12002 & PNNL-AGG-ICP-AES \\
\hline $7440-50-8$ & Copper & $<3.12 \mathrm{E} 1$ & ug/g dry & 3.12E1 & 6/13/08 & 8E12002 & PNNL-AGG-ICP-AES \\
\hline 7439-89-6 & Iron & $1.16 \mathrm{E} 4$ & ug/g dry & 8.95E1 & 6/13/08 & 8E12002 & PNNL-AGG-ICP-AES \\
\hline 7440-09-7 & Potassium & $9.46 \mathrm{E} 2$ & ug/g dry & 8.31E1 & 6/13/08 & 8E12002 & PNNL-AGG-ICP-AES \\
\hline 7439-95-4 & Magnesium & 3.07E3 & ug/g dry & 2.77E1 & 6/13/08 & 8E12002 & PNNL-AGG-ICP-AES \\
\hline 7439-96-5 & Manganese & $1.91 \mathrm{E} 2$ & ug/g dry & $1.05 \mathrm{E} 0$ & 6/13/08 & 8E12002 & PNNL-AGG-ICP-AES \\
\hline $7440-02-0$ & Nickel & $<7.21 \mathrm{E} 0$ & ug/g dry & $7.21 \mathrm{E} 0$ & 6/13/08 & 8E12002 & PNNL-AGG-ICP-AES \\
\hline $7782-49-2$ & Selenium & $<1.22 \mathrm{E} 2$ & ug/g dry & $1.22 \mathrm{E} 2$ & 6/13/08 & 8E12002 & PNNL-AGG-ICP-AES \\
\hline $7440-62-2$ & Vanadium & $1.58 \mathrm{E} 1$ & ug/g dry & $3.26 \mathrm{E} 0$ & 6/13/08 & 8E12002 & PNNL-AGG-ICP-AES \\
\hline
\end{tabular}


Total Metals by PNNL-AGG-ICP-AES/Acid Extract

\begin{tabular}{|c|c|c|c|c|c|c|c|}
\hline CAS \# & Analyte & Results & Units & EQL & Analyzed & Batch & Method \\
\hline HEIS No. & B1T1C4 & \multicolumn{3}{|c|}{ Lab ID: $\quad$ 0802018-03 } & & & \\
\hline $7440-66-6$ & Zinc & $2.56 \mathrm{E} 1$ & ug/g dry & $5.49 \mathrm{E} 0$ & 6/13/08 & 8E12002 & PNNL-AGG-ICP-AES \\
\hline $7440-23-5$ & Sodium & $<9.39 \mathrm{E} 2$ & ug/g dry & 9.39E2 & 6/13/08 & 8E12002 & PNNL-AGG-ICP-AES \\
\hline HEIS No. & B1T1H0 & \multicolumn{3}{|c|}{ Lab ID: $\quad$ 0802022-01 } & & & \\
\hline $7429-90-5$ & Aluminum & 5.83E3 & ug/g dry & 3.89E1 & 6/13/08 & 8E12002 & PNNL-AGG-ICP-AES \\
\hline $7440-39-3$ & Barium & $6.58 \mathrm{E} 1$ & ug/g dry & 3.71E0 & 6/13/08 & 8E12002 & PNNL-AGG-ICP-AES \\
\hline $7440-70-2$ & Calcium & $9.88 \mathrm{E} 3$ & ug/g dry & $1.45 \mathrm{E} 2$ & 6/13/08 & 8E12002 & PNNL-AGG-ICP-AES \\
\hline $7440-48-4$ & Cobalt & $<8.07 \mathrm{E} 0$ & ug/g dry & 8.07E0 & 6/13/08 & 8E12002 & PNNL-AGG-ICP-AES \\
\hline $7440-47-3$ & Chromium & $1.00 \mathrm{E} 1$ & ug/g dry & 3.23E0 & 6/13/08 & 8E12002 & PNNL-AGG-ICP-AES \\
\hline $7440-50-8$ & Copper & $<3.45 \mathrm{E} 1$ & ug/g dry & $3.45 \mathrm{E} 1$ & 6/13/08 & 8E12002 & PNNL-AGG-ICP-AES \\
\hline 7439-89-6 & Iron & $1.03 \mathrm{E} 4$ & ug/g dry & $9.88 \mathrm{E} 1$ & 6/13/08 & 8E12002 & PNNL-AGG-ICP-AES \\
\hline $7440-09-7$ & Potassium & $1.45 \mathrm{E} 3$ & ug/g dry & $9.17 \mathrm{E} 1$ & 6/13/08 & 8E12002 & PNNL-AGG-ICP-AES \\
\hline $7439-95-4$ & Magnesium & 4.38E3 & ug/g dry & 3.06E1 & 6/13/08 & 8E12002 & PNNL-AGG-ICP-AES \\
\hline $7439-96-5$ & Manganese & $2.84 \mathrm{E} 2$ & ug/g dry & $1.16 \mathrm{E} 0$ & 6/13/08 & 8E12002 & PNNL-AGG-ICP-AES \\
\hline $7440-02-0$ & Nickel & $1.30 \mathrm{E} 1$ & ug/g dry & 7.95E0 & 6/13/08 & 8E12002 & PNNL-AGG-ICP-AES \\
\hline $7782-49-2$ & Selenium & $<1.35 \mathrm{E} 2$ & ug/g dry & $1.35 \mathrm{E} 2$ & 6/13/08 & 8E12002 & PNNL-AGG-ICP-AES \\
\hline $7440-62-2$ & Vanadium & $1.21 \mathrm{E} 1$ & ug/g dry & 3.60E0 & 6/13/08 & 8E12002 & PNNL-AGG-ICP-AES \\
\hline $7440-66-6$ & Zinc & $2.87 \mathrm{E} 1$ & ug/g dry & $6.06 \mathrm{E} 0$ & 6/13/08 & 8E12002 & PNNL-AGG-ICP-AES \\
\hline $7440-23-5$ & Sodium & $<1.04 \mathrm{E} 3$ & ug/g dry & $1.04 \mathrm{E} 3$ & 6/13/08 & 8E12002 & PNNL-AGG-ICP-AES \\
\hline HEIS No. & B1T1H2 & \multicolumn{3}{|c|}{ Lab ID: $\quad$ 0802022-04 } & & & \\
\hline $7429-90-5$ & Aluminum & 5.93E3 & ug/g dry & $5.26 \mathrm{E} 1$ & 6/13/08 & 8E12002 & PNNL-AGG-ICP-AES \\
\hline $7440-39-3$ & Barium & 6.83E1 & ug/g dry & $5.01 \mathrm{E} 0$ & 6/13/08 & 8E12002 & PNNL-AGG-ICP-AES \\
\hline $7440-70-2$ & Calcium & $1.02 \mathrm{E} 4$ & ug/g dry & $1.95 \mathrm{E} 2$ & 6/13/08 & 8E12002 & PNNL-AGG-ICP-AES \\
\hline $7440-48-4$ & Cobalt & $<1.09 \mathrm{E} 1$ & ug/g dry & 1.09E1 & 6/13/08 & 8E12002 & PNNL-AGG-ICP-AES \\
\hline $7440-47-3$ & Chromium & $1.05 \mathrm{E} 1$ & ug/g dry & 4.37Е0 & 6/13/08 & 8E12002 & PNNL-AGG-ICP-AES \\
\hline $7440-50-8$ & Copper & $<4.66 \mathrm{E} 1$ & ug/g dry & $4.66 \mathrm{E} 1$ & 6/13/08 & 8E12002 & PNNL-AGG-ICP-AES \\
\hline 7439-89-6 & Iron & $1.09 \mathrm{E} 4$ & ug/g dry & $1.34 \mathrm{E} 2$ & 6/13/08 & 8E12002 & PNNL-AGG-ICP-AES \\
\hline 7440-09-7 & Potassium & $1.43 \mathrm{E} 3$ & ug/g dry & $1.24 \mathrm{E} 2$ & 6/13/08 & 8E12002 & PNNL-AGG-ICP-AES \\
\hline 7439-95-4 & Magnesium & $4.48 \mathrm{E} 3$ & ug/g dry & 4.13E1 & 6/13/08 & 8E12002 & PNNL-AGG-ICP-AES \\
\hline 7439-96-5 & Manganese & $2.80 \mathrm{E} 2$ & ug/g dry & $1.56 \mathrm{E} 0$ & 6/13/08 & 8E12002 & PNNL-AGG-ICP-AES \\
\hline 7440-02-0 & Nickel & $1.41 \mathrm{E} 1$ & ug/g dry & $1.07 \mathrm{E} 1$ & 6/13/08 & 8E12002 & PNNL-AGG-ICP-AES \\
\hline $7782-49-2$ & Selenium & $<1.82 \mathrm{E} 2$ & ug/g dry & $1.82 \mathrm{E} 2$ & 6/13/08 & 8E12002 & PNNL-AGG-ICP-AES \\
\hline $7440-62-2$ & Vanadium & $1.25 \mathrm{E} 1$ & ug/g dry & 4.86Е0 & 6/13/08 & 8E12002 & PNNL-AGG-ICP-AES \\
\hline $7440-66-6$ & Zinc & 2.93E1 & ug/g dry & 8.19E0 & 6/13/08 & 8E12002 & PNNL-AGG-ICP-AES \\
\hline $7440-23-5$ & Sodium & $<1.40 \mathrm{E} 3$ & ug/g dry & $1.40 \mathrm{E} 3$ & 6/13/08 & 8E12002 & PNNL-AGG-ICP-AES \\
\hline HEIS No. & B1T1H4 & \multicolumn{3}{|c|}{ Lab ID: $\quad 0802022-07$} & & & \\
\hline $7429-90-5$ & Aluminum & $7.76 \mathrm{E} 3$ & ug/g dry & $4.45 \mathrm{E} 1$ & 6/13/08 & 8E12002 & PNNL-AGG-ICP-AES \\
\hline $7440-39-3$ & Barium & $8.88 \mathrm{E} 1$ & ug/g dry & 4.24E0 & 6/13/08 & 8E12002 & PNNL-AGG-ICP-AES \\
\hline $7440-70-2$ & Calcium & $1.04 \mathrm{E} 4$ & ug/g dry & $1.65 \mathrm{E} 2$ & 6/13/08 & 8E12002 & PNNL-AGG-ICP-AES \\
\hline $7440-48-4$ & Cobalt & $<9.23 \mathrm{E} 0$ & ug/g dry & $9.23 \mathrm{E} 0$ & 6/13/08 & 8E12002 & PNNL-AGG-ICP-AES \\
\hline $7440-47-3$ & Chromium & $1.51 \mathrm{E} 1$ & ug/g dry & 3.70E0 & 6/13/08 & 8E12002 & PNNL-AGG-ICP-AES \\
\hline $7440-50-8$ & Copper & $<3.94 \mathrm{E} 1$ & ug/g dry & $3.94 \mathrm{E} 1$ & 6/13/08 & 8E12002 & PNNL-AGG-ICP-AES \\
\hline 7439-89-6 & Iron & $1.27 \mathrm{E} 4$ & ug/g dry & 1.13E2 & 6/13/08 & 8E12002 & PNNL-AGG-ICP-AES \\
\hline 7440-09-7 & Potassium & 1.73E3 & ug/g dry & $1.05 \mathrm{E} 2$ & 6/13/08 & 8E12002 & PNNL-AGG-ICP-AES \\
\hline 7439-95-4 & Magnesium & $5.21 \mathrm{E} 3$ & ug/g dry & $3.50 \mathrm{E} 1$ & 6/13/08 & 8E12002 & PNNL-AGG-ICP-AES \\
\hline $7439-96-5$ & Manganese & 3.30E2 & ug/g dry & $1.32 \mathrm{E} 0$ & 6/13/08 & 8E12002 & PNNL-AGG-ICP-AES \\
\hline $7440-02-0$ & Nickel & 1.69E1 & ug/g dry & $9.10 \mathrm{E} 0$ & 6/13/08 & 8E12002 & PNNL-AGG-ICP-AES \\
\hline 7782-49-2 & Selenium & $<1.54 \mathrm{E} 2$ & ug/g dry & $1.54 \mathrm{E} 2$ & 6/13/08 & 8E12002 & PNNL-AGG-ICP-AES \\
\hline $7440-62-2$ & Vanadium & $1.34 \mathrm{E} 1$ & ug/g dry & $4.12 \mathrm{E} 0$ & 6/13/08 & 8E12002 & PNNL-AGG-ICP-AES \\
\hline
\end{tabular}


Total Metals by PNNL-AGG-ICP-AES/Acid Extract

\begin{tabular}{|c|c|c|c|c|c|c|c|}
\hline CAS \# & Analyte & Results & Units & EQL & Analyzed & Batch & Method \\
\hline HEIS No. & B1T1H4 & \multicolumn{3}{|c|}{ Lab ID: $\quad$ 0802022-07 } & & & \\
\hline $7440-66-6$ & Zinc & 4.18E1 & ug/g dry & $6.94 \mathrm{E} 0$ & 6/13/08 & 8E12002 & PNNL-AGG-ICP-AES \\
\hline $7440-23-5$ & Sodium & $<1.19 \mathrm{E} 3$ & ug/g dry & $1.19 \mathrm{E} 3$ & 6/13/08 & 8E12002 & PNNL-AGG-ICP-AES \\
\hline HEIS No. & B1T1H6 & \multicolumn{3}{|c|}{ Lab ID: $\quad$ 0802022-11 } & & & \\
\hline $7429-90-5$ & Aluminum & $6.72 \mathrm{E} 3$ & ug/g dry & 4.37E1 & 6/13/08 & 8E12002 & PNNL-AGG-ICP-AES \\
\hline $7440-39-3$ & Barium & 8.18E1 & ug/g dry & 4.16Е0 & 6/13/08 & 8E12002 & PNNL-AGG-ICP-AES \\
\hline $7440-70-2$ & Calcium & $1.20 \mathrm{E} 4$ & ug/g dry & $1.62 \mathrm{E} 2$ & 6/13/08 & 8E12002 & PNNL-AGG-ICP-AES \\
\hline $7440-48-4$ & Cobalt & $<9.06 \mathrm{E} 0$ & ug/g dry & $9.06 \mathrm{E} 0$ & 6/13/08 & 8E12002 & PNNL-AGG-ICP-AES \\
\hline $7440-47-3$ & Chromium & 8.06E0 & ug/g dry & 3.63Е0 & 6/13/08 & 8E12002 & PNNL-AGG-ICP-AES \\
\hline $7440-50-8$ & Copper & $<3.87 \mathrm{E} 1$ & ug/g dry & $3.87 \mathrm{E} 1$ & 6/13/08 & 8E12002 & PNNL-AGG-ICP-AES \\
\hline 7439-89-6 & Iron & $1.14 \mathrm{E} 4$ & ug/g dry & $1.11 \mathrm{E} 2$ & 6/13/08 & 8E12002 & PNNL-AGG-ICP-AES \\
\hline $7440-09-7$ & Potassium & $1.54 \mathrm{E} 3$ & ug/g dry & $1.03 \mathrm{E} 2$ & 6/13/08 & 8E12002 & PNNL-AGG-ICP-AES \\
\hline $7439-95-4$ & Magnesium & 4.86E3 & ug/g dry & 3.43E1 & 6/13/08 & 8E12002 & PNNL-AGG-ICP-AES \\
\hline $7439-96-5$ & Manganese & 3.19E2 & ug/g dry & $1.30 \mathrm{E} 0$ & 6/13/08 & 8E12002 & PNNL-AGG-ICP-AES \\
\hline $7440-02-0$ & Nickel & $1.05 \mathrm{E} 1$ & ug/g dry & 8.93E0 & 6/13/08 & 8E12002 & PNNL-AGG-ICP-AES \\
\hline $7782-49-2$ & Selenium & $<1.52 \mathrm{E} 2$ & ug/g dry & $1.52 \mathrm{E} 2$ & 6/13/08 & 8E12002 & PNNL-AGG-ICP-AES \\
\hline $7440-62-2$ & Vanadium & $1.11 \mathrm{E} 1$ & ug/g dry & 4.04E0 & 6/13/08 & 8E12002 & PNNL-AGG-ICP-AES \\
\hline $7440-66-6$ & Zinc & 3.37E1 & ug/g dry & $6.81 \mathrm{E} 0$ & 6/13/08 & 8E12002 & PNNL-AGG-ICP-AES \\
\hline $7440-23-5$ & Sodium & $<1.16 \mathrm{E} 3$ & ug/g dry & $1.16 \mathrm{E} 3$ & 6/13/08 & 8E12002 & PNNL-AGG-ICP-AES \\
\hline HEIS No. & B1T1D8 & \multicolumn{3}{|c|}{ Lab ID: $\quad$ 0802024-15 } & & & \\
\hline $7429-90-5$ & Aluminum & 4.25E3 & ug/g dry & 3.38E1 & 6/13/08 & 8E12002 & PNNL-AGG-ICP-AES \\
\hline $7440-39-3$ & Barium & 4.11E1 & ug/g dry & $3.22 \mathrm{E} 0$ & 6/13/08 & 8E12002 & PNNL-AGG-ICP-AES \\
\hline $7440-70-2$ & Calcium & $5.44 \mathrm{E} 3$ & ug/g dry & $1.26 \mathrm{E} 2$ & 6/13/08 & 8E12002 & PNNL-AGG-ICP-AES \\
\hline $7440-48-4$ & Cobalt & $<7.02 \mathrm{E} 0$ & ug/g dry & 7.02E0 & 6/13/08 & 8E12002 & PNNL-AGG-ICP-AES \\
\hline $7440-47-3$ & Chromium & 7.16Е0 & ug/g dry & $2.81 \mathrm{E} 0$ & 6/13/08 & 8E12002 & PNNL-AGG-ICP-AES \\
\hline $7440-50-8$ & Copper & $<3.00 \mathrm{E} 1$ & ug/g dry & $3.00 \mathrm{E} 1$ & 6/13/08 & 8E12002 & PNNL-AGG-ICP-AES \\
\hline 7439-89-6 & Iron & $7.54 \mathrm{E} 3$ & ug/g dry & 8.59E1 & 6/13/08 & 8E12002 & PNNL-AGG-ICP-AES \\
\hline 7440-09-7 & Potassium & $9.04 \mathrm{E} 2$ & ug/g dry & $7.98 \mathrm{E} 1$ & 6/13/08 & 8E12002 & PNNL-AGG-ICP-AES \\
\hline 7439-95-4 & Magnesium & 3.32E3 & ug/g dry & $2.66 \mathrm{E} 1$ & 6/13/08 & 8E12002 & PNNL-AGG-ICP-AES \\
\hline 7439-96-5 & Manganese & $1.91 \mathrm{E} 2$ & ug/g dry & $1.01 \mathrm{E} 0$ & 6/13/08 & 8E12002 & PNNL-AGG-ICP-AES \\
\hline 7440-02-0 & Nickel & $1.08 \mathrm{E} 1$ & ug/g dry & $6.92 \mathrm{E} 0$ & 6/13/08 & 8E12002 & PNNL-AGG-ICP-AES \\
\hline $7782-49-2$ & Selenium & $<1.17 \mathrm{E} 2$ & ug/g dry & $1.17 \mathrm{E} 2$ & 6/13/08 & 8E12002 & PNNL-AGG-ICP-AES \\
\hline $7440-62-2$ & Vanadium & 8.58E0 & ug/g dry & 3.13E0 & 6/13/08 & 8E12002 & PNNL-AGG-ICP-AES \\
\hline $7440-66-6$ & Zinc & $2.04 \mathrm{E} 1$ & ug/g dry & $5.27 \mathrm{E} 0$ & 6/13/08 & 8E12002 & PNNL-AGG-ICP-AES \\
\hline $7440-23-5$ & Sodium & $<9.01 \mathrm{E} 2$ & ug/g dry & $9.01 \mathrm{E} 2$ & 6/13/08 & 8E12002 & PNNL-AGG-ICP-AES \\
\hline HEIS No. & B1T1F0 & \multicolumn{3}{|c|}{ Lab ID: $\quad$ 0802024-18 } & & & \\
\hline $7429-90-5$ & Aluminum & 7.84E3 & ug/g dry & 4.36E1 & 6/13/08 & 8E12002 & PNNL-AGG-ICP-AES \\
\hline $7440-39-3$ & Barium & $6.77 \mathrm{E} 1$ & ug/g dry & 4.15E0 & 6/13/08 & 8E12002 & PNNL-AGG-ICP-AES \\
\hline $7440-70-2$ & Calcium & $7.08 \mathrm{E} 3$ & ug/g dry & $1.62 \mathrm{E} 2$ & 6/13/08 & 8E12002 & PNNL-AGG-ICP-AES \\
\hline $7440-48-4$ & Cobalt & $<9.04 \mathrm{E} 0$ & ug/g dry & $9.04 \mathrm{E} 0$ & 6/13/08 & 8E12002 & PNNL-AGG-ICP-AES \\
\hline $7440-47-3$ & Chromium & $1.50 \mathrm{E} 1$ & ug/g dry & 3.62E0 & 6/13/08 & 8E12002 & PNNL-AGG-ICP-AES \\
\hline $7440-50-8$ & Copper & $<3.86 \mathrm{E} 1$ & ug/g dry & $3.86 \mathrm{E} 1$ & 6/13/08 & 8E12002 & PNNL-AGG-ICP-AES \\
\hline 7439-89-6 & Iron & $1.33 \mathrm{E} 4$ & ug/g dry & $1.11 \mathrm{E} 2$ & 6/13/08 & 8E12002 & PNNL-AGG-ICP-AES \\
\hline 7440-09-7 & Potassium & $1.54 \mathrm{E} 3$ & ug/g dry & $1.03 \mathrm{E} 2$ & 6/13/08 & 8E12002 & PNNL-AGG-ICP-AES \\
\hline 7439-95-4 & Magnesium & 5.12E3 & ug/g dry & $3.43 \mathrm{E} 1$ & 6/13/08 & 8E12002 & PNNL-AGG-ICP-AES \\
\hline $7439-96-5$ & Manganese & 3.34E2 & ug/g dry & $1.30 \mathrm{E} 0$ & 6/13/08 & 8E12002 & PNNL-AGG-ICP-AES \\
\hline $7440-02-0$ & Nickel & $1.76 \mathrm{E} 1$ & ug/g dry & 8.91E0 & 6/13/08 & 8E12002 & PNNL-AGG-ICP-AES \\
\hline 7782-49-2 & Selenium & $<1.51 \mathrm{E} 2$ & ug/g dry & $1.51 \mathrm{E} 2$ & 6/13/08 & 8E12002 & PNNL-AGG-ICP-AES \\
\hline $7440-62-2$ & Vanadium & $1.58 \mathrm{E} 1$ & ug/g dry & 4.03E0 & 6/13/08 & 8E12002 & PNNL-AGG-ICP-AES \\
\hline
\end{tabular}


Total Metals by PNNL-AGG-ICP-AES/Acid Extract

\begin{tabular}{|c|c|c|c|c|c|c|c|}
\hline CAS \# & Analyte & Results & Units & EQL & Analyzed & Batch & Method \\
\hline HEIS No. & B1T1F0 & \multicolumn{3}{|c|}{ Lab ID: $\quad$ 0802024-18 } & & & \\
\hline 7440-66-6 & Zinc & 3.70E1 & ug/g dry & 6.79E0 & 6/13/08 & 8E12002 & PNNL-AGG-ICP-AES \\
\hline $7440-23-5$ & Sodium & $<1.16 \mathrm{E} 3$ & ug/g dry & 1.16E3 & 6/13/08 & 8E12002 & PNNL-AGG-ICP-AES \\
\hline HEIS No. & B1T1F6 & \multicolumn{3}{|c|}{ Lab ID: $\quad$ 0802024-27 } & & & \\
\hline 7429-90-5 & Aluminum & $6.22 \mathrm{E} 3$ & ug/g dry & 4.21E1 & 6/13/08 & 8E12002 & PNNL-AGG-ICP-AES \\
\hline 7440-39-3 & Barium & 6.65E1 & ug/g dry & 4.01E0 & 6/13/08 & 8E12002 & PNNL-AGG-ICP-AES \\
\hline $7440-70-2$ & Calcium & 9.71E3 & ug/g dry & $1.56 \mathrm{E} 2$ & 6/13/08 & 8E12002 & PNNL-AGG-ICP-AES \\
\hline $7440-48-4$ & Cobalt & $<8.73 \mathrm{E} 0$ & ug/g dry & 8.73E0 & 6/13/08 & 8E12002 & PNNL-AGG-ICP-AES \\
\hline $7440-47-3$ & Chromium & 1.13E1 & ug/g dry & 3.50E0 & 6/13/08 & 8E12002 & PNNL-AGG-ICP-AES \\
\hline $7440-50-8$ & Copper & $<3.73 \mathrm{E} 1$ & ug/g dry & 3.73E1 & 6/13/08 & 8E12002 & PNNL-AGG-ICP-AES \\
\hline 7439-89-6 & Iron & $1.07 \mathrm{E} 4$ & ug/g dry & $1.07 \mathrm{E} 2$ & 6/13/08 & 8E12002 & PNNL-AGG-ICP-AES \\
\hline 7440-09-7 & Potassium & $1.22 \mathrm{E} 3$ & ug/g dry & 9.92E1 & 6/13/08 & 8E12002 & PNNL-AGG-ICP-AES \\
\hline 7439-95-4 & Magnesium & 4.38E3 & ug/g dry & $3.31 \mathrm{E} 1$ & 6/13/08 & 8E12002 & PNNL-AGG-ICP-AES \\
\hline 7439-96-5 & Manganese & 2.65E2 & ug/g dry & $1.25 \mathrm{E} 0$ & 6/13/08 & 8E12002 & PNNL-AGG-ICP-AES \\
\hline 7440-02-0 & Nickel & $1.43 \mathrm{E} 1$ & ug/g dry & 8.60E0 & 6/13/08 & 8E12002 & PNNL-AGG-ICP-AES \\
\hline $7782-49-2$ & Selenium & $<1.46 \mathrm{E} 2$ & ug/g dry & $1.46 \mathrm{E} 2$ & 6/13/08 & 8E12002 & PNNL-AGG-ICP-AES \\
\hline $7440-62-2$ & Vanadium & $1.45 \mathrm{E} 1$ & ug/g dry & 3.89E0 & 6/13/08 & 8E12002 & PNNL-AGG-ICP-AES \\
\hline 7440-66-6 & Zinc & 2.89E1 & ug/g dry & $6.56 \mathrm{E} 0$ & 6/13/08 & 8E12002 & PNNL-AGG-ICP-AES \\
\hline $7440-23-5$ & Sodium & $<1.12 \mathrm{E} 3$ & ug/g dry & $1.12 \mathrm{E} 3$ & 6/13/08 & 8E12002 & PNNL-AGG-ICP-AES \\
\hline HEIS No. & B1T1M0 & \multicolumn{3}{|c|}{ Lab ID: $\quad$ 0802024-56 } & & & \\
\hline 7429-90-5 & Aluminum & 3.79E3 & ug/g dry & $3.54 \mathrm{E} 1$ & 6/13/08 & 8E12002 & PNNL-AGG-ICP-AES \\
\hline 7440-39-3 & Barium & 4.76E1 & ug/g dry & 3.37E0 & 6/13/08 & 8E12002 & PNNL-AGG-ICP-AES \\
\hline $7440-70-2$ & Calcium & 3.97E3 & ug/g dry & $1.31 \mathrm{E} 2$ & 6/13/08 & 8E12002 & PNNL-AGG-ICP-AES \\
\hline $7440-48-4$ & Cobalt & $<7.34 \mathrm{E} 0$ & ug/g dry & 7.34E0 & 6/13/08 & 8E12002 & PNNL-AGG-ICP-AES \\
\hline $7440-47-3$ & Chromium & 5.10E0 & ug/g dry & $2.94 \mathrm{E} 0$ & 6/13/08 & 8E12002 & PNNL-AGG-ICP-AES \\
\hline $7440-50-8$ & Copper & $<3.13 \mathrm{E} 1$ & ug/g dry & 3.13E1 & $6 / 13 / 08$ & 8E12002 & PNNL-AGG-ICP-AES \\
\hline 7439-89-6 & Iron & $1.08 \mathrm{E} 4$ & ug/g dry & 8.99E1 & 6/13/08 & 8E12002 & PNNL-AGG-ICP-AES \\
\hline 7440-09-7 & Potassium & 5.47E2 & ug/g dry & 8.35E1 & $6 / 13 / 08$ & 8E12002 & PNNL-AGG-ICP-AES \\
\hline 7439-95-4 & Magnesium & 2.38E3 & ug/g dry & $2.78 \mathrm{E} 1$ & 6/13/08 & 8E12002 & PNNL-AGG-ICP-AES \\
\hline 7439-96-5 & Manganese & $1.56 \mathrm{E} 2$ & ug/g dry & $1.05 \mathrm{E} 0$ & 6/13/08 & 8E12002 & PNNL-AGG-ICP-AES \\
\hline $7440-02-0$ & Nickel & $<7.24 \mathrm{E} 0$ & ug/g dry & 7.24E0 & 6/13/08 & 8E12002 & PNNL-AGG-ICP-AES \\
\hline $7782-49-2$ & Selenium & $<1.23 \mathrm{E} 2$ & ug/g dry & 1.23E2 & 6/13/08 & 8E12002 & PNNL-AGG-ICP-AES \\
\hline 7440-62-2 & Vanadium & 1.66E1 & ug/g dry & 3.27E0 & $6 / 13 / 08$ & 8E12002 & PNNL-AGG-ICP-AES \\
\hline 7440-66-6 & Zinc & 2.00E1 & ug/g dry & 5.52E0 & 6/13/08 & 8E12002 & PNNL-AGG-ICP-AES \\
\hline $7440-23-5$ & Sodium & $<9.43 \mathrm{E} 2$ & ug/g dry & 9.43E2 & 6/13/08 & 8E12002 & PNNL-AGG-ICP-AES \\
\hline
\end{tabular}




\section{Radionuclides by ICP-MS/Acid Extract}

\begin{tabular}{|c|c|c|c|c|c|c|c|}
\hline CAS \# & Analyte & Results & Units & EQL & Analyzed & Batch & Method \\
\hline HEIS No. & B1T170 & \multicolumn{3}{|c|}{ Lab ID: $\quad$ 0802013-12 } & & & \\
\hline \multirow[t]{2}{*}{ 14133-76-7 } & Technetium-99 & $<4.97 \mathrm{E}-3$ & ug/g dry & 4.97E-3 & $5 / 15 / 08$ & 8E15002 & PNNL-AGG-415 \\
\hline & Uranium 238 & 4.17E-1 & ug/g dry & 3.61E-2 & $5 / 15 / 08$ & 8E15002 & PNNL-AGG-415 \\
\hline HEIS No. & B1T172 & \multicolumn{3}{|c|}{ Lab ID: $\quad$ 0802013-14 } & & & \\
\hline \multirow[t]{2}{*}{ 14133-76-7 } & Technetium-99 & $<4.46 \mathrm{E}-3$ & ug/g dry & $4.46 \mathrm{E}-3$ & $5 / 15 / 08$ & 8E15002 & PNNL-AGG-415 \\
\hline & Uranium 238 & 4.33E-1 & ug/g dry & $3.24 \mathrm{E}-2$ & $5 / 15 / 08$ & 8E15002 & PNNL-AGG-415 \\
\hline HEIS No. & B1T152 & \multicolumn{3}{|c|}{ Lab ID: $\quad$ 0802014-10 } & & & \\
\hline \multirow[t]{2}{*}{$14133-76-7$} & Technetium-99 & $<4.23 \mathrm{E}-3$ & ug/g dry & $4.23 E-3$ & $5 / 16 / 08$ & 8E15002 & PNNL-AGG-415 \\
\hline & Uranium 238 & 3.36E-1 & ug/g dry & 3.07E-2 & $5 / 16 / 08$ & 8E15002 & PNNL-AGG-415 \\
\hline HEIS No. & B1T142 & \multicolumn{3}{|c|}{ Lab ID: $\quad$ 0802015-15 } & & & \\
\hline \multirow[t]{2}{*}{$14133-76-7$} & Technetium-99 & $<4.16 \mathrm{E}-3$ & ug/g dry & 4.16E-3 & $5 / 16 / 08$ & 8E15002 & PNNL-AGG-415 \\
\hline & Uranium 238 & $3.49 \mathrm{E}-1$ & ug/g dry & $3.02 \mathrm{E}-2$ & $5 / 16 / 08$ & 8E15002 & PNNL-AGG-415 \\
\hline \multirow{3}{*}{$\begin{array}{l}\text { HEIS No. } \\
14133-76-7\end{array}$} & B1T198 & \multicolumn{3}{|c|}{ Lab ID: $\quad$ 0802016-26 } & & & \\
\hline & Technetium-99 & $<4.41 \mathrm{E}-3$ & ug/g dry & $4.41 E-3$ & $5 / 16 / 08$ & 8E15002 & PNNL-AGG-415 \\
\hline & Uranium 238 & $3.28 \mathrm{E}-1$ & ug/g dry & $3.21 \mathrm{E}-2$ & $5 / 16 / 08$ & 8E15002 & PNNL-AGG-415 \\
\hline \multirow{3}{*}{$\begin{array}{l}\text { HEIS No. } \\
14133-76-7\end{array}$} & B1T1C4 & \multicolumn{3}{|c|}{ Lab ID: $\quad$ 0802018-03 } & & & \\
\hline & Technetium-99 & $<4.25 \mathrm{E}-3$ & ug/g dry & $4.25 \mathrm{E}-3$ & $5 / 16 / 08$ & 8E15002 & PNNL-AGG-415 \\
\hline & Uranium 238 & $4.52 \mathrm{E}-1$ & ug/g dry & $3.08 \mathrm{E}-2$ & $5 / 16 / 08$ & 8E15002 & PNNL-AGG-415 \\
\hline \multirow{3}{*}{$\begin{array}{l}\text { HEIS No. } \\
14133-76-7\end{array}$} & B1T1H0 & \multicolumn{3}{|c|}{ Lab ID: $\quad$ 0802022-01 } & & & \\
\hline & Technetium-99 & $<4.69 \mathrm{E}-3$ & ug/g dry & 4.69E-3 & $5 / 16 / 08$ & 8E15002 & PNNL-AGG-415 \\
\hline & Uranium 238 & 8.21E1 & ug/g dry & $3.40 \mathrm{E} 0$ & $5 / 16 / 08$ & 8E15002 & PNNL-AGG-415 \\
\hline \multirow{3}{*}{$\begin{array}{l}\text { HEIS No. } \\
14133-76-7\end{array}$} & B1T1H2 & \multicolumn{3}{|c|}{ Lab ID: $\quad$ 0802022-04 } & & & \\
\hline & Technetium-99 & $<6.33 \mathrm{E}-3$ & ug/g dry & 6.33E-3 & $5 / 16 / 08$ & 8E15002 & PNNL-AGG-415 \\
\hline & Uranium 238 & 8.73E1 & ug/g dry & $4.60 \mathrm{E} 0$ & $5 / 16 / 08$ & 8E15002 & PNNL-AGG-415 \\
\hline \multirow{3}{*}{$\begin{array}{l}\text { HEIS No. } \\
\text { 14133-76-7 }\end{array}$} & B1T1H4 & \multicolumn{3}{|c|}{ Lab ID: $\quad$ 0802022-07 } & & & \\
\hline & Technetium-99 & $<5.36 \mathrm{E}-3$ & ug/g dry & $5.36 \mathrm{E}-3$ & $5 / 16 / 08$ & 8E15002 & PNNL-AGG-415 \\
\hline & Uranium 238 & $2.28 \mathrm{E} 2$ & ug/g dry & 3.89E0 & $5 / 16 / 08$ & 8E15002 & PNNL-AGG-415 \\
\hline \multirow{3}{*}{$\begin{array}{l}\text { HEIS No. } \\
14133-76-7\end{array}$} & B1T1H6 & \multicolumn{3}{|c|}{ Lab ID: $\quad$ 0802022-11 } & & & \\
\hline & Technetium-99 & $<5.26 \mathrm{E}-3$ & ug/g dry & $5.26 \mathrm{E}-3$ & 5/16/08 & 8E15002 & PNNL-AGG-415 \\
\hline & Uranium 238 & 1.03E2 & ug/g dry & 3.82E0 & $5 / 16 / 08$ & 8E15002 & PNNL-AGG-415 \\
\hline \multirow{3}{*}{$\begin{array}{l}\text { HEIS No. } \\
14133-76-7\end{array}$} & B1T1D8 & \multicolumn{3}{|c|}{ Lab ID: $\quad$ 0802024-15 } & & & \\
\hline & Technetium-99 & $<4.07 \mathrm{E}-3$ & ug/g dry & 4.07E-3 & $5 / 16 / 08$ & 8E15002 & PNNL-AGG-415 \\
\hline & Uranium 238 & 2.39E-1 & ug/g dry & $2.96 \mathrm{E}-2$ & $5 / 16 / 08$ & 8E15002 & PNNL-AGG-415 \\
\hline \multirow{3}{*}{$\begin{array}{l}\text { HEIS No. } \\
14133-76-7\end{array}$} & B1T1F0 & \multicolumn{3}{|c|}{ Lab ID: $\quad$ 0802024-18 } & & & \\
\hline & Technetium-99 & $<5.25 \mathrm{E}-3$ & ug/g dry & $5.25 \mathrm{E}-3$ & $5 / 16 / 08$ & 8E15002 & PNNL-AGG-415 \\
\hline & Uranium 238 & $6.99 \mathrm{E}-1$ & ug/g dry & $3.81 \mathrm{E}-2$ & $5 / 16 / 08$ & 8E15002 & PNNL-AGG-415 \\
\hline HEIS No. & B1T1F6 & & b ID: & 2024-27 & & & \\
\hline $14133-76-7$ & Technetium-99 & $<5.07 \mathrm{E}-3$ & ug/g dry & 5.07E-3 & 5/16/08 & 8E15002 & PNNL-AGG-415 \\
\hline & Uranium 238 & 4.01E0 & ug/g dry & $3.68 \mathrm{E}-2$ & $5 / 16 / 08$ & 8E15002 & PNNL-AGG-415 \\
\hline HEIS No. & B1T1M0 & & b ID: & 2024-56 & & & \\
\hline $14133-76-7$ & Technetium-99 & $<4.26 \mathrm{E}-3$ & ug/g dry & $4.26 \mathrm{E}-3$ & $5 / 16 / 08$ & 8E15002 & PNNL-AGG-415 \\
\hline & Uranium 238 & $1.55 \mathrm{E} 0$ & ug/g dry & $3.09 \mathrm{E}-2$ & $5 / 16 / 08$ & 8E15002 & PNNL-AGG-415 \\
\hline
\end{tabular}




\section{Radionuclides by ICP-MS/Water Extract}

\begin{tabular}{|c|c|c|c|c|c|c|c|}
\hline CAS \# & Analyte & Results & Units & EQL & Analyzed & Batch & Method \\
\hline \multirow{3}{*}{$\begin{array}{l}\text { HEIS No. } \\
14133-76-7\end{array}$} & B1T170 & \multicolumn{3}{|c|}{ Lab ID: $\quad$ 0802013-12 } & & & \\
\hline & Technetium-99 & $<2.30 \mathrm{E}-5$ & ug/g dry & $2.30 \mathrm{E}-5$ & $4 / 15 / 08$ & 8D15001 & PNNL-AGG-415 \\
\hline & Uranium 238 & $3.02 \mathrm{E}-3$ & ug/g dry & $5.64 \mathrm{E}-4$ & $4 / 15 / 08$ & 8D15001 & PNNL-AGG-415 \\
\hline \multirow{3}{*}{$\begin{array}{l}\text { HEIS No. } \\
14133-76-7\end{array}$} & B1T172 & \multicolumn{3}{|c|}{ Lab ID: $\quad$ 0802013-14 } & & & \\
\hline & Technetium-99 & $<2.27 \mathrm{E}-5$ & ug/g dry & $2.27 \mathrm{E}-5$ & 4/15/08 & 8D15001 & PNNL-AGG-415 \\
\hline & Uranium 238 & $2.51 \mathrm{E}-3$ & ug/g dry & 5.57E-4 & $4 / 15 / 08$ & 8D15001 & PNNL-AGG-415 \\
\hline \multirow{3}{*}{$\begin{array}{l}\text { HEIS No. } \\
14133-76-7\end{array}$} & B1T152 & \multicolumn{3}{|c|}{ Lab ID: $\quad$ 0802014-10 } & & & \\
\hline & Technetium-99 & $<2.28 \mathrm{E}-5$ & ug/g dry & $2.28 \mathrm{E}-5$ & $4 / 15 / 08$ & 8D15001 & PNNL-AGG-415 \\
\hline & Uranium 238 & $<5.58 \mathrm{E}-4$ & ug/g dry & $5.58 \mathrm{E}-4$ & $4 / 15 / 08$ & 8D15001 & PNNL-AGG-415 \\
\hline \multirow{3}{*}{$\begin{array}{l}\text { HEIS No. } \\
14133-76-7\end{array}$} & B1T142 & \multicolumn{3}{|c|}{ Lab ID: $\quad$ 0802015-15 } & & & \\
\hline & Technetium-99 & $<2.31 \mathrm{E}-5$ & ug/g dry & $2.31 \mathrm{E}-5$ & $4 / 15 / 08$ & 8D15001 & PNNL-AGG-415 \\
\hline & Uranium 238 & $<5.67 \mathrm{E}-4$ & ug/g dry & 5.67E-4 & $4 / 15 / 08$ & 8D15001 & PNNL-AGG-415 \\
\hline \multirow{3}{*}{$\begin{array}{l}\text { HEIS No. } \\
14133-76-7\end{array}$} & B1T198 & \multicolumn{3}{|c|}{ Lab ID: $\quad$ 0802016-26 } & & & \\
\hline & Technetium-99 & $<2.30 \mathrm{E}-5$ & ug/g dry & $2.30 \mathrm{E}-5$ & $4 / 15 / 08$ & 8D15001 & PNNL-AGG-415 \\
\hline & Uranium 238 & $<5.63 \mathrm{E}-4$ & ug/g dry & $5.63 \mathrm{E}-4$ & $4 / 15 / 08$ & 8D15001 & PNNL-AGG-415 \\
\hline \multirow{3}{*}{$\begin{array}{l}\text { HEIS No. } \\
14133-76-7\end{array}$} & B1T1C4 & \multicolumn{3}{|c|}{ Lab ID: $\quad$ 0802018-03 } & & & \\
\hline & Technetium-99 & $<2.31 \mathrm{E}-5$ & ug/g dry & $2.31 \mathrm{E}-5$ & $4 / 15 / 08$ & 8D15001 & PNNL-AGG-415 \\
\hline & Uranium 238 & $1.55 \mathrm{E}-3$ & ug/g dry & $5.65 \mathrm{E}-4$ & $4 / 15 / 08$ & 8D15001 & PNNL-AGG-415 \\
\hline \multirow{3}{*}{$\begin{array}{l}\text { HEIS No. } \\
14133-76-7\end{array}$} & В1T1H0 & \multicolumn{3}{|c|}{ Lab ID: $\quad$ 0802022-01 } & & & \\
\hline & Technetium-99 & $1.40 \mathrm{E}-3$ & ug/g dry & $2.30 \mathrm{E}-5$ & $4 / 15 / 08$ & 8D15001 & PNNL-AGG-415 \\
\hline & Uranium 238 & 1.97E1 & ug/g dry & 1.13E0 & $4 / 15 / 08$ & 8D15001 & PNNL-AGG-415 \\
\hline \multirow{3}{*}{$\begin{array}{l}\text { HEIS No. } \\
14133-76-7\end{array}$} & B1T1H2 & \multicolumn{3}{|c|}{ Lab ID: $\quad$ 0802022-04 } & & & \\
\hline & Technetium-99 & $3.71 \mathrm{E}-4$ & ug/g dry & $2.28 \mathrm{E}-5$ & $4 / 15 / 08$ & 8D15001 & PNNL-AGG-415 \\
\hline & Uranium 238 & 3.29E1 & ug/g dry & $1.12 \mathrm{E} 0$ & $4 / 15 / 08$ & 8D15001 & PNNL-AGG-415 \\
\hline \multirow{3}{*}{$\begin{array}{l}\text { HEIS No. } \\
14133-76-7\end{array}$} & B1T1H4 & \multicolumn{3}{|c|}{ Lab ID: $\quad$ 0802022-07 } & & & \\
\hline & Technetium-99 & $2.19 \mathrm{E}-3$ & ug/g dry & $2.30 \mathrm{E}-5$ & $4 / 15 / 08$ & 8D15001 & PNNL-AGG-415 \\
\hline & Uranium 238 & $9.46 \mathrm{E} 1$ & ug/g dry & $1.13 \mathrm{E} 0$ & $4 / 15 / 08$ & 8D15001 & PNNL-AGG-415 \\
\hline \multirow{3}{*}{$\begin{array}{l}\text { HEIS No. } \\
14133-76-7\end{array}$} & B1T1H6 & \multicolumn{3}{|c|}{ Lab ID: $\quad$ 0802022-11 } & & & \\
\hline & Technetium-99 & $3.55 E-3$ & ug/g dry & $2.30 \mathrm{E}-5$ & $4 / 15 / 08$ & 8D15001 & PNNL-AGG-415 \\
\hline & Uranium 238 & 3.95E1 & ug/g dry & 1.13E0 & $4 / 15 / 08$ & 8D15001 & PNNL-AGG-415 \\
\hline \multirow{3}{*}{$\begin{array}{l}\text { HEIS No. } \\
14133-76-7\end{array}$} & B1T1D8 & \multicolumn{3}{|c|}{ Lab ID: $\quad$ 0802024-15 } & & & \\
\hline & Technetium-99 & $<2.31 \mathrm{E}-5$ & ug/g dry & $2.31 E-5$ & $4 / 15 / 08$ & 8D15001 & PNNL-AGG-415 \\
\hline & Uranium 238 & $1.38 \mathrm{E}-3$ & ug/g dry & $5.65 \mathrm{E}-4$ & $4 / 15 / 08$ & 8D15001 & PNNL-AGG-415 \\
\hline \multirow{3}{*}{$\begin{array}{l}\text { HEIS No. } \\
14133-76-7\end{array}$} & B1T1F0 & \multicolumn{3}{|c|}{ Lab ID: $\quad$ 0802024-18 } & & & \\
\hline & Technetium-99 & $<2.31 \mathrm{E}-5$ & ug/g dry & $2.31 \mathrm{E}-5$ & $4 / 15 / 08$ & 8D15001 & PNNL-AGG-415 \\
\hline & Uranium 238 & $1.16 \mathrm{E}-2$ & ug/g dry & $5.65 \mathrm{E}-4$ & $4 / 15 / 08$ & 8D15001 & PNNL-AGG-415 \\
\hline HEIS No. & B1T1F6 & & b ID: & 2024-27 & & & \\
\hline 14133-76-7 & Technetium-99 & $<2.31 \mathrm{E}-5$ & ug/g dry & $2.31 \mathrm{E}-5$ & $4 / 15 / 08$ & 8D15001 & PNNL-AGG-415 \\
\hline & Uranium 238 & $1.75 \mathrm{E}-1$ & ug/g dry & $5.65 \mathrm{E}-4$ & $4 / 15 / 08$ & 8D15001 & PNNL-AGG-415 \\
\hline HEIS No. & B1T1M0 & & b ID: & 2024-56 & & & \\
\hline $14133-76-7$ & Technetium-99 & $<2.33 \mathrm{E}-5$ & ug/g dry & $2.33 \mathrm{E}-5$ & $4 / 15 / 08$ & 8D15001 & PNNL-AGG-415 \\
\hline & Uranium 238 & $1.90 \mathrm{E}-2$ & ug/g dry & $5.72 \mathrm{E}-4$ & $4 / 15 / 08$ & 8D15001 & PNNL-AGG-415 \\
\hline
\end{tabular}


RCRA Metals By PNNL-AGG-415/Water Extract

\begin{tabular}{|c|c|c|c|c|c|c|c|}
\hline CAS \# & Analyte & Results & Units & EQL & Analyzed & Batch & Method \\
\hline HEIS No. & B1T170 & \multicolumn{3}{|c|}{ Lab ID: $\quad$ 0802013-12 } & & & \\
\hline $14378-38-2$ & Silver & $<1.07 \mathrm{E}-3$ & ug/g dry & $1.07 \mathrm{E}-3$ & $4 / 24 / 08$ & 8D07004 & PNNL-AGG-415 \\
\hline $14336-64-2$ & Cadmium & $<2.95 \mathrm{E}-4$ & ug/g dry & $2.95 \mathrm{E}-4$ & $4 / 24 / 08$ & 8D07004 & PNNL-AGG-415 \\
\hline $14265-72-6$ & Antimony & $<5.40 \mathrm{E}-4$ & ug/g dry & $5.40 \mathrm{E}-4$ & $4 / 24 / 08$ & 8D07004 & PNNL-AGG-415 \\
\hline HEIS No. & B1T172 & \multicolumn{3}{|c|}{ Lab ID: $\quad$ 0802013-14 } & & & \\
\hline $14378-38-2$ & Silver & $<1.06 \mathrm{E}-3$ & ug/g dry & $1.06 \mathrm{E}-3$ & $4 / 24 / 08$ & 8D07004 & PNNL-AGG-415 \\
\hline 14336-64-2 & Cadmium & $<2.92 \mathrm{E}-4$ & ug/g dry & $2.92 \mathrm{E}-4$ & $4 / 24 / 08$ & 8D07004 & PNNL-AGG-415 \\
\hline $14265-72-6$ & Antimony & $<5.34 \mathrm{E}-4$ & ug/g dry & $5.34 \mathrm{E}-4$ & $4 / 24 / 08$ & 8D07004 & PNNL-AGG-415 \\
\hline HEIS No. & B1T152 & \multicolumn{3}{|c|}{ Lab ID: $\quad$ 0802014-10 } & & & \\
\hline $14378-38-2$ & Silver & $<1.06 \mathrm{E}-3$ & ug/g dry & $1.06 \mathrm{E}-3$ & $4 / 24 / 08$ & 8D07004 & PNNL-AGG-415 \\
\hline $14336-64-2$ & Cadmium & $<2.92 \mathrm{E}-4$ & ug/g dry & $2.92 \mathrm{E}-4$ & $4 / 24 / 08$ & 8D07004 & PNNL-AGG-415 \\
\hline $14265-72-6$ & Antimony & $<5.35 \mathrm{E}-4$ & ug/g dry & $5.35 \mathrm{E}-4$ & $4 / 24 / 08$ & 8D07004 & PNNL-AGG-415 \\
\hline HEIS No. & B1T142 & \multicolumn{3}{|c|}{ Lab ID: $\quad$ 0802015-15 } & & & \\
\hline $14378-38-2$ & Silver & $<1.08 \mathrm{E}-3$ & ug/g dry & $1.08 \mathrm{E}-3$ & $4 / 24 / 08$ & 8D07004 & PNNL-AGG-415 \\
\hline $14336-64-2$ & Cadmium & $<2.97 \mathrm{E}-4$ & ug/g dry & $2.97 \mathrm{E}-4$ & $4 / 24 / 08$ & 8D07004 & PNNL-AGG-415 \\
\hline $14265-72-6$ & Antimony & $<5.43 \mathrm{E}-4$ & ug/g dry & $5.43 \mathrm{E}-4$ & $4 / 24 / 08$ & 8D07004 & PNNL-AGG-415 \\
\hline HEIS No. & B1T198 & \multicolumn{3}{|c|}{ Lab ID: $\quad$ 0802016-26 } & & & \\
\hline $14378-38-2$ & Silver & $<1.07 \mathrm{E}-3$ & ug/g dry & $1.07 \mathrm{E}-3$ & $4 / 24 / 08$ & 8D07004 & PNNL-AGG-415 \\
\hline 14336-64-2 & Cadmium & $<2.95 \mathrm{E}-4$ & ug/g dry & $2.95 \mathrm{E}-4$ & $4 / 24 / 08$ & 8D07004 & PNNL-AGG-415 \\
\hline $14265-72-6$ & Antimony & $<5.39 \mathrm{E}-4$ & ug/g dry & 5.39E-4 & $4 / 24 / 08$ & 8D07004 & PNNL-AGG-415 \\
\hline HEIS No. & B1T1C4 & \multicolumn{3}{|c|}{ Lab ID: $\quad$ 0802018-03 } & & & \\
\hline $14378-38-2$ & Silver & $<1.07 \mathrm{E}-3$ & ug/g dry & $1.07 \mathrm{E}-3$ & $4 / 24 / 08$ & 8D07004 & PNNL-AGG-415 \\
\hline 14336-64-2 & Cadmium & $<2.96 \mathrm{E}-4$ & ug/g dry & $2.96 \mathrm{E}-4$ & $4 / 24 / 08$ & 8D07004 & PNNL-AGG-415 \\
\hline $14265-72-6$ & Antimony & $<5.41 \mathrm{E}-4$ & ug/g dry & $5.41 \mathrm{E}-4$ & $4 / 24 / 08$ & 8D07004 & PNNL-AGG-415 \\
\hline HEIS No. & B1T1H0 & \multicolumn{3}{|c|}{ Lab ID: $\quad 0802022-01$} & & & \\
\hline $14378-38-2$ & Silver & $<1.07 \mathrm{E}-3$ & ug/g dry & $1.07 \mathrm{E}-3$ & $4 / 24 / 08$ & 8D07004 & PNNL-AGG-415 \\
\hline 14336-64-2 & Cadmium & $<2.95 \mathrm{E}-4$ & ug/g dry & $2.95 \mathrm{E}-4$ & $4 / 24 / 08$ & 8D07004 & PNNL-AGG-415 \\
\hline $14265-72-6$ & Antimony & $<5.39 \mathrm{E}-4$ & ug/g dry & $5.39 \mathrm{E}-4$ & $4 / 24 / 08$ & 8D07004 & PNNL-AGG-415 \\
\hline HEIS No. & B1T1H2 & \multicolumn{3}{|c|}{ Lab ID: $\quad$ 0802022-04 } & & & \\
\hline $14378-38-2$ & Silver & $<1.06 \mathrm{E}-3$ & ug/g dry & $1.06 \mathrm{E}-3$ & $4 / 24 / 08$ & 8D07004 & PNNL-AGG-415 \\
\hline 14336-64-2 & Cadmium & $<2.93 \mathrm{E}-4$ & ug/g dry & 2.93E-4 & $4 / 24 / 08$ & 8D07004 & PNNL-AGG-415 \\
\hline $14265-72-6$ & Antimony & $7.65 \mathrm{E}-4$ & ug/g dry & $5.36 \mathrm{E}-4$ & $4 / 24 / 08$ & 8D07004 & PNNL-AGG-415 \\
\hline HEIS No. & B1T1H4 & \multicolumn{3}{|c|}{ Lab ID: $\quad$ 0802022-07 } & & & \\
\hline $14378-38-2$ & Silver & $<1.07 \mathrm{E}-3$ & ug/g dry & $1.07 \mathrm{E}-3$ & $4 / 24 / 08$ & 8D07004 & PNNL-AGG-415 \\
\hline 14336-64-2 & Cadmium & 4.37E-4 & ug/g dry & $2.95 \mathrm{E}-4$ & $4 / 24 / 08$ & 8D07004 & PNNL-AGG-415 \\
\hline $14265-72-6$ & Antimony & $5.59 \mathrm{E}-4$ & ug/g dry & $5.40 \mathrm{E}-4$ & $4 / 24 / 08$ & 8D07004 & PNNL-AGG-415 \\
\hline HEIS No. & B1T1H6 & \multicolumn{3}{|c|}{ Lab ID: $\quad 0802022-11$} & & & \\
\hline $14378-38-2$ & Silver & $<1.07 \mathrm{E}-3$ & ug/g dry & $1.07 \mathrm{E}-3$ & $4 / 24 / 08$ & 8D07004 & PNNL-AGG-415 \\
\hline 14336-64-2 & Cadmium & $<2.95 \mathrm{E}-4$ & ug/g dry & $2.95 \mathrm{E}-4$ & $4 / 24 / 08$ & 8D07004 & PNNL-AGG-415 \\
\hline $14265-72-6$ & Antimony & $<5.40 \mathrm{E}-4$ & ug/g dry & $5.40 \mathrm{E}-4$ & $4 / 24 / 08$ & 8D07004 & PNNL-AGG-415 \\
\hline HEIS No. & B1T1D8 & \multicolumn{3}{|c|}{ Lab ID: $\quad$ 0802024-15 } & & & \\
\hline $14378-38-2$ & Silver & $<1.07 \mathrm{E}-3$ & ug/g dry & $1.07 \mathrm{E}-3$ & $4 / 24 / 08$ & 8D07004 & PNNL-AGG-415 \\
\hline 14336-64-2 & Cadmium & $<2.96 \mathrm{E}-4$ & ug/g dry & $2.96 \mathrm{E}-4$ & $4 / 24 / 08$ & 8D07004 & PNNL-AGG-415 \\
\hline $14265-72-6$ & Antimony & $<5.42 \mathrm{E}-4$ & ug/g dry & $5.42 \mathrm{E}-4$ & $4 / 24 / 08$ & 8D07004 & PNNL-AGG-415 \\
\hline HEIS No. & B1T1F0 & \multicolumn{3}{|c|}{ Lab ID: $\quad$ 0802024-18 } & & & \\
\hline $14378-38-2$ & Silver & $<1.07 \mathrm{E}-3$ & ug/g dry & $1.07 \mathrm{E}-3$ & $4 / 24 / 08$ & 8D07004 & PNNL-AGG-415 \\
\hline 14336-64-2 & Cadmium & $<2.96 \mathrm{E}-4$ & ug/g dry & $2.96 \mathrm{E}-4$ & $4 / 24 / 08$ & 8D07004 & PNNL-AGG-415 \\
\hline $14265-72-6$ & Antimony & $<5.41 \mathrm{E}-4$ & ug/g dry & $5.41 \mathrm{E}-4$ & $4 / 24 / 08$ & 8D07004 & PNNL-AGG-415 \\
\hline
\end{tabular}


RCRA Metals By PNNL-AGG-415/Water Extract

\begin{tabular}{|c|c|c|c|c|c|c|c|}
\hline CAS \# & Analyte & Results & Units & EQL & Analyzed & Batch & Method \\
\hline HEIS No. & B1T1F6 & \multicolumn{3}{|c|}{ Lab ID: $\quad$ 0802024-27 } & & & \\
\hline $14378-38-2$ & Silver & $<1.07 \mathrm{E}-3$ & ug/g dry & $1.07 \mathrm{E}-3$ & $4 / 24 / 08$ & 8D07004 & PNNL-AGG-415 \\
\hline $14336-64-2$ & Cadmium & $<2.96 \mathrm{E}-4$ & ug/g dry & $2.96 \mathrm{E}-4$ & $4 / 24 / 08$ & 8D07004 & PNNL-AGG-415 \\
\hline $14265-72-6$ & Antimony & $<5.42 \mathrm{E}-4$ & ug/g dry & $5.42 \mathrm{E}-4$ & $4 / 24 / 08$ & 8D07004 & PNNL-AGG-415 \\
\hline HEIS No. & B1T1M0 & \multicolumn{3}{|c|}{ Lab ID: $\quad$ 0802024-56 } & & & \\
\hline $14378-38-2$ & Silver & $<1.09 \mathrm{E}-3$ & ug/g dry & $1.09 \mathrm{E}-3$ & $4 / 24 / 08$ & 8D07004 & PNNL-AGG-415 \\
\hline $14336-64-2$ & Cadmium & $<2.99 \mathrm{E}-4$ & ug/g dry & $2.99 \mathrm{E}-4$ & $4 / 24 / 08$ & 8D07004 & PNNL-AGG-415 \\
\hline $14265-72-6$ & Antimony & $8.48 \mathrm{E}-4$ & ug/g dry & $5.48 \mathrm{E}-4$ & $4 / 24 / 08$ & 8D07004 & PNNL-AGG-415 \\
\hline
\end{tabular}


RCRA Metals By PNNL-AGG-415/Acid Extract

\begin{tabular}{|c|c|c|c|c|c|c|c|}
\hline CAS \# & Analyte & Results & Units & EQL & Analyzed & Batch & Method \\
\hline HEIS No. & B1T170 & \multicolumn{3}{|c|}{ Lab ID: $\quad$ 0802013-12 } & & & \\
\hline $14336-64-2$ & Cadmium & $7.84 \mathrm{E}-2$ & ug/g dry & 5.89E-2 & 6/03/08 & 8E15001 & PNNL-AGG-415 \\
\hline $14265-72-6$ & Antimony & $<9.53 \mathrm{E}-2$ & ug/g dry & $9.53 \mathrm{E}-2$ & 6/03/08 & 8E15001 & PNNL-AGG-415 \\
\hline HEIS No. & B1T172 & \multicolumn{3}{|c|}{ Lab ID: $\quad$ 0802013-14 } & & & \\
\hline $14336-64-2$ & Cadmium & $<5.29 \mathrm{E}-2$ & ug/g dry & $5.29 \mathrm{E}-2$ & 6/03/08 & 8E15001 & PNNL-AGG-415 \\
\hline $14265-72-6$ & Antimony & $<8.55 \mathrm{E}-2$ & ug/g dry & 8.55E-2 & $6 / 03 / 08$ & 8E15001 & PNNL-AGG-415 \\
\hline HEIS No. & B1T152 & \multicolumn{3}{|c|}{ Lab ID: $\quad$ 0802014-10 } & & & \\
\hline $14336-64-2$ & Cadmium & $5.41 \mathrm{E}-2$ & ug/g dry & $5.01 \mathrm{E}-2$ & $6 / 03 / 08$ & 8E15001 & PNNL-AGG-415 \\
\hline $14265-72-6$ & Antimony & $<8.11 \mathrm{E}-2$ & ug/g dry & 8.11E-2 & $6 / 03 / 08$ & 8E15001 & PNNL-AGG-415 \\
\hline HEIS No. & B1T142 & \multicolumn{3}{|c|}{ Lab ID: $\quad$ 0802015-15 } & & & \\
\hline $14336-64-2$ & Cadmium & $6.25 \mathrm{E}-2$ & ug/g dry & 4.93E-2 & 6/03/08 & 8E15001 & PNNL-AGG-415 \\
\hline $14265-72-6$ & Antimony & $<7.97 \mathrm{E}-2$ & ug/g dry & 7.97E-2 & 6/03/08 & 8E15001 & PNNL-AGG-415 \\
\hline HEIS No. & B1T198 & \multicolumn{3}{|c|}{ Lab ID: $\quad$ 0802016-26 } & & & \\
\hline $14336-64-2$ & Cadmium & $<5.23 \mathrm{E}-2$ & ug/g dry & $5.23 E-2$ & 6/03/08 & 8E15001 & PNNL-AGG-415 \\
\hline $14265-72-6$ & Antimony & $<8.46 \mathrm{E}-2$ & ug/g dry & $8.46 \mathrm{E}-2$ & 6/03/08 & 8E15001 & PNNL-AGG-415 \\
\hline HEIS No. & B1T1C4 & \multicolumn{3}{|c|}{ Lab ID: $\quad$ 0802018-03 } & & & \\
\hline 14336-64-2 & Cadmium & $6.13 \mathrm{E}-2$ & ug/g dry & 5.03E-2 & $6 / 03 / 08$ & 8E15001 & PNNL-AGG-415 \\
\hline $14265-72-6$ & Antimony & $<8.13 \mathrm{E}-2$ & ug/g dry & $8.13 E-2$ & $6 / 03 / 08$ & 8E15001 & PNNL-AGG-415 \\
\hline HEIS No. & B1T1H0 & \multicolumn{3}{|c|}{ Lab ID: $\quad$ 0802022-01 } & & & \\
\hline $14336-64-2$ & Cadmium & $8.28 \mathrm{E}-2$ & ug/g dry & $5.55 \mathrm{E}-2$ & 6/03/08 & 8E15001 & PNNL-AGG-415 \\
\hline $14265-72-6$ & Antimony & $<8.98 \mathrm{E}-2$ & ug/g dry & $8.98 \mathrm{E}-2$ & 6/03/08 & 8E15001 & PNNL-AGG-415 \\
\hline HEIS No. & B1T1H2 & \multicolumn{3}{|c|}{ Lab ID: $\quad$ 0802022-04 } & & & \\
\hline $14336-64-2$ & Cadmium & $1.03 \mathrm{E}-1$ & ug/g dry & $7.50 \mathrm{E}-2$ & 6/03/08 & 8E15001 & PNNL-AGG-415 \\
\hline $14265-72-6$ & Antimony & $<1.21 \mathrm{E}-1$ & ug/g dry & $1.21 \mathrm{E}-1$ & 6/03/08 & 8E15001 & PNNL-AGG-415 \\
\hline HEIS No. & B1T1H4 & \multicolumn{3}{|c|}{ Lab ID: $\quad$ 0802022-07 } & & & \\
\hline $14336-64-2$ & Cadmium & $1.14 \mathrm{E}-1$ & ug/g dry & 6.35E-2 & 6/03/08 & 8E15001 & PNNL-AGG-415 \\
\hline $14265-72-6$ & Antimony & $<1.03 \mathrm{E}-1$ & ug/g dry & $1.03 \mathrm{E}-1$ & 6/03/08 & 8E15001 & PNNL-AGG-415 \\
\hline HEIS No. & В1T1H6 & \multicolumn{3}{|c|}{ Lab ID: $\quad$ 0802022-11 } & & & \\
\hline 14336-64-2 & Cadmium & $1.03 \mathrm{E}-1$ & ug/g dry & $6.23 E-2$ & 6/03/08 & 8E15001 & PNNL-AGG-415 \\
\hline $14265-72-6$ & Antimony & $<1.01 \mathrm{E}-1$ & ug/g dry & $1.01 \mathrm{E}-1$ & 6/03/08 & 8E15001 & PNNL-AGG-415 \\
\hline HEIS No. & B1T1D8 & \multicolumn{3}{|c|}{ Lab ID: $\quad$ 0802024-15 } & & & \\
\hline 14336-64-2 & Cadmium & $<4.83 \mathrm{E}-2$ & ug/g dry & 4.83E-2 & 6/03/08 & 8E15001 & PNNL-AGG-415 \\
\hline $14265-72-6$ & Antimony & $<7.81 \mathrm{E}-2$ & ug/g dry & $7.81 \mathrm{E}-2$ & 6/03/08 & 8E15001 & PNNL-AGG-415 \\
\hline HEIS No. & B1T1F0 & \multicolumn{3}{|c|}{ Lab ID: $\quad$ 0802024-18 } & & & \\
\hline $14336-64-2$ & Cadmium & $1.15 \mathrm{E}-1$ & ug/g dry & $6.22 \mathrm{E}-2$ & 6/03/08 & 8E15001 & PNNL-AGG-415 \\
\hline $14265-72-6$ & Antimony & $<1.01 \mathrm{E}-1$ & ug/g dry & $1.01 \mathrm{E}-1$ & 6/03/08 & 8E15001 & PNNL-AGG-415 \\
\hline HEIS No. & B1T1F6 & \multicolumn{3}{|c|}{ Lab ID: $\quad$ 0802024-27 } & & & \\
\hline 14336-64-2 & Cadmium & $1.04 \mathrm{E}-1$ & ug/g dry & $6.00 \mathrm{E}-2$ & 6/03/08 & 8E15001 & PNNL-AGG-415 \\
\hline $14265-72-6$ & Antimony & $<9.71 \mathrm{E}-2$ & ug/g dry & $9.71 \mathrm{E}-2$ & 6/03/08 & 8E15001 & PNNL-AGG-415 \\
\hline HEIS No. & B1T1M0 & \multicolumn{3}{|c|}{ Lab ID: $\quad$ 0802024-56 } & & & \\
\hline 14336-64-2 & Cadmium & $5.34 \mathrm{E}-2$ & ug/g dry & $5.05 \mathrm{E}-2$ & 6/03/08 & 8E15001 & PNNL-AGG-415 \\
\hline $14265-72-6$ & Antimony & $<8.17 \mathrm{E}-2$ & ug/g dry & 8.17E-2 & 6/03/08 & 8E15001 & PNNL-AGG-415 \\
\hline
\end{tabular}




\section{GEA/Soil}

\begin{tabular}{|c|c|c|c|c|c|c|c|c|}
\hline CAS \# & Analyte & Results & Units & MDA & UNC & Analyzed & Batch & Method \\
\hline HEIS No. & B1T170 & \multicolumn{3}{|c|}{ Lab ID: $\quad$ 0802013-12 } & & & & \\
\hline 10198-40-0 & Cobalt-60 & $<2.71 \mathrm{E}-1$ & pCi/g dry & $2.71 \mathrm{E}-1$ & & $4 / 01 / 08$ & $8 C 25009$ & AGG-RRL-001 \\
\hline $10045-97-3$ & Cesium-137 & $<3.25 \mathrm{E}-1$ & pCi/g dry & 3.25E-1 & & $4 / 01 / 08$ & 8C25009 & AGG-RRL-001 \\
\hline 14683-23-9 & Europium-152 & $<1.20 \mathrm{E} 0$ & pCi/g dry & $1.20 \mathrm{E} 0$ & & $4 / 01 / 08$ & $8 C 25009$ & AGG-RRL-001 \\
\hline 15585-10-1 & Europium-154 & $<6.64 \mathrm{E}-1$ & pCi/g dry & 6.64E-1 & & $4 / 01 / 08$ & 8C25009 & AGG-RRL-001 \\
\hline 14391-16-3 & Europium-155 & $<1.14 \mathrm{E} 0$ & pCi/g dry & $1.14 \mathrm{E} 0$ & & $4 / 01 / 08$ & 8C25009 & AGG-RRL-001 \\
\hline HEIS No. & B1T152 & \multicolumn{3}{|c|}{ Lab ID: $\quad$ 0802014-10 } & & & & \\
\hline 10198-40-0 & Cobalt-60 & $<2.36 \mathrm{E}-1$ & pCi/g dry & 2.36E-1 & & $4 / 01 / 08$ & $8 C 25009$ & AGG-RRL-001 \\
\hline $10045-97-3$ & Cesium-137 & $<2.92 \mathrm{E}-1$ & pCi/g dry & $2.92 \mathrm{E}-1$ & & $4 / 01 / 08$ & 8C25009 & AGG-RRL-001 \\
\hline 14683-23-9 & Europium-152 & $<1.01 \mathrm{E} 0$ & pCi/g dry & $1.01 \mathrm{E} 0$ & & $4 / 01 / 08$ & $8 C 25009$ & AGG-RRL-001 \\
\hline $15585-10-1$ & Europium-154 & $<6.04 \mathrm{E}-1$ & pCi/g dry & $6.04 \mathrm{E}-1$ & & $4 / 01 / 08$ & $8 C 25009$ & AGG-RRL-001 \\
\hline 14391-16-3 & Europium-155 & $<9.71 \mathrm{E}-1$ & pCi/g dry & $9.71 \mathrm{E}-1$ & & $4 / 01 / 08$ & $8 C 25009$ & AGG-RRL-001 \\
\hline HEIS No. & $\begin{array}{l}\text { B1T198 } \\
\end{array}$ & \multicolumn{3}{|c|}{ Lab ID: $\quad$ 0802016-26 } & & & & \\
\hline 10198-40-0 & Cobalt-60 & $<2.39 \mathrm{E}-1$ & pCi/g dry & 2.39E-1 & & $4 / 01 / 08$ & 8C25009 & AGG-RRL-001 \\
\hline $10045-97-3$ & Cesium-137 & $<2.83 \mathrm{E}-1$ & pCi/g dry & $2.83 \mathrm{E}-1$ & & $4 / 01 / 08$ & 8C25009 & AGG-RRL-001 \\
\hline 14683-23-9 & Europium-152 & $<1.07 \mathrm{E} 0$ & pCi/g dry & 1.07E0 & & $4 / 01 / 08$ & 8C25009 & AGG-RRL-001 \\
\hline 15585-10-1 & Europium-154 & $<5.88 \mathrm{E}-1$ & pCi/g dry & $5.88 \mathrm{E}-1$ & & $4 / 01 / 08$ & $8 C 25009$ & AGG-RRL-001 \\
\hline 14391-16-3 & Europium-155 & $<9.85 \mathrm{E}-1$ & pCi/g dry & $9.85 \mathrm{E}-1$ & & $4 / 01 / 08$ & 8C25009 & AGG-RRL-001 \\
\hline HEIS No. & B1T1C4 & \multicolumn{3}{|c|}{ Lab ID: $\quad$ 0802018-03 } & & & & \\
\hline 10198-40-0 & Cobalt-60 & $<1.65 \mathrm{E}-1$ & pCi/g dry & 1.65E-1 & & $4 / 02 / 08$ & 8C25009 & AGG-RRL-001 \\
\hline $10045-97-3$ & Cesium-137 & $<2.02 \mathrm{E}-1$ & pCi/g dry & $2.02 \mathrm{E}-1$ & & $4 / 02 / 08$ & $8 C 25009$ & AGG-RRL-001 \\
\hline 14683-23-9 & Europium-152 & $<7.32 \mathrm{E}-1$ & pCi/g dry & 7.32E-1 & & $4 / 02 / 08$ & 8C25009 & AGG-RRL-001 \\
\hline 15585-10-1 & Europium-154 & $<4.38 \mathrm{E}-1$ & pCi/g dry & $4.38 \mathrm{E}-1$ & & $4 / 02 / 08$ & $8 C 25009$ & AGG-RRL-001 \\
\hline 14391-16-3 & Europium-155 & $<7.03 \mathrm{E}-1$ & pCi/g dry & 7.03E-1 & & $4 / 02 / 08$ & 8C25009 & AGG-RRL-001 \\
\hline HEIS No. & B1T1H2 & \multicolumn{3}{|c|}{ Lab ID: $\quad$ 0802022-04 } & & & & \\
\hline $10198-40-0$ & Cobalt-60 & $<2.02 \mathrm{E}-1$ & pCi/g dry & $2.02 \mathrm{E}-1$ & & $4 / 01 / 08$ & $8 C 25009$ & AGG-RRL-001 \\
\hline $10045-97-3$ & Cesium-137 & $<2.35 \mathrm{E}-1$ & pCi/g dry & 2.35E-1 & & $4 / 01 / 08$ & 8C25009 & AGG-RRL-001 \\
\hline $14683-23-9$ & Europium-152 & $<8.47 \mathrm{E}-1$ & pCi/g dry & 8.47E-1 & & $4 / 01 / 08$ & 8C25009 & AGG-RRL-001 \\
\hline $15585-10-1$ & Europium-154 & $<5.98 \mathrm{E}-1$ & pCi/g dry & $5.98 \mathrm{E}-1$ & & $4 / 01 / 08$ & 8C25009 & AGG-RRL-001 \\
\hline 14391-16-3 & Europium-155 & $<1.15 \mathrm{E} 0$ & pCi/g dry & $1.15 \mathrm{E} 0$ & & $4 / 01 / 08$ & 8C25009 & AGG-RRL-001 \\
\hline HEIS No. & B1T1H4 & \multicolumn{3}{|c|}{ Lab ID: $\quad$ 0802022-07 } & & & & \\
\hline 10198-40-0 & Cobalt-60 & $<3.08 \mathrm{E}-1$ & pCi/g dry & $3.08 \mathrm{E}-1$ & & $4 / 01 / 08$ & 8C25009 & AGG-RRL-001 \\
\hline $10045-97-3$ & Cesium-137 & $<4.03 \mathrm{E}-1$ & pCi/g dry & 4.03E-1 & & $4 / 01 / 08$ & 8C25009 & AGG-RRL-001 \\
\hline $14683-23-9$ & Europium-152 & $<1.49 \mathrm{E} 0$ & pCi/g dry & 1.49E0 & & $4 / 01 / 08$ & $8 C 25009$ & AGG-RRL-001 \\
\hline 15585-10-1 & Europium-154 & $<9.70 \mathrm{E}-1$ & pCi/g dry & $9.70 \mathrm{E}-1$ & & $4 / 01 / 08$ & $8 C 25009$ & AGG-RRL-001 \\
\hline 14391-16-3 & Europium-155 & $<3.01 \mathrm{E} 0$ & pCi/g dry & $3.01 \mathrm{E} 0$ & & $4 / 01 / 08$ & $8 C 25009$ & AGG-RRL-001 \\
\hline HEIS No. & B1T1H6 & \multicolumn{3}{|c|}{ Lab ID: $\quad$ 0802022-11 } & & & & \\
\hline $10198-40-0$ & Cobalt-60 & $<2.50 \mathrm{E}-1$ & pCi/g dry & $2.50 \mathrm{E}-1$ & & $4 / 01 / 08$ & 8C25009 & AGG-RRL-001 \\
\hline $10045-97-3$ & Cesium-137 & $<2.96 \mathrm{E}-1$ & pCi/g dry & 2.96E-1 & & $4 / 01 / 08$ & $8 C 25009$ & AGG-RRL-001 \\
\hline 14683-23-9 & Europium-152 & $<1.05 \mathrm{E} 0$ & pCi/g dry & $1.05 \mathrm{E} 0$ & & $4 / 01 / 08$ & 8C25009 & AGG-RRL-001 \\
\hline $15585-10-1$ & Europium-154 & $<7.14 \mathrm{E}-1$ & pCi/g dry & 7.14E-1 & & $4 / 01 / 08$ & 8C25009 & AGG-RRL-001 \\
\hline 14391-16-3 & Europium-155 & $<1.40 \mathrm{E} 0$ & pCi/g dry & $1.40 \mathrm{E} 0$ & & $4 / 01 / 08$ & 8C25009 & AGG-RRL-001 \\
\hline HEIS No. & B1T1F0 & \multicolumn{3}{|c|}{ Lab ID: $\quad$ 0802024-18 } & & & & \\
\hline 10198-40-0 & Cobalt-60 & $<2.92 \mathrm{E}-1$ & pCi/g dry & 2.92E-1 & & $4 / 02 / 08$ & 8C25009 & AGG-RRL-001 \\
\hline $10045-97-3$ & Cesium-137 & $<3.27 \mathrm{E}-1$ & pCi/g dry & $3.27 \mathrm{E}-1$ & & $4 / 02 / 08$ & 8C25009 & AGG-RRL-001 \\
\hline 14683-23-9 & Europium-152 & $<1.28 \mathrm{E} 0$ & pCi/g dry & $1.28 \mathrm{E} 0$ & & $4 / 02 / 08$ & 8C25009 & AGG-RRL-001 \\
\hline 15585-10-1 & Europium-154 & $<7.22 \mathrm{E}-1$ & pCi/g dry & $7.22 \mathrm{E}-1$ & & $4 / 02 / 08$ & 8C25009 & AGG-RRL-001 \\
\hline 14391-16-3 & Europium-155 & $<1.24 \mathrm{E} 0$ & pCi/g dry & $1.24 \mathrm{E} 0$ & & $4 / 02 / 08$ & 8C25009 & AGG-RRL-001 \\
\hline
\end{tabular}




\section{GEA/Soil}

\begin{tabular}{|c|c|c|c|c|c|c|c|c|}
\hline CAS \# & Analyte & Results & Units & MDA & UNC & Analyzed & Batch & Method \\
\hline HEIS No. & B1T1F6 & \multicolumn{4}{|c|}{ Lab ID: $\quad$ 0802024-27 } & & & \\
\hline $10198-40-0$ & Cobalt-60 & $<2.39 \mathrm{E}-1$ & pCi/g dry & 2.39E-1 & & $4 / 02 / 08$ & $8 C 25009$ & AGG-RRL-001 \\
\hline $10045-97-3$ & Cesium-137 & $<3.04 \mathrm{E}-1$ & pCi/g dry & 3.04E-1 & & $4 / 02 / 08$ & $8 C 25009$ & AGG-RRL-001 \\
\hline $14683-23-9$ & Europium-152 & $<1.11 \mathrm{E} 0$ & $\mathrm{pCi} / \mathrm{g}$ dry & $1.11 \mathrm{E} 0$ & & $4 / 02 / 08$ & 8C25009 & AGG-RRL-001 \\
\hline $15585-10-1$ & Europium-154 & $<6.30 \mathrm{E}-1$ & pCi/g dry & 6.30E-1 & & $4 / 02 / 08$ & $8 C 25009$ & AGG-RRL-001 \\
\hline $14391-16-3$ & Europium-155 & $<1.04 \mathrm{E} 0$ & pCi/g dry & $1.04 \mathrm{E} 0$ & & $4 / 02 / 08$ & $8 C 25009$ & AGG-RRL-001 \\
\hline HEIS No. & B1T1M0 & \multicolumn{4}{|c|}{ Lab ID: $\quad$ 0802024-56 } & & & \\
\hline $10198-40-0$ & Cobalt-60 & $<1.77 \mathrm{E}-1$ & pCi/g dry & 1.77E-1 & & $4 / 02 / 08$ & 8C25009 & AGG-RRL-001 \\
\hline $10045-97-3$ & Cesium-137 & $<2.16 \mathrm{E}-1$ & pCi/g dry & $2.16 \mathrm{E}-1$ & & $4 / 02 / 08$ & $8 C 25009$ & AGG-RRL-001 \\
\hline $14683-23-9$ & Europium-152 & $<7.79 \mathrm{E}-1$ & pCi/g dry & 7.79E-1 & & $4 / 02 / 08$ & 8C25009 & AGG-RRL-001 \\
\hline 15585-10-1 & Europium-154 & $<4.82 \mathrm{E}-1$ & pCi/g dry & 4.82E-1 & & $4 / 02 / 08$ & 8C25009 & AGG-RRL-001 \\
\hline $14391-16-3$ & Europium-155 & $<8.01 \mathrm{E}-1$ & pCi/g dry & 8.01E-1 & & $4 / 02 / 08$ & $8 C 25009$ & AGG-RRL-001 \\
\hline
\end{tabular}


Total Alpha Total Beta/Acid Extract

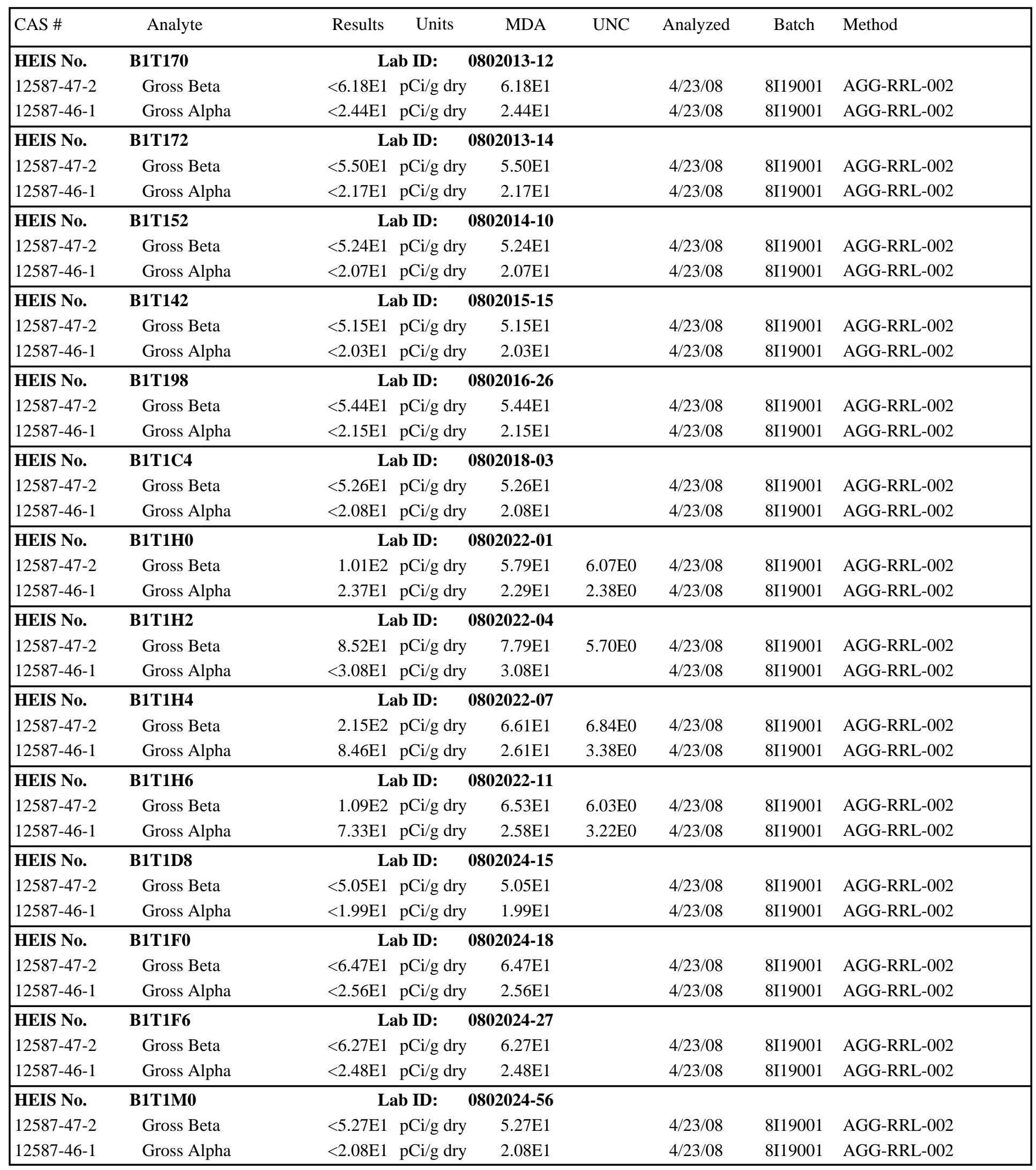


Wet Chemistry - Quality Control

Environmental Science Laboratory

\begin{tabular}{|lrrrrrrrrrrr}
\hline & & Reporting & & Spike & Source & & \%REC & & RPD & & \\
Analyte & Result & Limit & Units & Level & Result & \%REC & Limits & RPD & Limit & Notes \\
\hline
\end{tabular}

Batch 8C28004 - 1:1 Water Extract (pH_EC_Alk)

\begin{tabular}{|c|c|c|c|c|c|c|}
\hline \multirow{2}{*}{$\begin{array}{l}\text { Blank (8C28004-BLK1) } \\
\text { Specific Conductance (EC) }\end{array}$} & \multicolumn{6}{|c|}{ Prepared \& Analyzed: 03/28/08 } \\
\hline & $<1.00 \mathrm{E}-2$ & $1.00 \mathrm{E}-2$ & $\mathrm{mS} / \mathrm{cm}$ & & & \\
\hline Duplicate (8C28004-DUP1) & \multicolumn{3}{|c|}{ Source: 0802013-12 } & \multicolumn{3}{|l|}{ Prepared \& Analyzed: 03/28/08 } \\
\hline Specific Conductance (EC) & $<1.00 \mathrm{E}-2$ & $1.00 \mathrm{E}-2$ & $\mathrm{mS} / \mathrm{cm}$ & $3.15 \mathrm{E}-1$ & & 35 \\
\hline Duplicate (8C28004-DUP2) & \multicolumn{3}{|c|}{ Source: 0802024-18 } & \multicolumn{3}{|l|}{ Prepared \& Analyzed: 03/28/08 } \\
\hline Specific Conductance (EC) & $4.15 \mathrm{E}-1$ & $1.00 \mathrm{E}-2$ & $\mathrm{mS} / \mathrm{cm}$ & $4.60 \mathrm{E}-1$ & 10.3 & 35 \\
\hline $\mathrm{pH}$ & 8.19E0 & N/A & pH Units & 8.13E0 & 0.735 & 35 \\
\hline
\end{tabular}

Batch 8C31001 - 1:1 Water Extract (pH_EC_Alk)

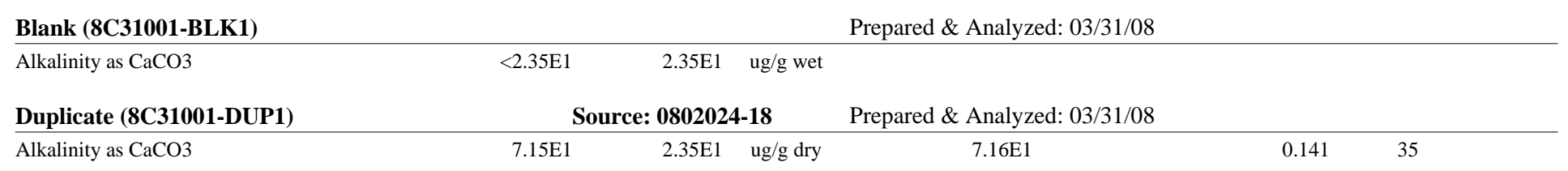


Anions by Ion Chromatography - Quality Control

Environmental Science Laboratory

\begin{tabular}{|c|c|c|c|c|c|c|c|c|c|c|}
\hline Analyte & Result & $\begin{array}{r}\text { orting } \\
\text { Limit }\end{array}$ & Units & $\begin{array}{l}\text { Spike } \\
\text { Level }\end{array}$ & $\begin{array}{l}\text { Source } \\
\text { Result }\end{array}$ & \%REC & $\begin{array}{l}\text { \%REC } \\
\text { Limits }\end{array}$ & RPD & $\begin{array}{l}\text { RPD } \\
\text { Limit }\end{array}$ & Notes \\
\hline
\end{tabular}

Batch 8C28005 - 1:1 Water Extract (IC)

\begin{tabular}{|c|c|c|c|c|c|c|c|c|c|}
\hline \multirow{2}{*}{$\begin{array}{l}\text { Blank (8C28005-BLK1) } \\
\text { Fluoride }\end{array}$} & \multicolumn{9}{|c|}{ Prepared: 03/28/08 Analyzed: 03/29/08 } \\
\hline & $<2.00 \mathrm{E}-1$ & $2.00 \mathrm{E}-1$ & ug/g wet & & & & & & \\
\hline Chloride & $<5.00 \mathrm{E}-1$ & $5.00 \mathrm{E}-1$ & " & & & & & & \\
\hline Nitrite & $<1.00 \mathrm{E} 0$ & $1.00 \mathrm{E} 0$ & $"$ & & & & & & \\
\hline Bromide & $<1.00 \mathrm{E} 0$ & $1.00 \mathrm{E} 0$ & $"$ & & & & & & \\
\hline Nitrate & $<1.00 \mathrm{E} 0$ & $1.00 \mathrm{E} 0$ & $"$ & & & & & & \\
\hline Sulfate & $<1.50 \mathrm{E} 0$ & $1.50 \mathrm{E} 0$ & $"$ & & & & & & \\
\hline Phosphate & $<1.50 \mathrm{E} 0$ & $1.50 \mathrm{E} 0$ & $"$ & & & & & & \\
\hline LCS (8C28005-BS1) & \multicolumn{9}{|c|}{ Prepared: 03/28/08 Analyzed: 03/29/08 } \\
\hline Fluoride & $2.07 \mathrm{E} 0$ & $2.00 \mathrm{E}-1$ & ug/g wet & $2.00 \mathrm{E} 0$ & & 103 & $80-120$ & & \\
\hline Chloride & $4.87 \mathrm{E} 0$ & $5.00 \mathrm{E}-1$ & $"$ & $5.00 \mathrm{E} 0$ & & 97.4 & $80-120$ & & \\
\hline Nitrite & $1.05 \mathrm{E} 1$ & $1.00 \mathrm{E} 0$ & $"$ & $1.00 \mathrm{E} 1$ & & 105 & $80-120$ & & \\
\hline Bromide & $9.68 \mathrm{E} 0$ & $1.00 \mathrm{E} 0$ & " & $1.00 \mathrm{E} 1$ & & 96.8 & $80-120$ & & \\
\hline Nitrate & $1.04 \mathrm{E} 1$ & $1.00 \mathrm{E} 0$ & $"$ & $1.00 \mathrm{E} 1$ & & 104 & $80-120$ & & \\
\hline Sulfate & $1.51 \mathrm{E} 1$ & $1.50 \mathrm{E} 0$ & " & $1.51 \mathrm{E} 1$ & & 99.9 & $80-120$ & & \\
\hline Phosphate & $1.46 \mathrm{E} 1$ & $1.50 \mathrm{E} 0$ & $"$ & $1.51 \mathrm{E} 1$ & & 97.0 & $80-120$ & & \\
\hline Duplicate (8C28005-DUP1) & \multicolumn{3}{|c|}{ Source: 0802024-18 } & \multicolumn{6}{|c|}{ Prepared: 03/28/08 Analyzed: 03/29/08 } \\
\hline Fluoride & 4.33E-1 & $2.00 \mathrm{E}-1$ & ug/g dry & & 4.11E-1 & & & 5.32 & 35 \\
\hline Chloride & $1.47 \mathrm{E} 1$ & $5.01 \mathrm{E}-1$ & $"$ & & 1.69E1 & & & 13.6 & 35 \\
\hline Nitrite & $<1.00 \mathrm{E} 0$ & $1.00 \mathrm{E} 0$ & " & & ND & & & & 35 \\
\hline Bromide & $<1.00 \mathrm{E} 0$ & $1.00 \mathrm{E} 0$ & $"$ & & ND & & & & 35 \\
\hline Nitrate & $1.52 \mathrm{E} 1$ & $1.00 \mathrm{E} 0$ & $"$ & & $1.86 \mathrm{E} 1$ & & & 19.8 & 35 \\
\hline Sulfate & $<1.50 \mathrm{E} 0$ & $1.50 \mathrm{E} 0$ & $"$ & & $9.91 \mathrm{E} 1$ & & & & 35 \\
\hline Phosphate & $<1.50 \mathrm{E} 0$ & $1.50 \mathrm{E} 0$ & $"$ & & ND & & & & 35 \\
\hline Post Spike (8C28005-PS1) & \multicolumn{3}{|c|}{ Source: 0802013-12 } & \multicolumn{4}{|c|}{ Prepared \& Analyzed: 03/28/08 } & & \\
\hline Fluoride & $4.26 \mathrm{E}-1$ & N/A & $\mathrm{ug} / \mathrm{mL}$ & $4.00 \mathrm{E}-1$ & $3.80 \mathrm{E}-2$ & 97 & $75-125$ & & \\
\hline Chloride & $2.28 \mathrm{E} 0$ & N/A & " & $1.00 \mathrm{E} 0$ & $1.32 \mathrm{E} 0$ & 95.9 & $75-125$ & & \\
\hline Nitrite & $1.95 \mathrm{E} 0$ & N/A & $"$ & $2.00 \mathrm{E} 0$ & ND & 97.7 & $75-125$ & & \\
\hline Bromide & $1.87 \mathrm{E} 0$ & N/A & $"$ & $2.00 \mathrm{E} 0$ & ND & 93.5 & $75-125$ & & \\
\hline Nitrate & 2.65E0 & N/A & $"$ & $2.00 \mathrm{E} 0$ & 8.81E-1 & 88.2 & $75-125$ & & \\
\hline Phosphate & 2.79E0 & N/A & $"$ & $3.00 \mathrm{E} 0$ & ND & 93.1 & $75-125$ & & \\
\hline
\end{tabular}


Total Metals by PNNL-AGG-ICP-AES/Water Extract - Quality Control

Environmental Science Laboratory

\begin{tabular}{|lrrrrrrrrrr}
\hline & & Reporting & & Spike & Source & & \%REC & & RPD & \\
Analyte & Result & Limit & Units & Level & Result & \%REC & Limits & RPD & Limit & Notes \\
\hline
\end{tabular}

Batch 8D28004 - 1:1 Water Extract (ICP/ICPMS)

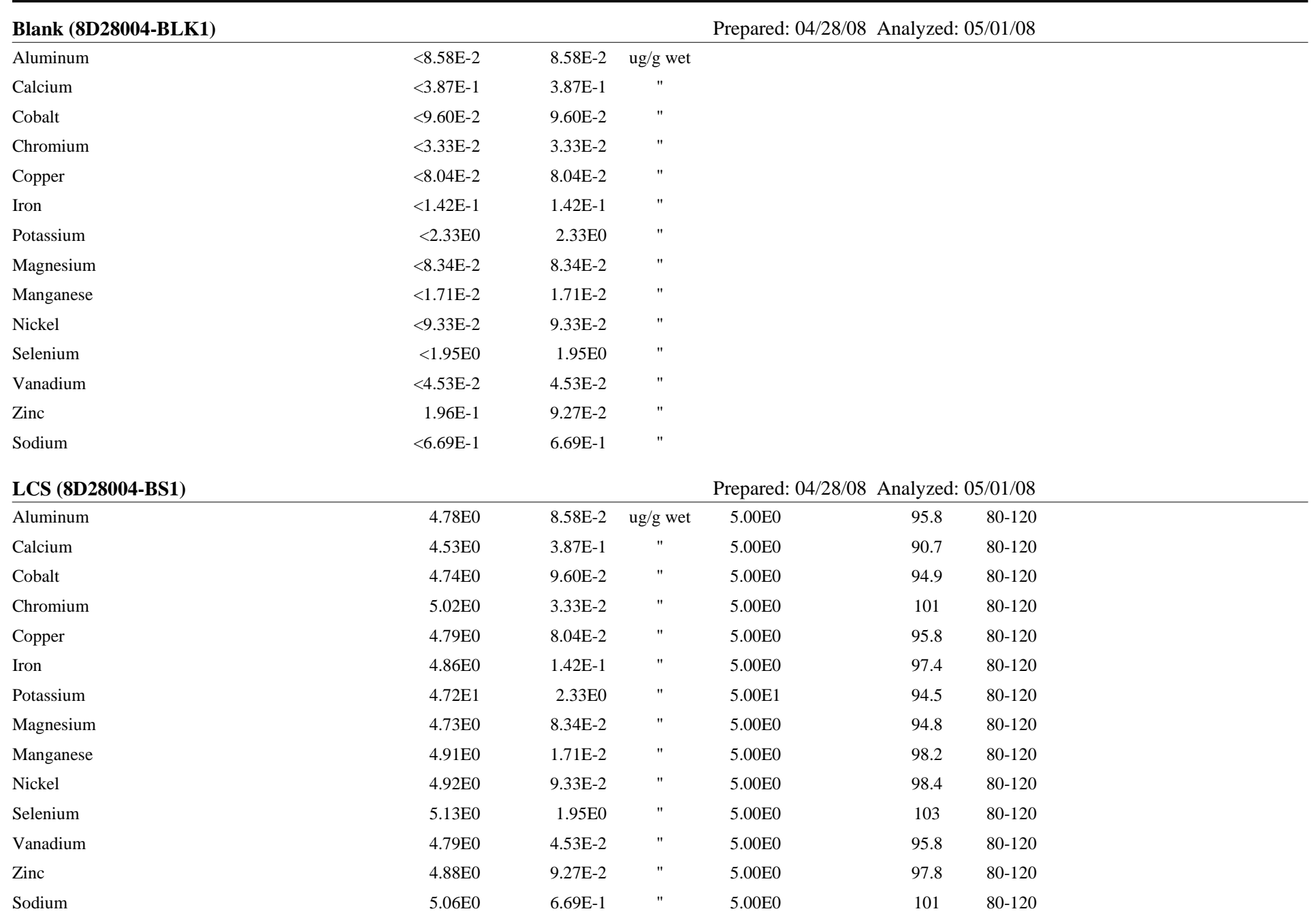


Total Metals by PNNL-AGG-ICP-AES/Water Extract - Quality Control

Environmental Science Laboratory

\begin{tabular}{|lrrrrrrrrrrr}
\hline & & Reporting & & Spike & Source & & & \%REC & & RPD & \\
Analyte & Result & Limit & Units & Level & Result & \%REC & Limits & RPD & Limit & Notes \\
\hline
\end{tabular}

Batch 8D28004 - 1:1 Water Extract (ICP/ICPMS)

\begin{tabular}{|c|c|c|c|c|c|c|c|c|c|}
\hline \multirow{2}{*}{$\begin{array}{l}\text { Duplicate (8D28004-DUP1) } \\
\text { Aluminum }\end{array}$} & \multicolumn{3}{|c|}{ Source: 0802024-18 } & \multicolumn{4}{|c|}{ Prepared: 04/28/08 Analyzed: 05/01/08 } & & \multirow[b]{2}{*}{35} \\
\hline & $<8.59 \mathrm{E}-2$ & $8.59 \mathrm{E}-2$ & ug/g dry & & ND & & & & \\
\hline Calcium & 3.31E1 & 3.87E-1 & $"$ & & 3.75E1 & & & 12.5 & 35 \\
\hline Cobalt & $<9.61 \mathrm{E}-2$ & $9.61 \mathrm{E}-2$ & $"$ & & ND & & & & 35 \\
\hline Chromium & $<3.33 \mathrm{E}-2$ & 3.33E-2 & $"$ & & ND & & & & 35 \\
\hline Copper & $<8.05 \mathrm{E}-2$ & $8.05 \mathrm{E}-2$ & $"$ & & ND & & & & 35 \\
\hline Iron & $<1.42 \mathrm{E}-1$ & $1.42 \mathrm{E}-1$ & $"$ & & ND & & & & 35 \\
\hline Potassium & $6.28 \mathrm{E} 0$ & 2.33E0 & $"$ & & $6.01 \mathrm{E} 0$ & & & 4.45 & 35 \\
\hline Magnesium & $9.26 \mathrm{E} 0$ & 8.35E-2 & $"$ & & $1.04 \mathrm{E} 1$ & & & 12.0 & 35 \\
\hline Manganese & $<1.71 \mathrm{E}-2$ & $1.71 \mathrm{E}-2$ & $"$ & & $\mathrm{ND}$ & & & & 35 \\
\hline Nickel & $<9.34 \mathrm{E}-2$ & $9.34 \mathrm{E}-2$ & $"$ & & ND & & & & 35 \\
\hline Selenium & $<1.95 \mathrm{E} 0$ & $1.95 \mathrm{E} 0$ & $"$ & & ND & & & & 35 \\
\hline Vanadium & $<4.53 \mathrm{E}-2$ & $4.53 \mathrm{E}-2$ & $"$ & & ND & & & & 35 \\
\hline Zinc & $<9.28 \mathrm{E}-2$ & $9.28 \mathrm{E}-2$ & $"$ & & ND & & & & 35 \\
\hline Sodium & $2.41 \mathrm{E} 1$ & $6.70 \mathrm{E}-1$ & $"$ & & $2.59 \mathrm{E} 1$ & & & 7.03 & 35 \\
\hline Post Spike (8D28004-PS1) & \multicolumn{3}{|c|}{ Source: 0802022-11 } & \multicolumn{2}{|c|}{ Prepared: 04/28/08 } & \multicolumn{2}{|c|}{ Analyzed: 05/01/08 } & & \\
\hline Aluminum & $5.40 \mathrm{E} 2$ & N/A & ug/L & $5.00 \mathrm{E} 2$ & 3.38E1 & 101 & $75-125$ & & \\
\hline Calcium & $5.57 \mathrm{E} 4$ & N/A & $"$ & 5.00E2 & $5.34 \mathrm{E} 4$ & 464 & $75-125$ & & \\
\hline Cobalt & $2.41 \mathrm{E} 2$ & N/A & $"$ & $2.50 \mathrm{E} 2$ & $3.22 \mathrm{E} 0$ & 95.2 & $75-125$ & & \\
\hline Chromium & 1.17E2 & N/A & $"$ & 1.25E2 & ND & 103 & $75-125$ & & \\
\hline Copper & $5.30 \mathrm{E} 2$ & N/A & $"$ & $5.00 \mathrm{E} 2$ & 3.38E1 & 99.3 & $75-125$ & & \\
\hline Iron & 5.05E2 & N/A & $"$ & $5.00 \mathrm{E} 2$ & ND & 101 & $75-125$ & & \\
\hline Potassium & $6.29 \mathrm{E} 3$ & N/A & $"$ & $1.25 \mathrm{E} 3$ & 4.95E3 & 107 & $75-125$ & & \\
\hline Magnesium & $3.02 \mathrm{E} 4$ & N/A & $"$ & $5.00 \mathrm{E} 2$ & 2.89E4 & 249 & 75-125 & & \\
\hline Manganese & 2.69E2 & N/A & $"$ & $2.50 \mathrm{E} 2$ & 1.66E1 & 101 & 75-125 & & \\
\hline Nickel & 4.87E2 & N/A & " & $5.00 \mathrm{E} 2$ & $1.85 \mathrm{E} 0$ & 97 & $75-125$ & & \\
\hline Selenium & $5.61 \mathrm{E} 2$ & N/A & $"$ & $5.00 \mathrm{E} 2$ & 2.25E1 & 108 & $75-125$ & & \\
\hline Vanadium & $1.24 \mathrm{E} 2$ & N/A & $"$ & $2.50 \mathrm{E} 2$ & ND & 98.1 & $75-125$ & & \\
\hline Zinc & $3.36 \mathrm{E} 2$ & N/A & $"$ & $2.50 \mathrm{E} 2$ & 3.68E1 & 120 & $75-125$ & & \\
\hline Sodium & $3.02 \mathrm{E} 4$ & N/A & $"$ & $5.00 \mathrm{E} 2$ & $2.87 \mathrm{E} 4$ & 298 & $75-125$ & & \\
\hline
\end{tabular}


Total Metals by PNNL-AGG-ICP-AES/Acid Extract - Quality Control

Environmental Science Laboratory

\begin{tabular}{|c|c|c|c|c|c|c|c|c|c|c|}
\hline Analyte & Result & $\begin{array}{r}\text { orting } \\
\text { Limit }\end{array}$ & Units & $\begin{array}{l}\text { Spike } \\
\text { Level }\end{array}$ & $\begin{array}{l}\text { Source } \\
\text { Result }\end{array}$ & \%REC & $\begin{array}{l}\text { \%REC } \\
\text { Limits }\end{array}$ & RPD & $\begin{array}{l}\text { RPD } \\
\text { Limit }\end{array}$ & Notes \\
\hline
\end{tabular}

Batch 8E12002 - ASTM D 5198 (ICP/ICPMS)

\begin{tabular}{|c|c|c|c|c|}
\hline Blank (8E12002-BLK1) & & & & Prepared: 05/12/08 Analyzed: 06/13/08 \\
\hline Aluminum & $<5.07 \mathrm{E}-1$ & $5.07 \mathrm{E}-1$ & ug/g wet & \\
\hline Barium & $<4.83 \mathrm{E}-2$ & $4.83 \mathrm{E}-2$ & $"$ & \\
\hline Calcium & $<1.88 \mathrm{E} 0$ & $1.88 \mathrm{E} 0$ & $"$ & \\
\hline Cobalt & $<1.05 \mathrm{E}-1$ & $1.05 \mathrm{E}-1$ & $"$ & \\
\hline Chromium & $<4.21 \mathrm{E}-2$ & $4.21 \mathrm{E}-2$ & $"$ & \\
\hline Copper & $<4.49 \mathrm{E}-1$ & $4.49 \mathrm{E}-1$ & $"$ & \\
\hline Iron & $<1.29 \mathrm{E} 0$ & $1.29 \mathrm{E} 0$ & $"$ & \\
\hline Potassium & $<1.19 \mathrm{E} 0$ & $1.19 \mathrm{E} 0$ & $"$ & \\
\hline Magnesium & $<3.99 \mathrm{E}-1$ & $3.99 \mathrm{E}-1$ & $"$ & \\
\hline Manganese & $<1.51 \mathrm{E}-2$ & $1.51 \mathrm{E}-2$ & $"$ & \\
\hline Nickel & $<1.04 \mathrm{E}-1$ & $1.04 \mathrm{E}-1$ & " & \\
\hline Selenium & $<1.76 \mathrm{E} 0$ & $1.76 \mathrm{E} 0$ & $"$ & \\
\hline Vanadium & $<4.69 \mathrm{E}-2$ & $4.69 \mathrm{E}-2$ & $"$ & \\
\hline Zinc & $<7.90 \mathrm{E}-2$ & $7.90 \mathrm{E}-2$ & " & \\
\hline Sodium & $<1.35 \mathrm{E} 1$ & $1.35 \mathrm{E} 1$ & $"$ & \\
\hline Blank (8E12002-BLK2) & & & & Prepared: 05/12/08 Analyzed: 06/13/08 \\
\hline Aluminum & $<5.10 \mathrm{E}-1$ & $5.10 \mathrm{E}-1$ & ug/g wet & \\
\hline Barium & $<4.86 \mathrm{E}-2$ & $4.86 \mathrm{E}-2$ & " & \\
\hline Calcium & $<1.90 \mathrm{E} 0$ & $1.90 \mathrm{E} 0$ & " & \\
\hline Cobalt & $<1.06 \mathrm{E}-1$ & $1.06 \mathrm{E}-1$ & $"$ & \\
\hline Chromium & $<4.24 \mathrm{E}-2$ & $4.24 \mathrm{E}-2$ & $"$ & \\
\hline Copper & $<4.52 \mathrm{E}-1$ & $4.52 \mathrm{E}-1$ & $"$ & \\
\hline Iron & $<1.30 \mathrm{E} 0$ & $1.30 \mathrm{E} 0$ & " & \\
\hline Potassium & $<1.20 \mathrm{E} 0$ & $1.20 \mathrm{E} 0$ & $"$ & \\
\hline Magnesium & $<4.01 \mathrm{E}-1$ & $4.01 \mathrm{E}-1$ & " & \\
\hline Manganese & $<1.52 \mathrm{E}-2$ & $1.52 \mathrm{E}-2$ & $"$ & \\
\hline Nickel & $<1.04 \mathrm{E}-1$ & $1.04 \mathrm{E}-1$ & $"$ & \\
\hline Selenium & $<1.77 \mathrm{E} 0$ & $1.77 \mathrm{E} 0$ & $"$ & \\
\hline Vanadium & $<4.72 \mathrm{E}-2$ & $4.72 \mathrm{E}-2$ & $"$ & \\
\hline Zinc & $<7.96 \mathrm{E}-2$ & $7.96 \mathrm{E}-2$ & $"$ & \\
\hline Sodium & $<1.36 \mathrm{E} 1$ & $1.36 \mathrm{E} 1$ & $"$ & \\
\hline
\end{tabular}


Total Metals by PNNL-AGG-ICP-AES/Acid Extract - Quality Control

Environmental Science Laboratory

\begin{tabular}{|c|c|c|c|c|c|c|c|c|c|c|}
\hline & & porting & & Spike & Source & & \%REC & & RPD & \\
\hline Analyte & Result & Limit & Units & Level & Result & \%REC & Limits & RPD & Limit & Notes \\
\hline
\end{tabular}

Batch 8E12002 - ASTM D 5198 (ICP/ICPMS)

\begin{tabular}{|c|c|c|c|c|c|c|}
\hline \multirow{2}{*}{$\begin{array}{l}\text { LCS (8E12002-BS1) } \\
\text { Aluminum }\end{array}$} & \multicolumn{6}{|c|}{ Prepared: 05/12/08 Analyzed: 06/13/08 } \\
\hline & 4.90E0 & $1.43 \mathrm{E}-1$ & ug/g wet & $5.00 \mathrm{E} 0$ & 98.1 & $80-120$ \\
\hline Barium & $5.26 \mathrm{E} 0$ & $1.36 \mathrm{E}-2$ & $"$ & $5.00 \mathrm{E} 0$ & 105 & $80-120$ \\
\hline Calcium & 5.10E0 & $5.31 \mathrm{E}-1$ & $"$ & $5.00 \mathrm{E} 0$ & 102 & $80-120$ \\
\hline Cobalt & 5.18E0 & $2.97 \mathrm{E}-2$ & $"$ & $5.00 \mathrm{E} 0$ & 104 & $80-120$ \\
\hline Chromium & 5.25E0 & $1.19 \mathrm{E}-2$ & $"$ & $5.00 \mathrm{E} 0$ & 105 & $80-120$ \\
\hline Copper & $5.28 \mathrm{E} 0$ & $1.27 \mathrm{E}-1$ & $"$ & $5.00 \mathrm{E} 0$ & 106 & $80-120$ \\
\hline Iron & 5.35E0 & 3.63E-1 & $"$ & $5.00 \mathrm{E} 0$ & 107 & $80-120$ \\
\hline Potassium & $5.26 \mathrm{E} 1$ & 3.37E-1 & $"$ & $5.00 \mathrm{E} 1$ & 105 & $80-120$ \\
\hline Magnesium & $4.90 \mathrm{E} 0$ & $1.12 \mathrm{E}-1$ & $"$ & $5.00 \mathrm{E} 0$ & 98.0 & $80-120$ \\
\hline Manganese & 5.33E0 & $4.25 \mathrm{E}-3$ & $"$ & $5.00 \mathrm{E} 0$ & 107 & $80-120$ \\
\hline Nickel & 5.29E0 & $2.92 \mathrm{E}-2$ & $"$ & $5.00 \mathrm{E} 0$ & 106 & $80-120$ \\
\hline Selenium & 4.57E0 & $4.96 \mathrm{E}-1$ & $"$ & $5.00 \mathrm{E} 0$ & 91.4 & $80-120$ \\
\hline Vanadium & 5.29E0 & $1.32 \mathrm{E}-2$ & $"$ & $5.00 \mathrm{E} 0$ & 106 & $80-120$ \\
\hline Zinc & 4.83E0 & $2.23 \mathrm{E}-2$ & $"$ & $5.00 \mathrm{E} 0$ & 96.7 & $80-120$ \\
\hline Sodium & $5.74 \mathrm{E} 0$ & $3.80 \mathrm{E} 0$ & $"$ & $5.00 \mathrm{E} 0$ & 115 & $80-120$ \\
\hline LCS (8E12002-BS2) & \multicolumn{6}{|c|}{ Prepared: 05/12/08 Analyzed: 06/13/08 } \\
\hline Aluminum & 4.83E0 & $1.44 \mathrm{E}-1$ & ug/g wet & $5.00 \mathrm{E} 0$ & 96.6 & $80-120$ \\
\hline Barium & $5.07 \mathrm{E} 0$ & $1.37 \mathrm{E}-2$ & " & $5.00 \mathrm{E} 0$ & 101 & $80-120$ \\
\hline Calcium & $4.90 \mathrm{E} 0$ & $5.33 \mathrm{E}-1$ & $"$ & $5.00 \mathrm{E} 0$ & 98.0 & $80-120$ \\
\hline Cobalt & $5.08 \mathrm{E} 0$ & $2.98 \mathrm{E}-2$ & $"$ & $5.00 \mathrm{E} 0$ & 102 & $80-120$ \\
\hline Chromium & 5.23E0 & $1.19 \mathrm{E}-2$ & $"$ & $5.00 \mathrm{E} 0$ & 105 & $80-120$ \\
\hline Copper & 5.18E0 & $1.27 \mathrm{E}-1$ & $"$ & $5.00 \mathrm{E} 0$ & 104 & $80-120$ \\
\hline Iron & 5.27E0 & $3.65 \mathrm{E}-1$ & $"$ & $5.00 \mathrm{E} 0$ & 105 & $80-120$ \\
\hline Potassium & $5.06 \mathrm{E} 1$ & $3.38 \mathrm{E}-1$ & $"$ & $5.00 \mathrm{E} 1$ & 101 & $80-120$ \\
\hline Magnesium & $4.82 \mathrm{E} 0$ & $1.13 \mathrm{E}-1$ & " & $5.00 \mathrm{E} 0$ & 96.4 & $80-120$ \\
\hline Manganese & $5.24 \mathrm{E} 0$ & $4.27 \mathrm{E}-3$ & $"$ & $5.00 \mathrm{E} 0$ & 105 & $80-120$ \\
\hline Nickel & 5.17E0 & $2.93 E-2$ & $"$ & $5.00 \mathrm{E} 0$ & 103 & $80-120$ \\
\hline Selenium & 4.93E0 & $4.98 \mathrm{E}-1$ & $"$ & $5.00 \mathrm{E} 0$ & 98.7 & $80-120$ \\
\hline Vanadium & $5.21 \mathrm{E} 0$ & $1.33 E-2$ & $"$ & $5.00 \mathrm{E} 0$ & 104 & $80-120$ \\
\hline Zinc & $4.81 \mathrm{E} 0$ & $2.24 \mathrm{E}-2$ & $"$ & $5.00 \mathrm{E} 0$ & 96.3 & $80-120$ \\
\hline Sodium & 5.46E0 & 3.82E0 & $"$ & $5.00 \mathrm{E} 0$ & 109 & $80-120$ \\
\hline
\end{tabular}


Total Metals by PNNL-AGG-ICP-AES/Acid Extract - Quality Control

Environmental Science Laboratory

\begin{tabular}{|lrrrrrrrrrrr}
\hline & & Reporting & & Spike & Source & & & \%REC & & RPD & \\
Analyte & Result & Limit & Units & Level & Result & \%REC & Limits & RPD & Limit & Notes \\
\hline
\end{tabular}

Batch 8E12002 - ASTM D 5198 (ICP/ICPMS)

\begin{tabular}{|c|c|c|c|c|c|c|}
\hline \multirow{2}{*}{$\begin{array}{l}\text { Duplicate (8E12002-DUP1) } \\
\text { Aluminum }\end{array}$} & \multicolumn{3}{|c|}{ Source: 0802024-18 } & \multirow{2}{*}{$\begin{array}{r}\text { Prepared: 05/12/08 } \\
7.84 \mathrm{E} 3\end{array}$} & \multicolumn{2}{|l|}{ Analyzed: 06/13/08 } \\
\hline & $8.00 \mathrm{E} 3$ & 4.27E1 & ug/g dry & & 2.02 & 35 \\
\hline Barium & $6.76 \mathrm{E} 1$ & 4.07E0 & $"$ & $6.77 \mathrm{E} 1$ & 0.147 & 35 \\
\hline Calcium & $7.76 \mathrm{E} 3$ & $1.59 \mathrm{E} 2$ & $"$ & $7.08 \mathrm{E} 3$ & 9.07 & 35 \\
\hline Cobalt & $<8.86 \mathrm{E} 0$ & $8.86 \mathrm{E} 0$ & $"$ & ND & & 35 \\
\hline Chromium & $1.45 \mathrm{E} 1$ & $3.55 \mathrm{E} 0$ & $"$ & $1.50 \mathrm{E} 1$ & 3.42 & 35 \\
\hline Copper & $<3.78 \mathrm{E} 1$ & 3.78E1 & $"$ & ND & & 35 \\
\hline Iron & $1.36 \mathrm{E} 4$ & $1.09 \mathrm{E} 2$ & $"$ & 1.33E4 & 2.44 & 35 \\
\hline Potassium & 1.57E3 & $1.01 \mathrm{E} 2$ & $"$ & $1.54 \mathrm{E} 3$ & 1.91 & 35 \\
\hline Magnesium & $5.24 \mathrm{E} 3$ & 3.36E1 & $"$ & 5.12E3 & 2.40 & 35 \\
\hline Manganese & 3.69E2 & 1.27E0 & $"$ & 3.34E2 & 10.1 & 35 \\
\hline Nickel & $1.79 \mathrm{E} 1$ & 8.73E0 & $"$ & $1.76 \mathrm{E} 1$ & 1.79 & 35 \\
\hline Selenium & $<1.48 \mathrm{E} 2$ & $1.48 \mathrm{E} 2$ & $"$ & ND & & 35 \\
\hline Vanadium & $1.60 \mathrm{E} 1$ & 3.95E0 & $"$ & $1.58 \mathrm{E} 1$ & 1.01 & 35 \\
\hline Zinc & $3.75 \mathrm{E} 1$ & $6.66 \mathrm{E} 0$ & $"$ & $3.70 \mathrm{E} 1$ & 1.33 & 35 \\
\hline Sodium & $<1.14 \mathrm{E} 3$ & $1.14 \mathrm{E} 3$ & $"$ & ND & & 35 \\
\hline Duplicate (8E12002-DUP2) & \multicolumn{3}{|c|}{ Source: 0802028-03 } & Prepared: 05/12/08 & Analyzed: 06/13/08 & \\
\hline Aluminum & $1.08 \mathrm{E} 4$ & 4.32E1 & ug/g dry & 9.98E3 & 7.44 & 35 \\
\hline Barium & 8.15E1 & 4.12E0 & $"$ & 7.27E1 & 11.4 & 35 \\
\hline Calcium & $1.02 \mathrm{E} 4$ & $1.61 \mathrm{E} 2$ & $"$ & $9.80 \mathrm{E} 3$ & 4.49 & 35 \\
\hline Cobalt & $9.71 \mathrm{E} 0$ & 8.97E0 & $"$ & ND & & 35 \\
\hline Chromium & $2.06 \mathrm{E} 1$ & 3.59E0 & $"$ & $1.82 \mathrm{E} 1$ & 12.0 & 35 \\
\hline Copper & $<3.83 \mathrm{E} 1$ & 3.83E1 & $"$ & ND & & 35 \\
\hline Iron & $1.64 \mathrm{E} 4$ & $1.10 \mathrm{E} 2$ & " & $1.52 \mathrm{E} 4$ & 7.91 & 35 \\
\hline Potassium & $1.94 \mathrm{E} 3$ & $1.02 \mathrm{E} 2$ & $"$ & $1.84 \mathrm{E} 3$ & 5.48 & 35 \\
\hline Magnesium & $6.56 \mathrm{E} 3$ & $3.40 \mathrm{E} 1$ & " & $6.06 \mathrm{E} 3$ & 8.00 & 35 \\
\hline Manganese & 4.13E2 & $1.29 \mathrm{E} 0$ & $"$ & 4.00E2 & 3.24 & 35 \\
\hline Nickel & $2.38 \mathrm{E} 1$ & 8.84E0 & $"$ & $2.15 \mathrm{E} 1$ & 10.3 & 35 \\
\hline Selenium & $<1.50 \mathrm{E} 2$ & $1.50 \mathrm{E} 2$ & $"$ & ND & & 35 \\
\hline Vanadium & $1.95 \mathrm{E} 1$ & 4.00E0 & $"$ & $1.91 \mathrm{E} 1$ & 1.96 & 35 \\
\hline Zinc & 4.67E1 & $6.74 \mathrm{E} 0$ & $"$ & 4.61E1 & 1.27 & 35 \\
\hline Sodium & $<1.15 \mathrm{E} 3$ & $1.15 \mathrm{E} 3$ & $"$ & ND & & 35 \\
\hline
\end{tabular}


Total Metals by PNNL-AGG-ICP-AES/Acid Extract - Quality Control

Environmental Science Laboratory

\begin{tabular}{|lrrrrrrrrrr}
\hline & & Reporting & & Spike & Source & & \%REC & & RPD & \\
Analyte & Result & Limit & Units & Level & Result & \%REC & Limits & RPD & Limit & Notes \\
\hline
\end{tabular}

Batch 8E12002 - ASTM D 5198 (ICP/ICPMS)

\begin{tabular}{|c|c|c|c|c|c|c|c|}
\hline \multirow{2}{*}{$\begin{array}{l}\text { Post Spike (8E12002-PS1) } \\
\text { Aluminum }\end{array}$} & \multicolumn{3}{|c|}{ Source: 0802024-18 } & \multicolumn{4}{|c|}{ Prepared: 05/12/08 Analyzed: 06/13/08 } \\
\hline & $1.89 \mathrm{E} 4$ & N/A & $\mathrm{ug} / \mathrm{L}$ & $5.00 \mathrm{E} 2$ & $1.78 \mathrm{E} 4$ & 227 & $75-125$ \\
\hline Barium & 3.82E2 & N/A & $"$ & $2.50 \mathrm{E} 2$ & $1.53 \mathrm{E} 2$ & 91.5 & $75-125$ \\
\hline Calcium & $1.71 \mathrm{E} 4$ & N/A & $"$ & $5.00 \mathrm{E} 2$ & $1.61 \mathrm{E} 4$ & 212 & $75-125$ \\
\hline Cobalt & 2.59E2 & N/A & $"$ & $2.50 \mathrm{E} 2$ & $1.66 \mathrm{E} 1$ & 96.9 & 75-125 \\
\hline Chromium & $1.55 \mathrm{E} 2$ & N/A & $"$ & $1.25 \mathrm{E} 2$ & $3.40 \mathrm{E} 1$ & 97.1 & $75-125$ \\
\hline Copper & 5.13E2 & N/A & $"$ & $5.00 \mathrm{E} 2$ & $2.82 \mathrm{E} 1$ & 97 & $75-125$ \\
\hline Iron & 3.17E4 & N/A & $"$ & $5.00 \mathrm{E} 2$ & 3.02E4 & 316 & $75-125$ \\
\hline Potassium & 4.72E3 & N/A & $"$ & $1.25 \mathrm{E} 3$ & $3.50 \mathrm{E} 3$ & 98 & $75-125$ \\
\hline Magnesium & $1.26 \mathrm{E} 4$ & N/A & $"$ & $5.00 \mathrm{E} 2$ & $1.16 \mathrm{E} 4$ & 192 & 75-125 \\
\hline Manganese & $1.03 \mathrm{E} 3$ & N/A & $"$ & $2.50 \mathrm{E} 2$ & 7.57E2 & 111 & $75-125$ \\
\hline Nickel & 5.32E2 & N/A & $"$ & $5.00 \mathrm{E} 2$ & 3.99E1 & 98.5 & $75-125$ \\
\hline Selenium & 5.17E2 & N/A & $"$ & $5.00 \mathrm{E} 2$ & ND & 117 & $75-125$ \\
\hline Vanadium & 2.83E2 & N/A & $"$ & $2.50 \mathrm{E} 2$ & 3.59E1 & 99 & $75-125$ \\
\hline Zinc & 3.31E2 & N/A & $"$ & $2.50 \mathrm{E} 2$ & 8.39E1 & 98.8 & $75-125$ \\
\hline Sodium & 8.53E2 & N/A & $"$ & $5.00 \mathrm{E} 2$ & $3.88 \mathrm{E} 2$ & 92.9 & 75-125 \\
\hline
\end{tabular}


Radionuclides by ICP-MS/Acid Extract - Quality Control

Environmental Science Laboratory

\begin{tabular}{|lrrrrrrrrrr}
\hline & & Reporting & & Spike & Source & & \%REC & & RPD & \\
Analyte & Result & Limit & Units & Level & Result & \%REC & Limits & RPD & Limit & Notes \\
\hline
\end{tabular}

Batch 8E15002 - ASTM D 5198 (ICP/ICPMS)

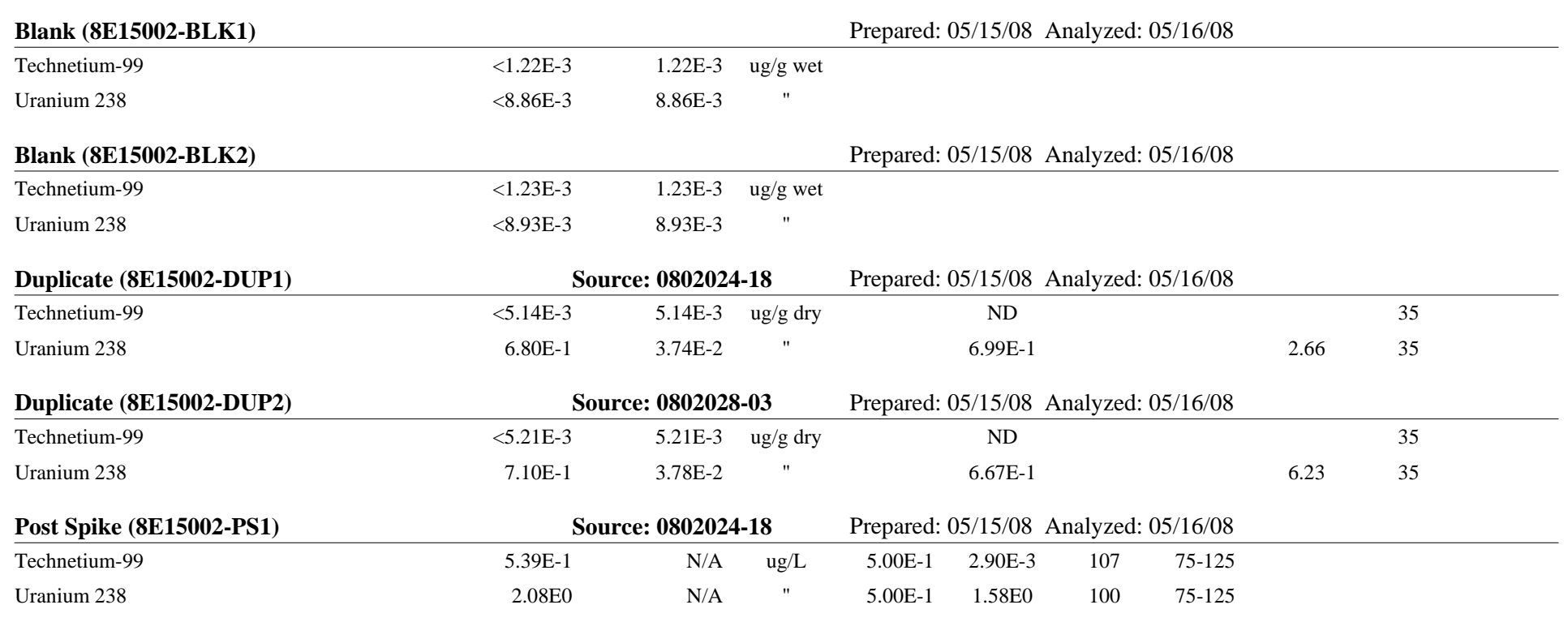




\section{Radionuclides by ICP-MS/Water Extract - Quality Control \\ Environmental Science Laboratory}

\begin{tabular}{|lrrrrrrrrrr}
\hline & & Reporting & & Spike & Source & & \%REC & & RPD & \\
Analyte & Result & Limit & Units & Level & Result & \%REC & Limits & RPD & Limit & Notes \\
\hline
\end{tabular}

Batch 8D15001 - 1:1 Water Extract (ICP/ICPMS)

\begin{tabular}{|c|c|c|c|c|c|c|c|c|}
\hline \multirow{2}{*}{$\frac{\text { Blank (8D15001-BLK1) }}{\text { Technetium-99 }}$} & \multicolumn{8}{|c|}{ Prepared \& Analyzed: 04/15/08 } \\
\hline & $<2.30 \mathrm{E}-5$ & $2.30 \mathrm{E}-5$ & ug/g wet & & & & & \\
\hline Uranium 238 & $<5.64 \mathrm{E}-4$ & 5.64E-4 & " & & & & & \\
\hline Blank (8D15001-BLK2) & \multicolumn{8}{|c|}{ Prepared \& Analyzed: 04/15/08 } \\
\hline Technetium-99 & $<2.30 \mathrm{E}-5$ & $2.30 \mathrm{E}-5$ & ug/g wet & & & & & \\
\hline Uranium 238 & $<5.64 \mathrm{E}-4$ & $5.64 \mathrm{E}-4$ & " & & & & & \\
\hline Duplicate (8D15001-DUP2) & \multicolumn{3}{|c|}{ Source: 0802024-18 } & \multicolumn{5}{|c|}{ Prepared \& Analyzed: 04/15/08 } \\
\hline Technetium-99 & $<2.30 \mathrm{E}-5$ & $2.30 \mathrm{E}-5$ & ug/g dry & & ND & & & 35 \\
\hline Uranium 238 & $7.68 \mathrm{E}-3$ & $5.64 \mathrm{E}-4$ & " & & $1.16 \mathrm{E}-2$ & & 40.8 & 35 \\
\hline Post Spike (8D15001-PS2) & \multicolumn{3}{|c|}{ Source: 0802024-18 } & \multicolumn{5}{|c|}{ Prepared \& Analyzed: 04/15/08 } \\
\hline Technetium-99 & 5.05E-1 & N/A & ug/L & $5.00 \mathrm{E}-1$ & $1.72 \mathrm{E}-3$ & 101 & $75-125$ & \\
\hline Uranium 238 & $3.02 \mathrm{E} 0$ & N/A & $"$ & $5.00 \mathrm{E}-1$ & 2.32E0 & 140 & $75-125$ & \\
\hline
\end{tabular}


RCRA Metals By PNNL-AGG-415/Water Extract - Quality Control

Environmental Science Laboratory

\begin{tabular}{|lrrrrrrrrrrr}
\hline & & Reporting & & Spike & Source & & \%REC & & RPD & & \\
Analyte & Result & Limit & Units & Level & Result & \%REC & Limits & RPD & Limit & Notes \\
\hline
\end{tabular}

Batch 8D07004 - 1:1 Water Extract (ICP/ICPMS)

\begin{tabular}{lllllllr} 
Post Spike (8D07004-PS2) & \multicolumn{3}{c}{ Source: 0802024-18 } & \multicolumn{3}{c}{ Prepared: 04/07/08 } & Analyzed: 04/24/08 \\
\hline Silver & $4.70 \mathrm{E} 0$ & N/A & ug/L & $5.00 \mathrm{E} 0$ & $5.15 \mathrm{E}-3$ & 93.8 & $75-125$ \\
Cadmium & $4.88 \mathrm{E} 0$ & N/A & $"$ & $5.00 \mathrm{E} 0$ & $4.18 \mathrm{E}-2$ & 96.7 & $75-125$ \\
Antimony & $4.94 \mathrm{E} 0$ & N/A & $"$ & $5.00 \mathrm{E} 0$ & $9.67 \mathrm{E}-2$ & 96.9 & $75-125$
\end{tabular}


RCRA Metals By PNNL-AGG-415/Acid Extract - Quality Control

Environmental Science Laboratory

\begin{tabular}{|c|c|c|c|c|c|c|c|c|c|c|}
\hline Analyte & Result & $\begin{array}{r}\text { orting } \\
\text { Limit }\end{array}$ & Units & $\begin{array}{l}\text { Spike } \\
\text { Level }\end{array}$ & $\begin{array}{l}\text { Source } \\
\text { Result }\end{array}$ & \%REC & $\begin{array}{l}\text { \%REC } \\
\text { Limits }\end{array}$ & RPD & $\begin{array}{l}\text { RPD } \\
\text { Limit }\end{array}$ & Notes \\
\hline
\end{tabular}

Batch 8E15001 - ASTM D 5198 (ICP/ICPMS)

\begin{tabular}{|c|c|c|c|c|c|c|c|c|c|}
\hline \multirow{2}{*}{$\begin{array}{l}\text { Blank (8E15001-BLK1) } \\
\text { Cadmium }\end{array}$} & \multicolumn{9}{|c|}{ Prepared: 05/14/08 Analyzed: 06/03/08 } \\
\hline & $<1.45 \mathrm{E}-2$ & $1.45 \mathrm{E}-2$ & ug/g wet & & & & & & \\
\hline Antimony & $<2.34 \mathrm{E}-2$ & $2.34 \mathrm{E}-2$ & $"$ & & & & & & \\
\hline Blank (8E15001-BLK2) & \multicolumn{9}{|c|}{ Prepared: 05/14/08 Analyzed: 06/03/08 } \\
\hline Cadmium & $<1.46 \mathrm{E}-2$ & $1.46 \mathrm{E}-2$ & ug/g wet & & & & & & \\
\hline Antimony & $<2.36 \mathrm{E}-2$ & $2.36 \mathrm{E}-2$ & $"$ & & & & & & \\
\hline LCS (8E15001-BS1) & \multicolumn{9}{|c|}{ Prepared: 05/14/08 Analyzed: 06/03/08 } \\
\hline Cadmium & $4.58 \mathrm{E} 0$ & 7.14E-2 & ug/g wet & 5.00E0 & & 91.6 & $80-120$ & & \\
\hline Antimony & $4.55 \mathrm{E} 0$ & $1.15 \mathrm{E}-1$ & $"$ & $5.00 \mathrm{E} 0$ & & 91.1 & $80-120$ & & \\
\hline LCS (8E15001-BS2) & \multicolumn{9}{|c|}{ Prepared: 05/14/08 Analyzed: 06/03/08 } \\
\hline Cadmium & 4.65E0 & 7.17E-2 & ug/g wet & 5.00E0 & & 92.9 & $80-120$ & & \\
\hline Antimony & $4.48 \mathrm{E} 0$ & $1.16 \mathrm{E}-1$ & $"$ & $5.00 \mathrm{E} 0$ & & 89.6 & $80-120$ & & \\
\hline Duplicate (8E15001-DUP1) & \multicolumn{3}{|c|}{ Source: 0802024-18 } & \multicolumn{6}{|c|}{ Prepared: 05/14/08 Analyzed: 06/03/08 } \\
\hline Cadmium & $1.07 \mathrm{E}-1$ & $6.10 \mathrm{E}-2$ & ug/g dry & & $1.15 \mathrm{E}-1$ & & & 7.80 & 35 \\
\hline Antimony & $<9.86 \mathrm{E}-2$ & $9.86 \mathrm{E}-2$ & $"$ & & ND & & & & 35 \\
\hline Duplicate (8E15001-DUP2) & \multicolumn{3}{|c|}{ Source: 0802028-03 } & \multicolumn{4}{|c|}{ Prepared: 05/14/08 Analyzed: 06/03/08 } & & \\
\hline Cadmium & $1.58 \mathrm{E}-1$ & 6.17E-2 & ug/g dry & & $1.64 \mathrm{E}-1$ & & & 4.01 & 35 \\
\hline Antimony & $<9.97 \mathrm{E}-2$ & $9.97 \mathrm{E}-2$ & $"$ & & ND & & & & 35 \\
\hline Post Spike (8E15001-PS1) & \multicolumn{3}{|c|}{ Source: 0802024-18 } & \multicolumn{4}{|c|}{ Prepared: 05/14/08 Analyzed: 06/03/08 } & & \\
\hline Cadmium & 4.59E0 & N/A & $\mathrm{ug} / \mathrm{L}$ & 5.00E0 & $2.62 \mathrm{E}-1$ & 86.6 & $75-125$ & & \\
\hline Antimony & 3.99E0 & N/A & " & $5.00 \mathrm{E} 0$ & $2.60 \mathrm{E}-2$ & 79.3 & $75-125$ & & \\
\hline
\end{tabular}


Total Alpha Total Beta/Acid Extract - Quality Control

Environmental Science Laboratory

\begin{tabular}{|lrrrrrrrrrr}
\hline & & Reporting & & Spike & Source & & \%REC & & RPD & \\
Analyte & Result & Limit & Units & Level & Result & \%REC & Limits & RPD & Limit & Notes \\
\hline
\end{tabular}

Batch 8I19001 - ASTM D 5198 (RadChem)

\begin{tabular}{|c|c|c|c|c|c|}
\hline \multirow{2}{*}{$\begin{array}{l}\text { Blank (8I19001-BLK1) } \\
\text { Gross Beta }\end{array}$} & \multicolumn{5}{|c|}{ Prepared: 04/22/08 Analyzed: 04/23/08 } \\
\hline & $<1.47 \mathrm{E} 1$ & $1.47 \mathrm{E} 1 \mathrm{pCi} / \mathrm{g}$ wet & & & \\
\hline Gross Alpha & $<5.81 \mathrm{E} 0$ & $5.81 \mathrm{E} 0$ & & & \\
\hline Duplicate (8I19001-DUP1) & \multicolumn{2}{|c|}{ Source: 0802024-18 } & Prepared: 04/22/08 & Analyzed: 04/23/08 & \\
\hline Gross Beta & $<6.39 \mathrm{E} 1$ & 6.39E1 pCi/g dry & ND & & 35 \\
\hline Gross Alpha & $<2.52 \mathrm{E} 1$ & $2.52 \mathrm{E} 1$ & ND & & 35 \\
\hline
\end{tabular}




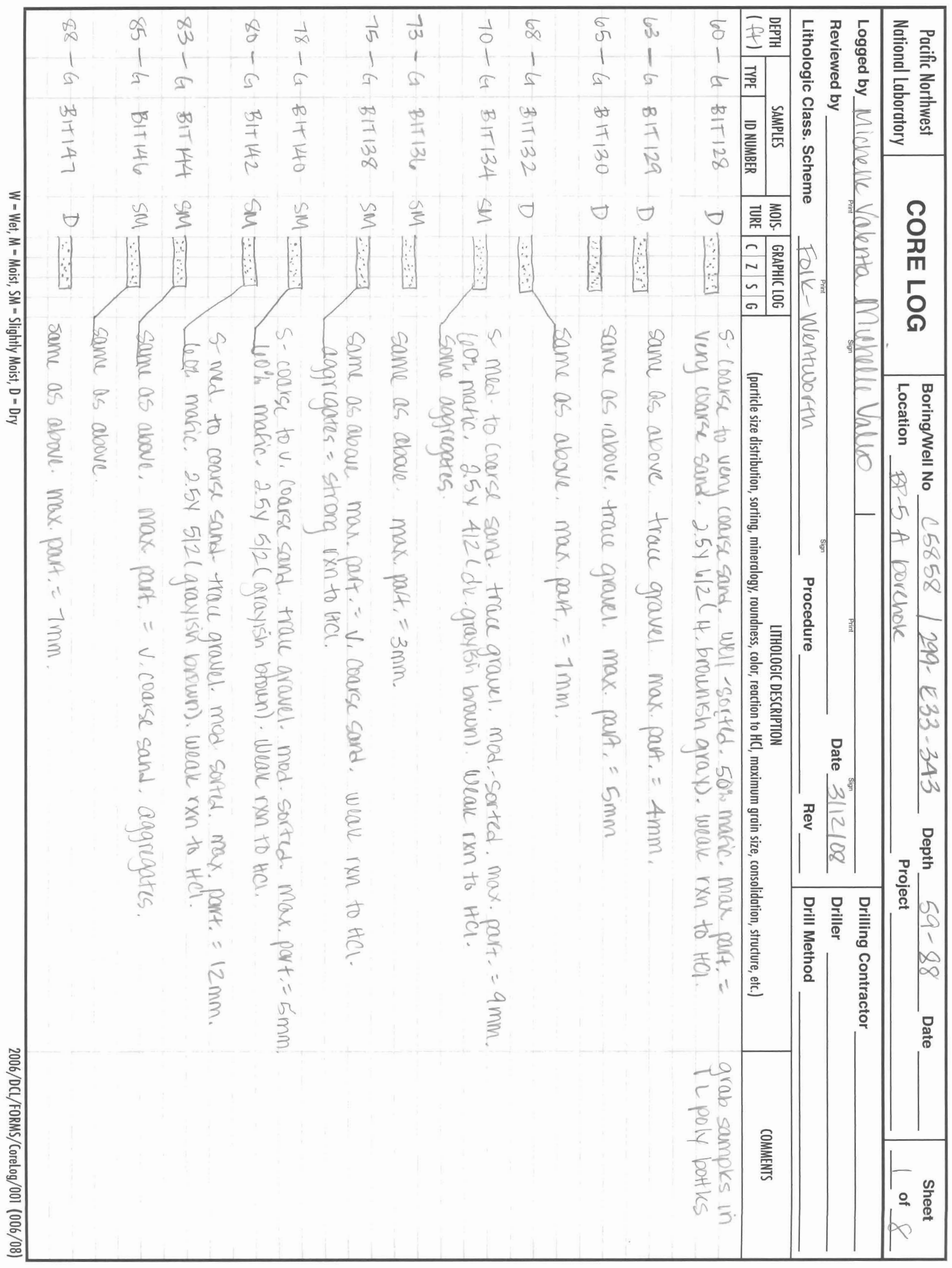




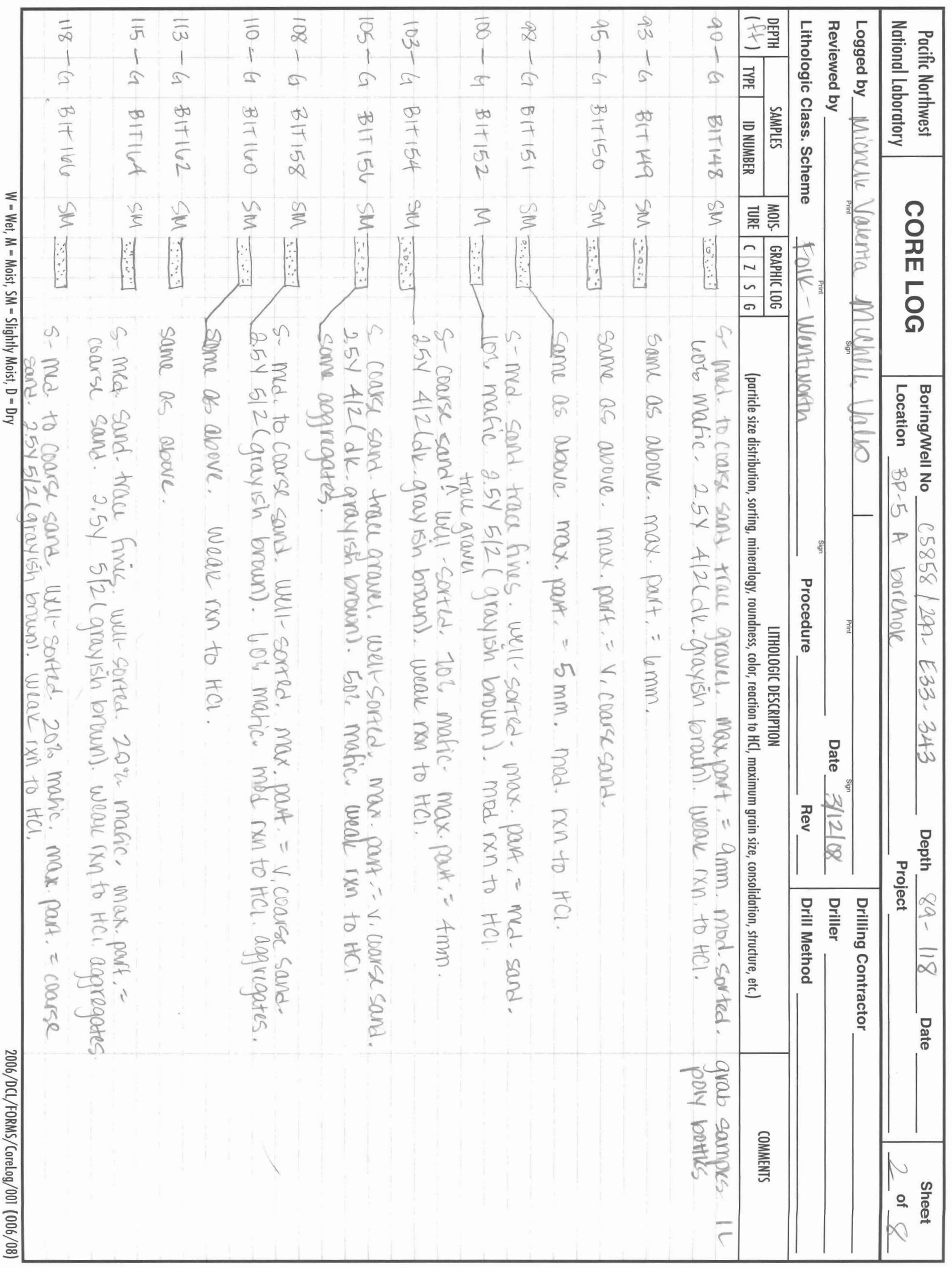




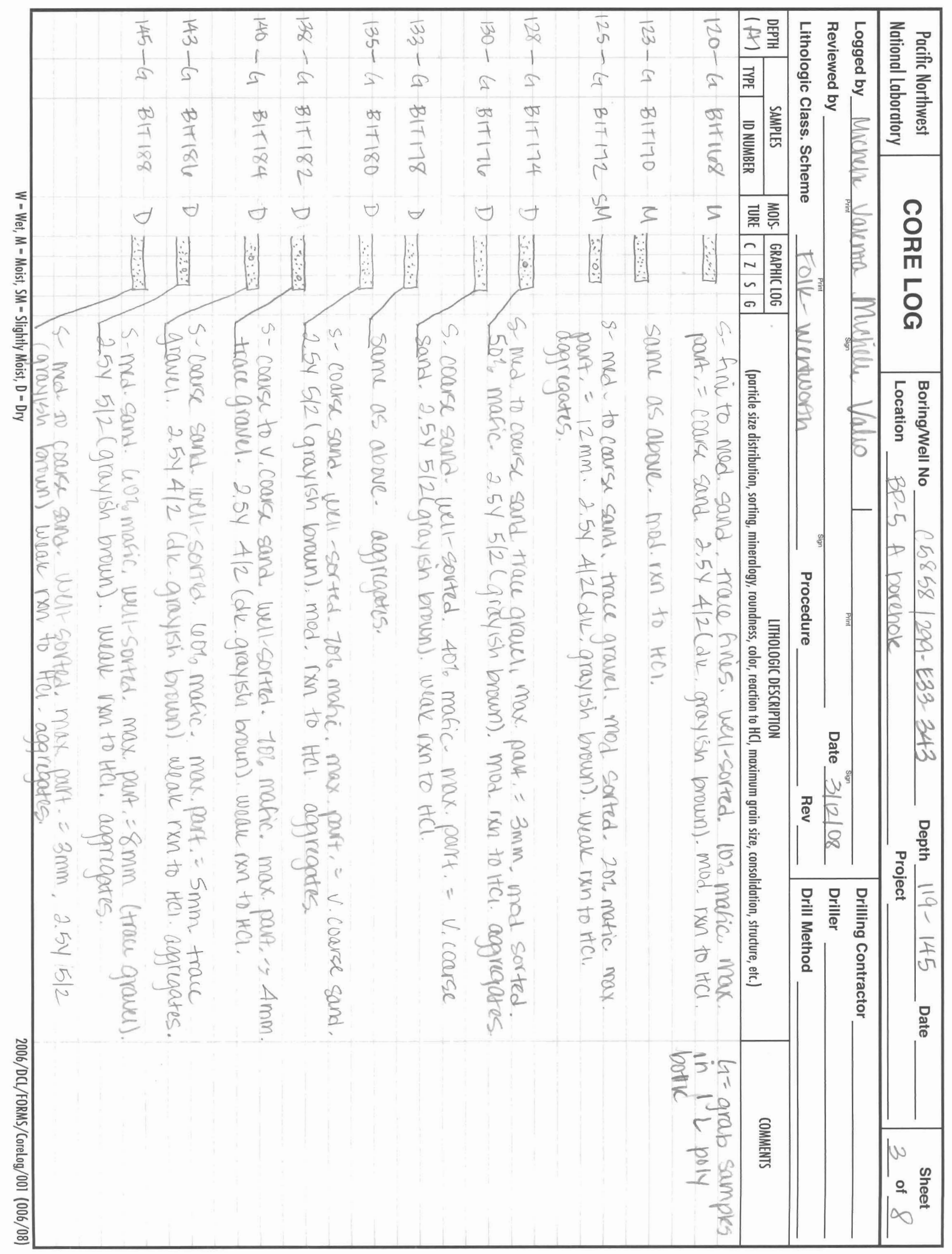




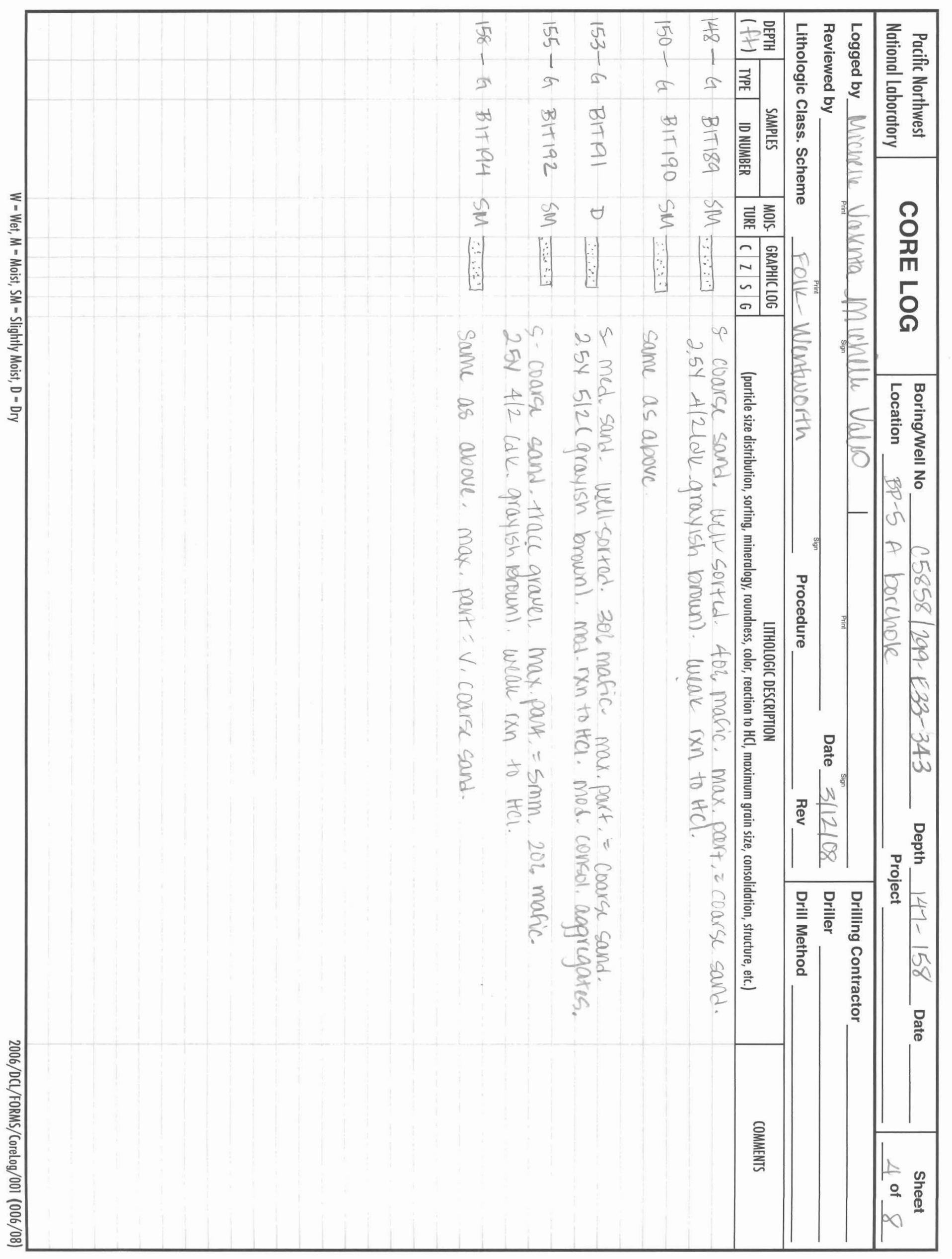




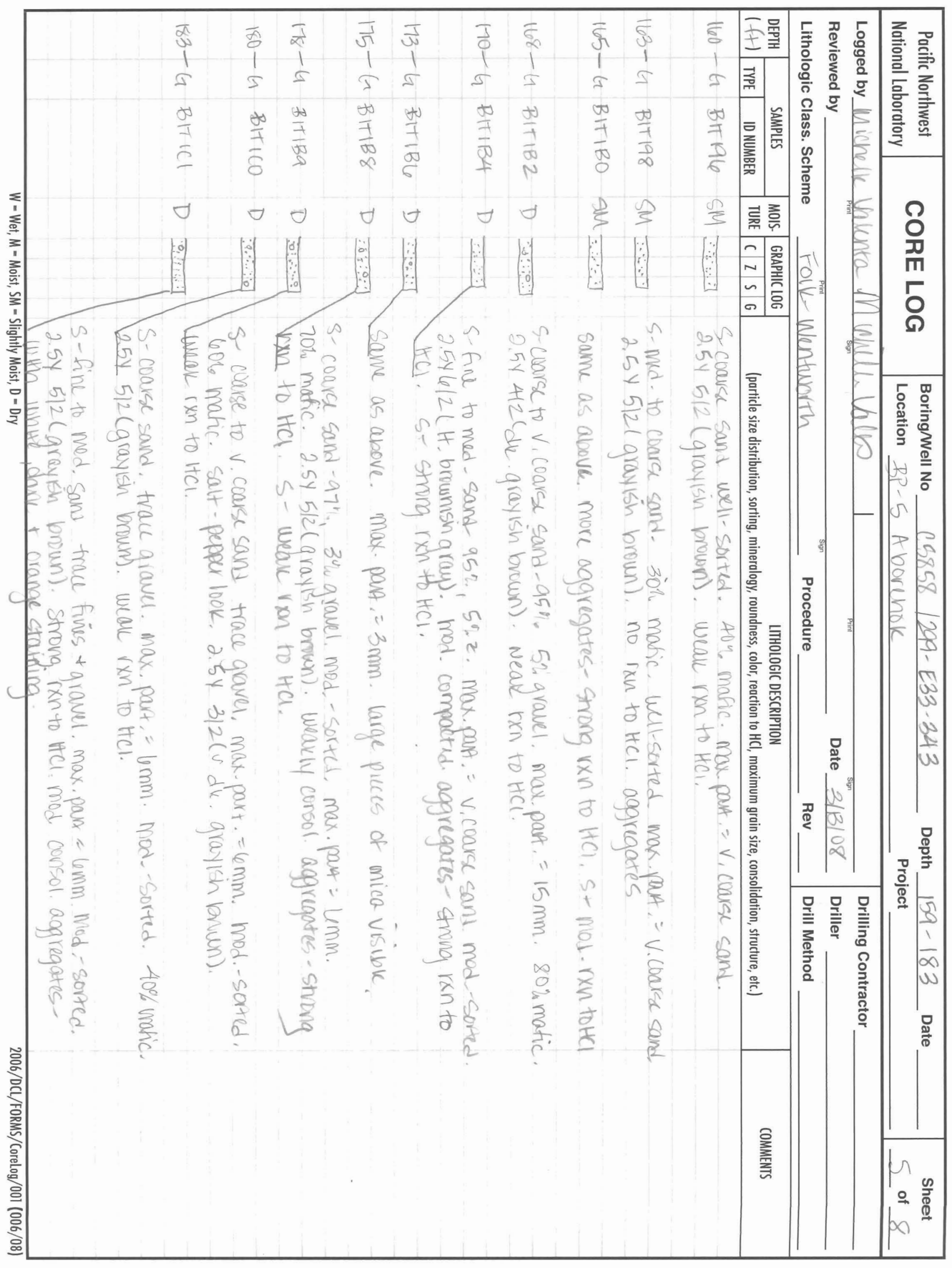




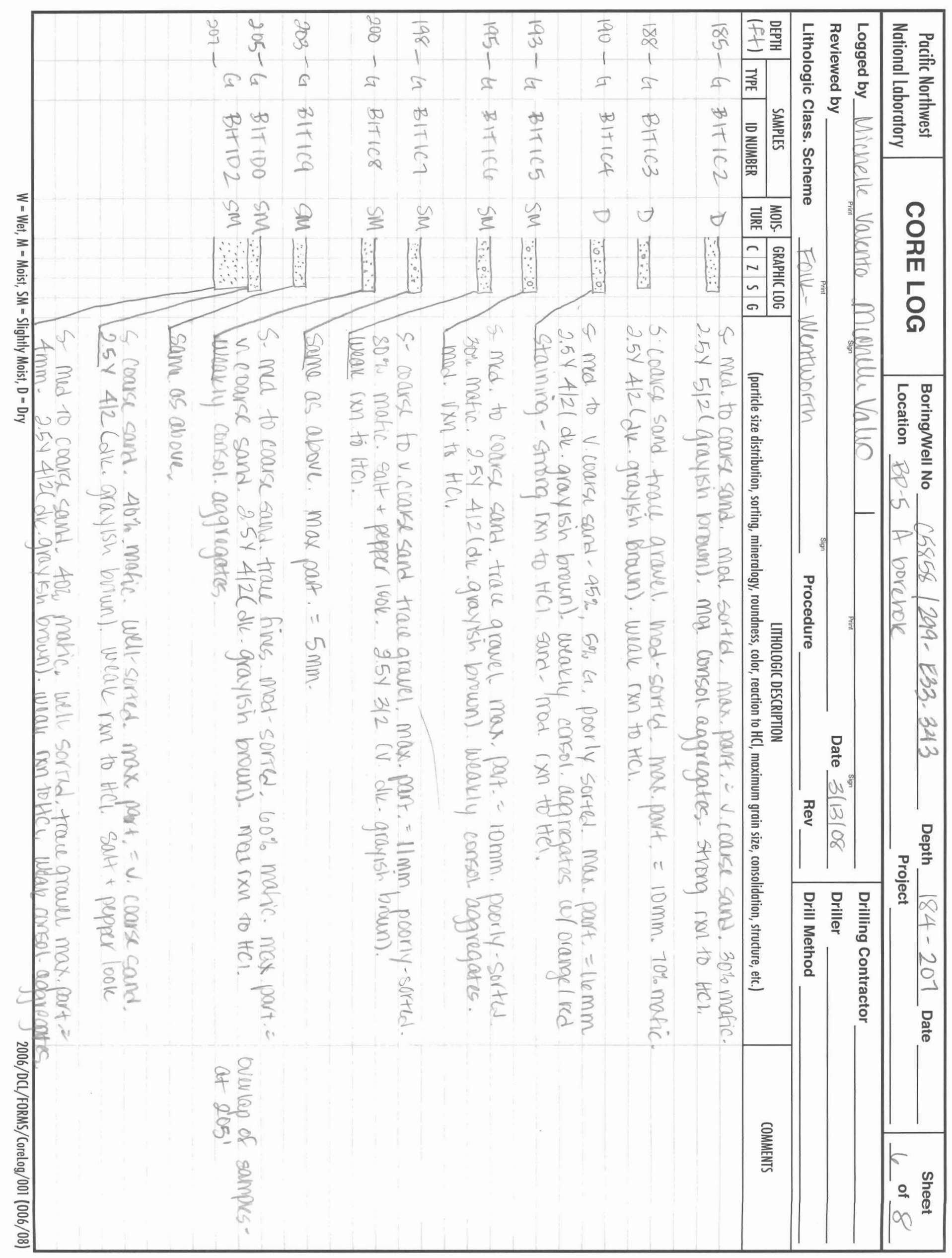




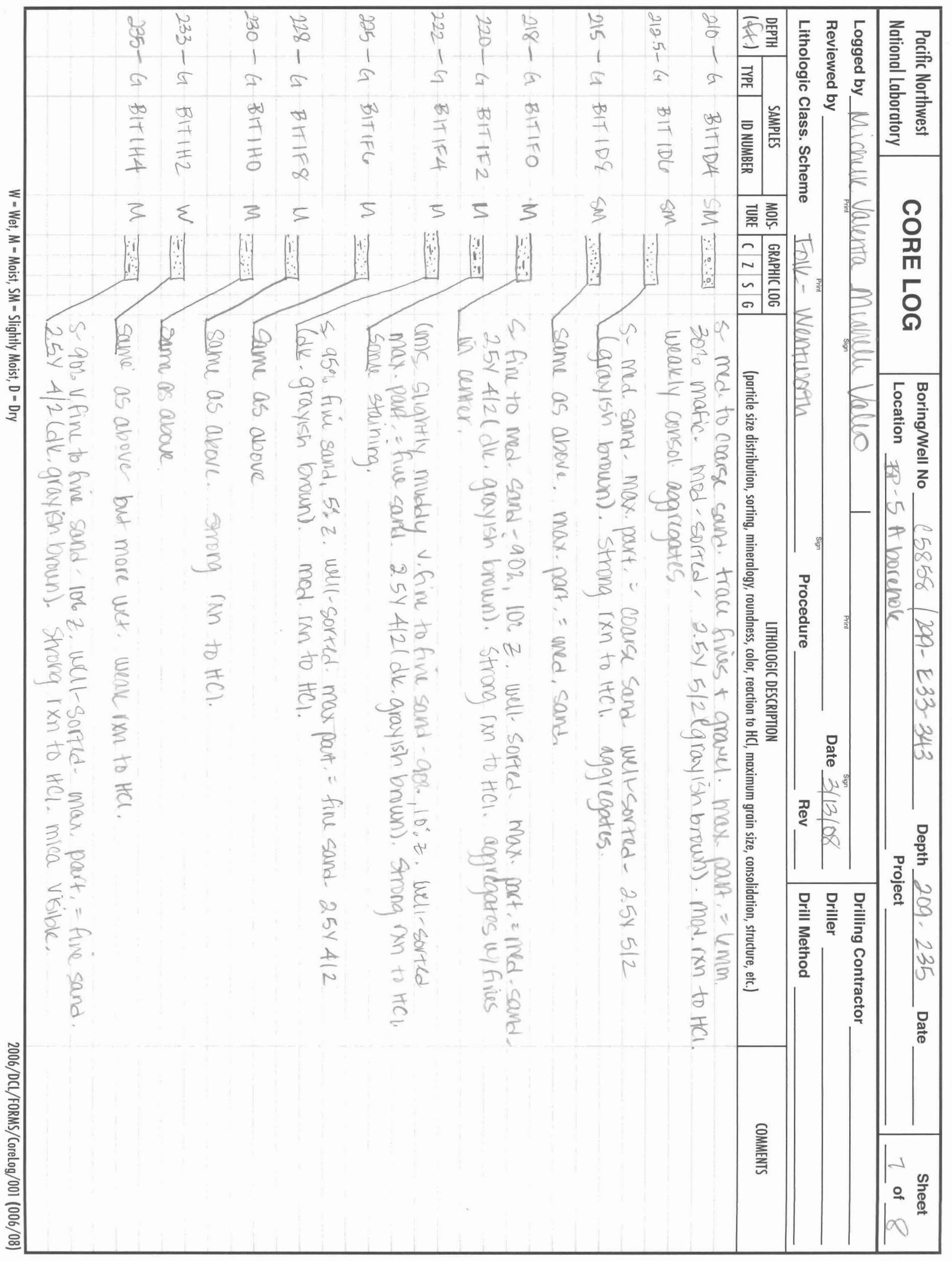




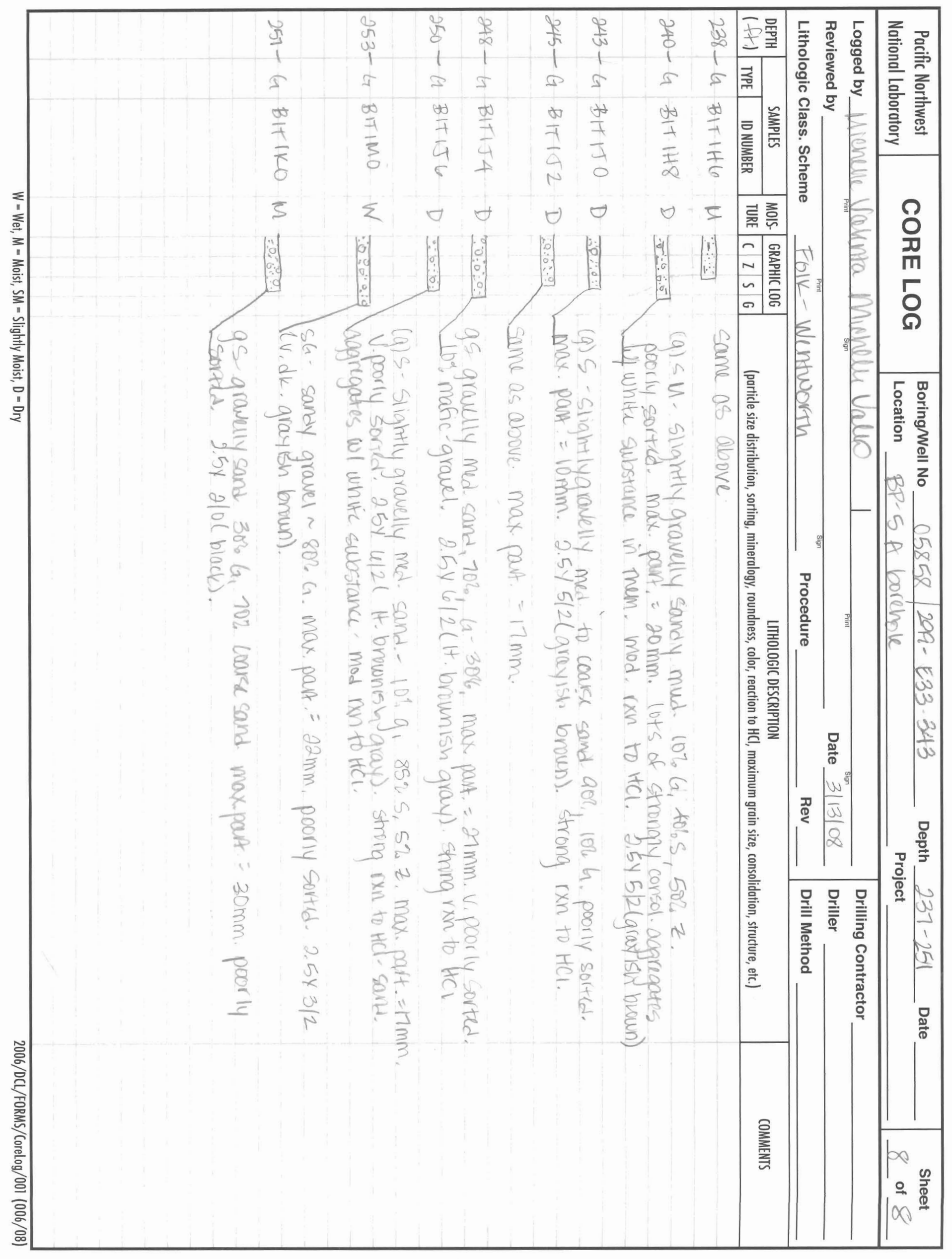




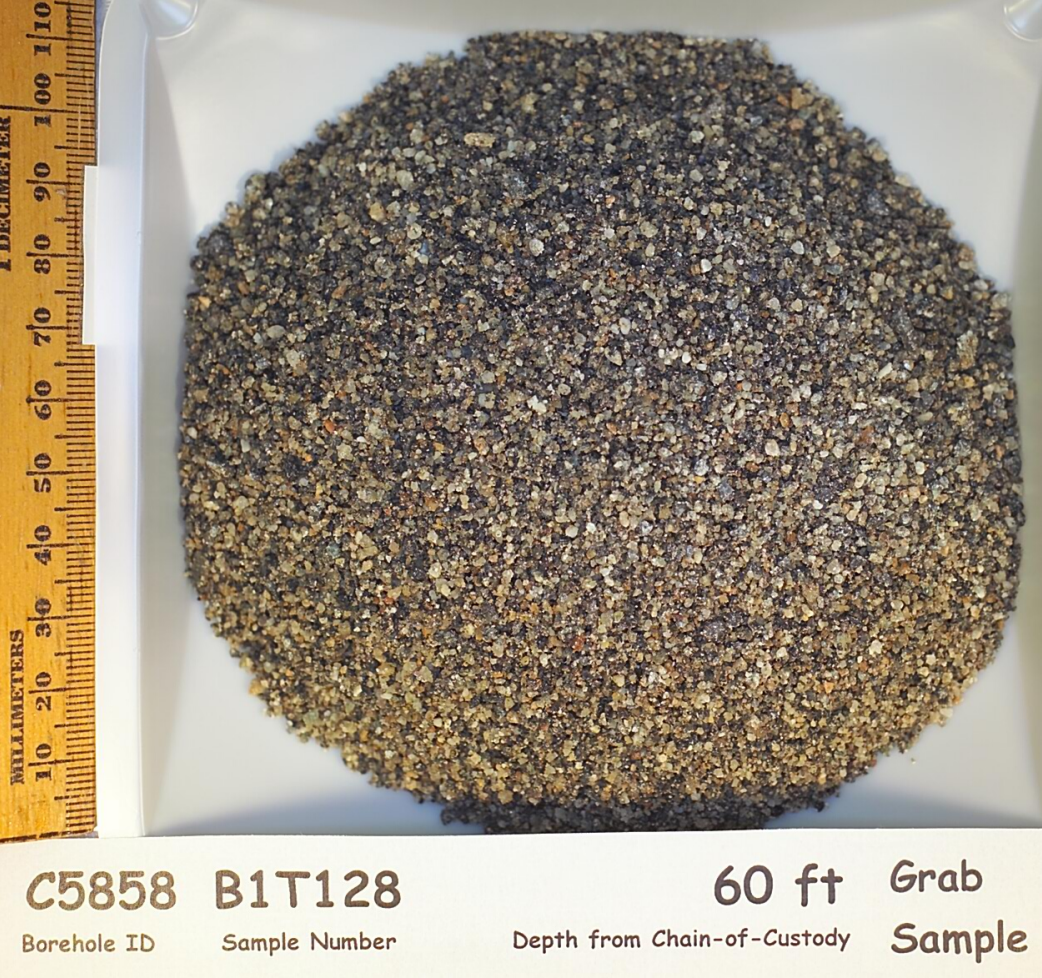




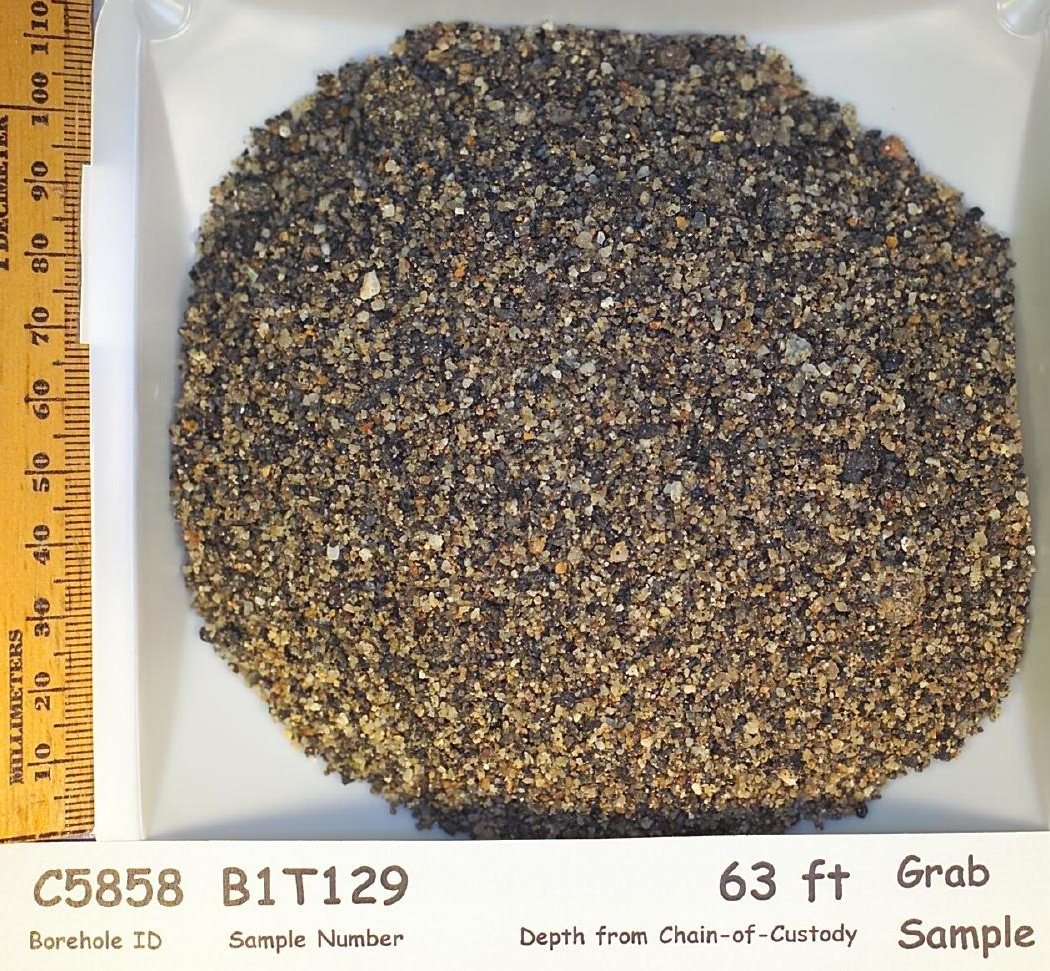




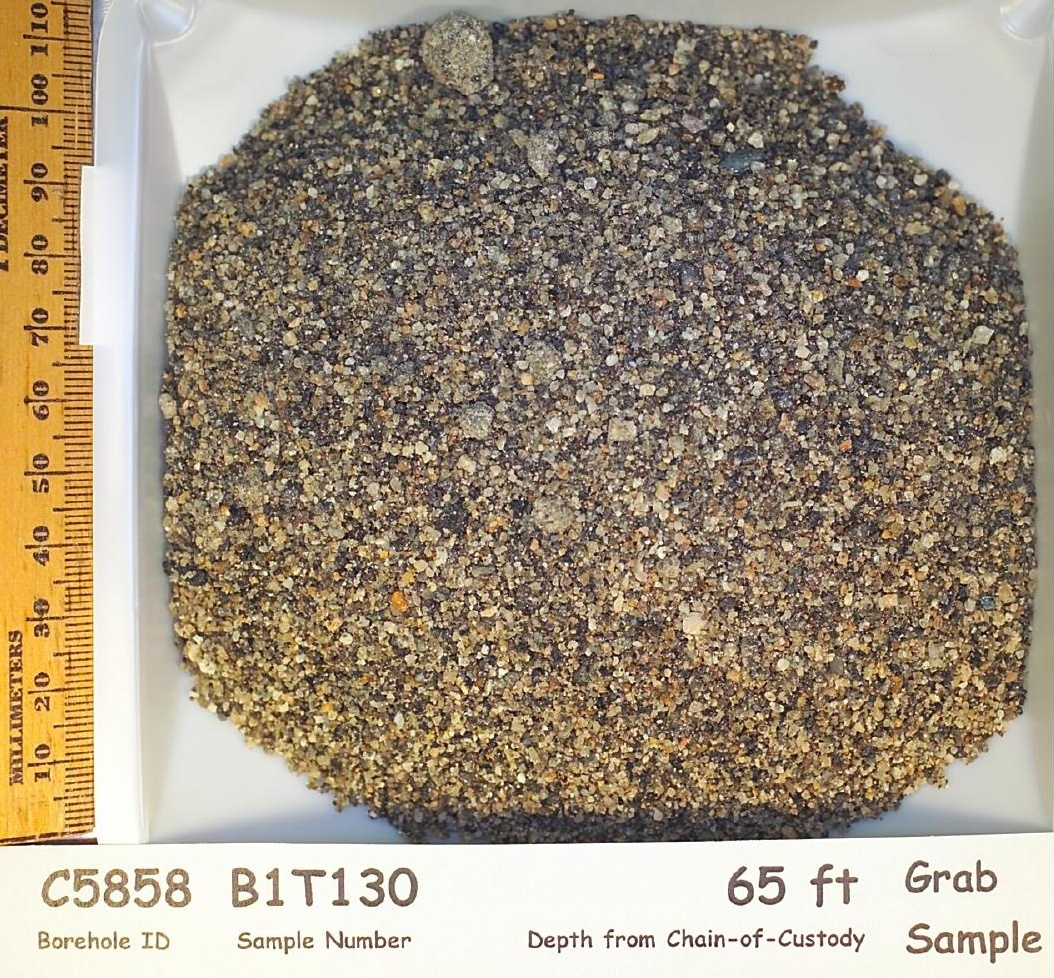




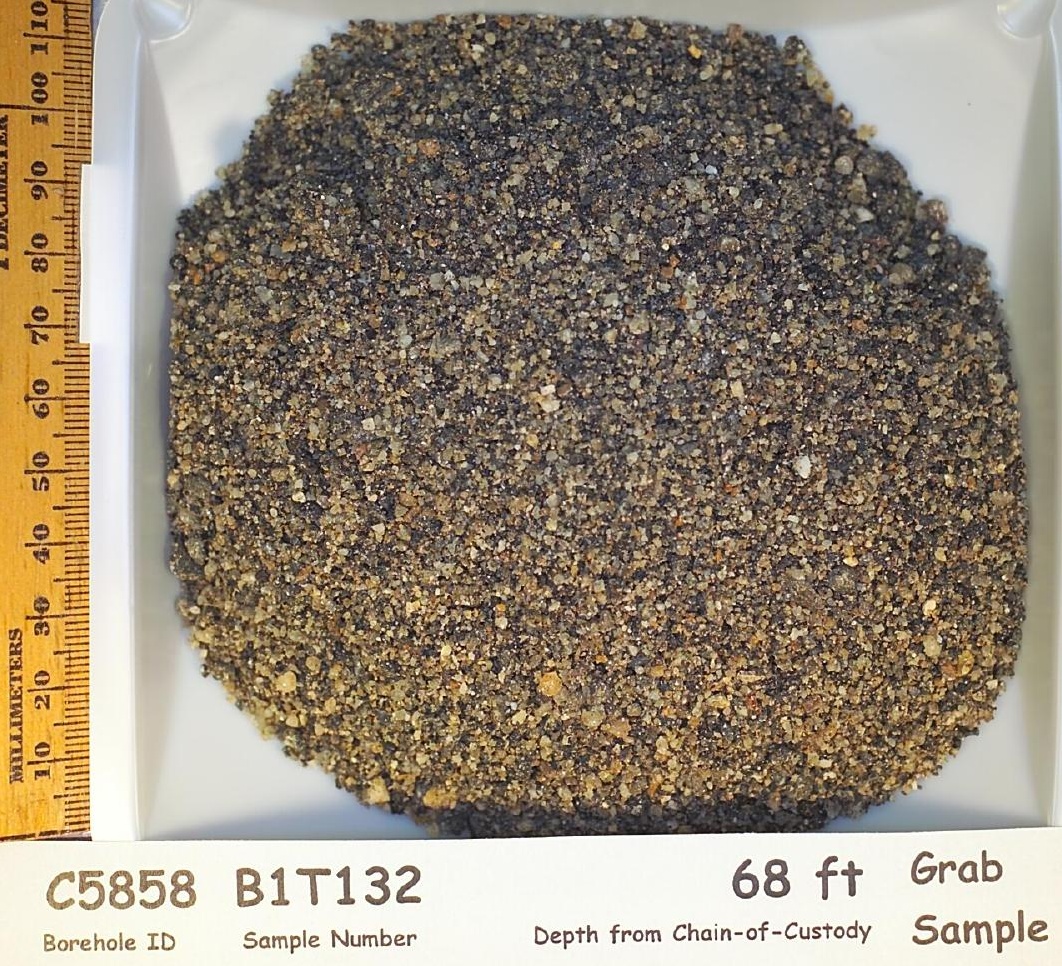




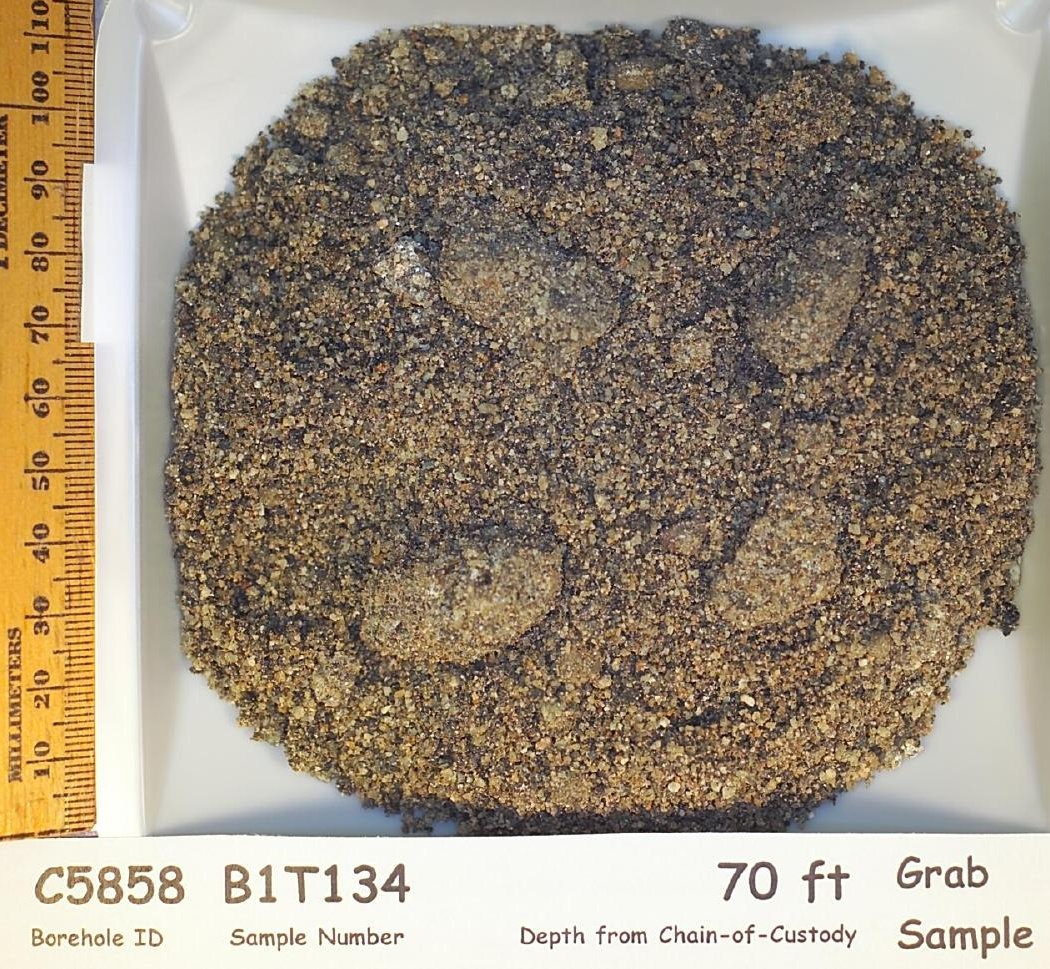




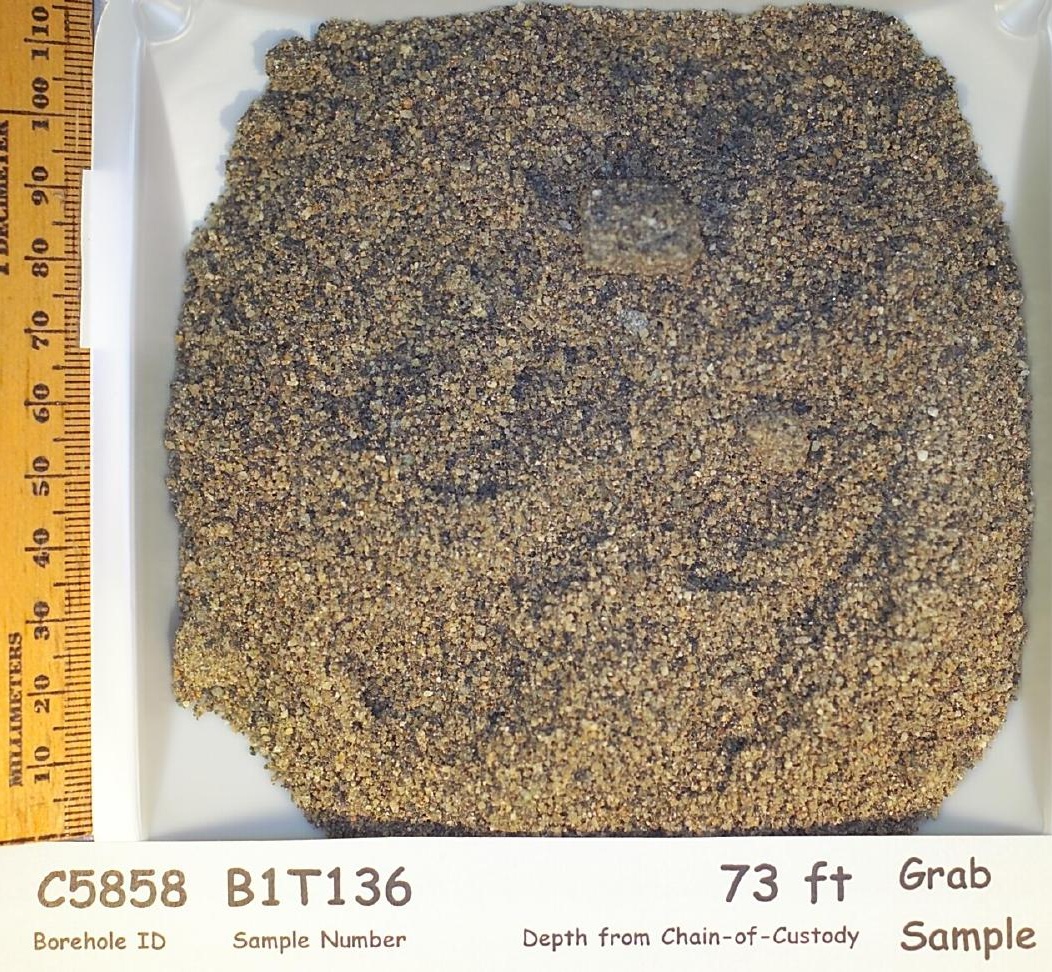




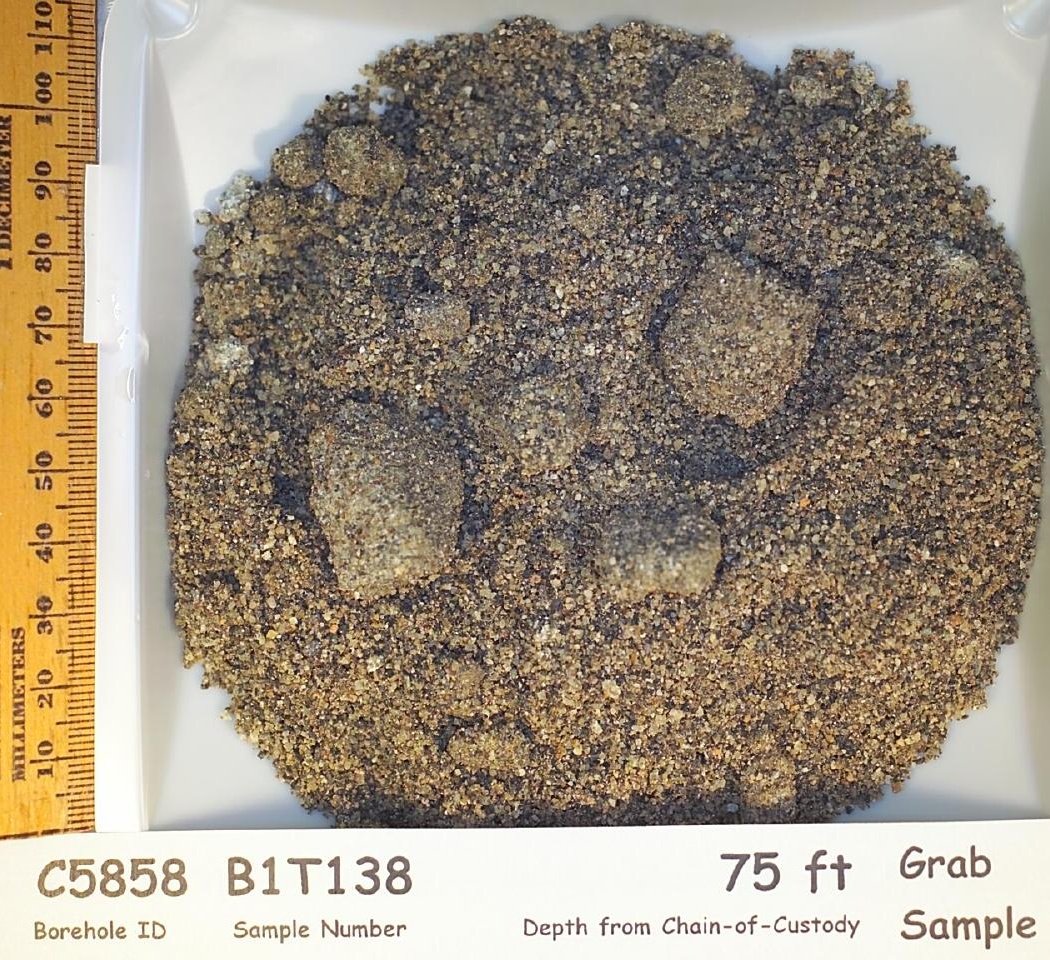




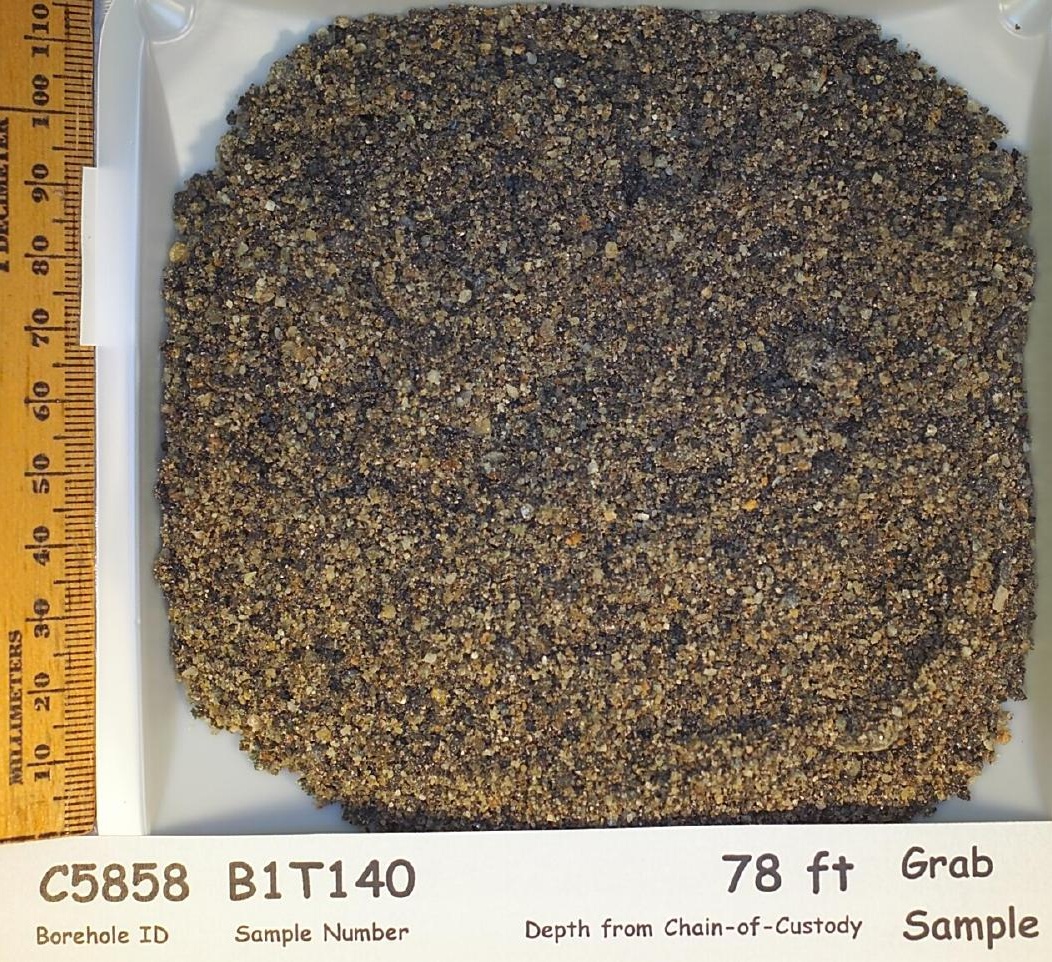




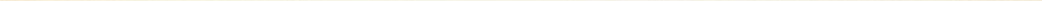




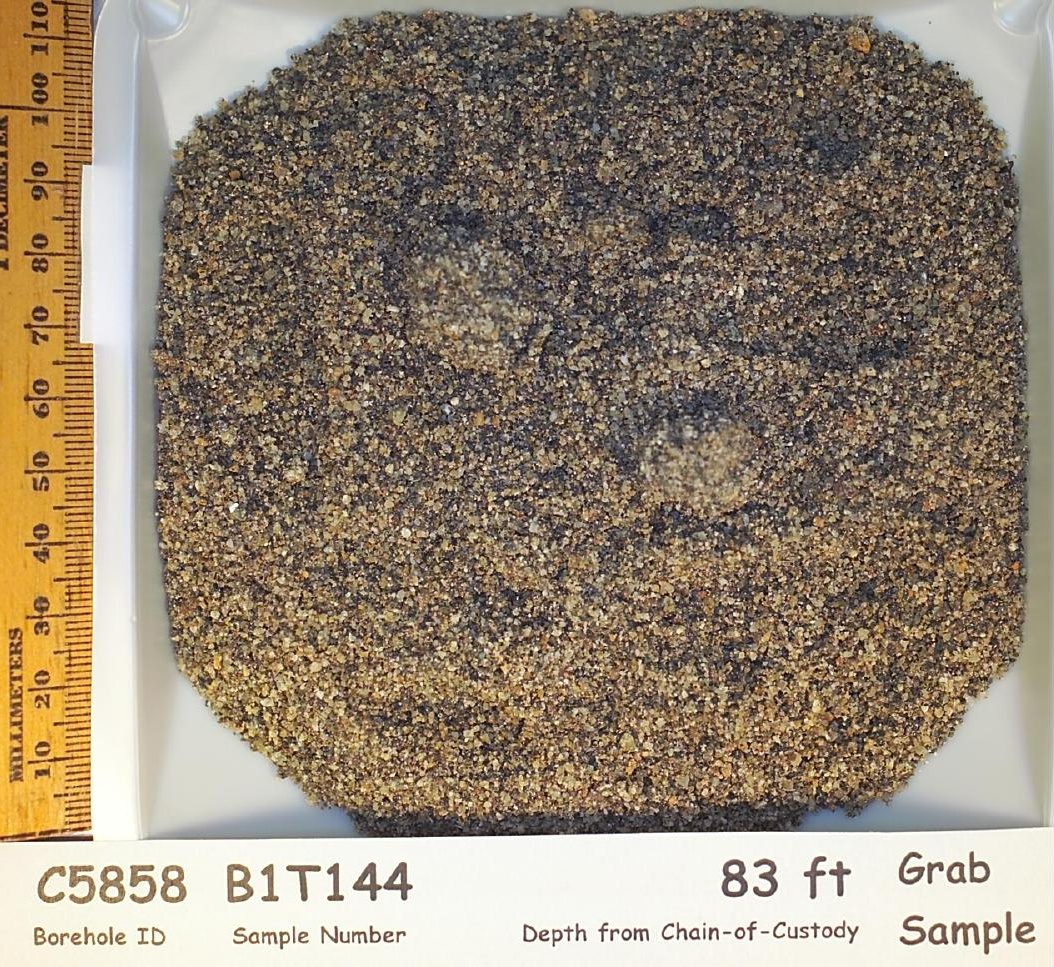




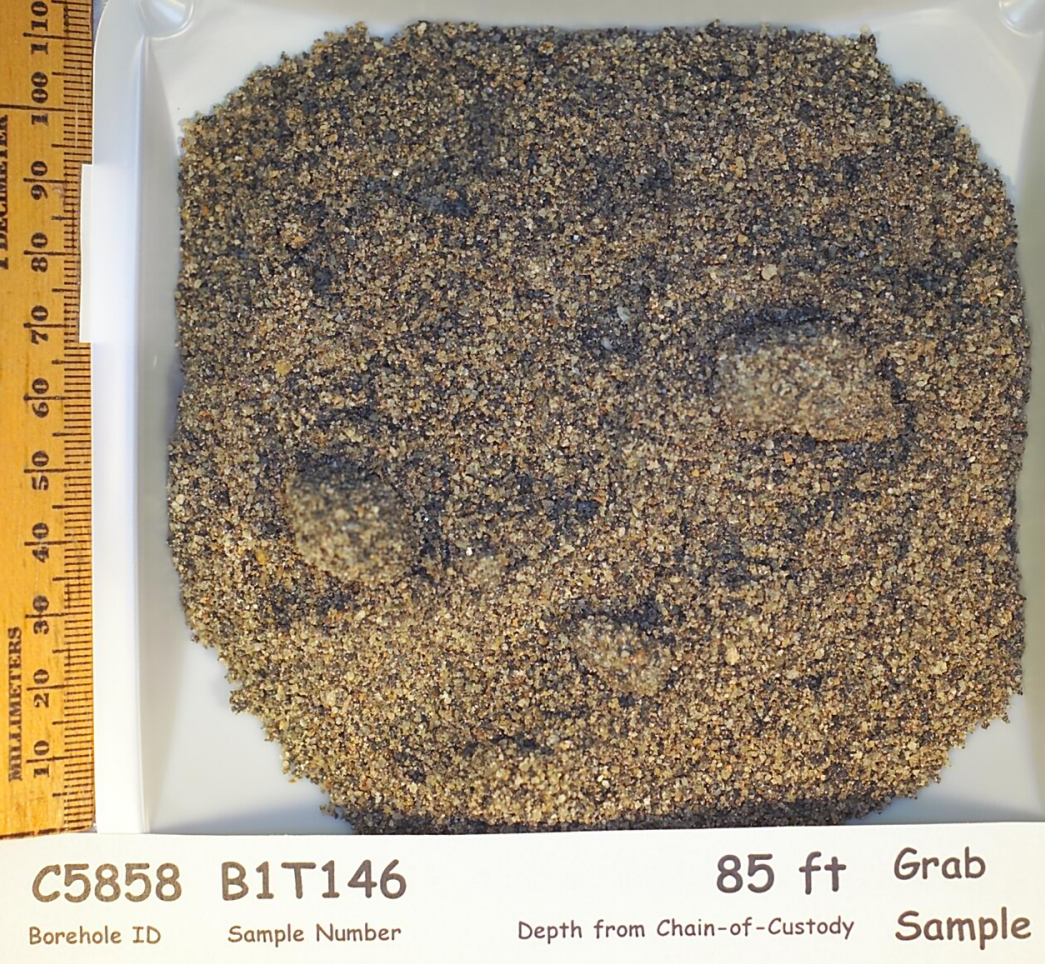




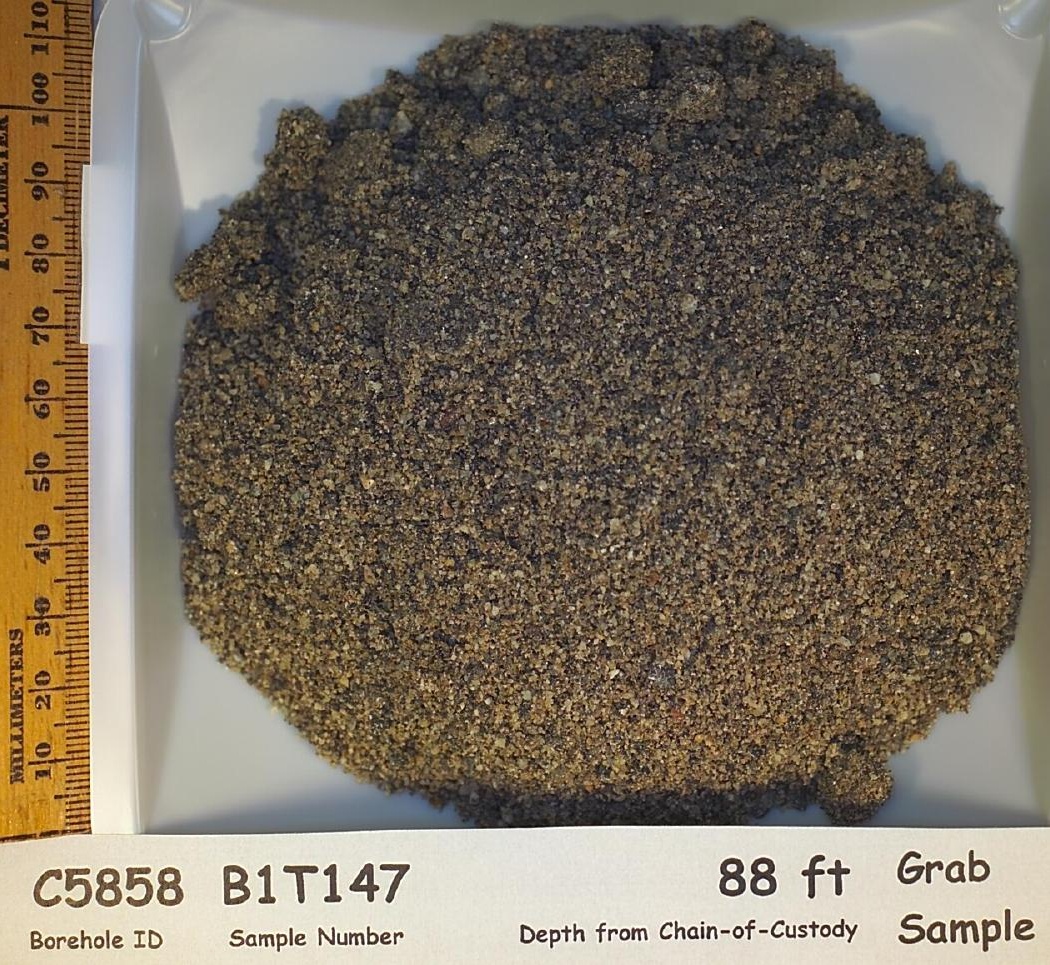




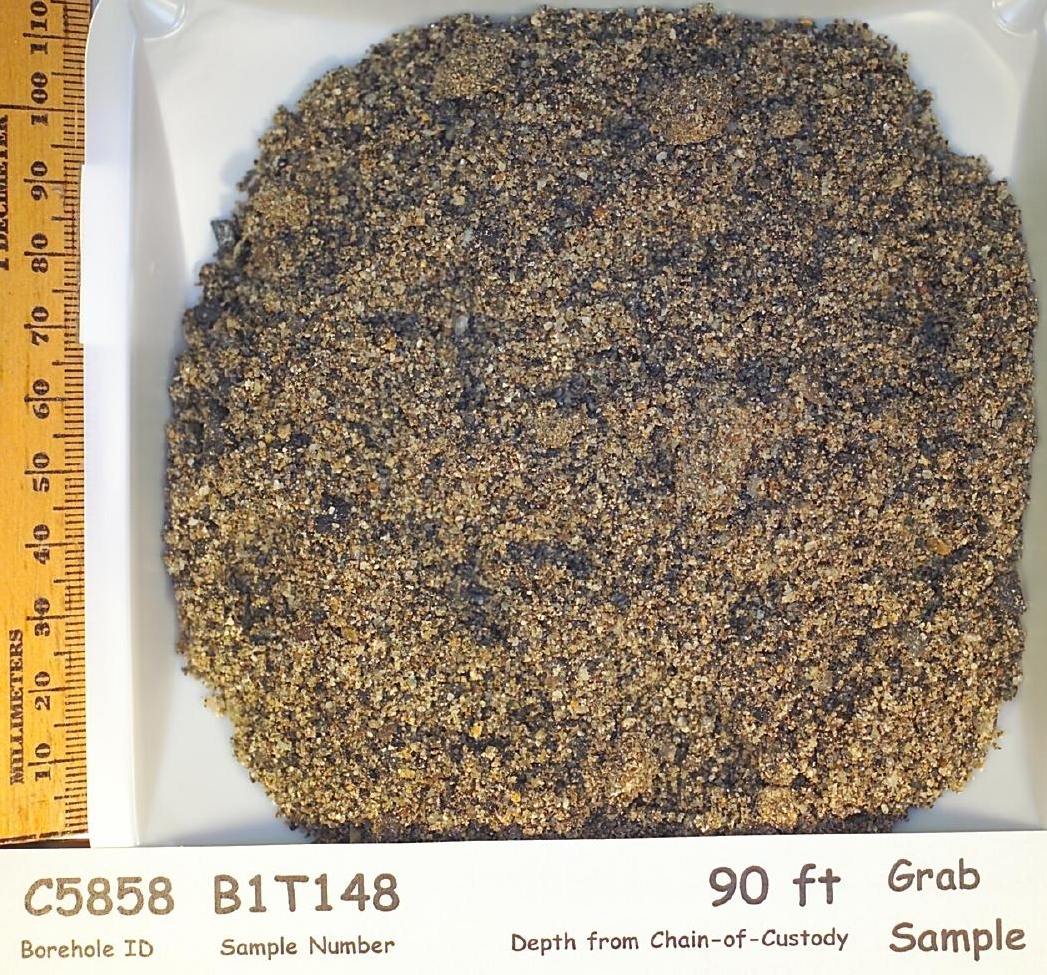




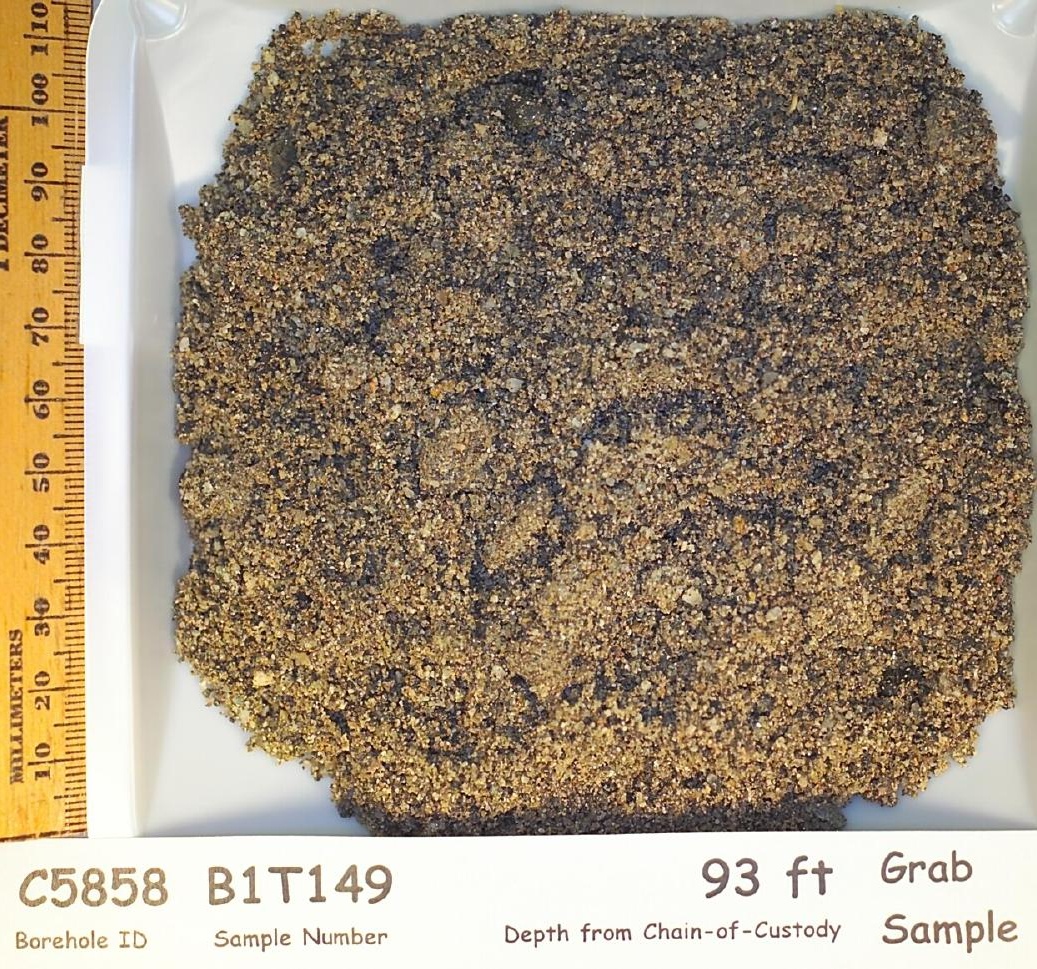




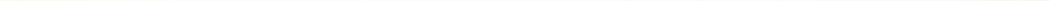




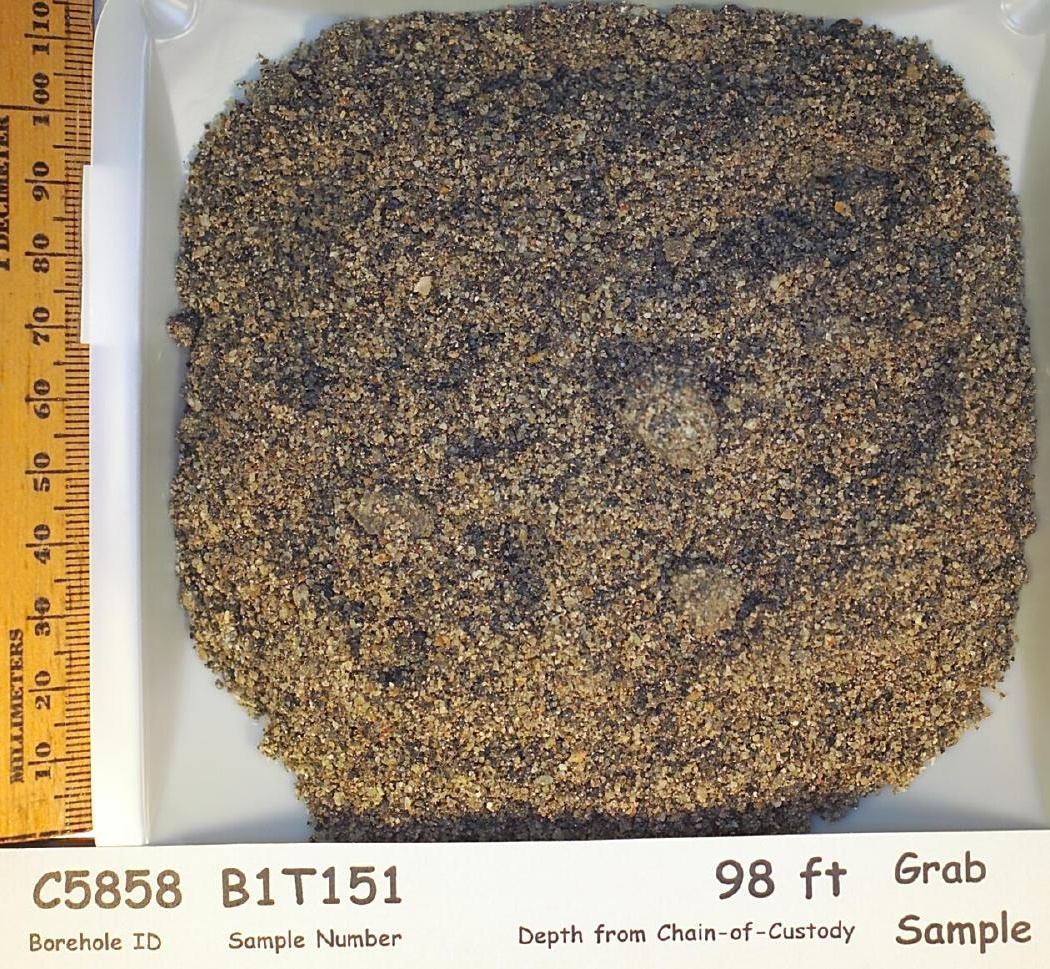




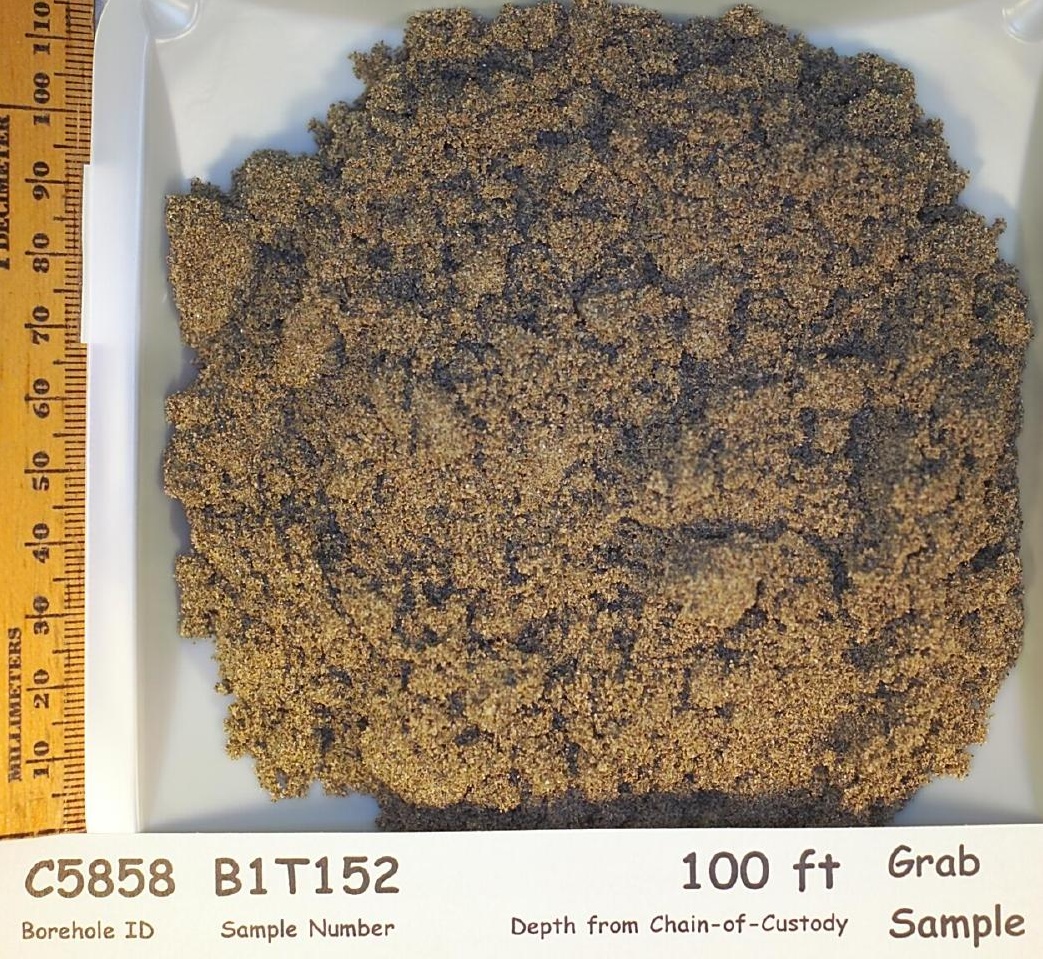




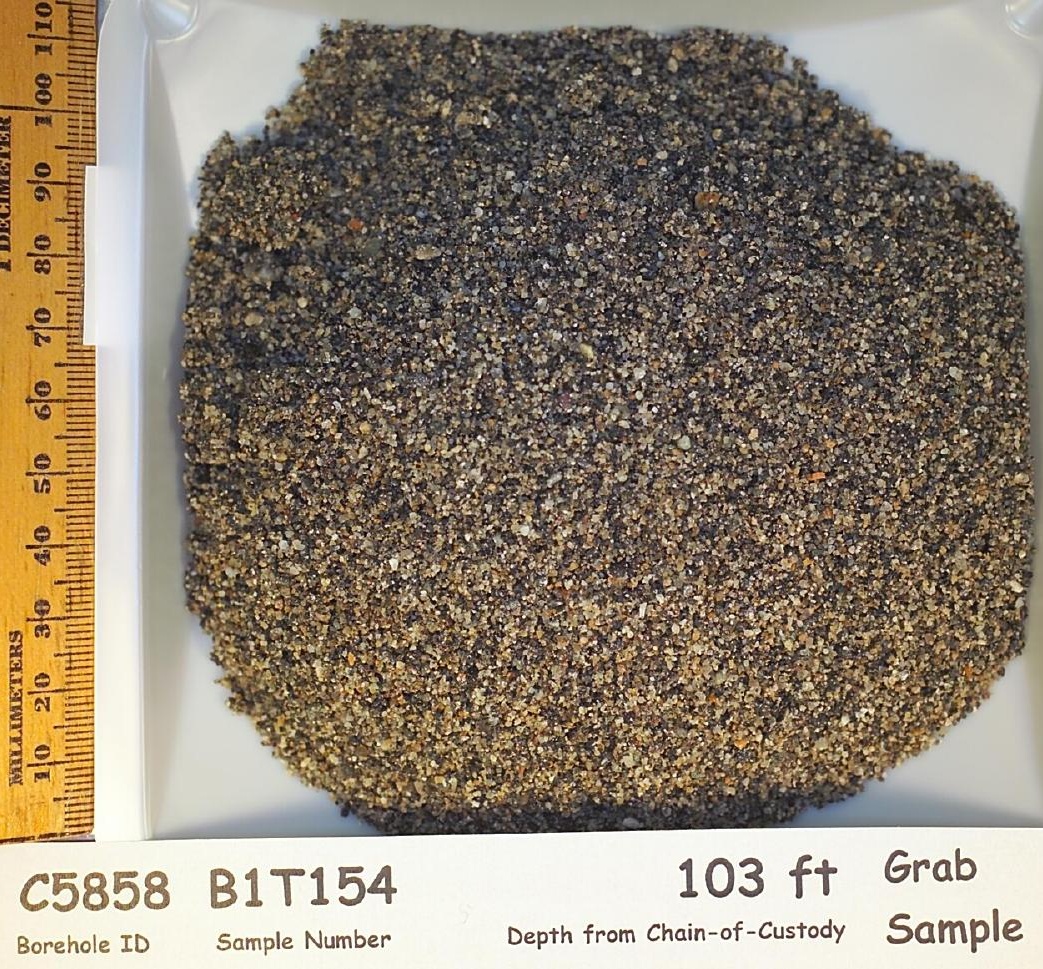




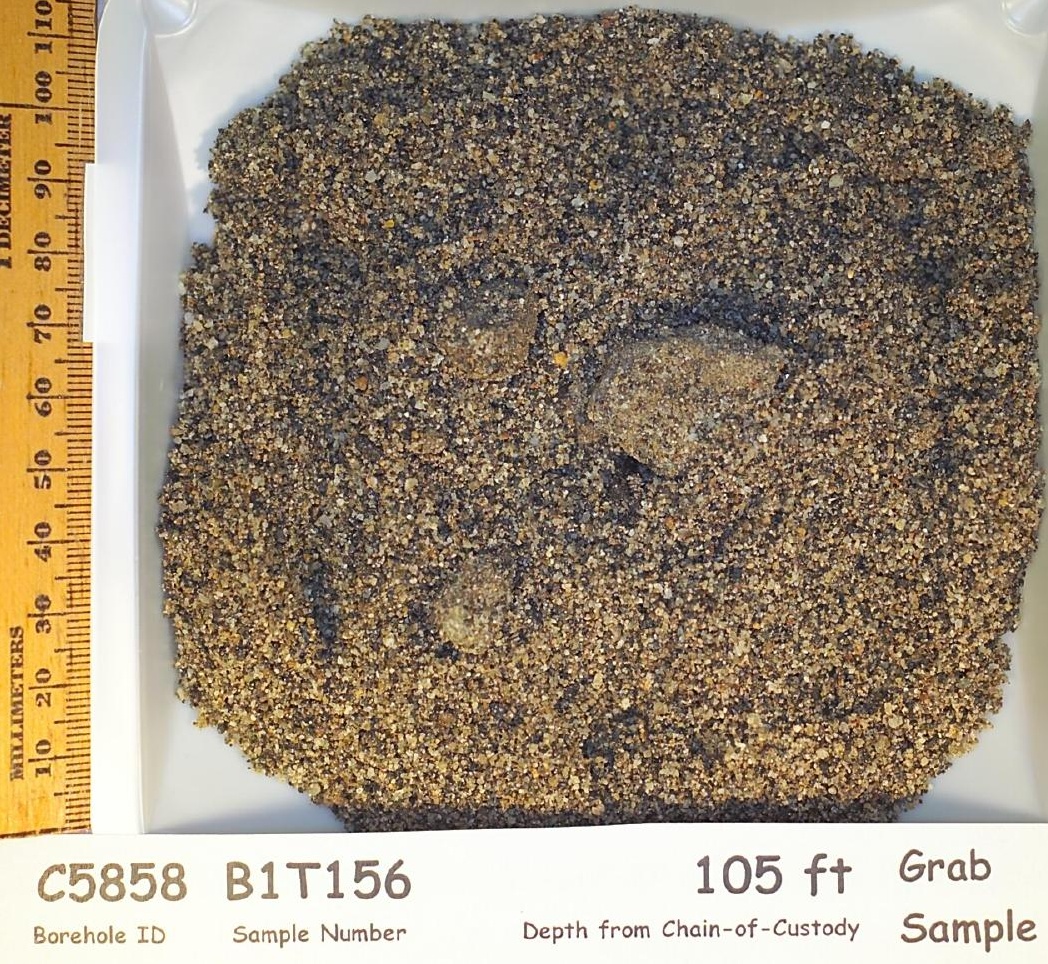




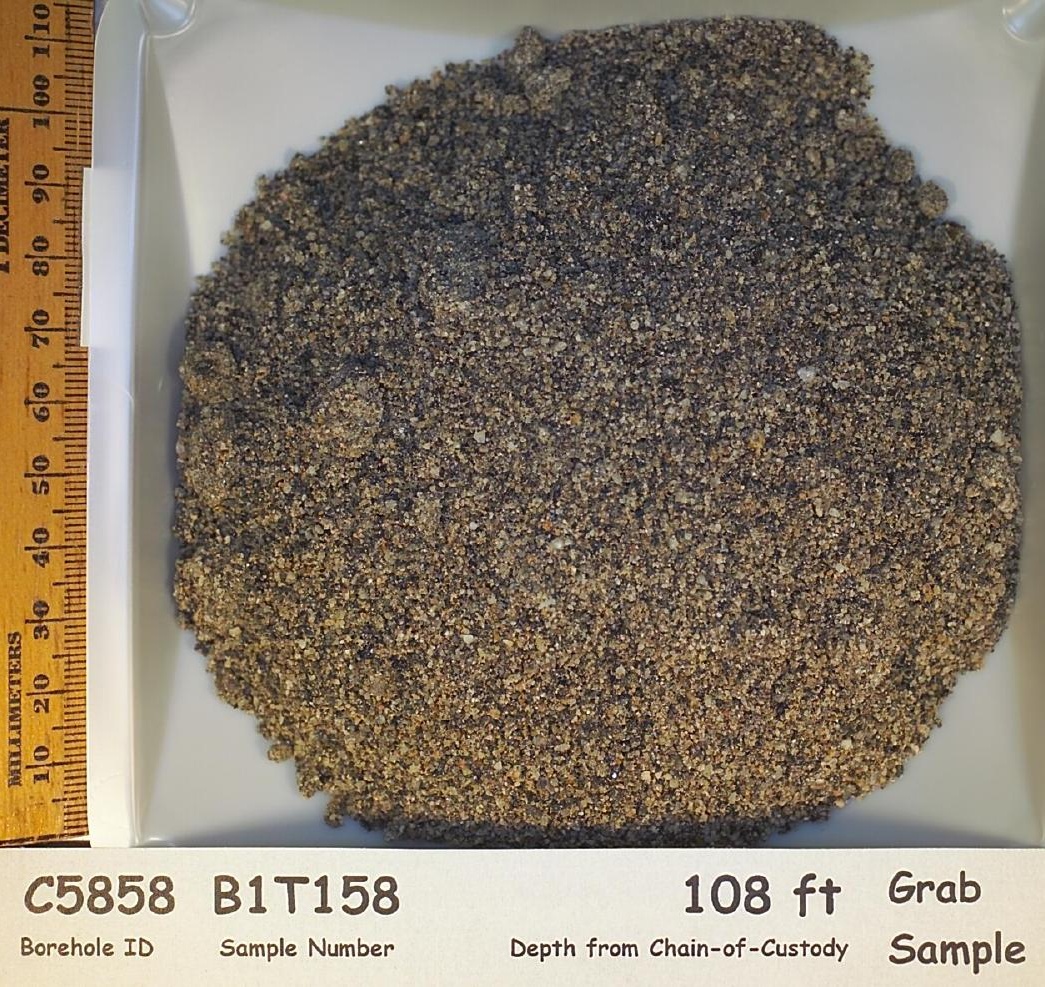




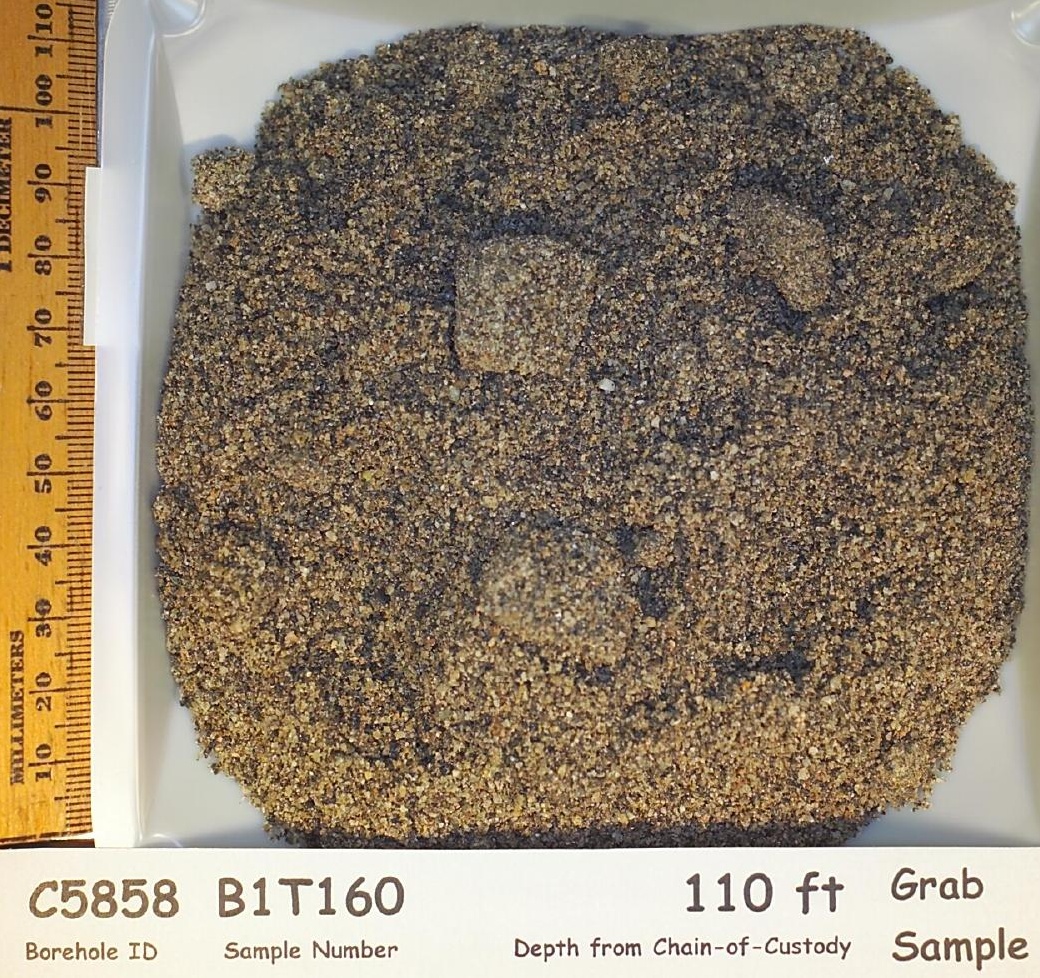




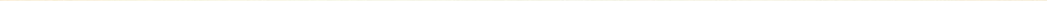




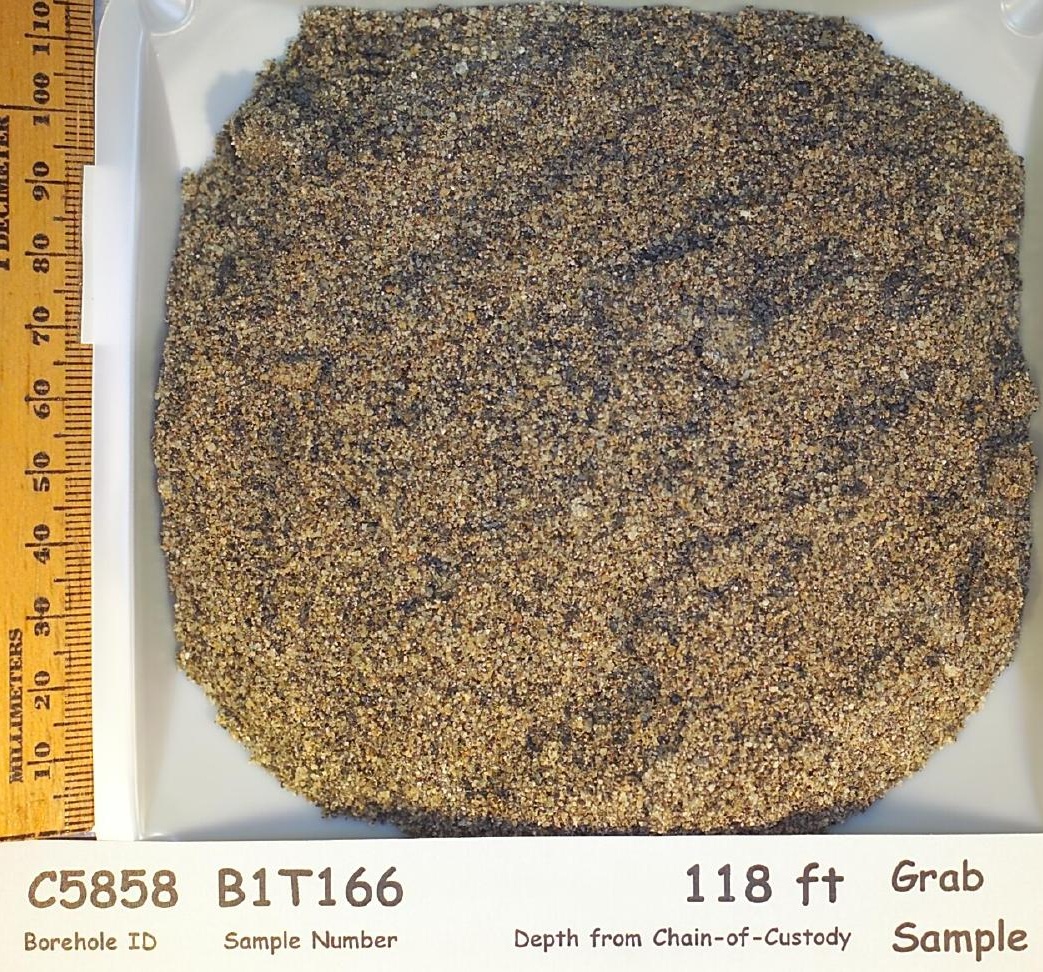




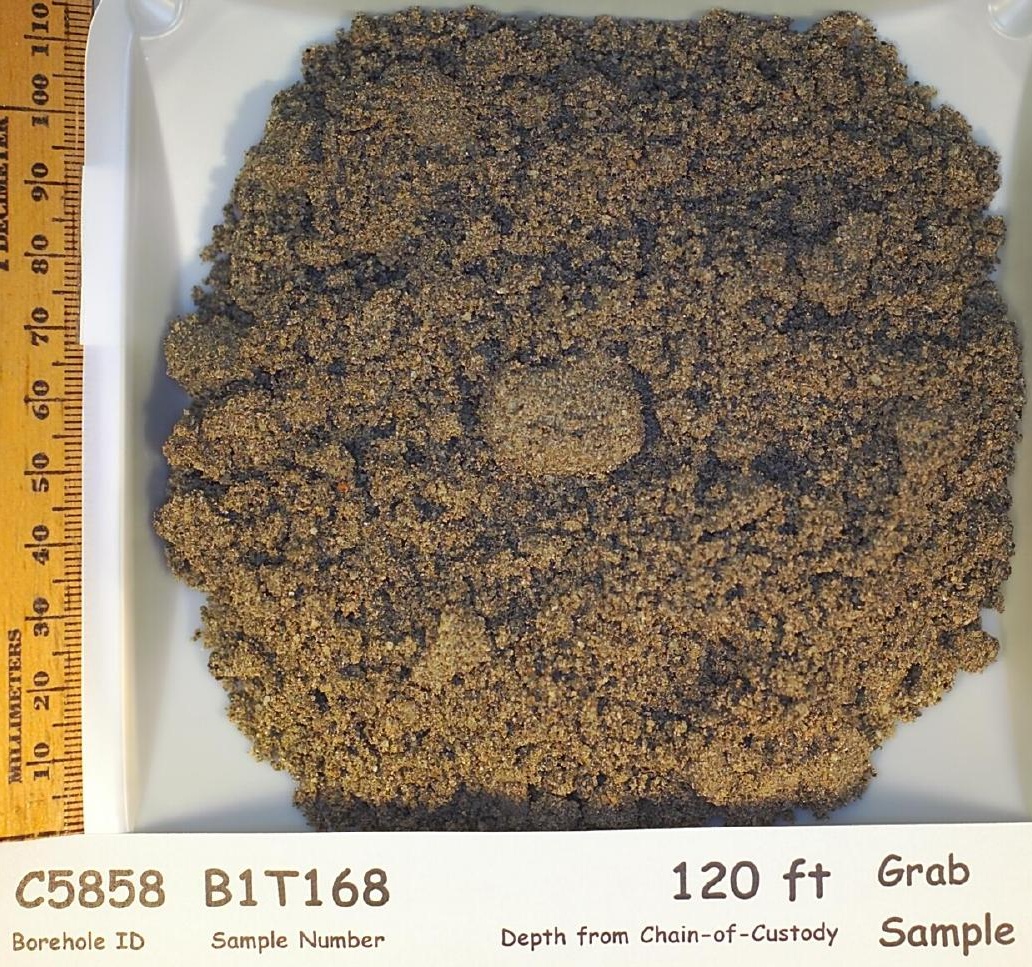




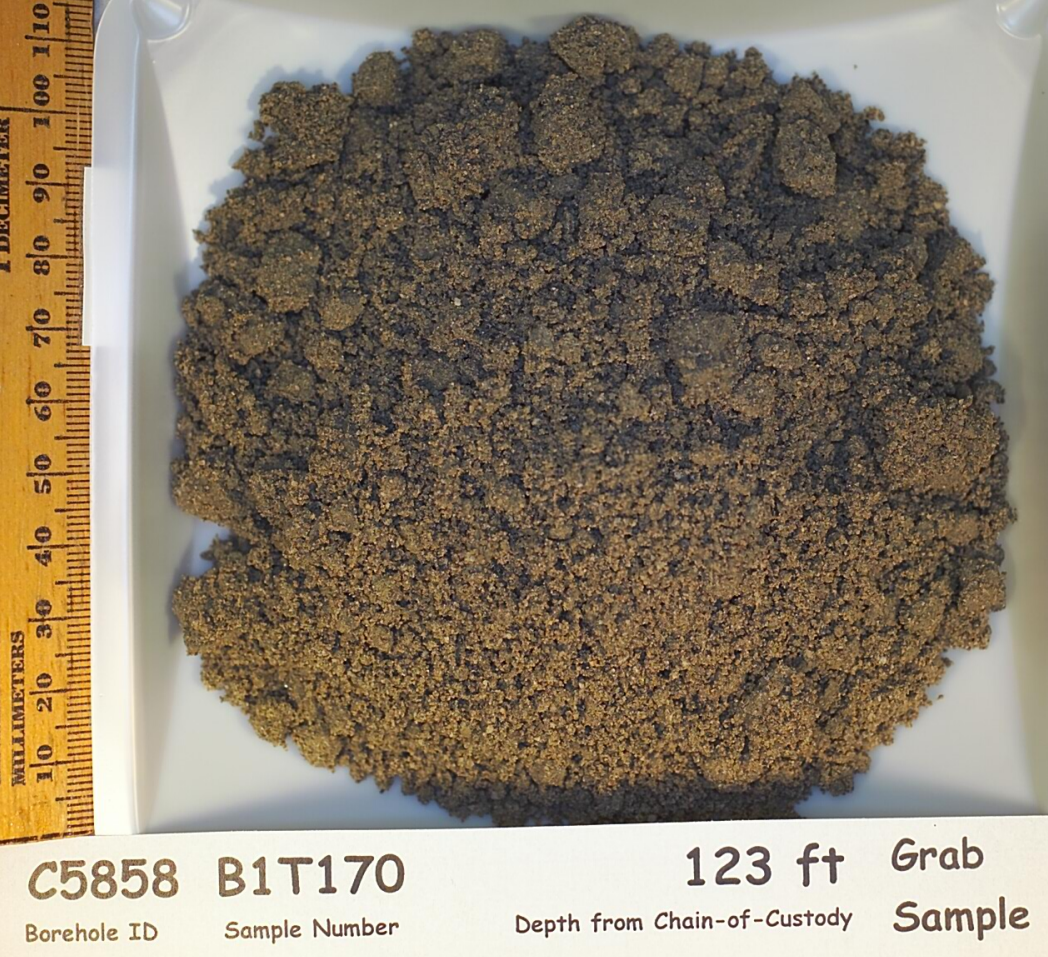




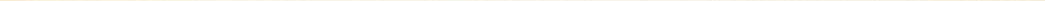




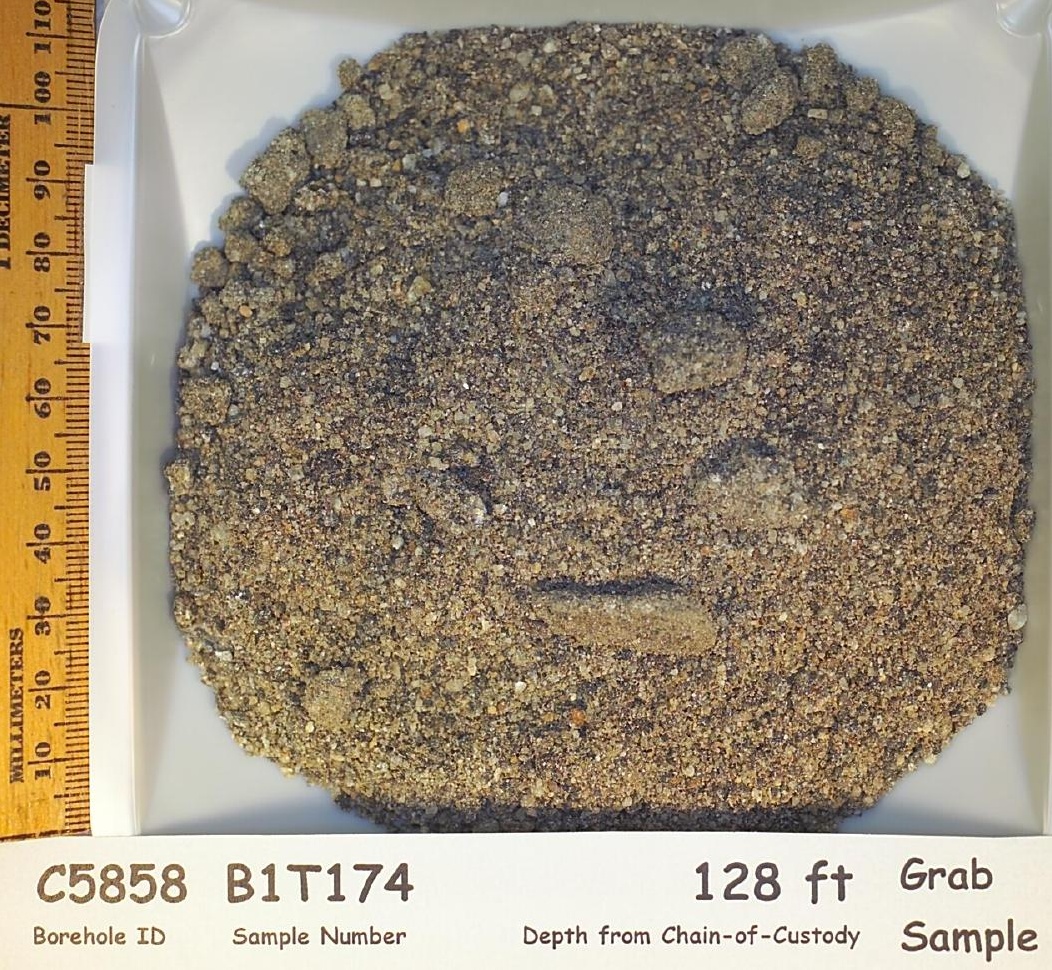




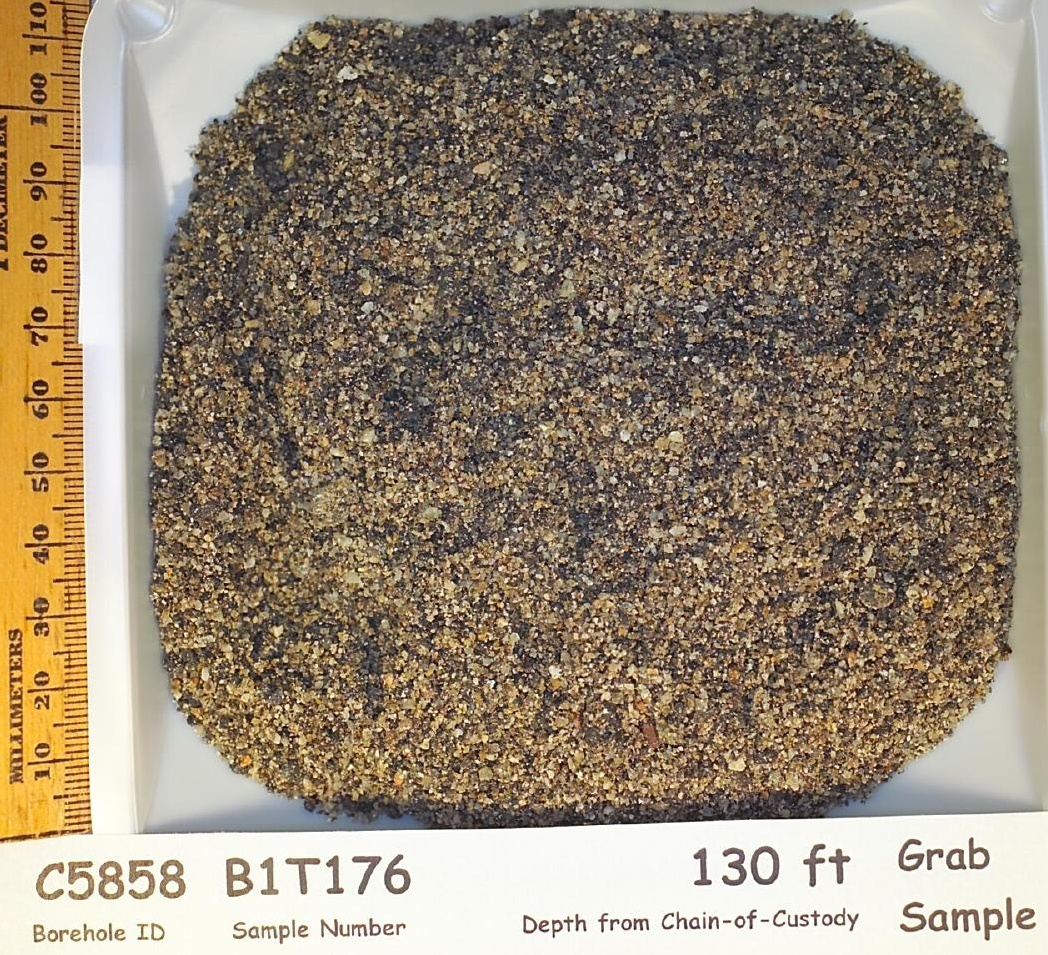




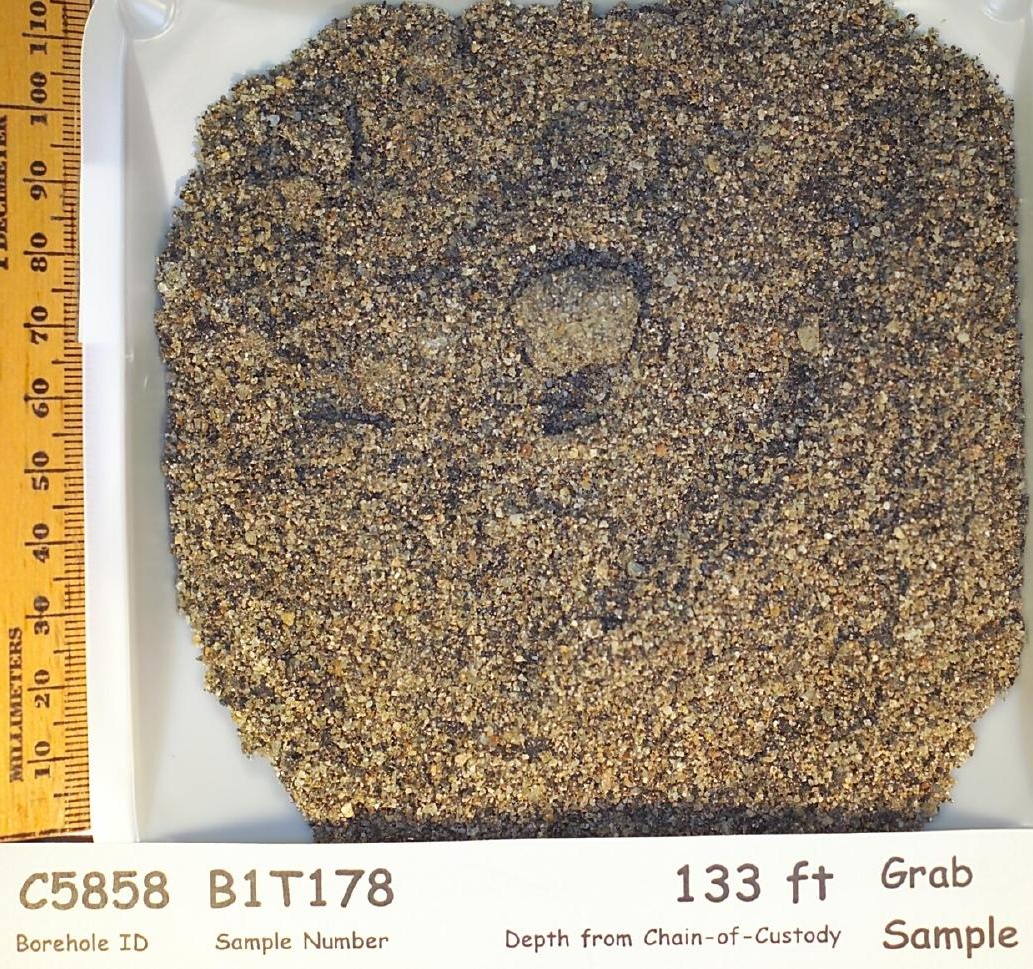




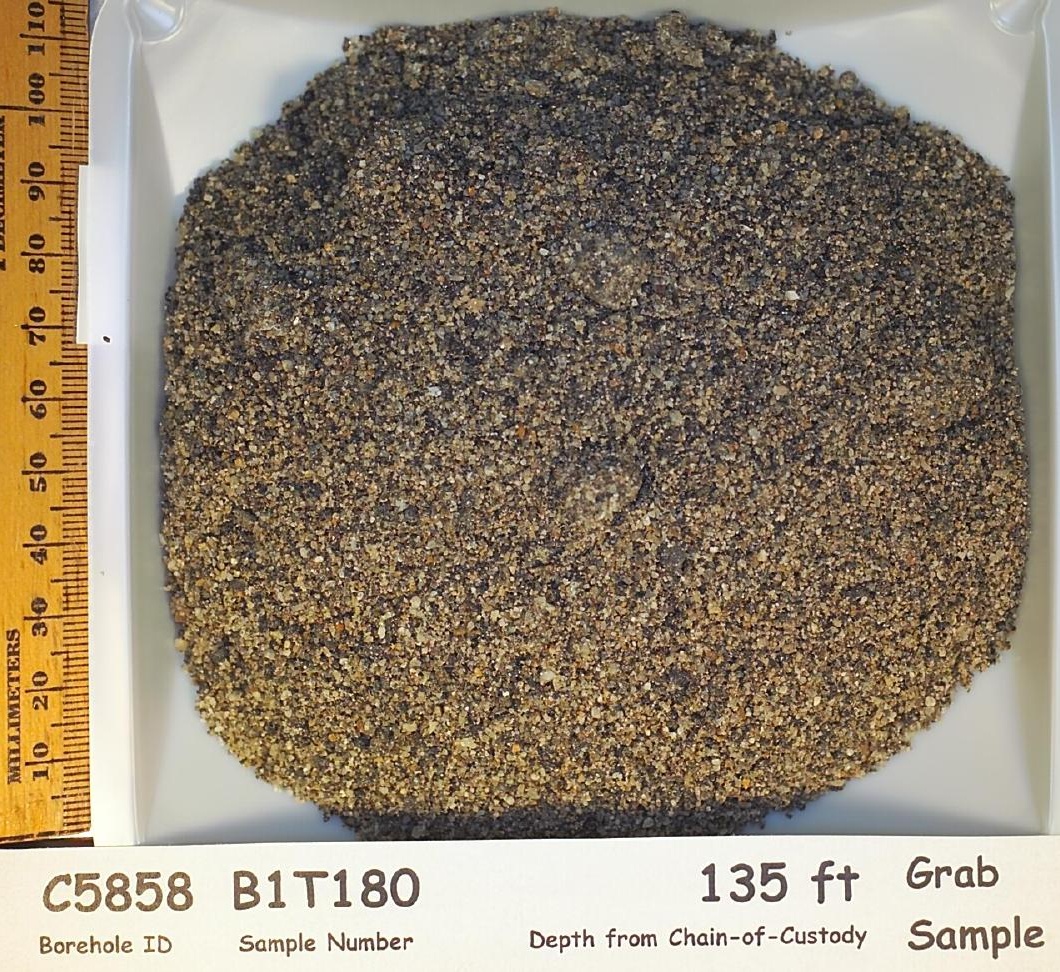




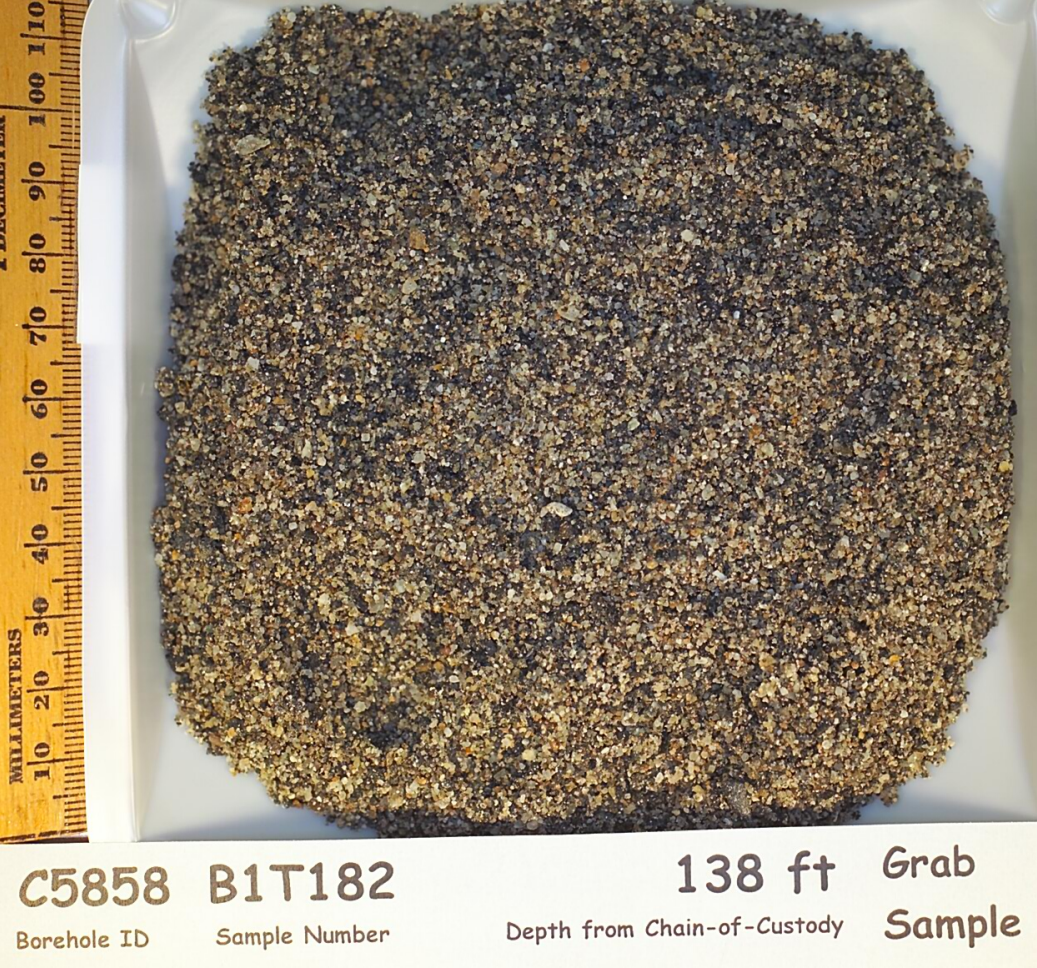




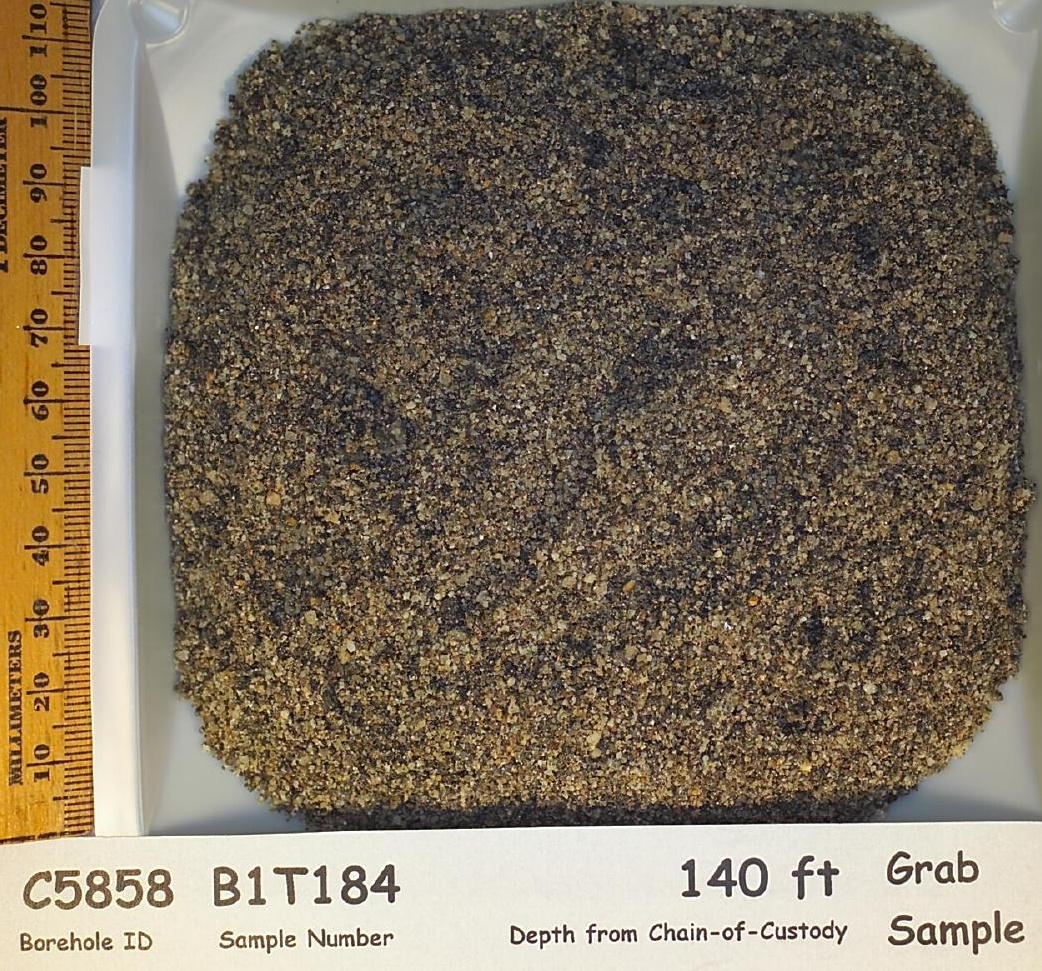




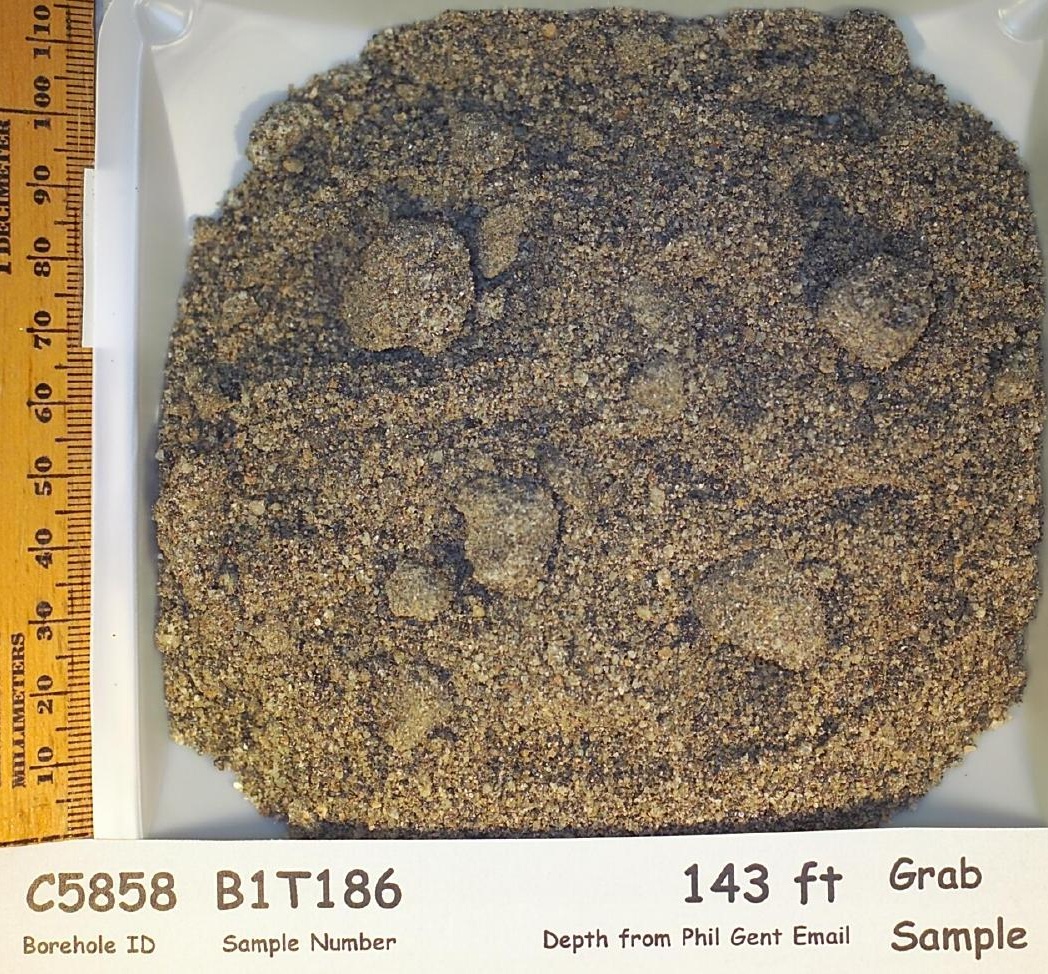




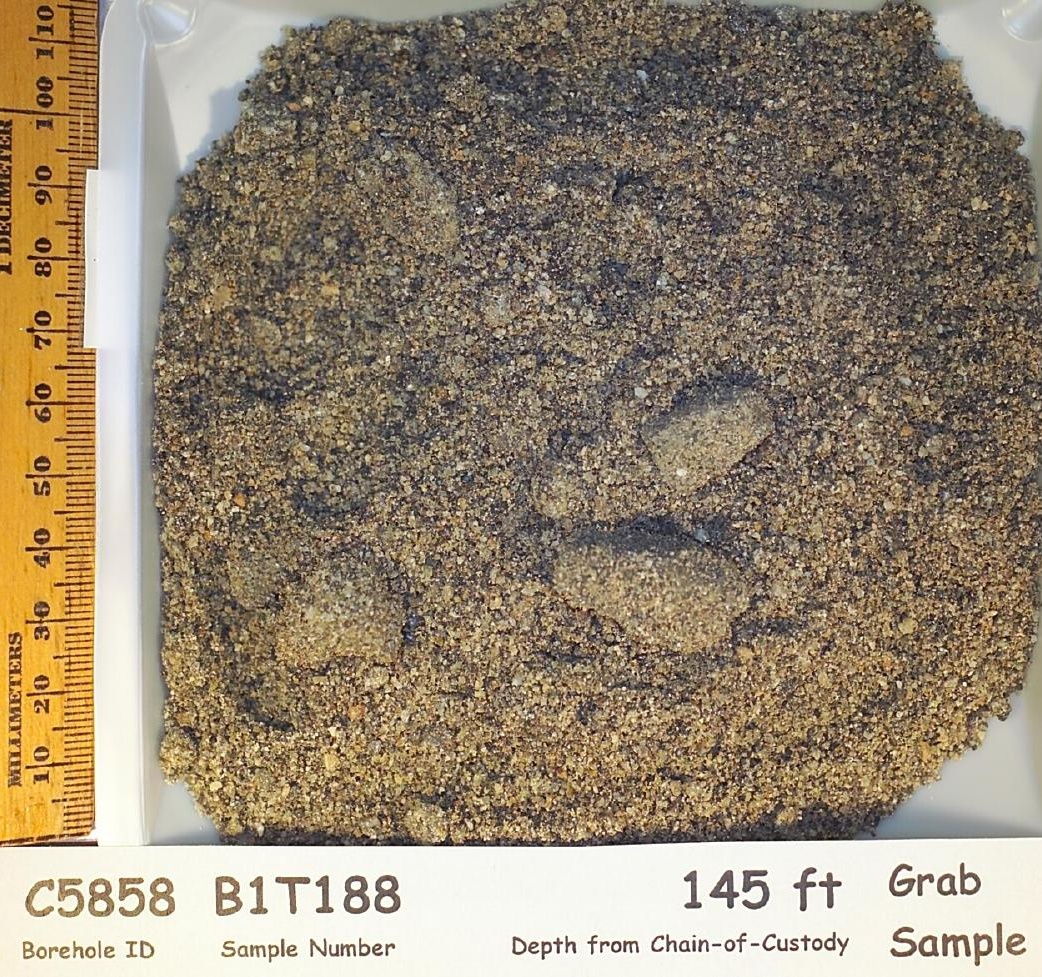




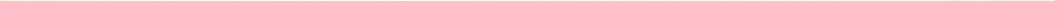




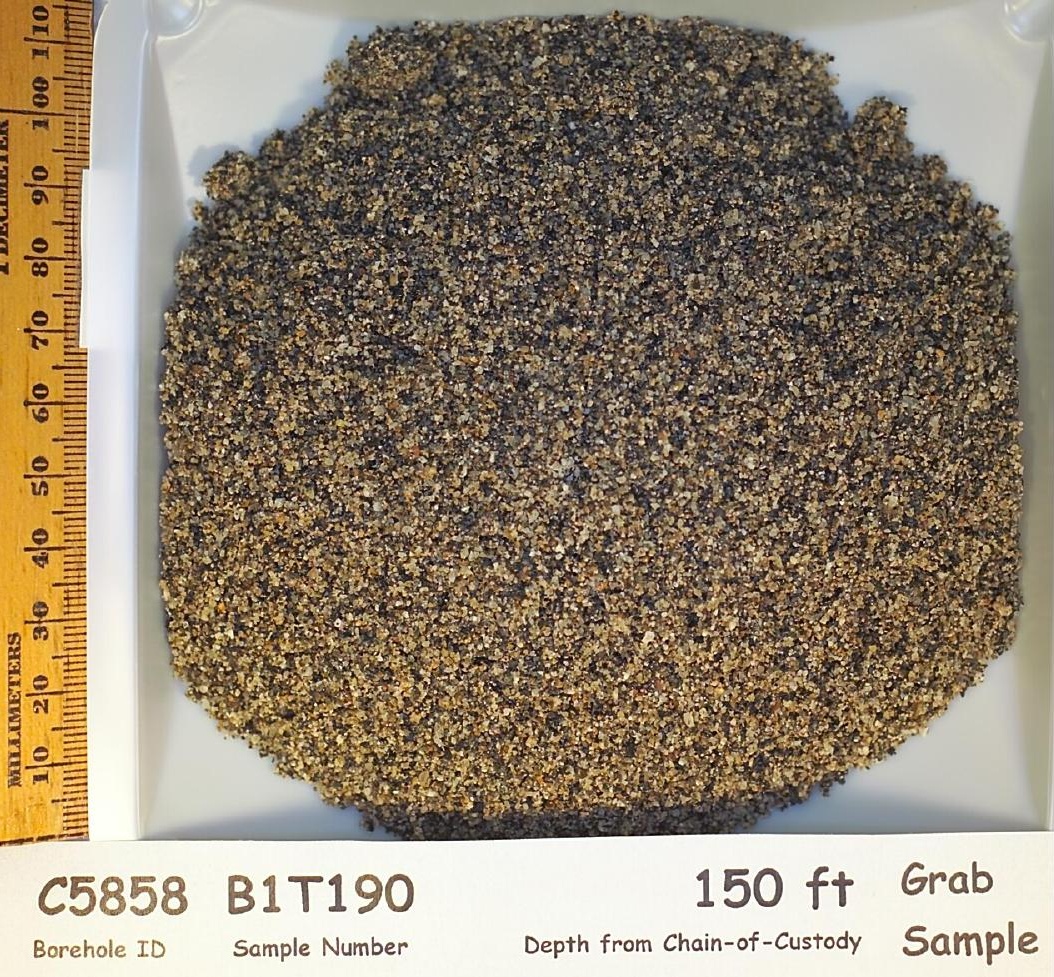




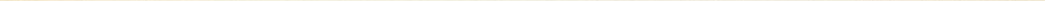




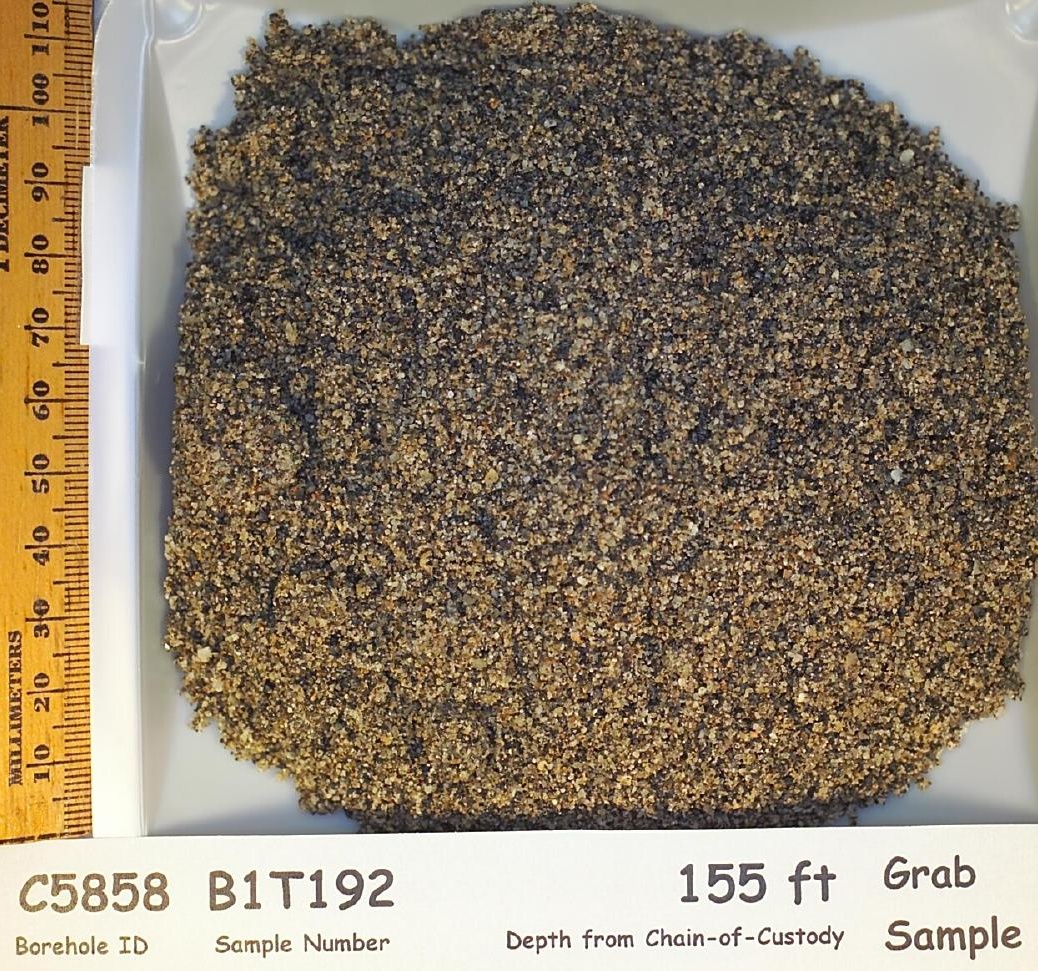




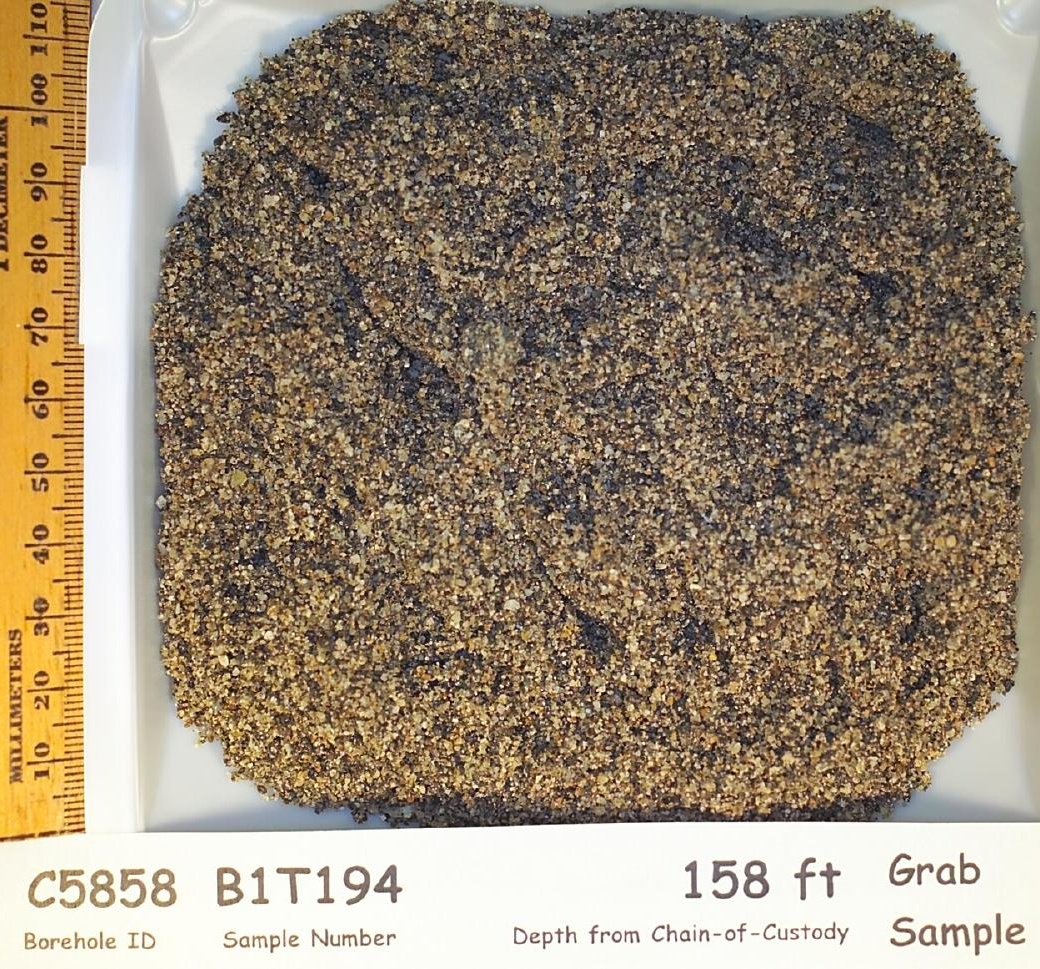




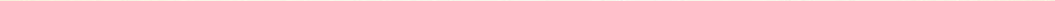




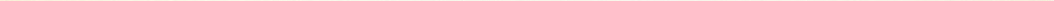




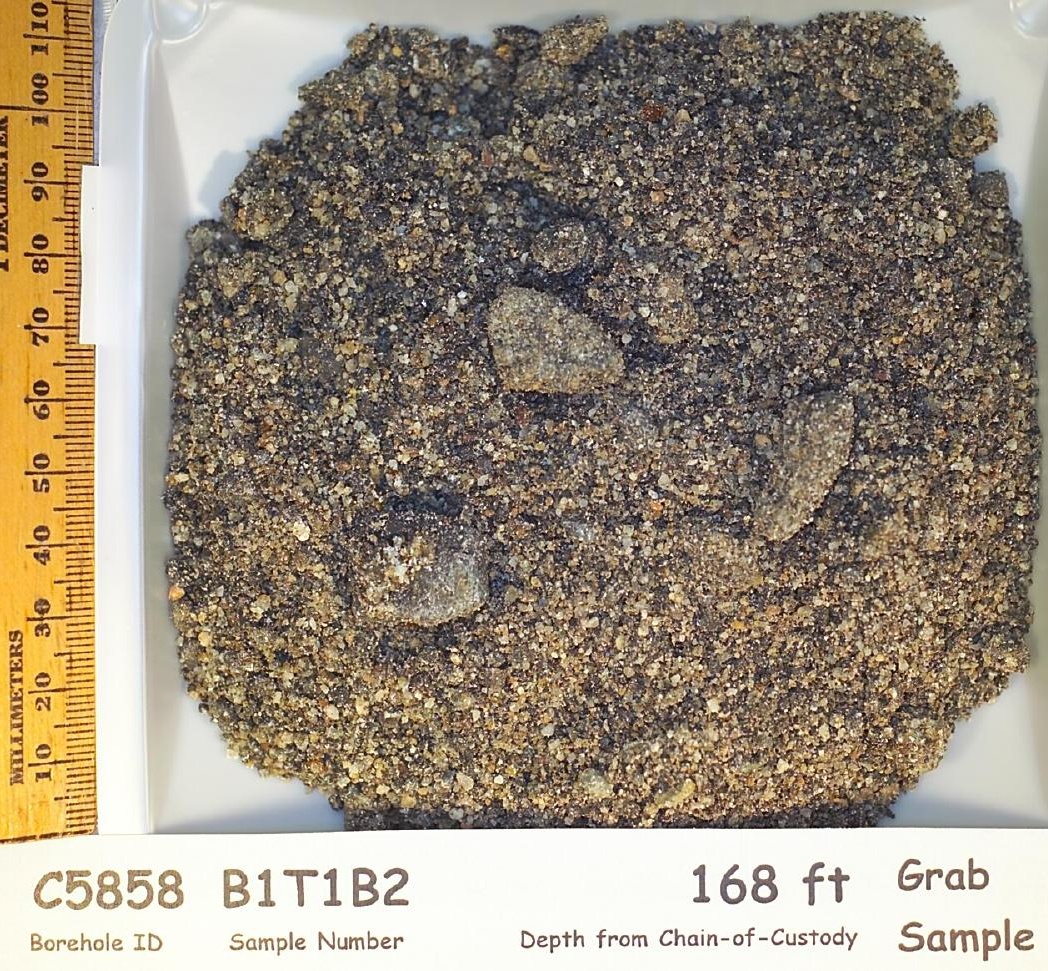




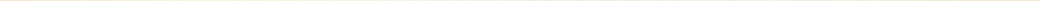




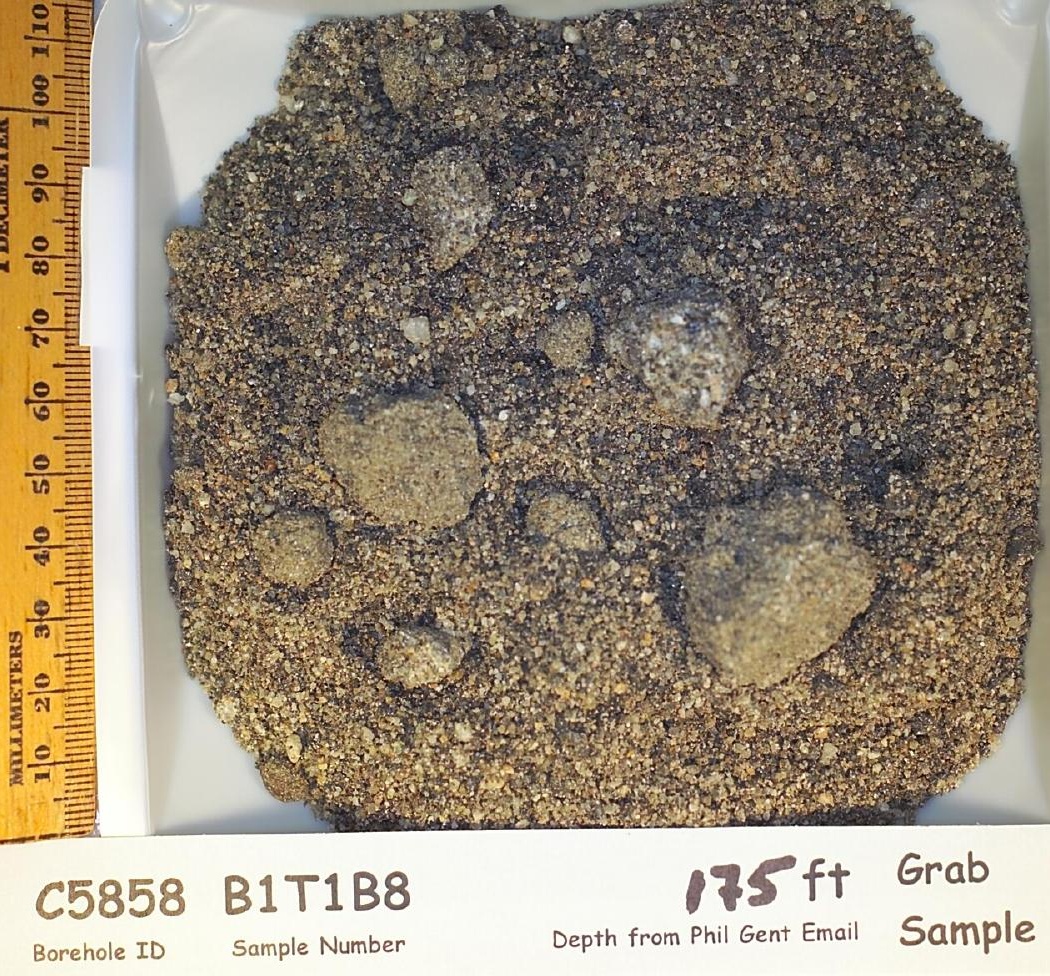




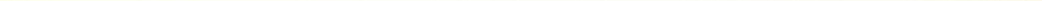




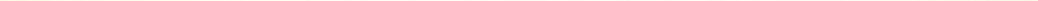




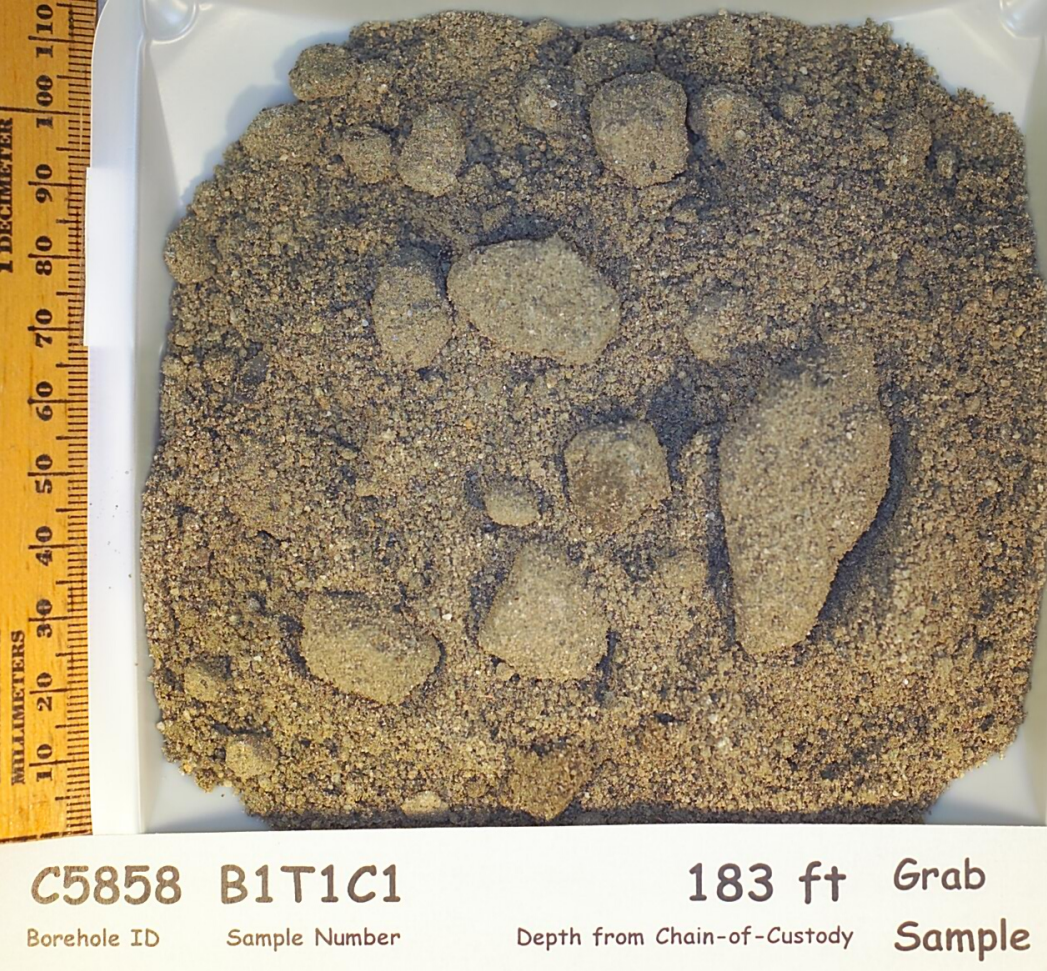




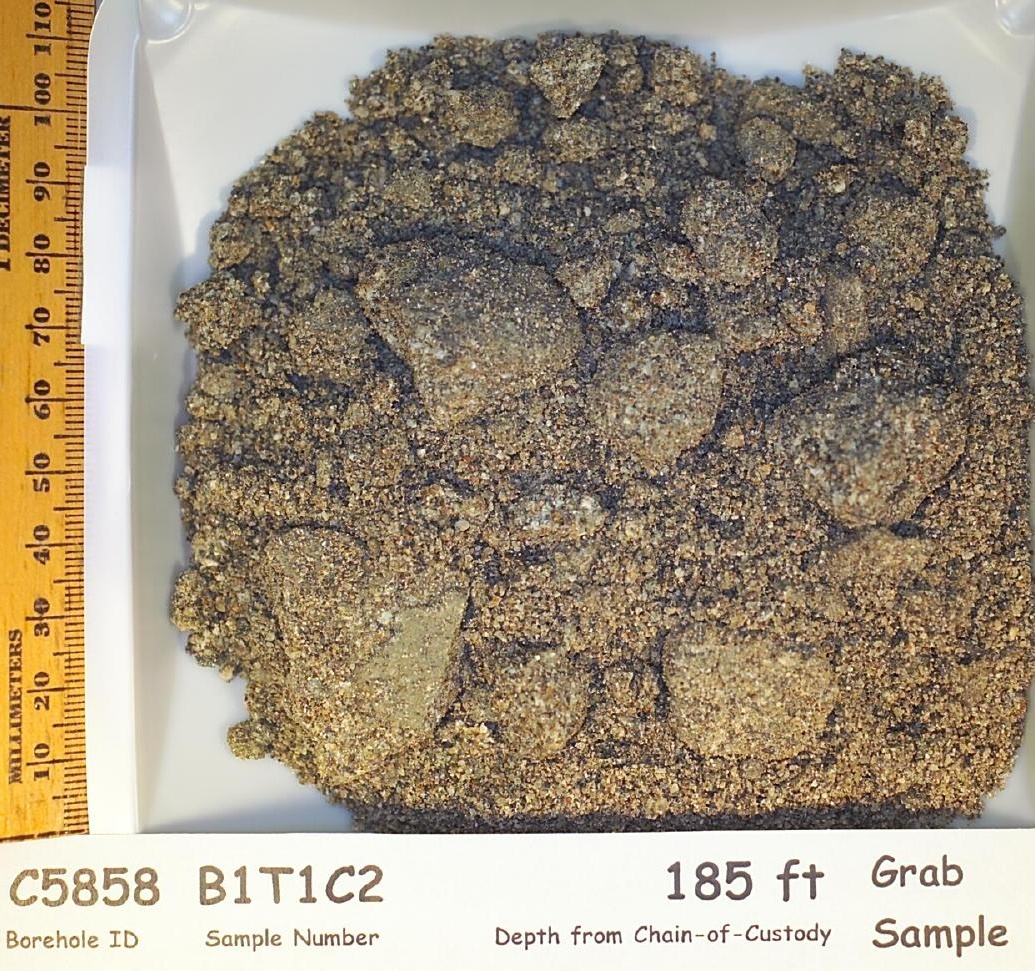




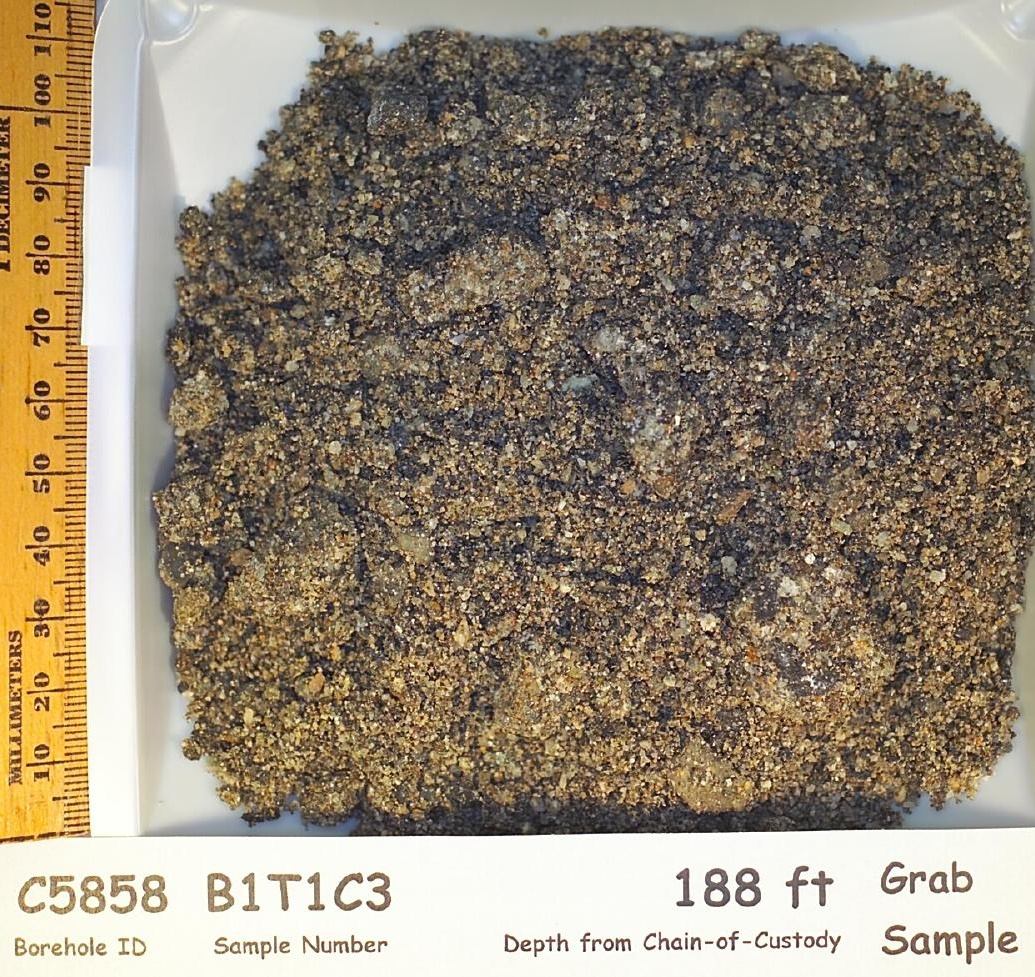




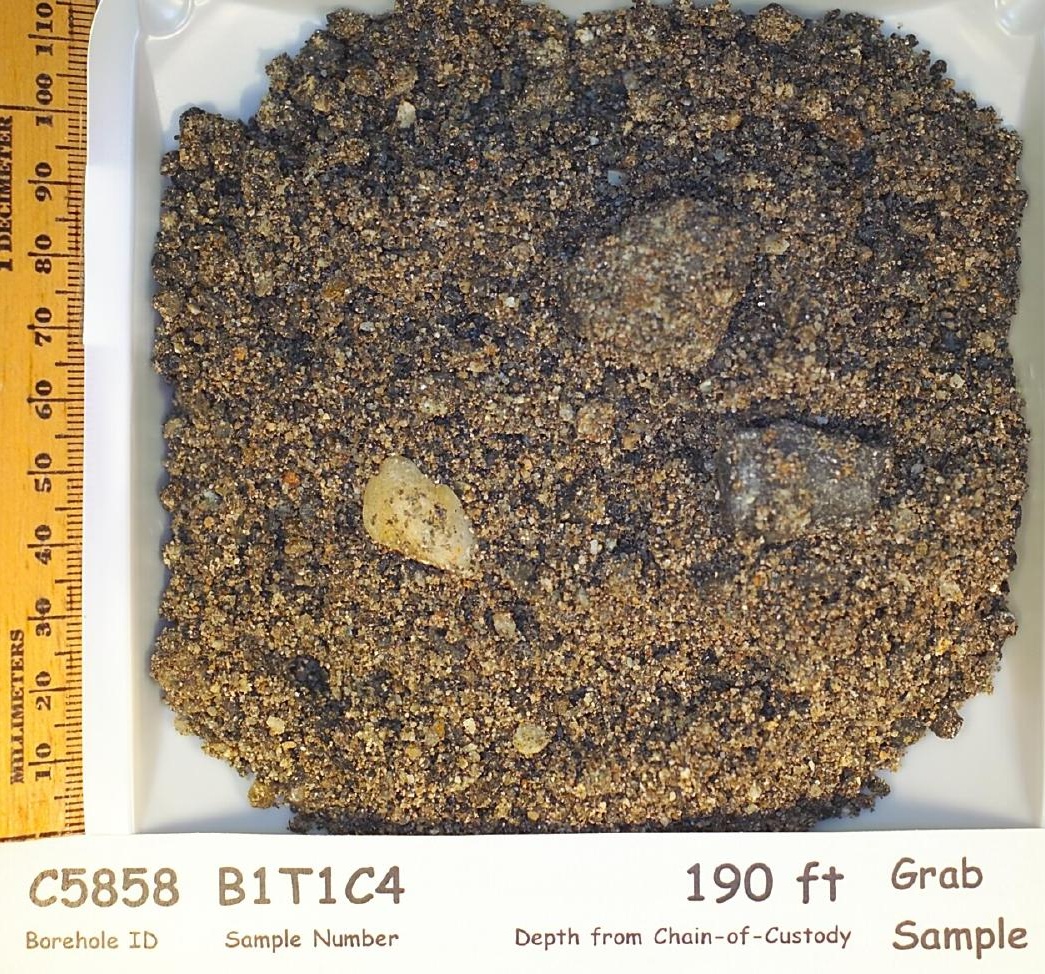




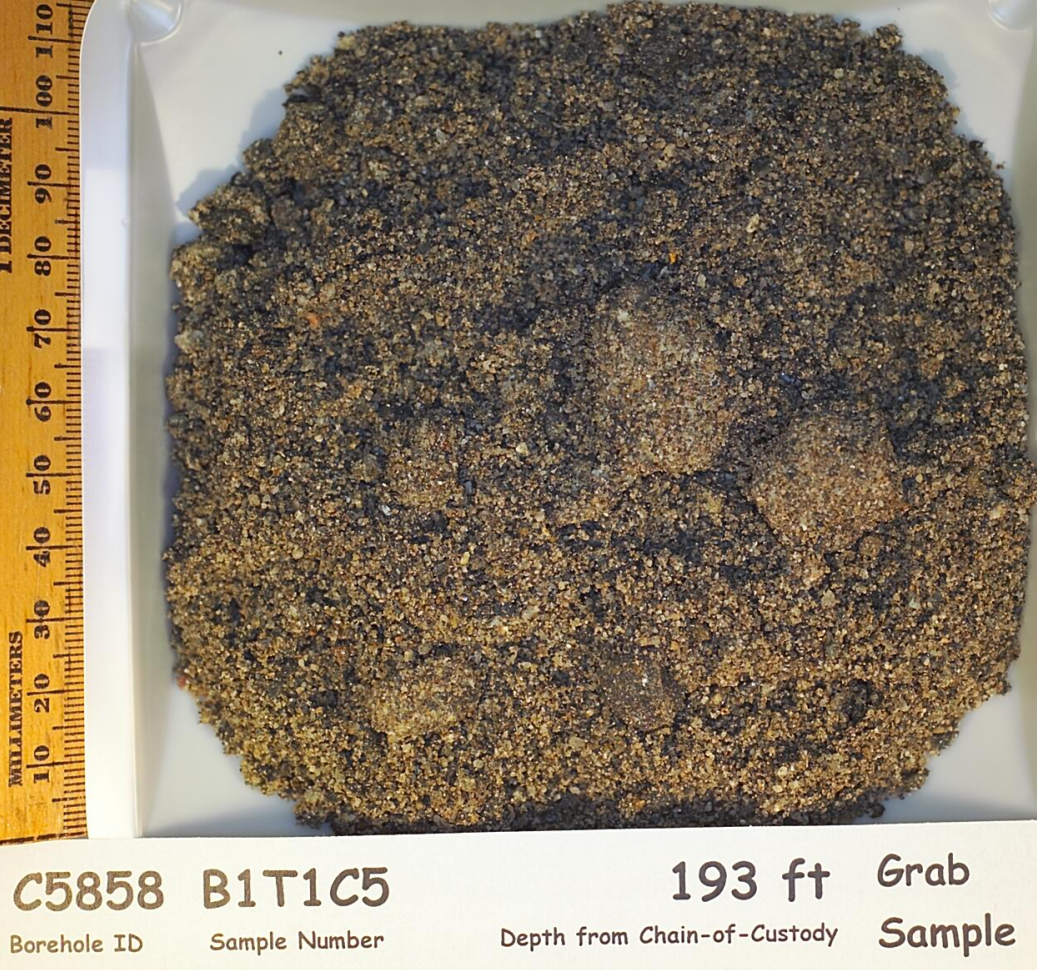




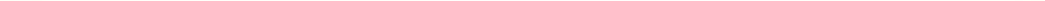




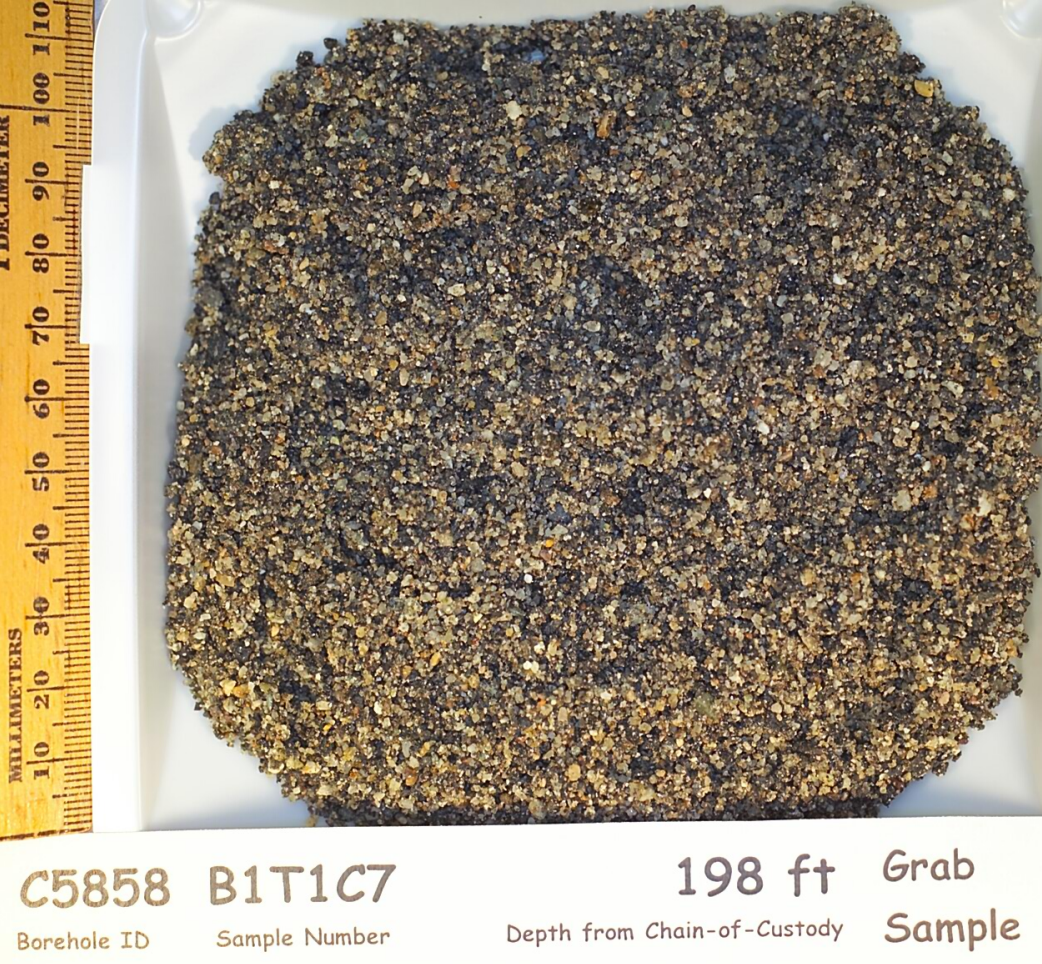




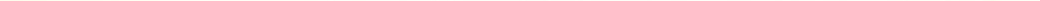




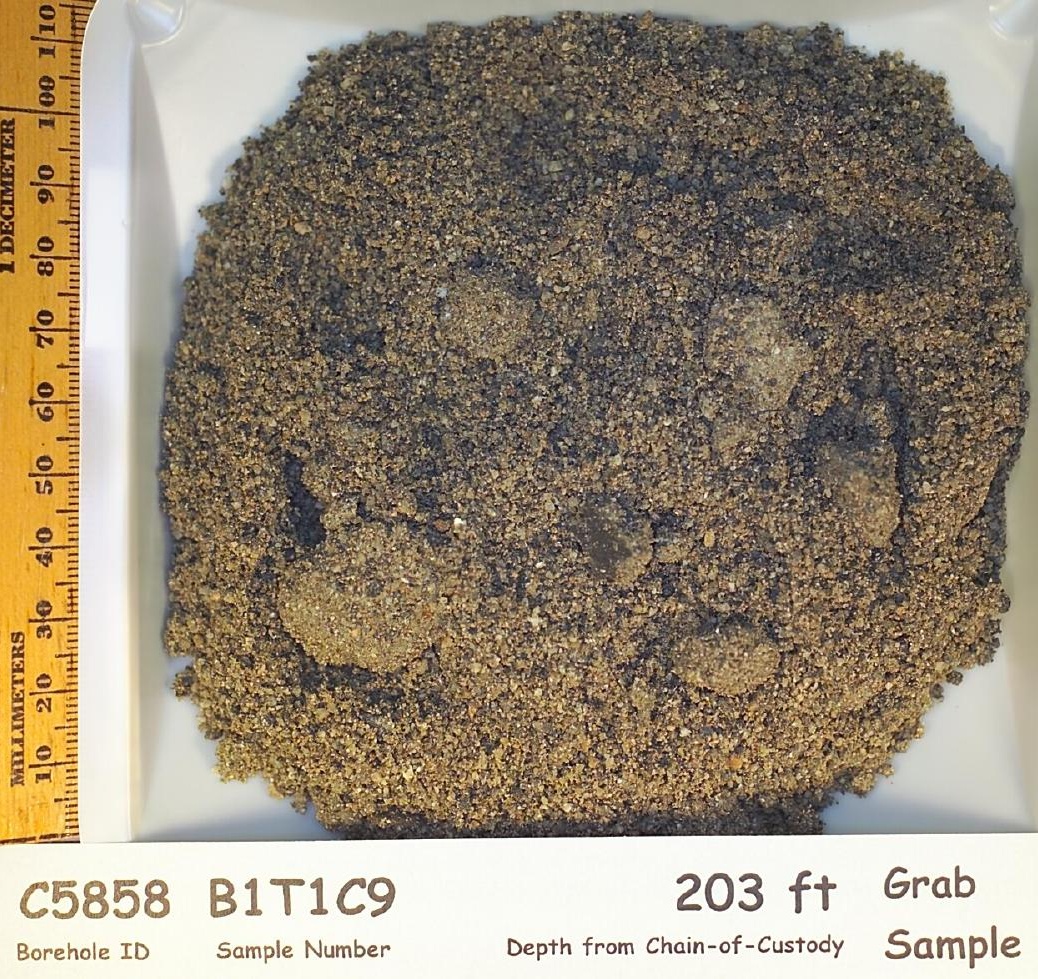




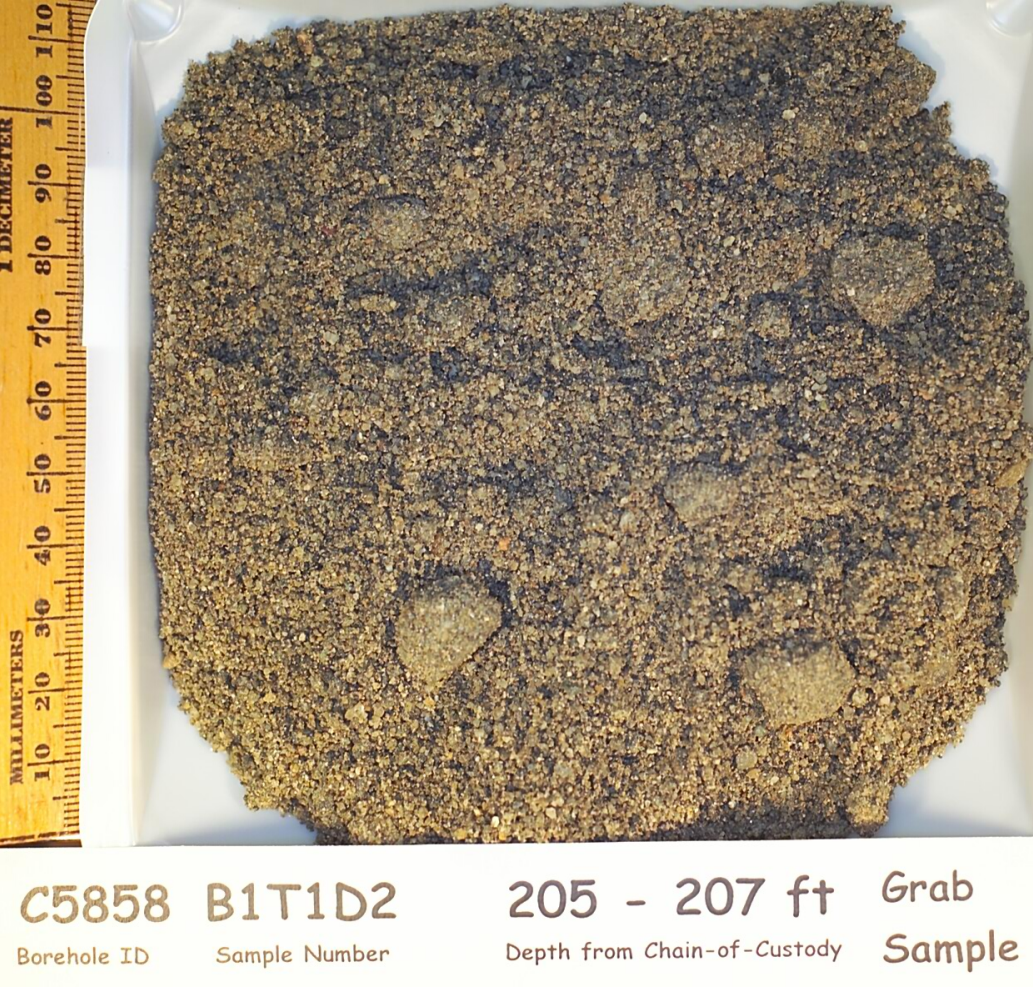




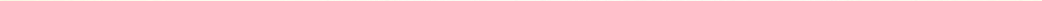




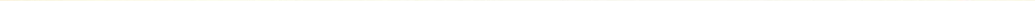




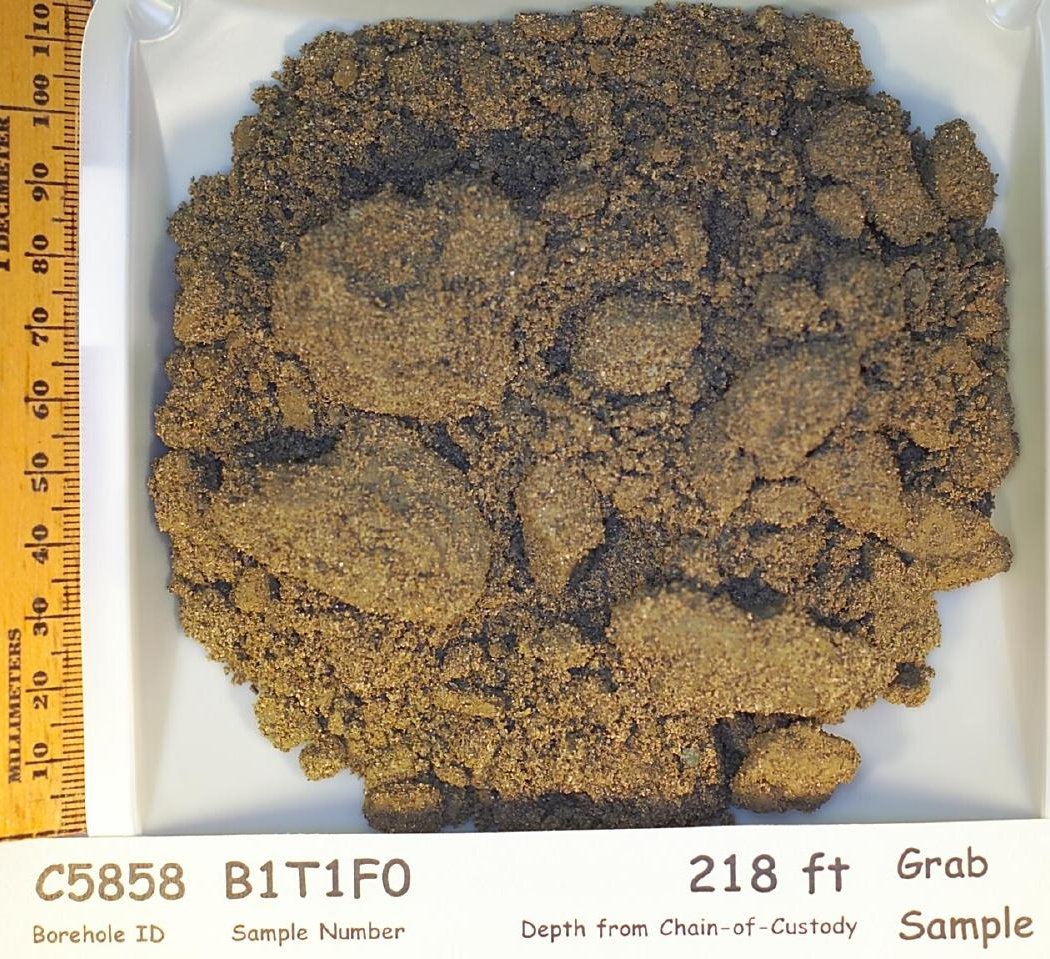




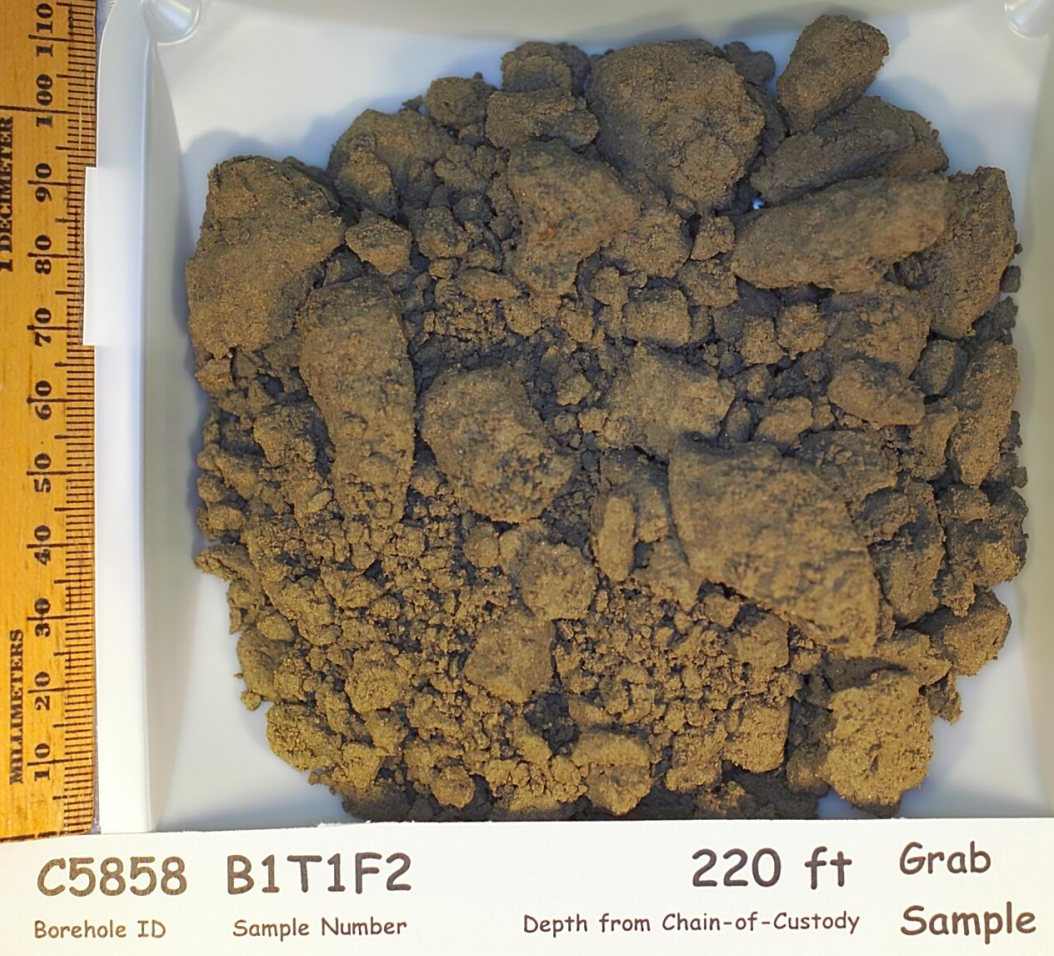




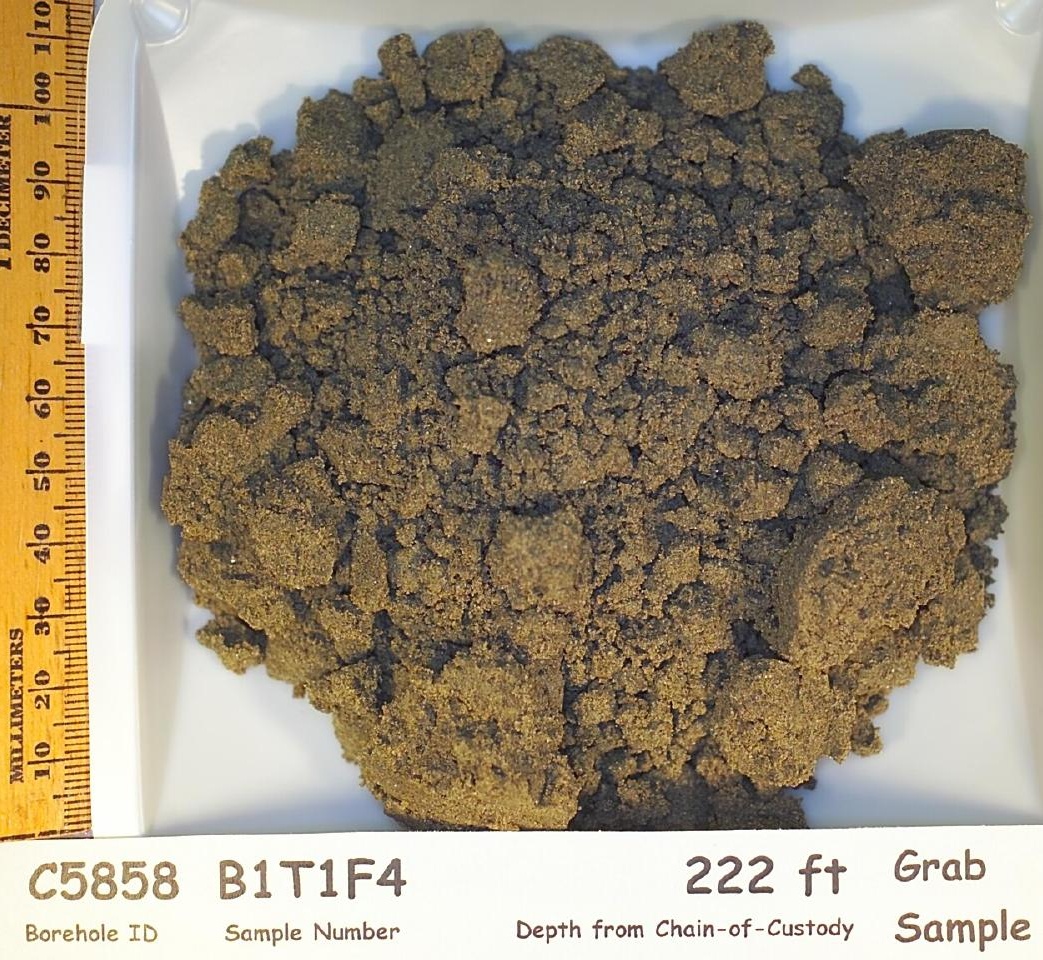




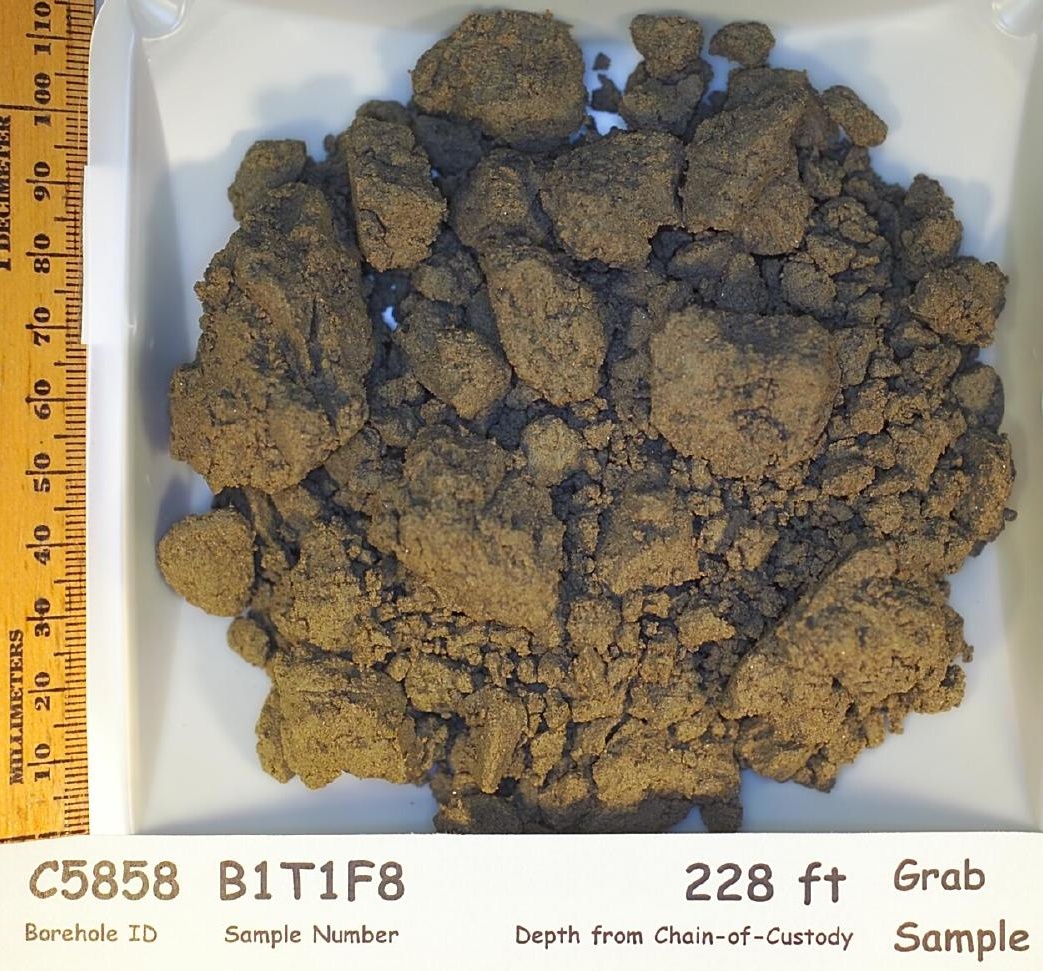




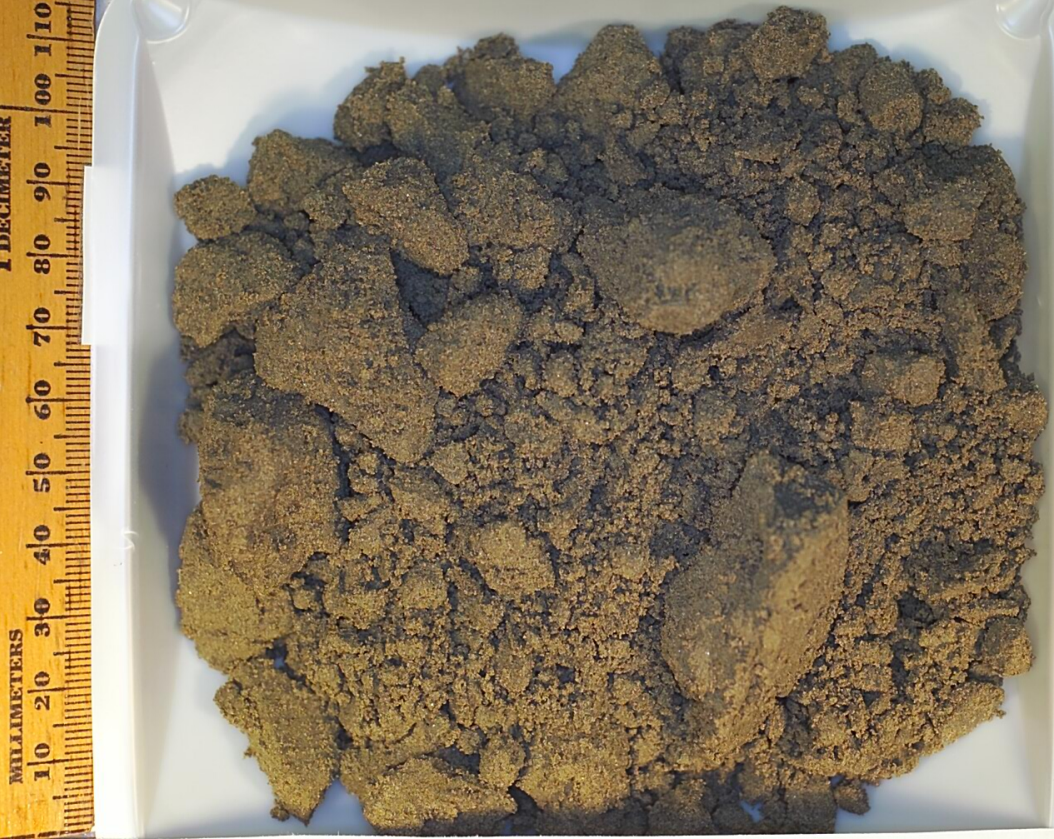

\section{C5858}

Borehole ID
B1T1HO

Sample Number
$230 \mathrm{ft}$

Depth from Chain-of-Custody
Grab Sample 


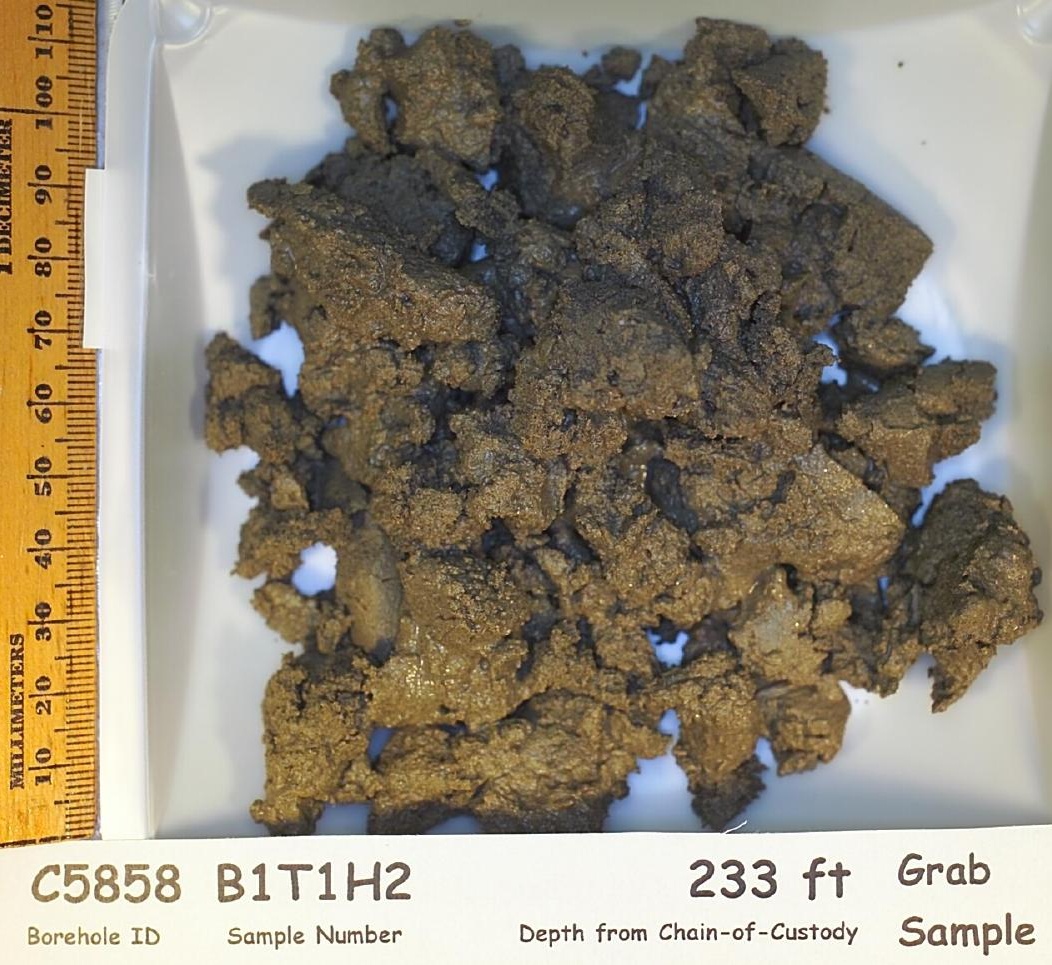




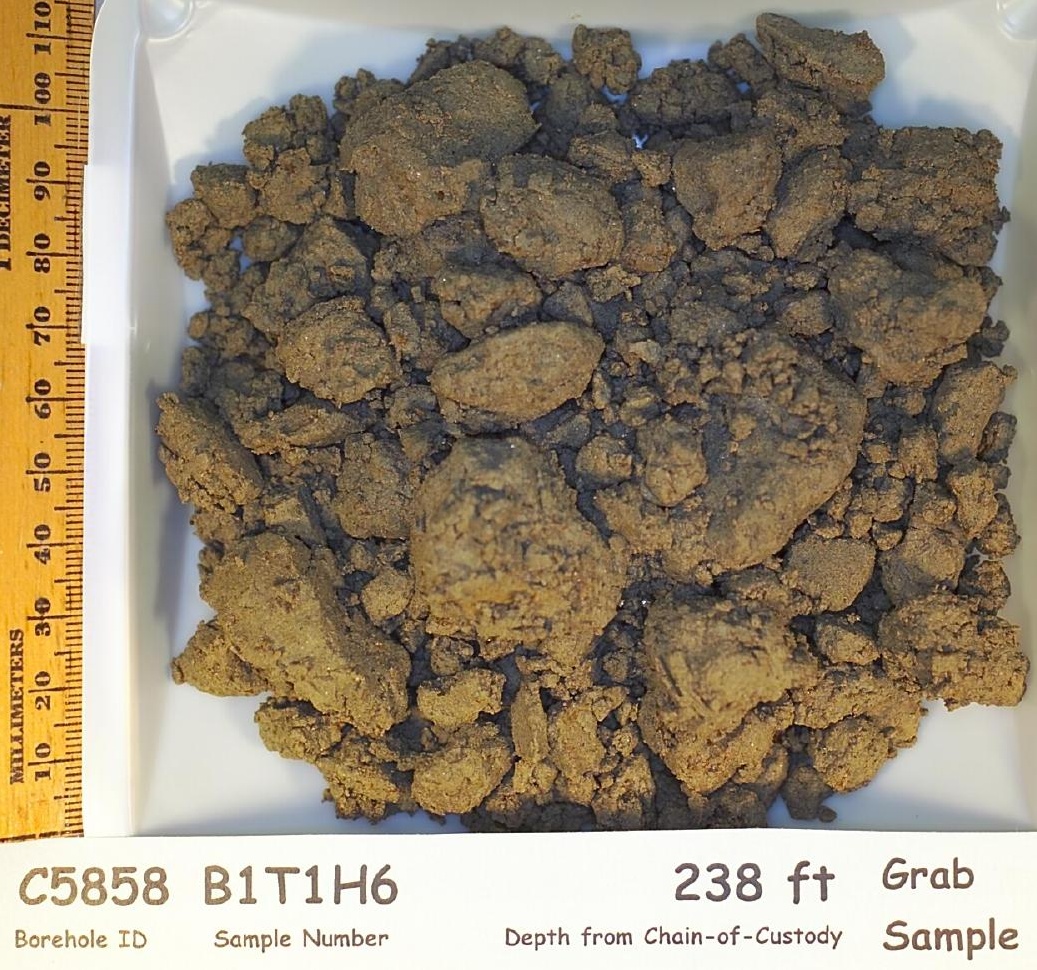




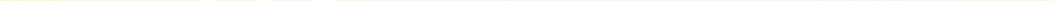




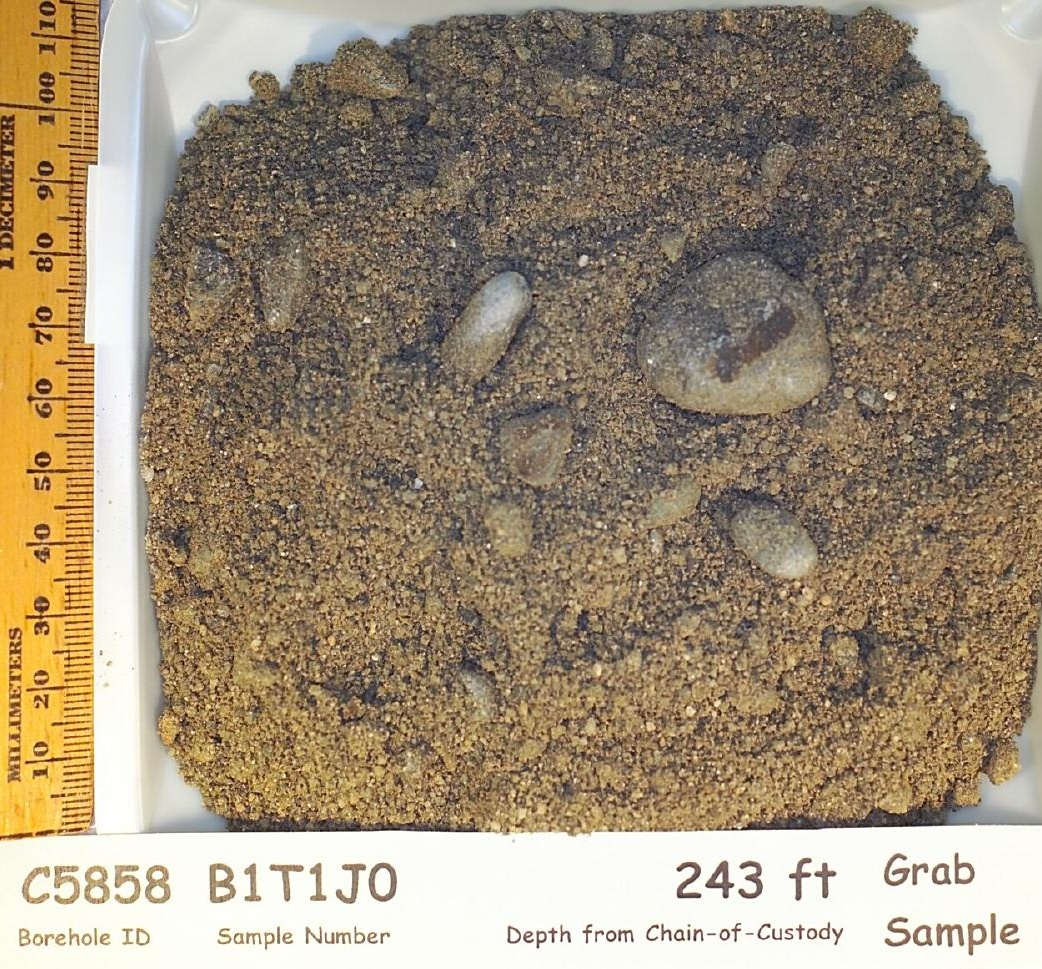




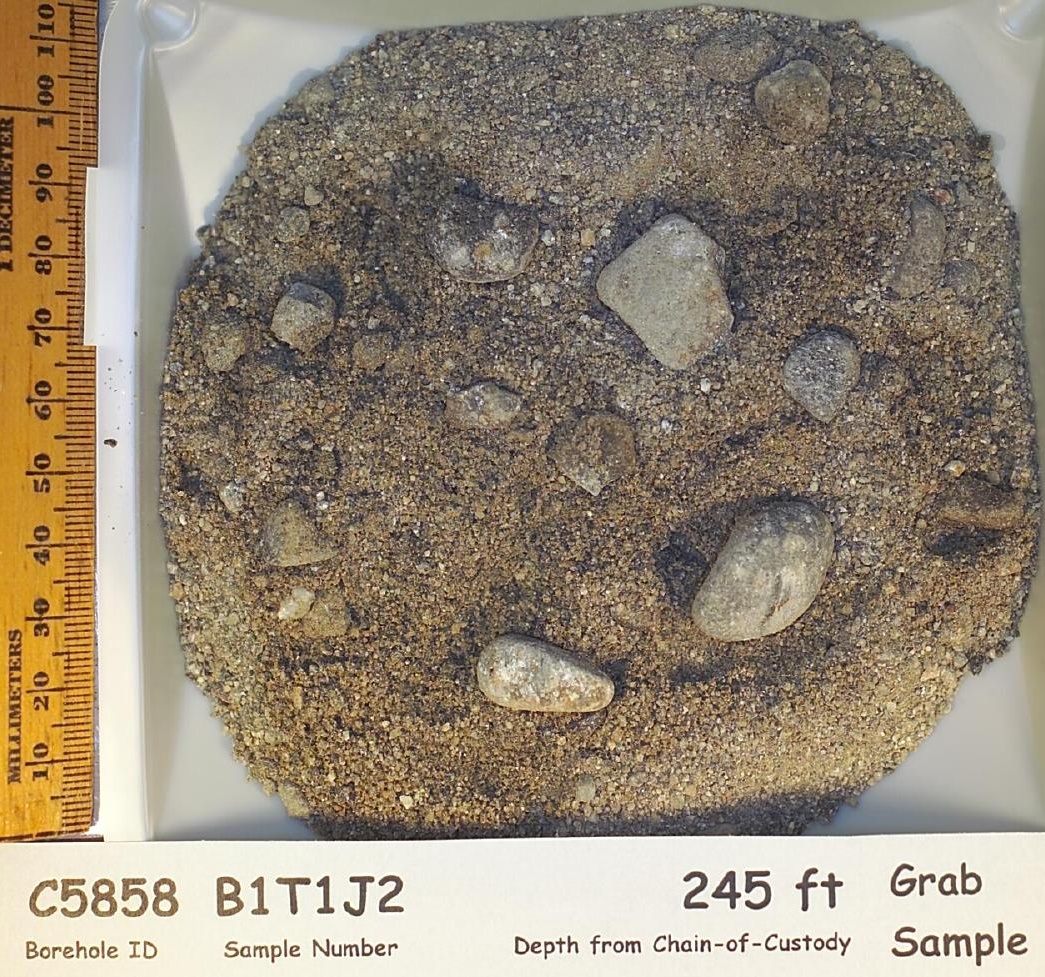




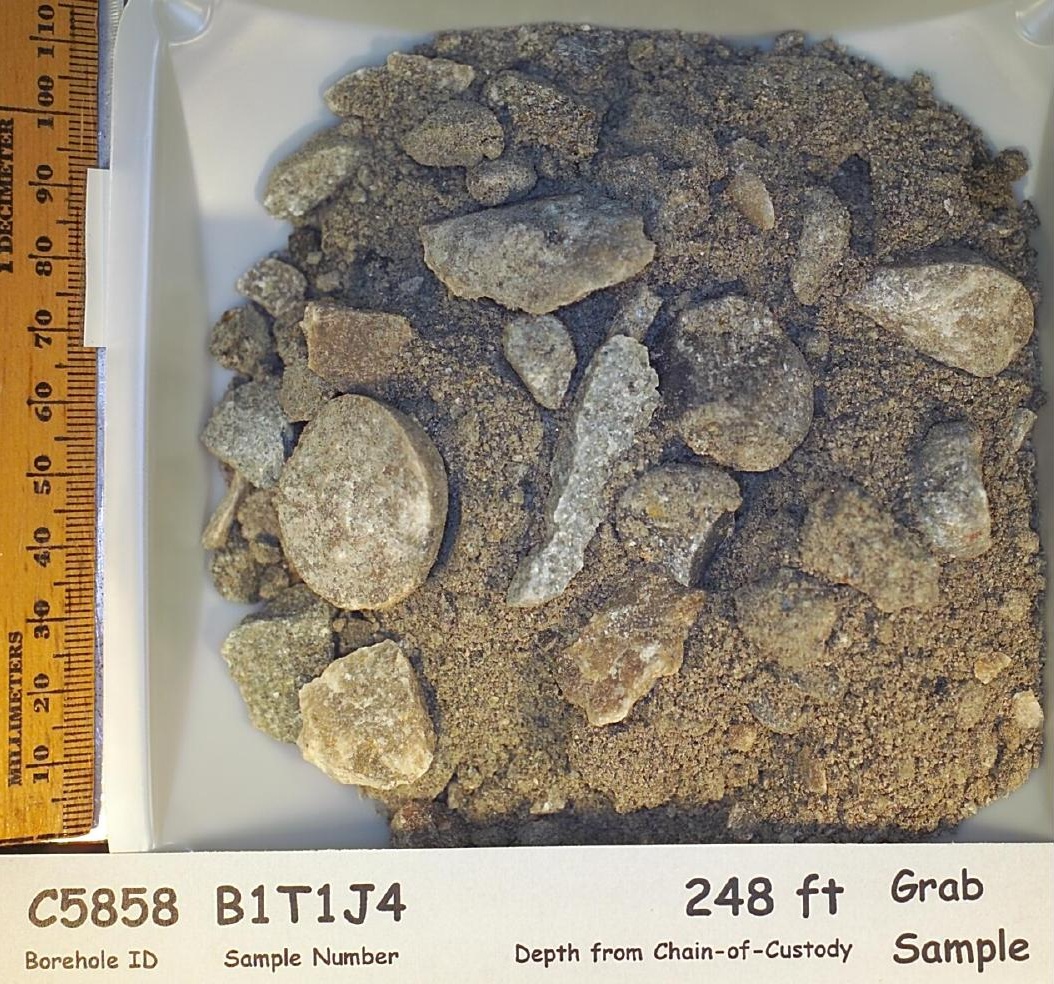




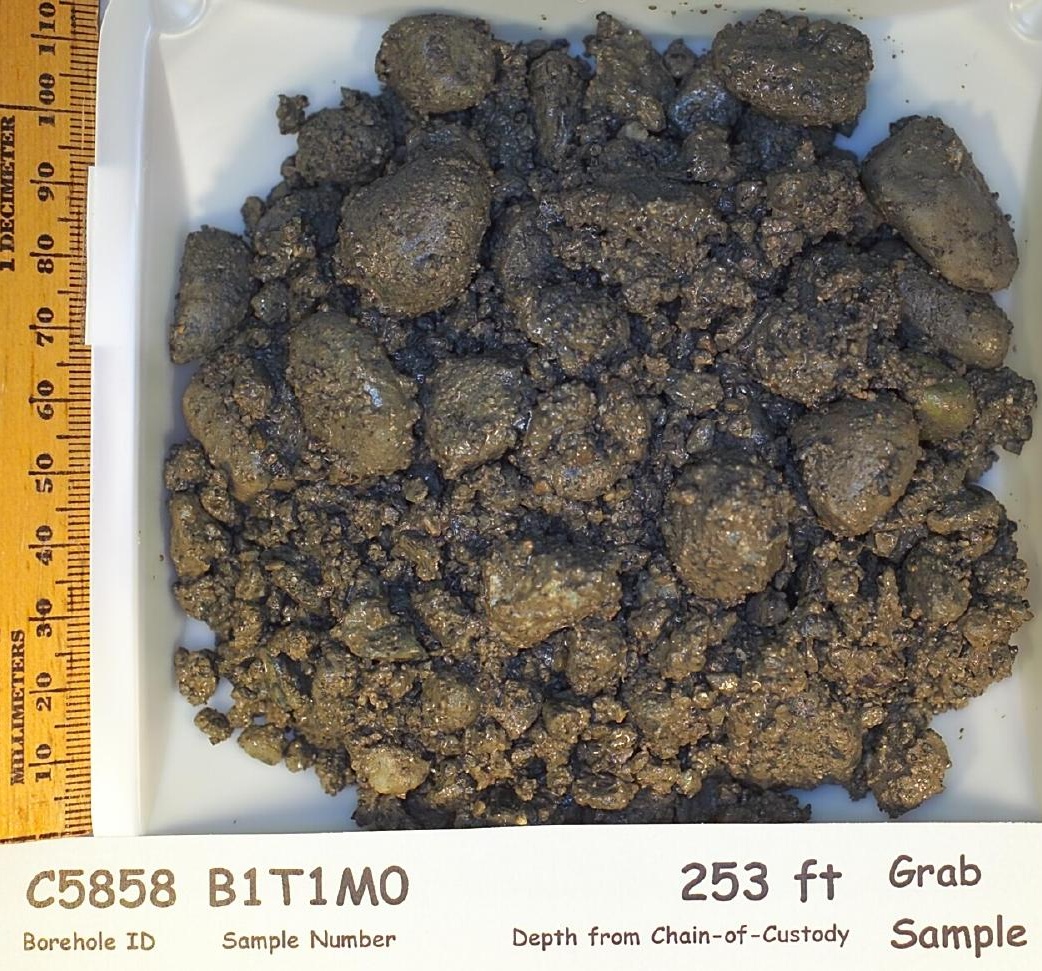




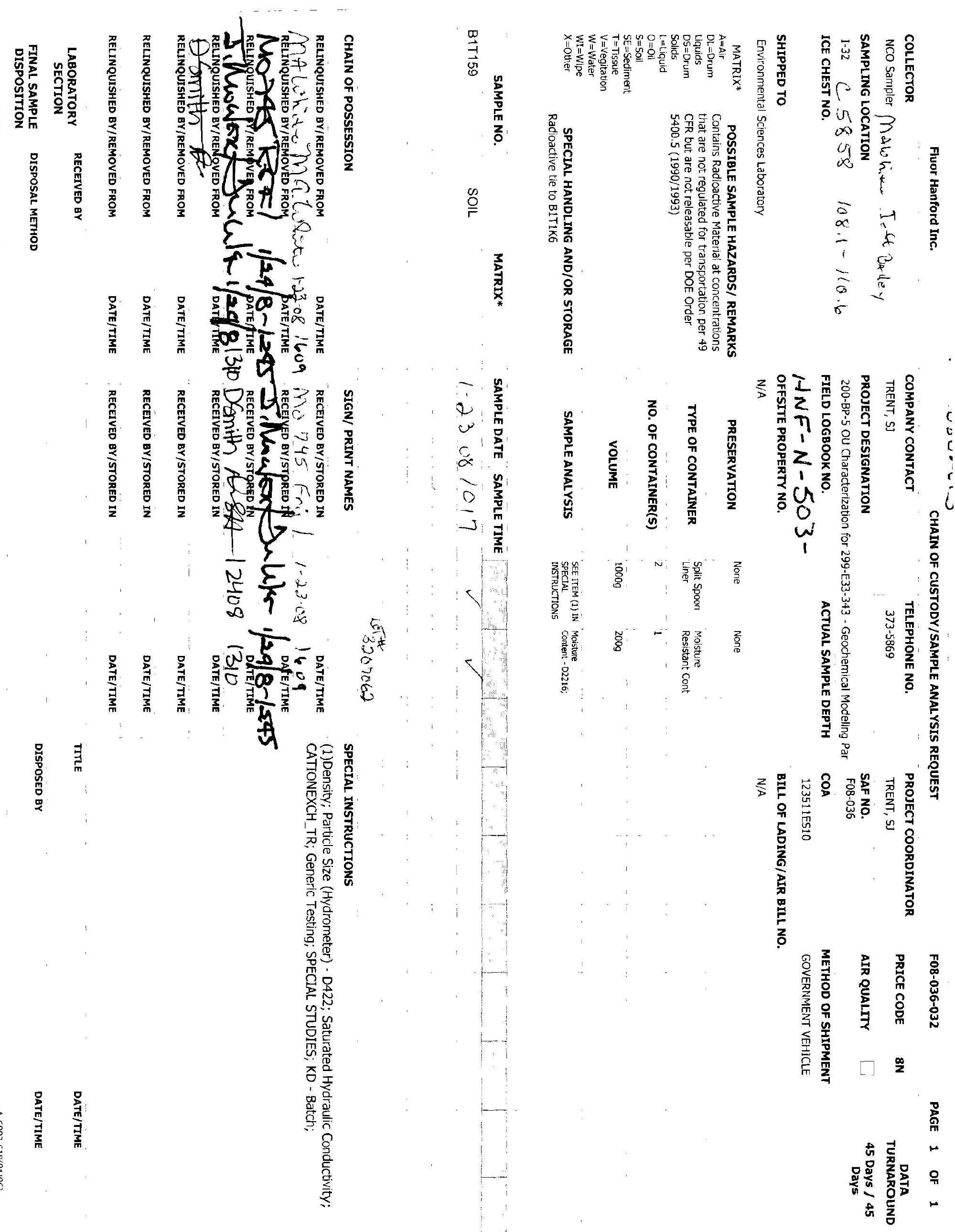




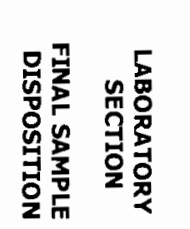

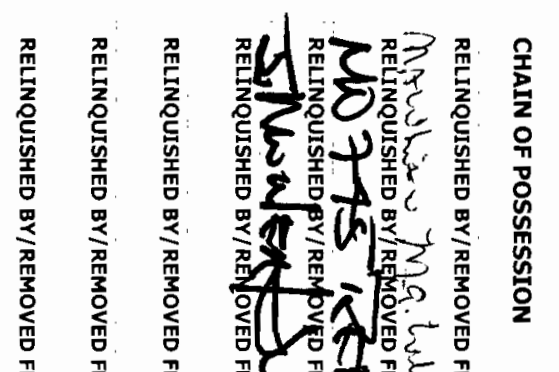

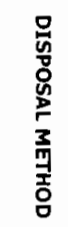

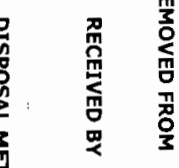

舅

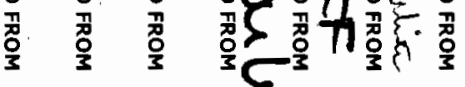

年

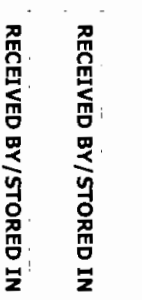

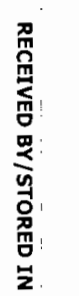

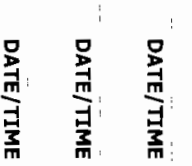

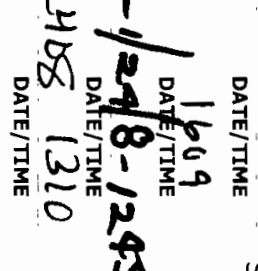

$\stackrel{\text { ㅁ }}{\vec{g}}$

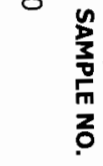

$\stackrel{\infty}{\stackrel{\rho}{=}}$

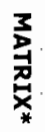

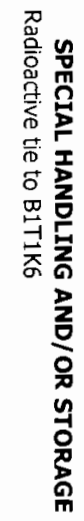

$\begin{array}{ll}\overline{1} & 0 \\ \omega & \frac{3}{5} \\ \omega & 0 \\ 0 & 0\end{array}$

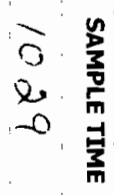

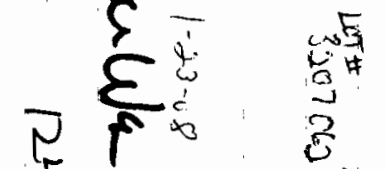

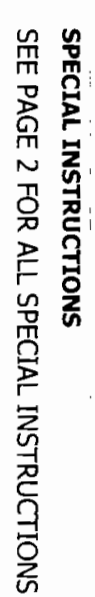

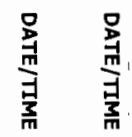




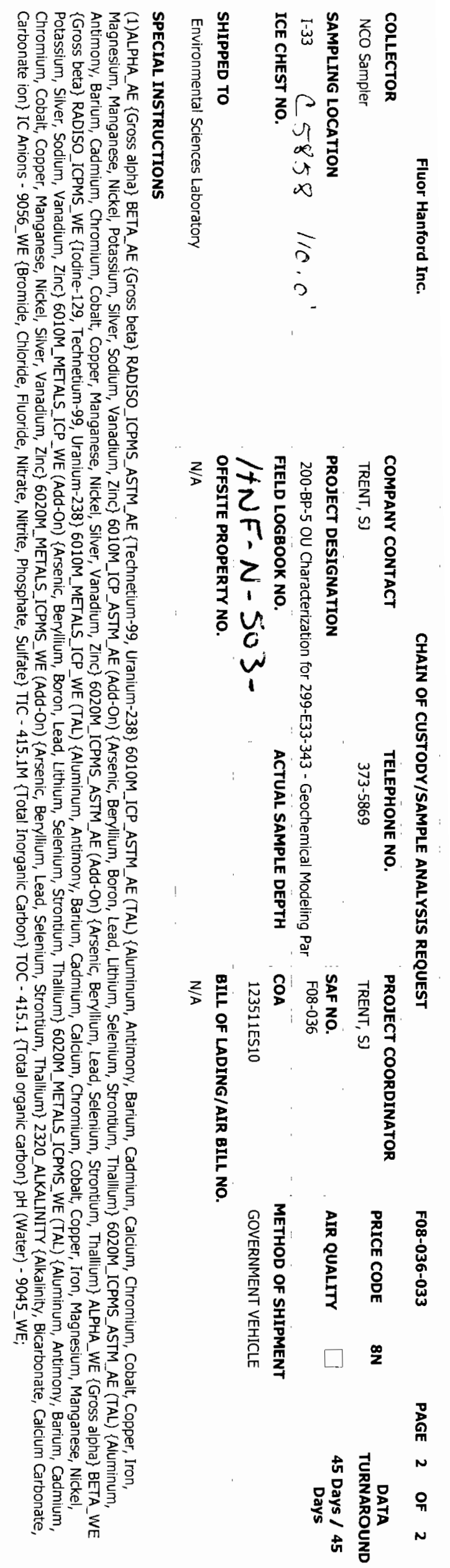



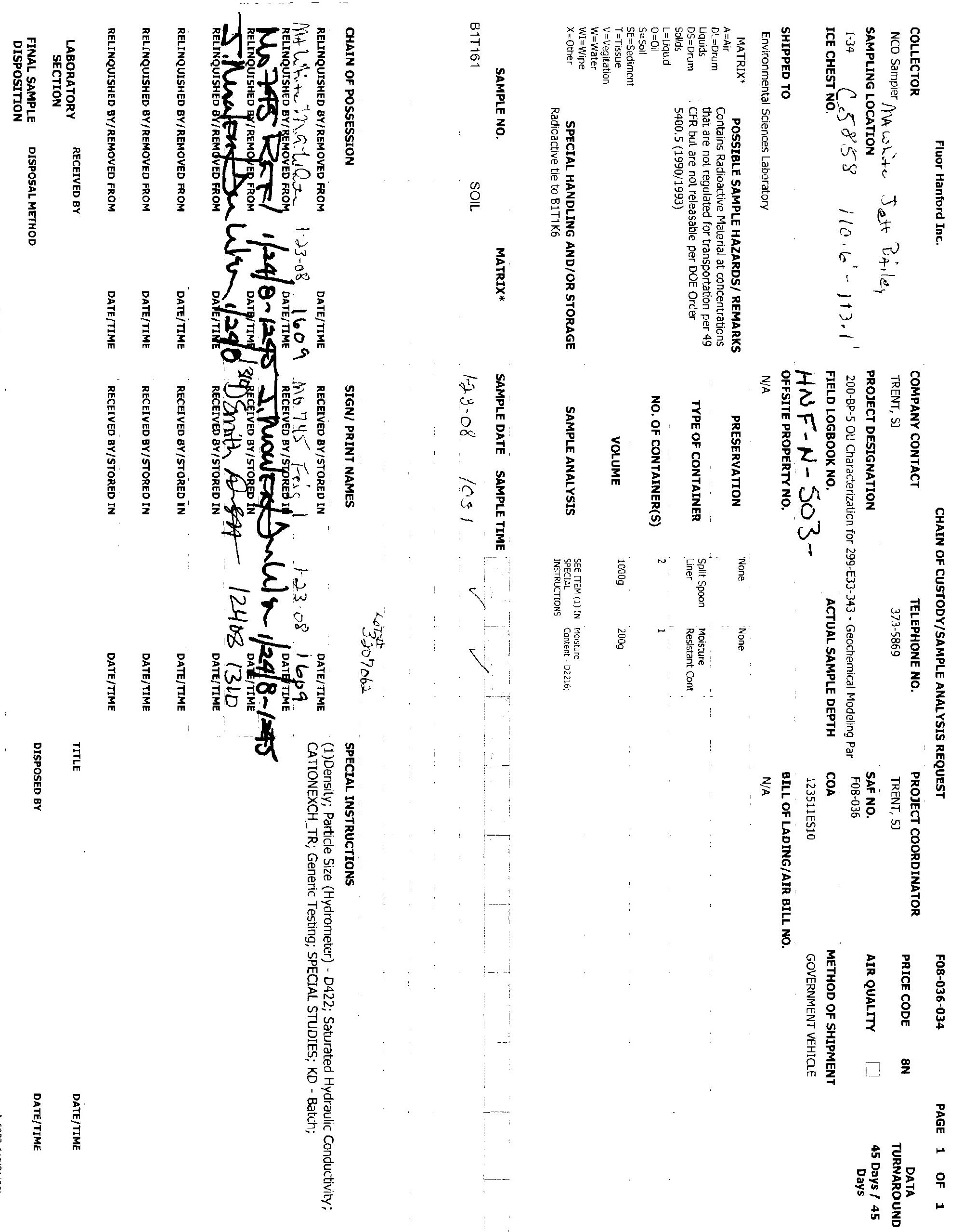
严

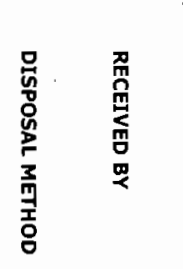

ת
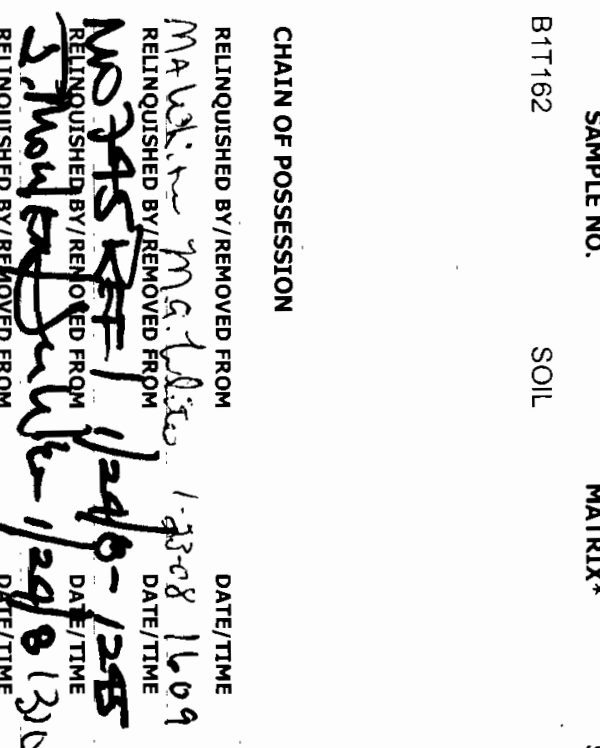

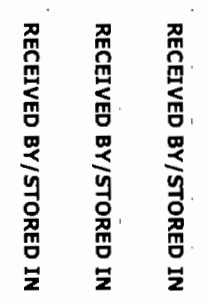
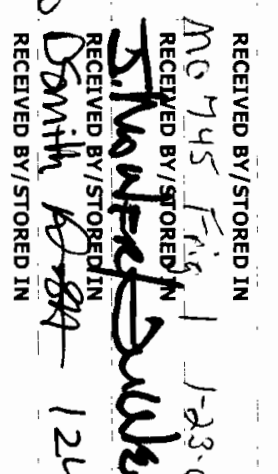

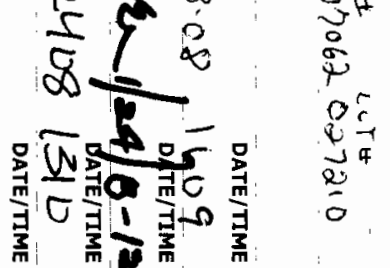

总
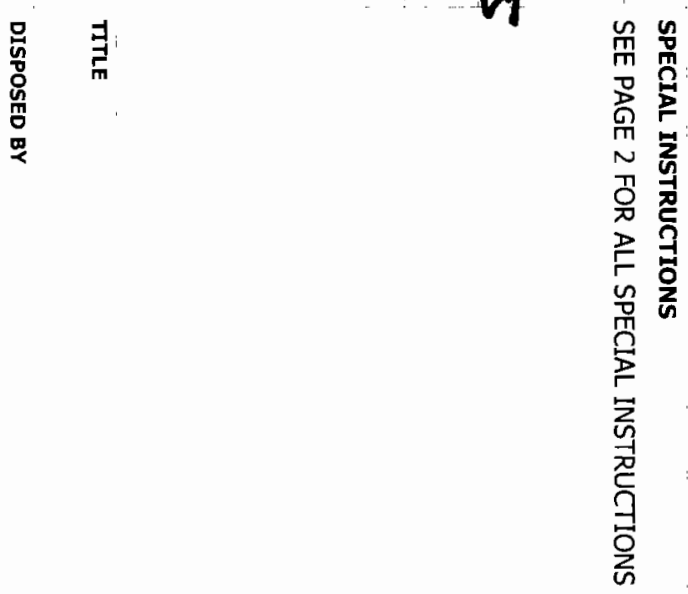

$\stackrel{\infty}{\stackrel{\rho}{\Gamma}}$

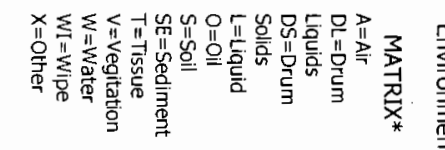

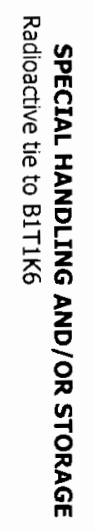

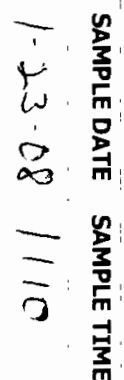

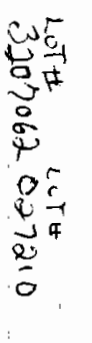

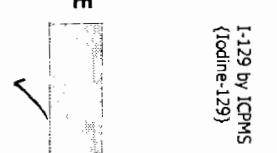

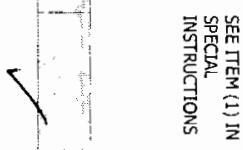

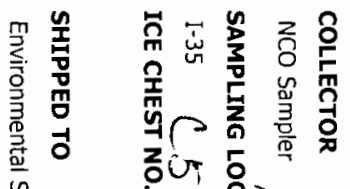

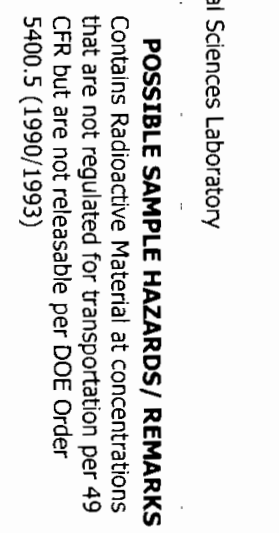

的

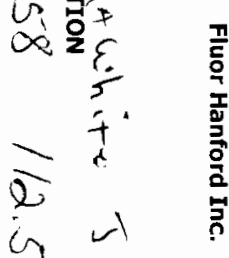
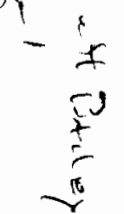

z 육

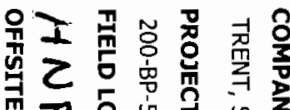

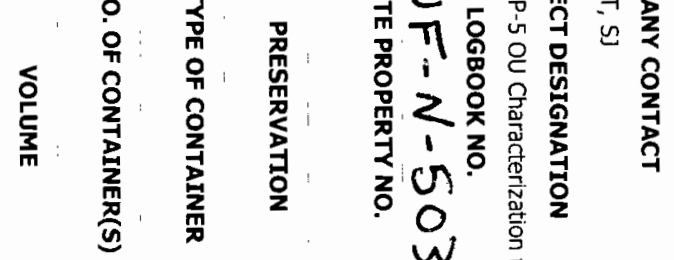

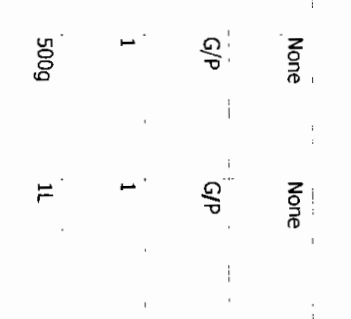




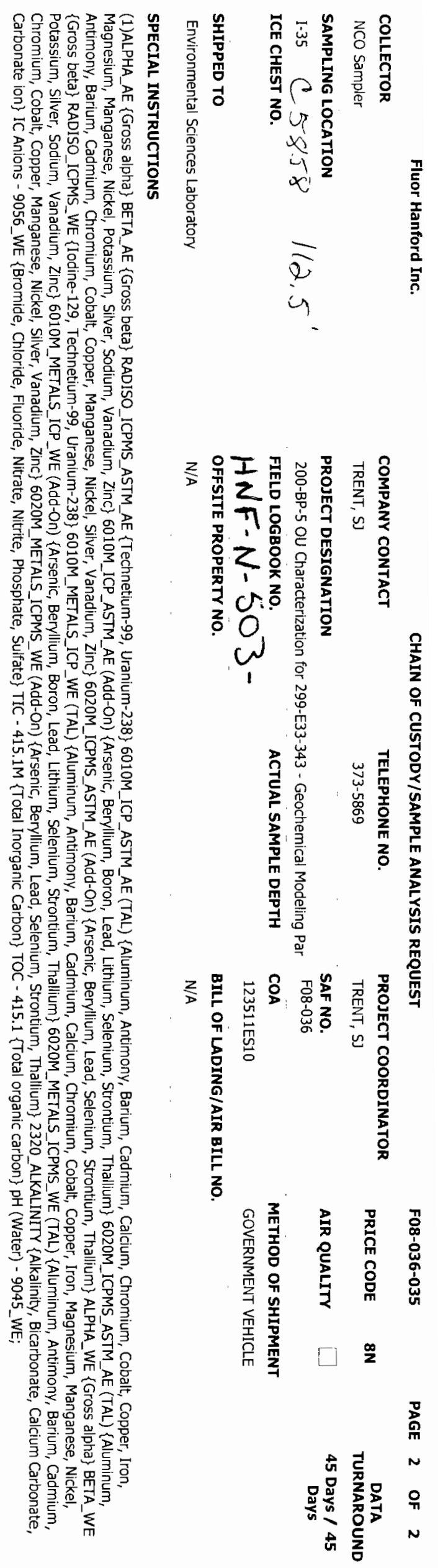




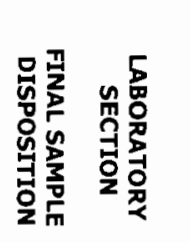

11
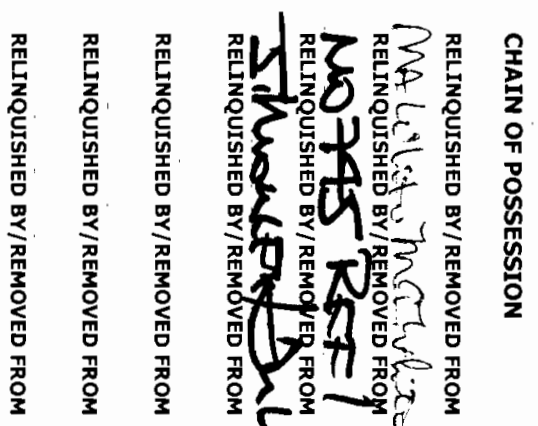

,

w

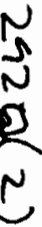

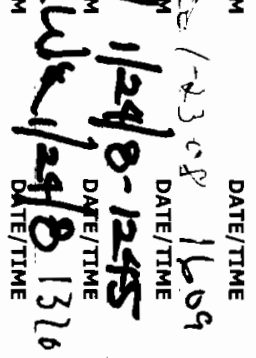

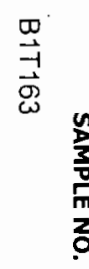

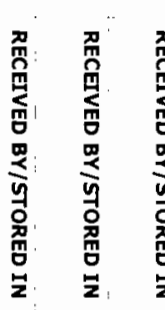

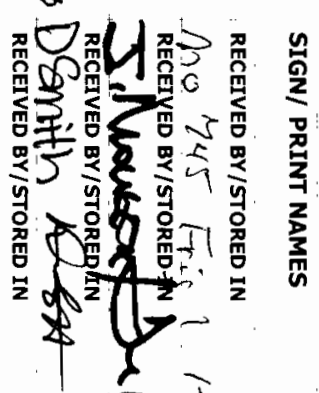

虽品品
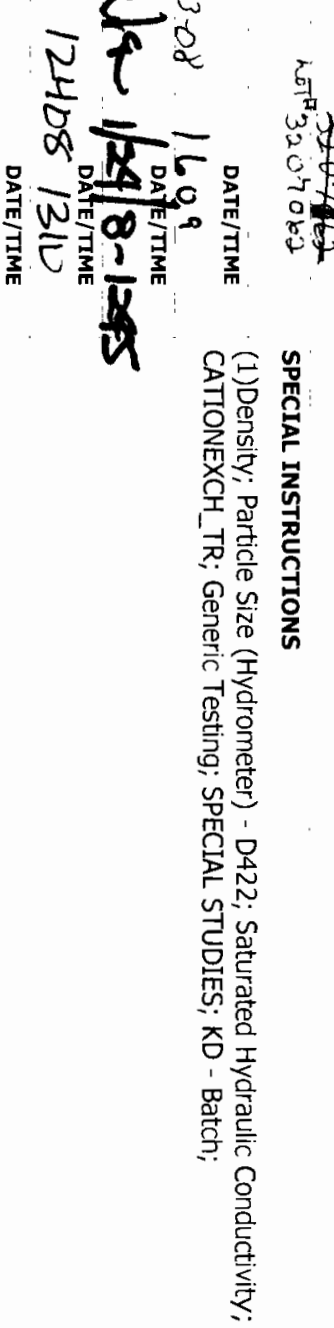

$\stackrel{0}{\circ}$

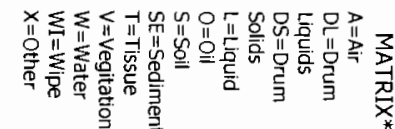

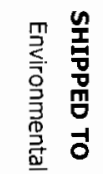

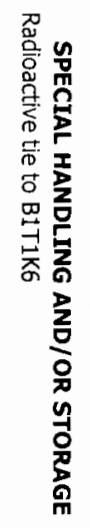

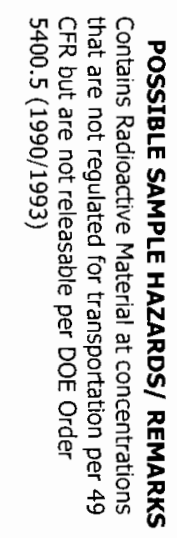

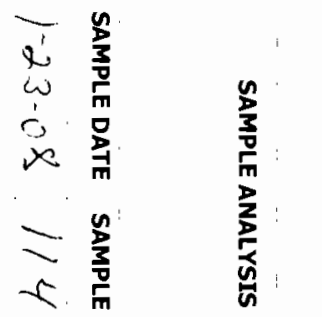

\& 글

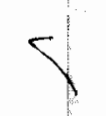

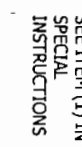

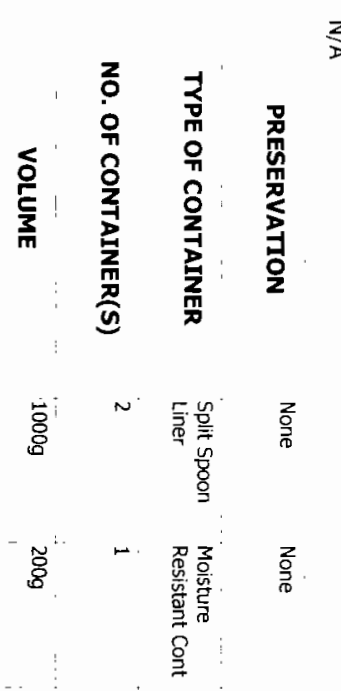

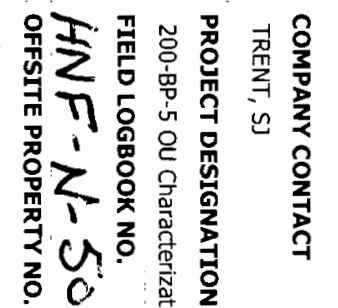

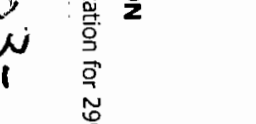

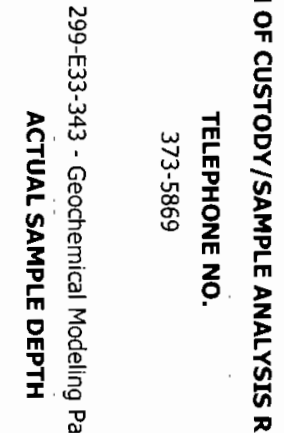

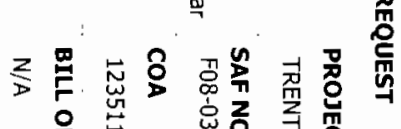

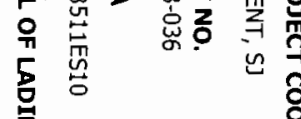

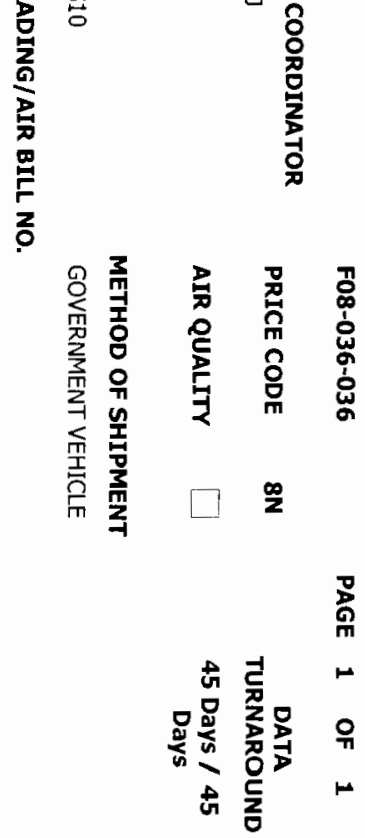




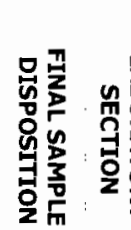

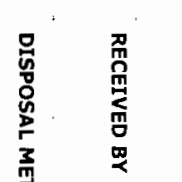
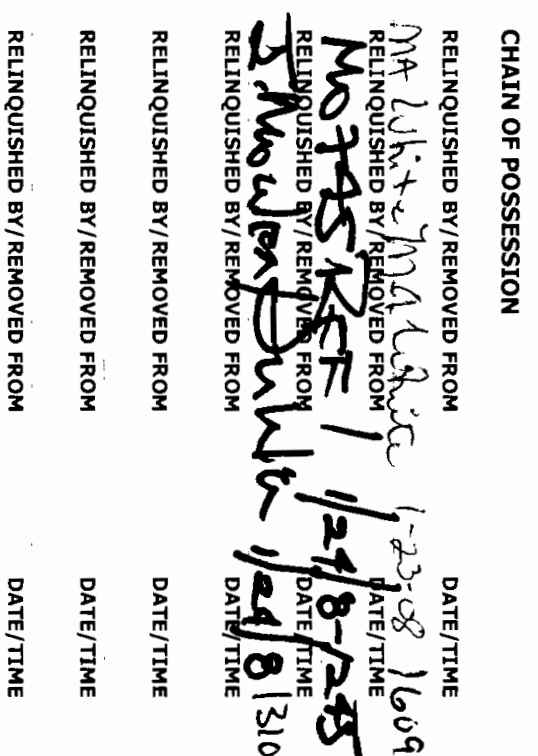

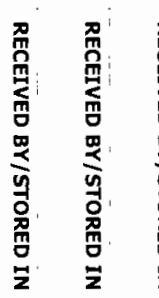

录

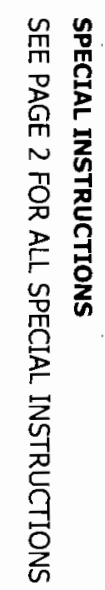

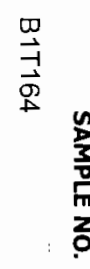

$\stackrel{\rho}{\circ}$

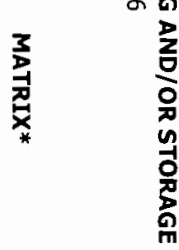

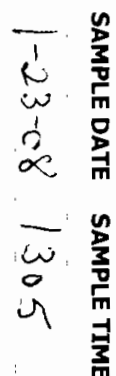

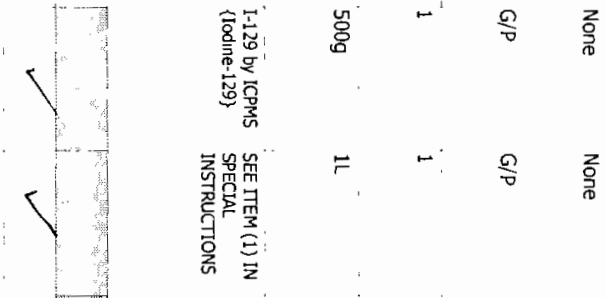

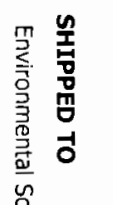

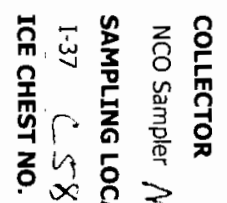

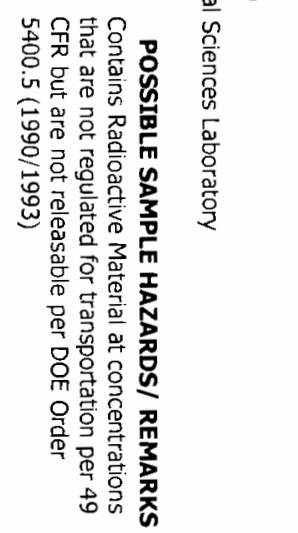

证家

«

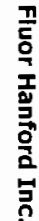

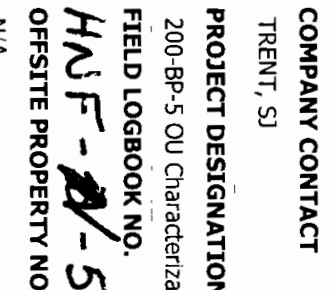

W ${ }^{0}{ }^{\frac{\mathrm{a}}{\circ}}{ }^{2}$

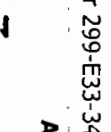

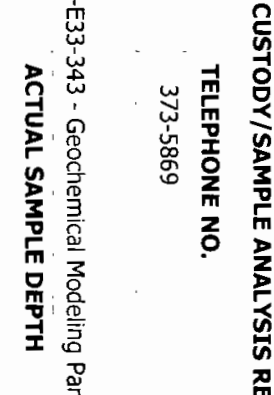

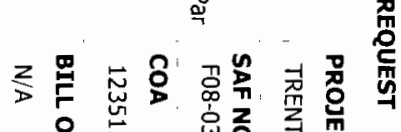

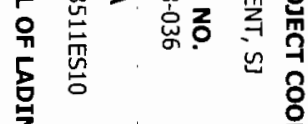

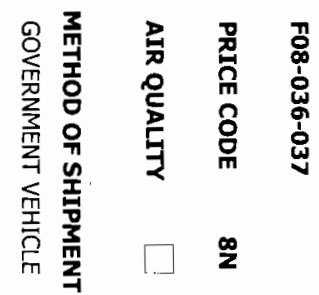




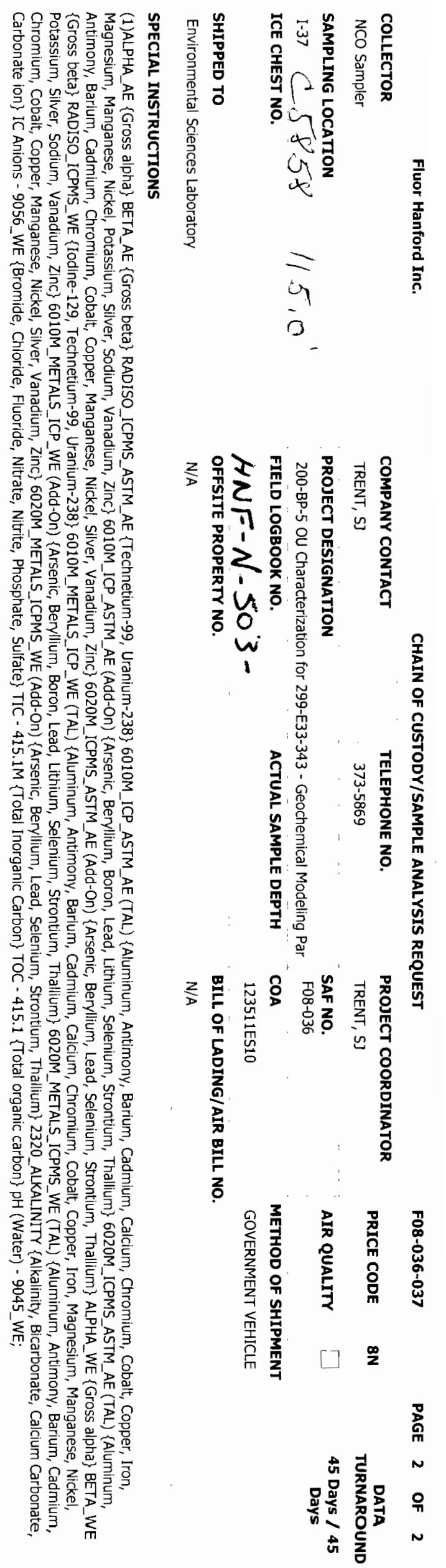




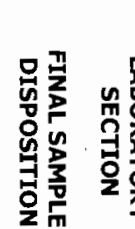
量
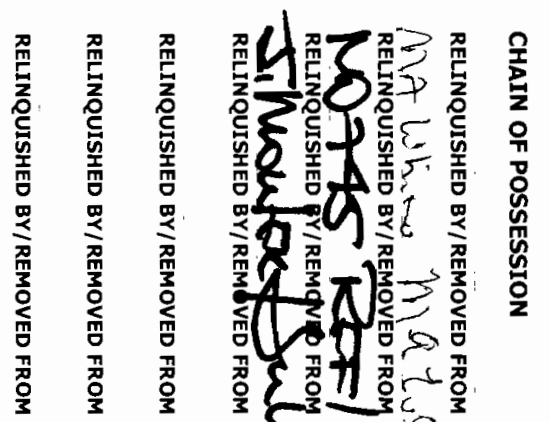

$$
\text { D }
$$
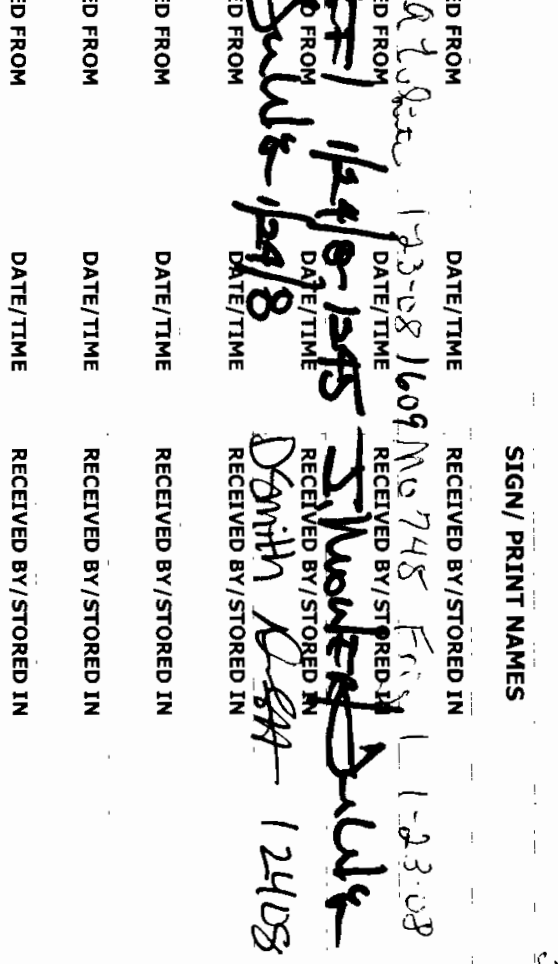
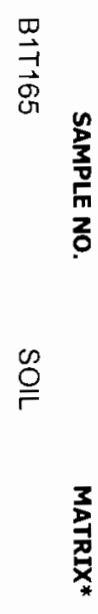

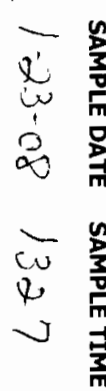

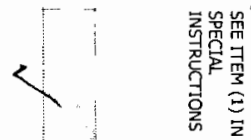

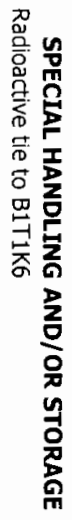

哭 省
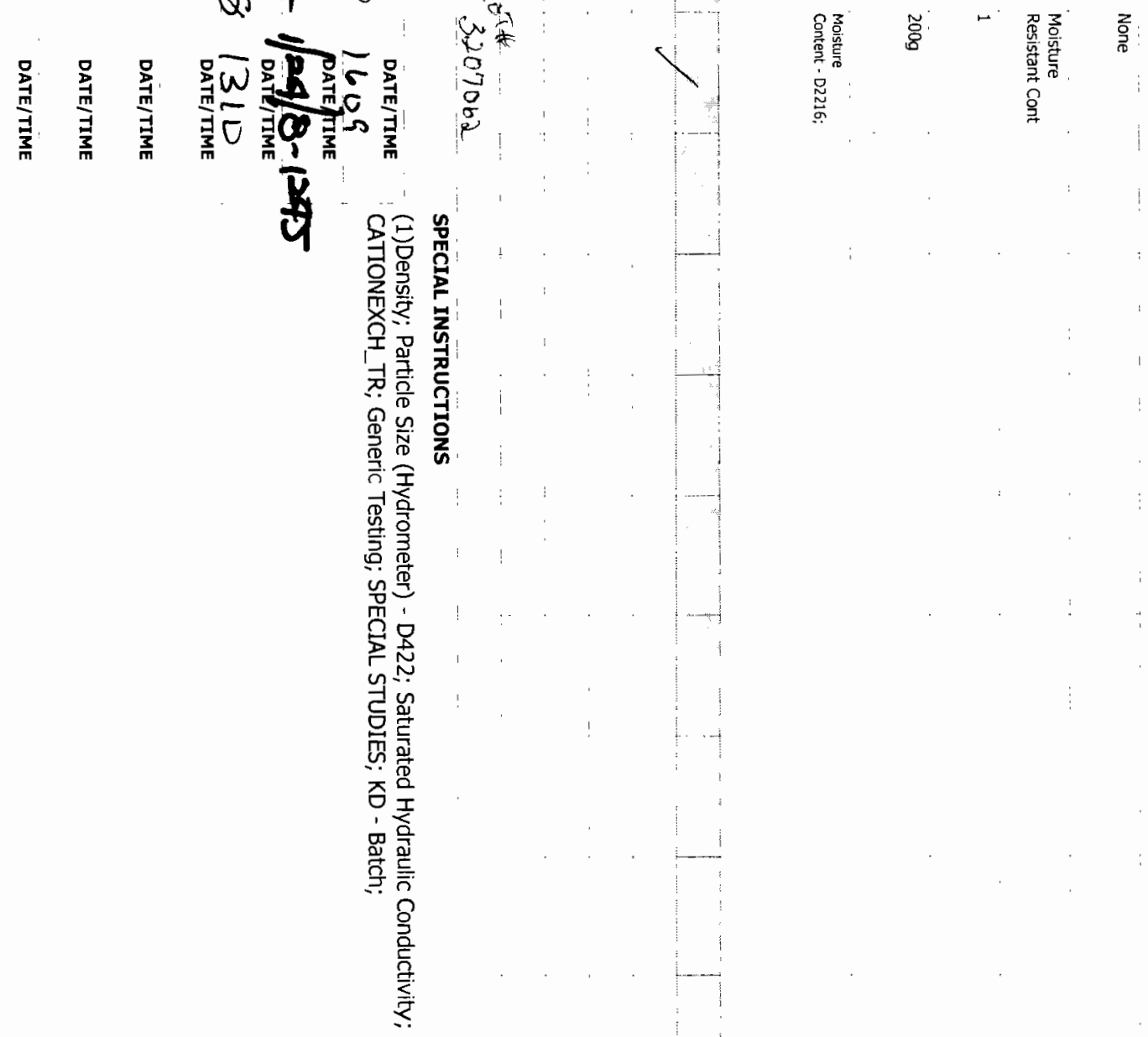

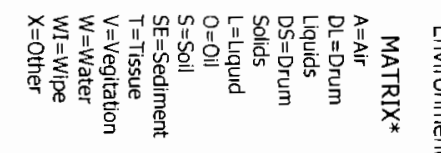

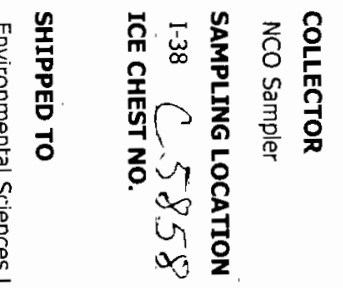

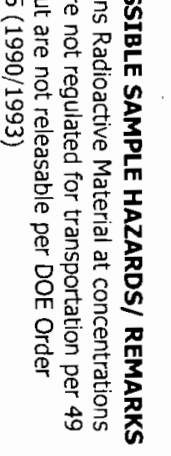

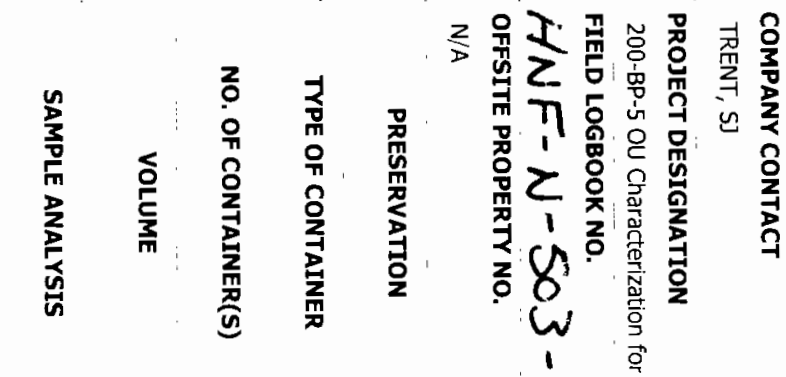

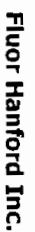

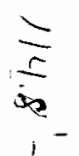

I

$\omega$

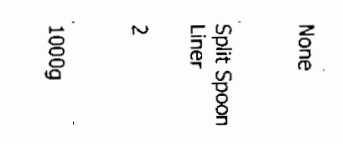
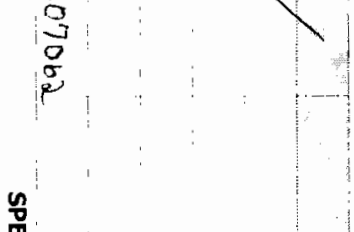

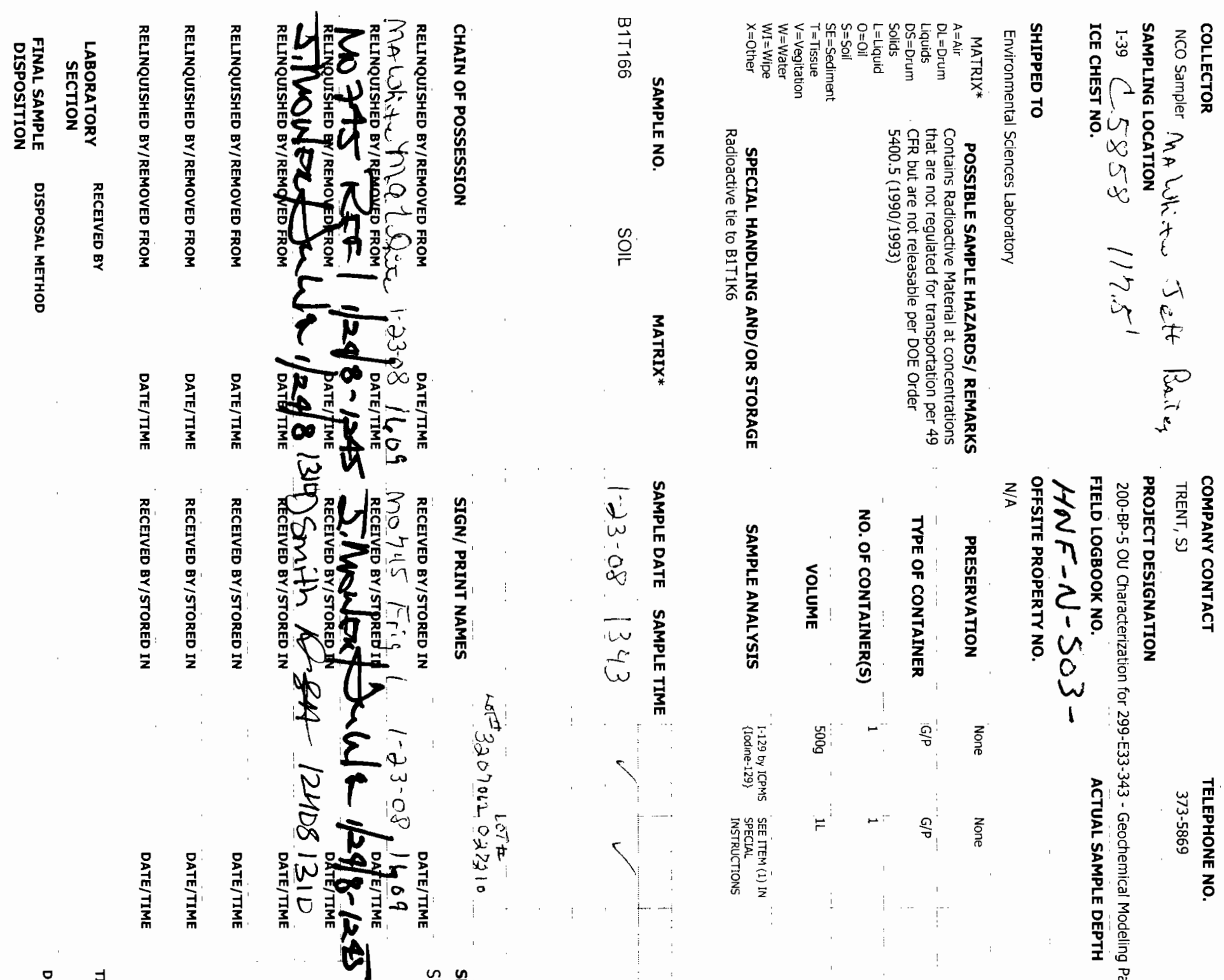

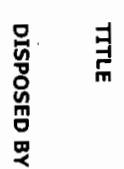
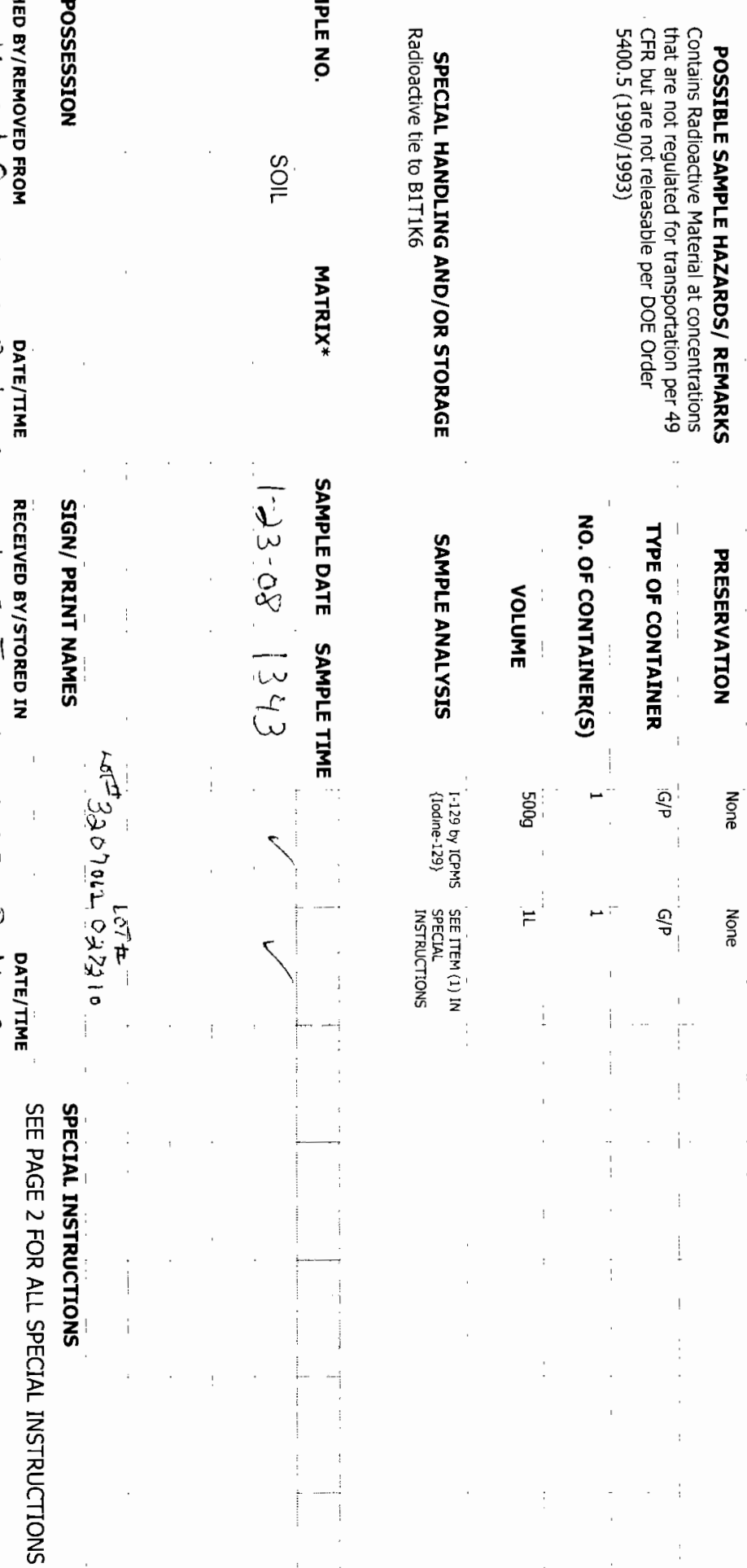

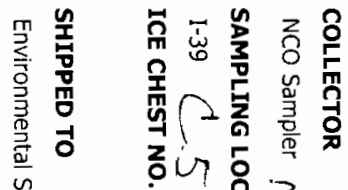
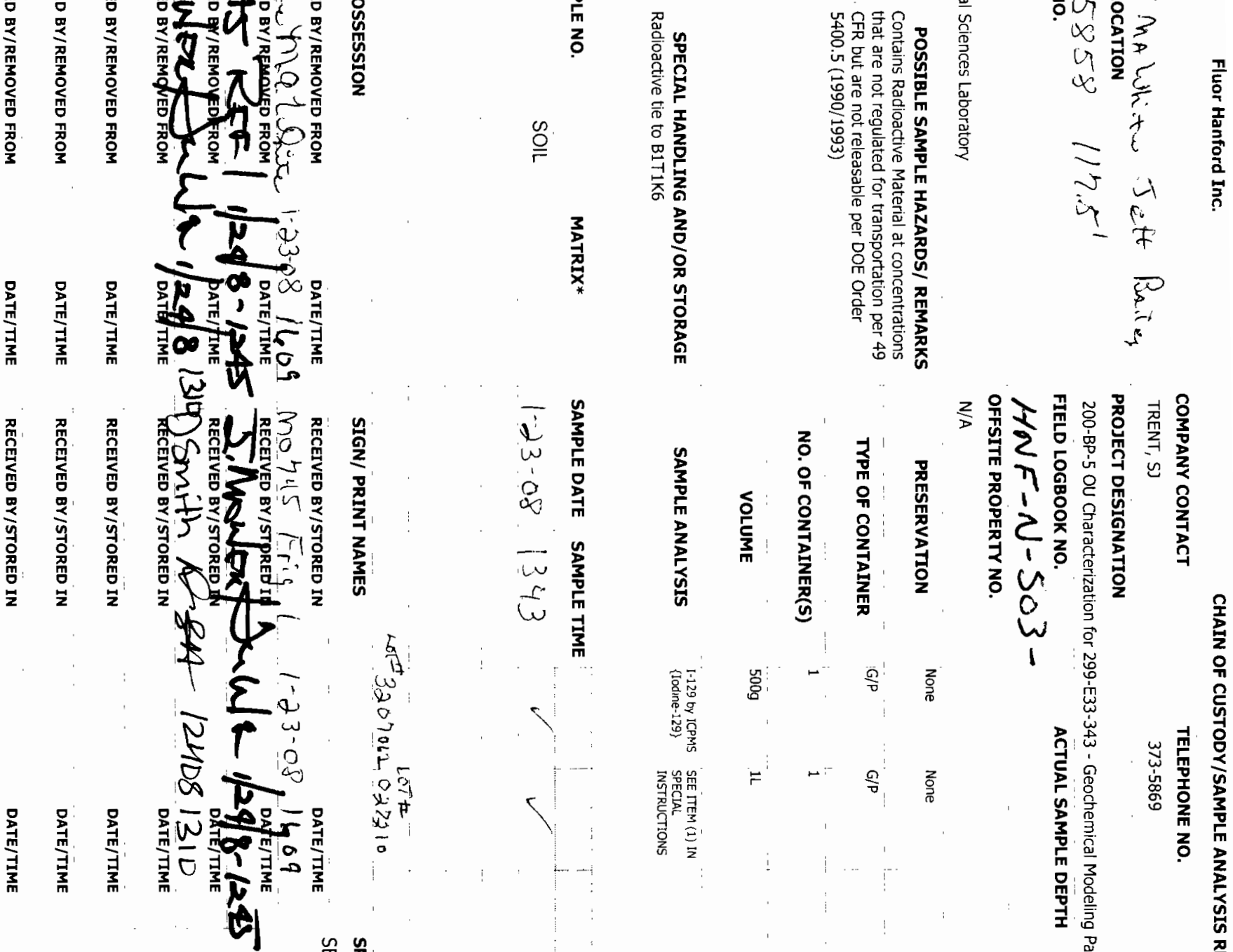

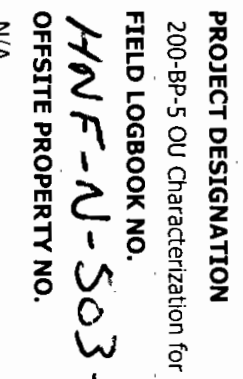

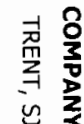

옥

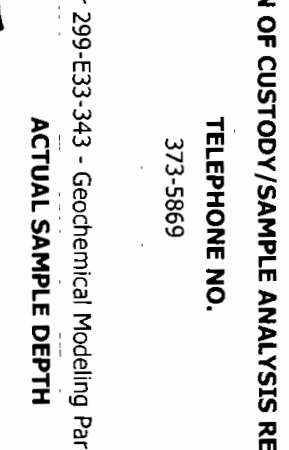

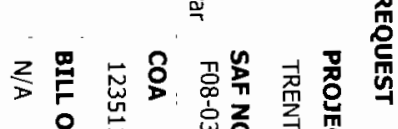

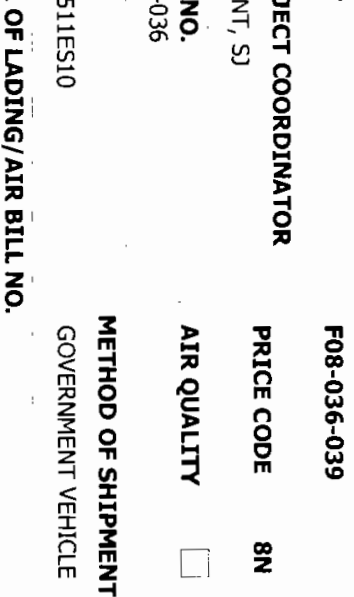

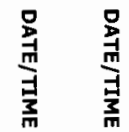

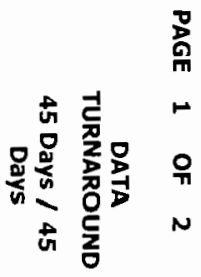




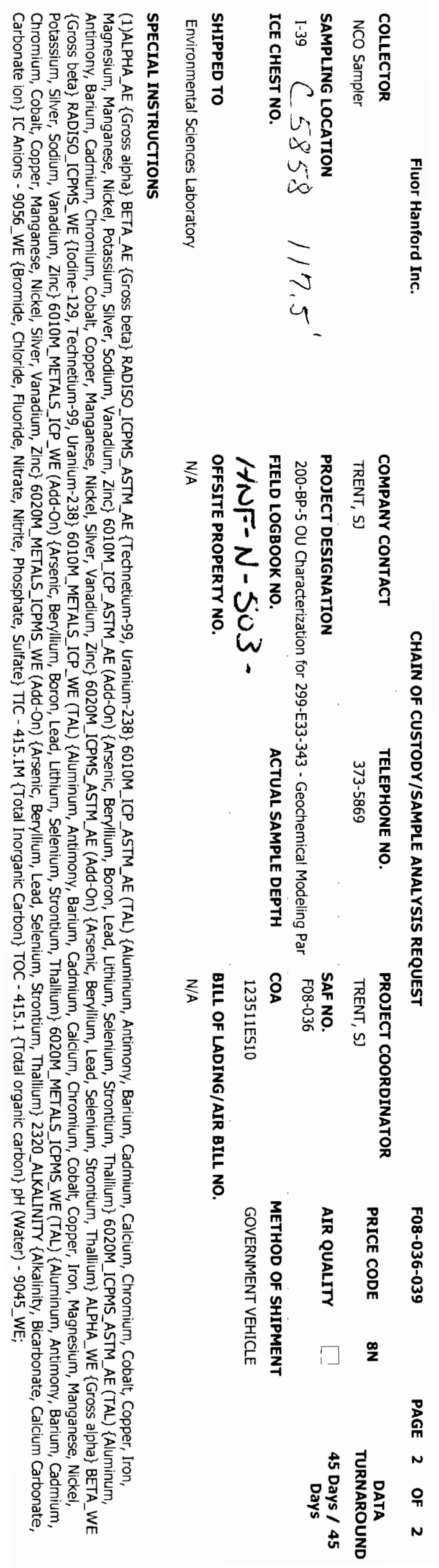




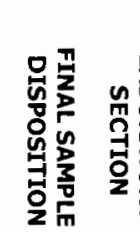

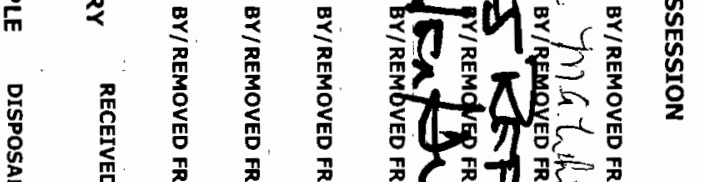
骨

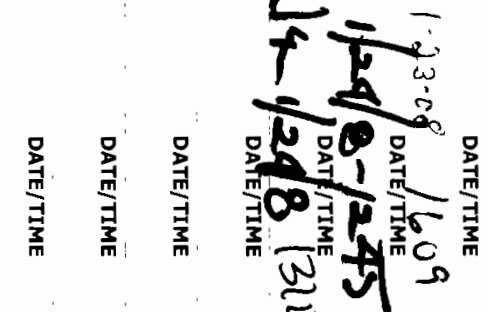

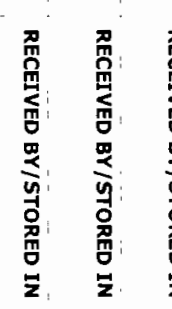

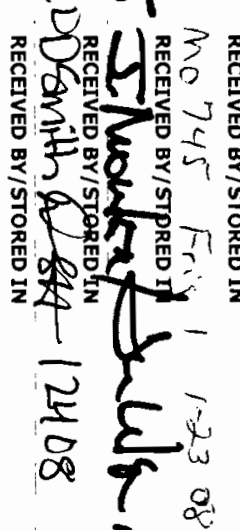

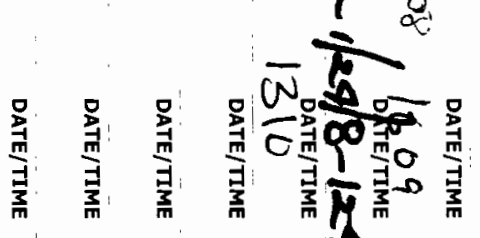

鸟

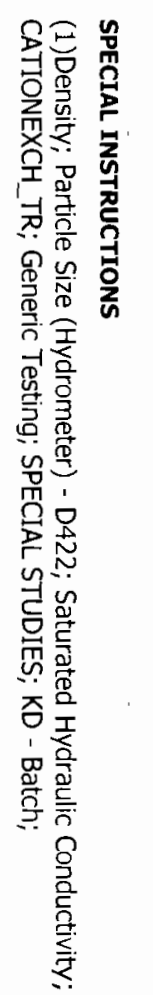

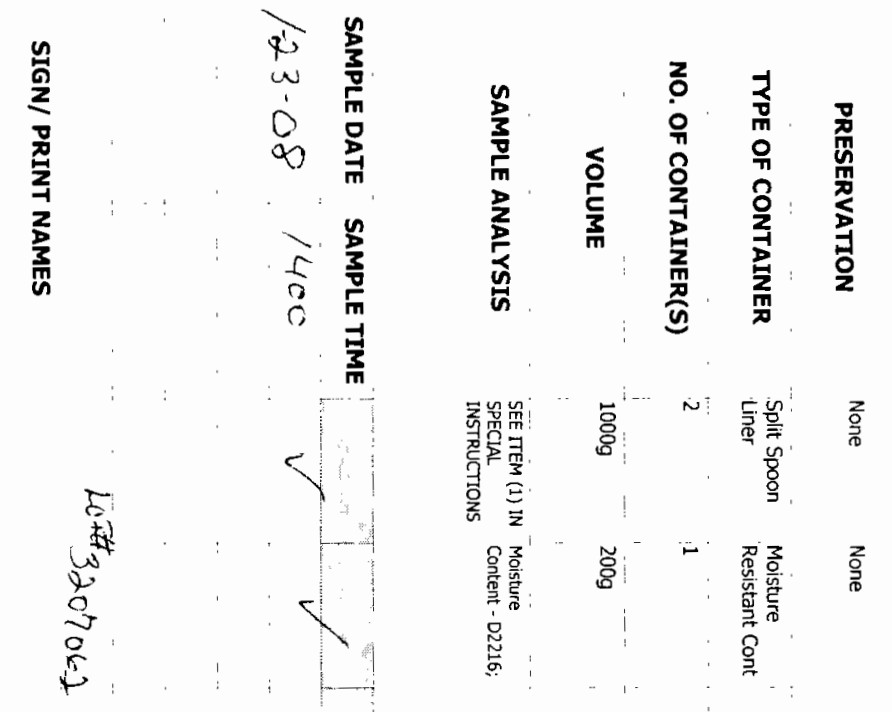
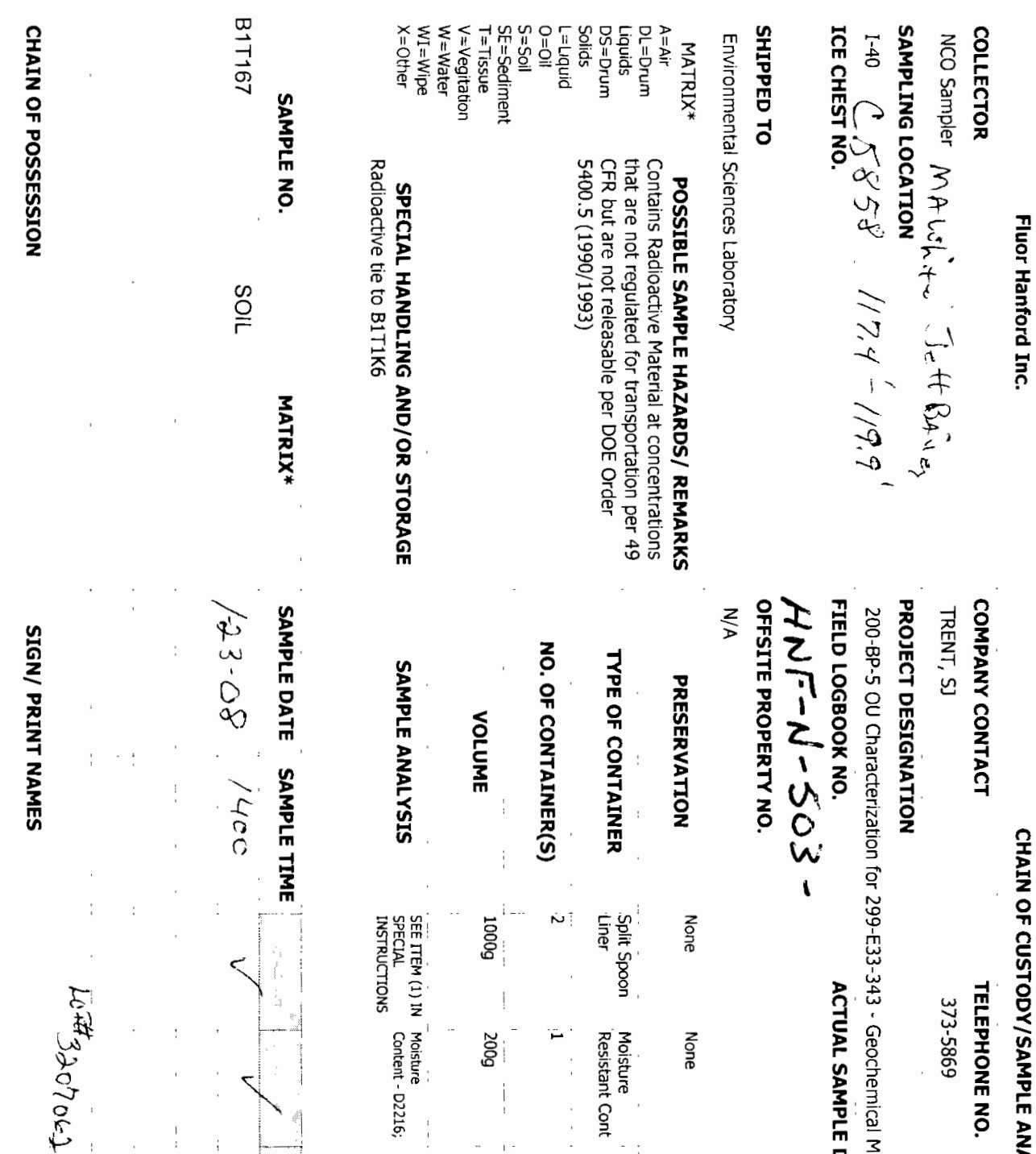

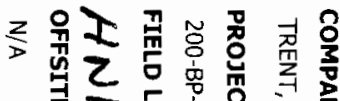

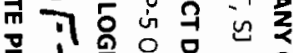

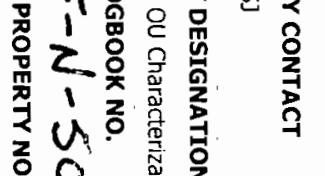

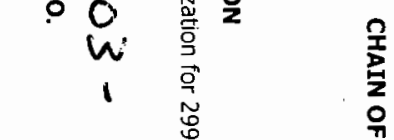

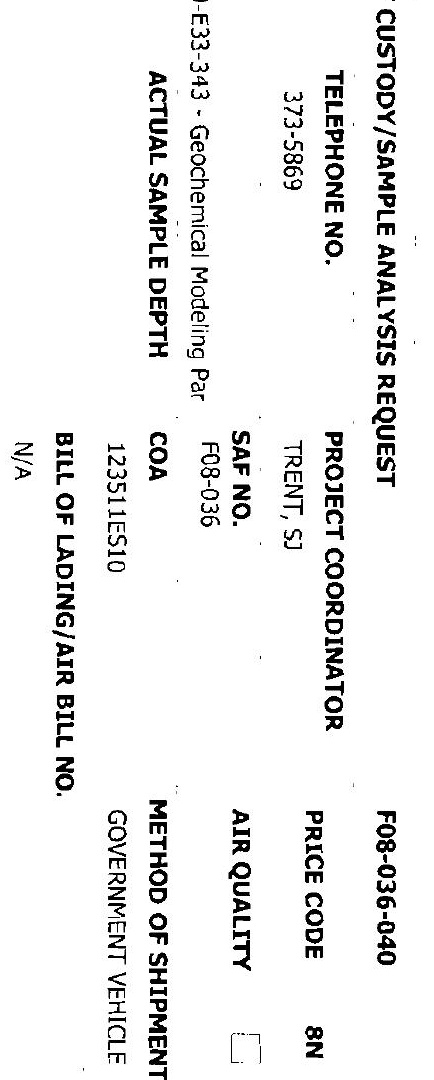
予

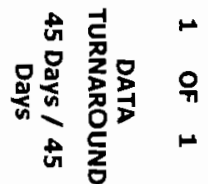




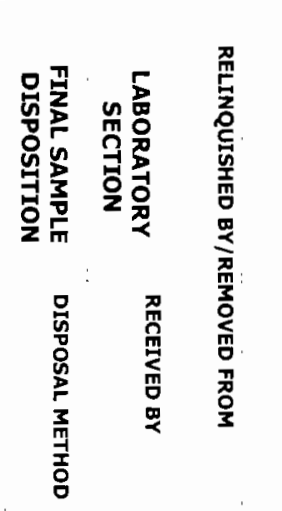

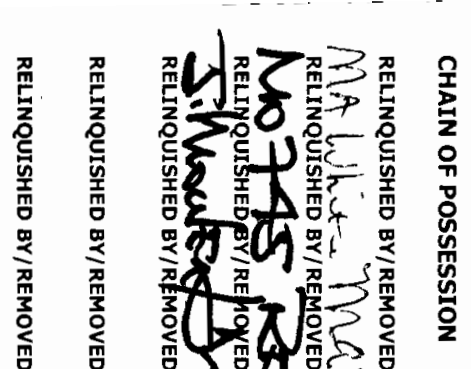

告

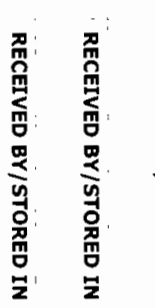

寻

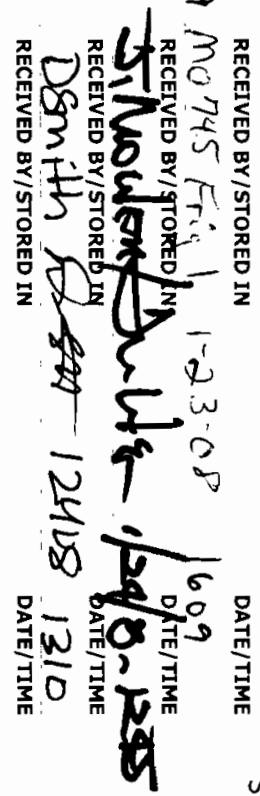

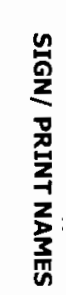

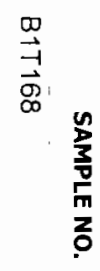

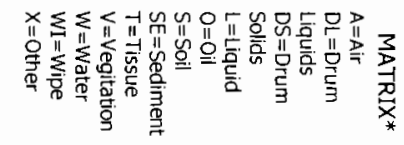

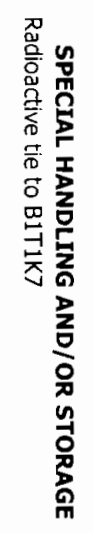

尊 管

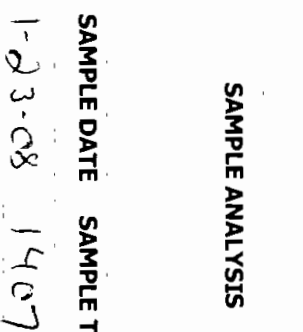

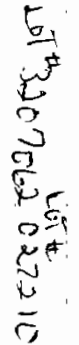

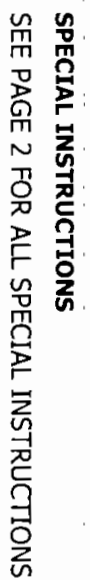

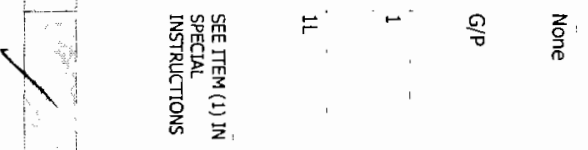

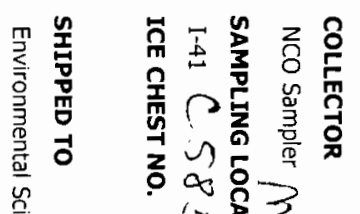
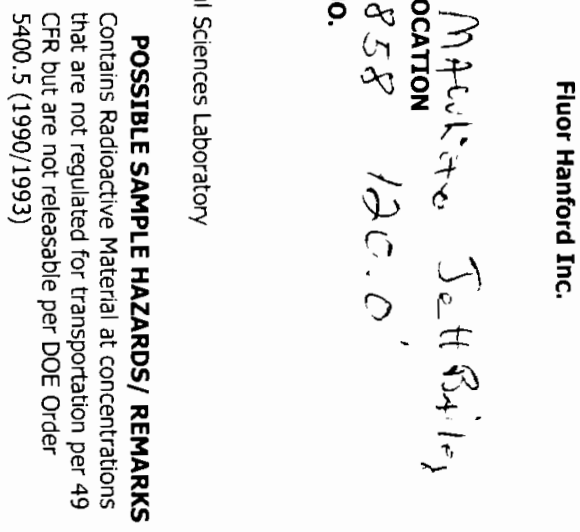

욕

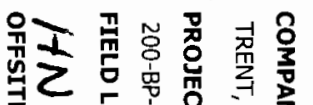

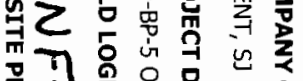

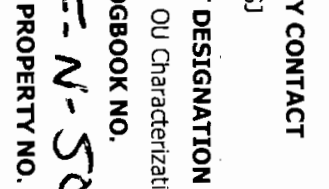
$\begin{array}{lll}0 & \stackrel{2}{2} & \frac{0}{1} \\ W_{1} & \stackrel{0}{0} & \end{array}$

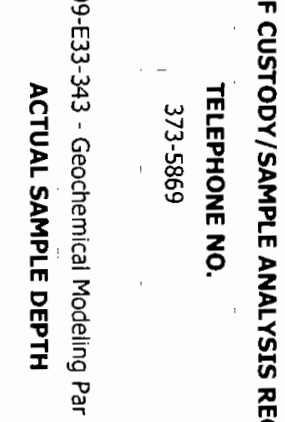

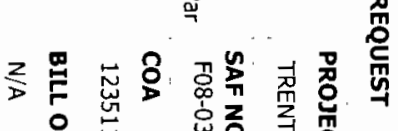
은 䎡 总 


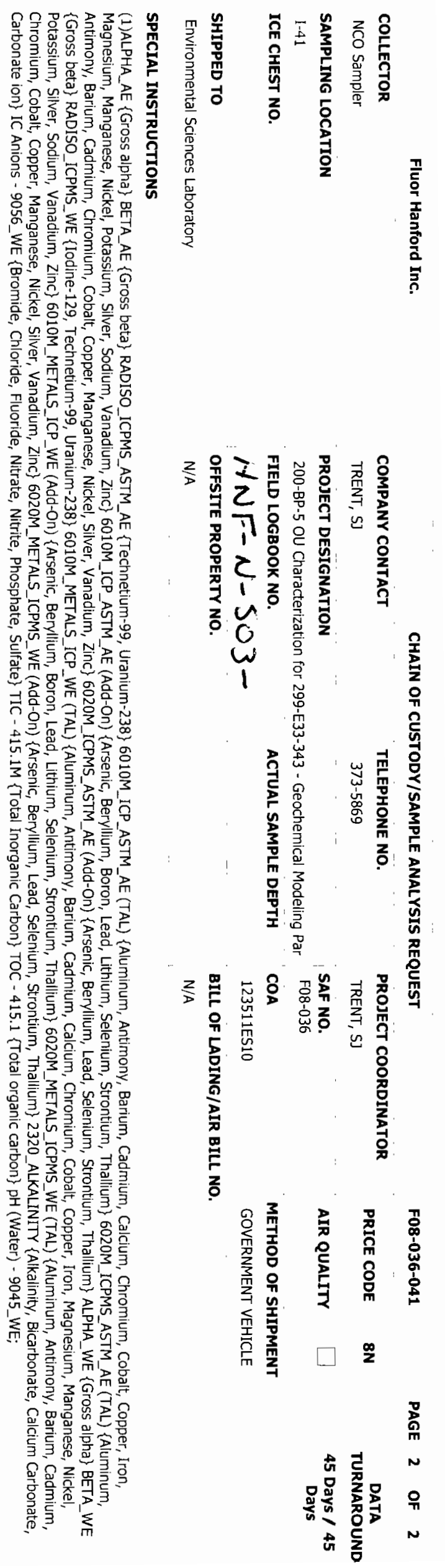




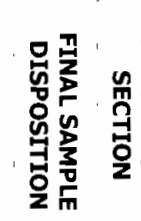

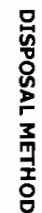

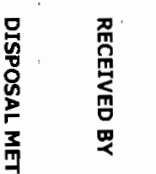

甭$$
\text { , }
$$
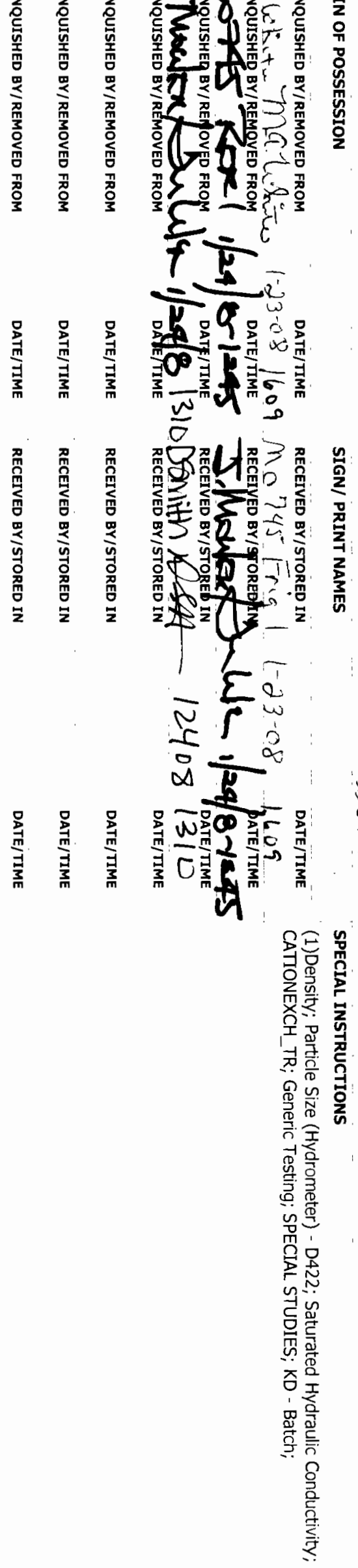

吾

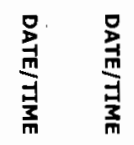
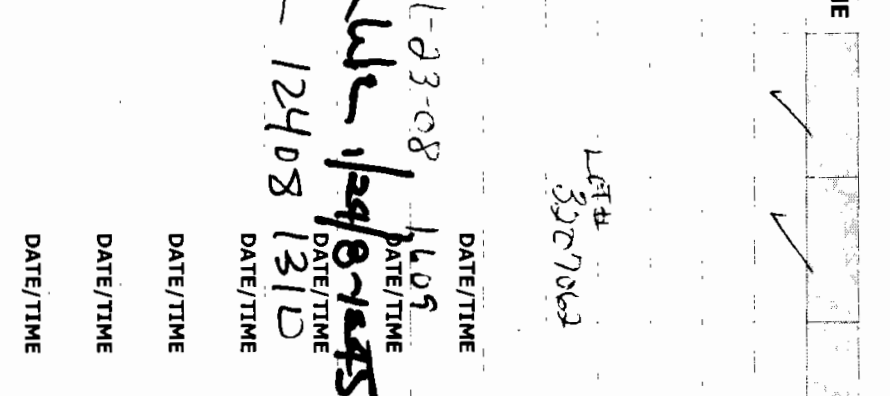$$
\text { . }
$$

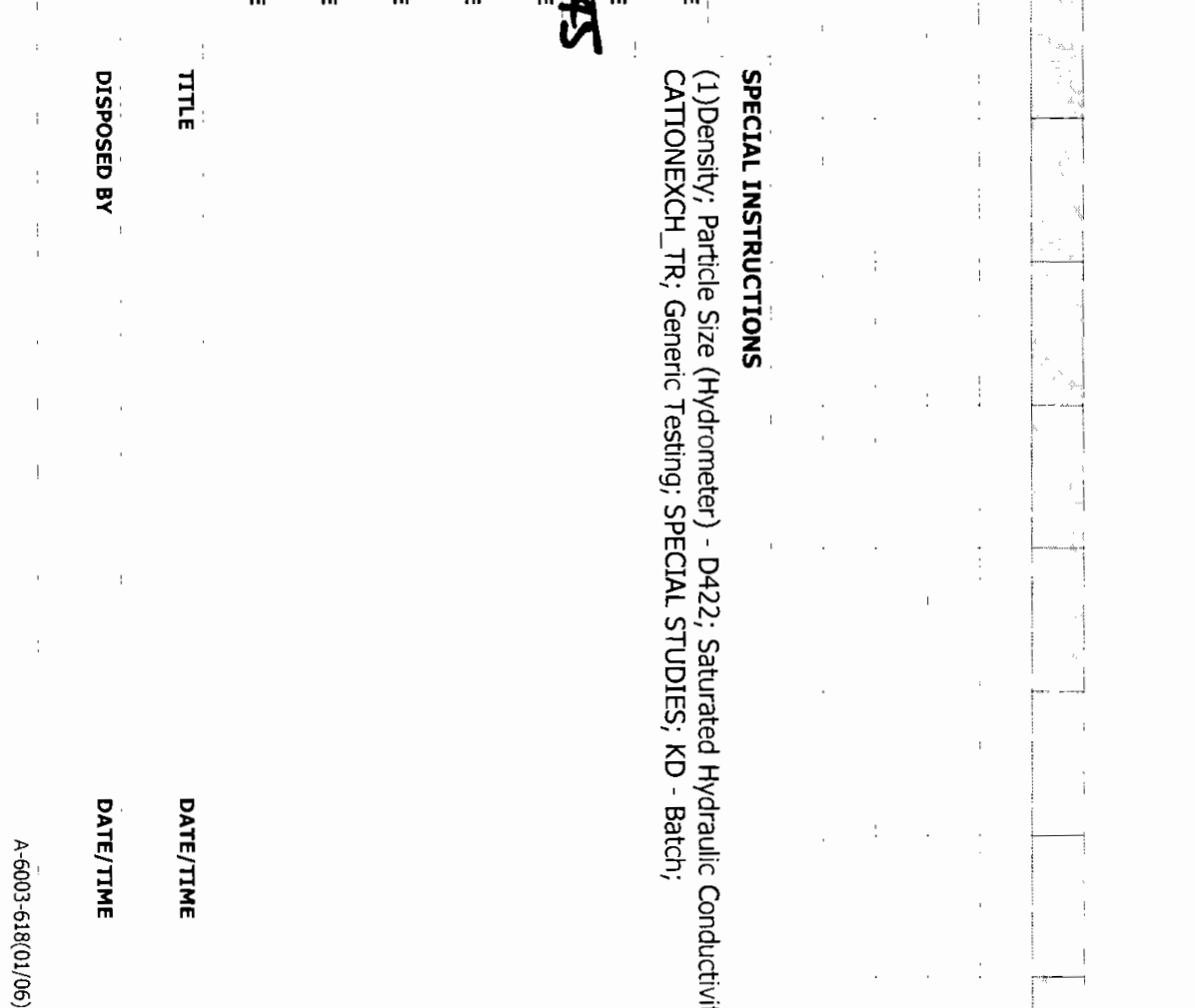

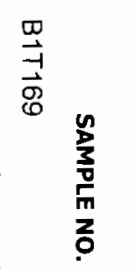

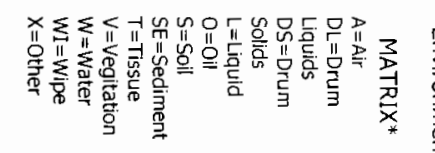

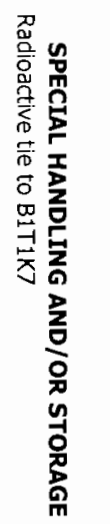

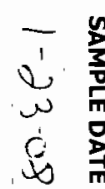

$\begin{array}{ll}2 & \frac{n}{3} \\ y & \frac{3}{7} \\ 0 & \frac{1}{3}\end{array}$

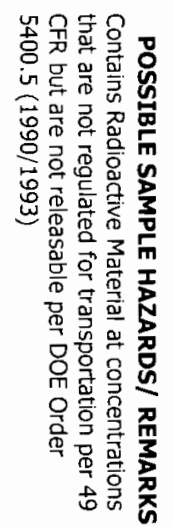

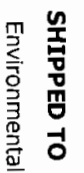

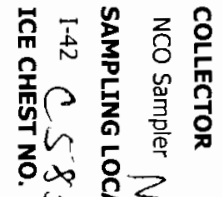

$\stackrel{0}{\circ}$

丞

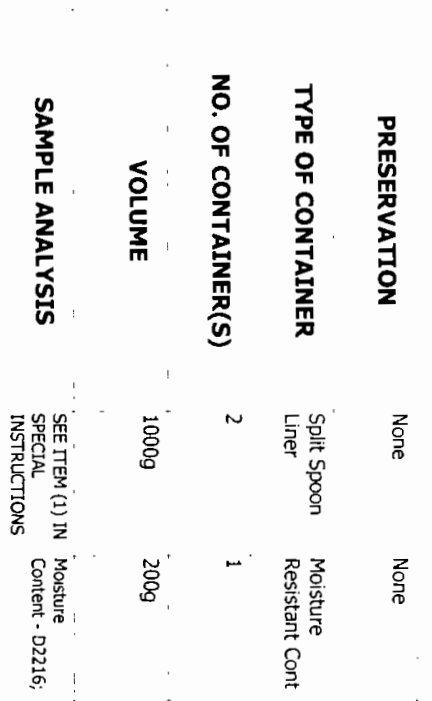

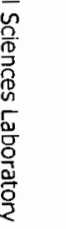$$
\text { 论 }
$$

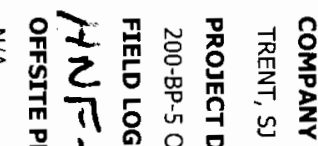
z $u_{0} \frac{0}{0}$

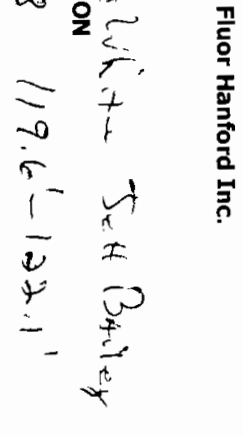

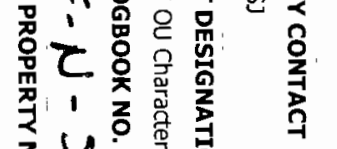

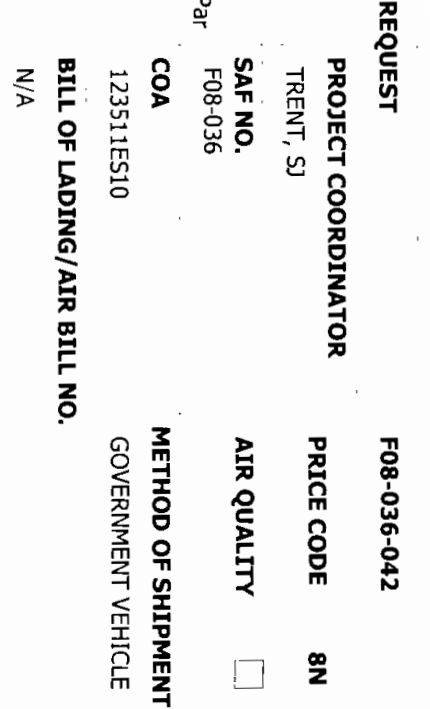

旁

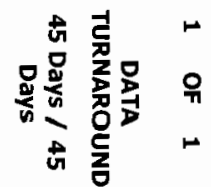




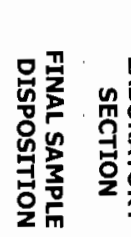

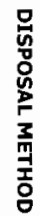

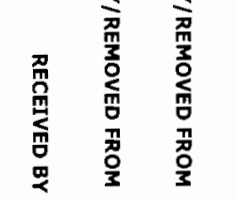

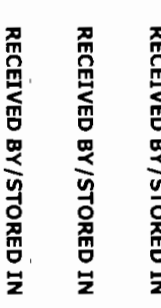

趸

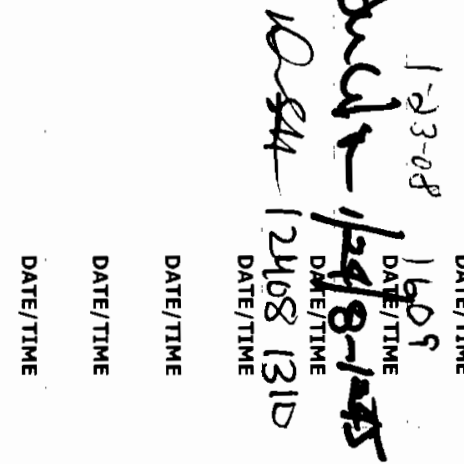

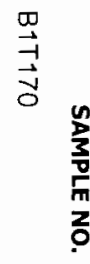

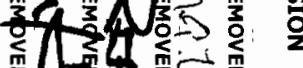

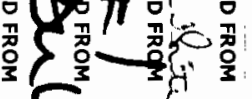

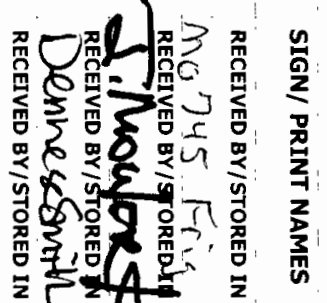

这皆

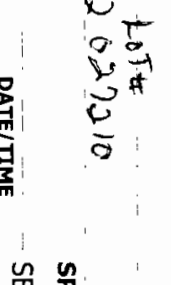

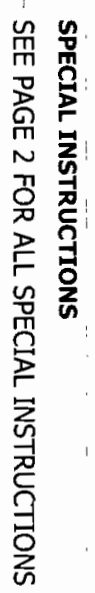

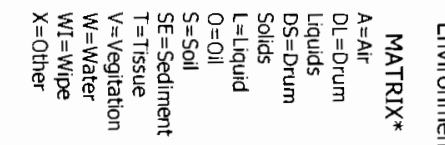

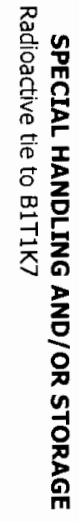
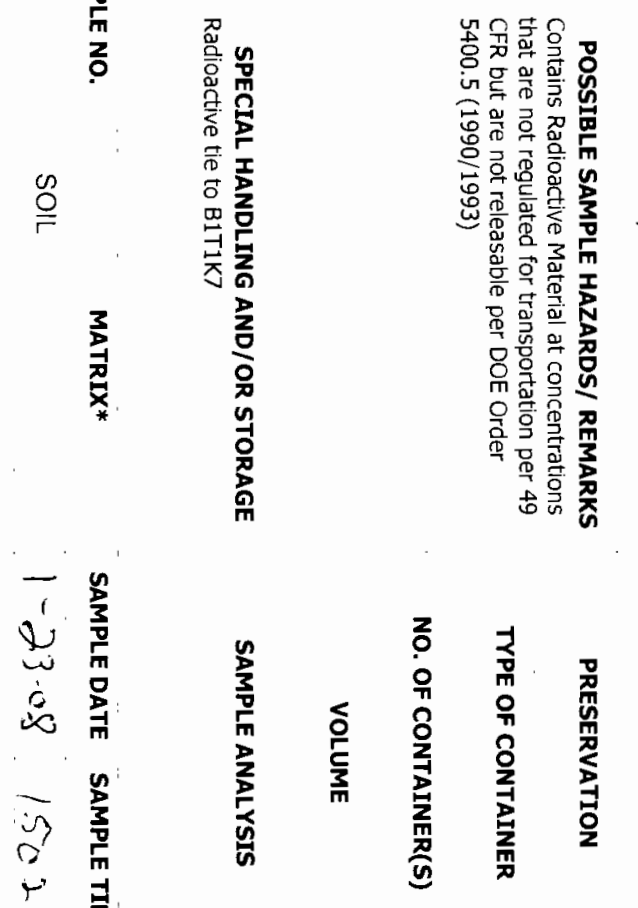

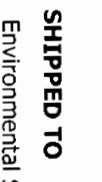

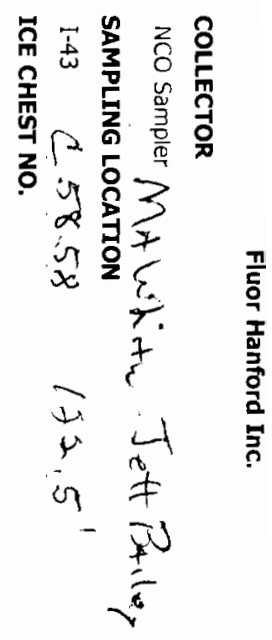

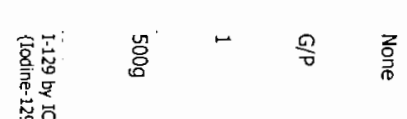

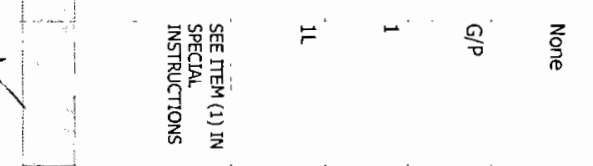

$z$ 위

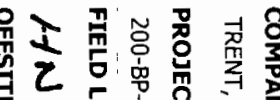

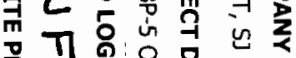
꽁

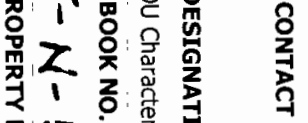
उ

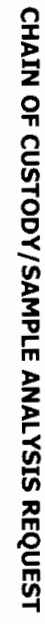

z

率

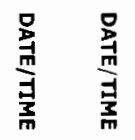




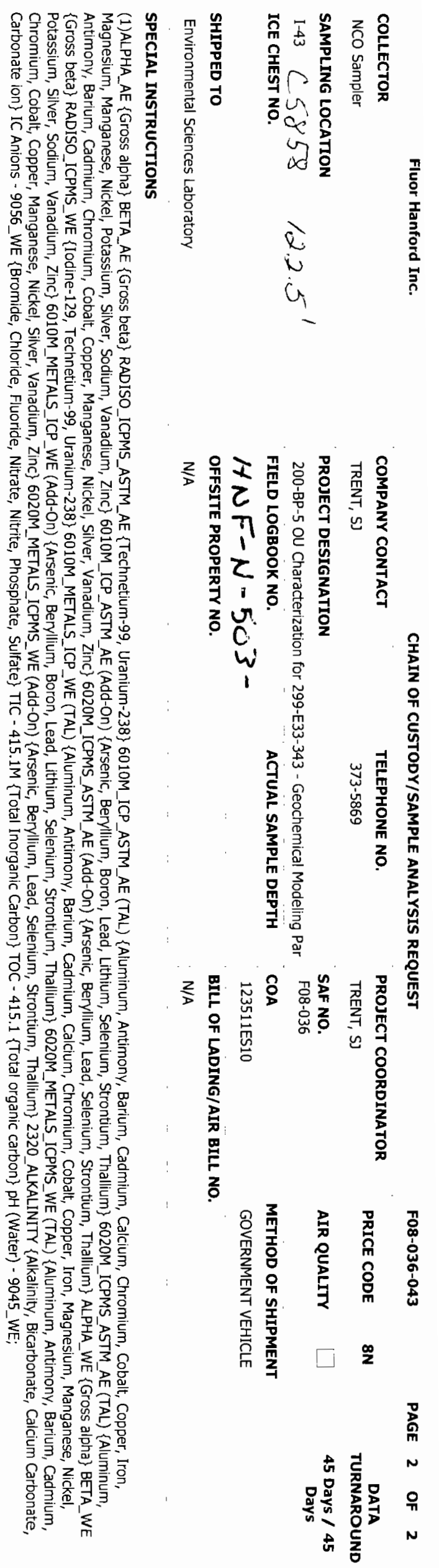




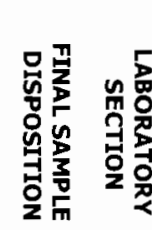
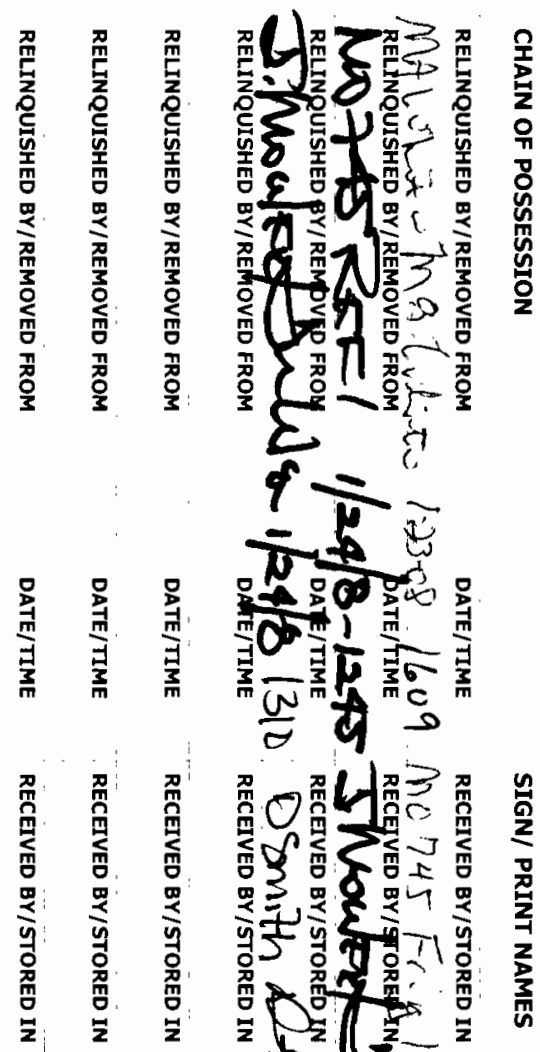

$\stackrel{O}{\stackrel{O}{ }}$

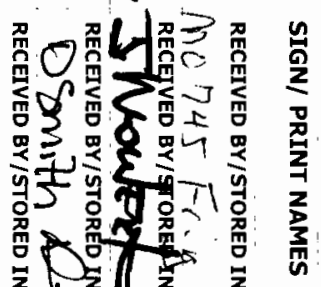

일

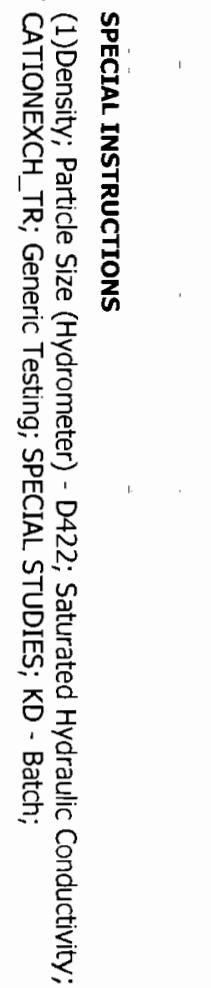

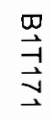

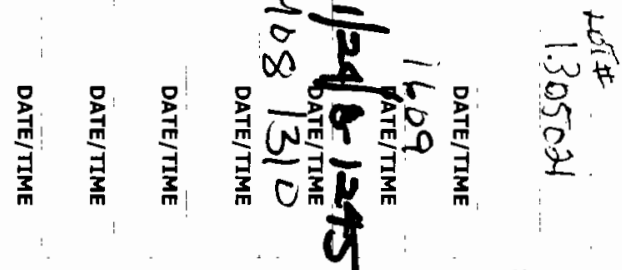

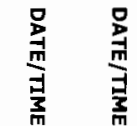

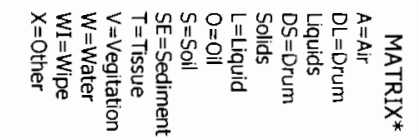

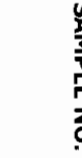

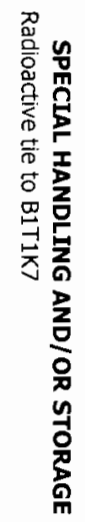

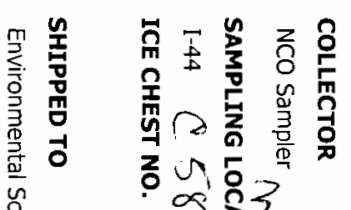

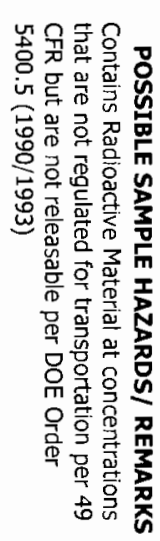

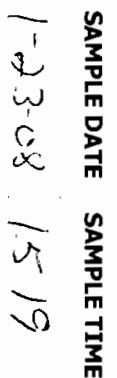

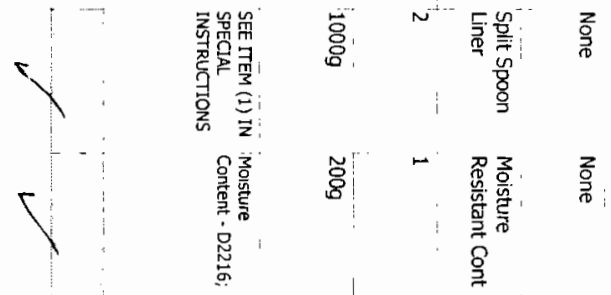

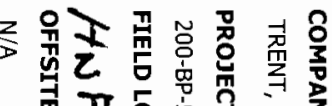
$\infty$ ?

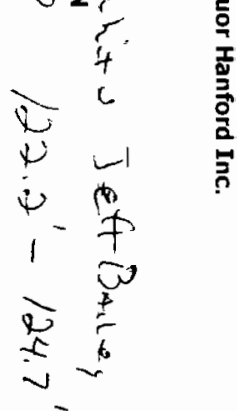

Tा 5 in

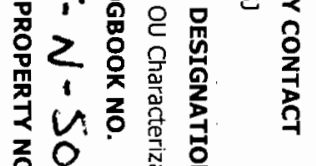

苛悹

承

帝

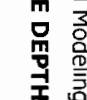

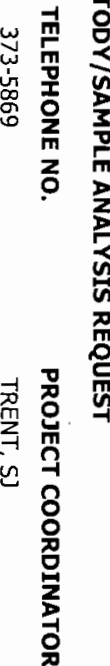

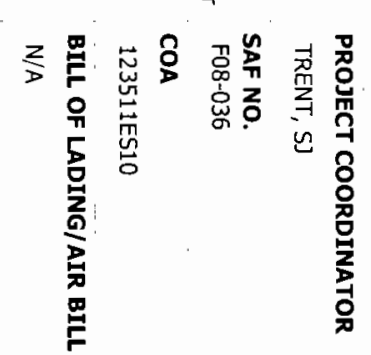

굴

일

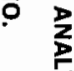

zo

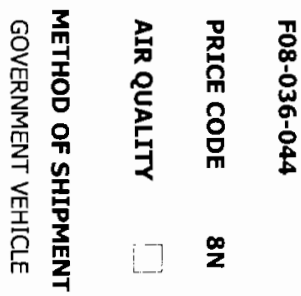

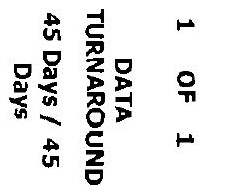




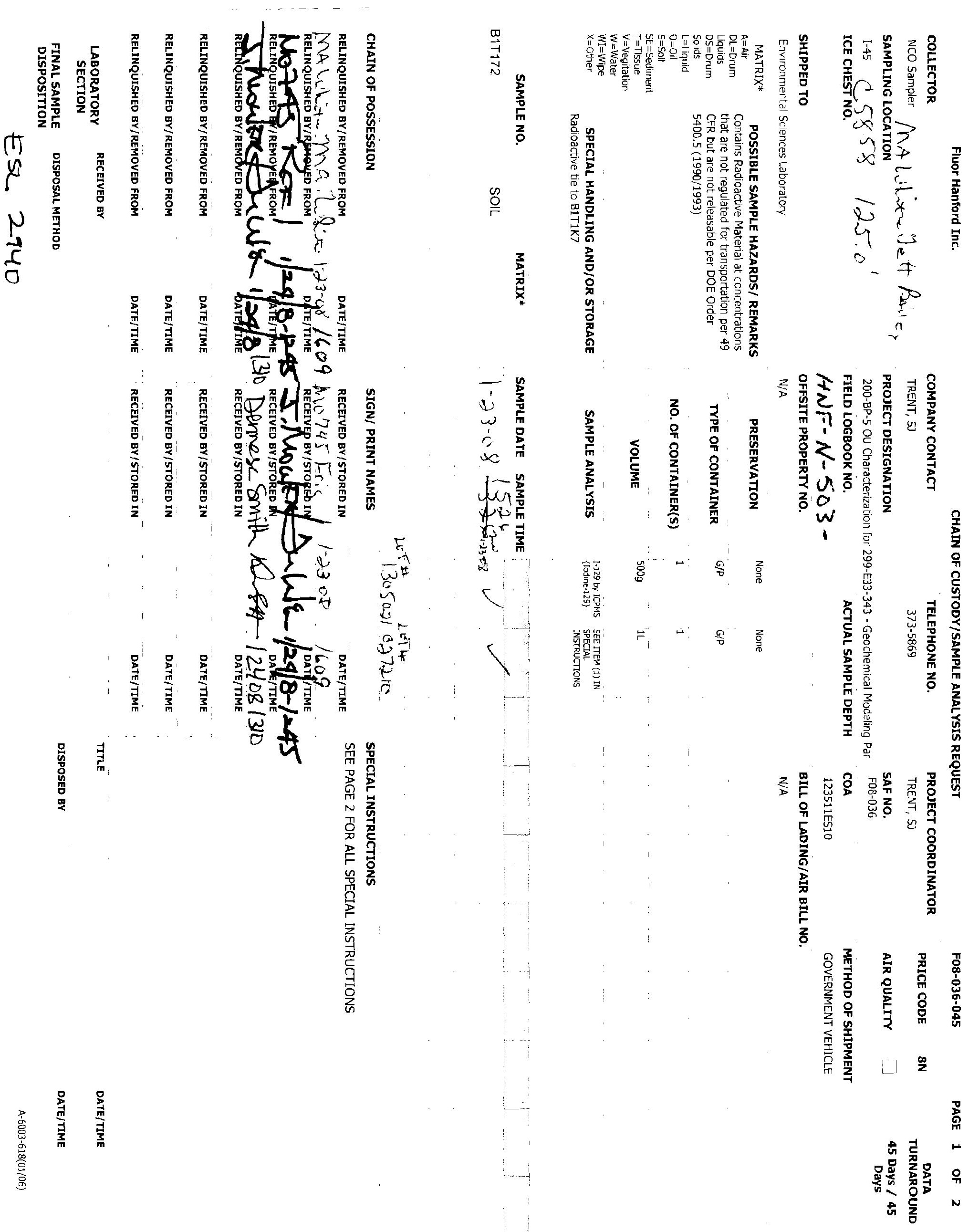




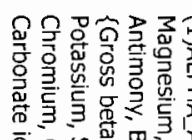

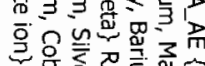

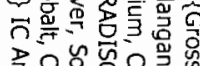

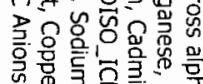

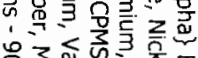

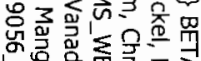

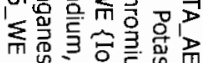

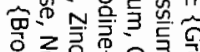

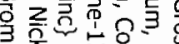

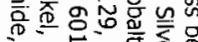

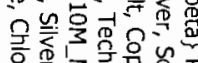

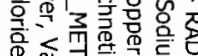

끌

음.

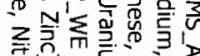

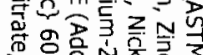

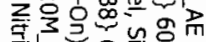

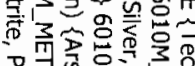

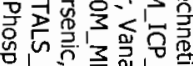

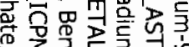

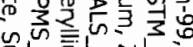

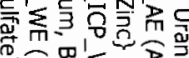

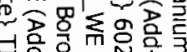

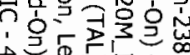

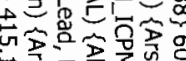

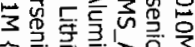

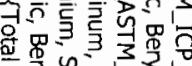

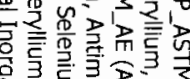

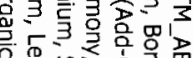

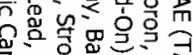

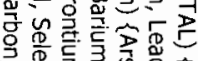

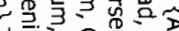

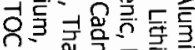

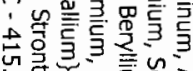

记

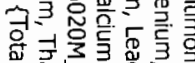

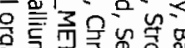

을

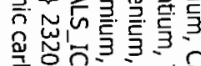

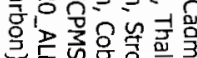

모닐

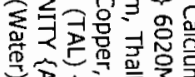

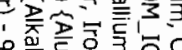

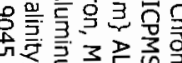

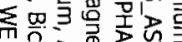

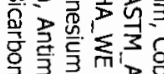

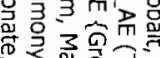

23

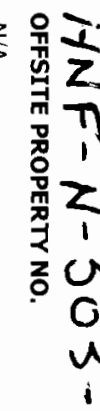

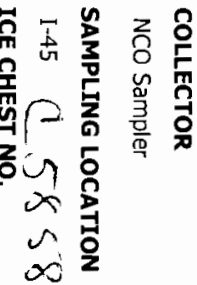

रे

in

3

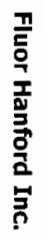

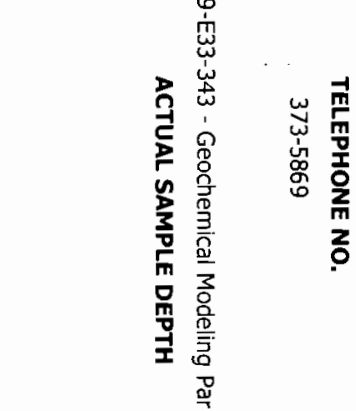

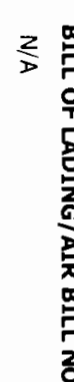

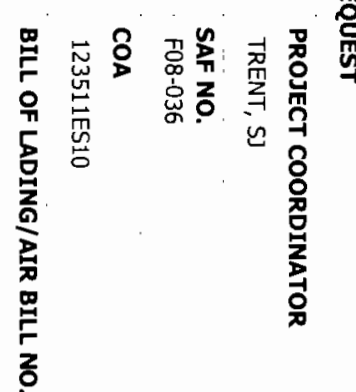

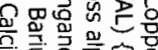

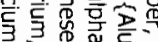

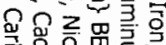

올

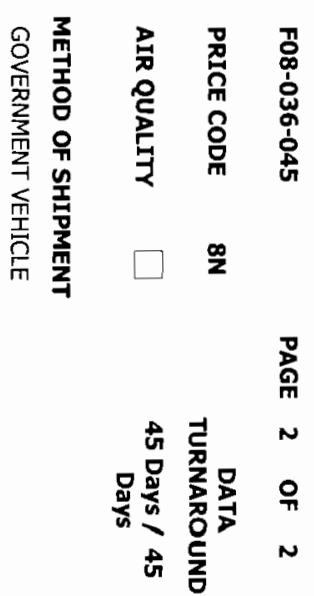



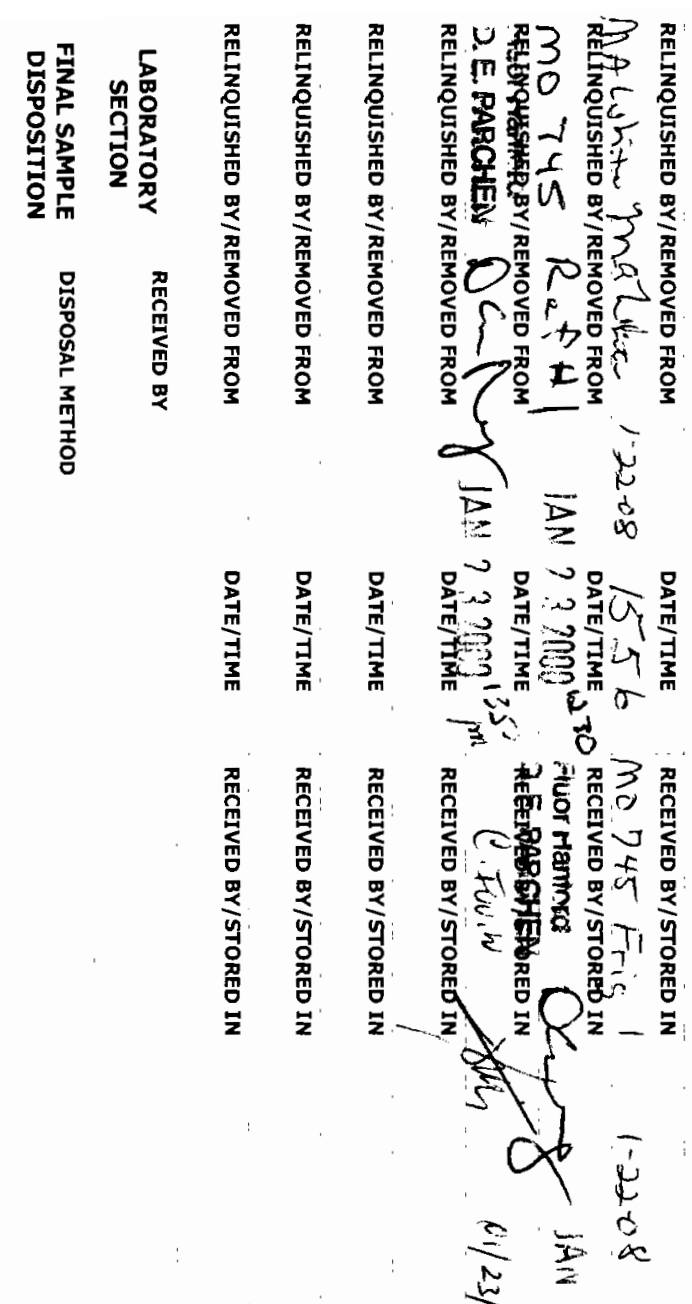

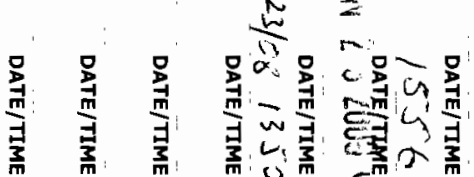

寻

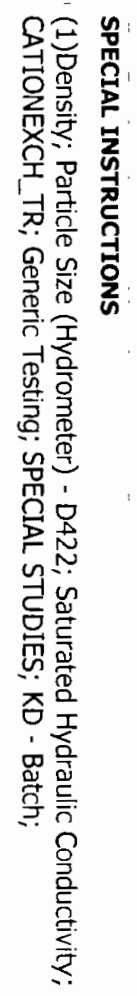

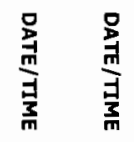

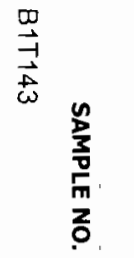

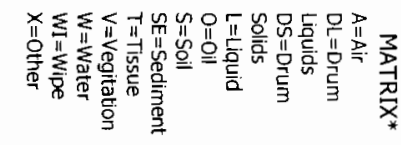

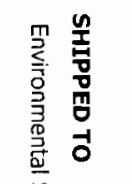

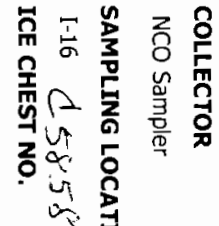

$\stackrel{\infty}{\circ}$

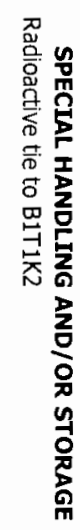

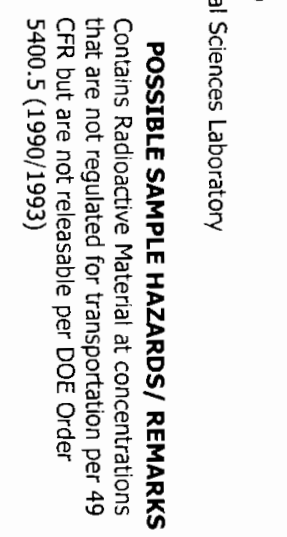

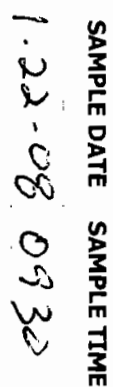

$\frac{6}{7}$

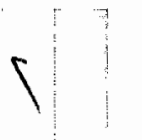

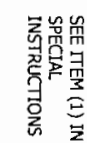

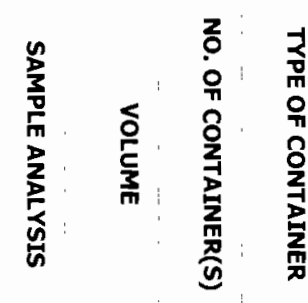

족

뀰

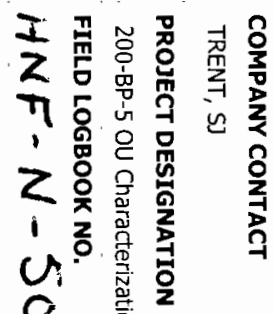

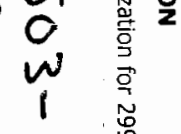

$\frac{n}{2}$

00

w

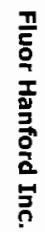

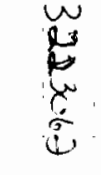

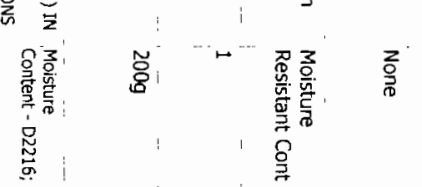

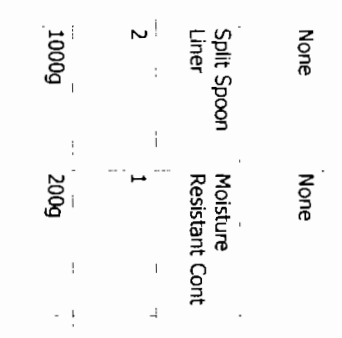




\section{\|\|}
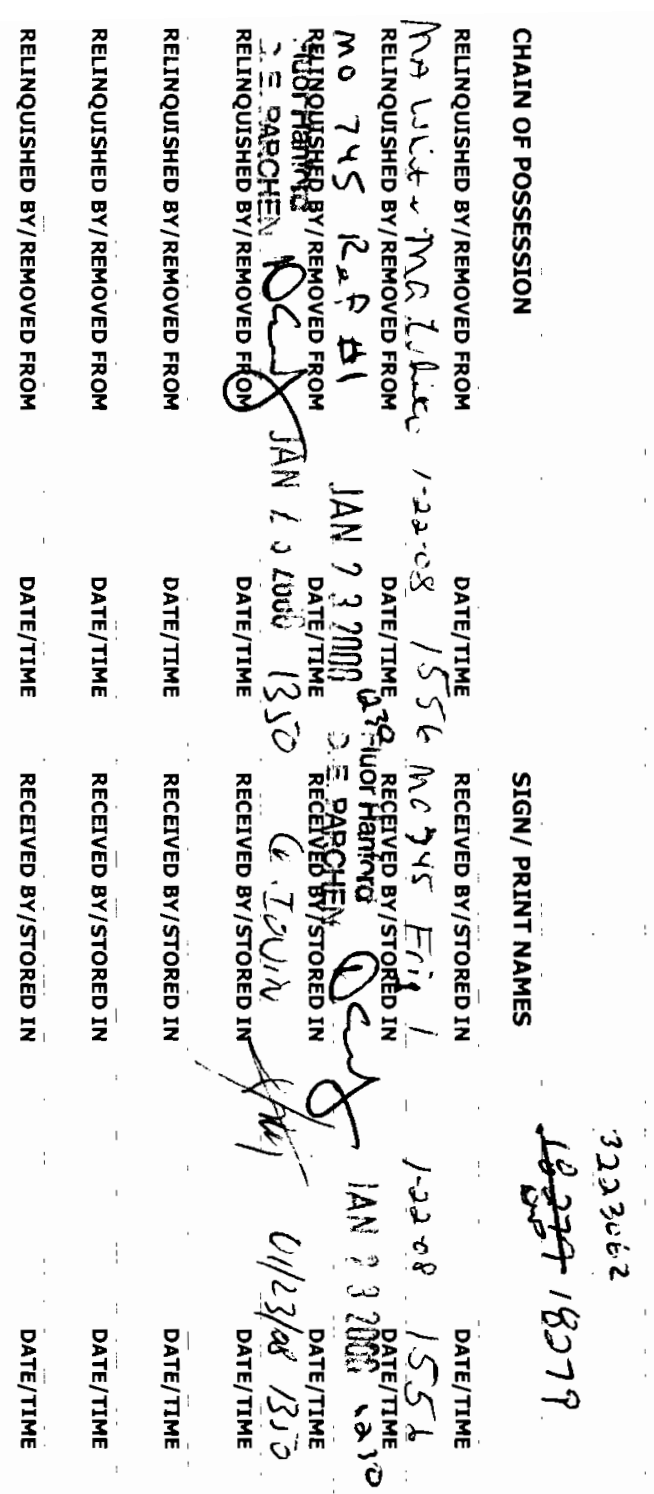

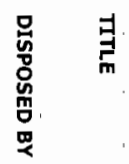

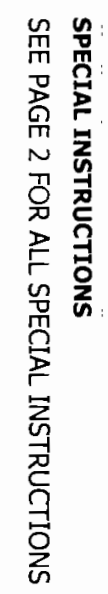

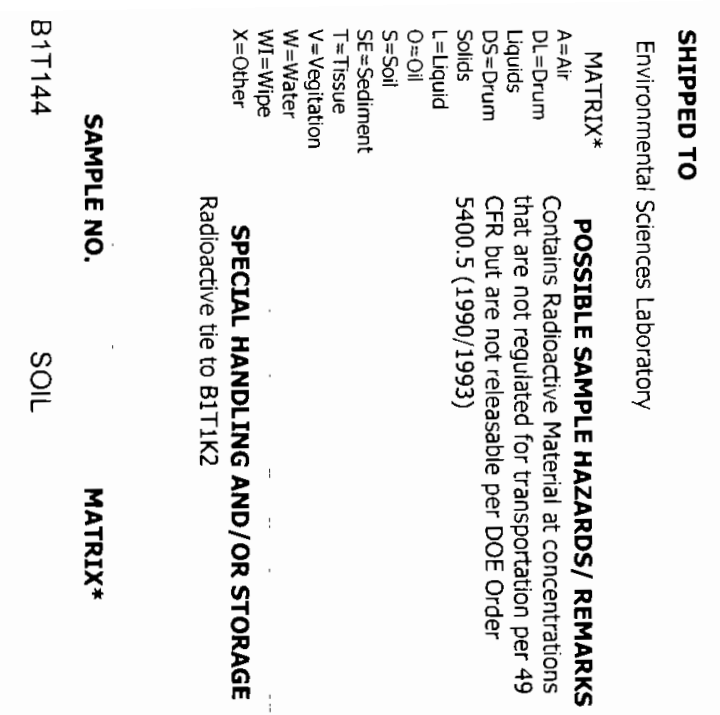

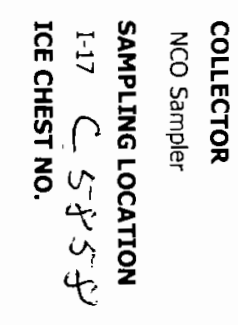

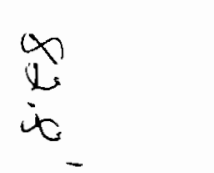

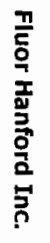
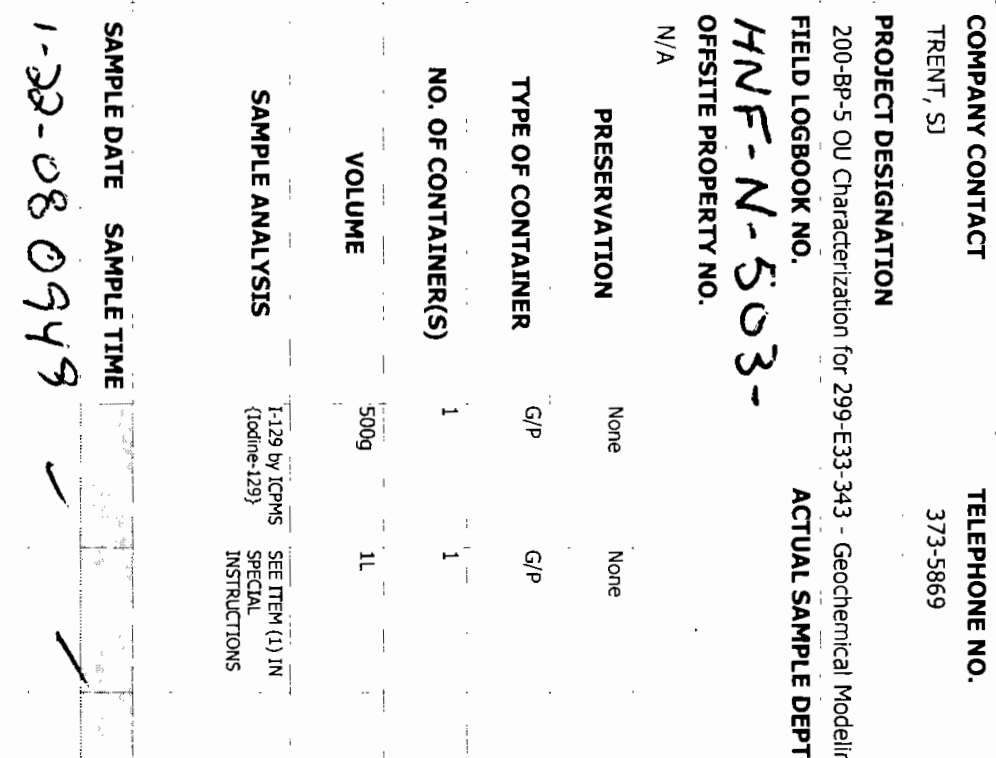

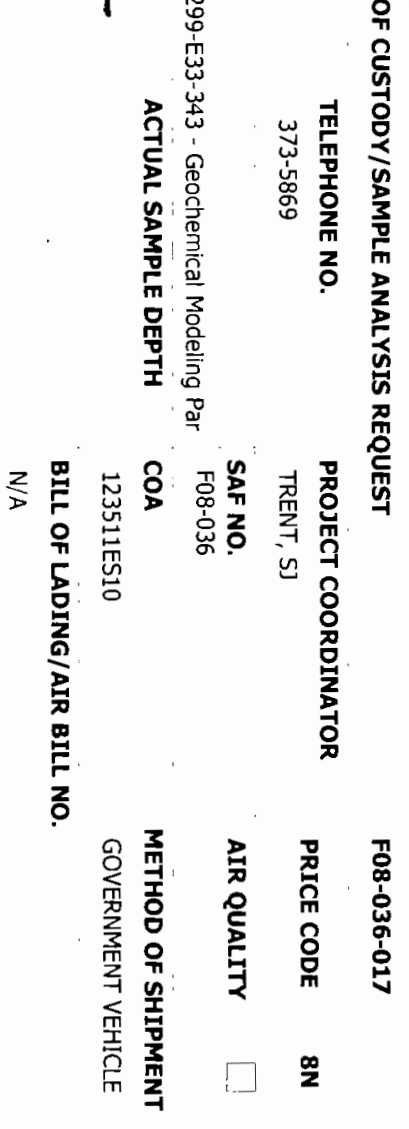

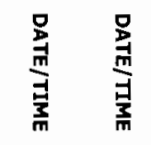

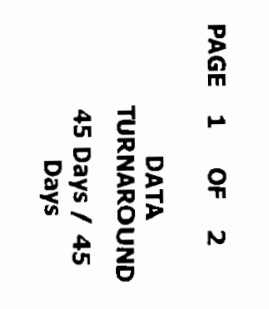




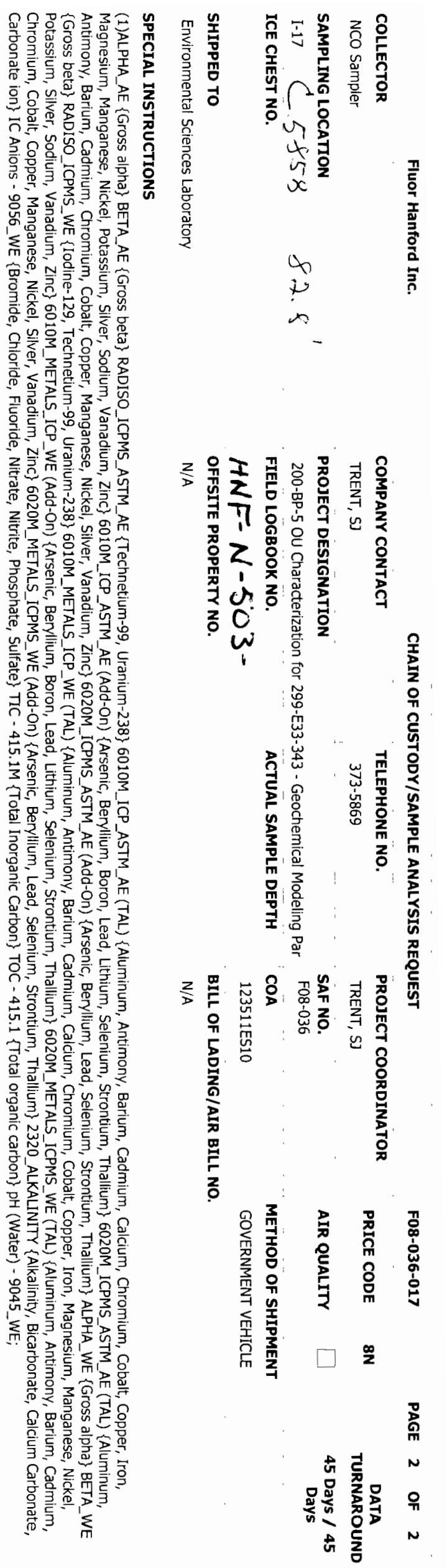




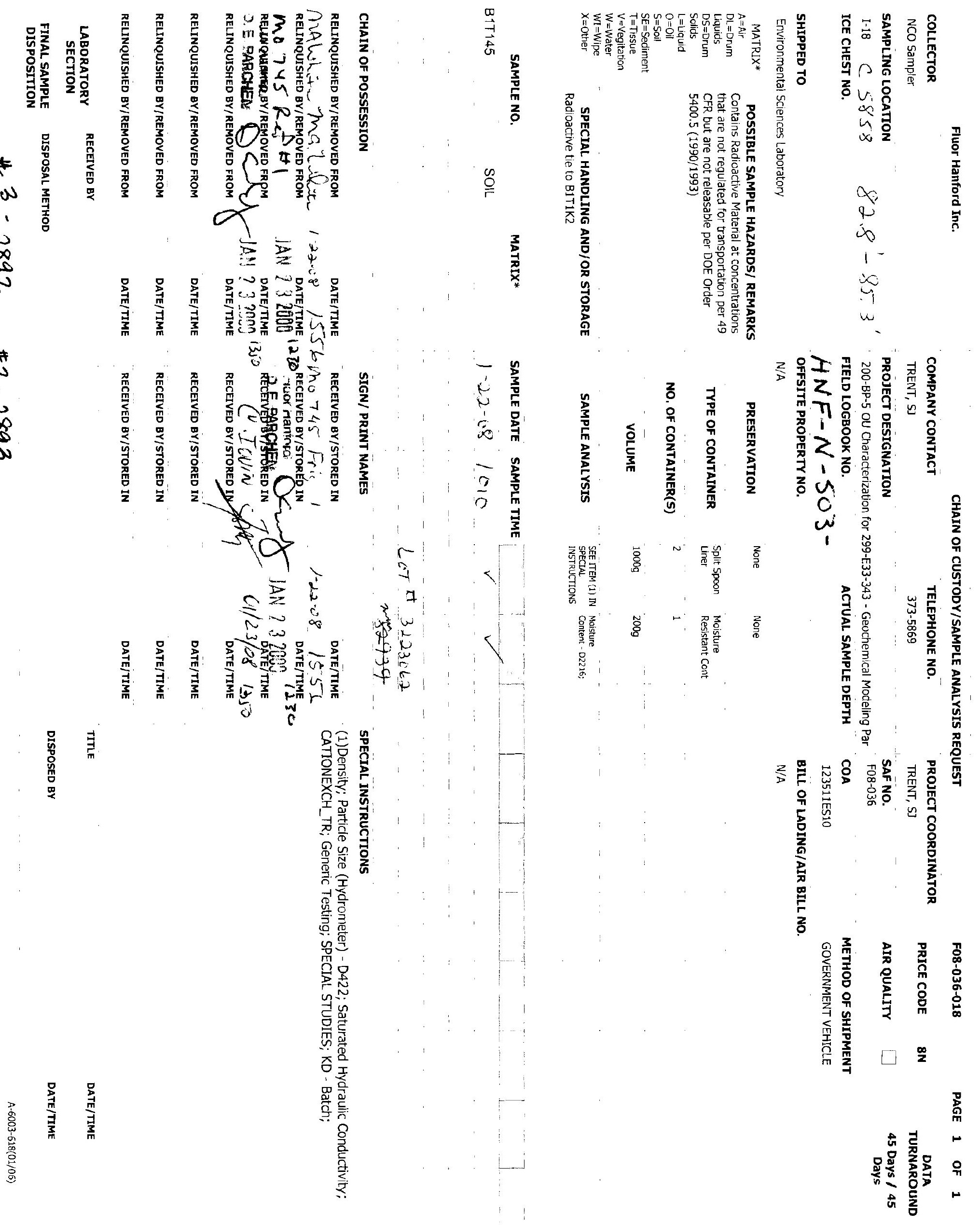




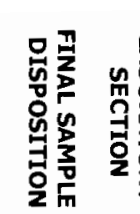

量 㟨

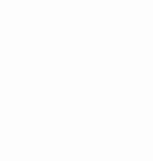

尊品

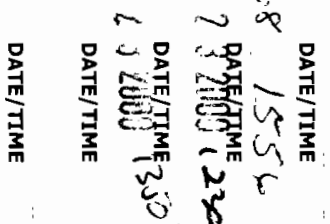

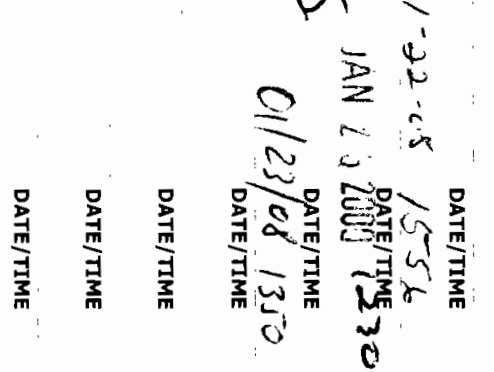

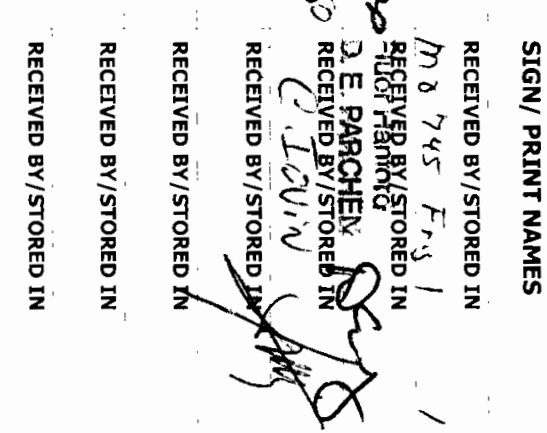

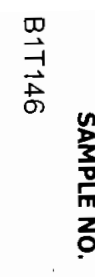

$\stackrel{\circ}{\stackrel{0}{\Gamma}}$

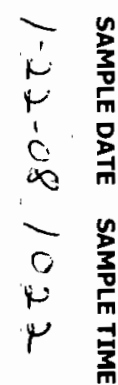

全

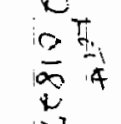

I

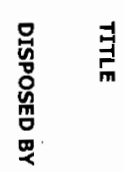

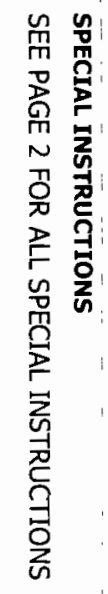

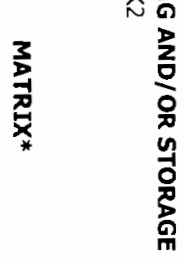

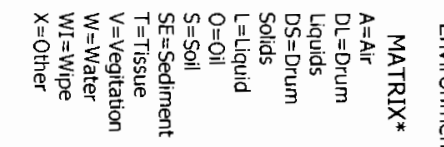

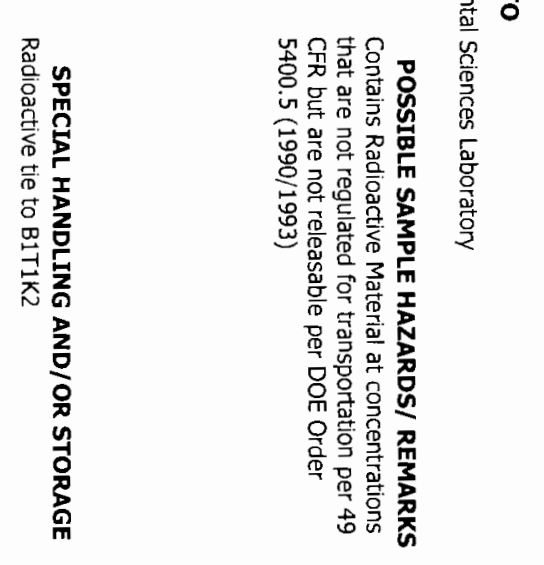

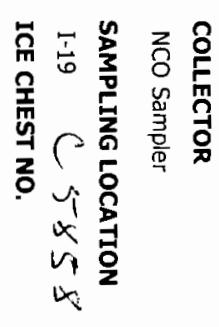

in

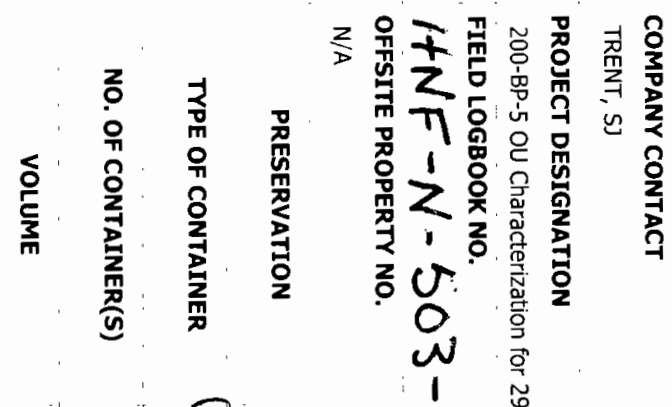

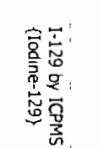

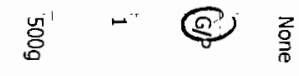

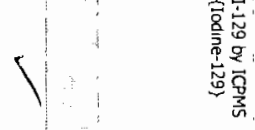

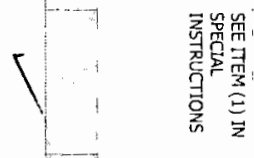

$\frac{1}{2} \frac{0}{1}$

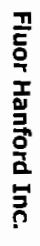

올

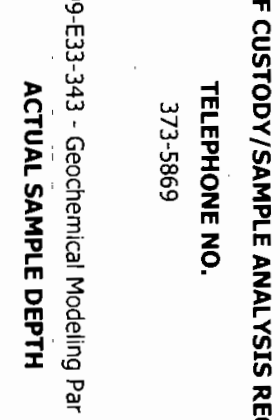

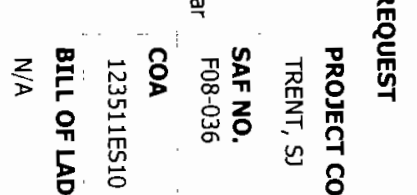

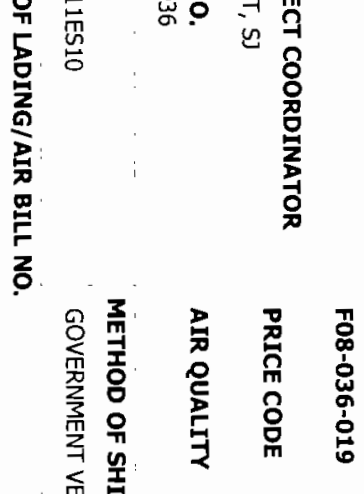

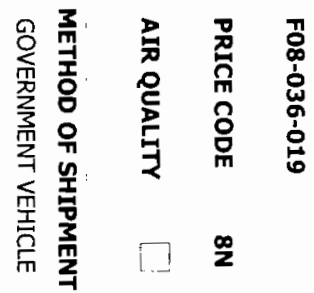

วั

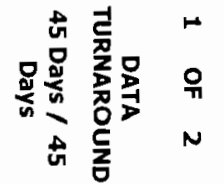




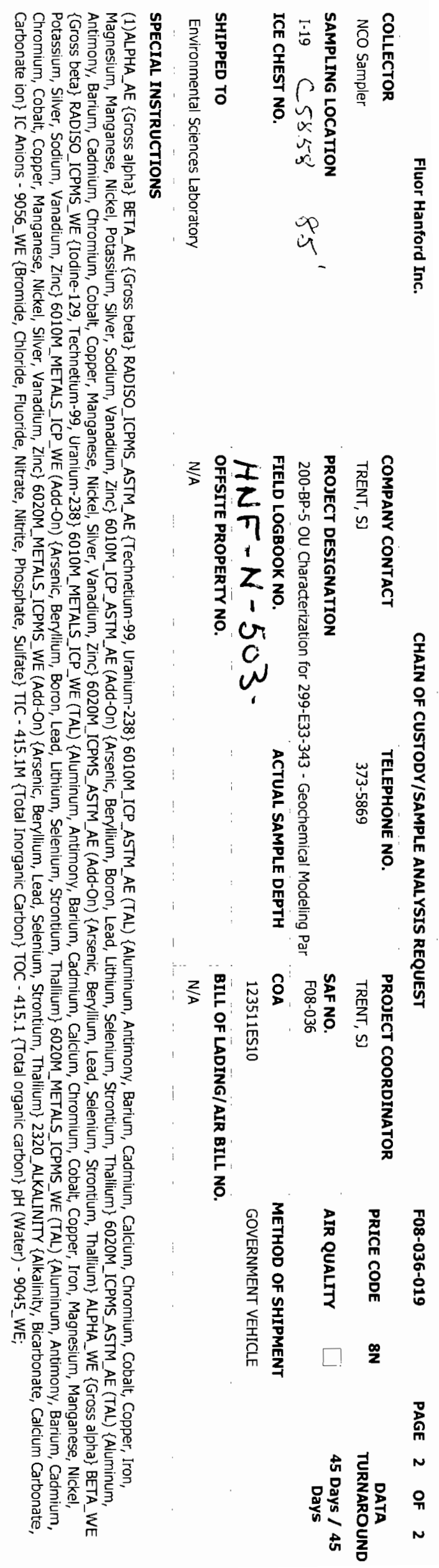




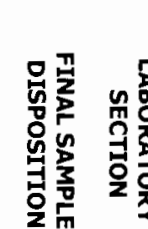
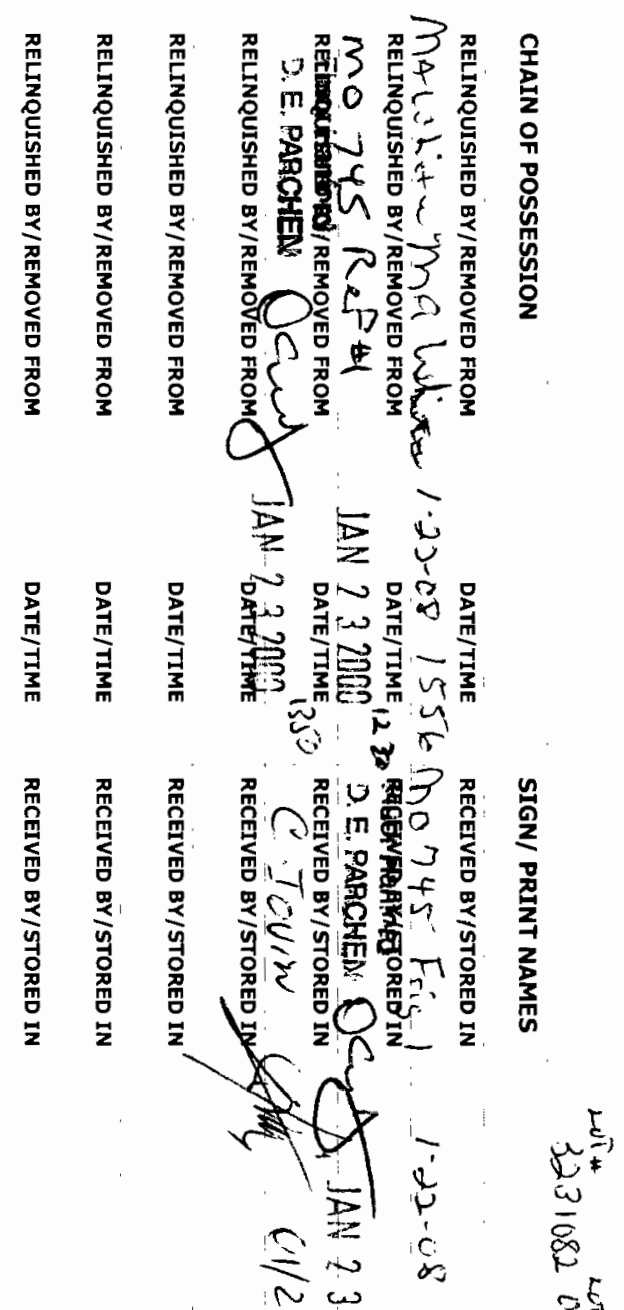

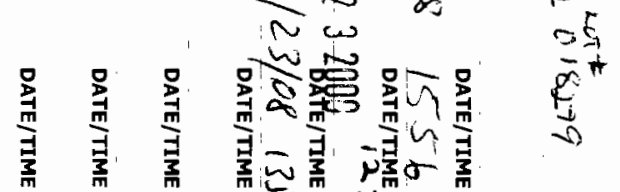

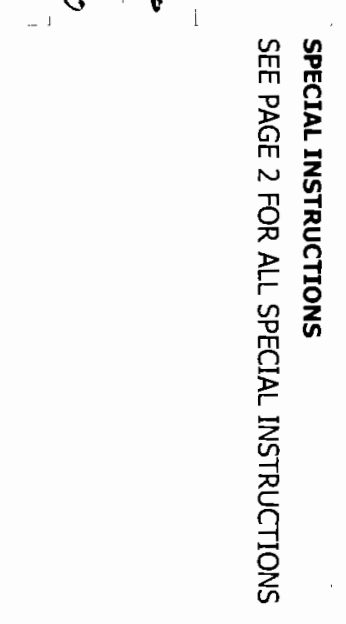

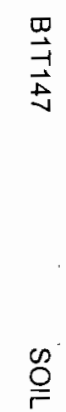

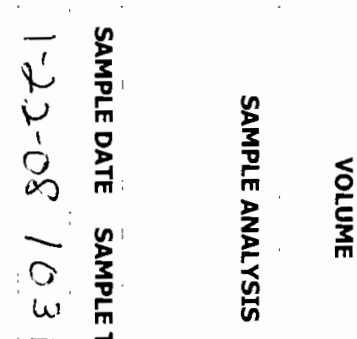

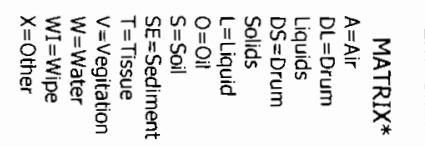

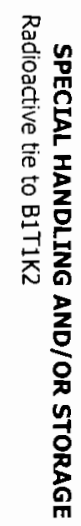
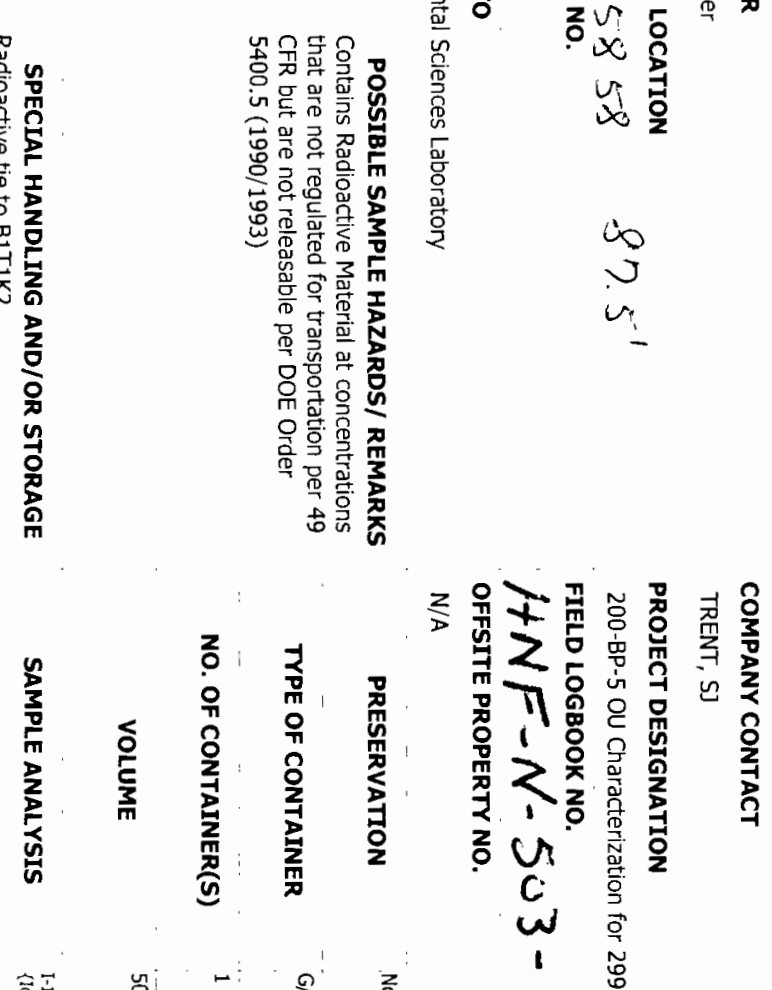

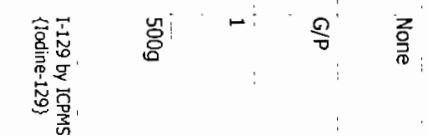

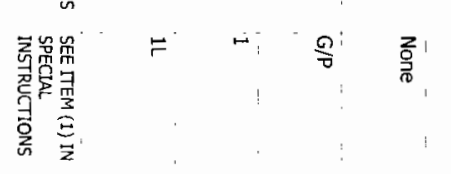

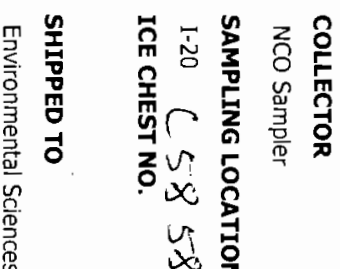

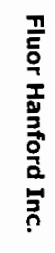

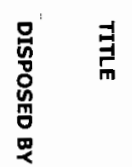

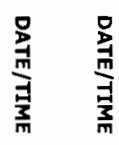

然 


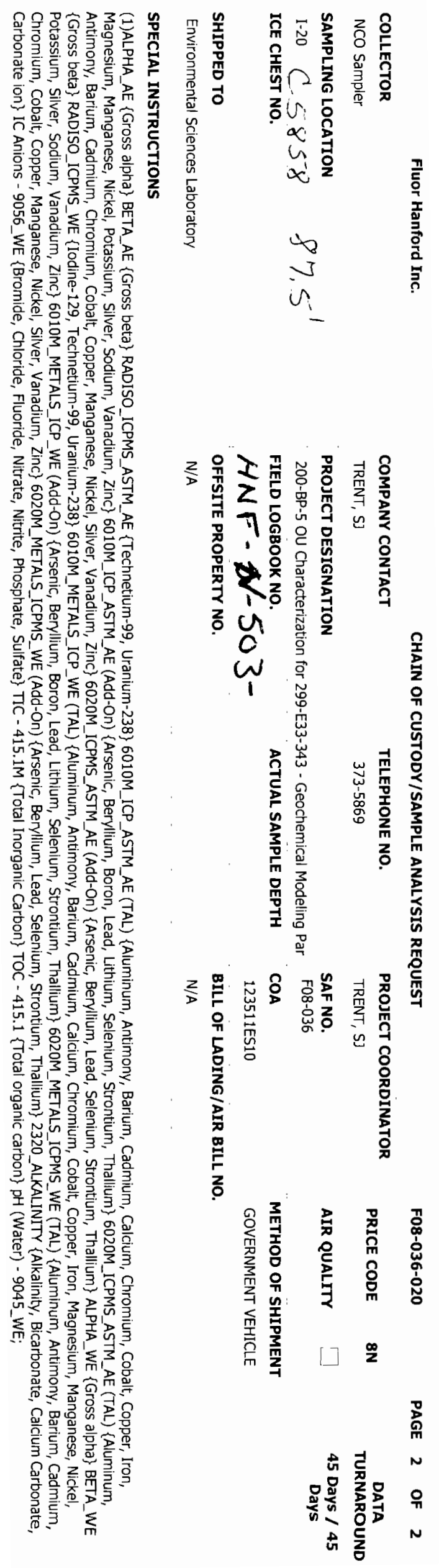




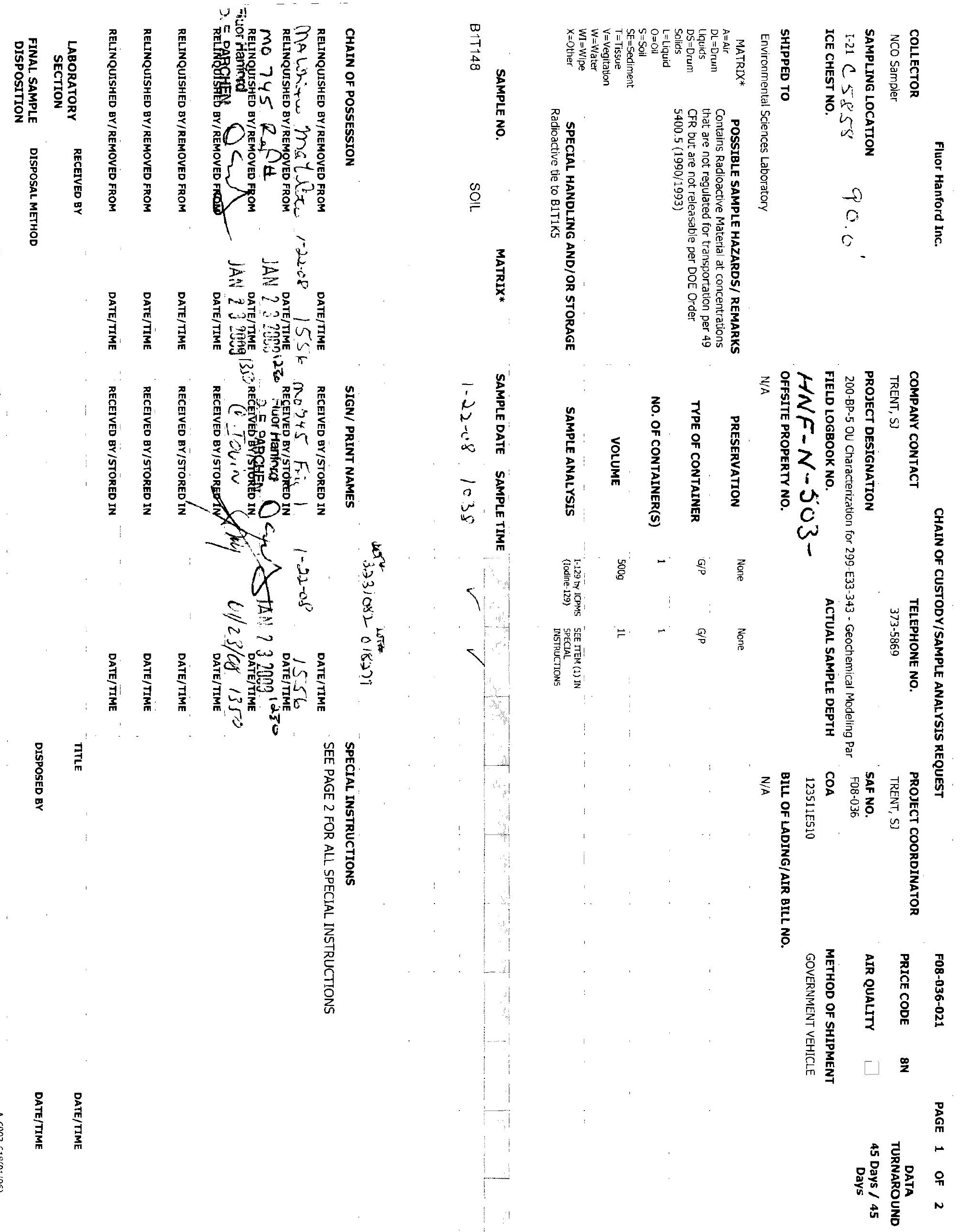




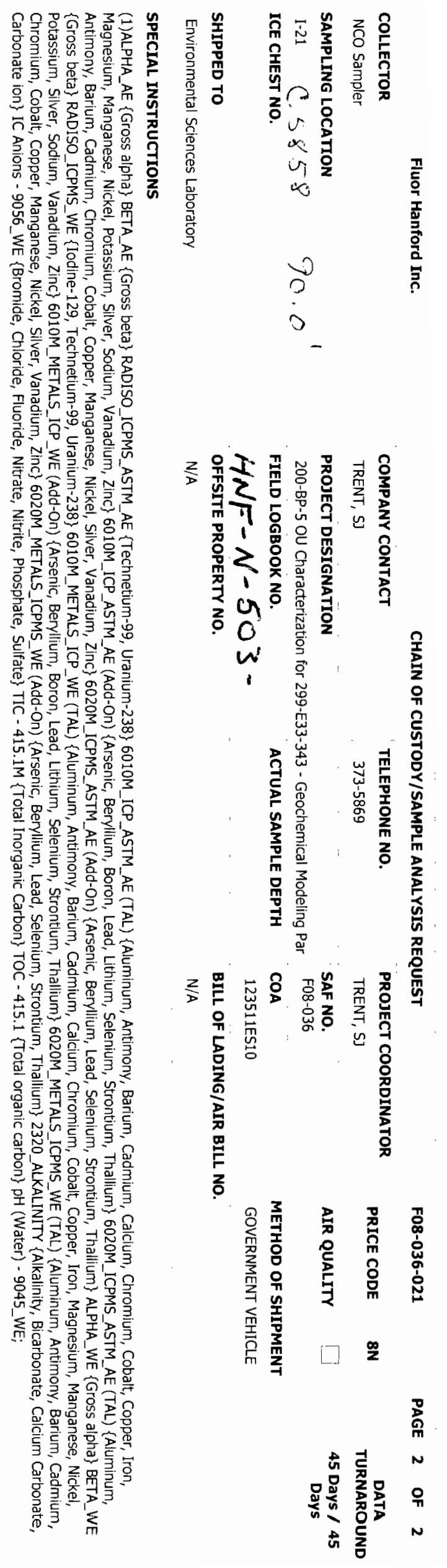



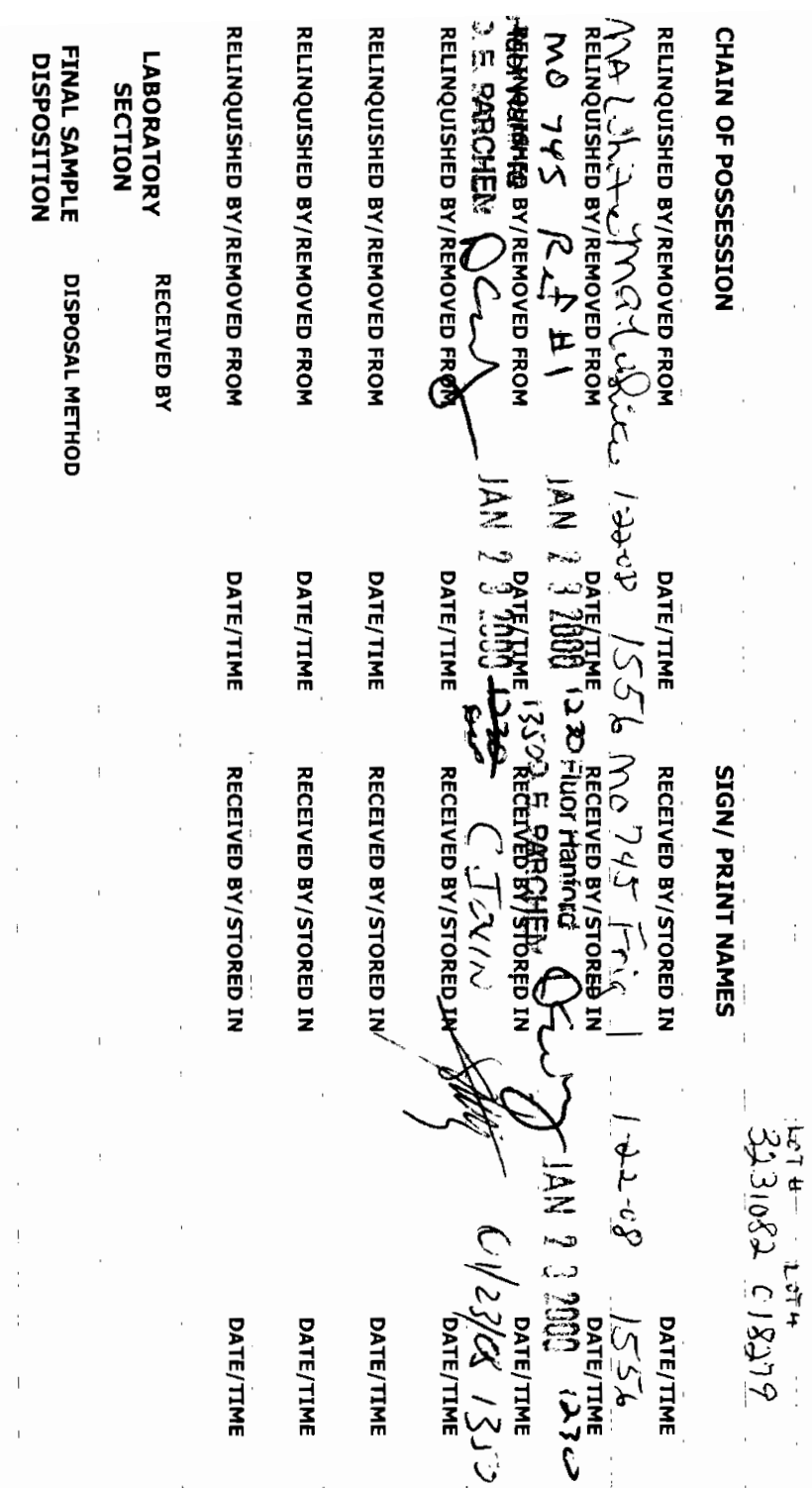

of $\bar{y}$ \%

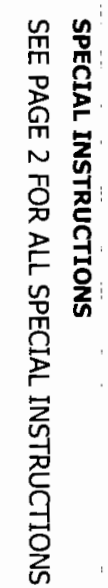

$\infty$
$\vec{A}$
$\overrightarrow{0}$

$\stackrel{9}{\circ}$

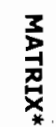

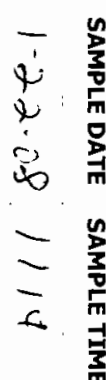

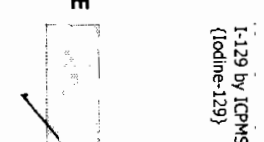

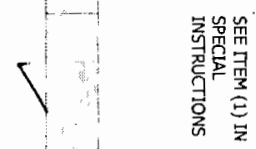

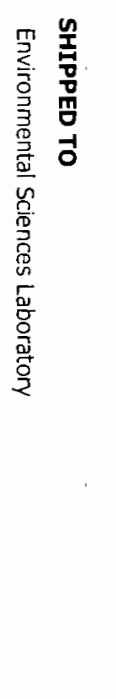

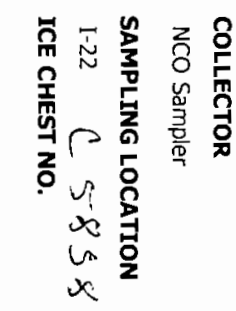

2

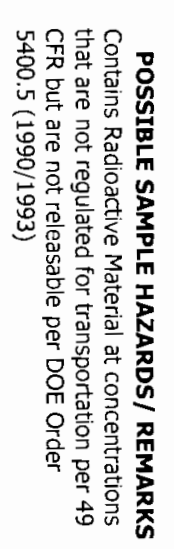

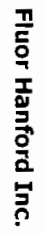

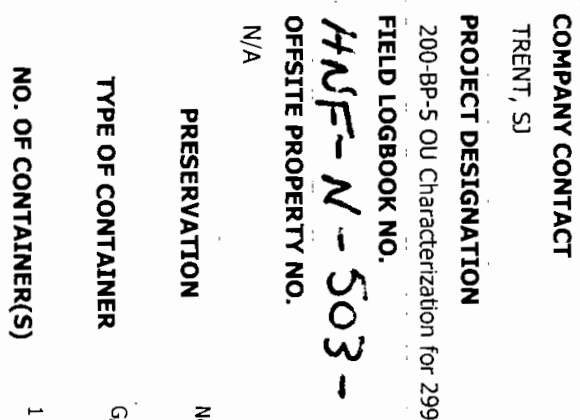

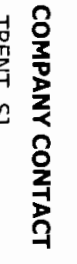

울
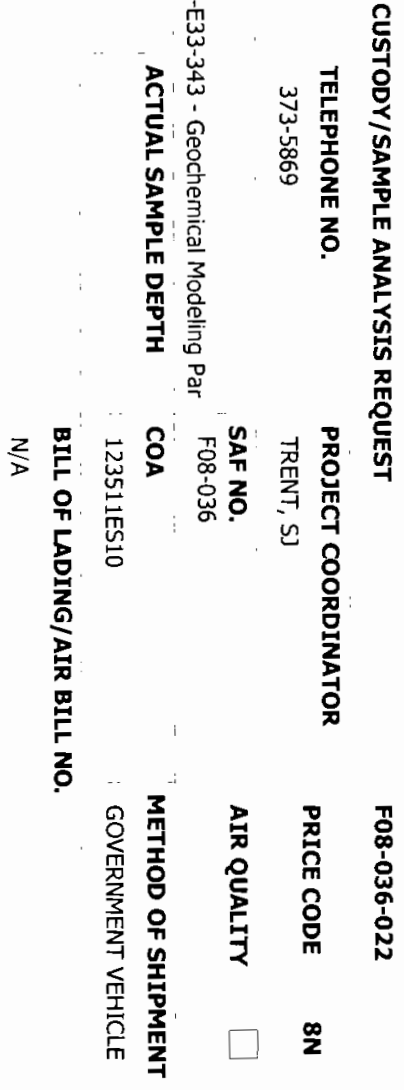

荈品

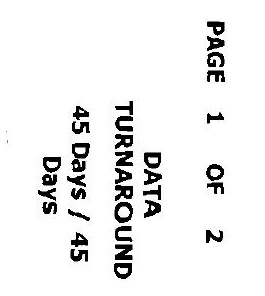




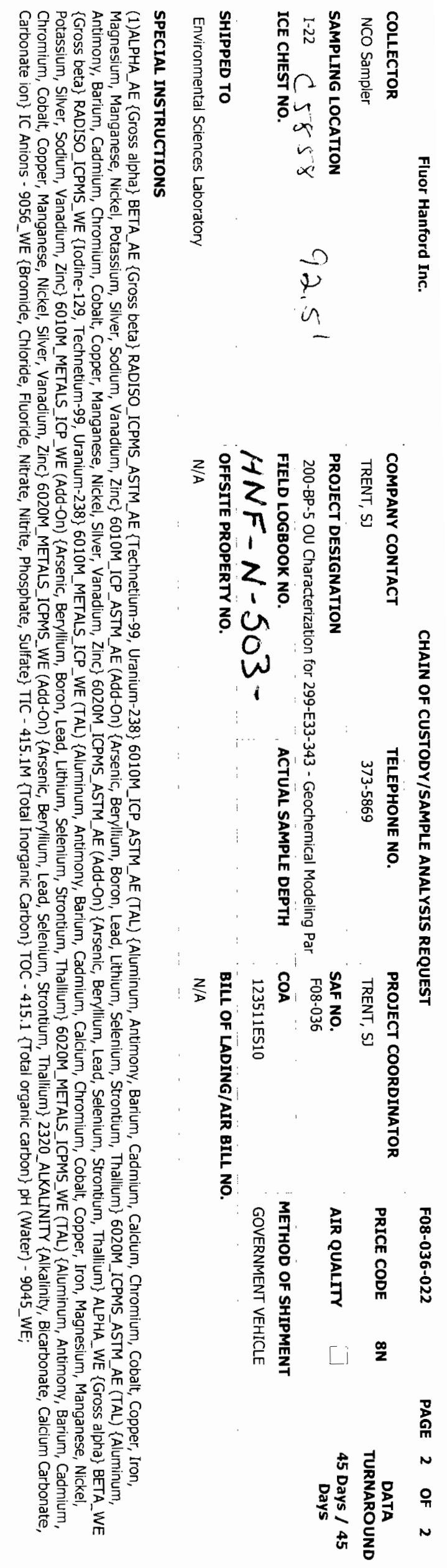




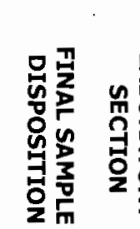

量

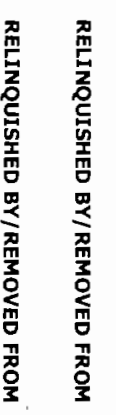

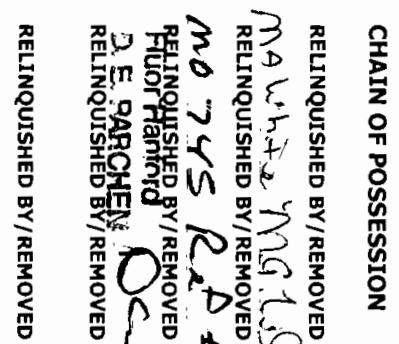

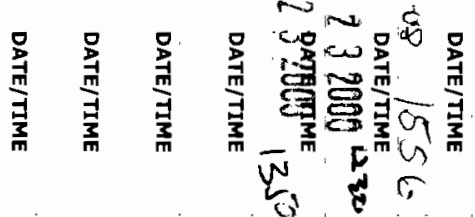

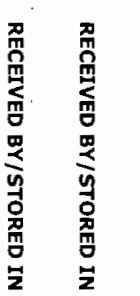

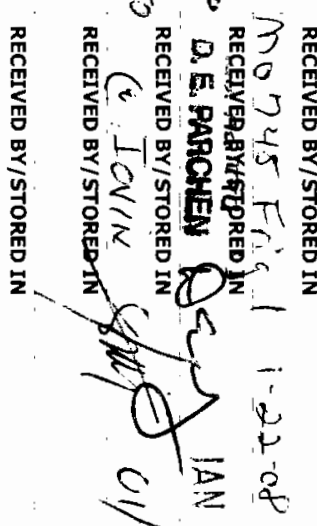

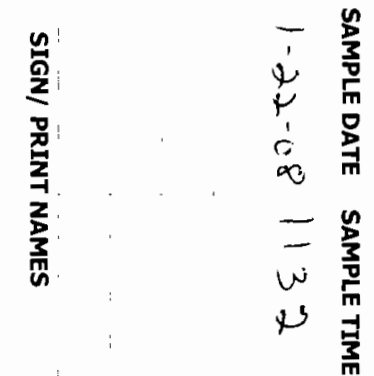

$w$
$w$
$w$
0
0
0
0
0

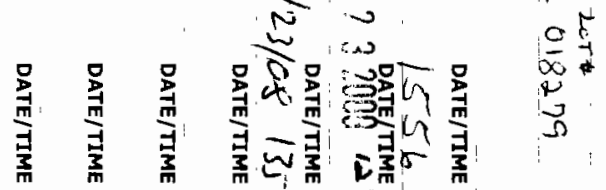

量寻

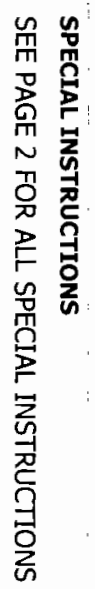

$\stackrel{\stackrel{\rho}{ }}{\Gamma}$
D
$\stackrel{\vec{J}}{\vec{H}}$
ज़

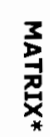

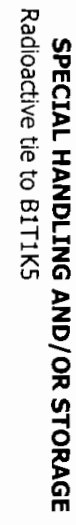

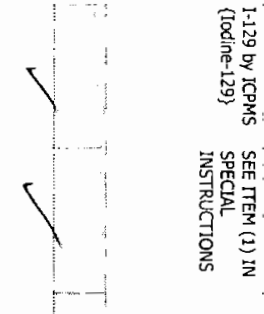

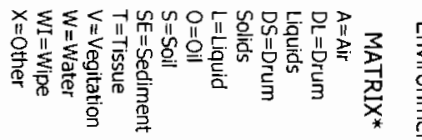

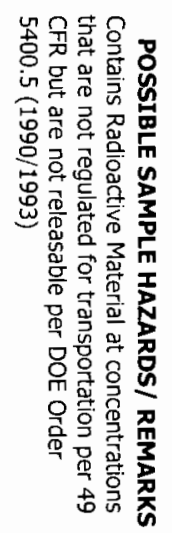

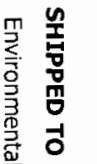

罂

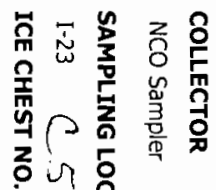

i

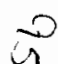

in.

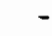

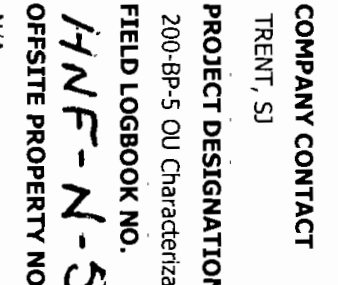

w

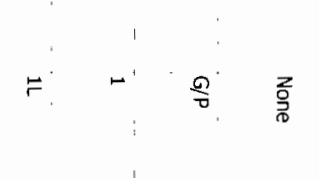
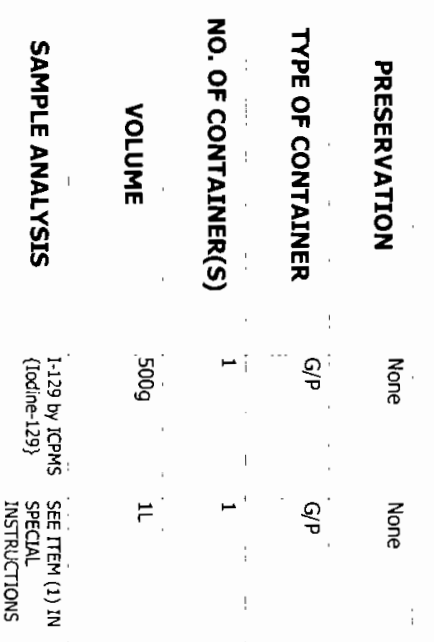

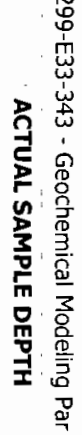

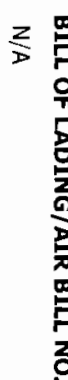

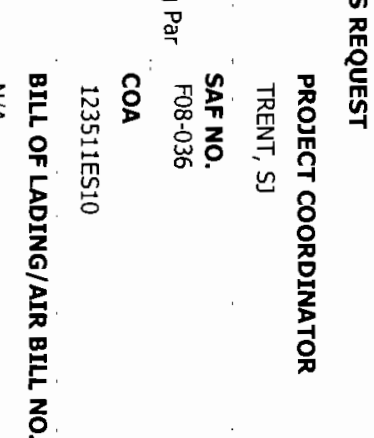

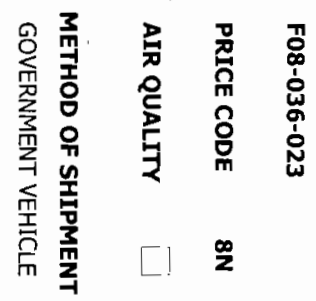

芦

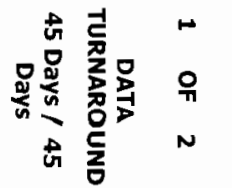




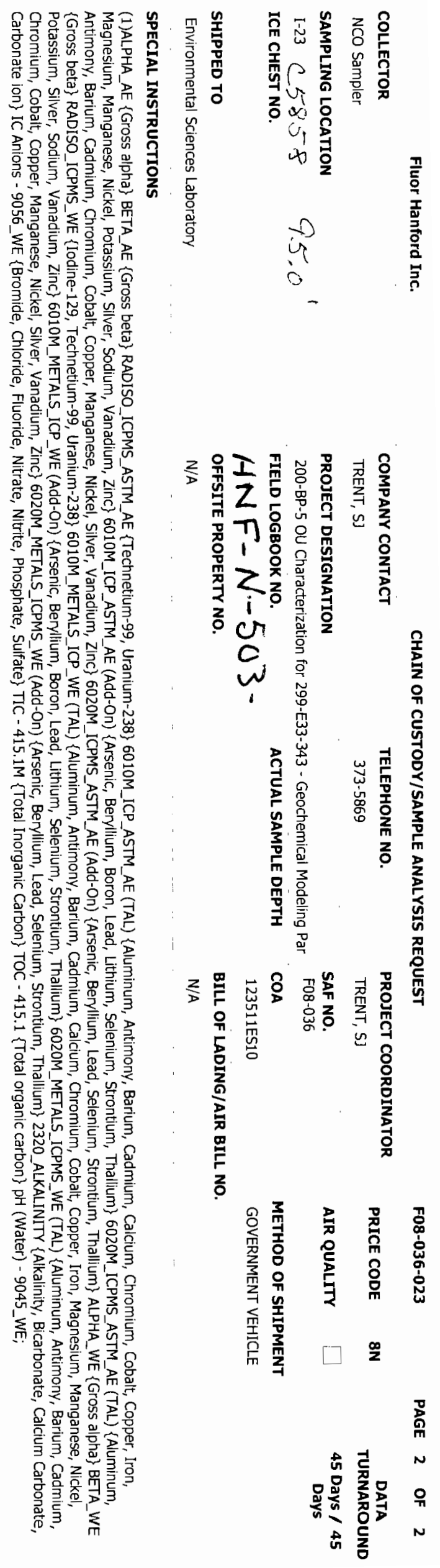




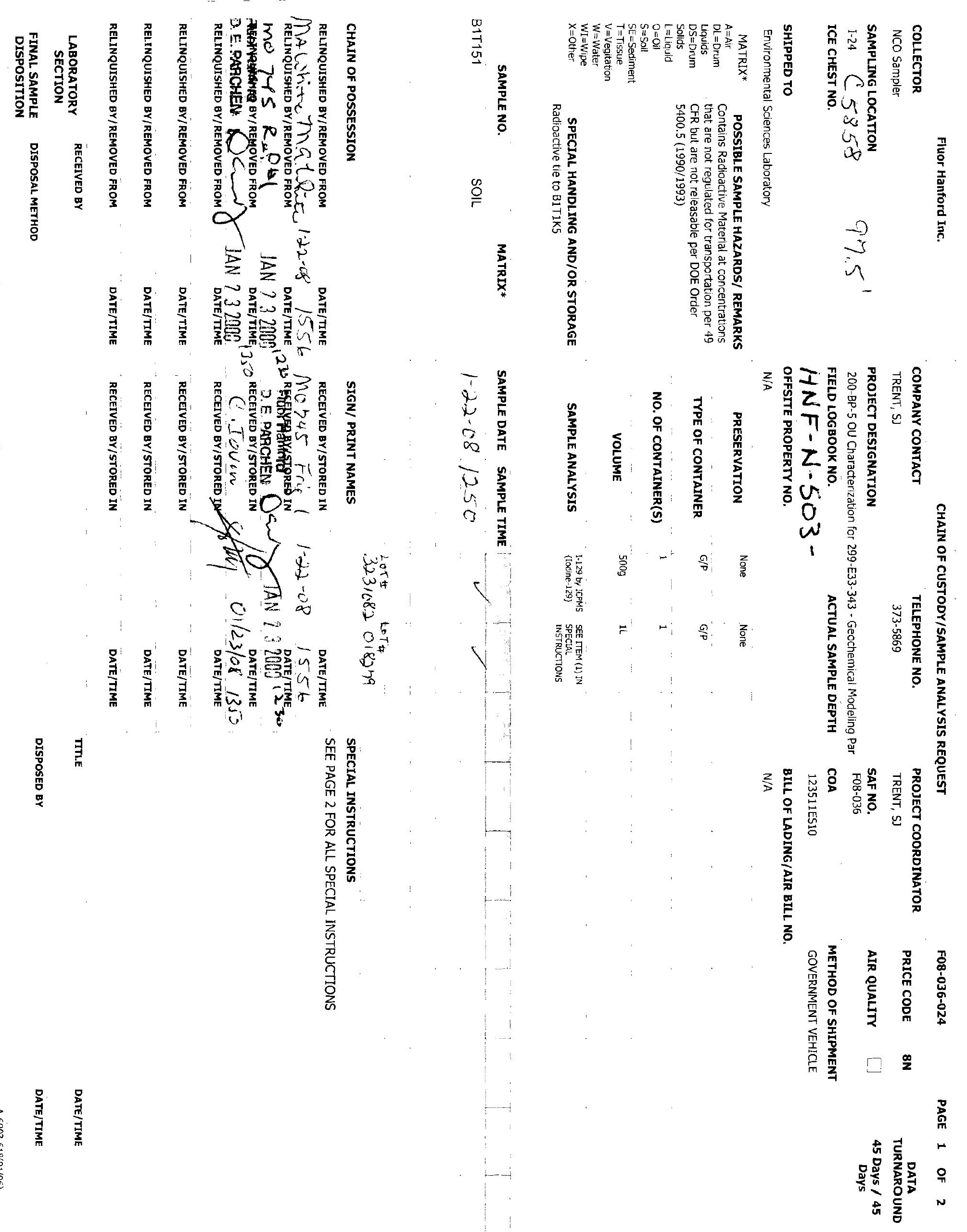




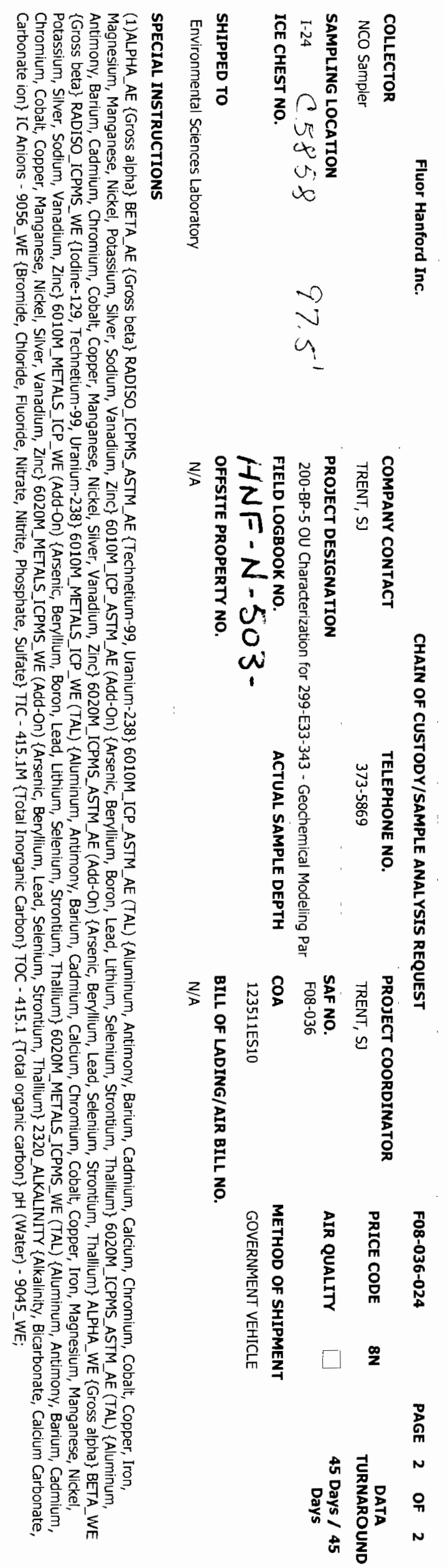




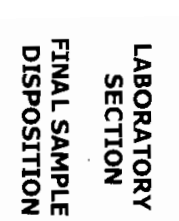

in

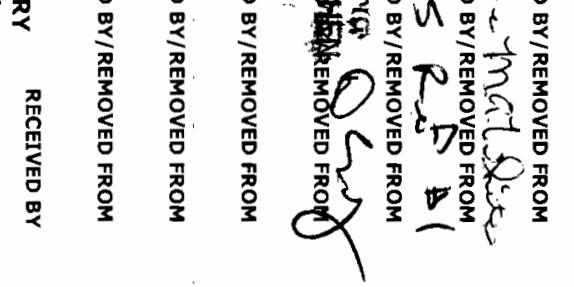

豆竞

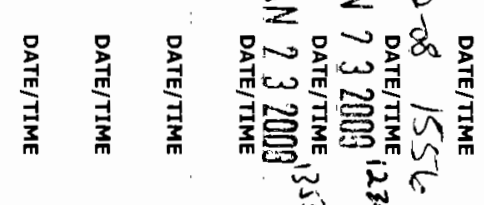
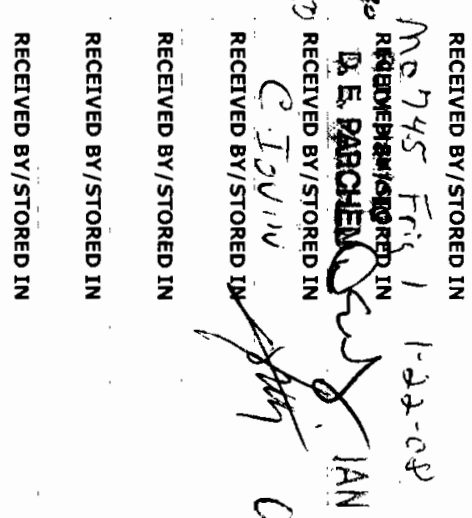

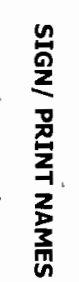

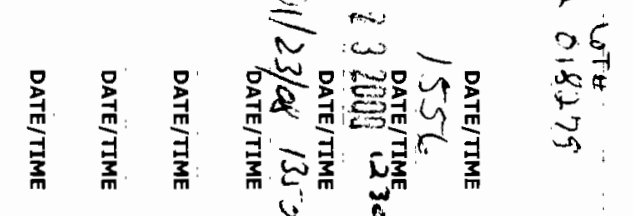

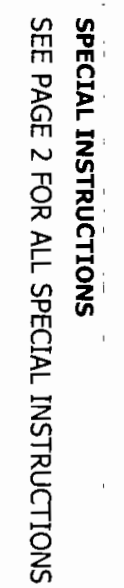

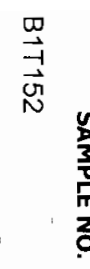

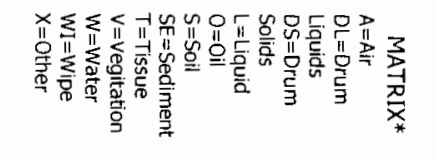

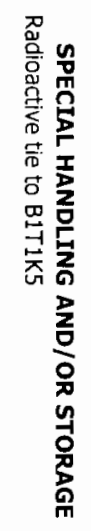

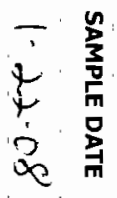

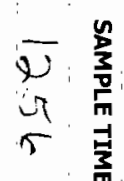

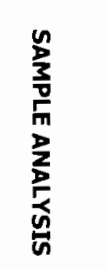

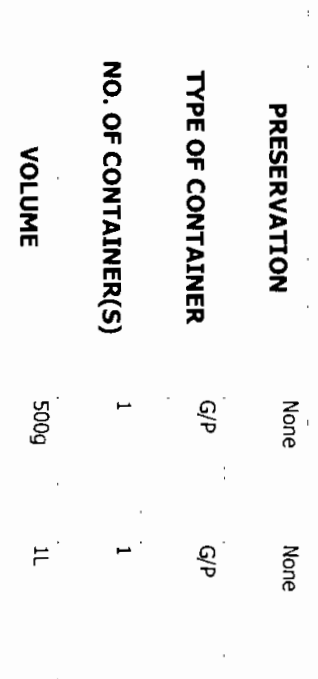

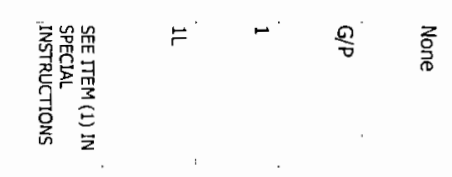

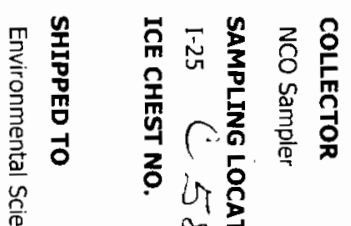

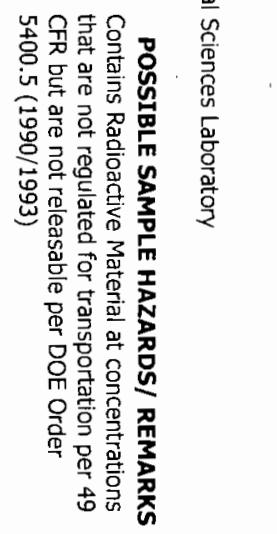

용을

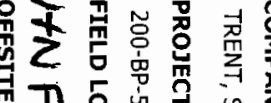

기요의

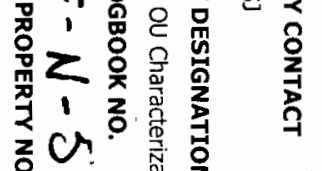

w

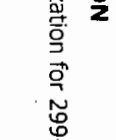



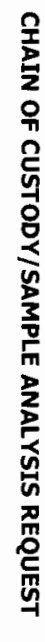

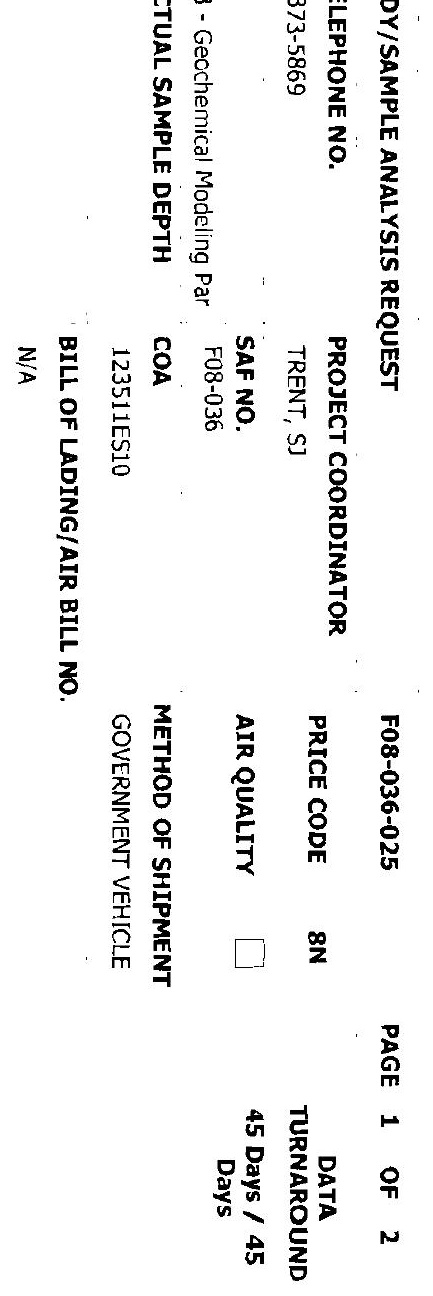

רั

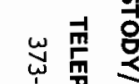




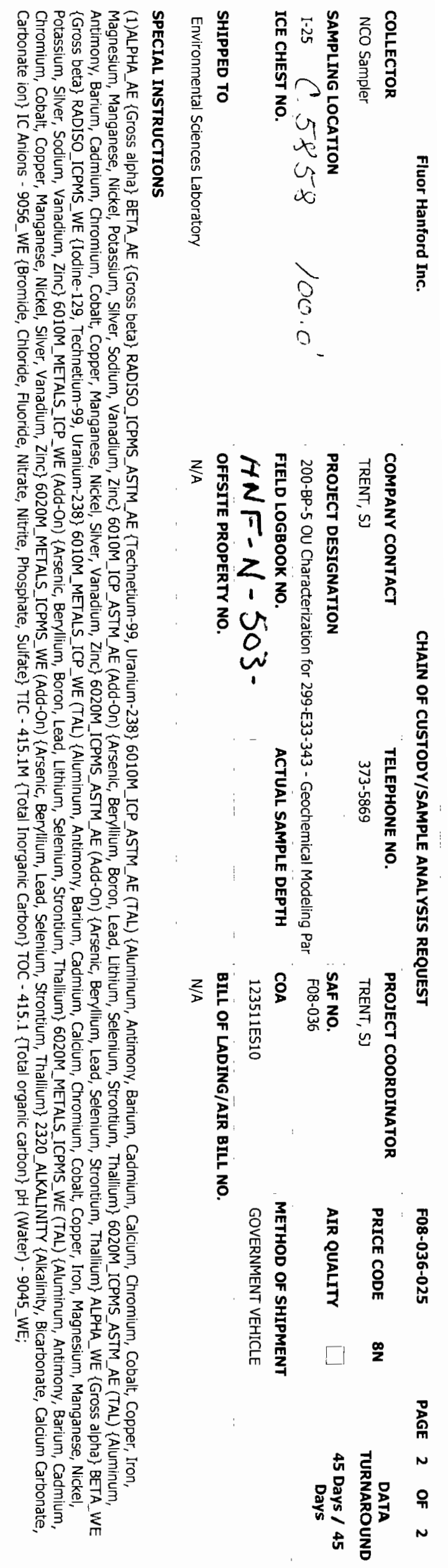


员率

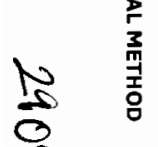

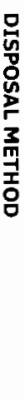

I

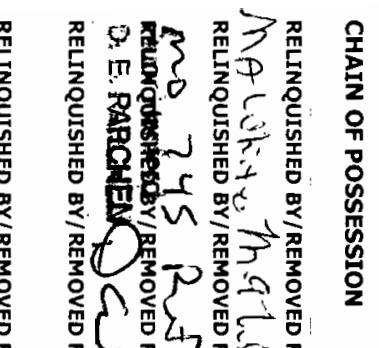

N

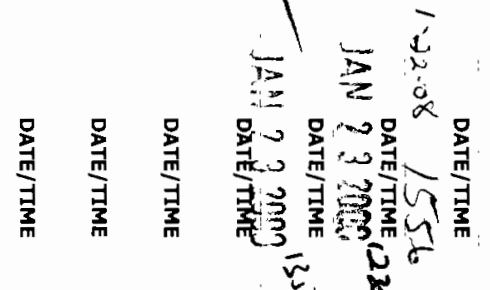

$\#$

N

$\stackrel{\text { W }}{\vec{\exists}}$

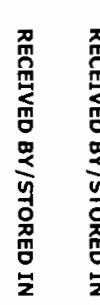

$\stackrel{\mathscr{P}}{\Gamma}$
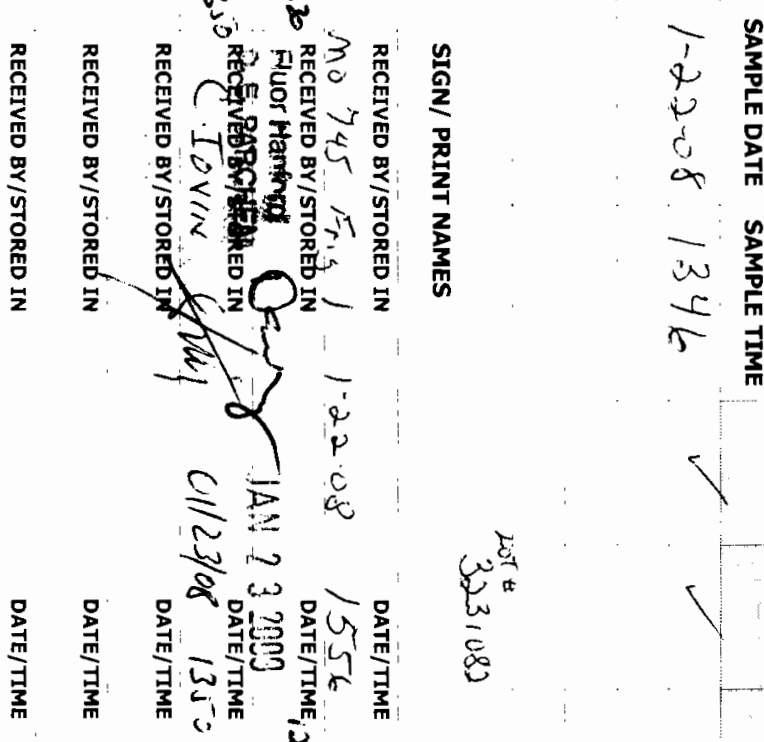

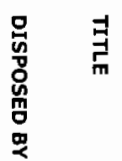
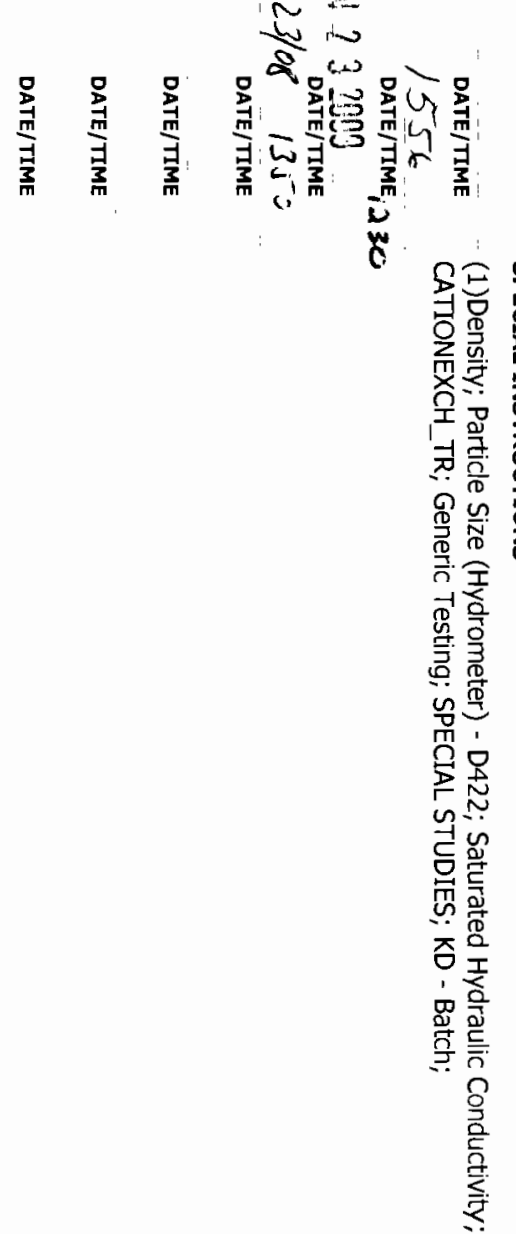

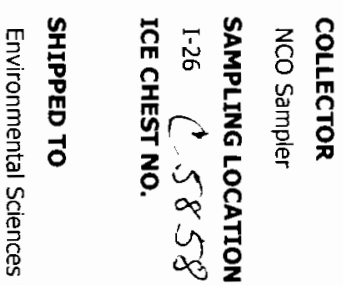

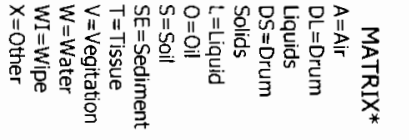

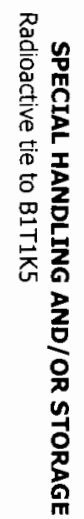

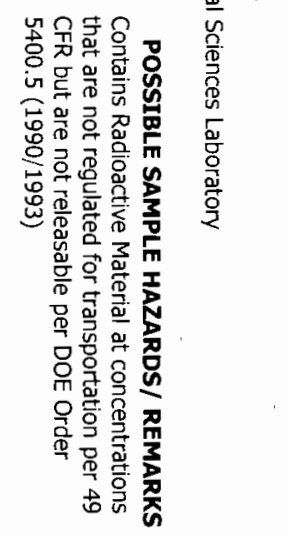

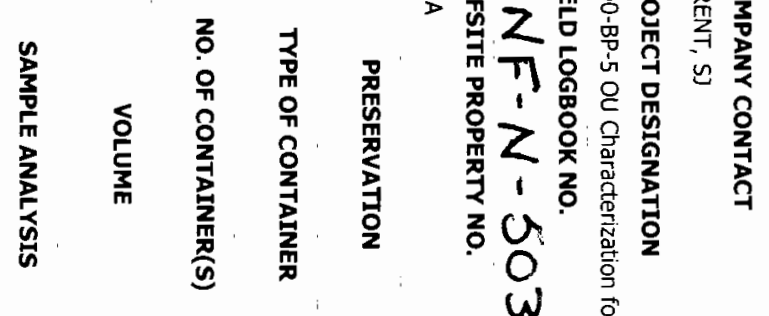

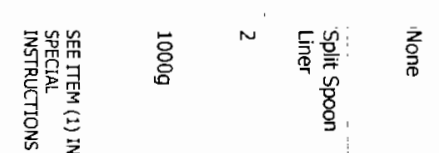

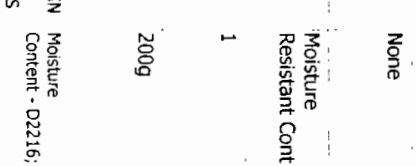

$\rightarrow$

is

$\lambda^{1}$

3

$+$

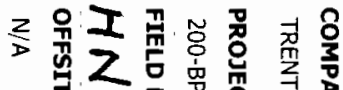

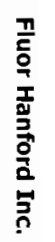

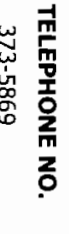
$\vec{O}$
$\tilde{0}$
0

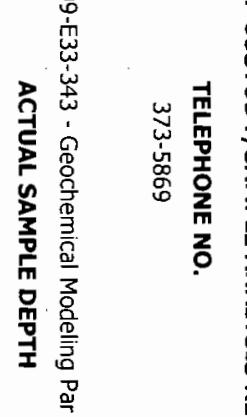

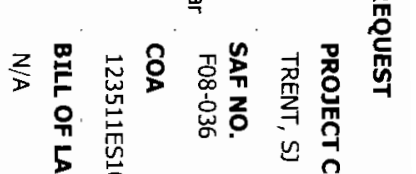

点
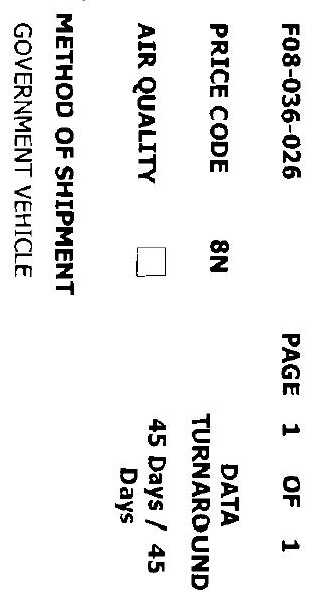

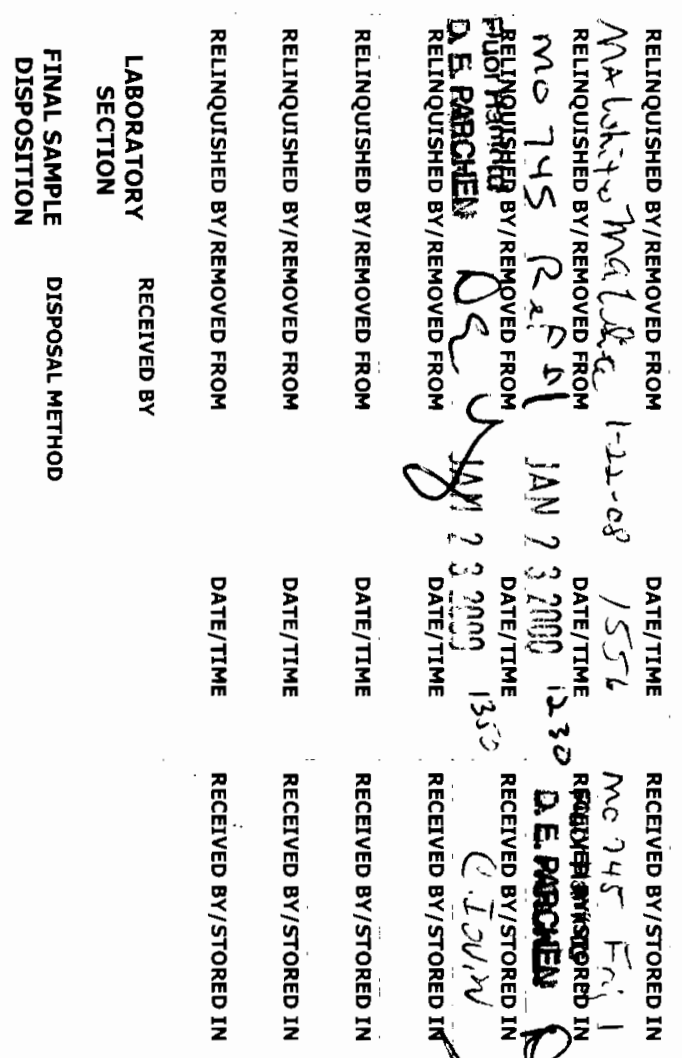

1.

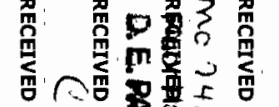

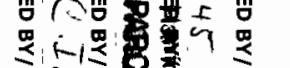

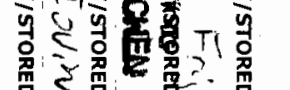

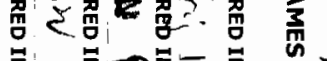

- ${ }^{2} 0^{2}-\frac{5}{5}$

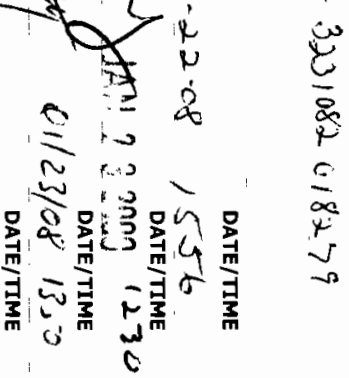

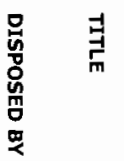

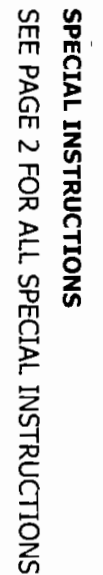

$\stackrel{\square}{\vec{\exists}}$

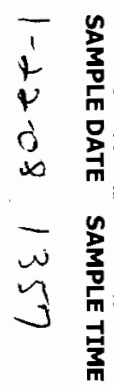

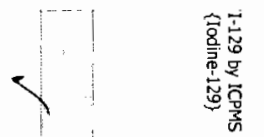

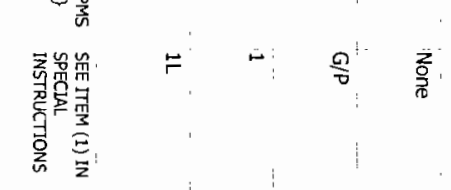

$\stackrel{\mathscr{O}}{=}$

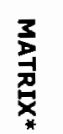

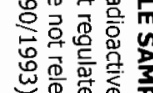

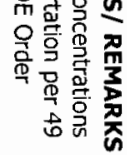

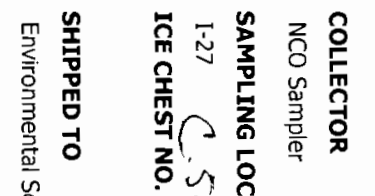

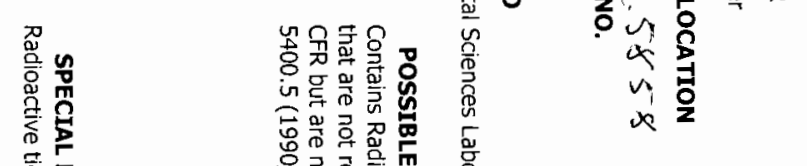

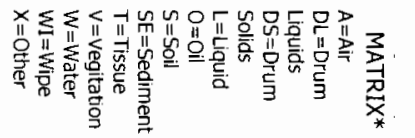

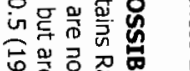

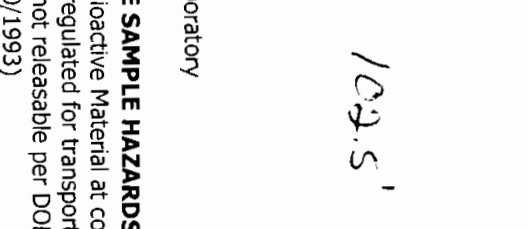

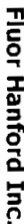

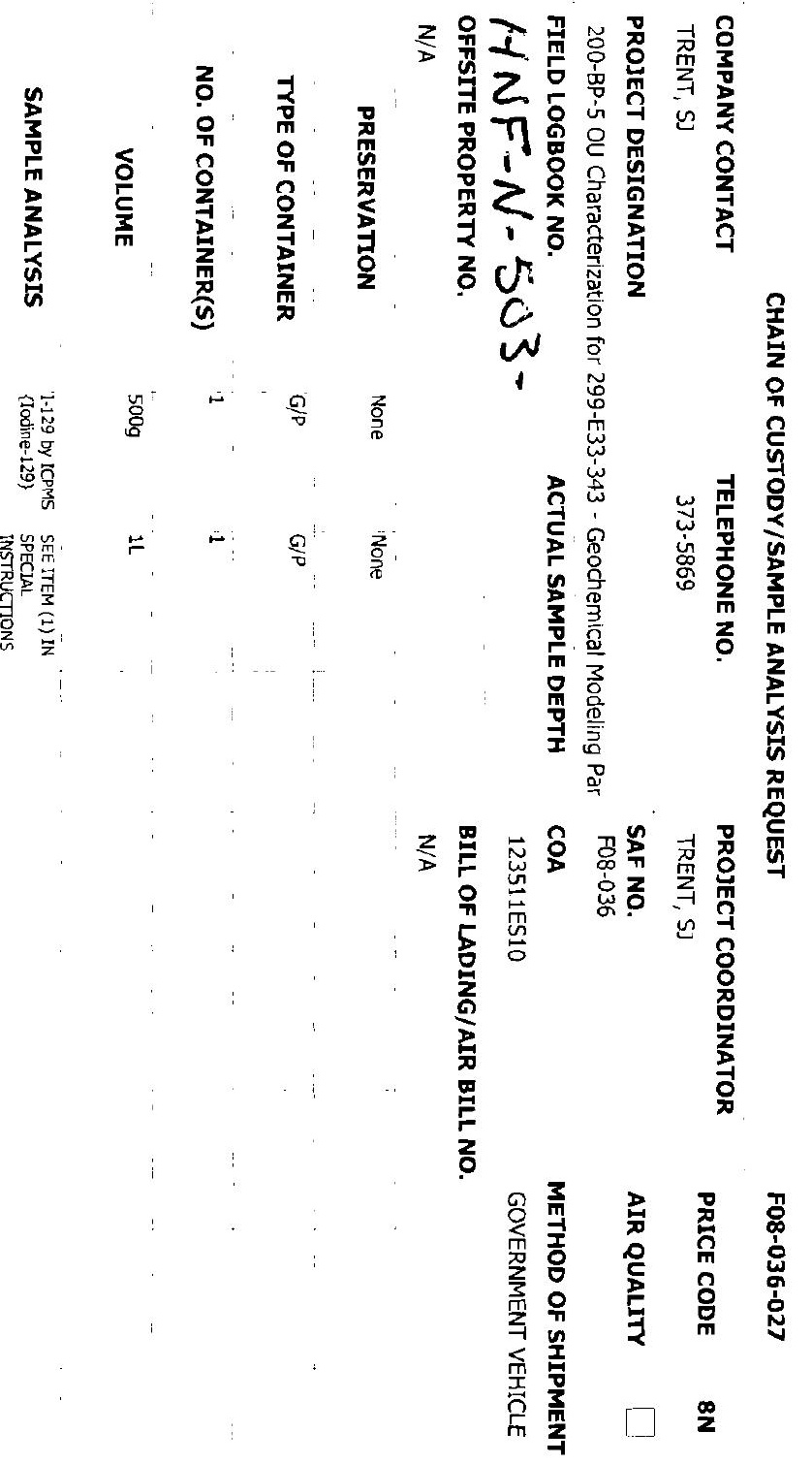




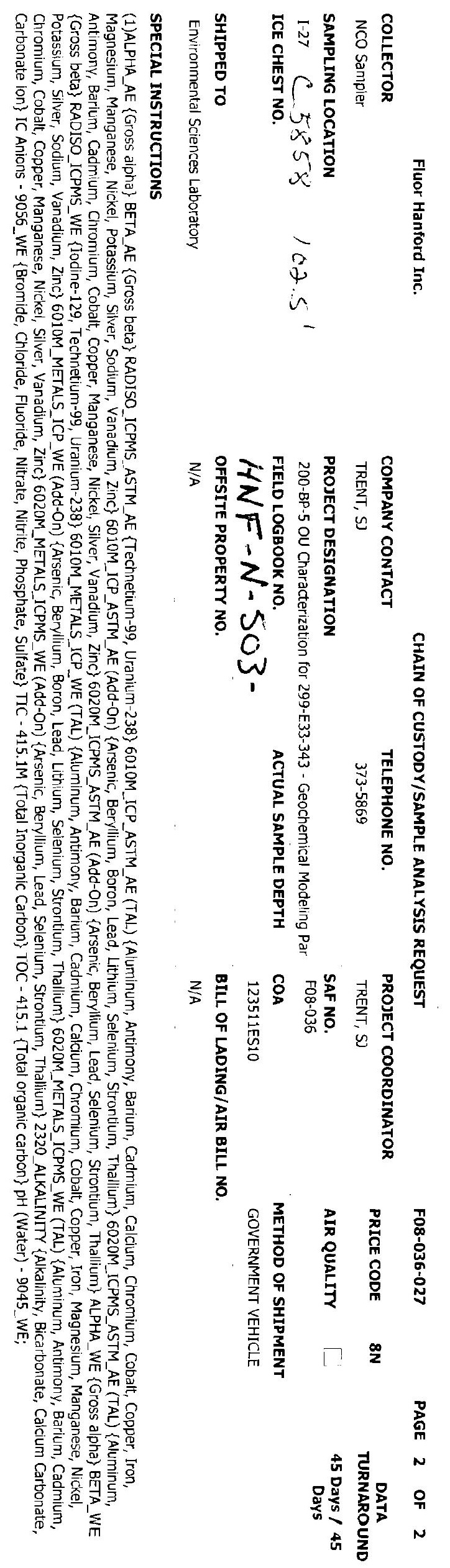




\section{翟嫼}
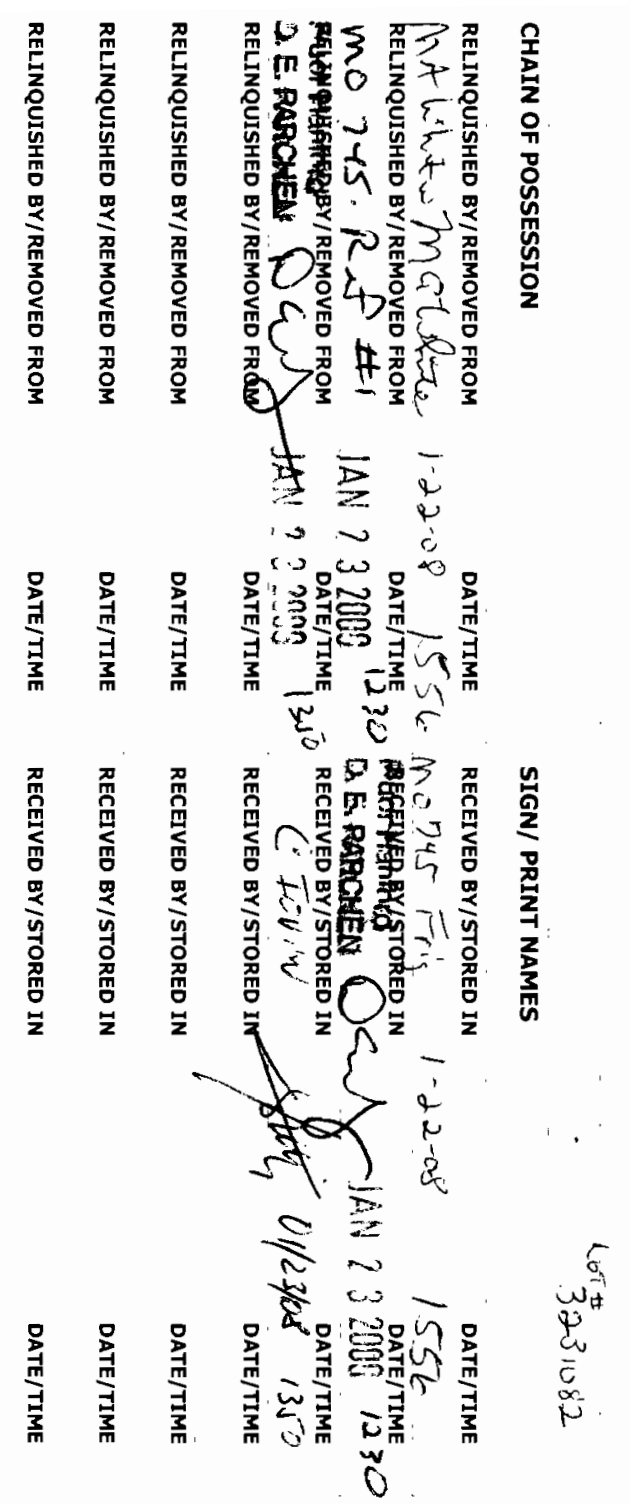

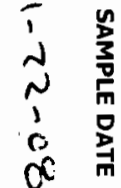

章

量

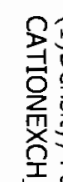

尽言

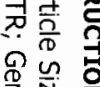

중

그ㄱㅗㅗ

ํㅗㄹ 을

盗

官足

穿嵒

思

증 종

맘옴
竞

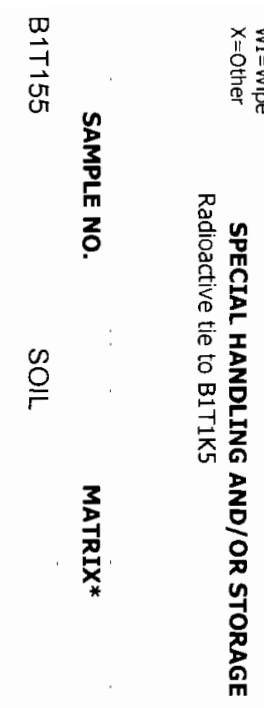

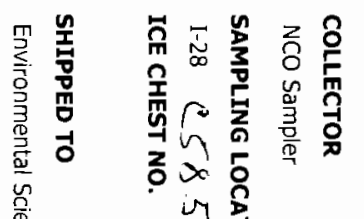

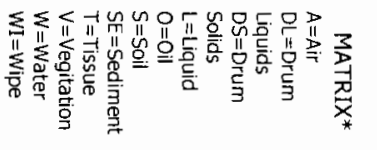

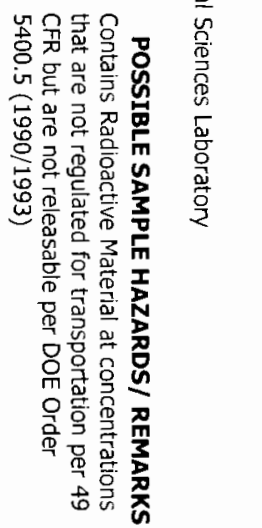

必

$\overline{3}$

5

is

I

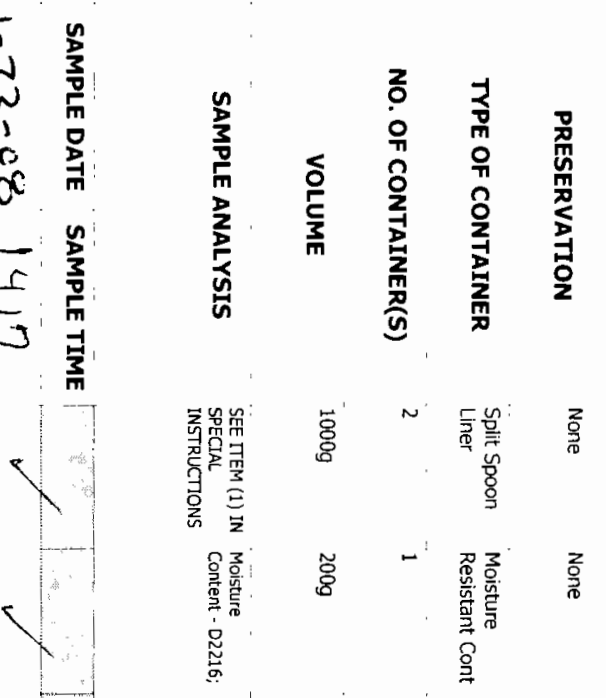

\section{z}

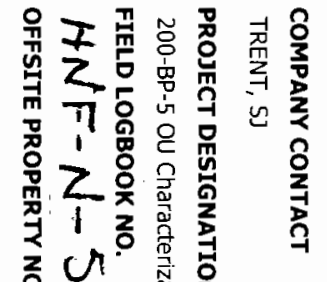

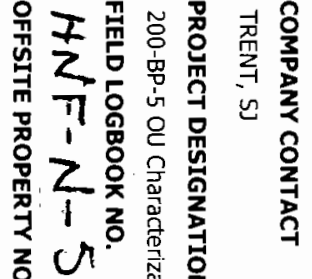

豪

$\begin{array}{cc}0 & 0 \\ 1 & 0 \\ 1 & 0\end{array}$

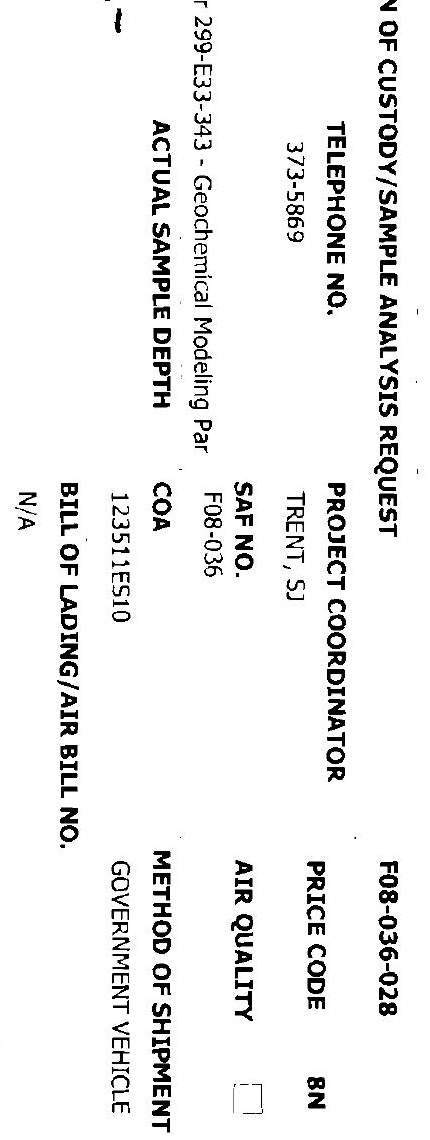

总

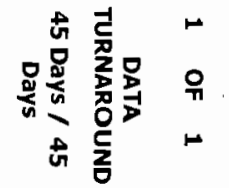



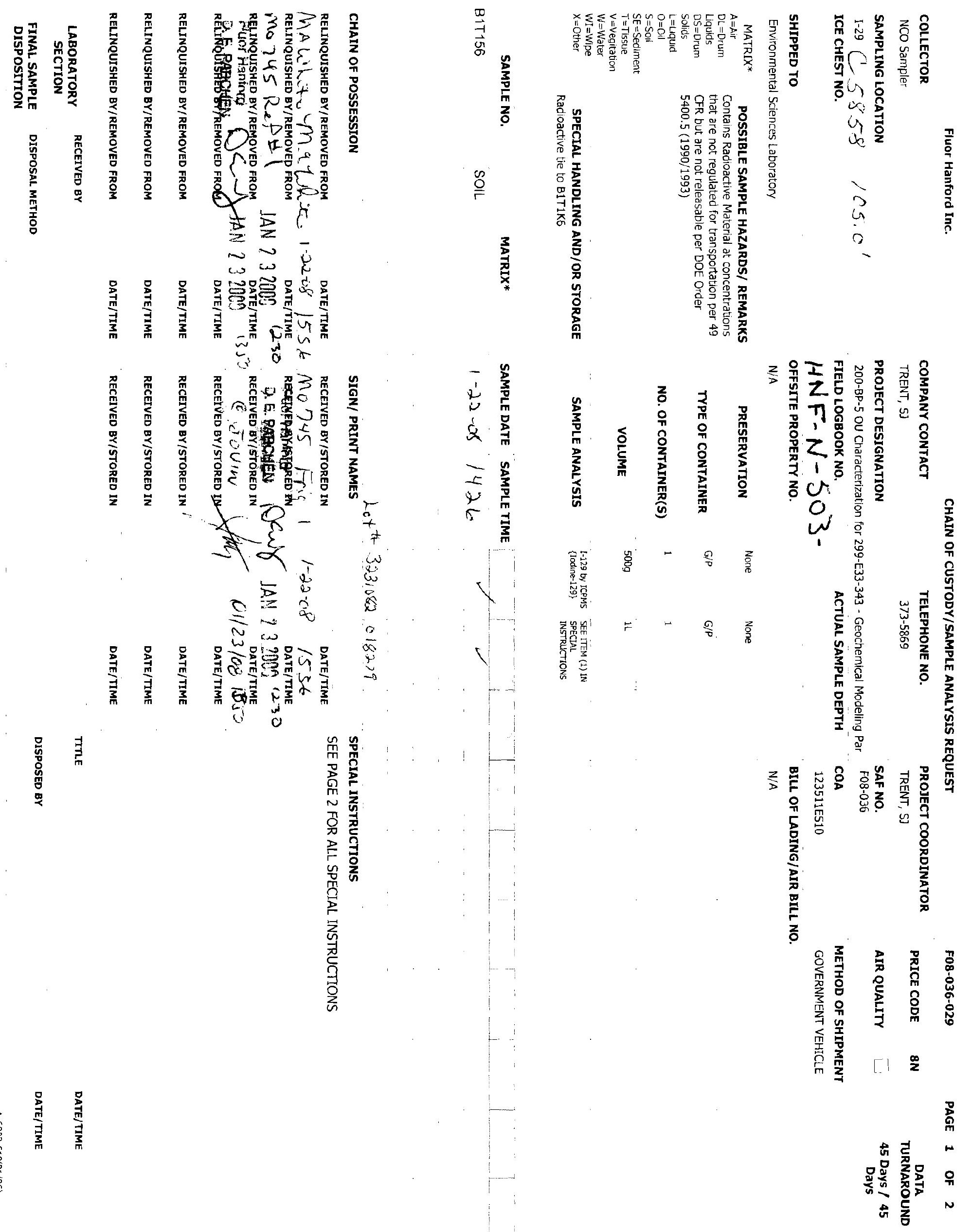


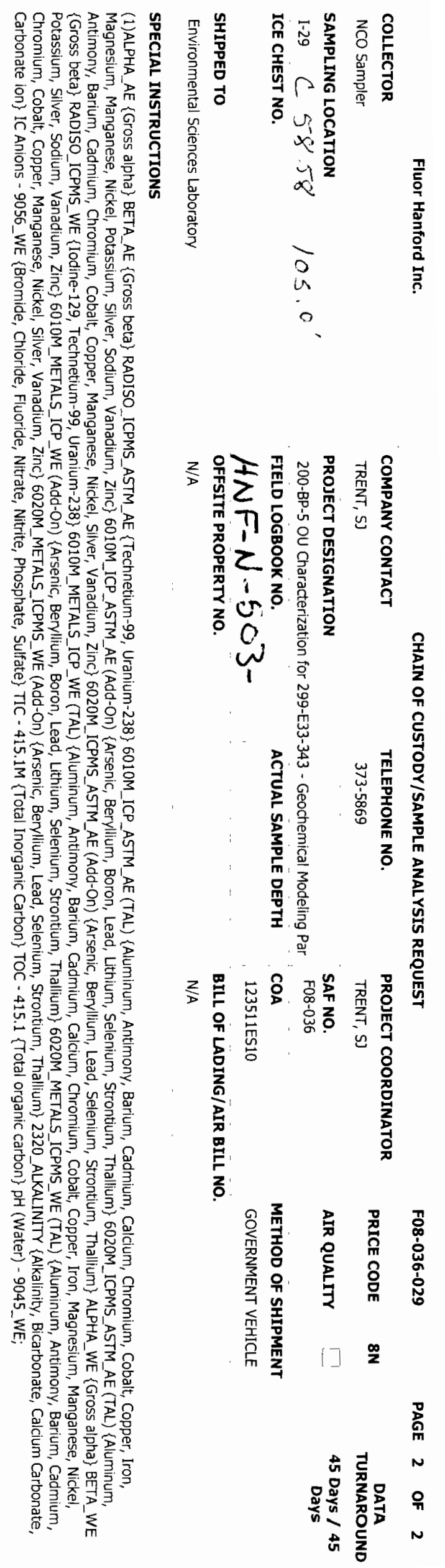




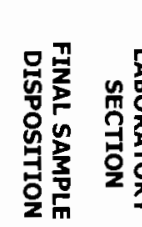

$\frac{1}{5}$

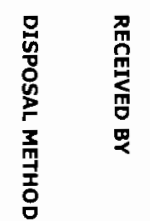

$t$

$\frac{8}{1}$

$\neq$

N

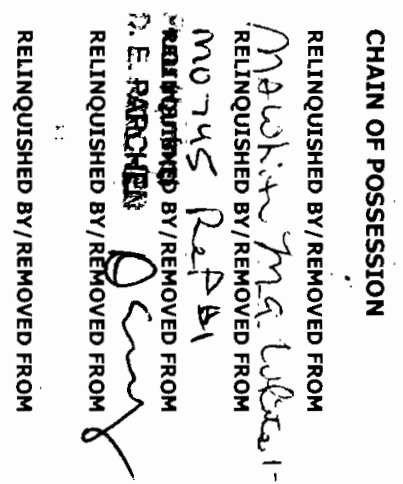

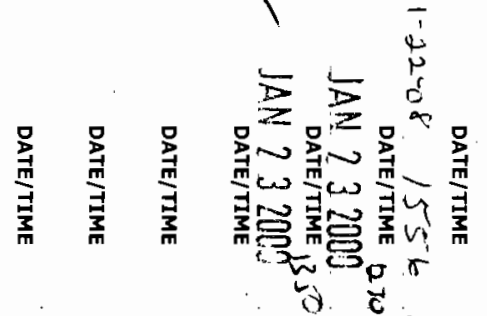

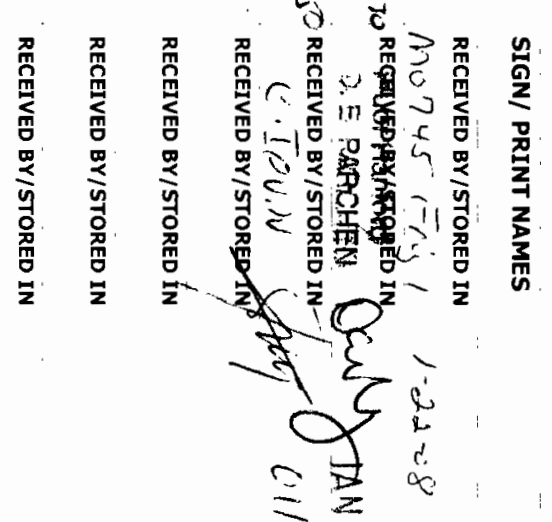

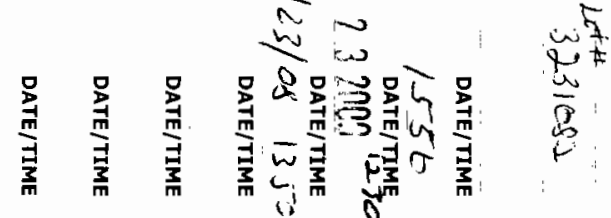

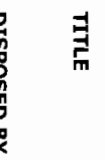

$\underset{\substack{\frac{2}{m} \\ \frac{m}{3}}}{\frac{9}{3}}$

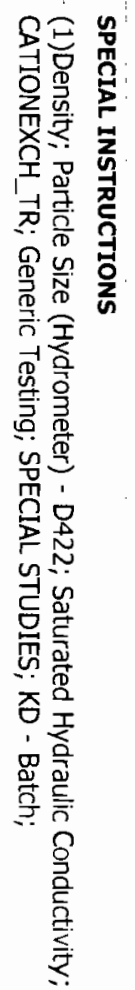

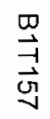

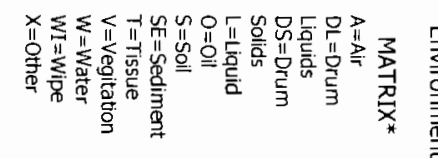

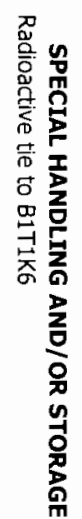

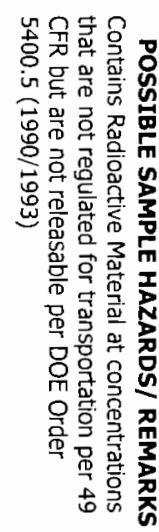

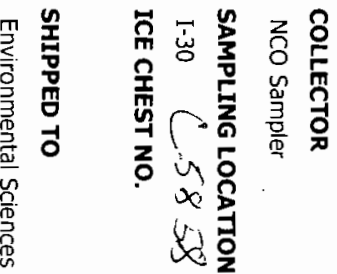

$\stackrel{g}{\circ}$

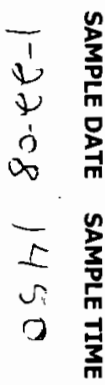

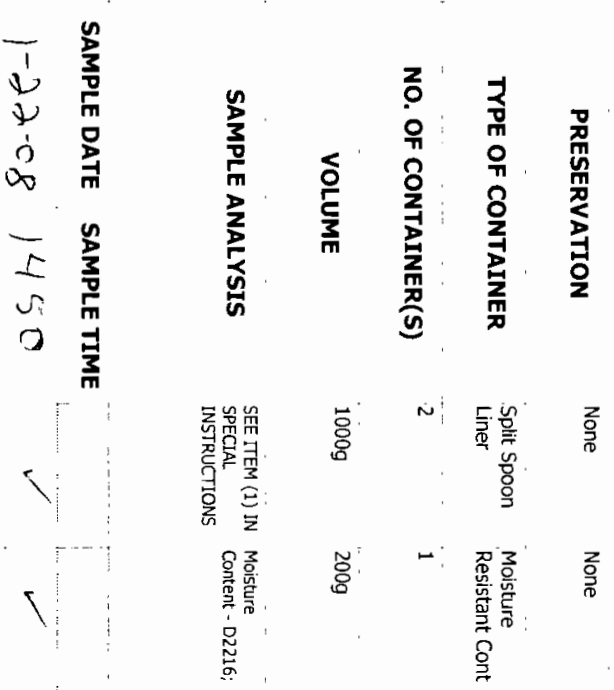

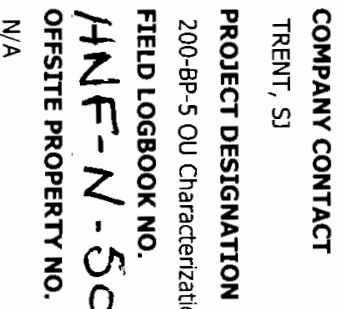
w

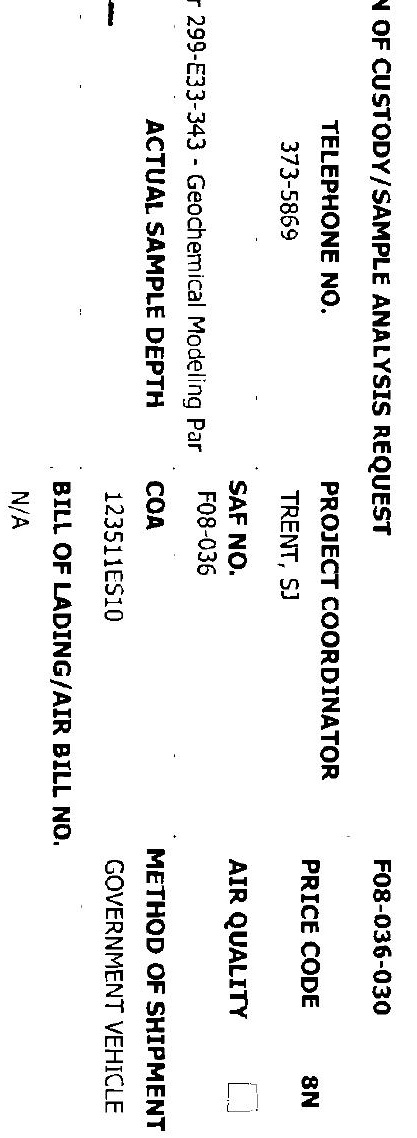




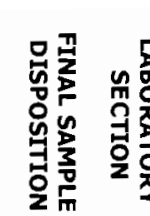

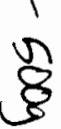

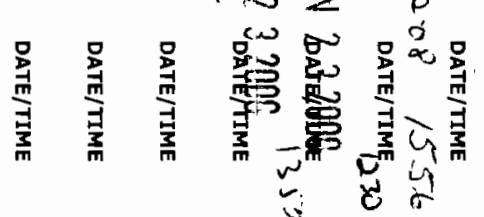

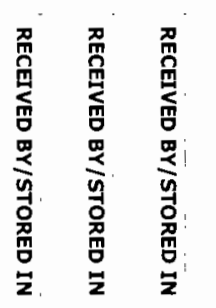

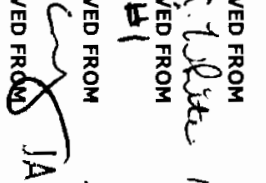

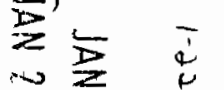

$\frac{1}{3}$

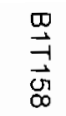

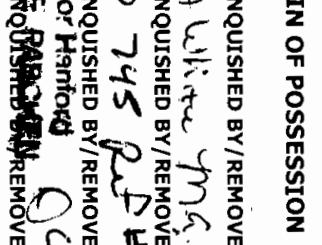

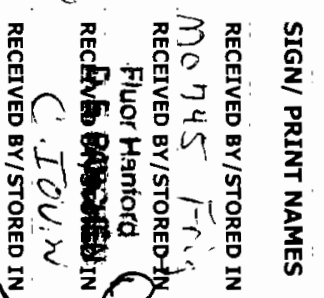

$\int \varepsilon^{-}$

So i i

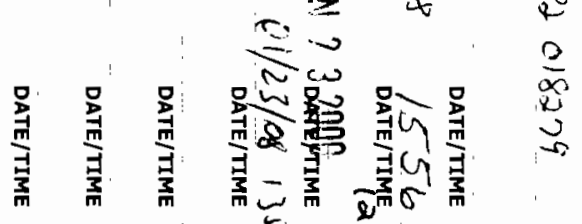

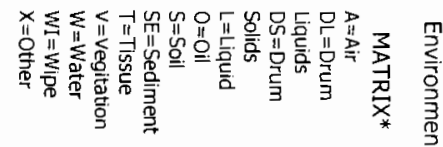

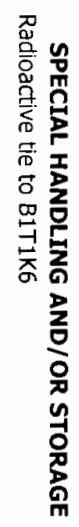

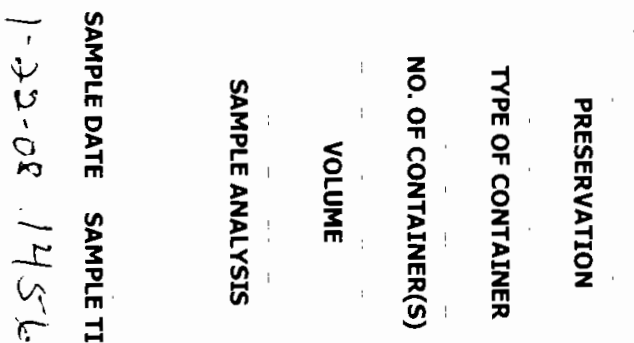

농 유

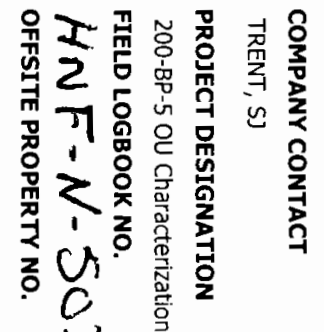

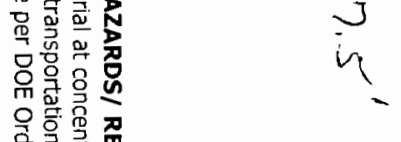

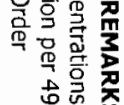

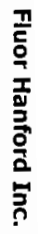
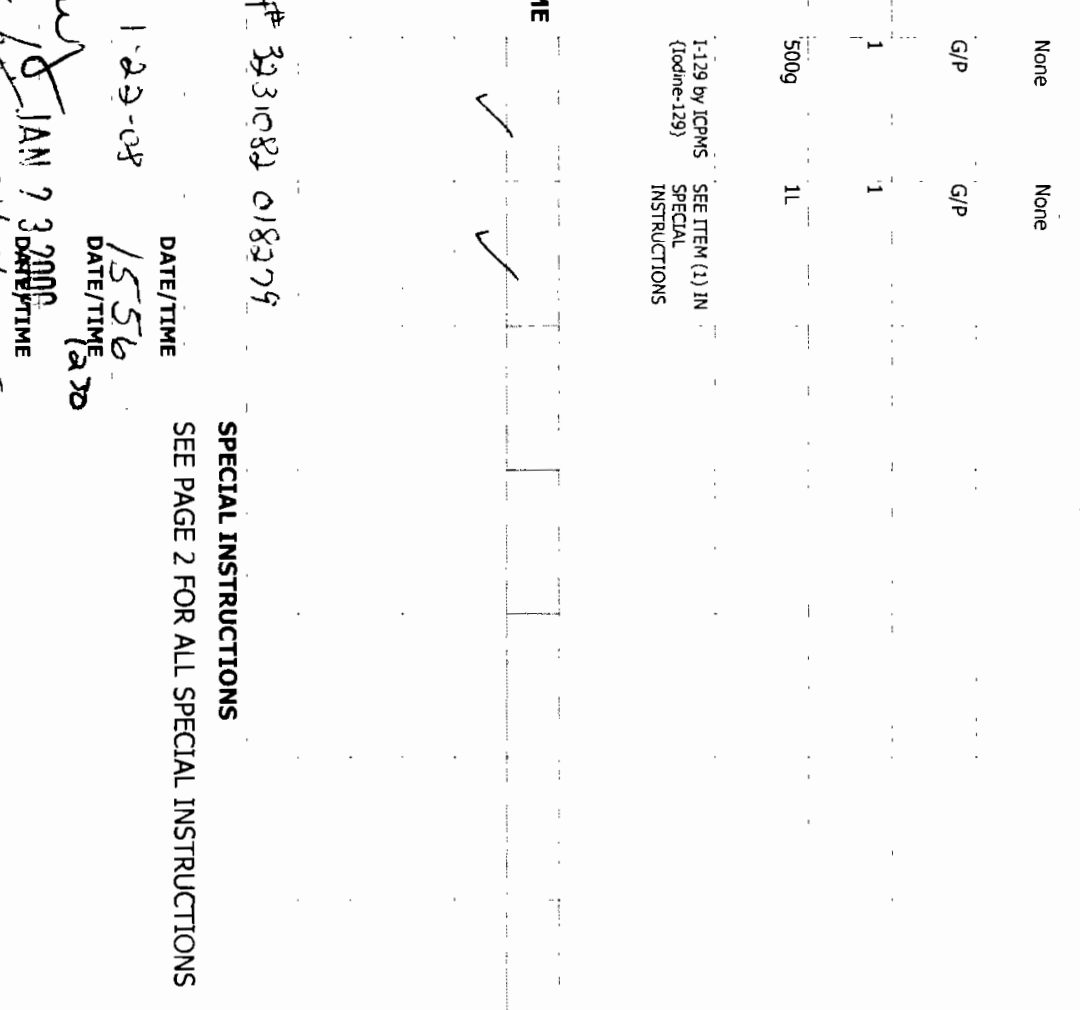

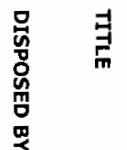

$$
\text { - ज वे. }
$$

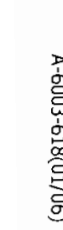

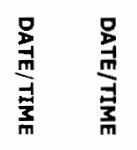




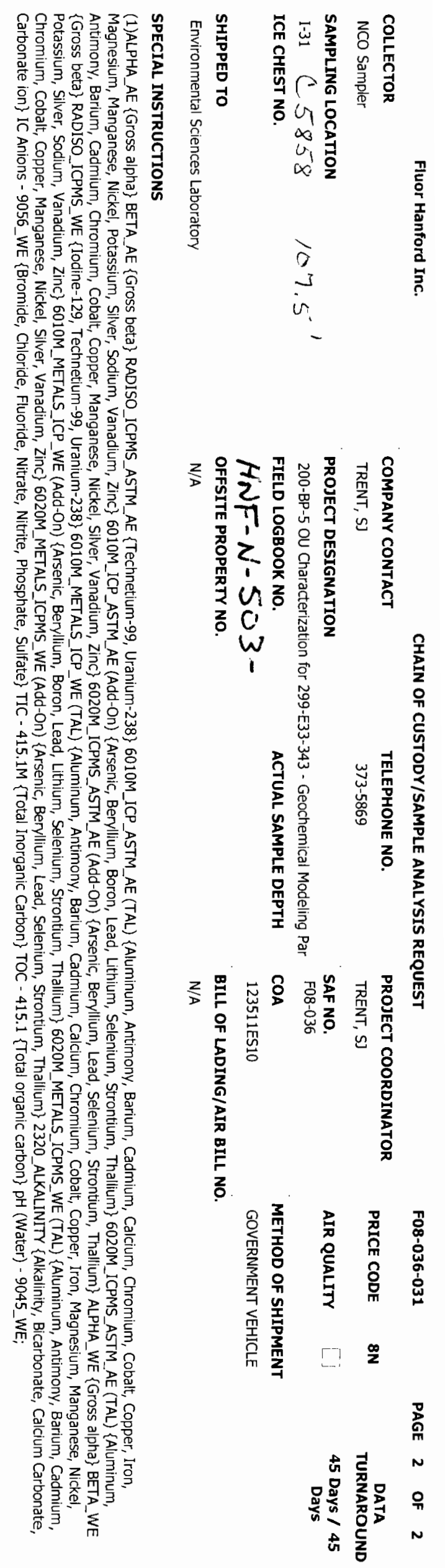




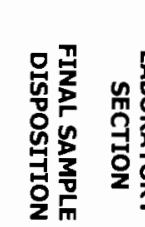

党

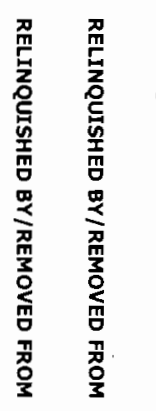

品

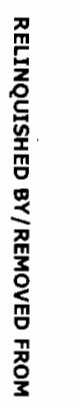

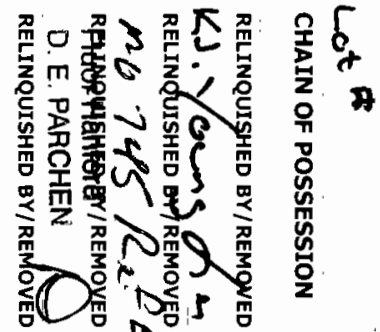

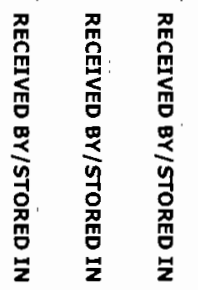

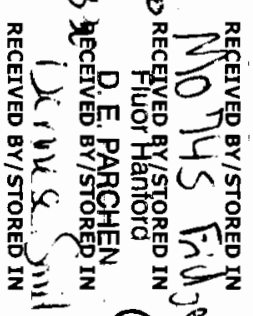

$$
\left.\begin{array}{l}
= \\
\sum \\
\sum
\end{array}\right\} \frac{1}{5}
$$

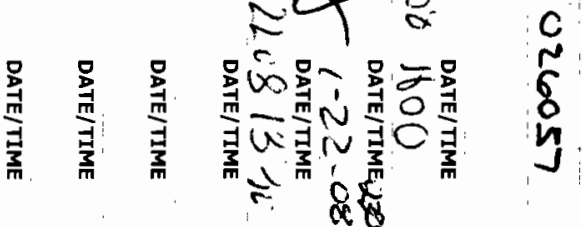

$\underset{\pi}{\vec{G}}$

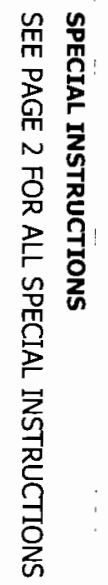

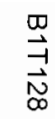

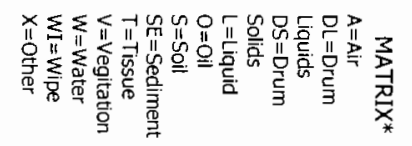

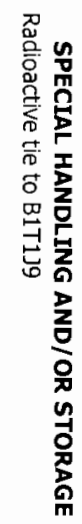

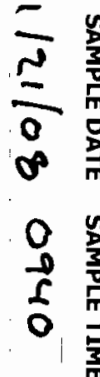

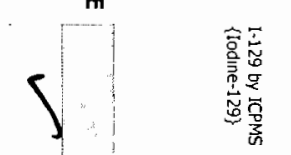

\&)

$\frac{2}{8}-\frac{5}{8}-\frac{2}{\circ}$

$\frac{2}{2}$

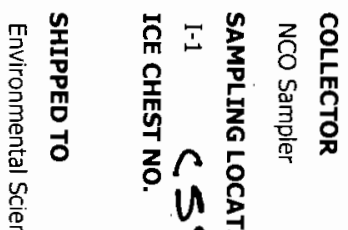

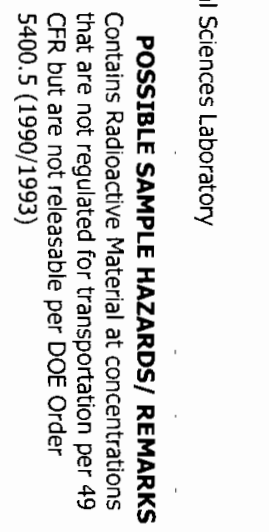

$\omega^{0}$

0

0

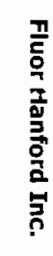
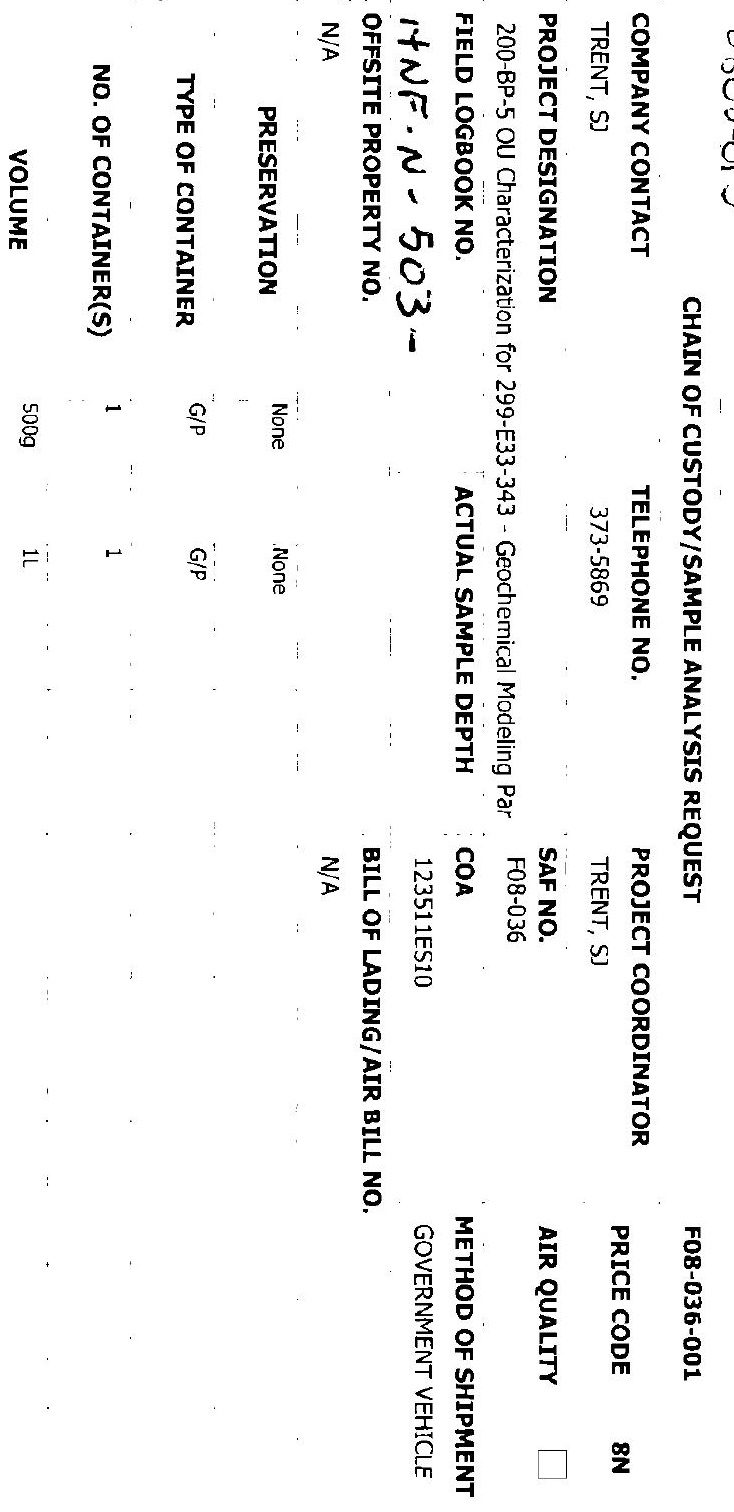

ลั่

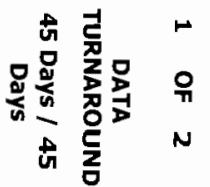




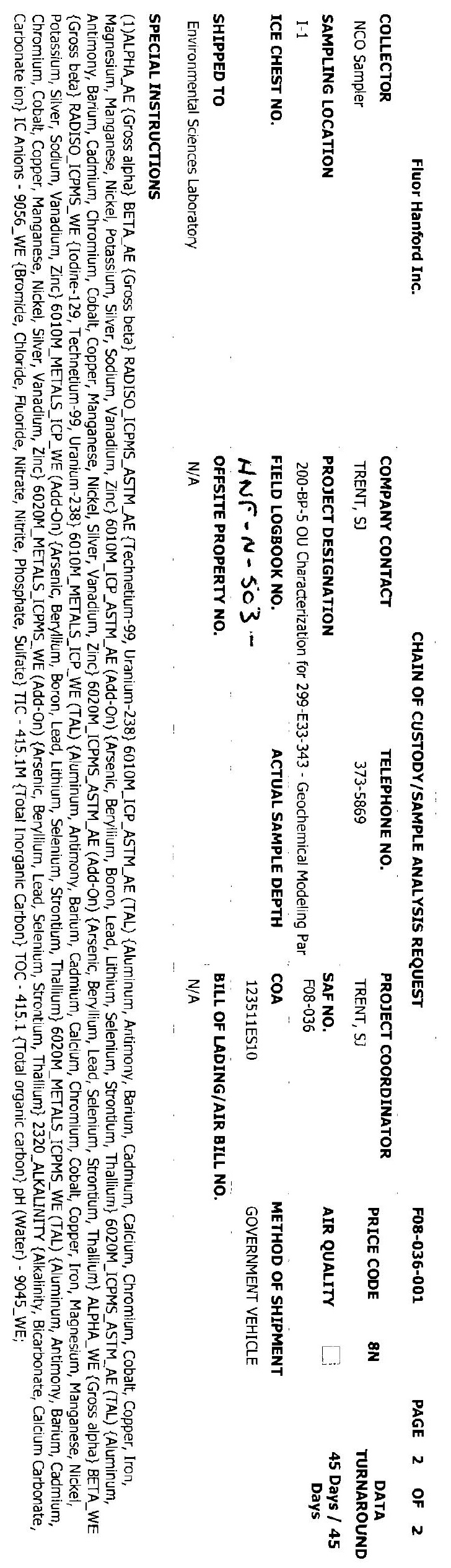




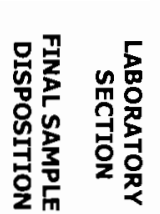

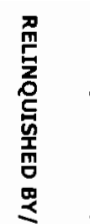

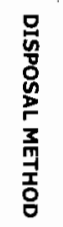

1

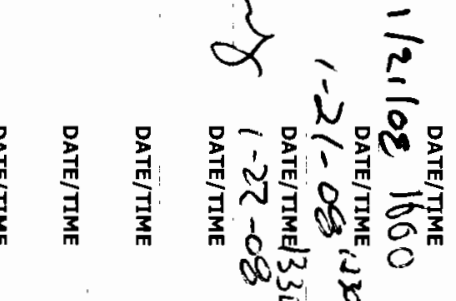

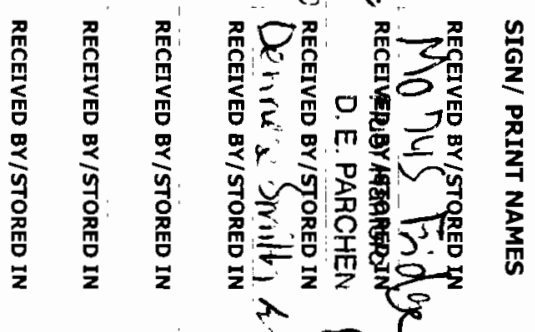

$D^{\circ}$

A $\Omega$

iq $\frac{T}{\frac{T}{2}}$

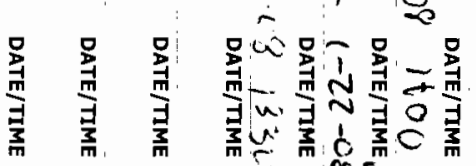

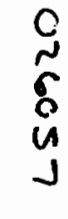

!

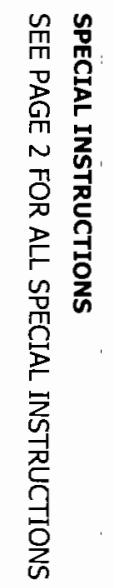

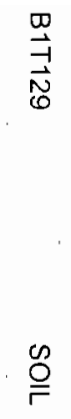

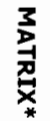

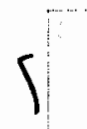

1

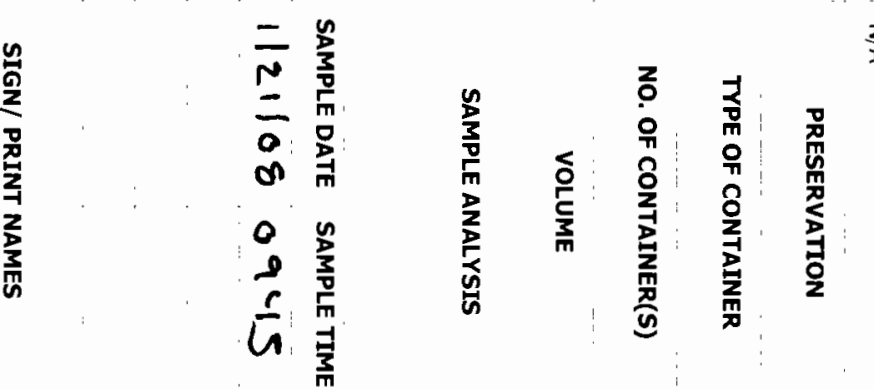

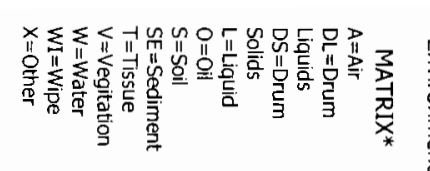

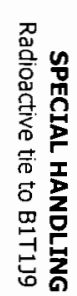

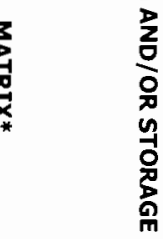




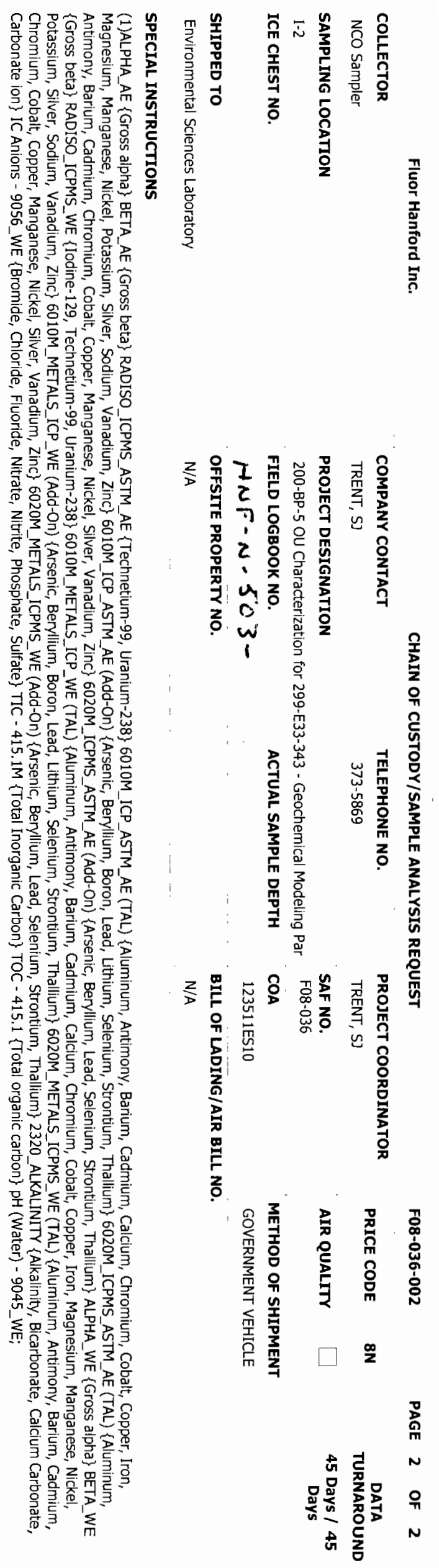




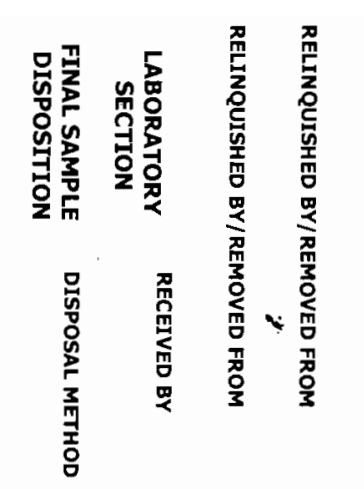

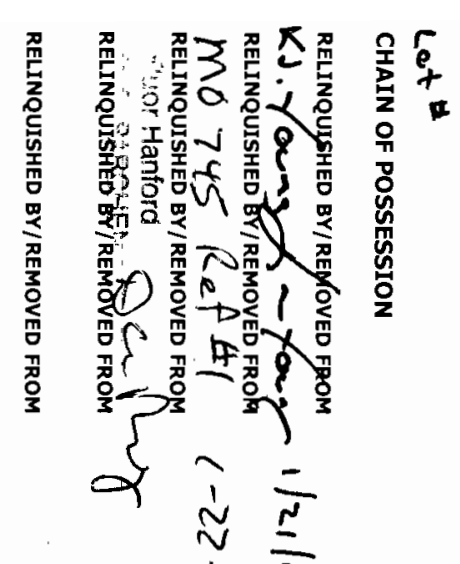

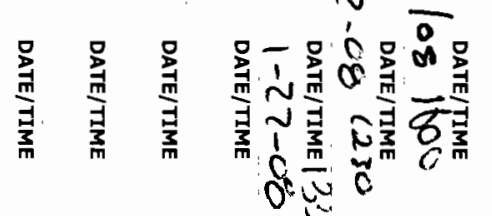

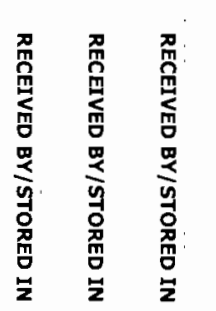

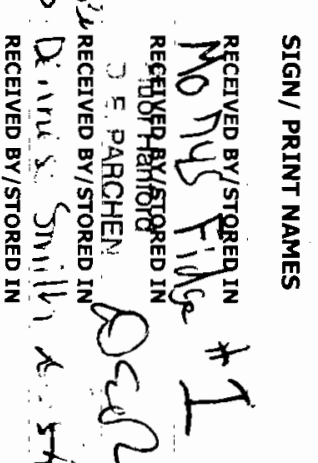

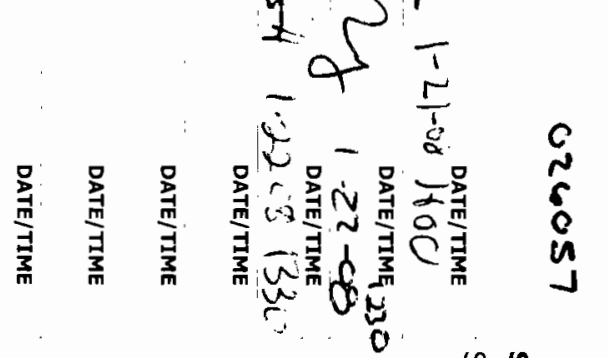

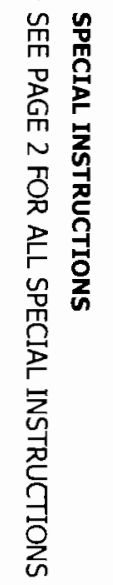

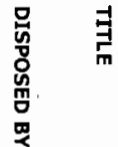

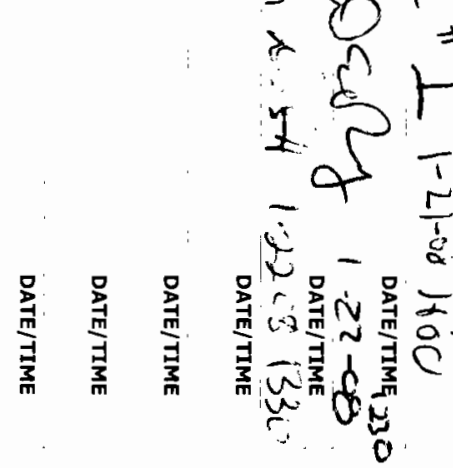
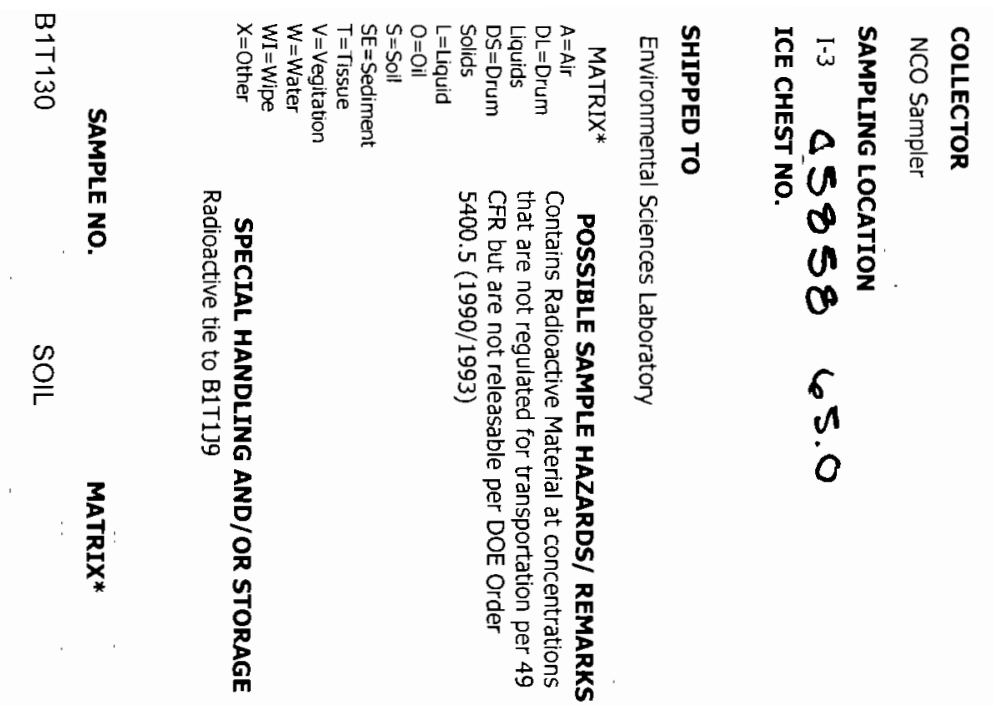

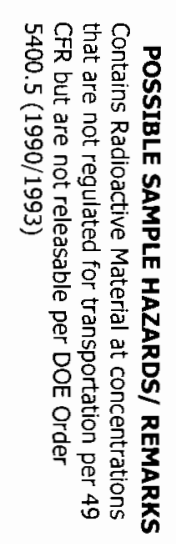

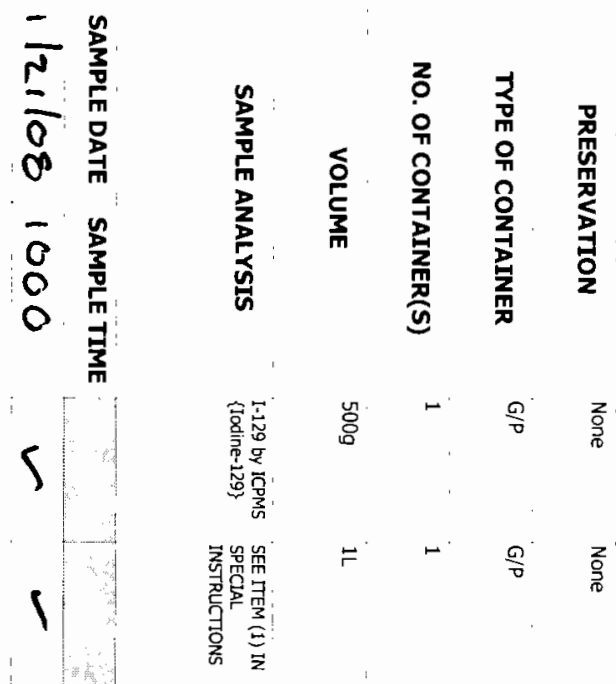

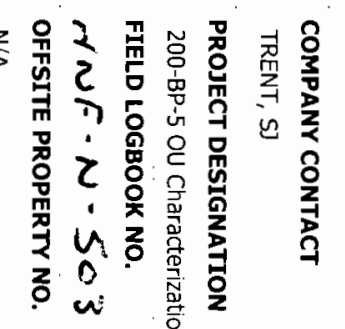

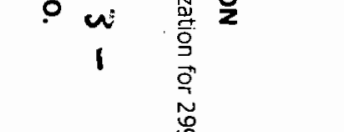

$\stackrel{\infty}{\stackrel{\rho}{ }}$

$\underset{3}{\stackrel{3}{2}}$

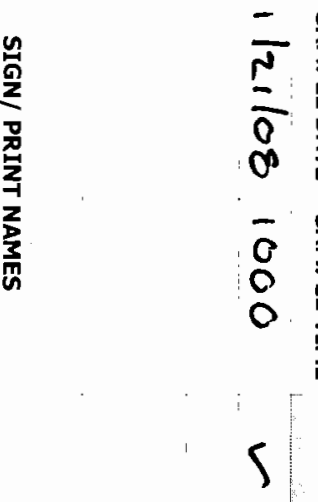

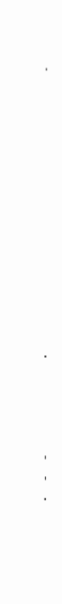

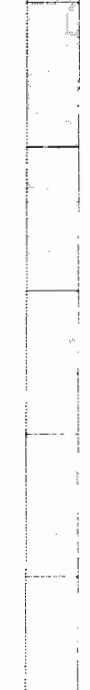

굴

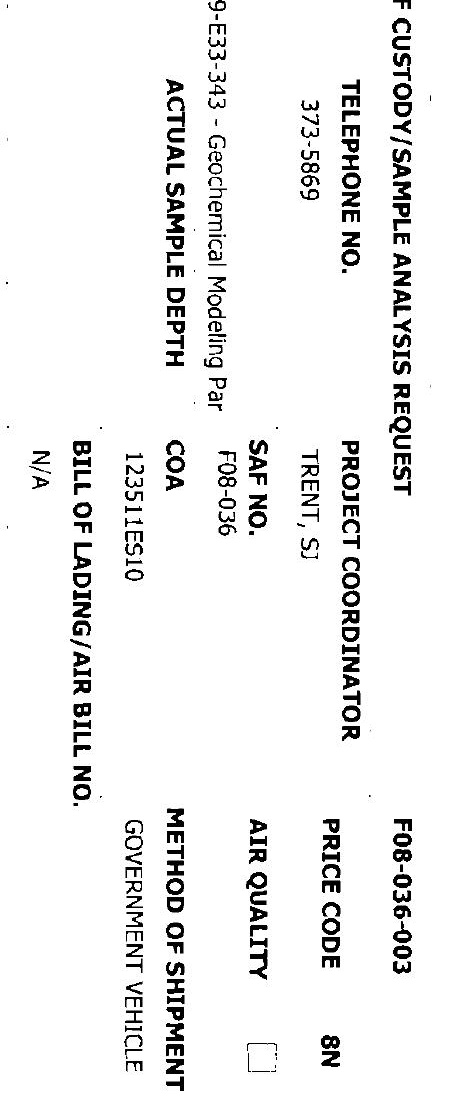

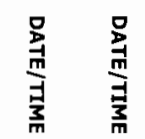

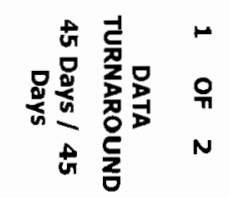




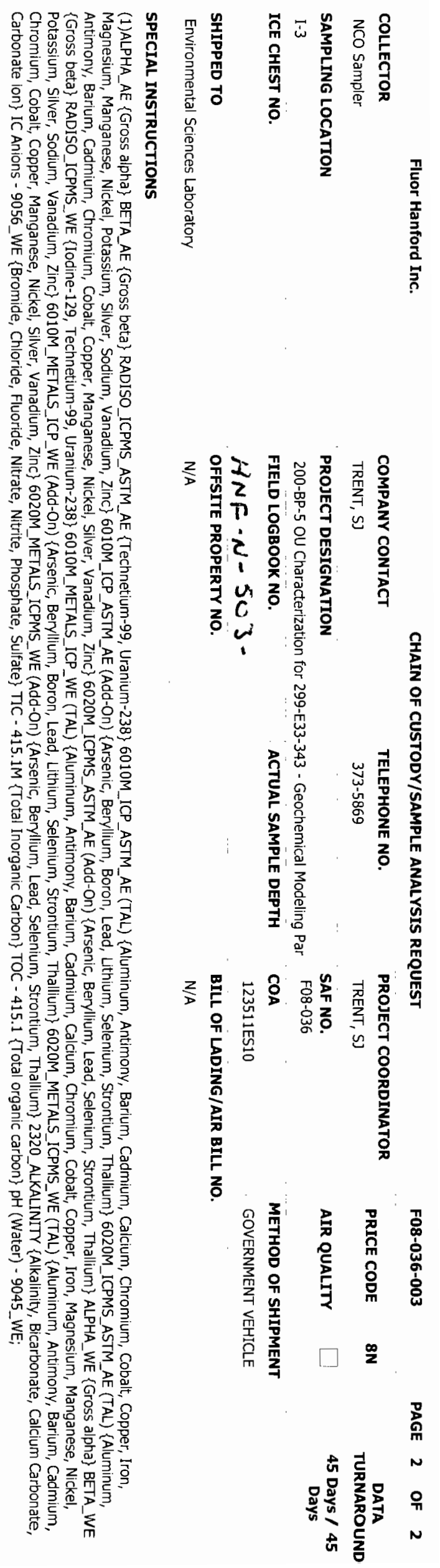




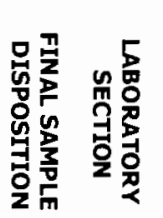

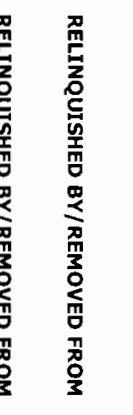

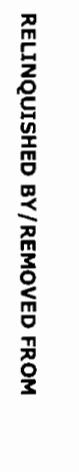

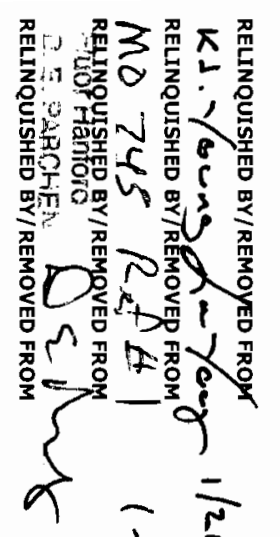

尊 总

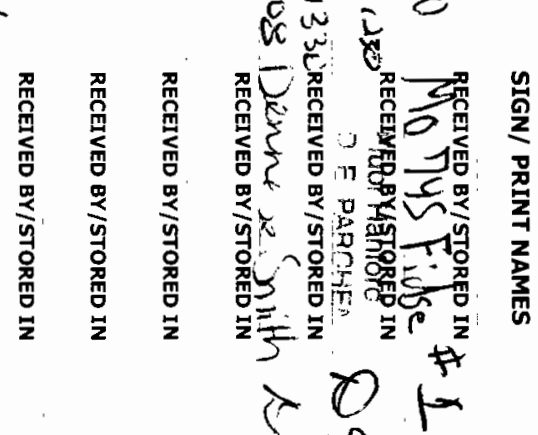

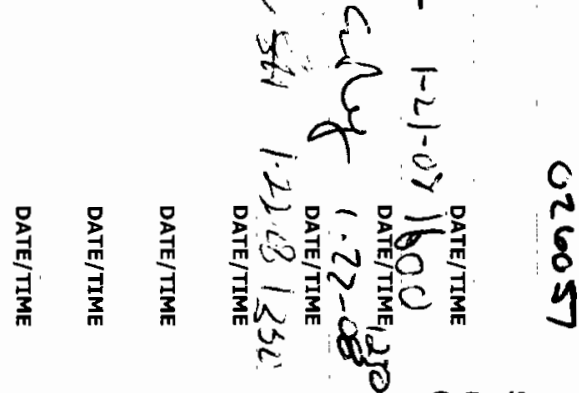

总 寻

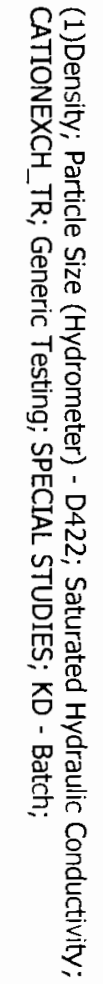

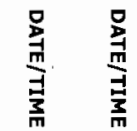

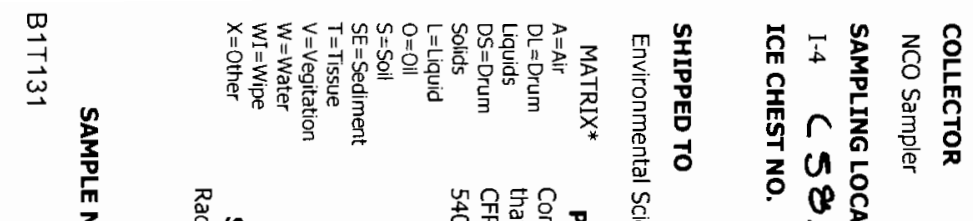

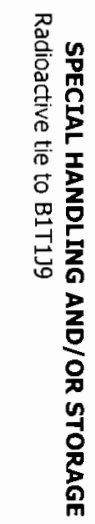

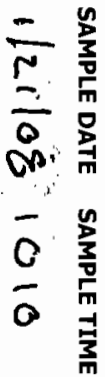

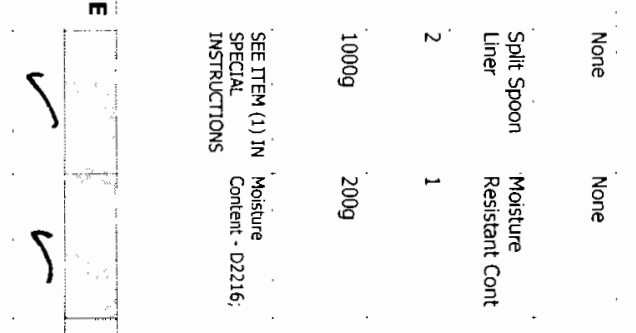

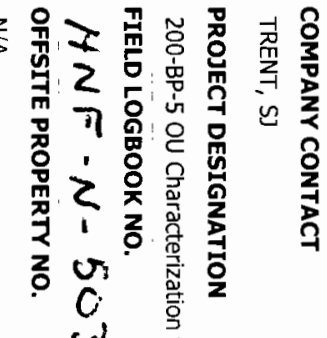

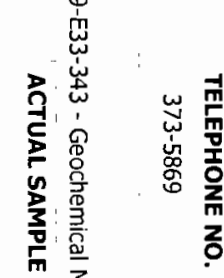

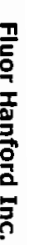

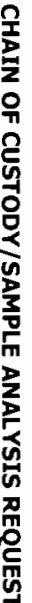

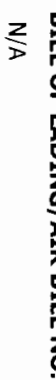

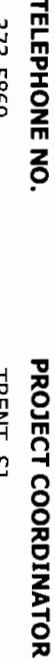

赌

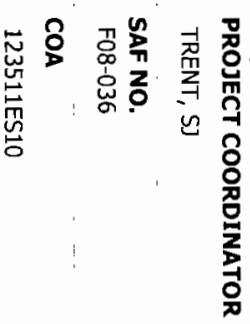
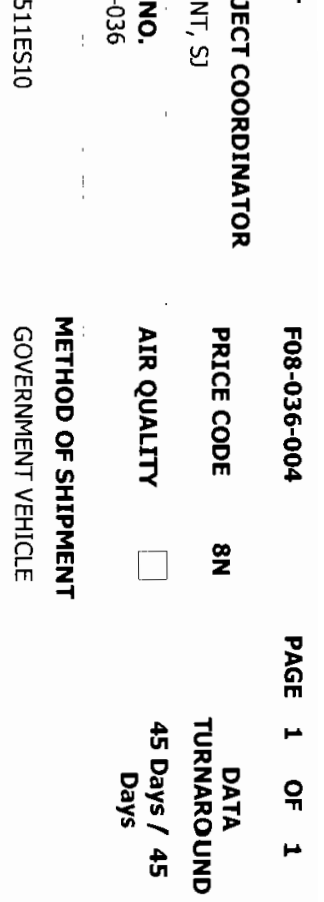


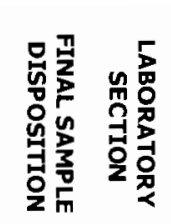
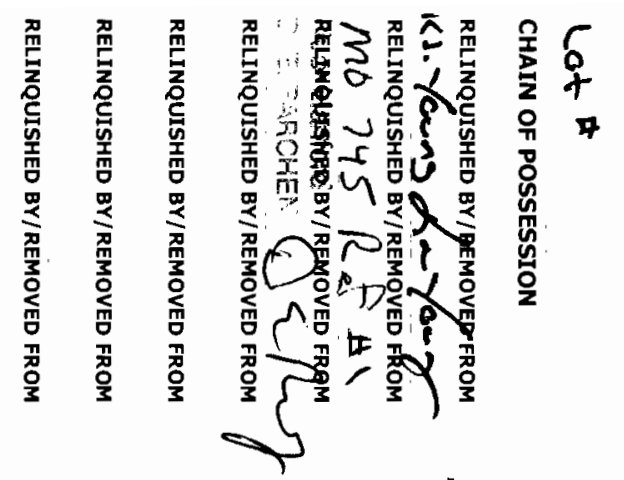

草

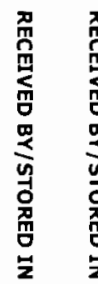

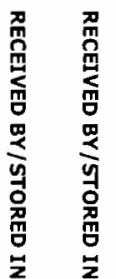

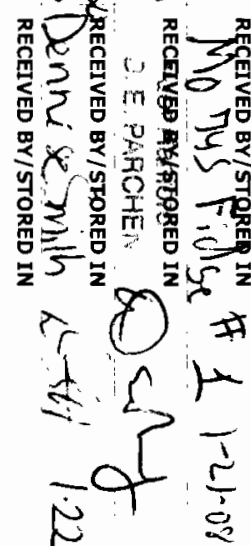

总羔煎

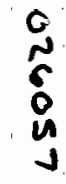

of

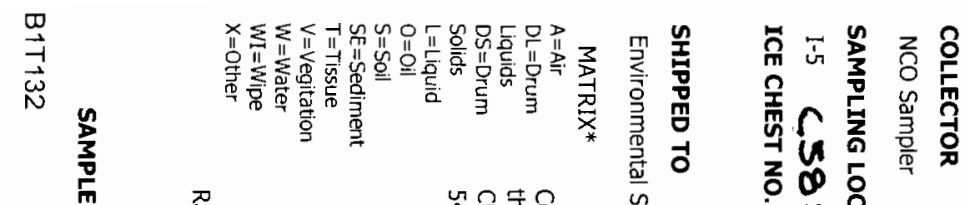

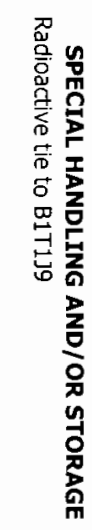

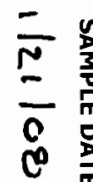

$\begin{array}{cc}\frac{n}{3} \\ 0 & \frac{3}{3} \\ 0 & \frac{1}{3} \\ \frac{3}{m}\end{array}$

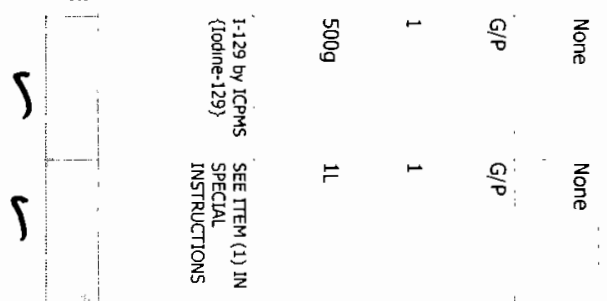

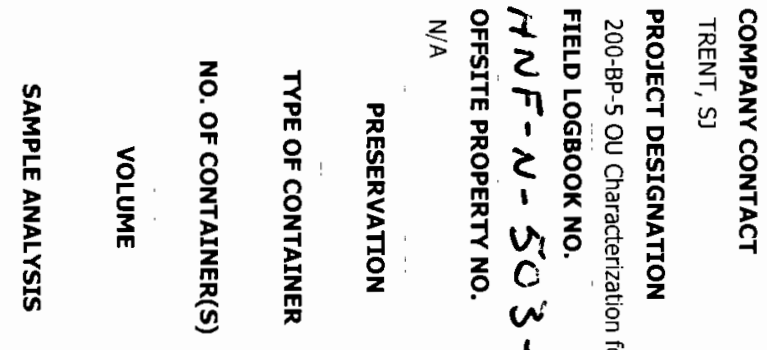

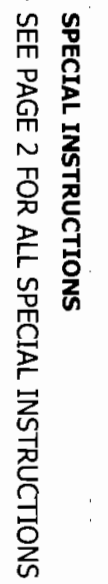

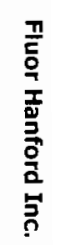

号

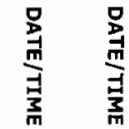




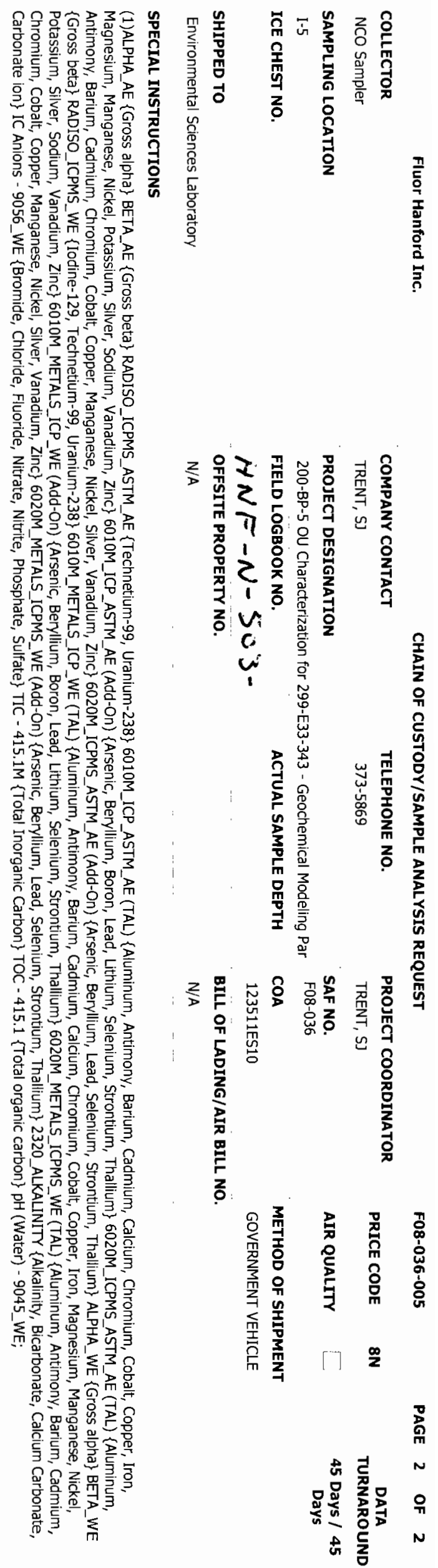




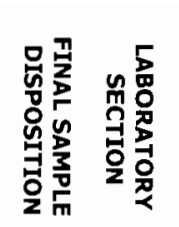

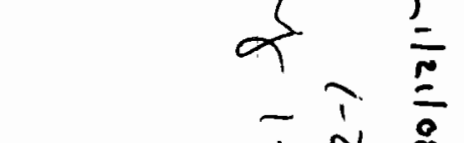

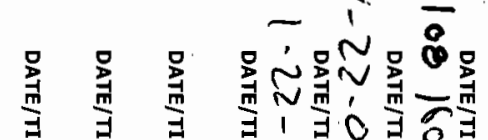

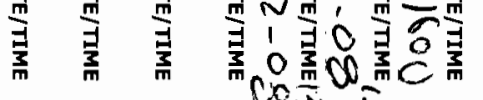
जै yु?

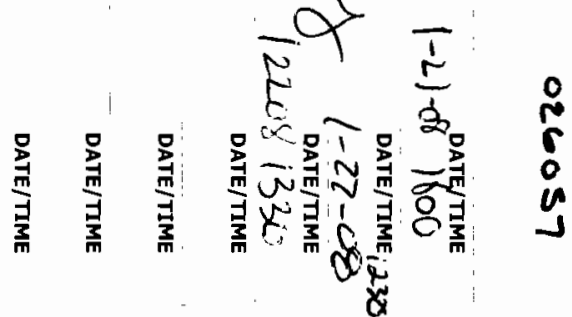
$\underset{m}{\exists}$

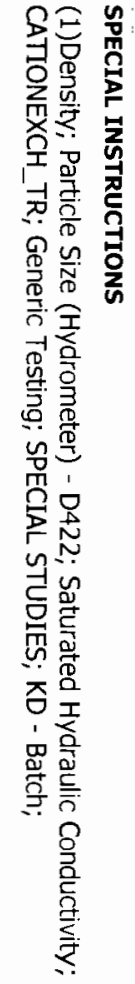

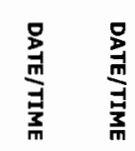
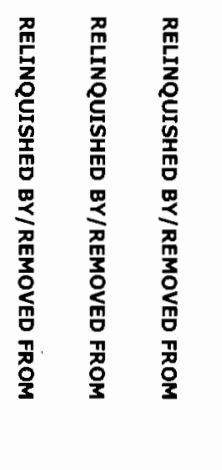

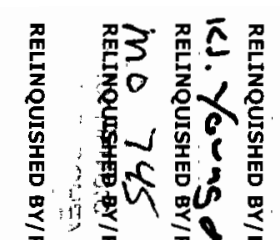

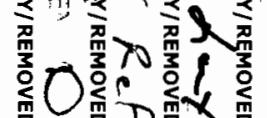

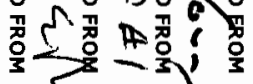

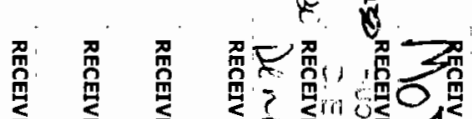
ㄴ.

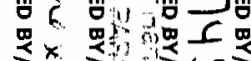

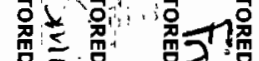
声

-

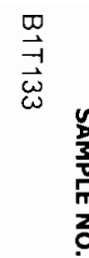

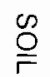

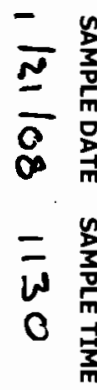

1

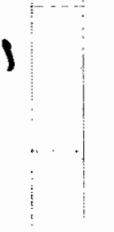

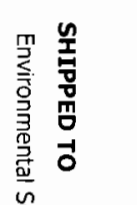

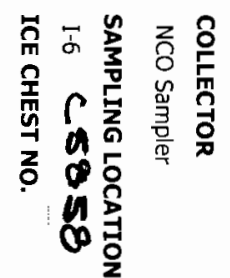

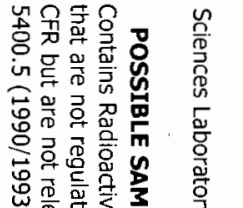

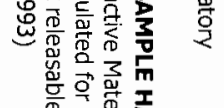

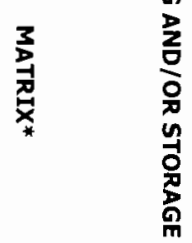

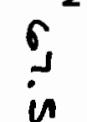

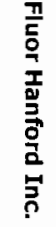

$\frac{5}{0}$

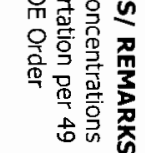

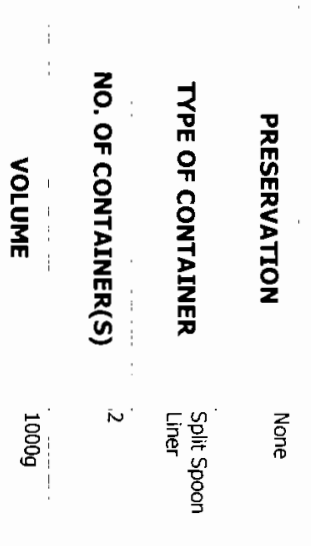

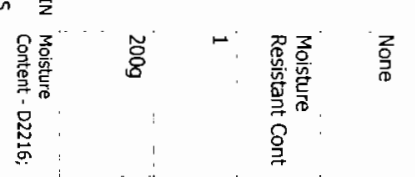

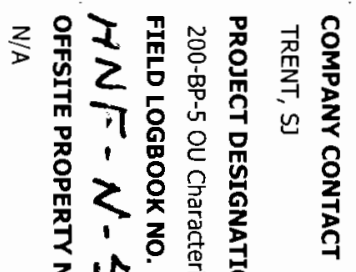
0 年 w<smiles>Ic1ccccc1</smiles>

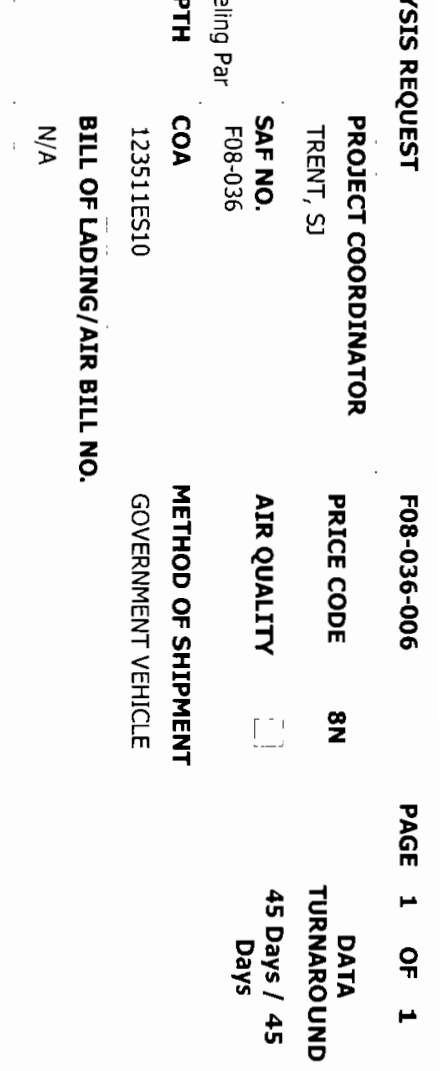




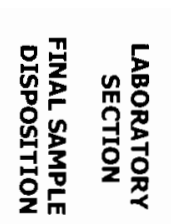

I

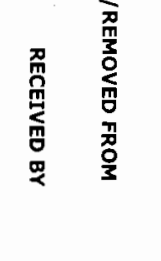

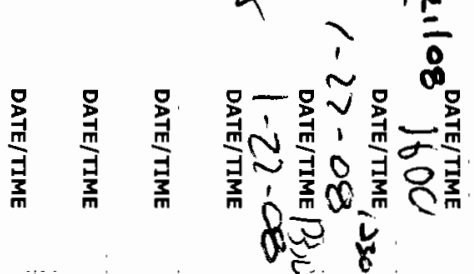

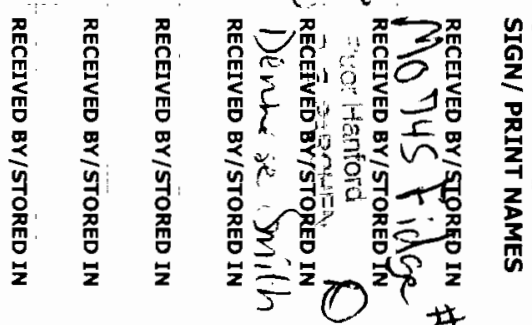

$>n+$

过

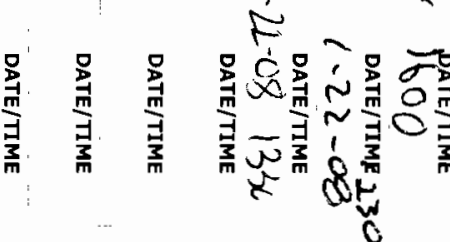

翠

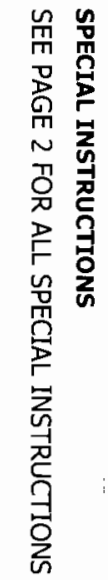

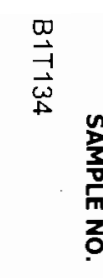

$\stackrel{8}{ }$

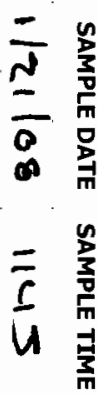

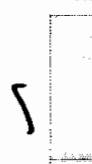

S

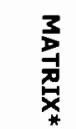

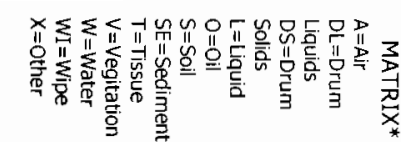

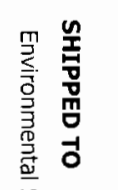

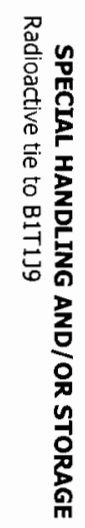

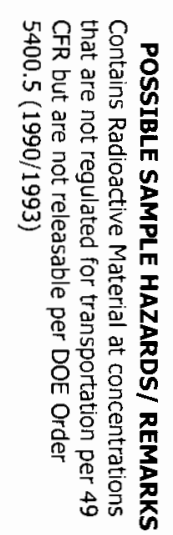

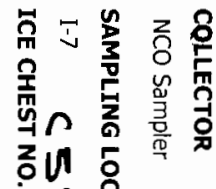

u

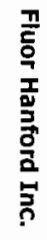
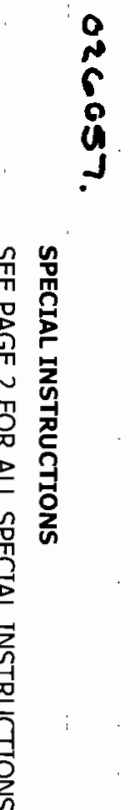

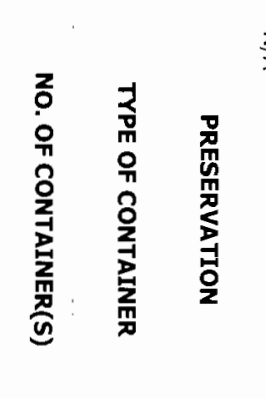

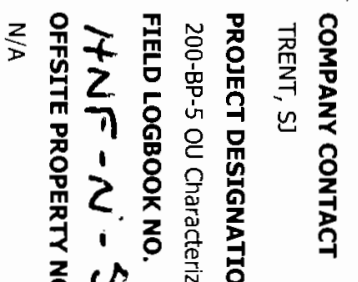

c. ${ }^{w} \overrightarrow{\bar{g}}^{2}$

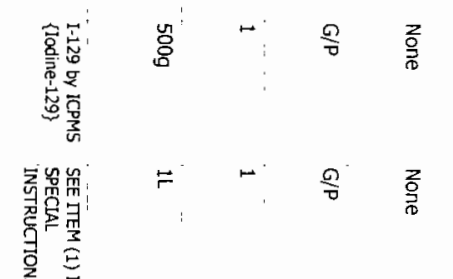

疍
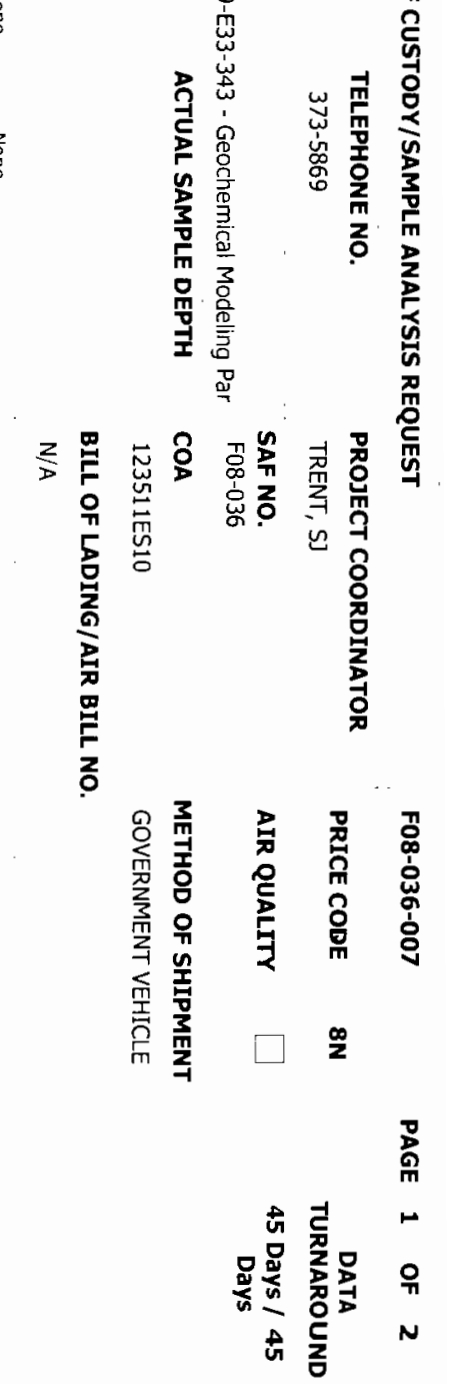


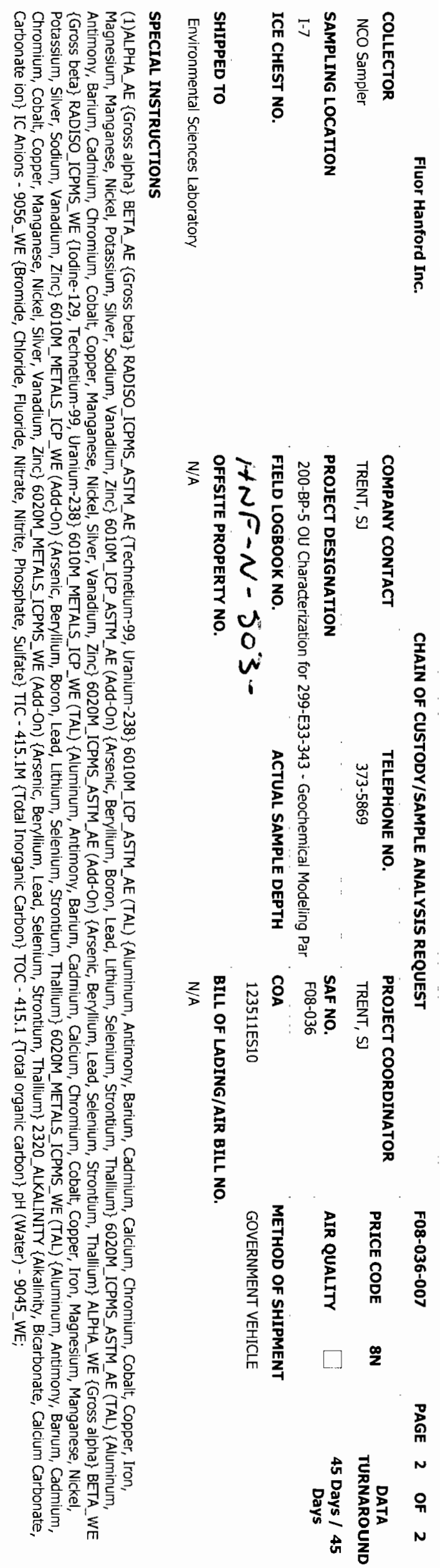




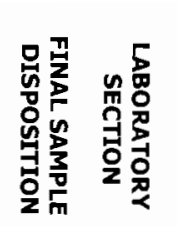
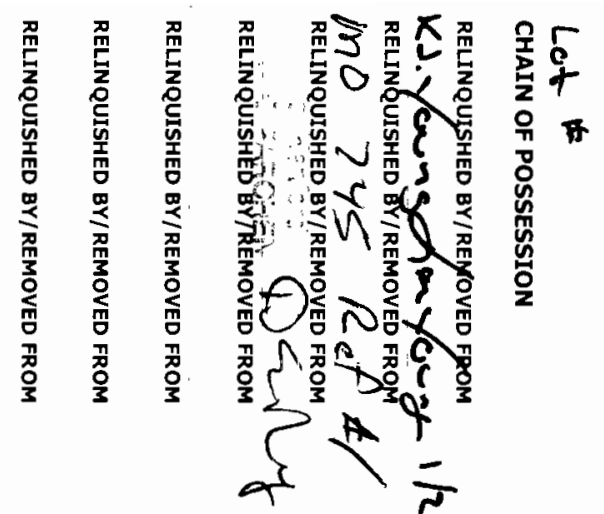

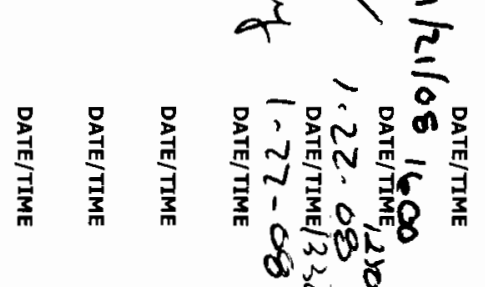
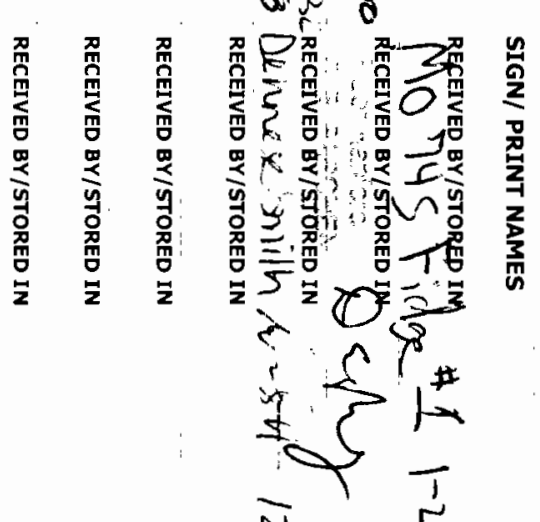

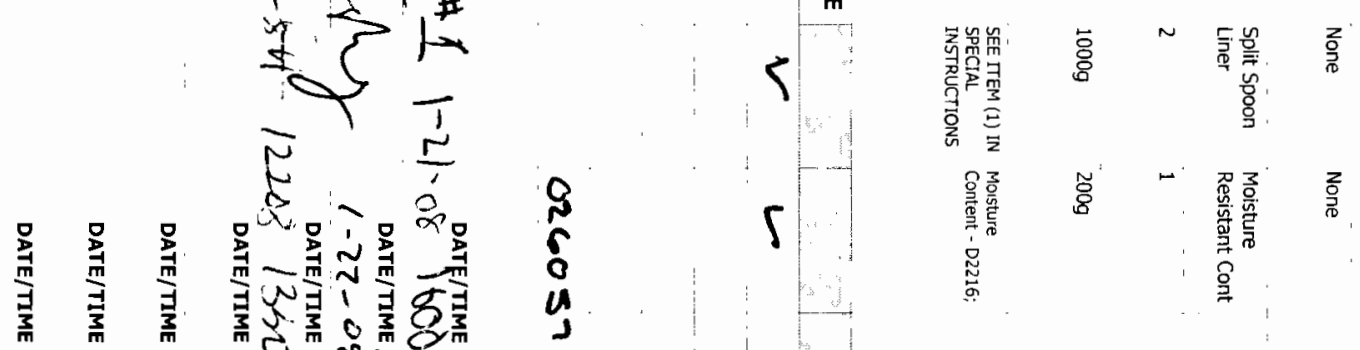
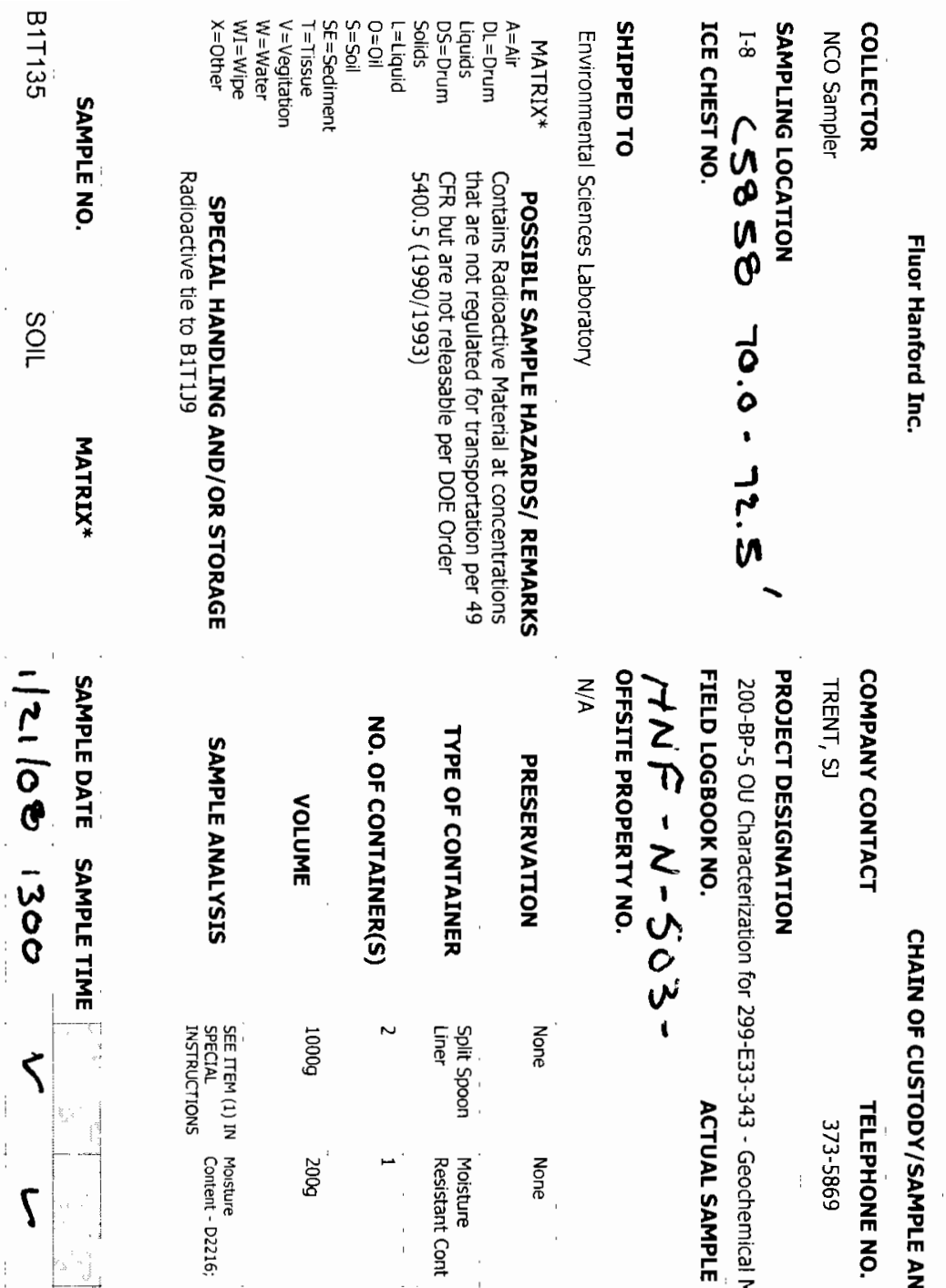

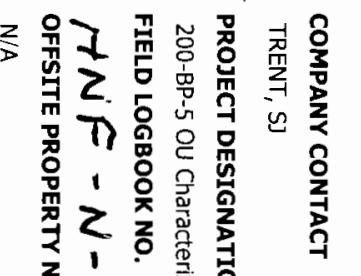
$\begin{array}{ll}u & \\ 0 & 0 \\ w & 0\end{array}$

?⿳亠口子丸

递 辛

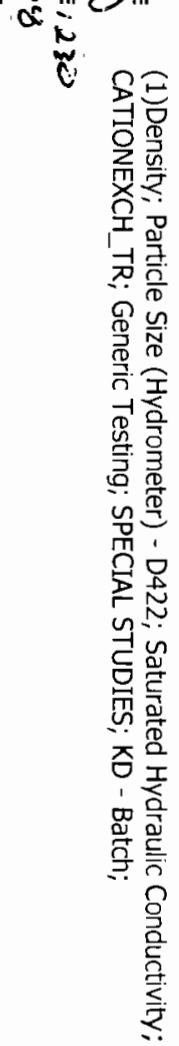

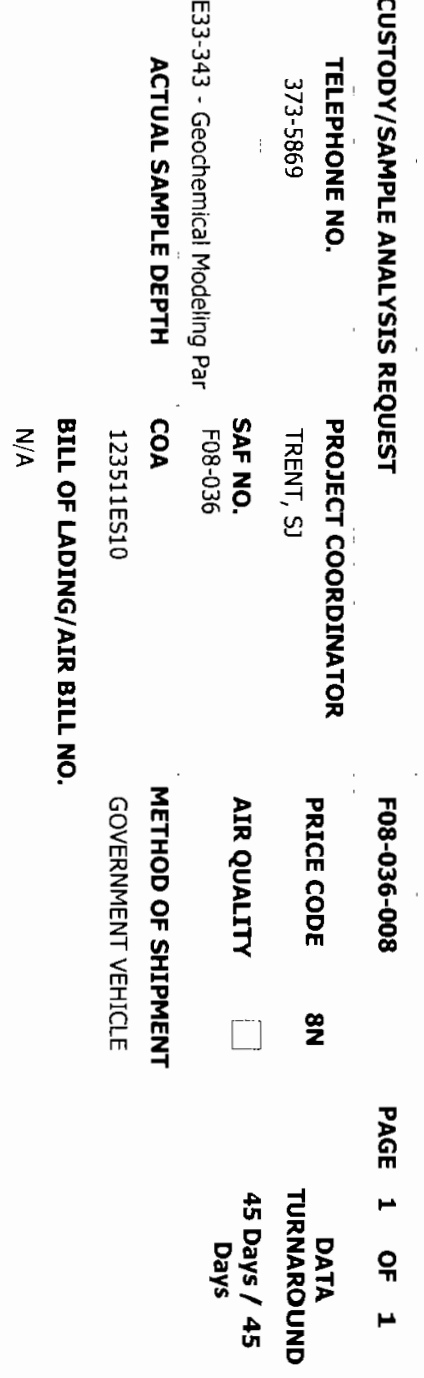




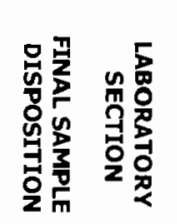

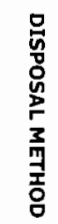

㟨

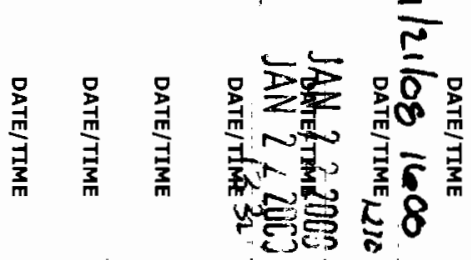

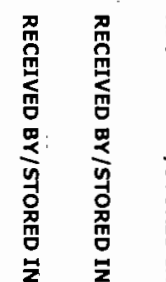

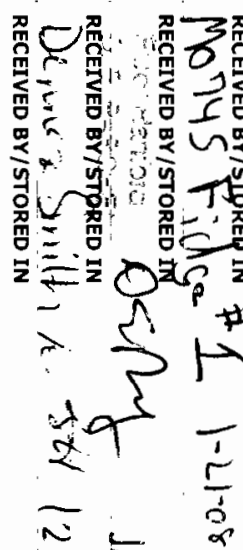

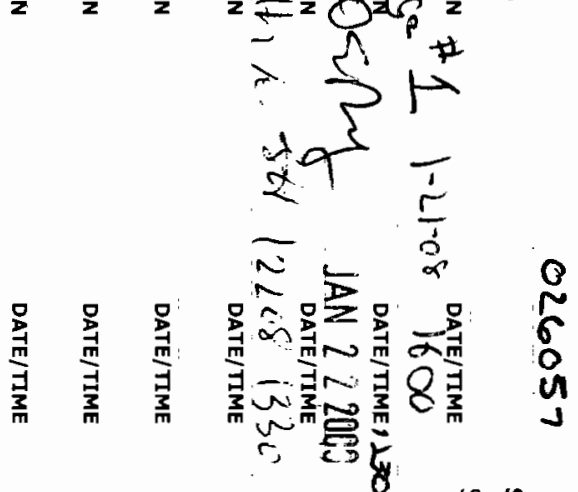

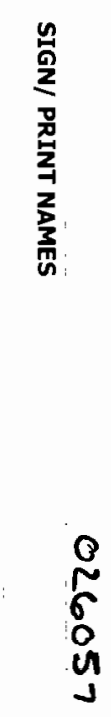

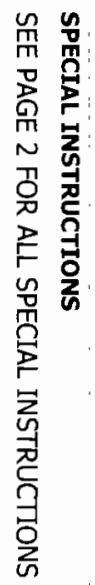

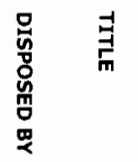

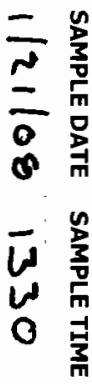

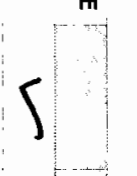

s

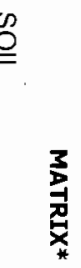

*

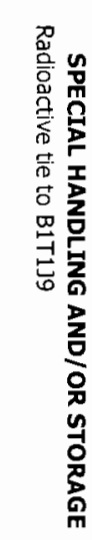

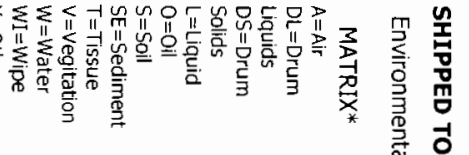

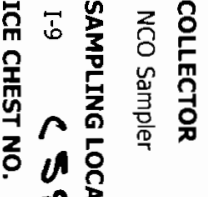

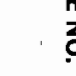

$\stackrel{\varrho}{\rightleftharpoons}$

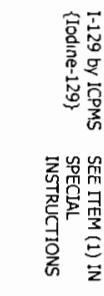

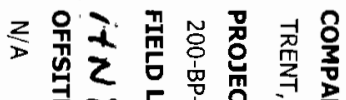

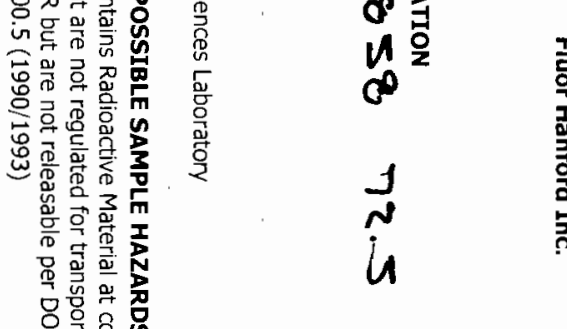

뮹

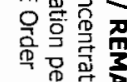

政视

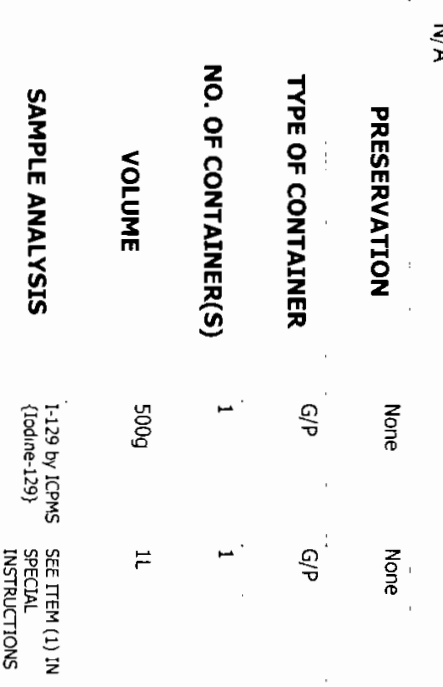

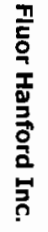




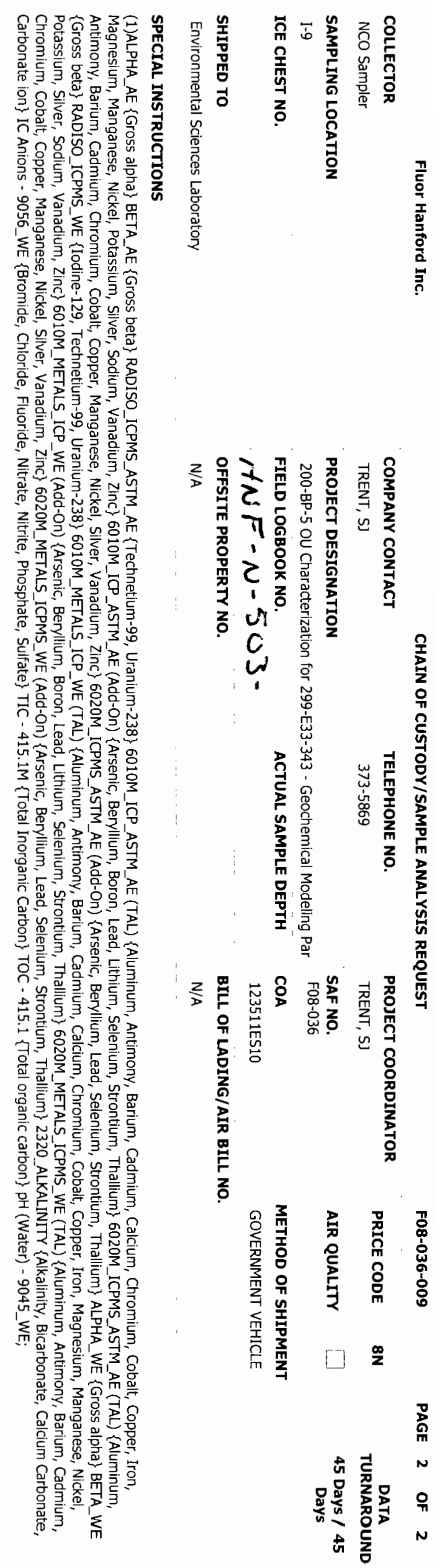




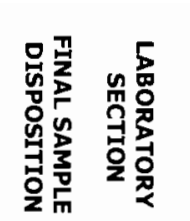
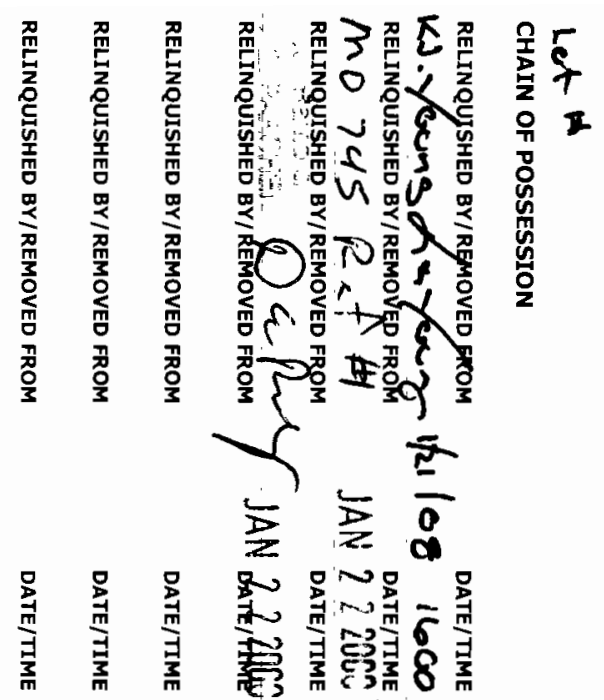

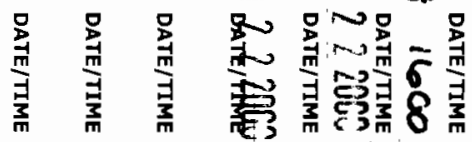
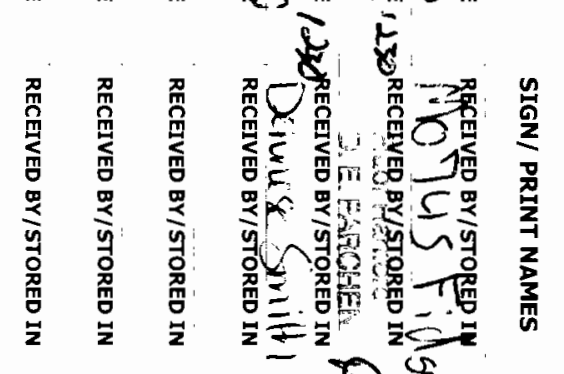

$\stackrel{\square}{\vec{\exists}}$

$\stackrel{\infty}{\circ}$

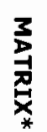

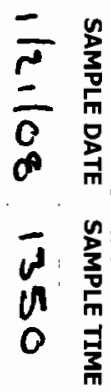
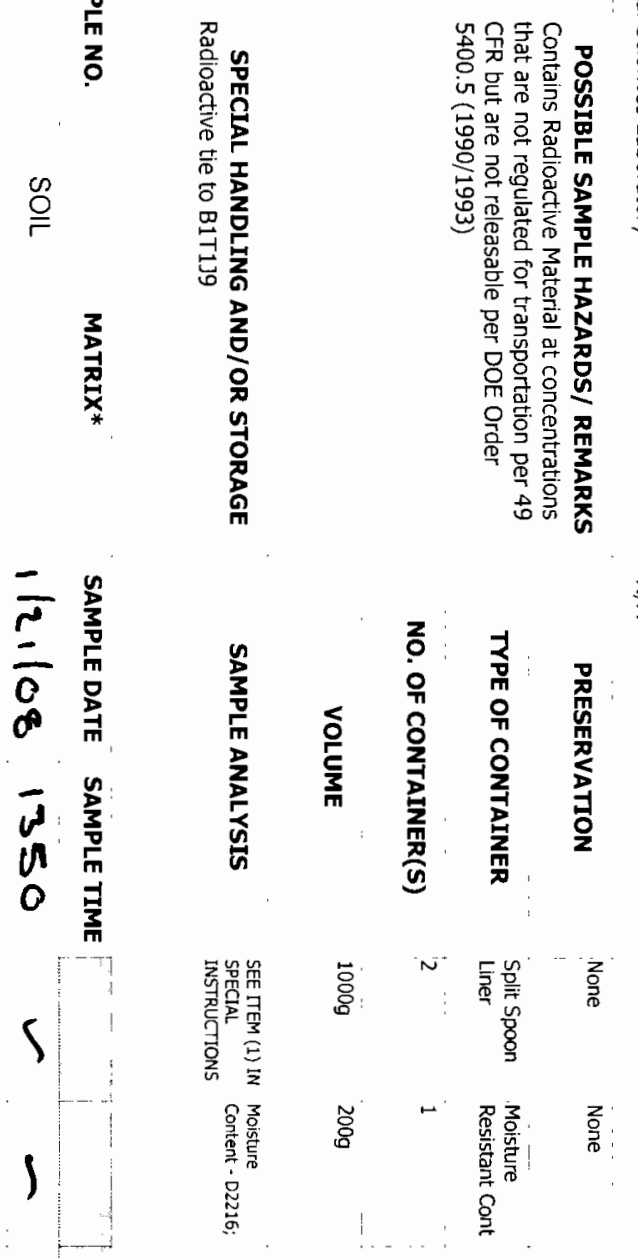

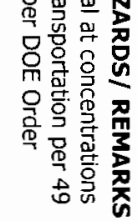

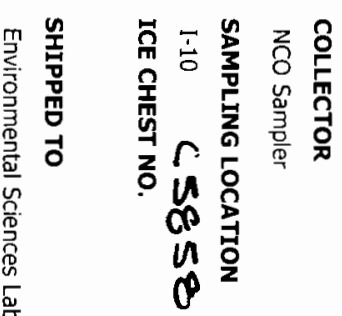

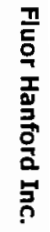

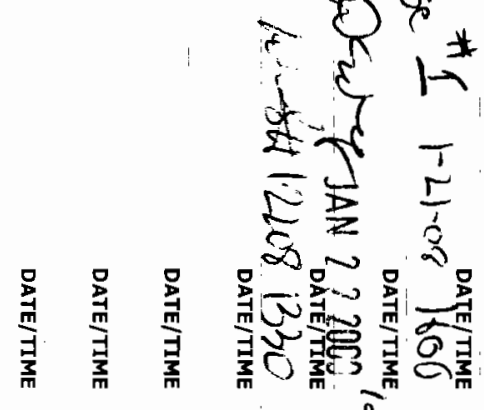

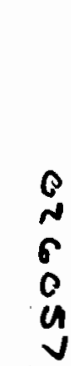

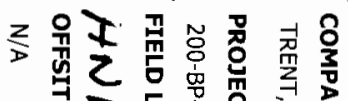

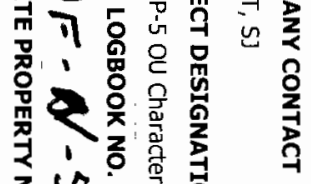
zúl
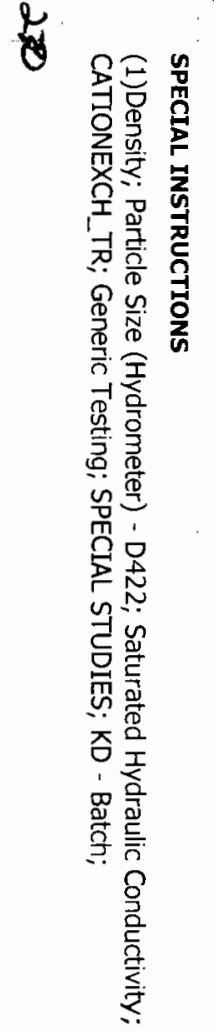<smiles>[H]</smiles>

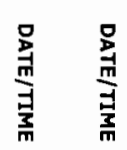




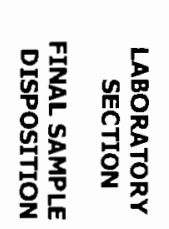
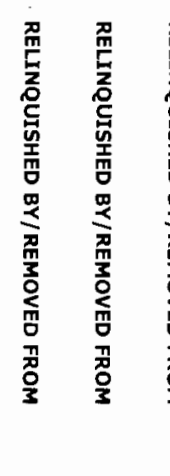

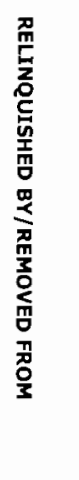
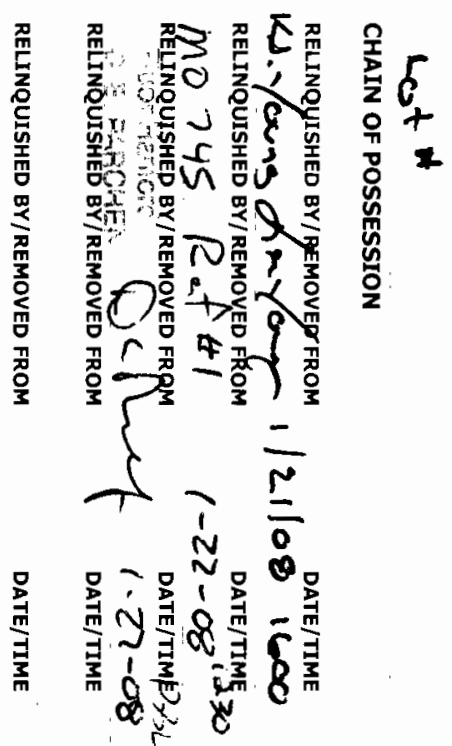

总总
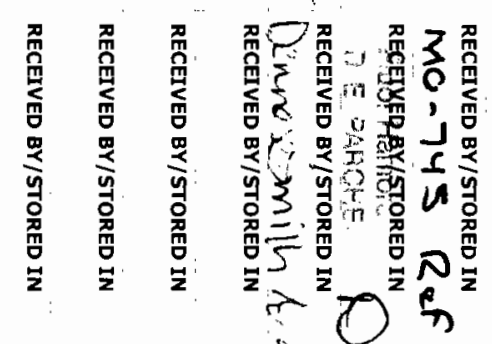

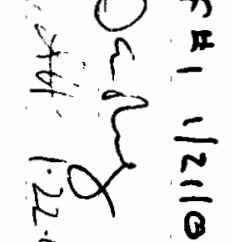

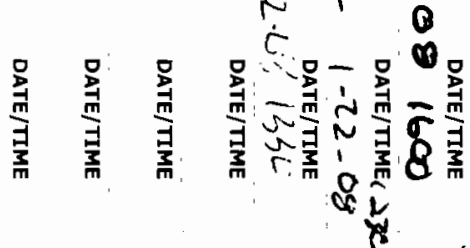
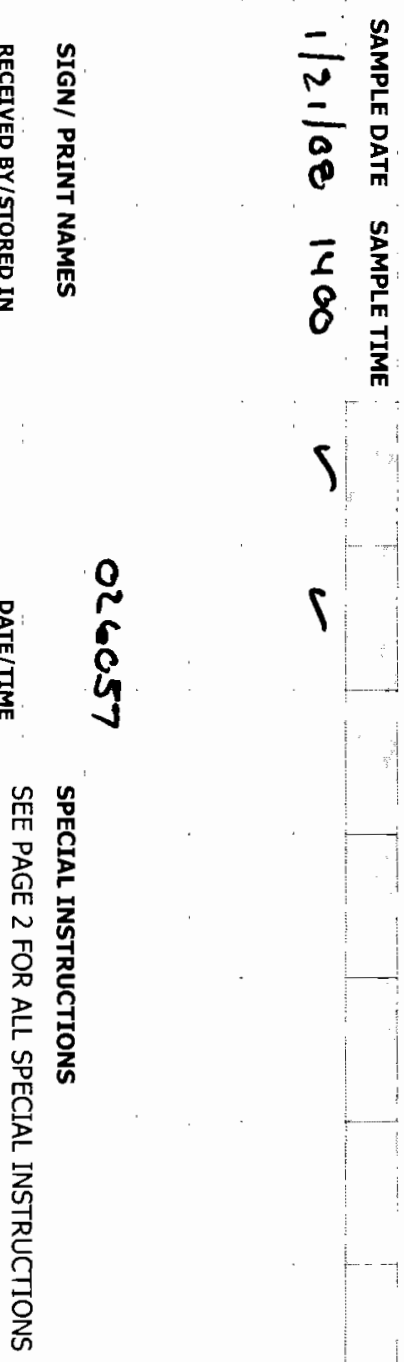

量

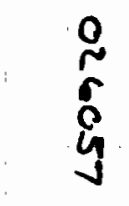

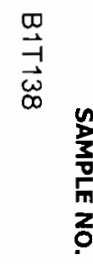

$\stackrel{\infty}{\circ}$

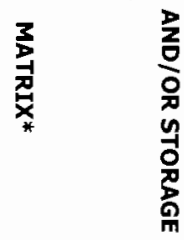

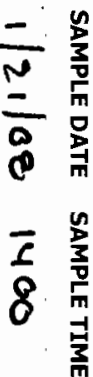

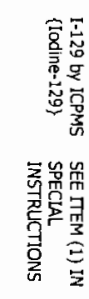

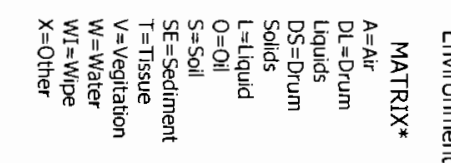

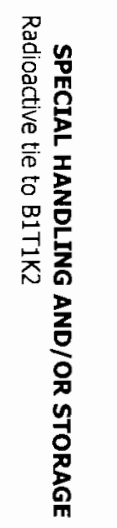

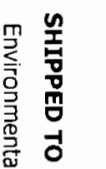

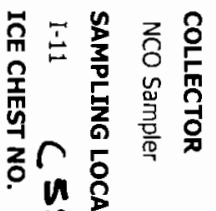

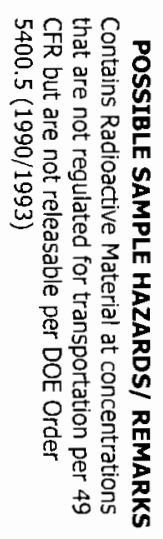

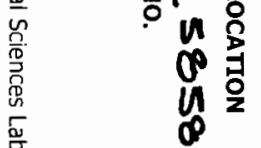

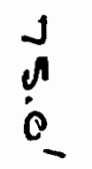

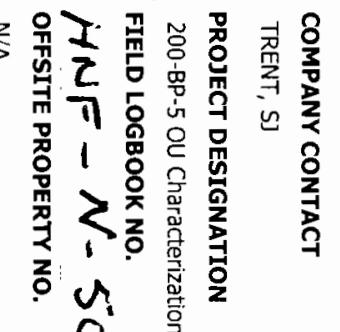

愛 


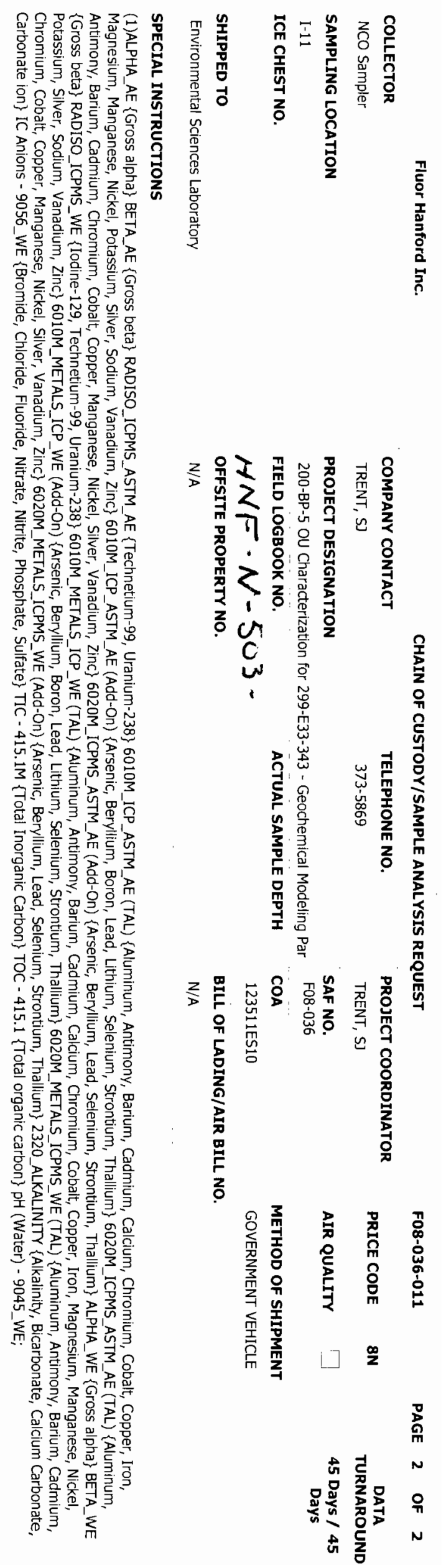




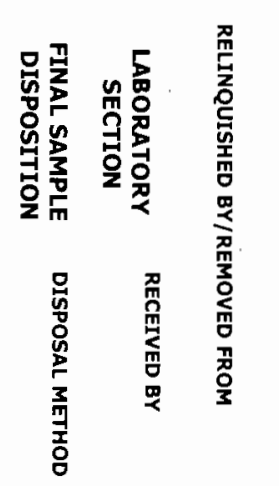

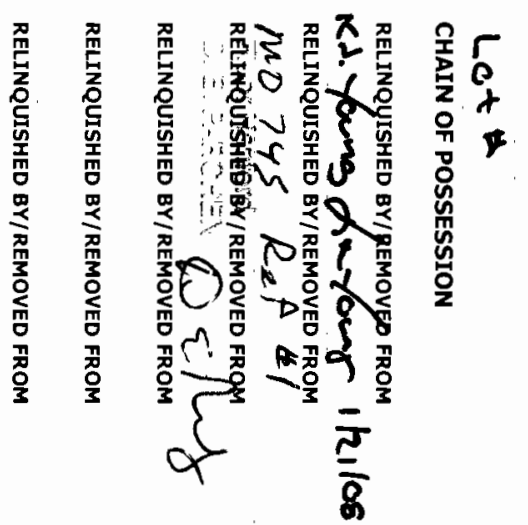

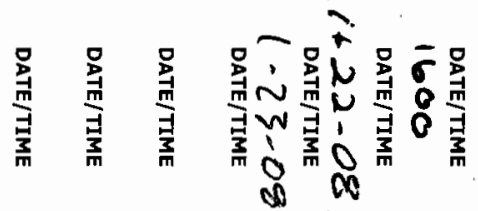
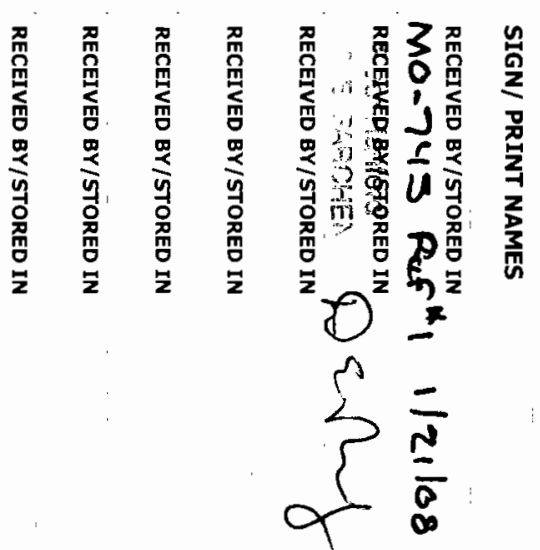

总量

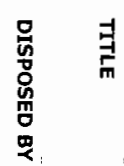

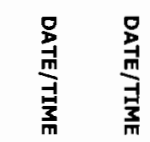

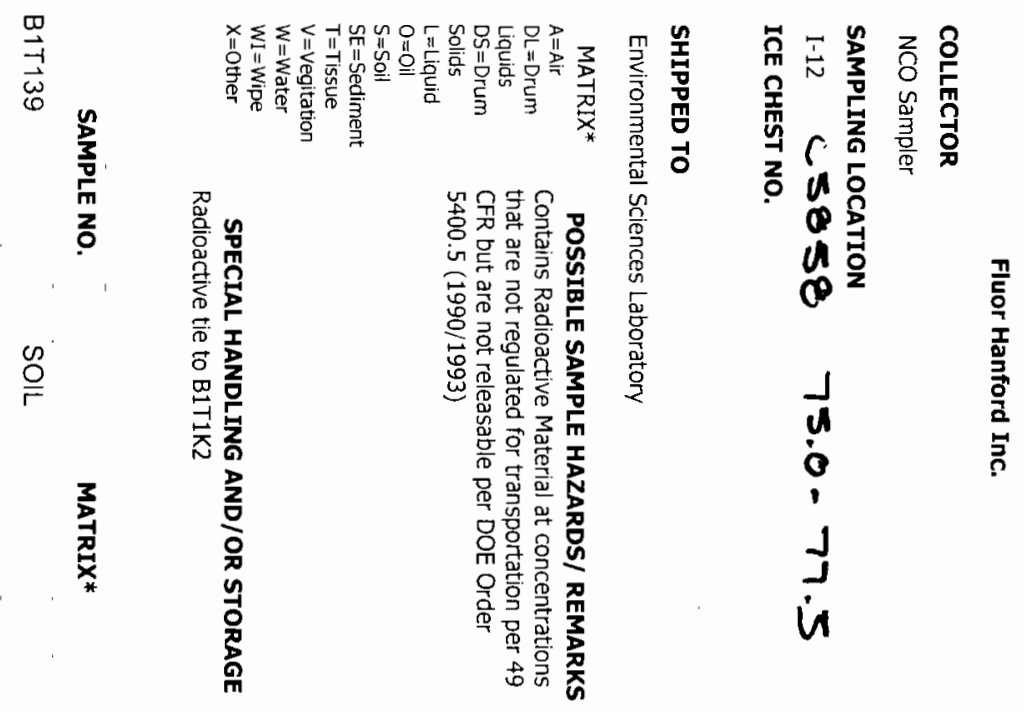

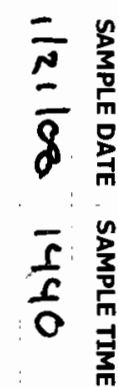

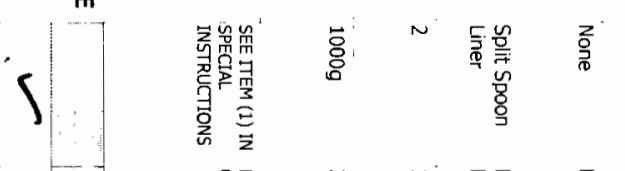

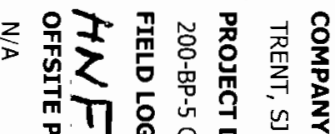

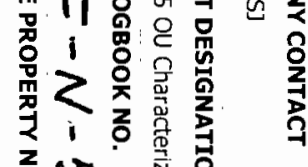

o 올

c

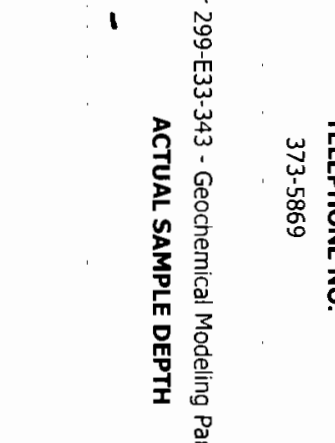

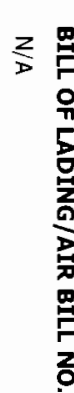

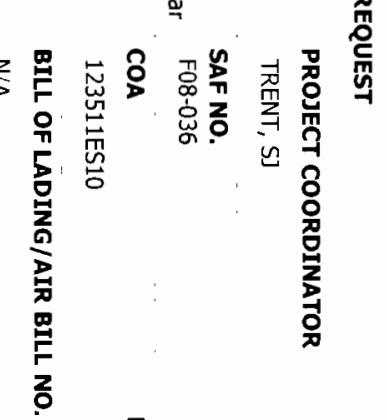

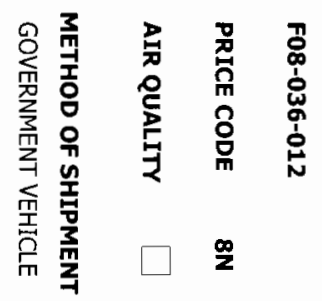

วั

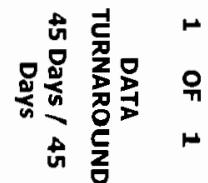




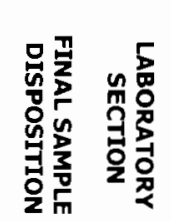

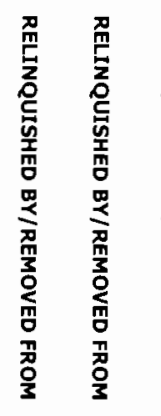

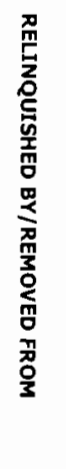

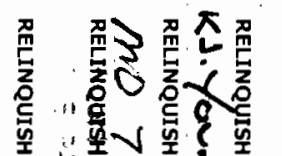

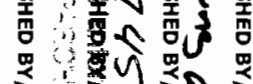

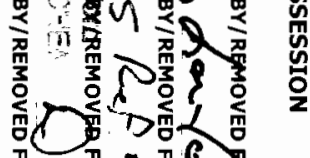

㞸<smiles>C/C=C\CC1CC1</smiles>

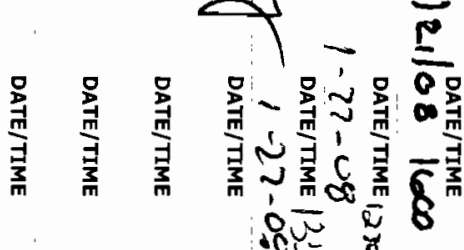

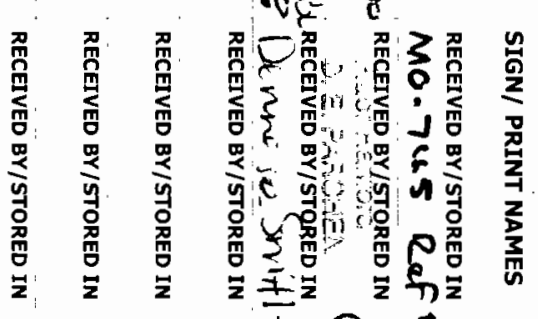

\%

$\underset{\pi}{\pi} \frac{-}{\frac{N}{0}}$

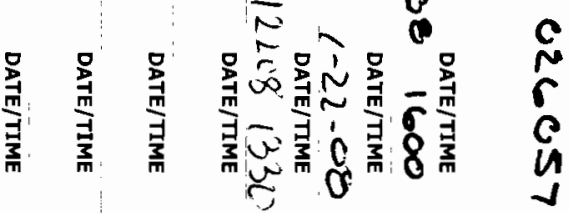

品 $\vec{\exists}$

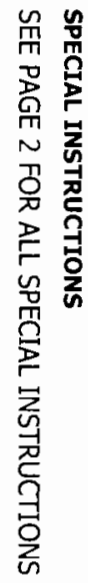

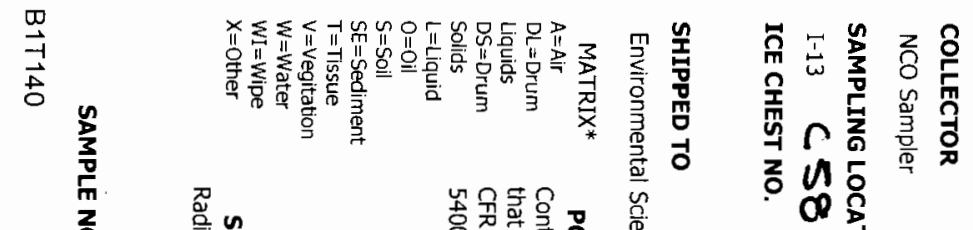

un

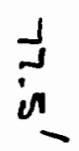

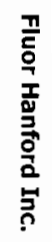

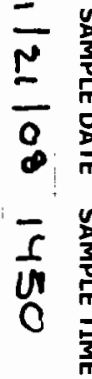

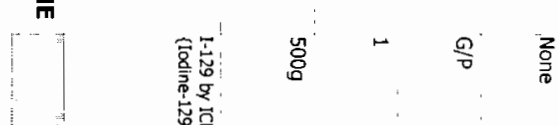

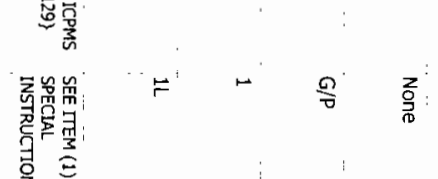

$\underset{>}{2}$

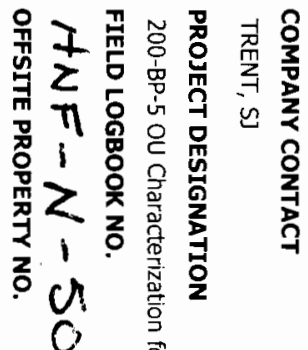

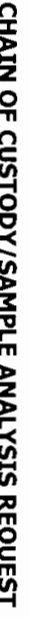

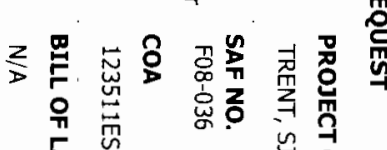

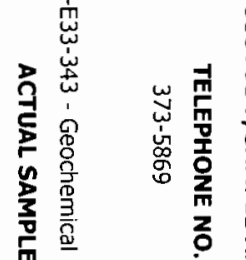

용

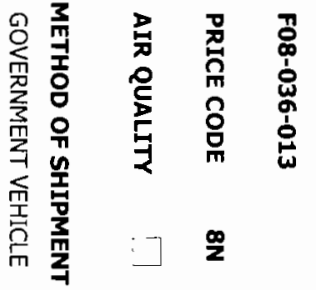

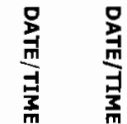

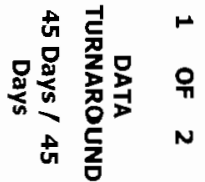




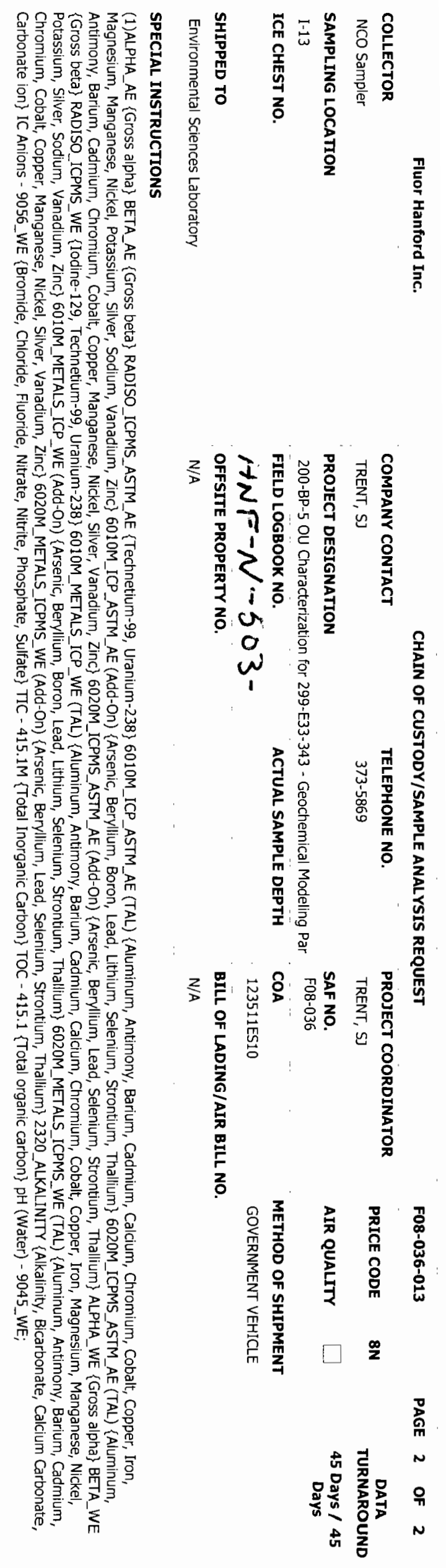




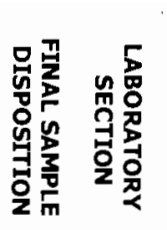
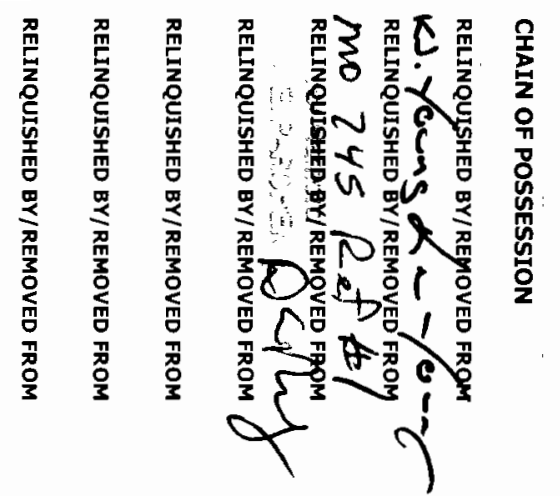

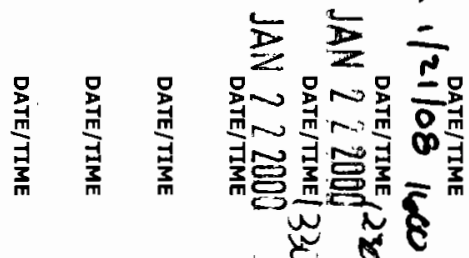
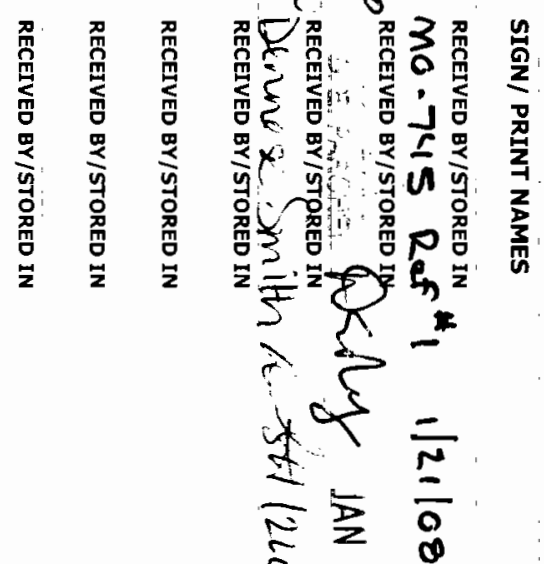

政事

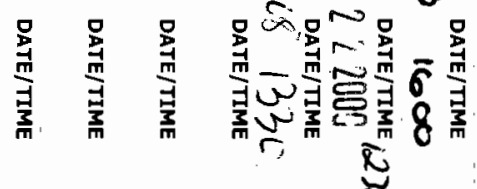

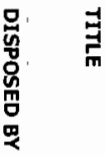

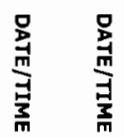

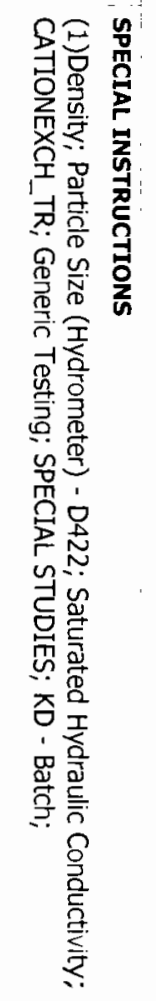

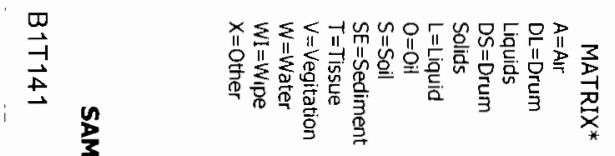

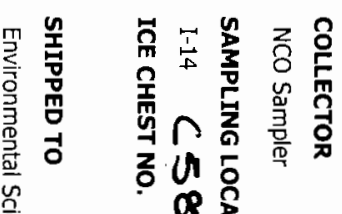
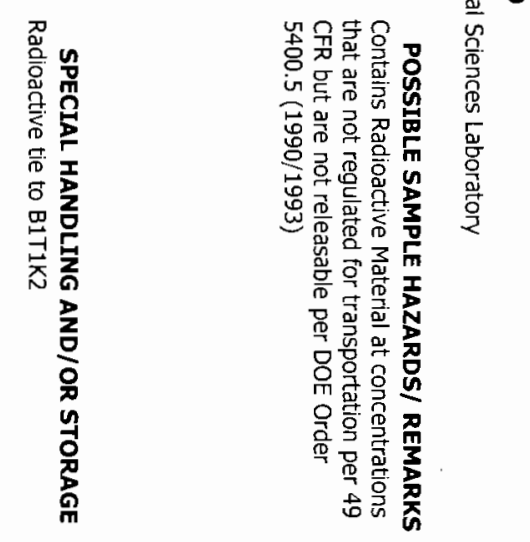

伎京

$\stackrel{\infty}{\circ}$

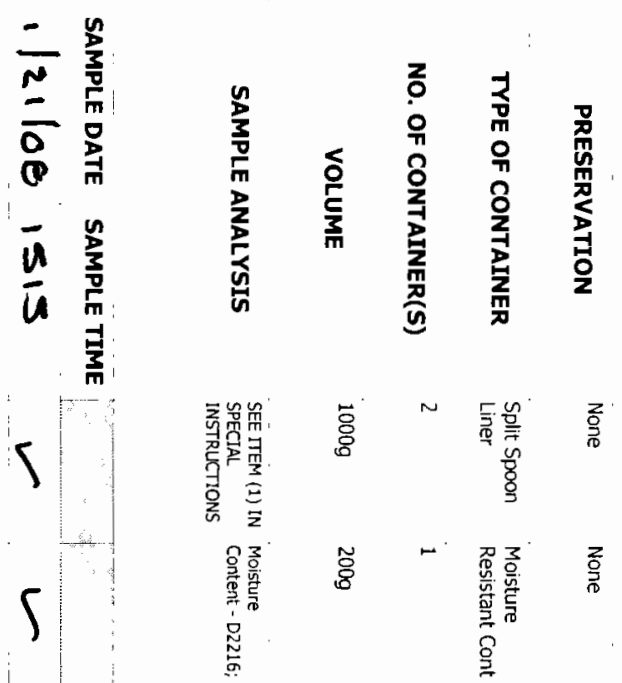

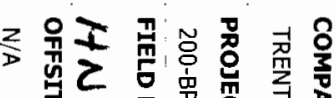

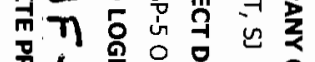

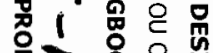

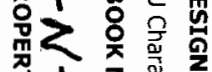

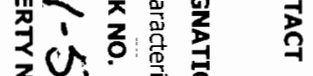
zô

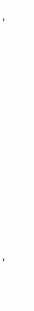
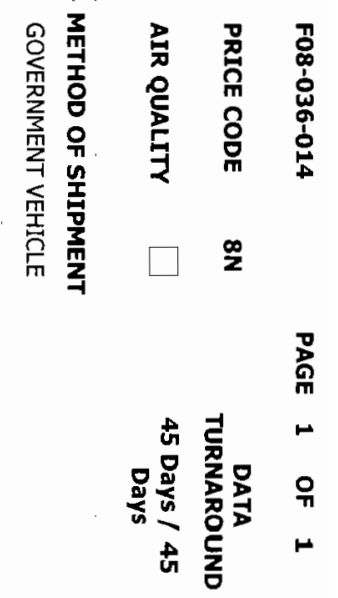


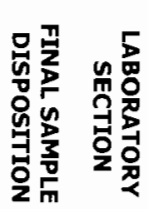

号

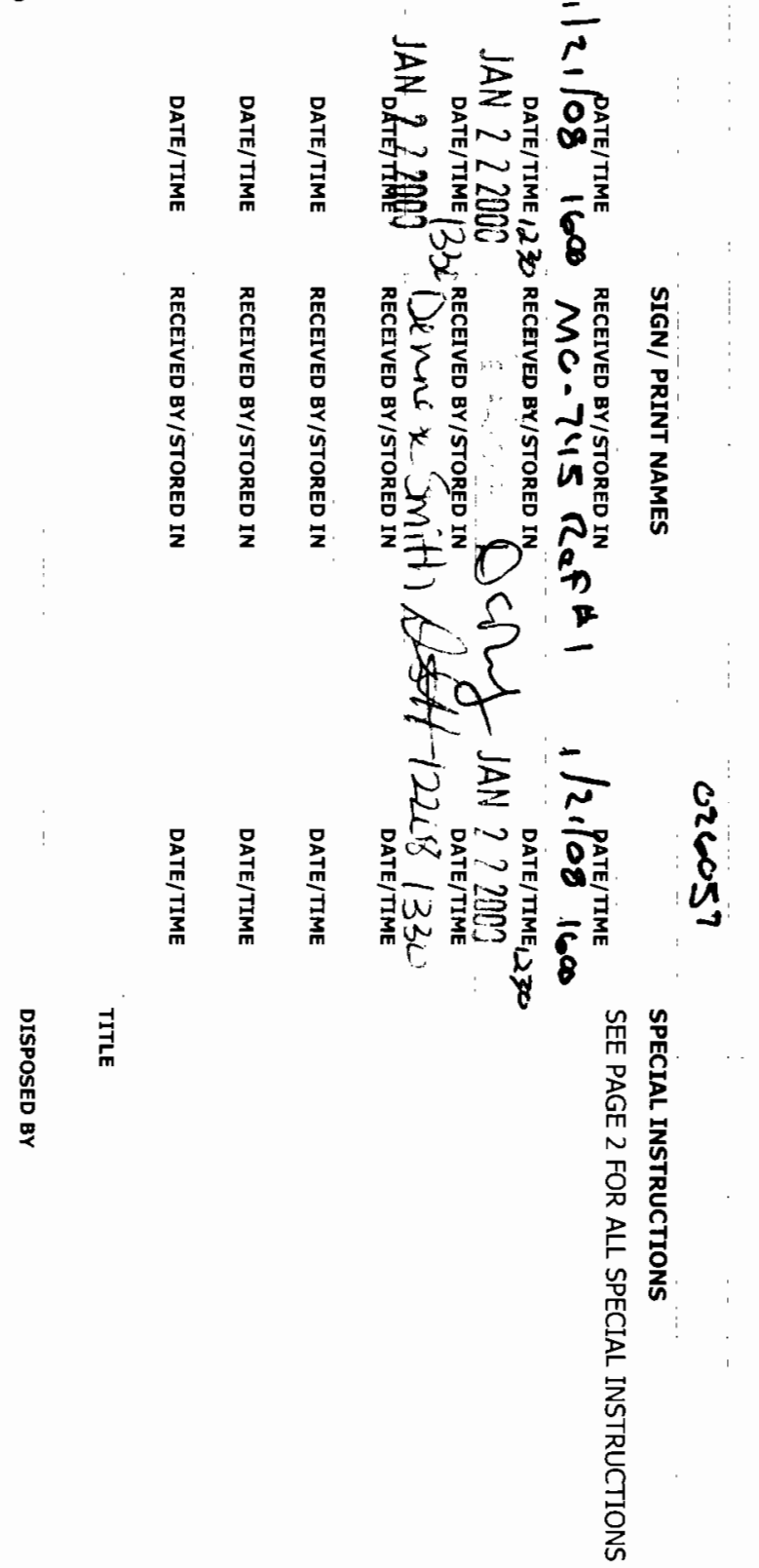

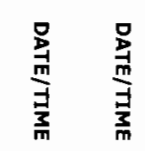
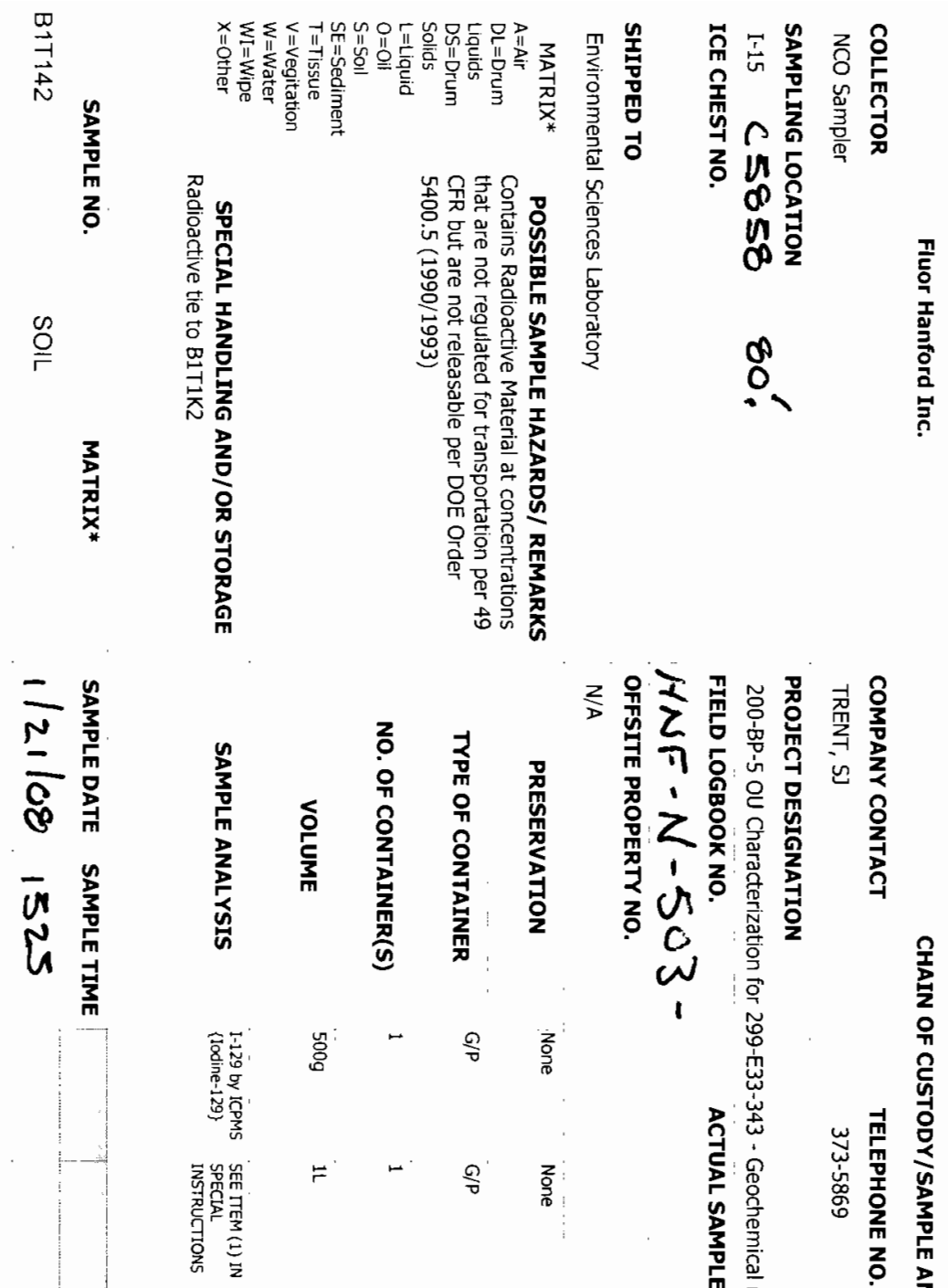

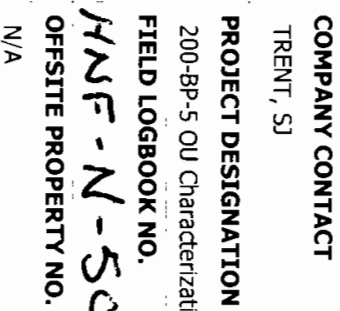
c

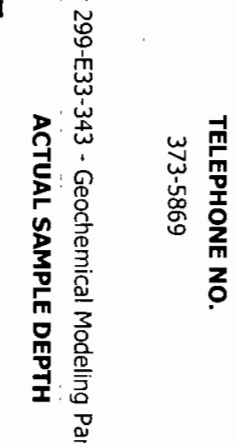

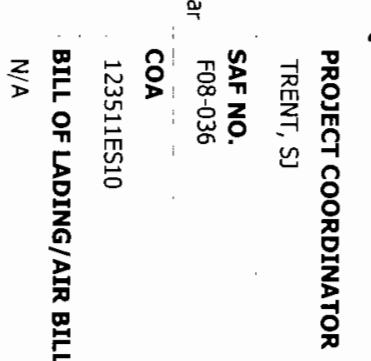

z

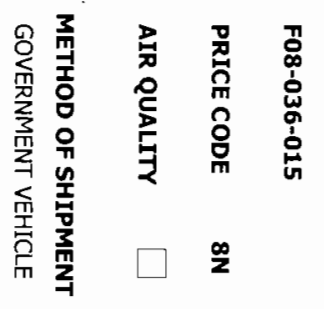




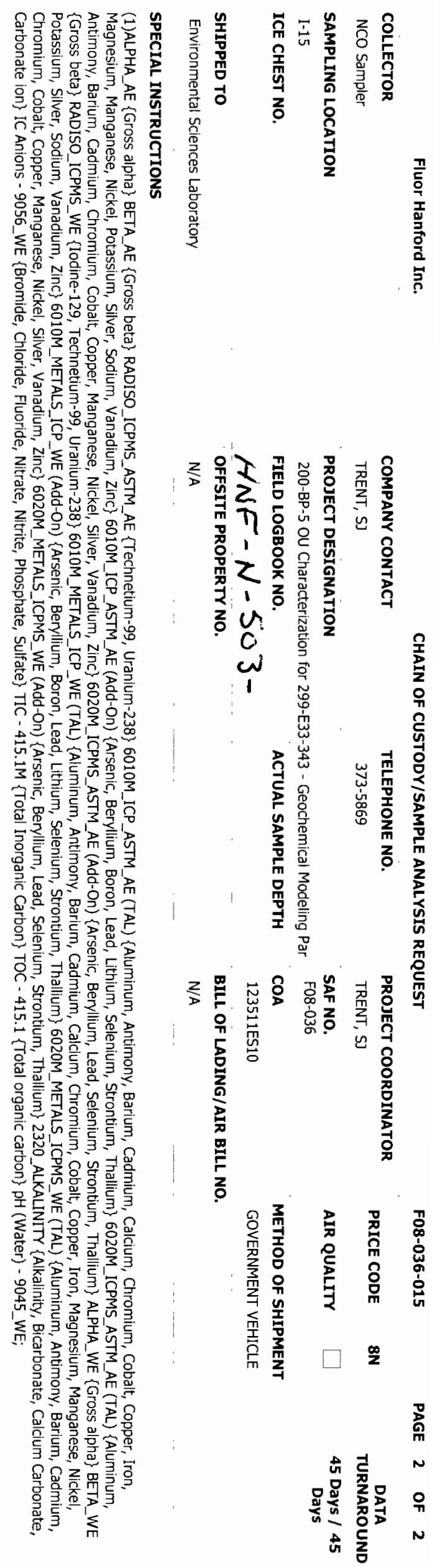




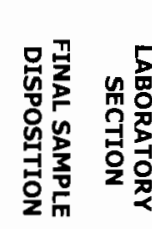

몸

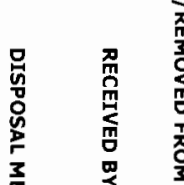

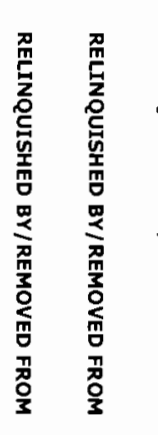

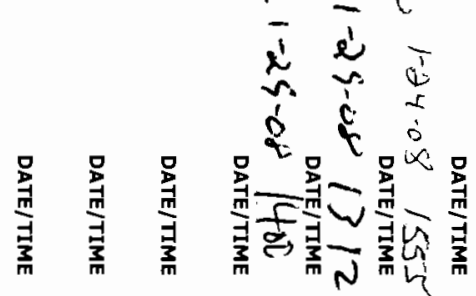

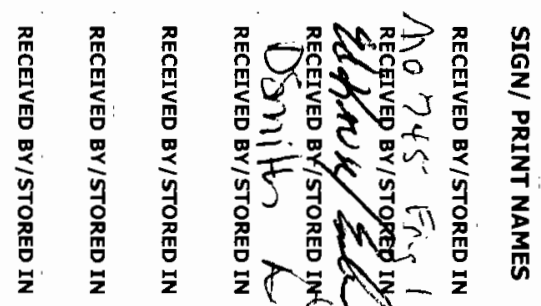

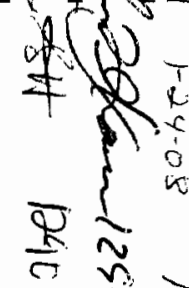

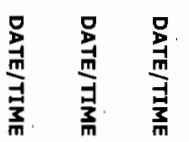

量

숨

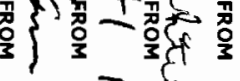

$\stackrel{\square}{\overrightarrow{\vec{a}}}$

$\stackrel{\text { O }}{\Gamma}$

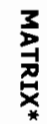

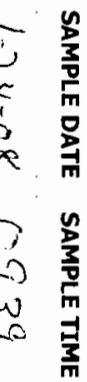

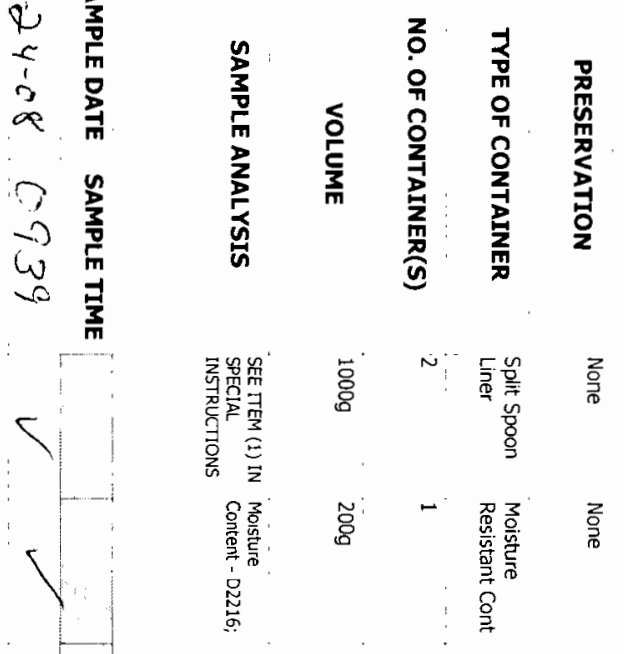

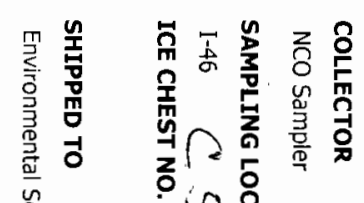

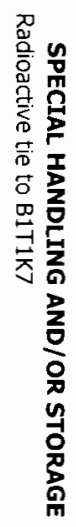

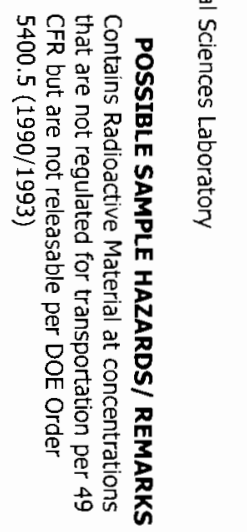

乐苜

ए)

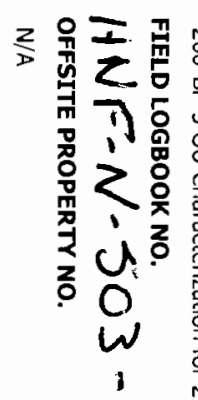

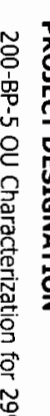

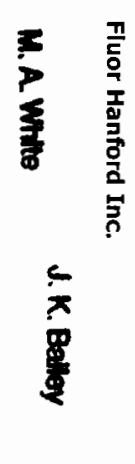

$\vdots$
$\vdots$
$\vdots$
0
4
0
0
0

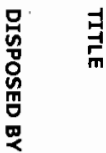

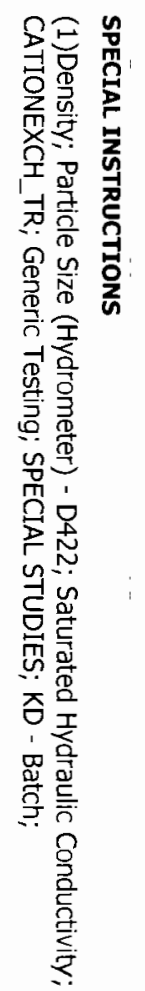<smiles>C1CCCCC1</smiles>
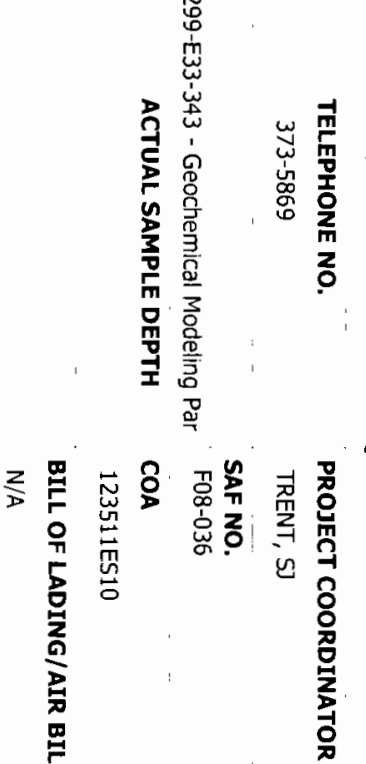

zo

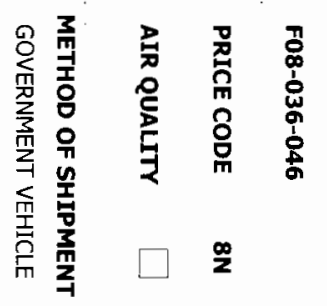

尊 


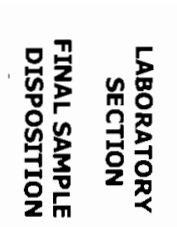
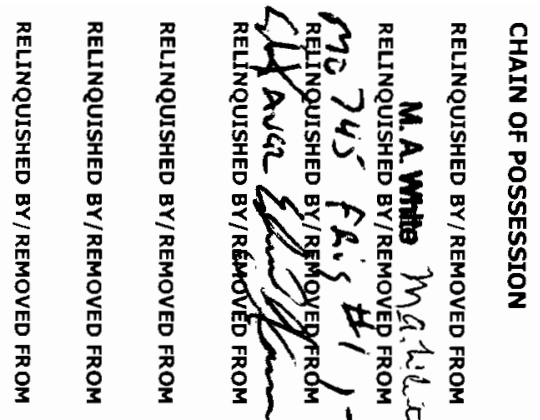

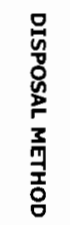

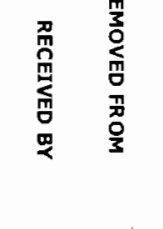

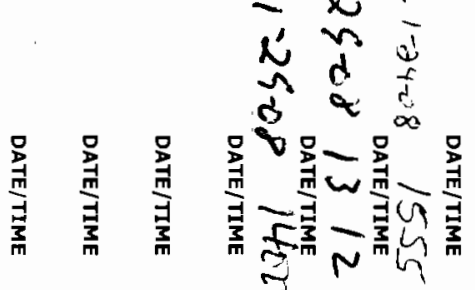

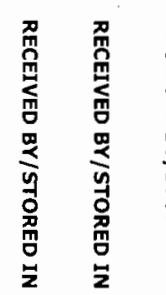
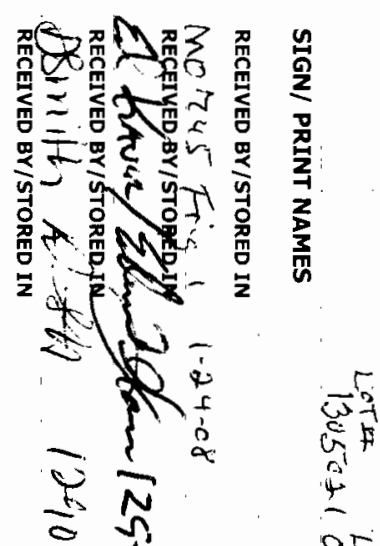

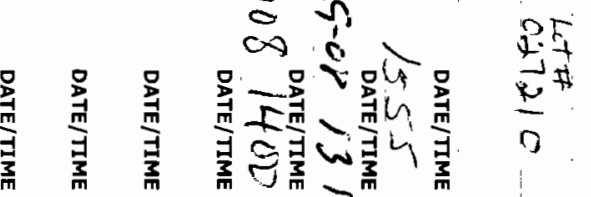
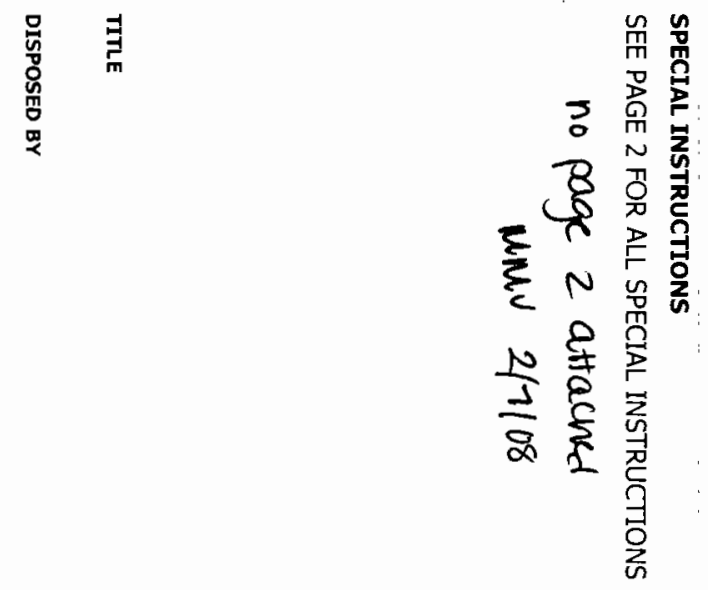

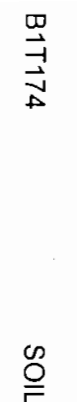

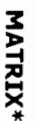

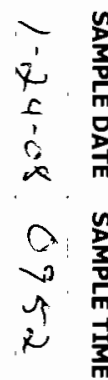

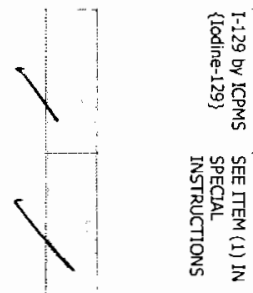

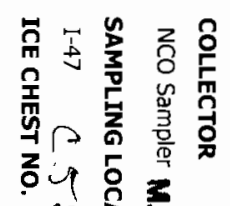

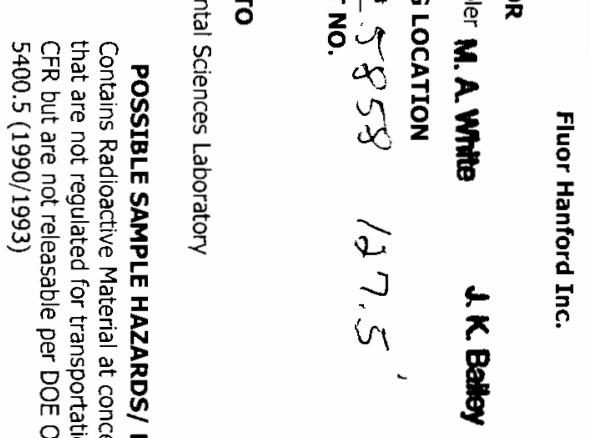

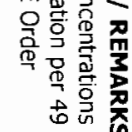

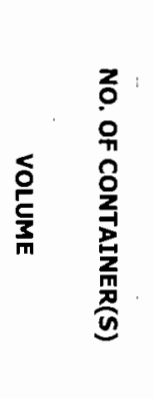

जัํํㅁ

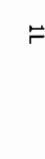

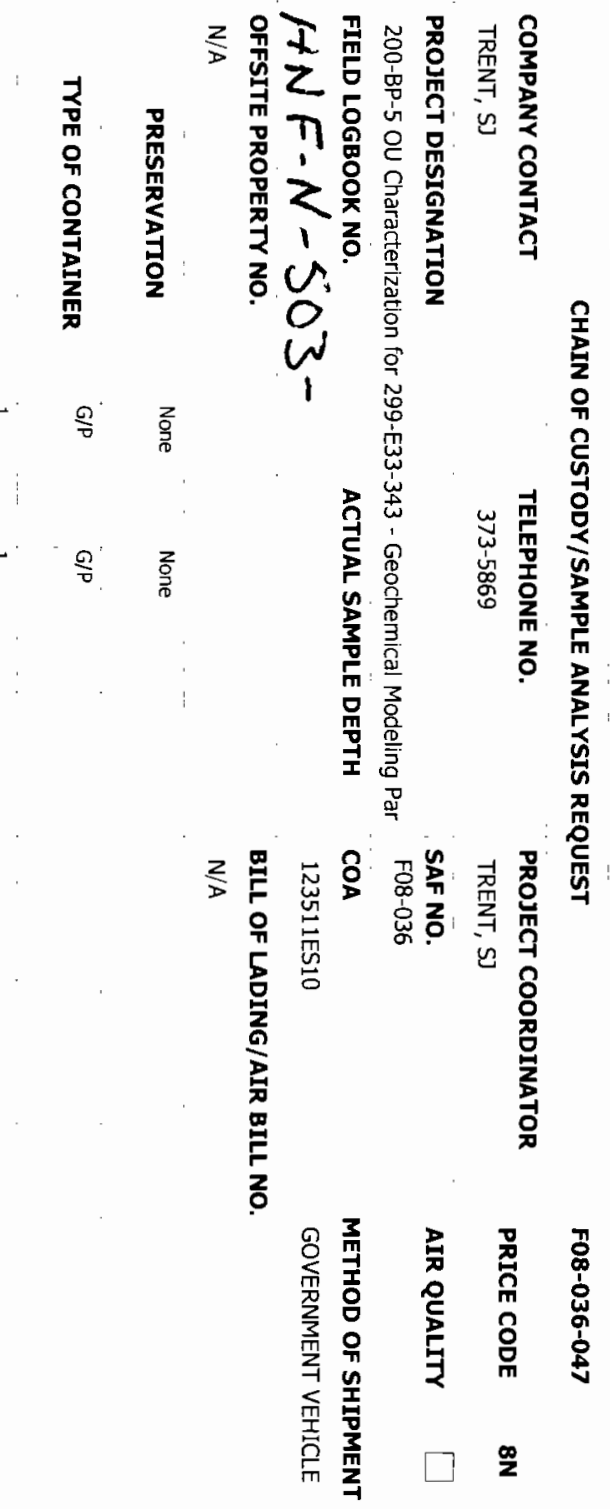




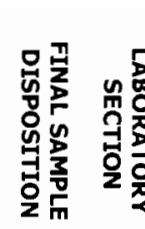

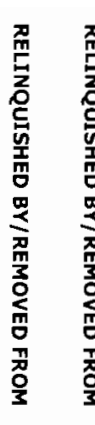

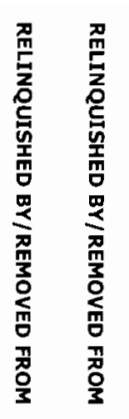

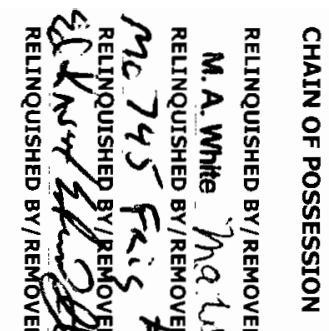

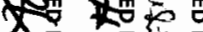

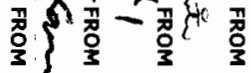

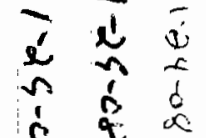

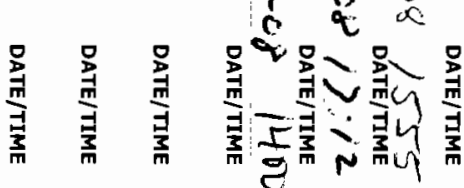

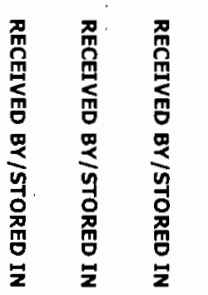
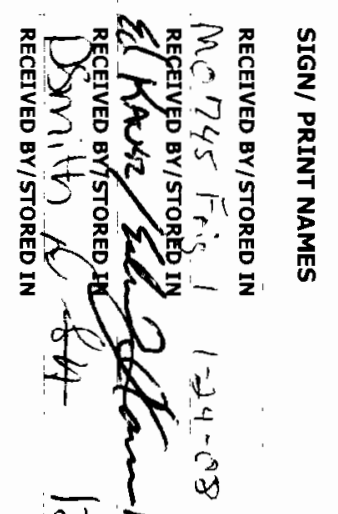

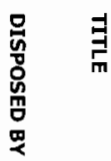

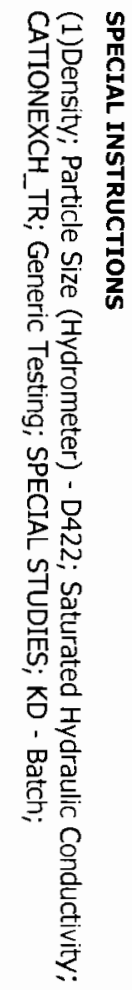

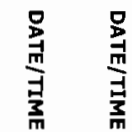

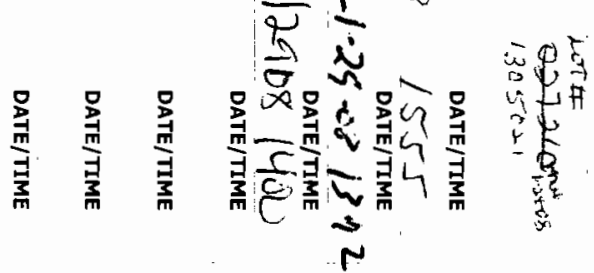

$\underset{\vec{G}}{\vec{\exists}}$

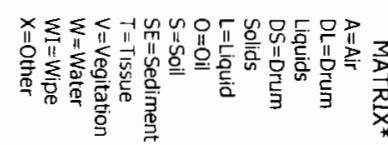

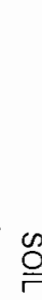

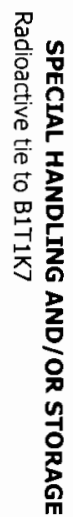

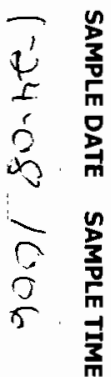

盖酸

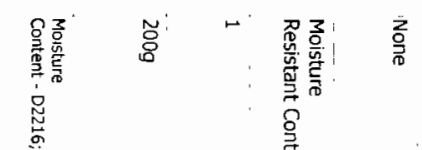

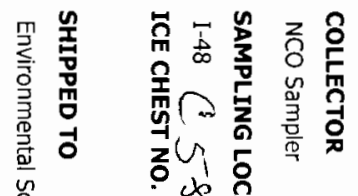
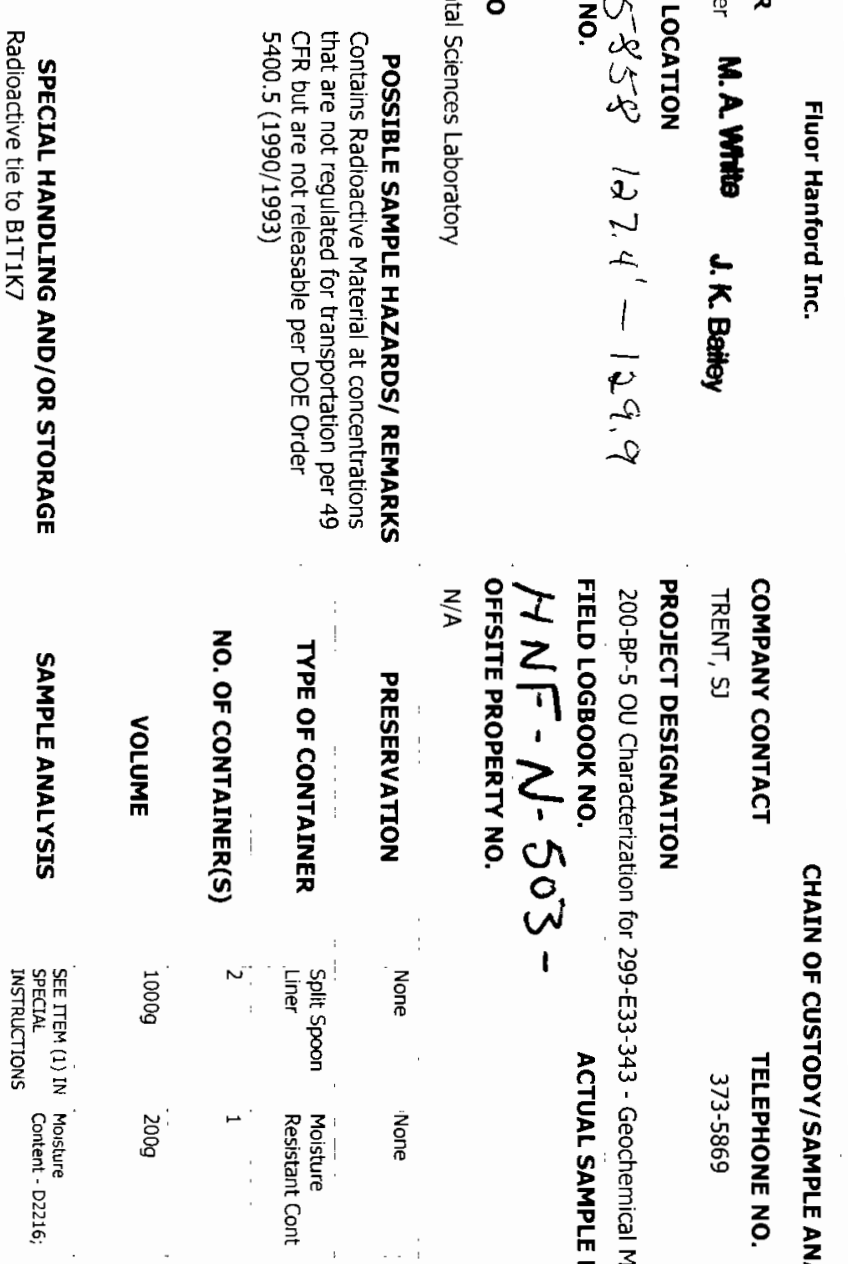

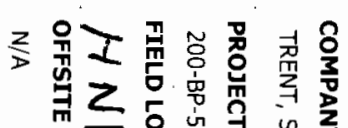

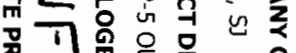

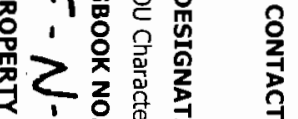
उc 它 1 ํํㅇ

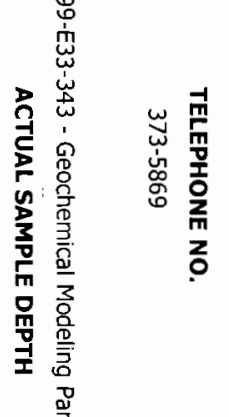

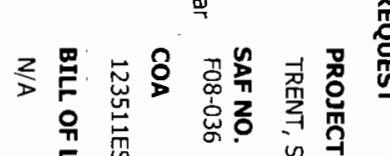

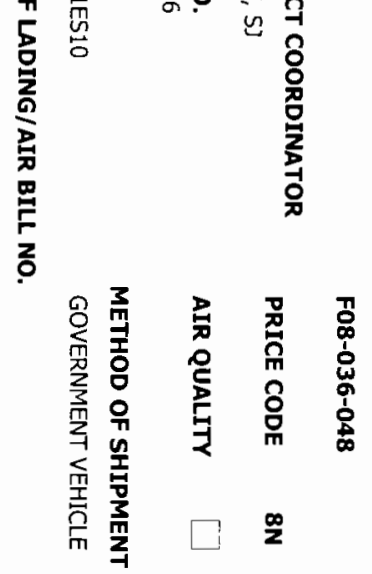
울

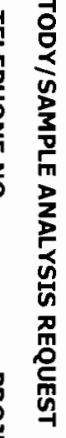

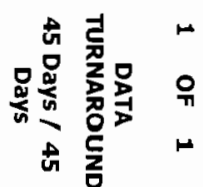



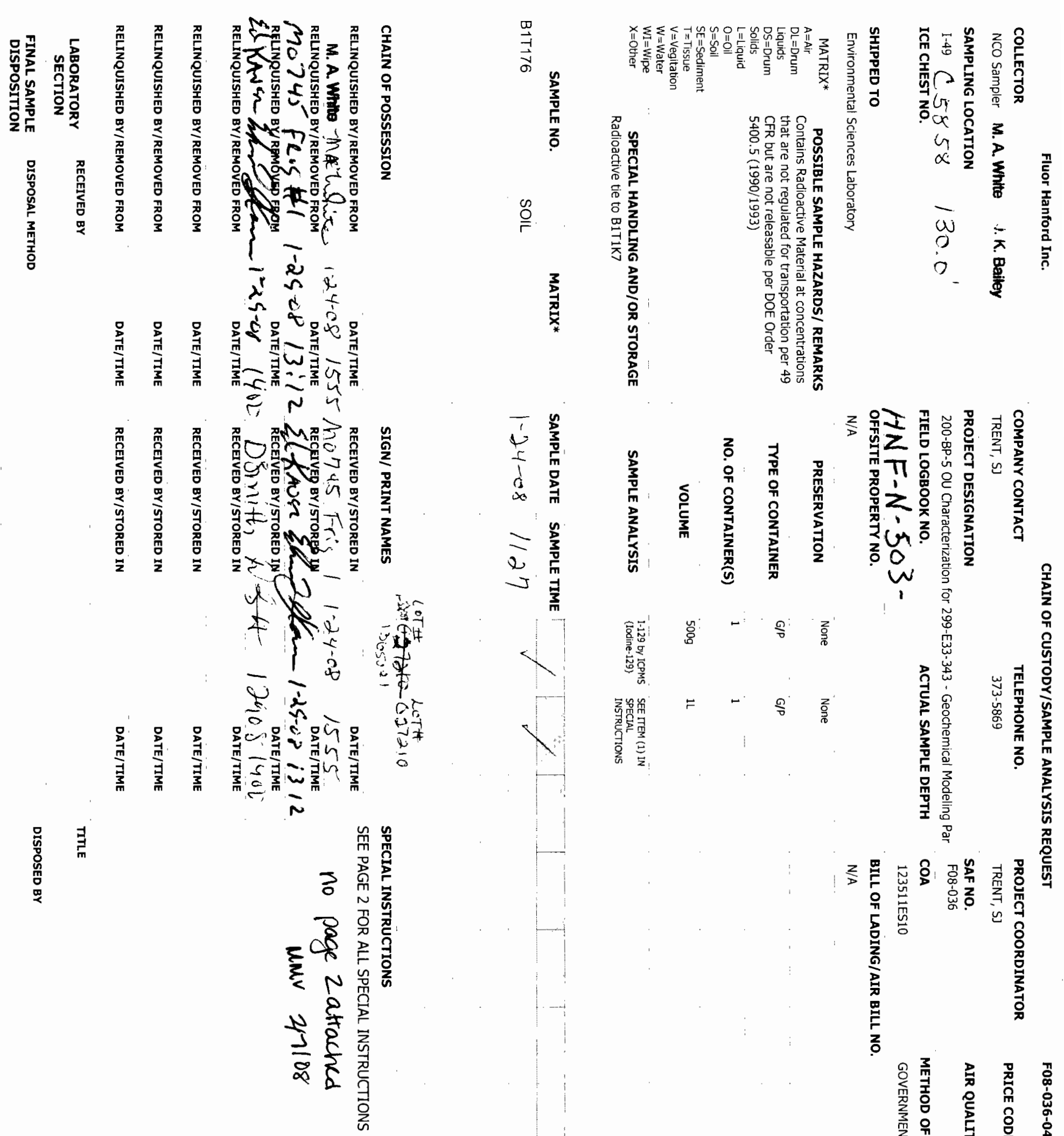

量薏 


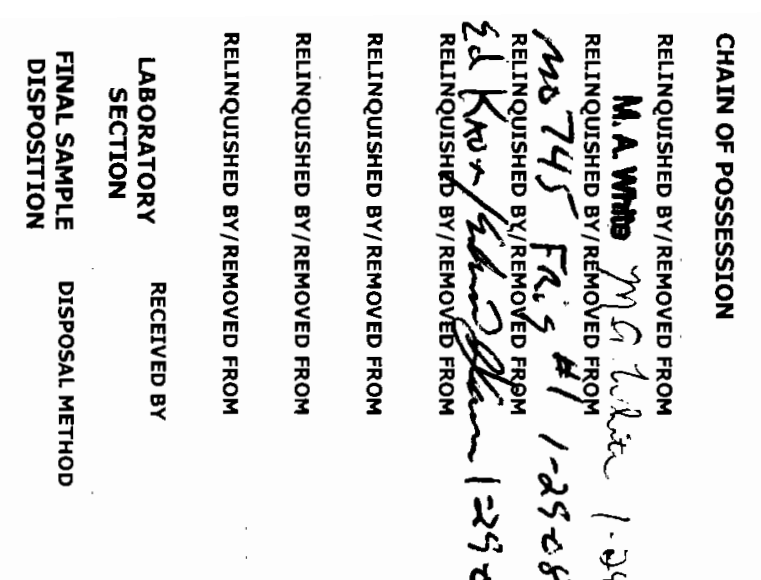

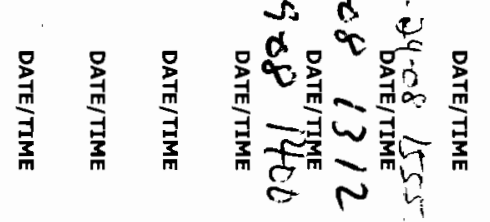

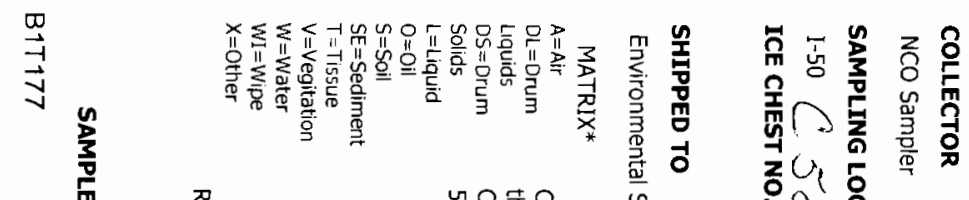
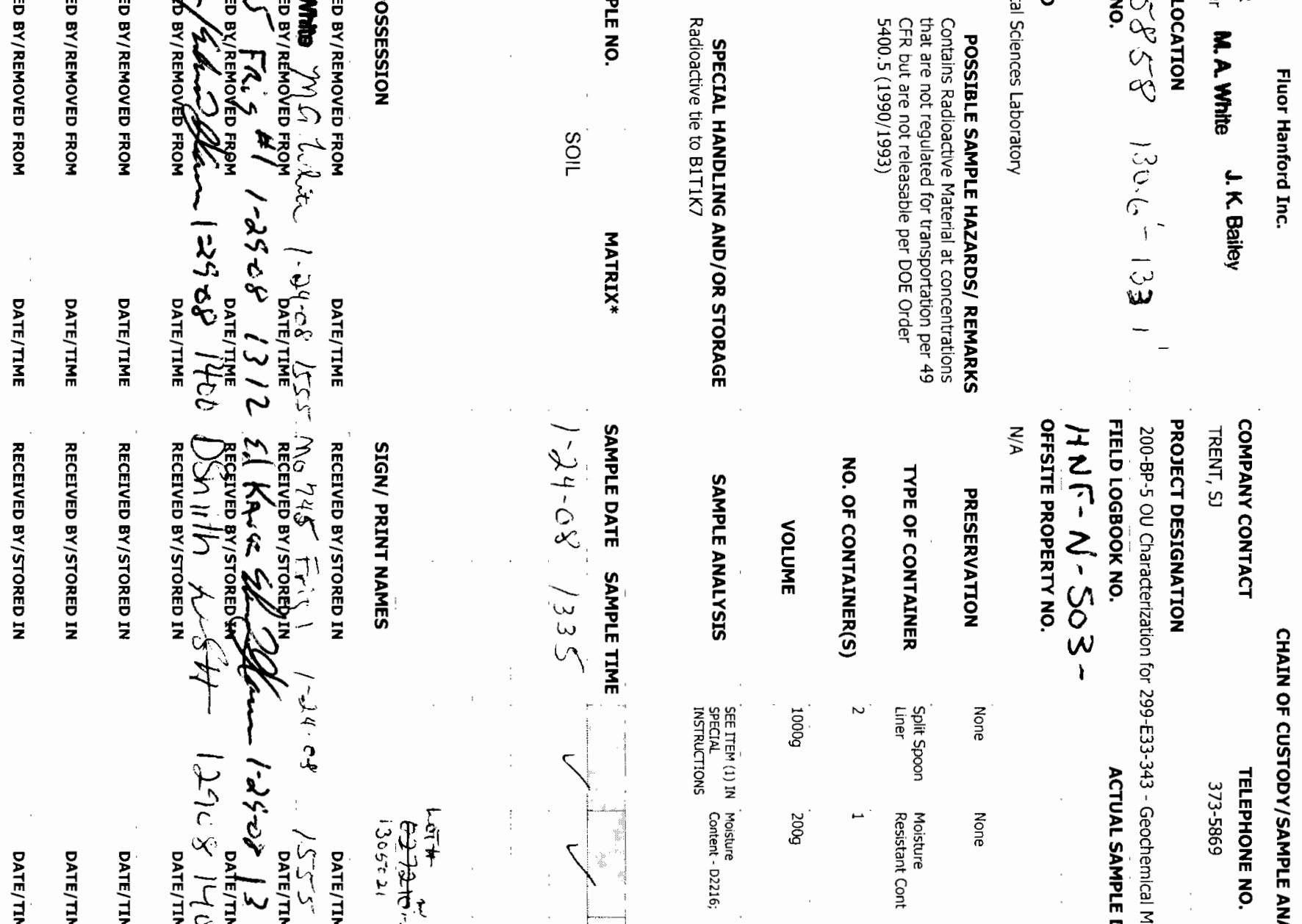

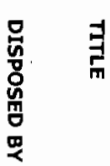
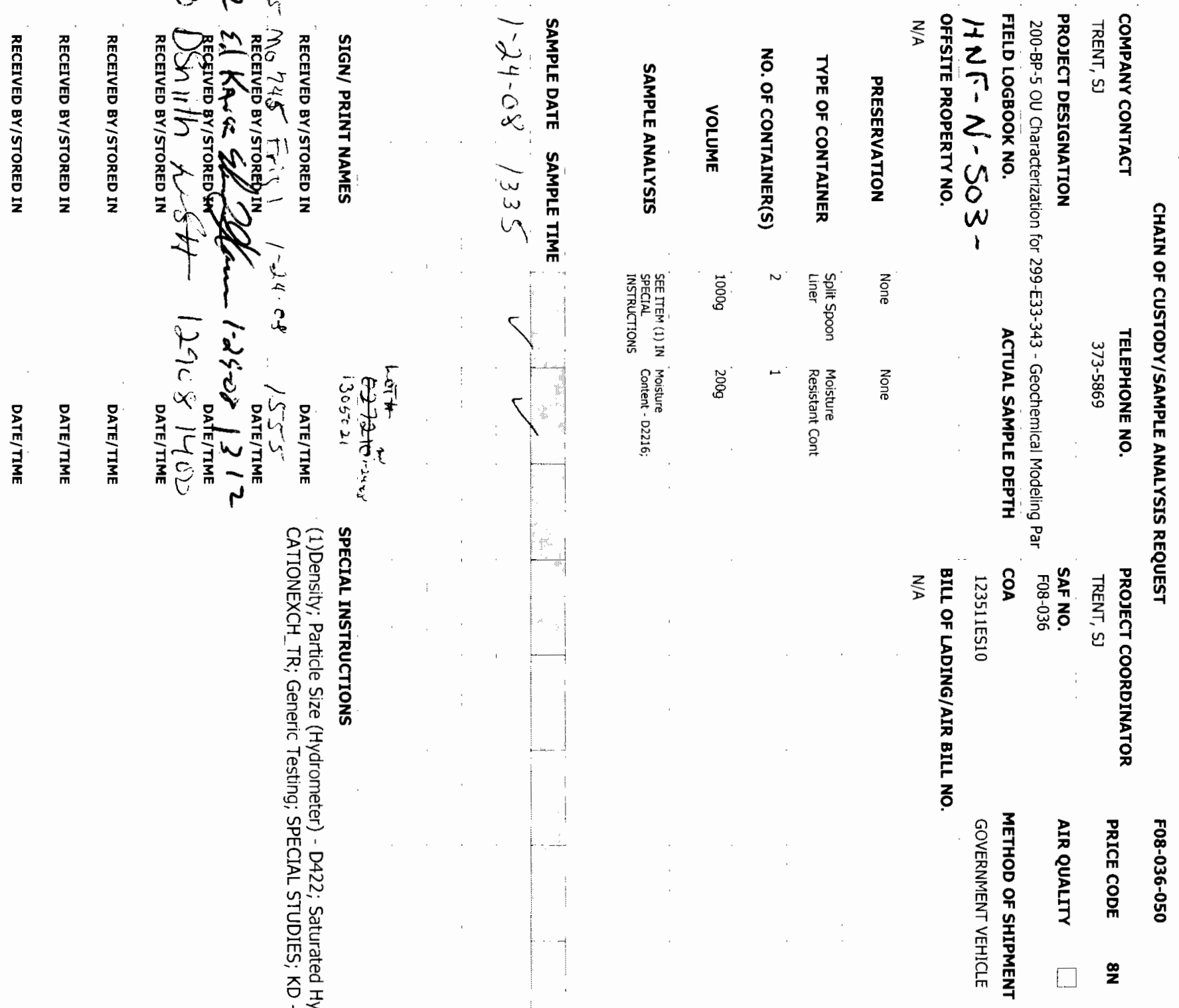

量

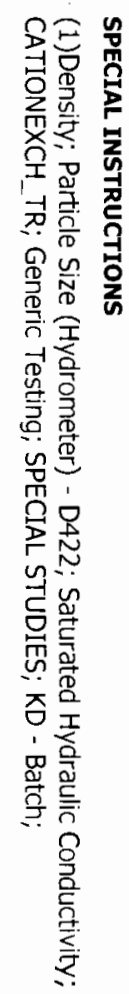




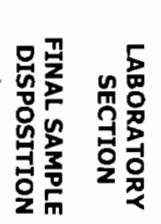

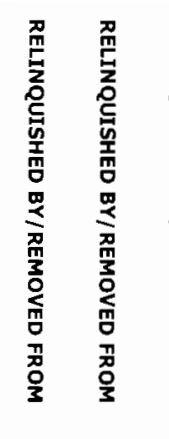

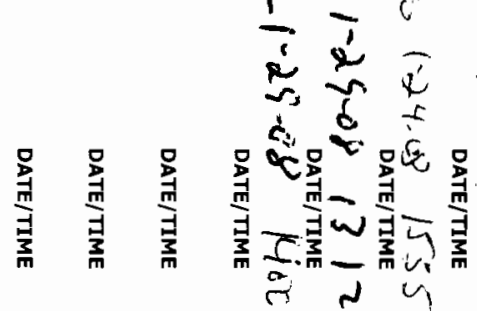

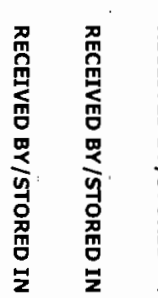

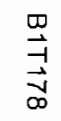

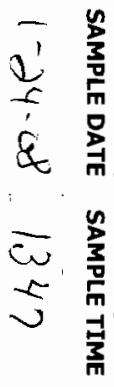

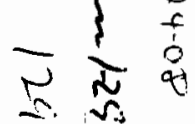

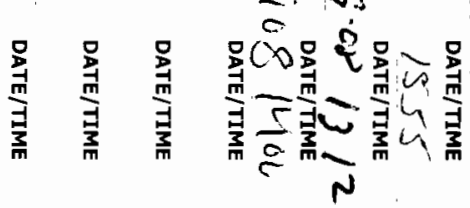
है

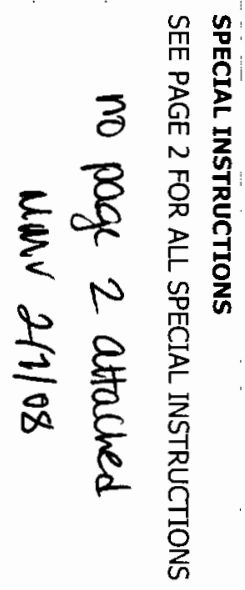

$\stackrel{\circ}{\rightleftharpoons}$

$\underset{*}{\stackrel{3}{x}}$

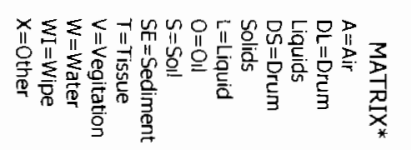

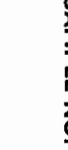

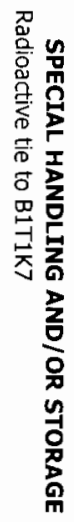

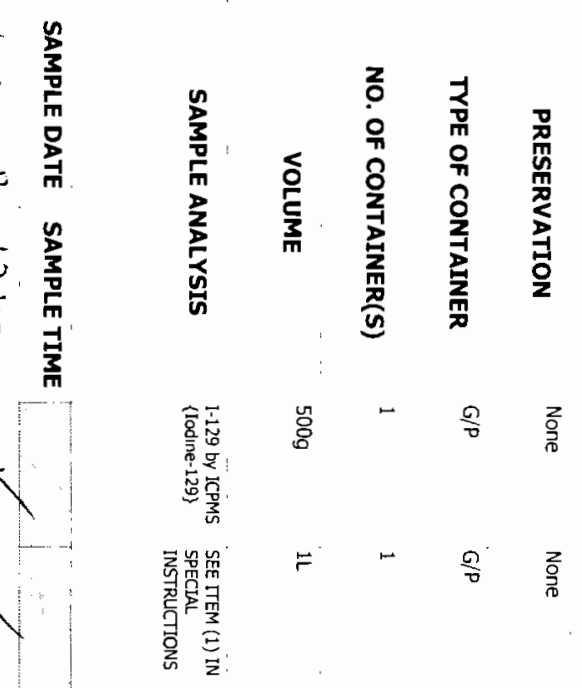

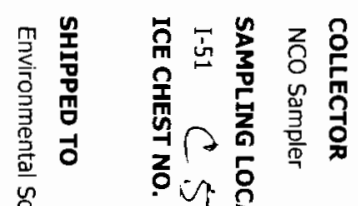

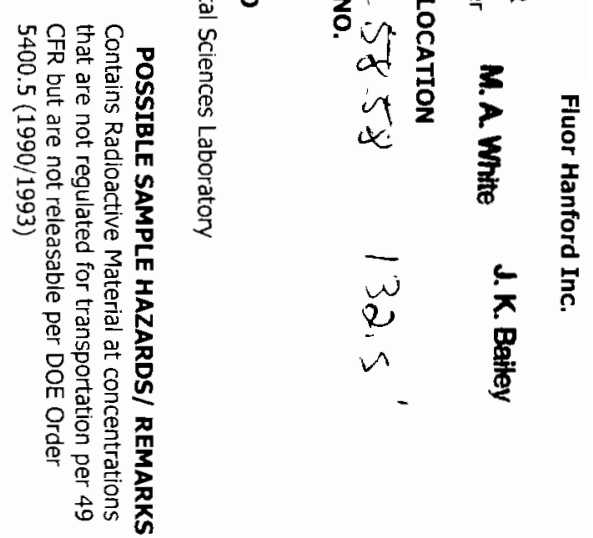

z

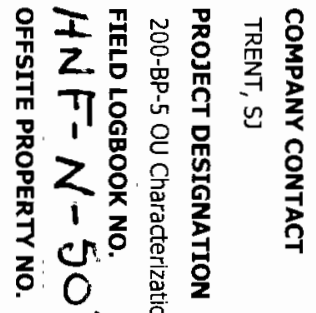

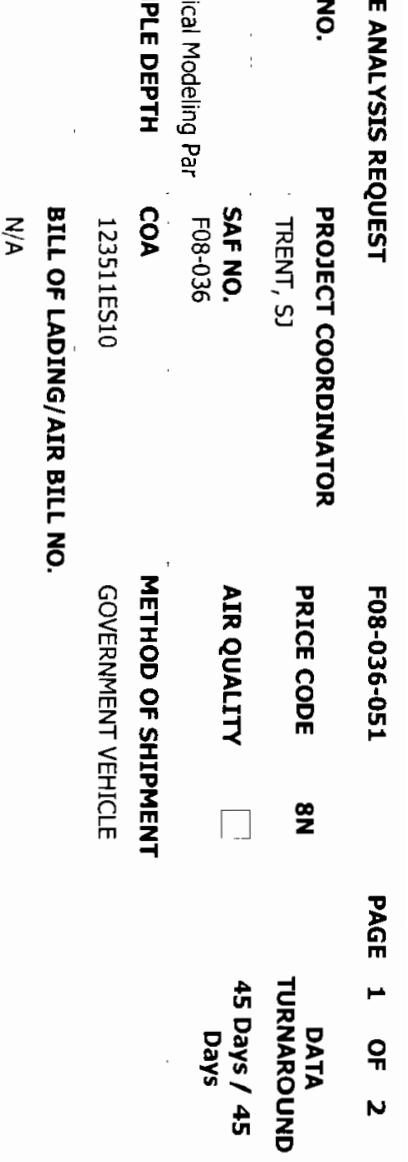




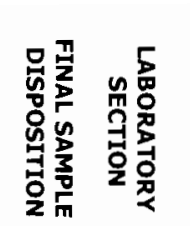

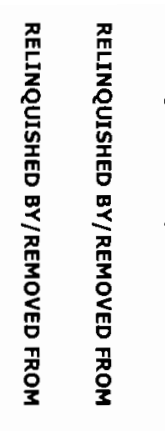

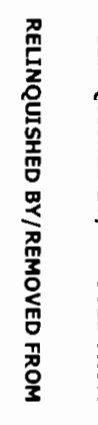

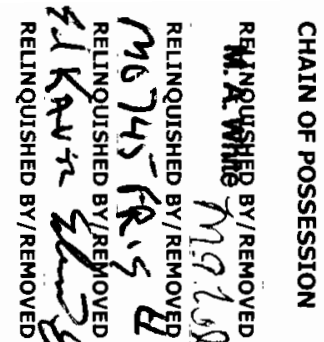

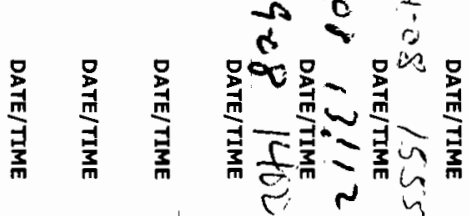

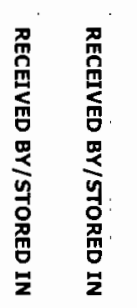

然

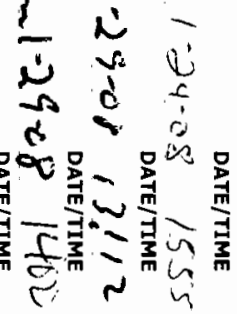

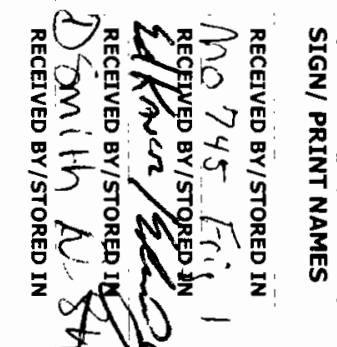

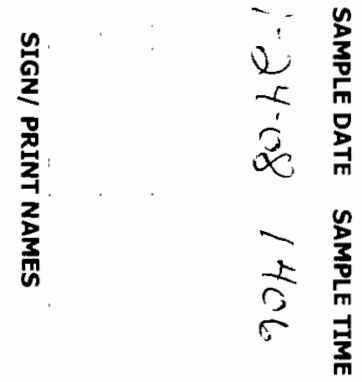

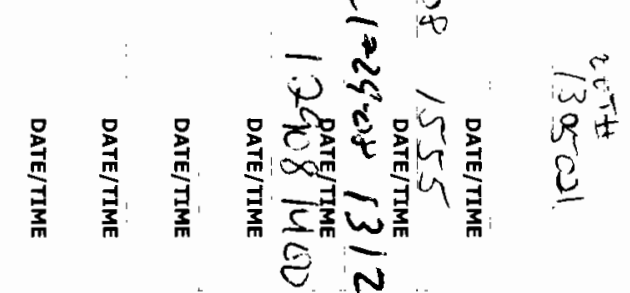
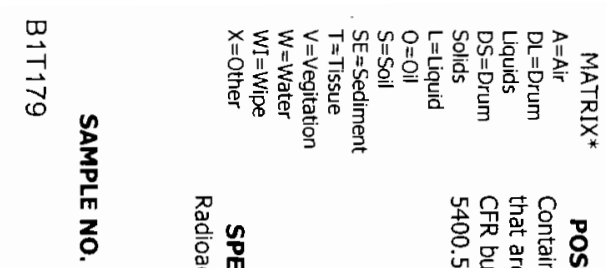

$\stackrel{2}{\circ}$
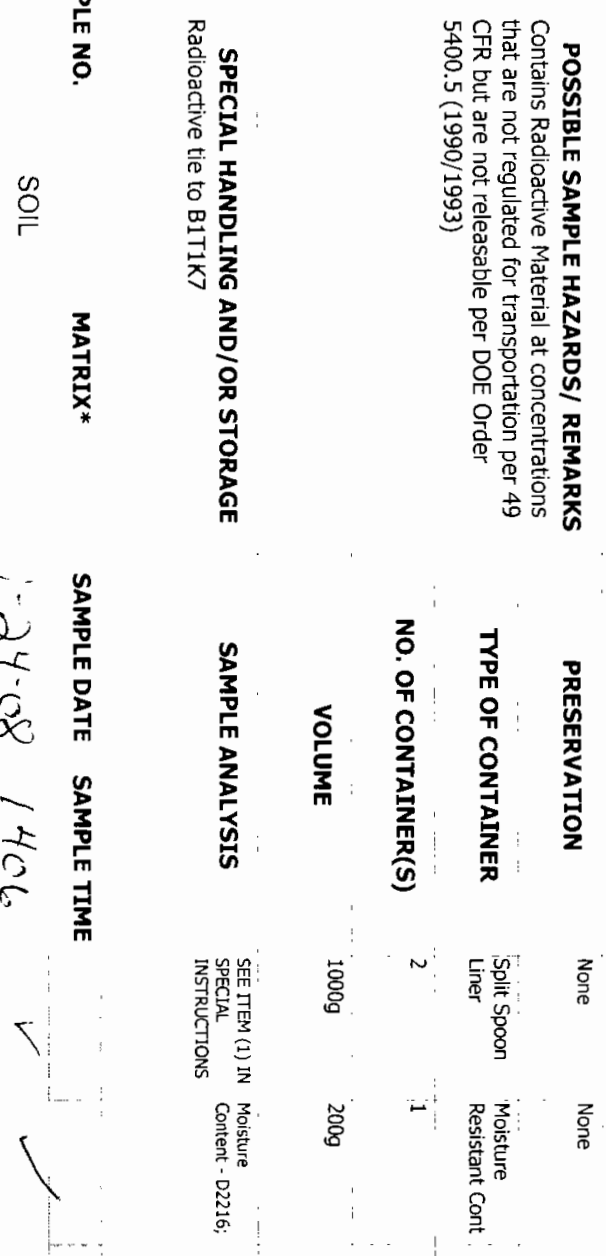

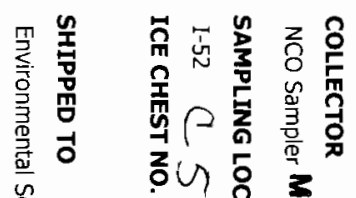
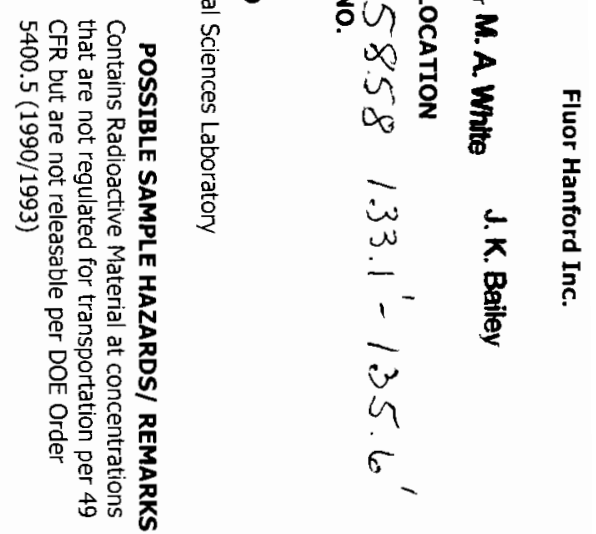

亭

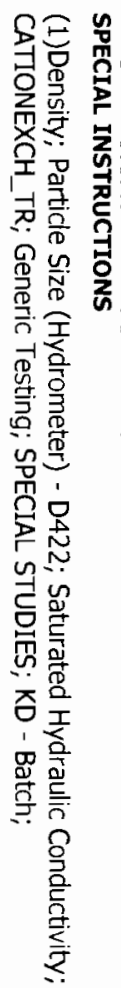

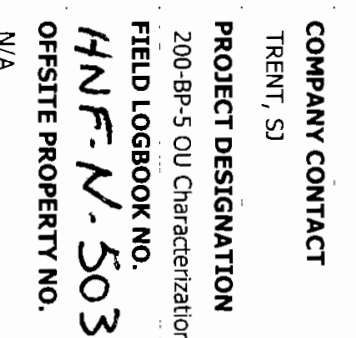

o

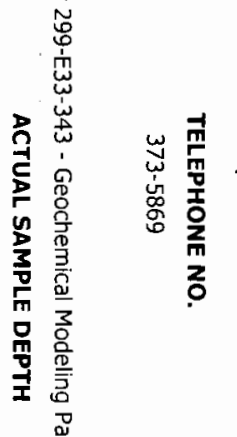

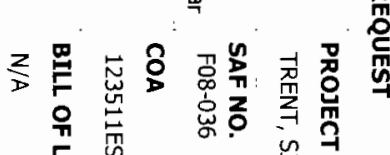

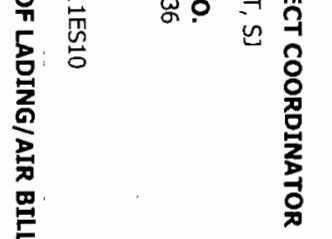

z

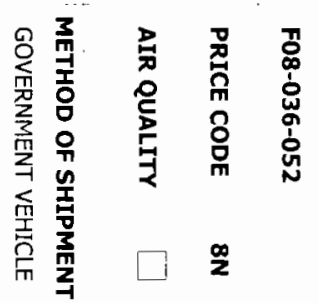

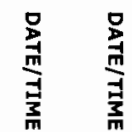




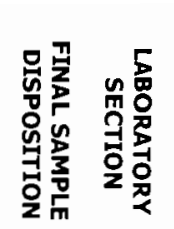
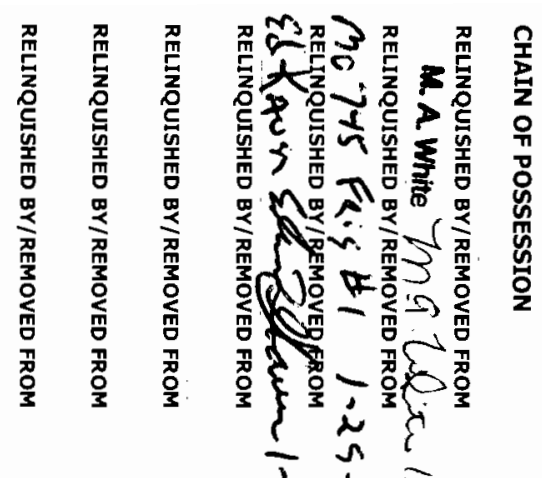

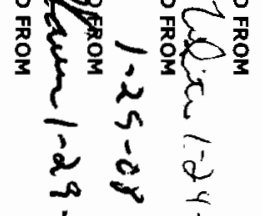

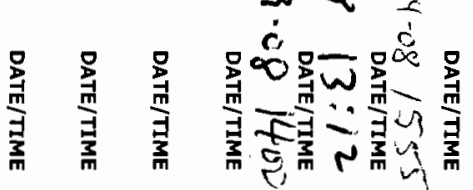
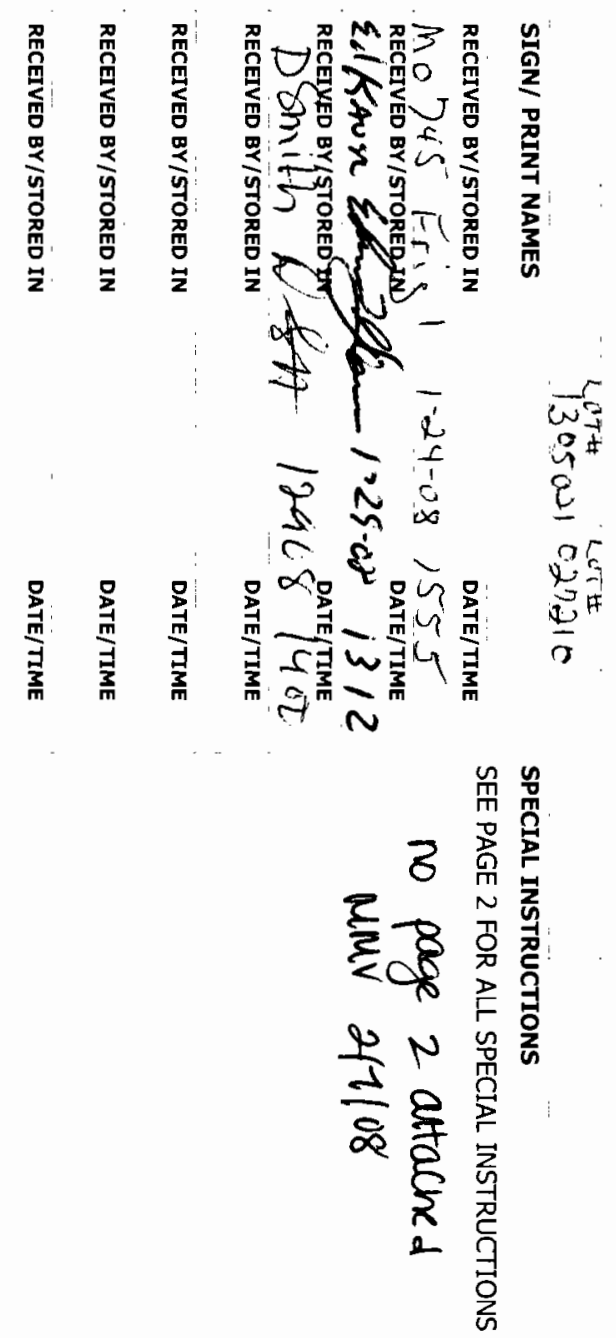

量

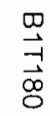

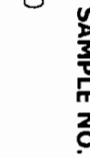

$\stackrel{\infty}{\stackrel{O}{ }}$

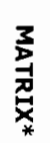

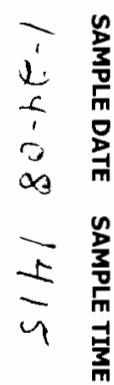

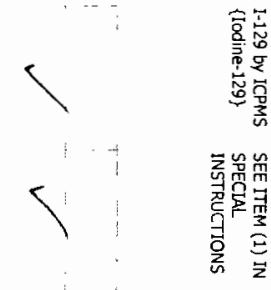

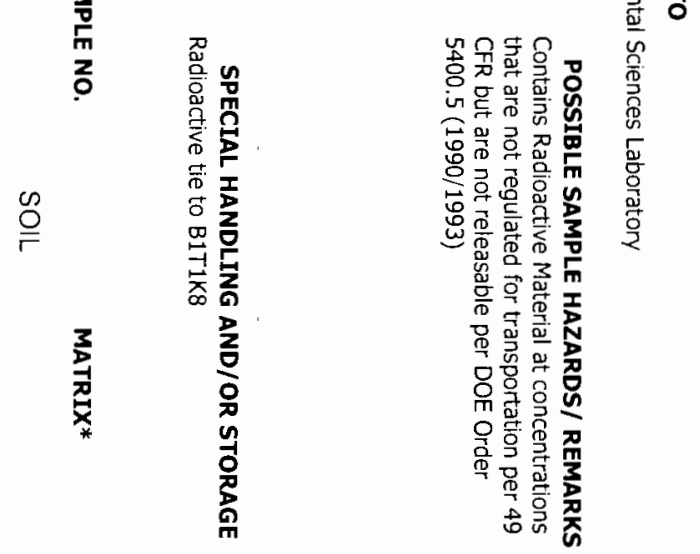

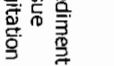

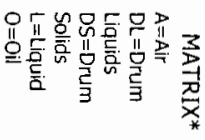

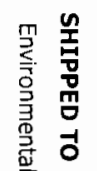

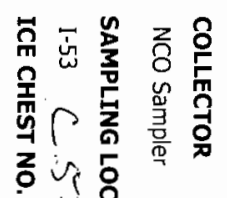

的要

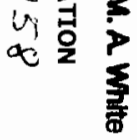

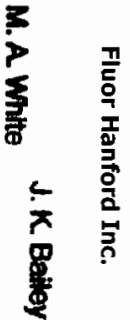

8 要

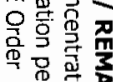

故密
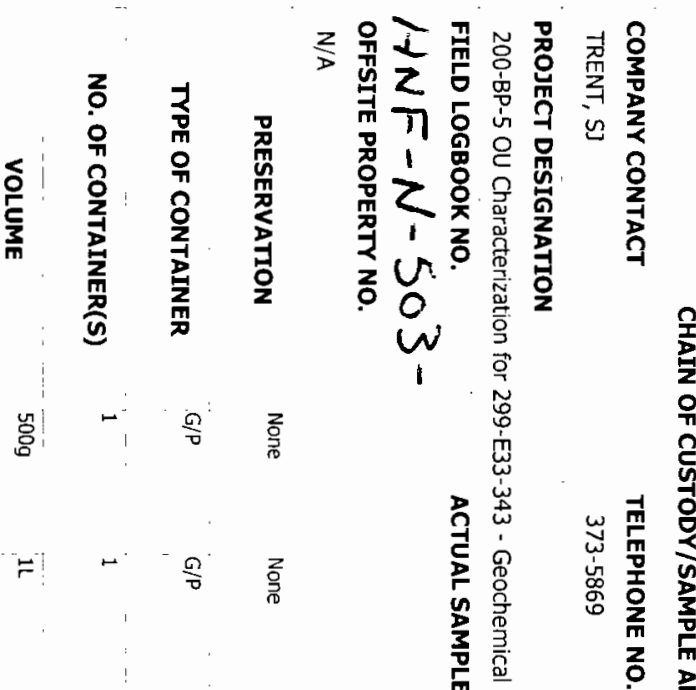

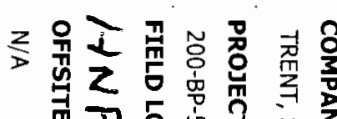

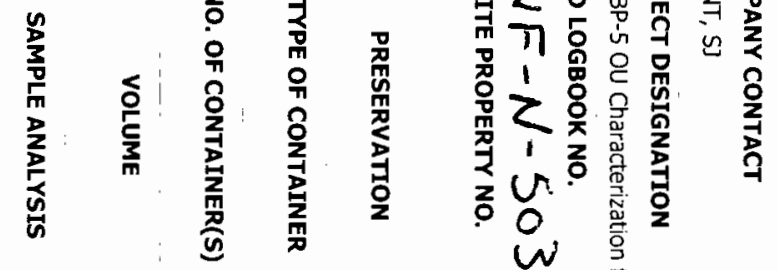

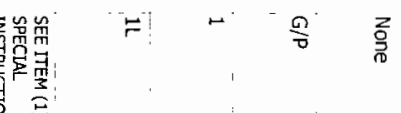
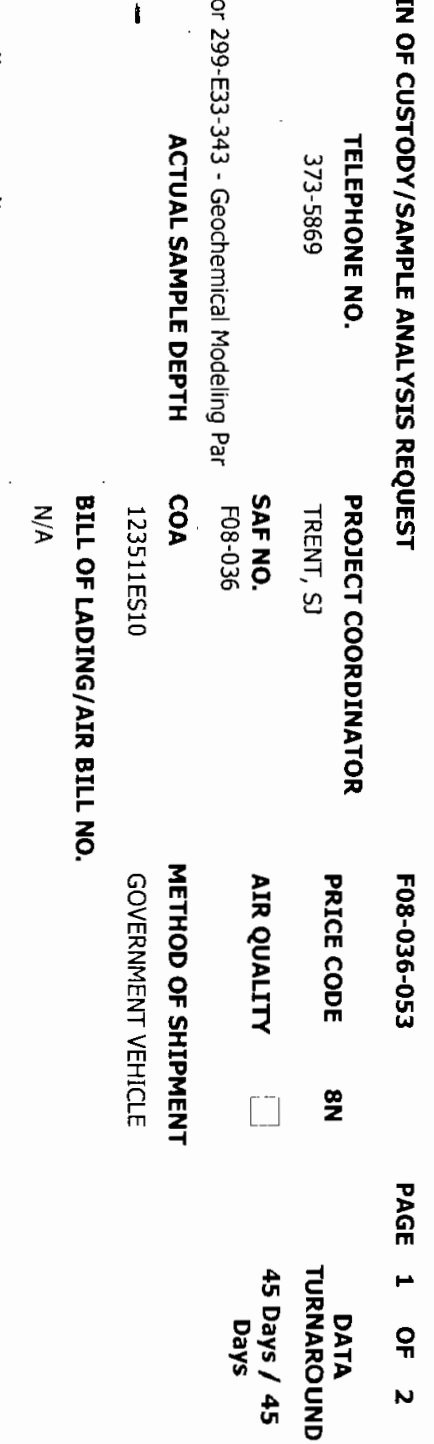


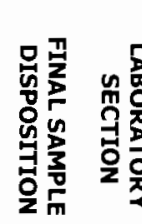
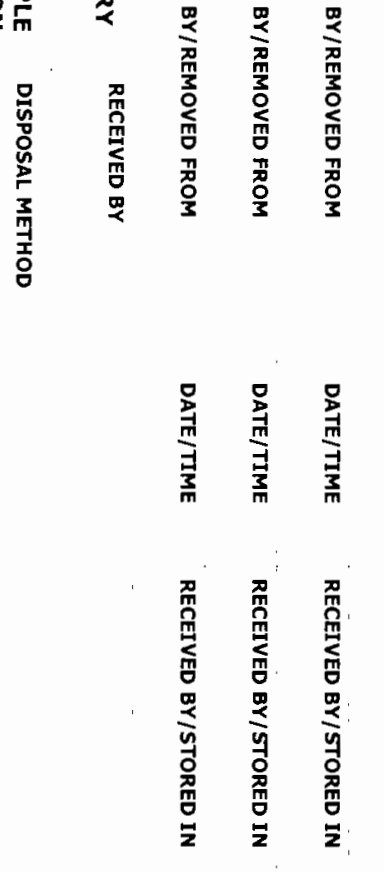

L

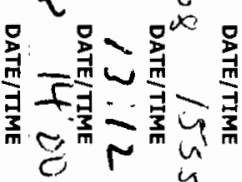

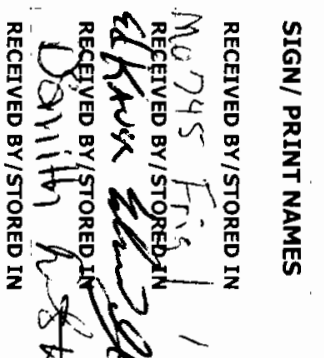

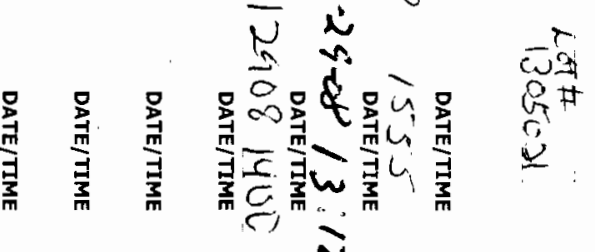

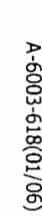

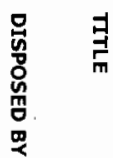

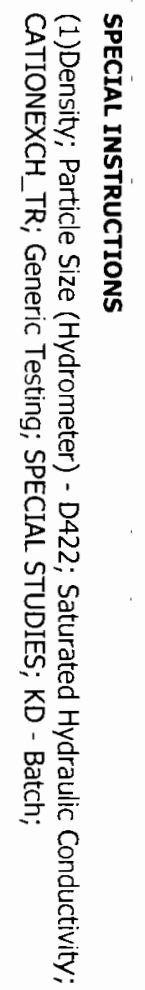

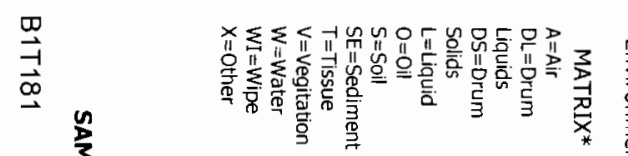

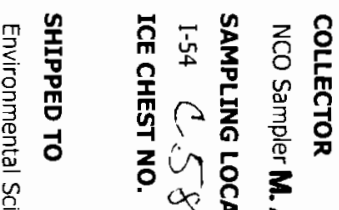

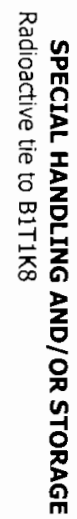

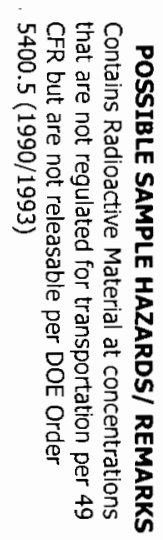

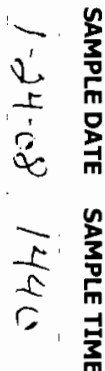

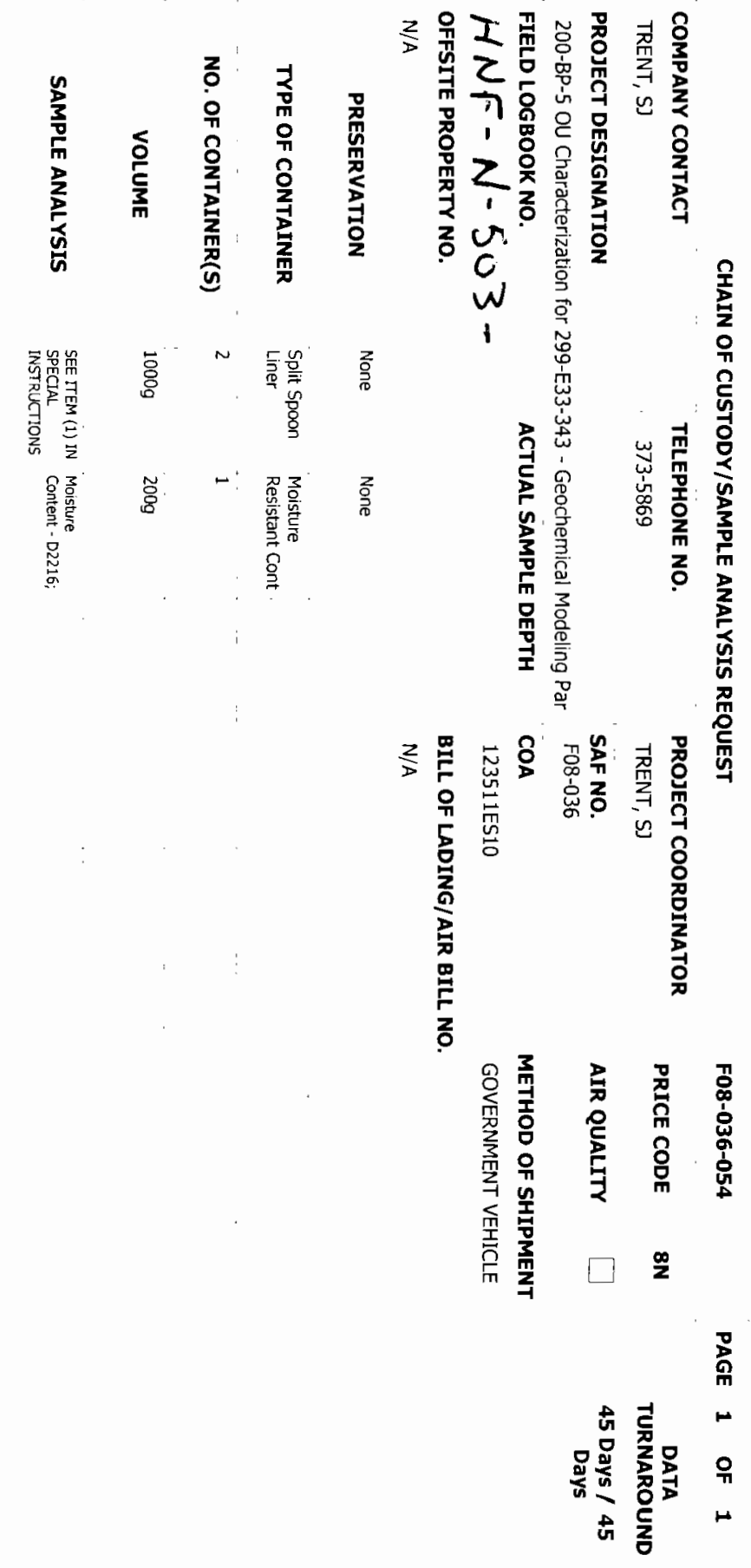




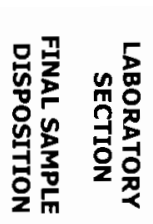

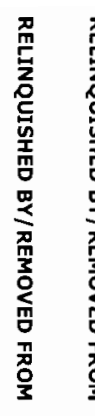

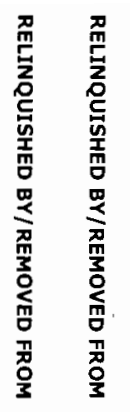

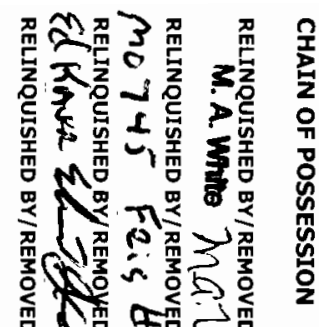

男

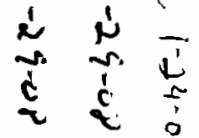

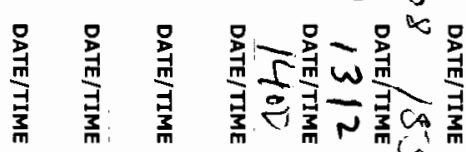

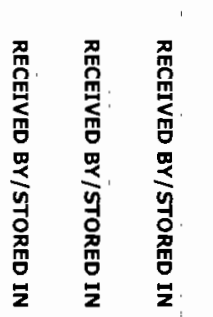
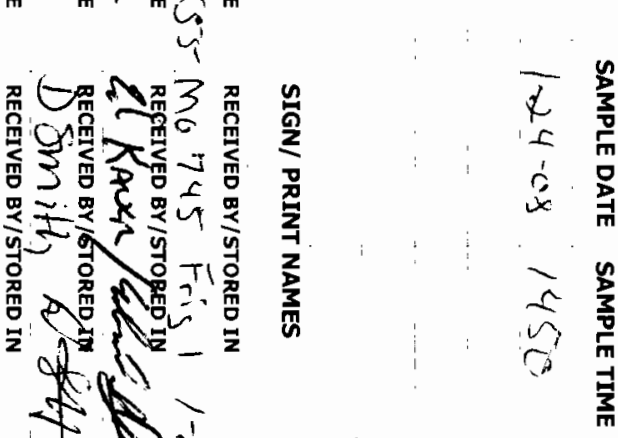

$-15$

\& $\infty$ क

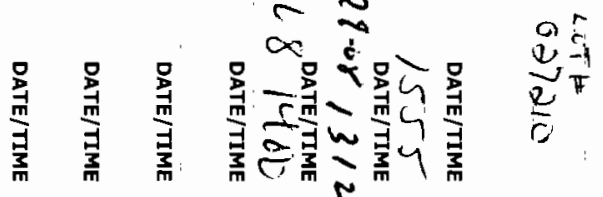

总

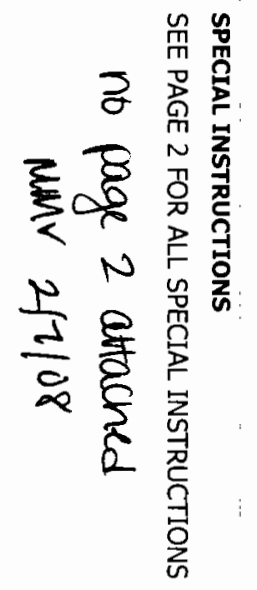

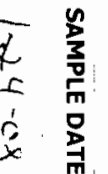

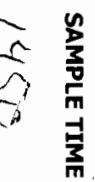

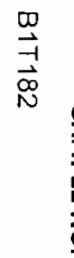

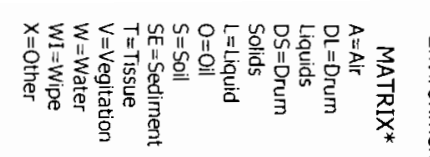

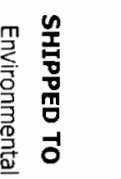

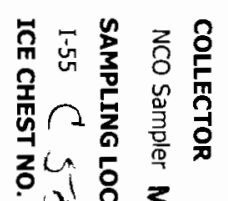

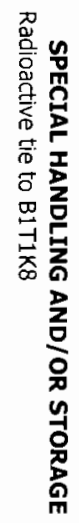

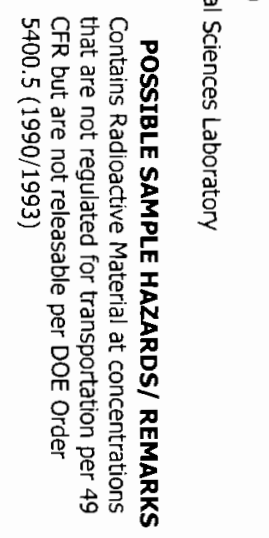

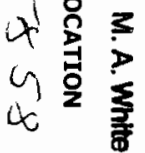

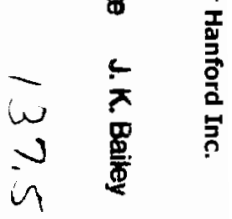

$>$

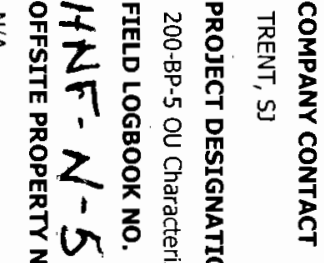
zu w
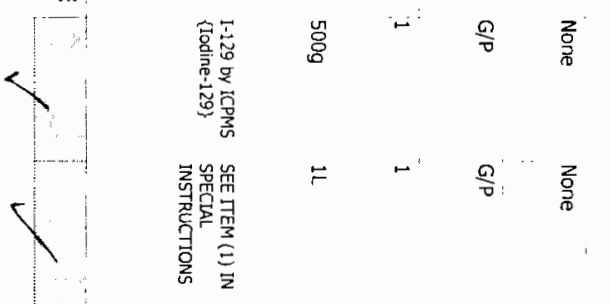


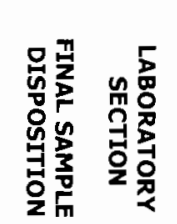
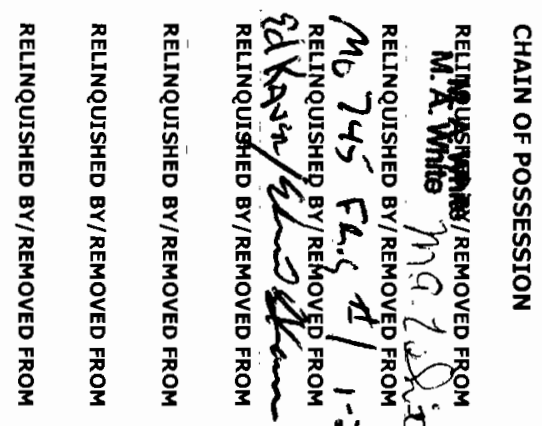

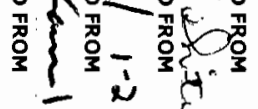
ì
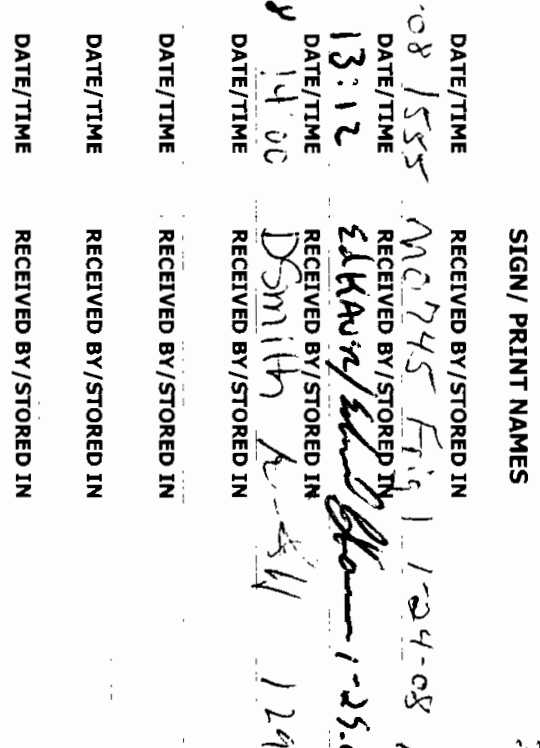

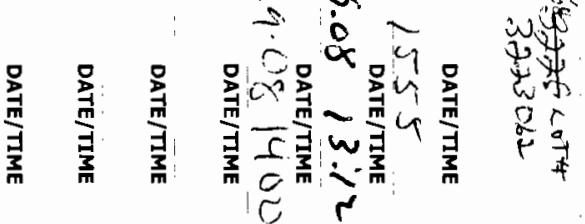
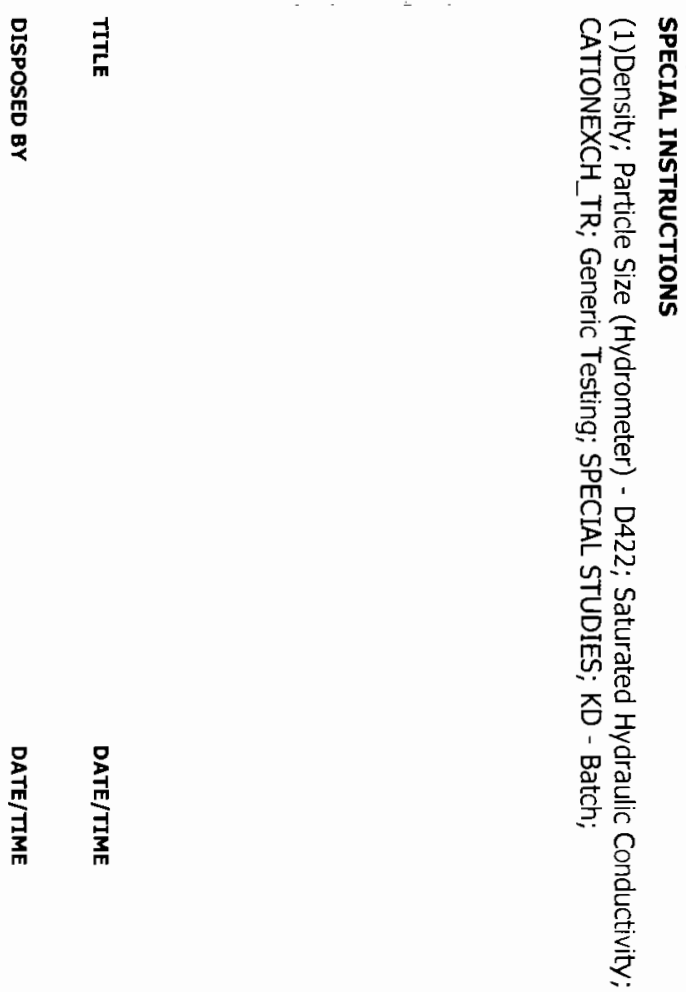

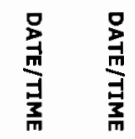
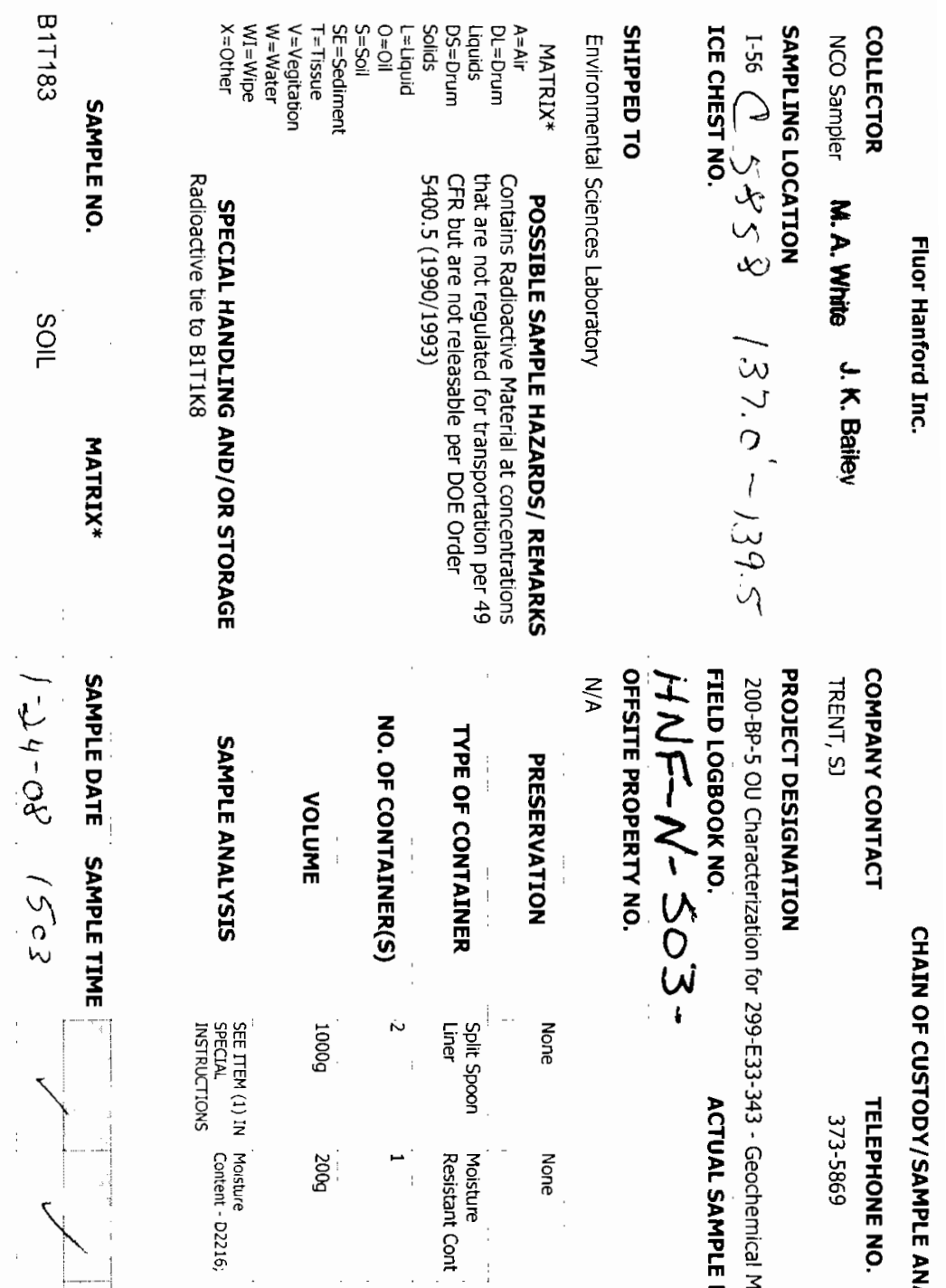

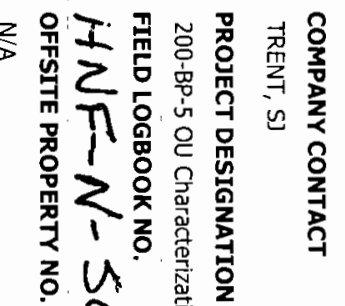

$\begin{array}{ll}0 & { }^{2} \\ \omega & \stackrel{9}{\circ}\end{array}$

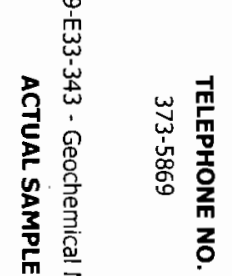

学墨点
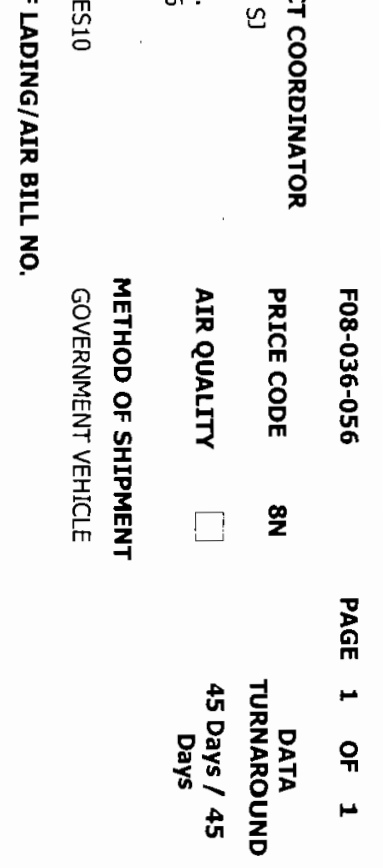


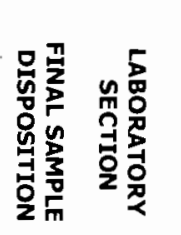

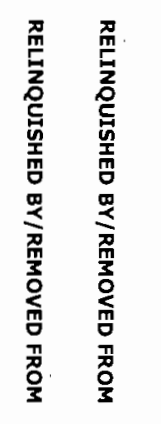

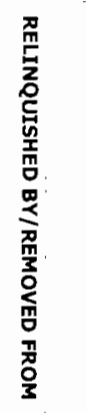

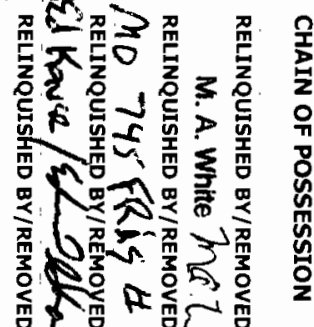

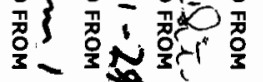

$\stackrel{i}{\dot{0}} \bar{k}$

$\dot{\alpha}=\frac{1}{b}$

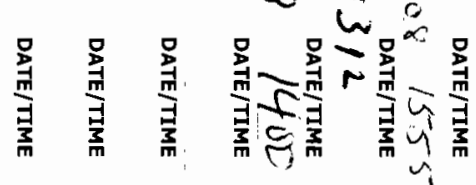

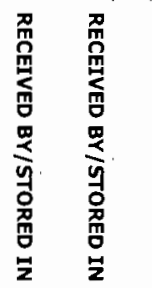

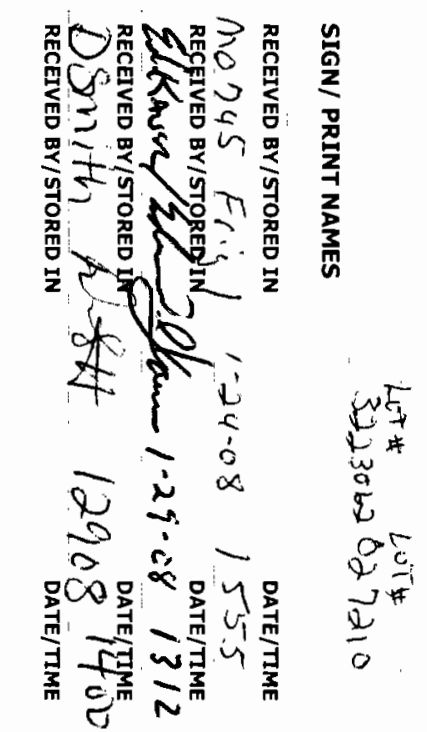

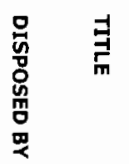

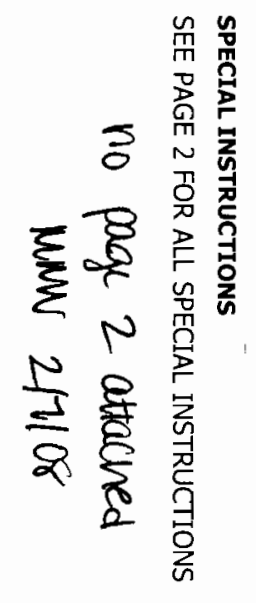

疍是

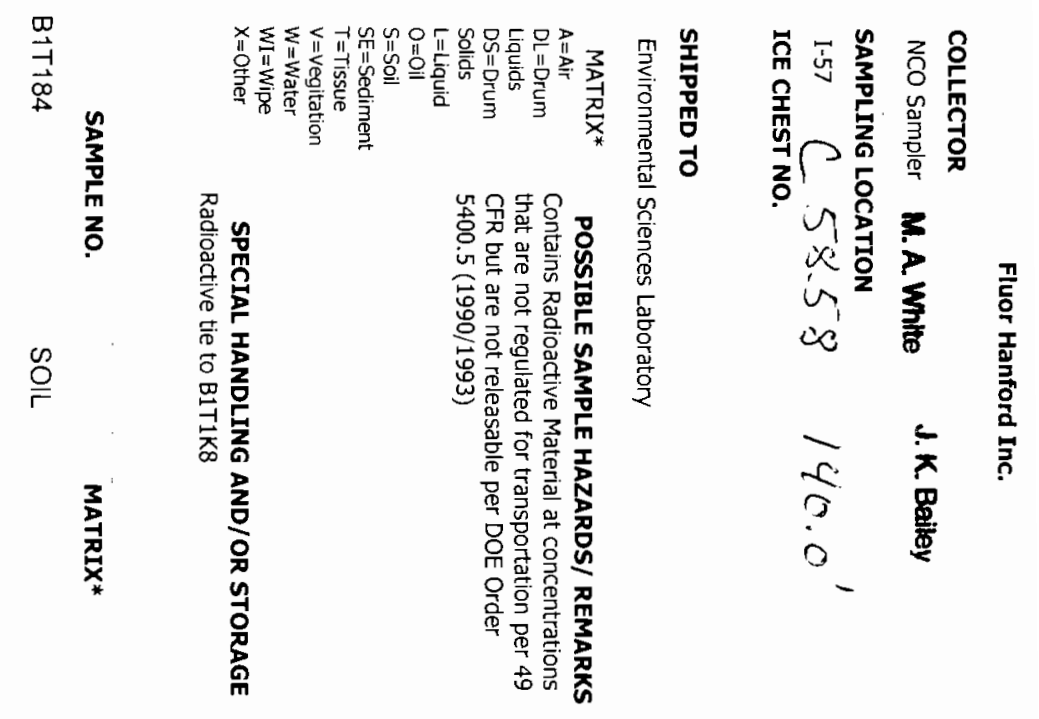

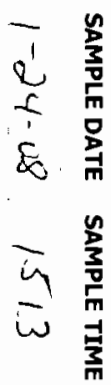

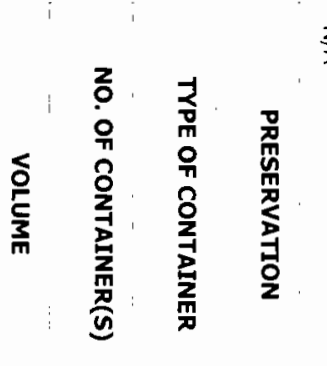

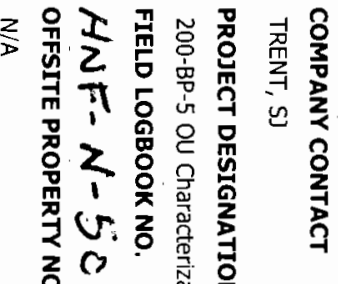

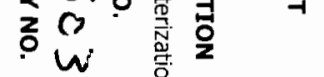

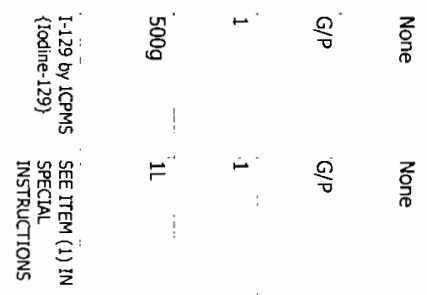

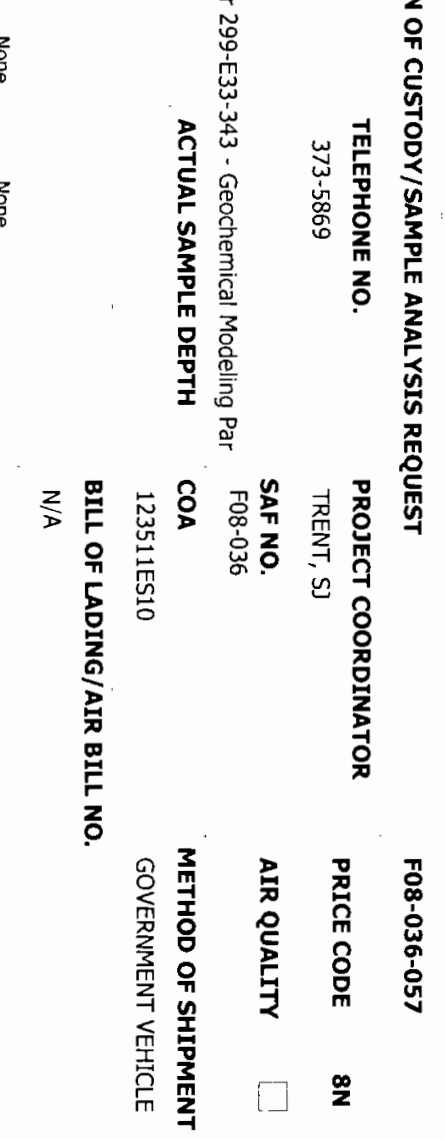




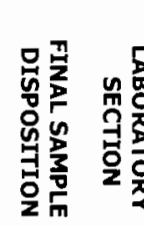

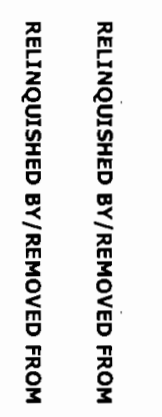

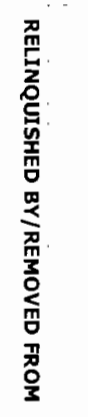

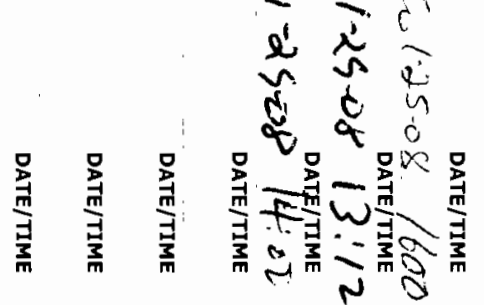

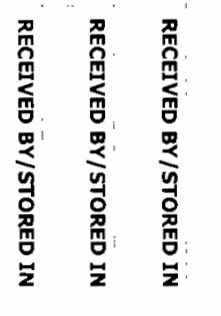

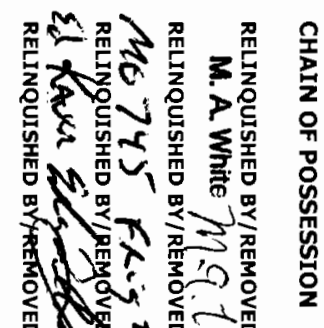

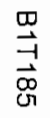

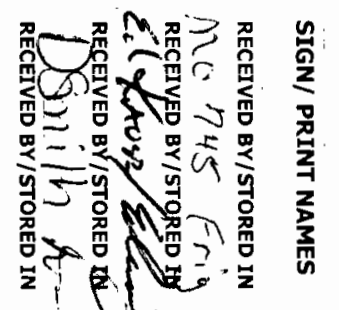

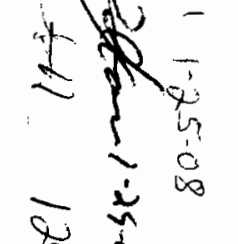

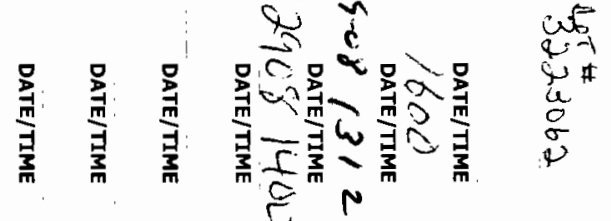

$\begin{array}{ll}\text { 品 } & \exists \\ \text { 声 } \\ \text { 品 } \\ \text { 品 }\end{array}$

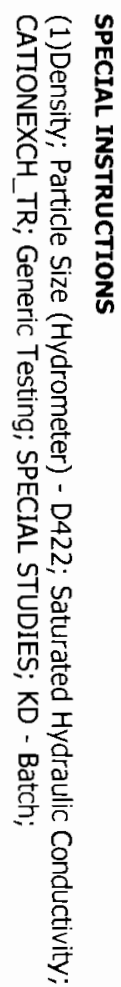

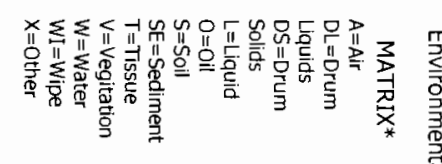

商

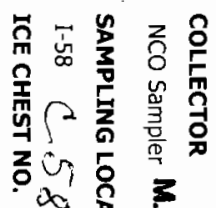

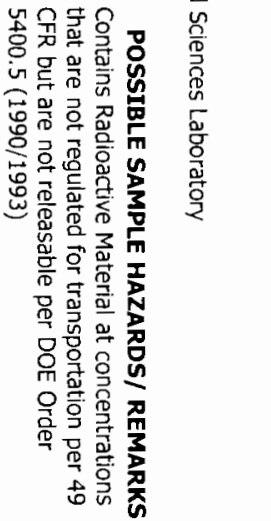

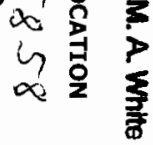

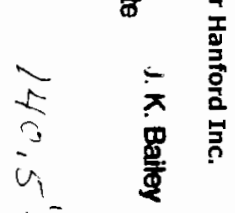

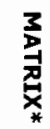

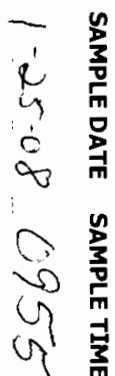

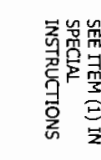

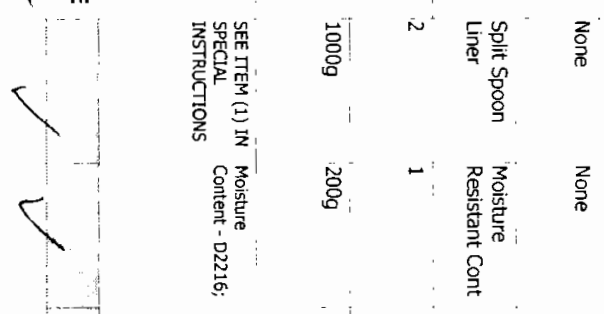

z

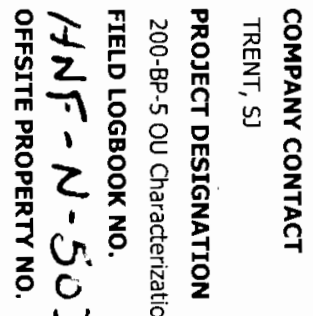

$\frac{1}{0}$
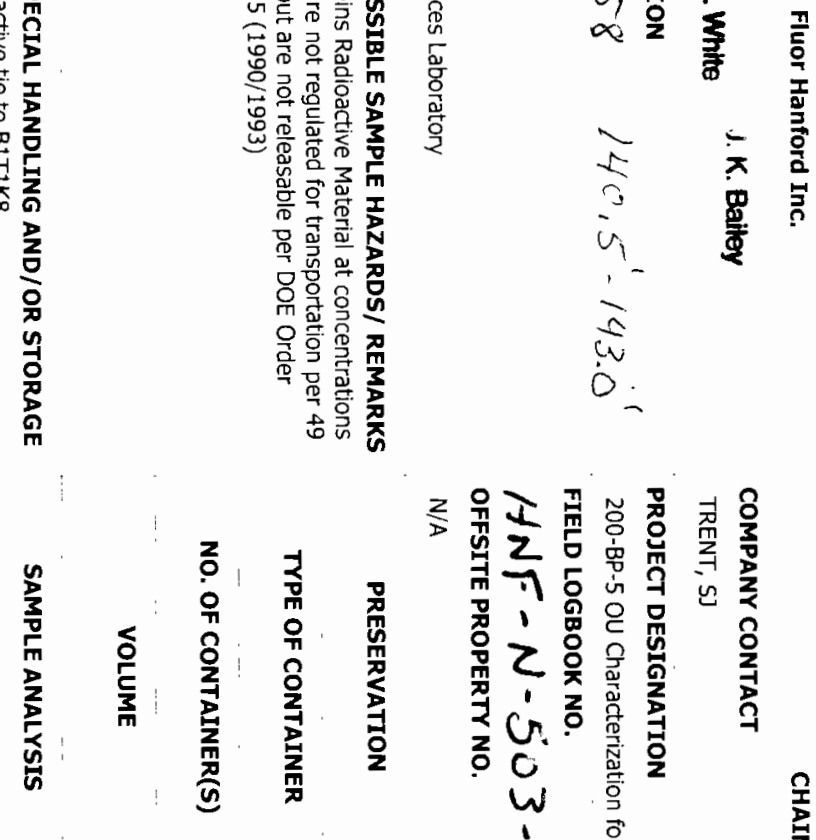

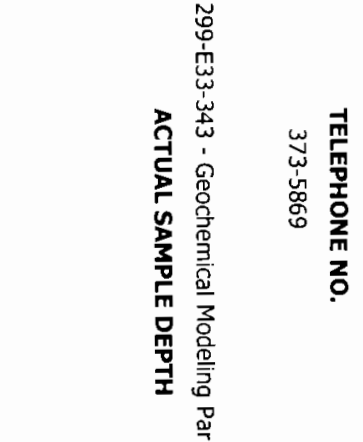

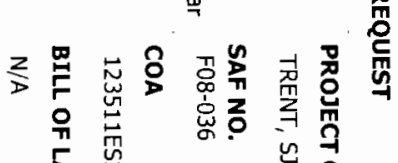

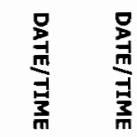



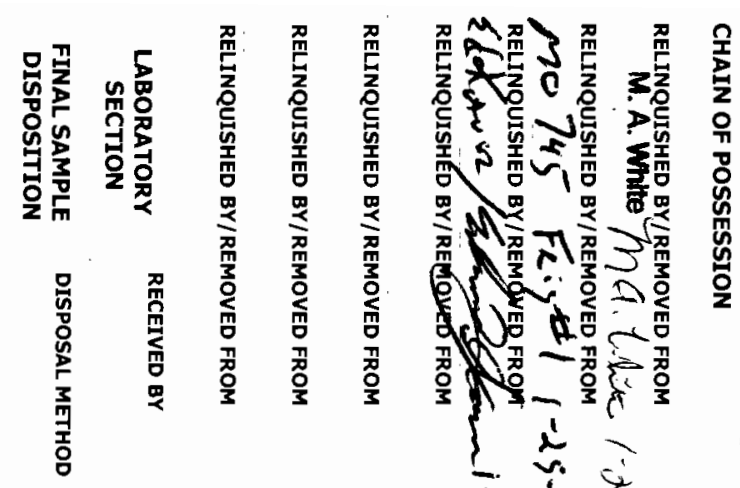

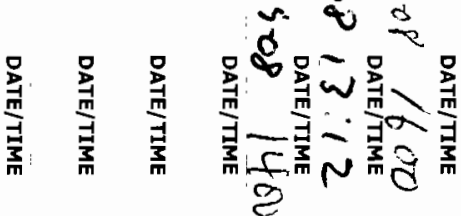
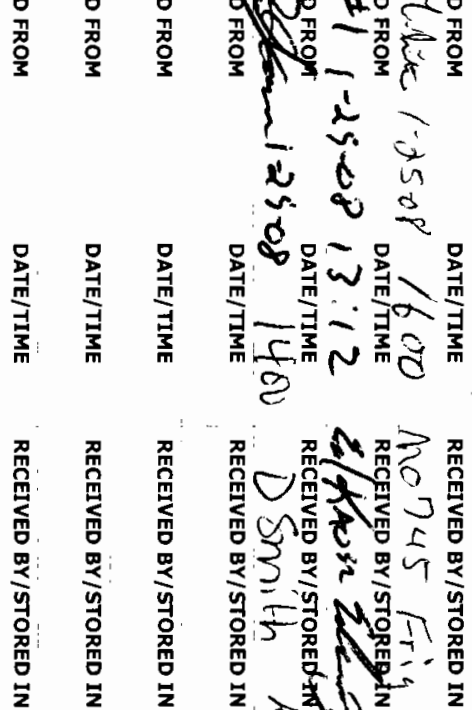

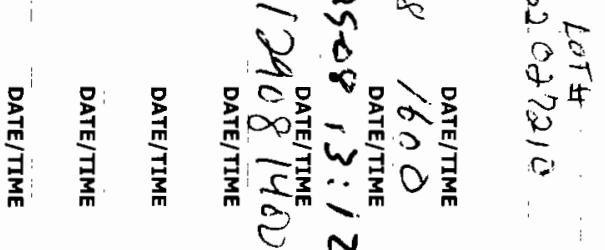
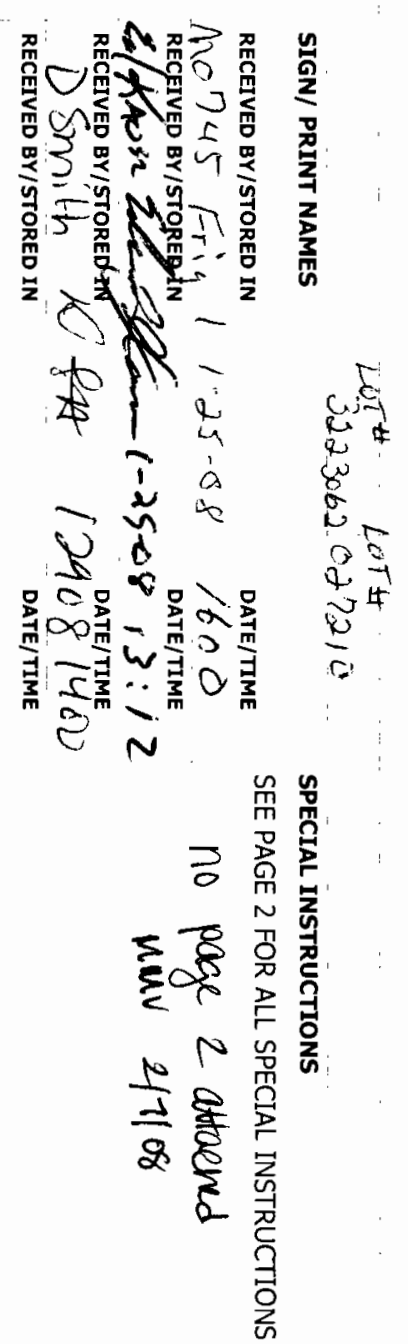

$\stackrel{\mathbb{0}}{\vec{\exists}}$

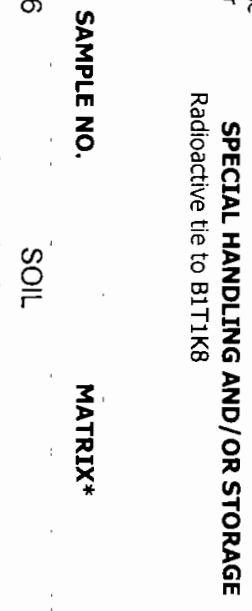

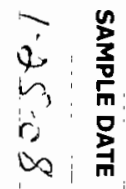

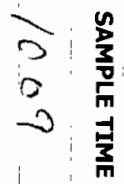

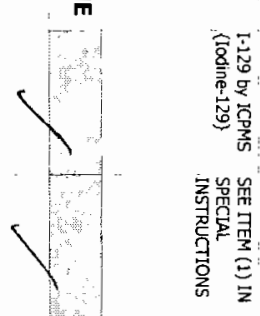

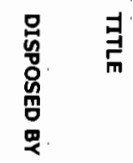

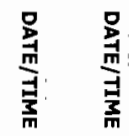

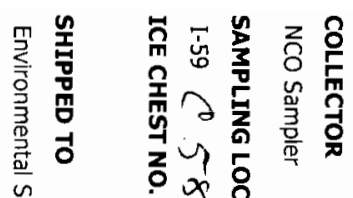

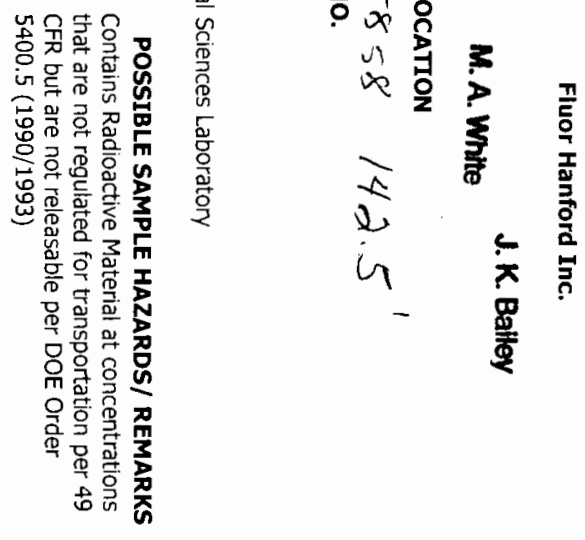

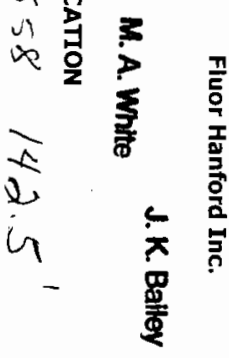

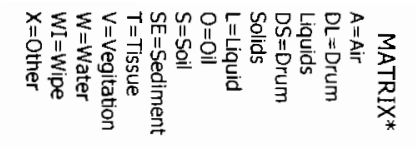
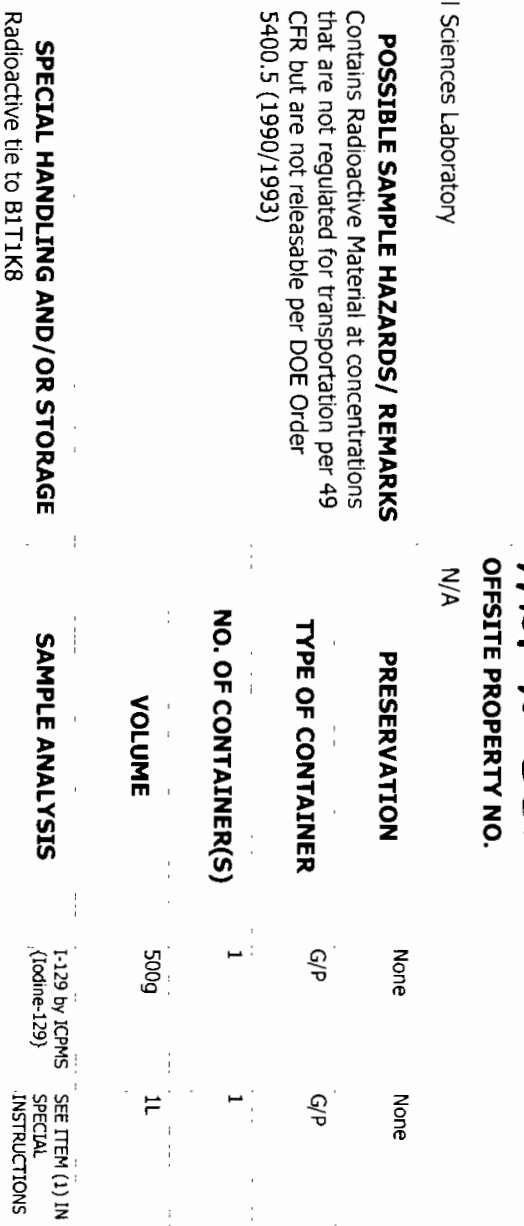

농 윅

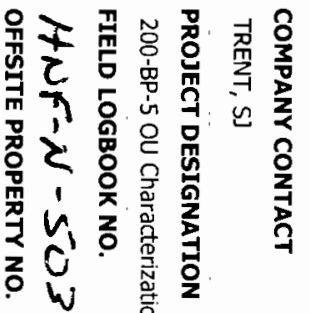

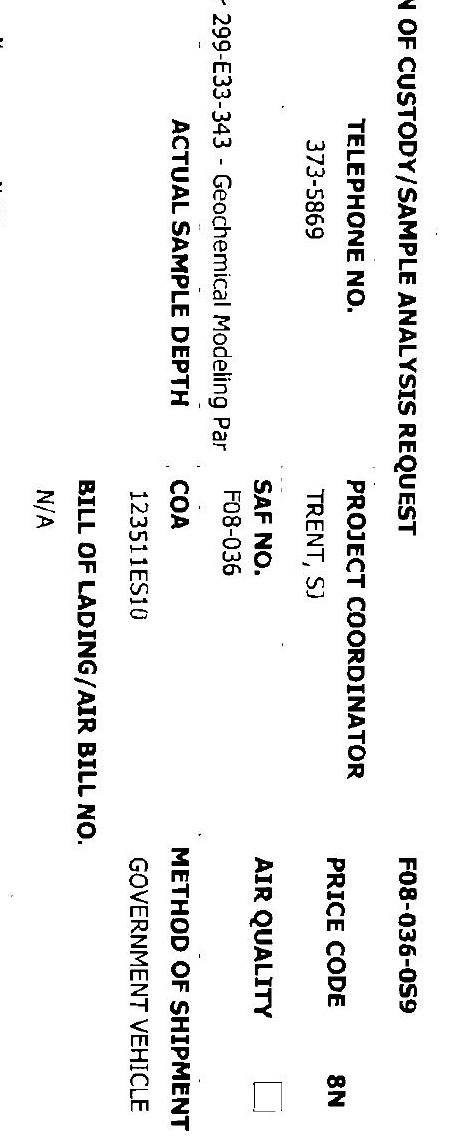



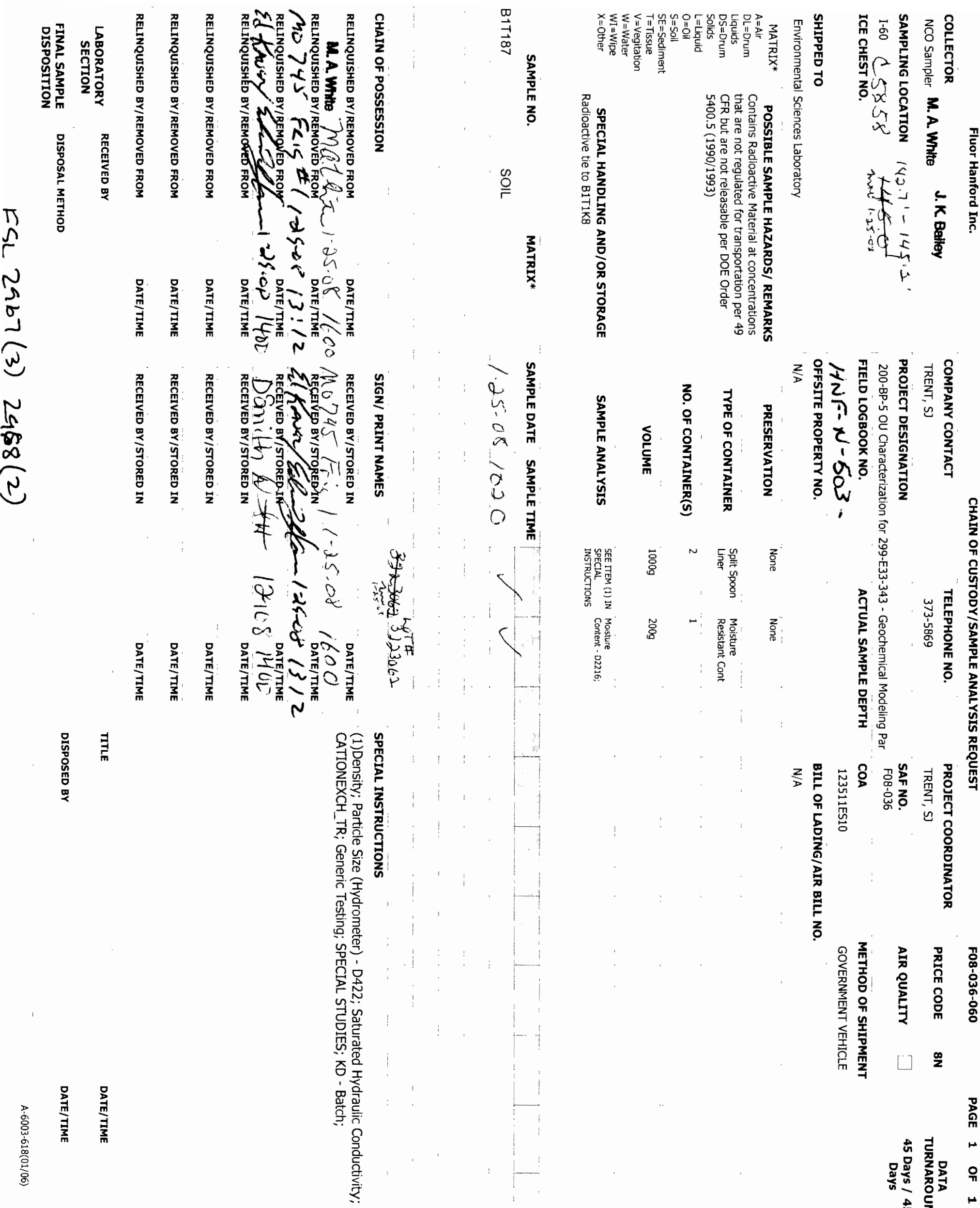


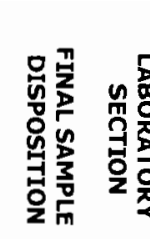

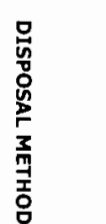
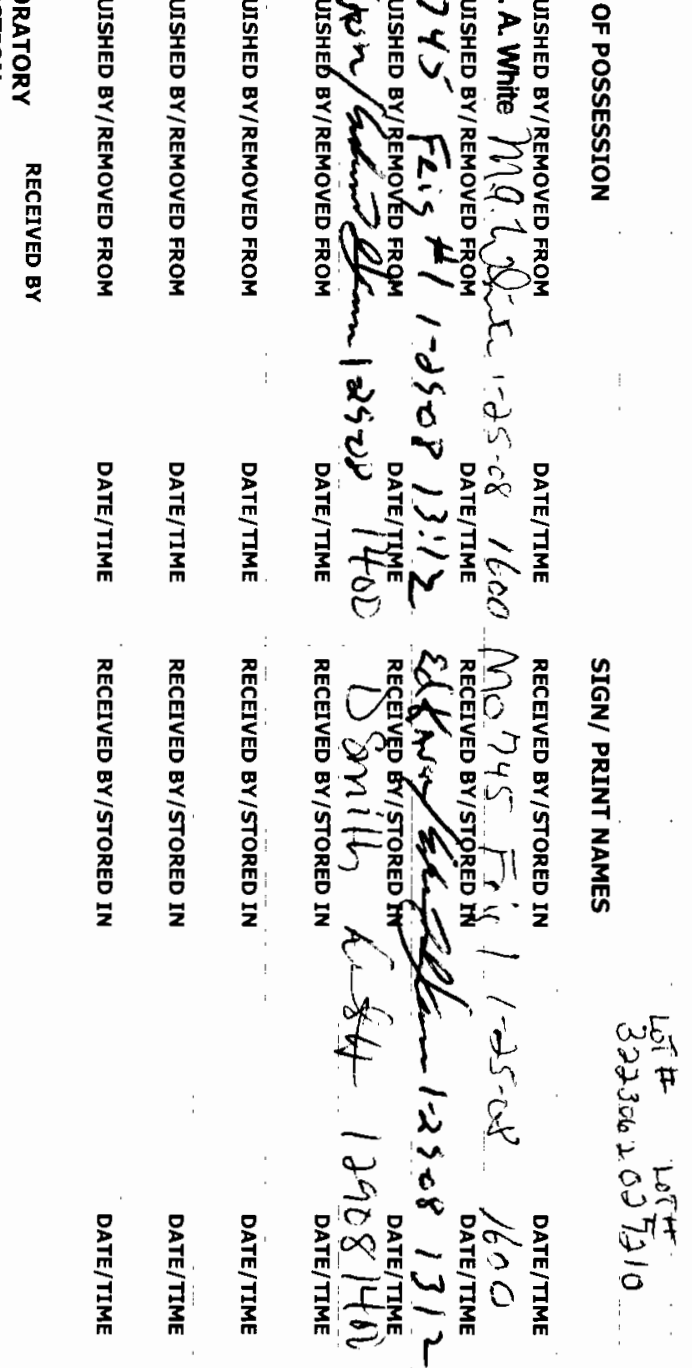

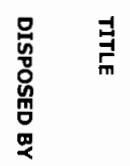

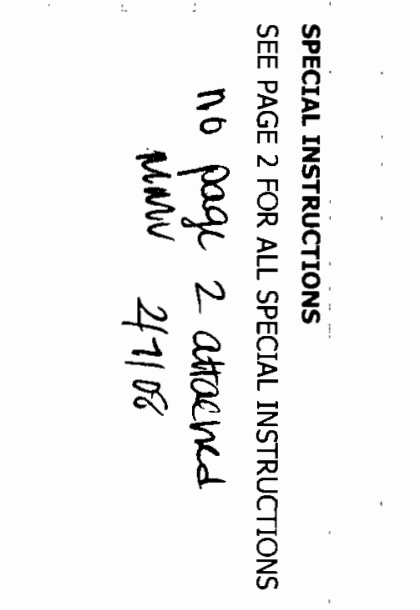

竞 $\stackrel{\infty}{=}$

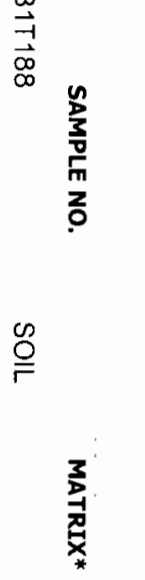

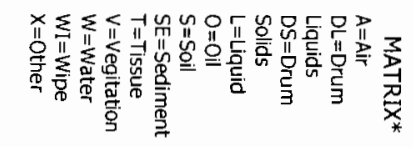

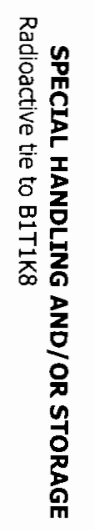

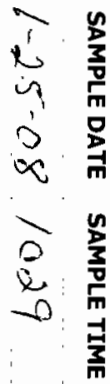

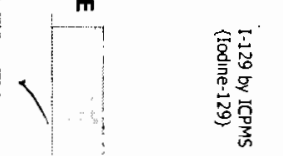

亳

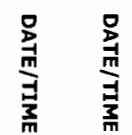




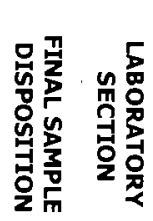

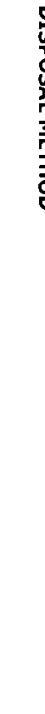

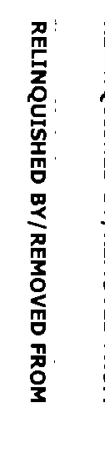

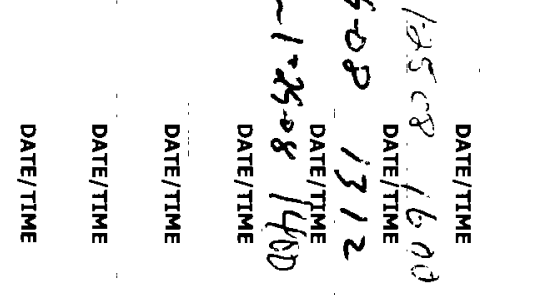

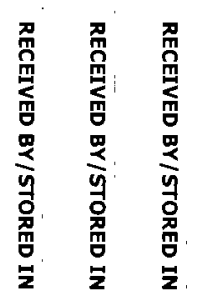

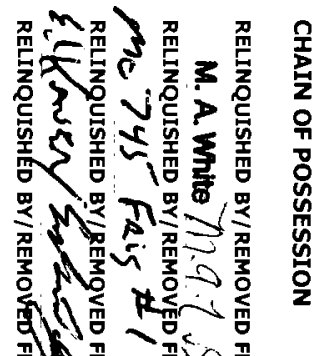

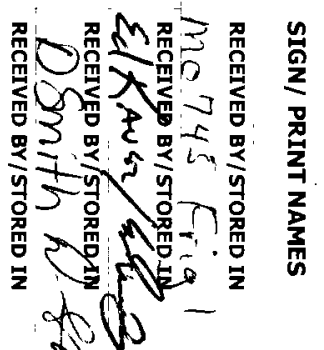

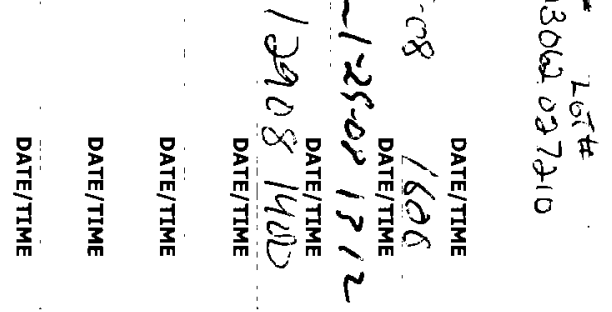

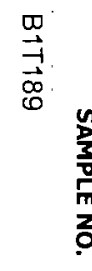

$\stackrel{\text { g }}{\risingdotseq}$

$\underset{*}{\stackrel{3}{3}}$

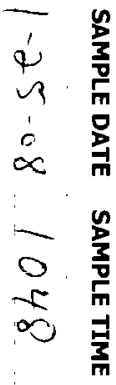

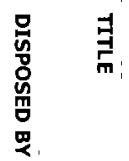

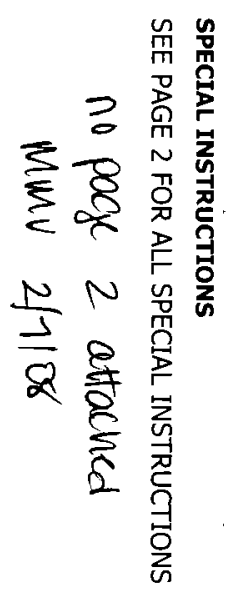

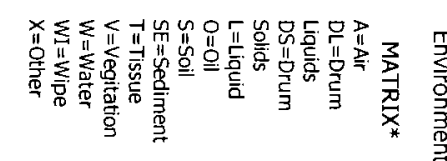

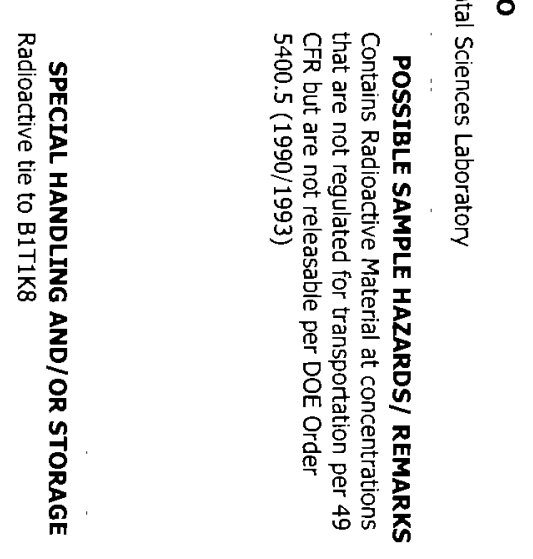

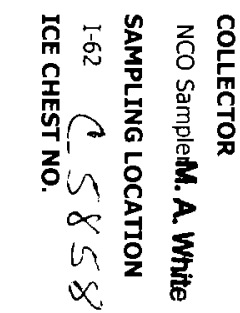

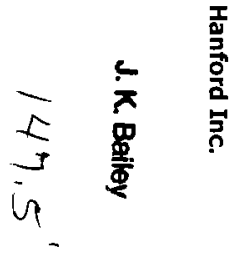

i

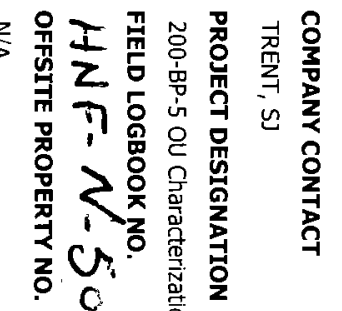

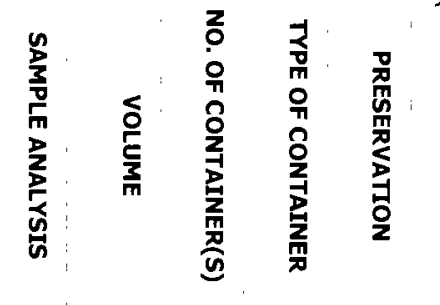

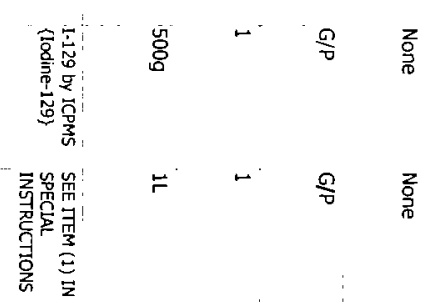

C $\begin{gathered}\mathrm{a} \\ \mathrm{W}\end{gathered}$

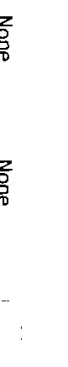

总

索苫

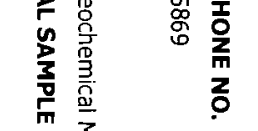

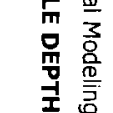

골

-7 ํํㅇ

岀 $\frac{\pi}{7} \frac{7}{y}$

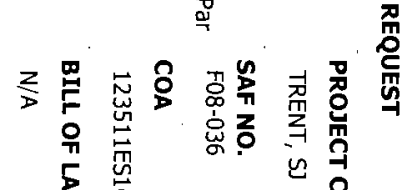

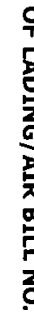

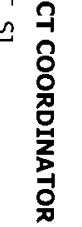

o
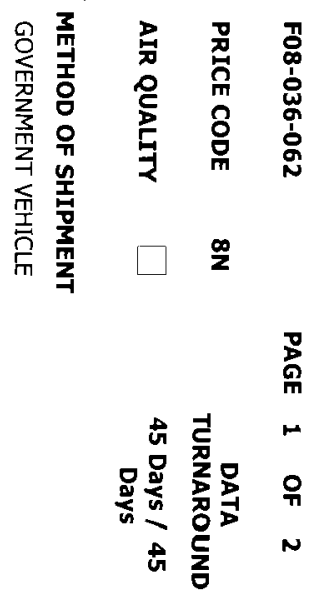


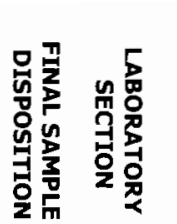

11

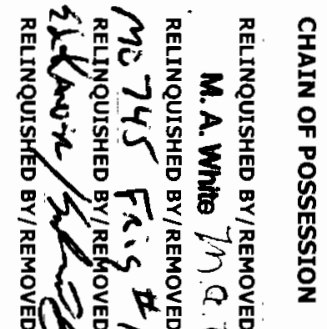

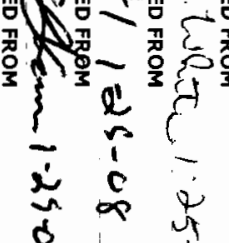

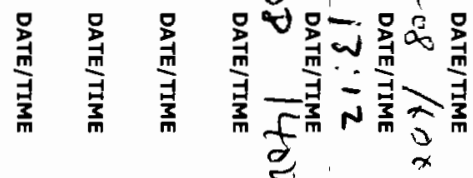

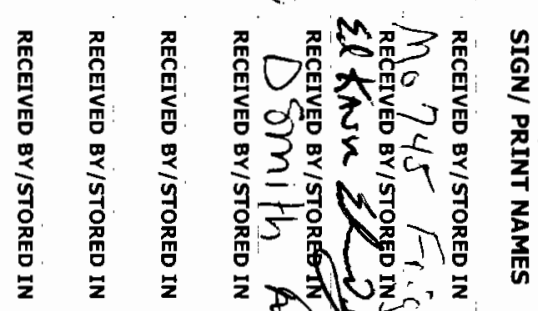

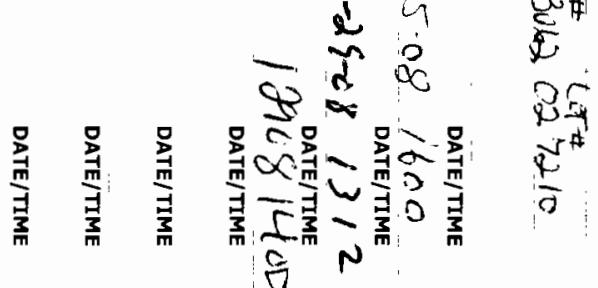

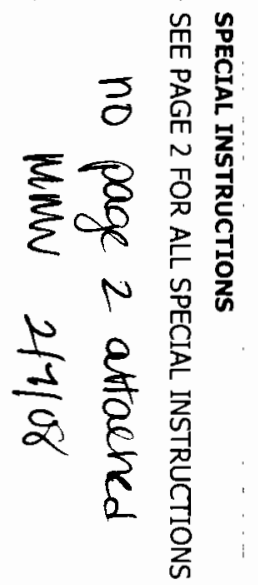

\section{高}<smiles>C1CCCC1</smiles>

妥

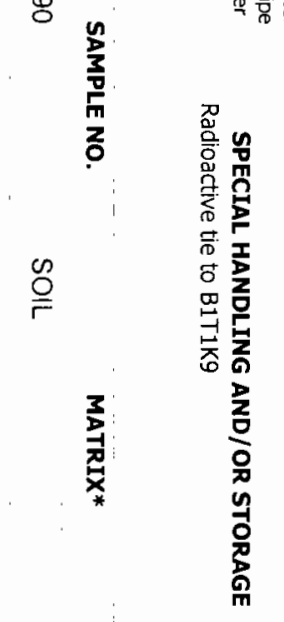

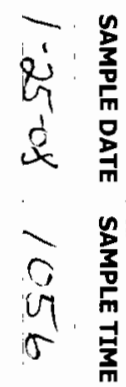

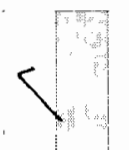

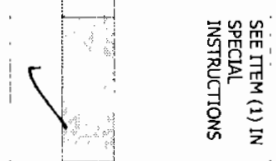

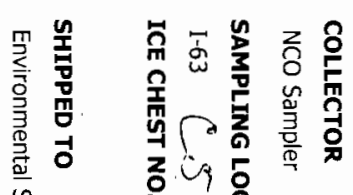
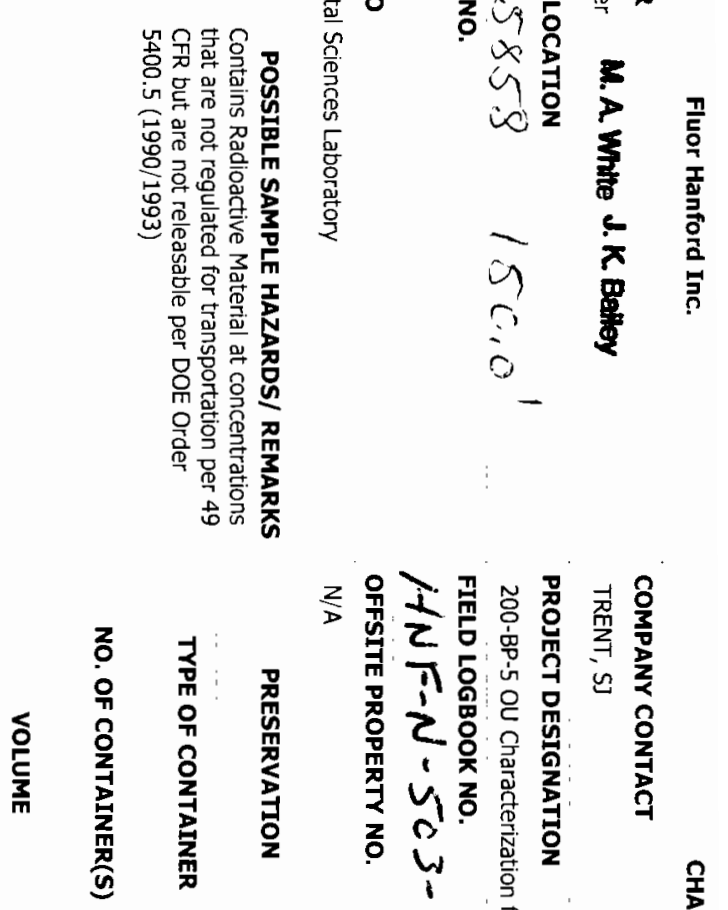

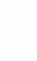
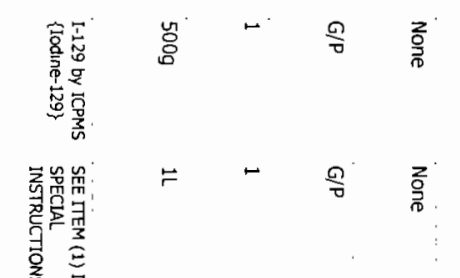

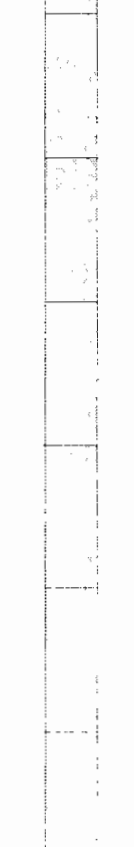

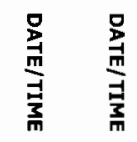




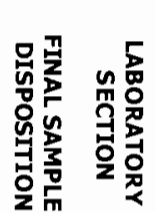

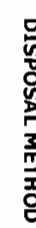

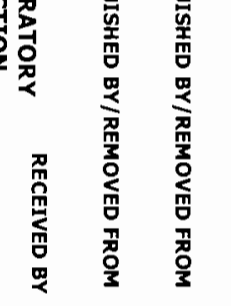

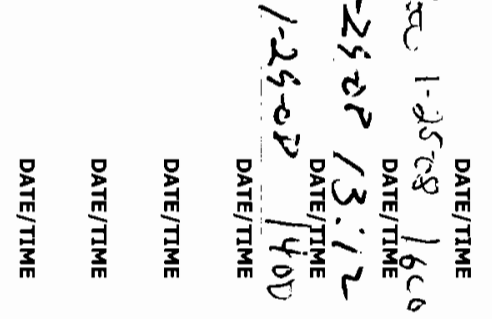
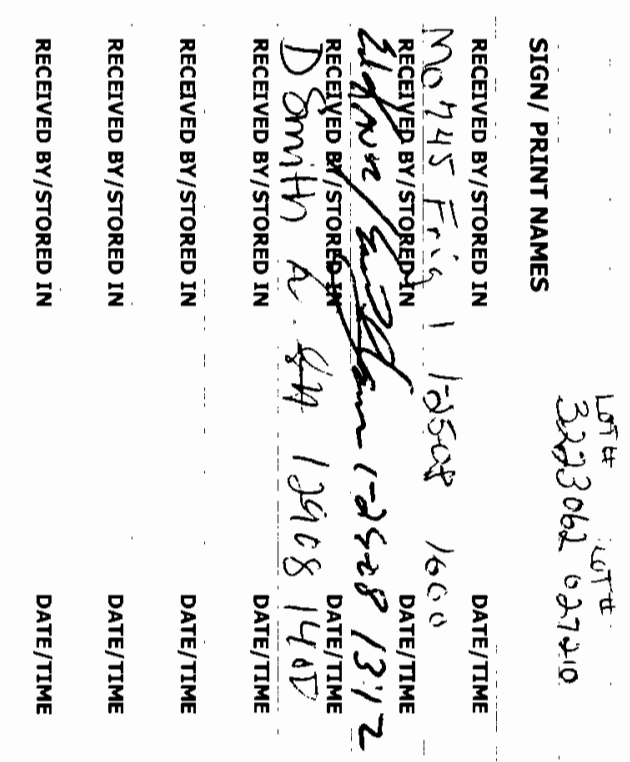

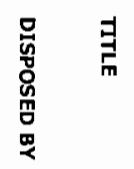
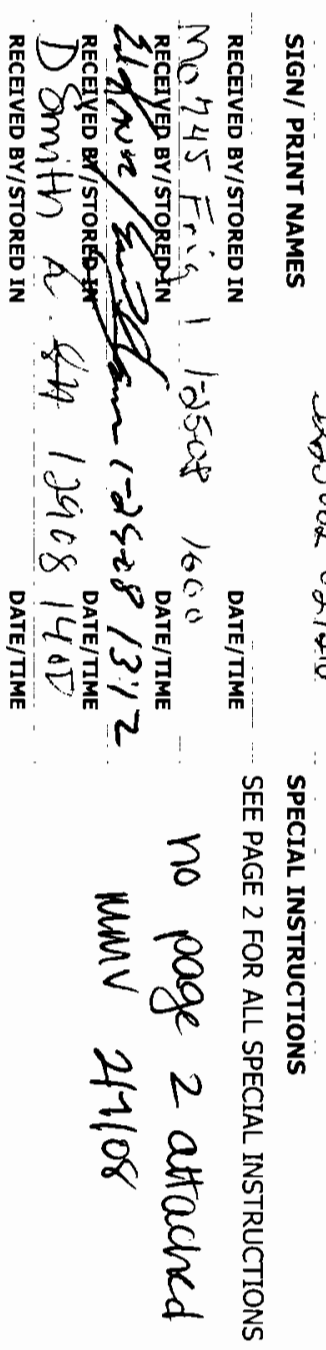

$\stackrel{\varpi}{\vec{\exists}}$

$\stackrel{8}{\circ}$

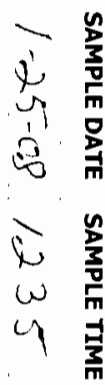

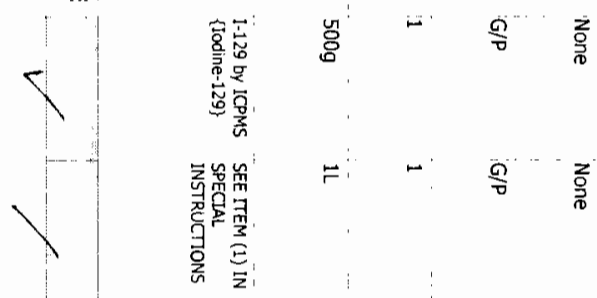

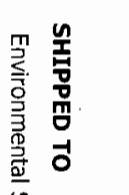

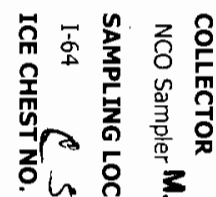

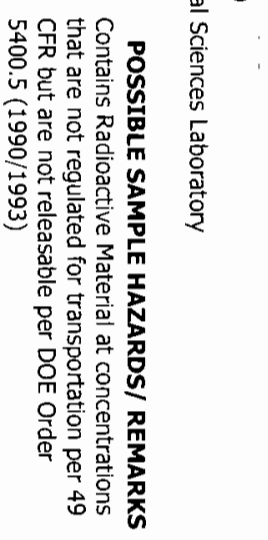

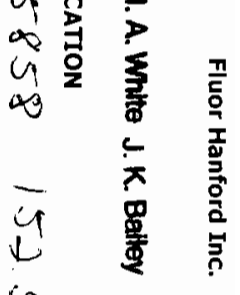

국윅

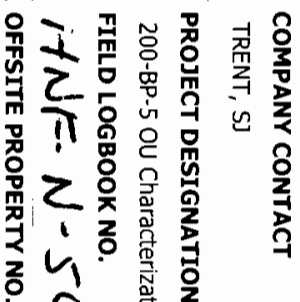

골

1
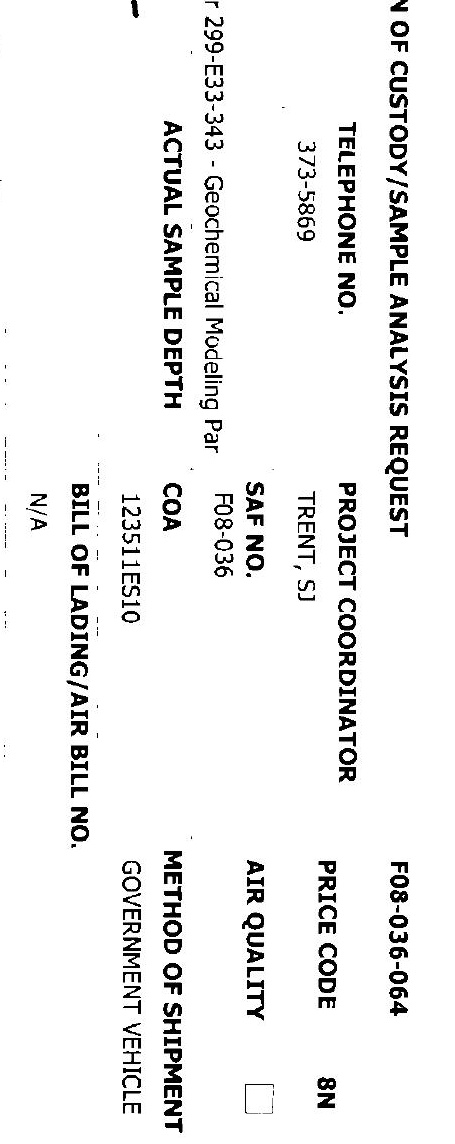

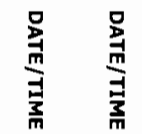

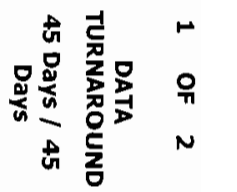




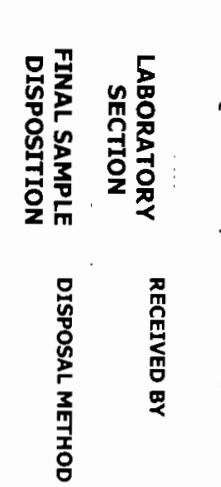

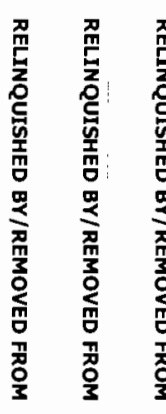

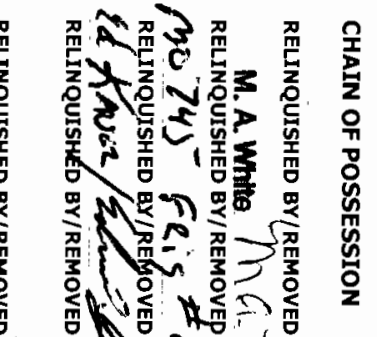

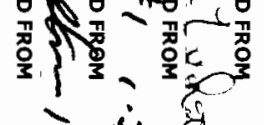
i $i$

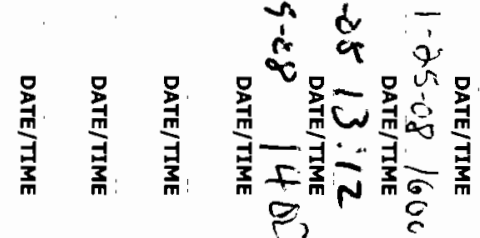

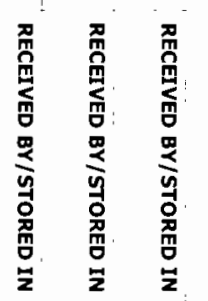
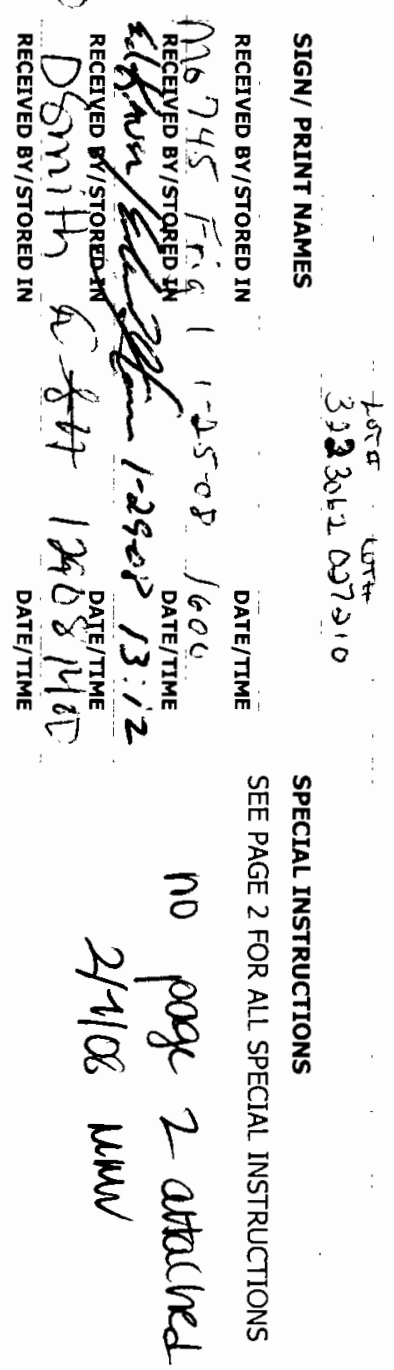

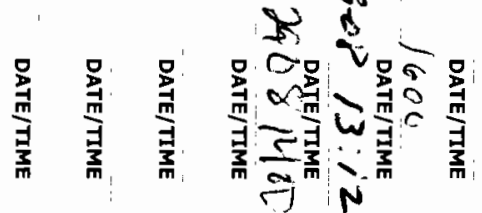

$\underset{\vec{N}}{\overrightarrow{1}}$

$\stackrel{0}{\circ}$

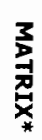

U.

w $\frac{2}{\frac{3}{2}}$

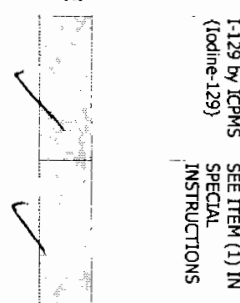

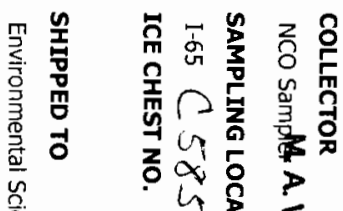

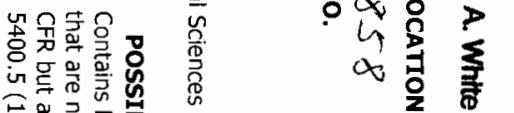

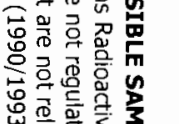

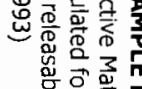

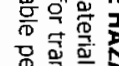

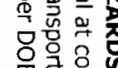

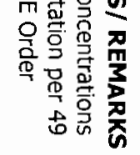

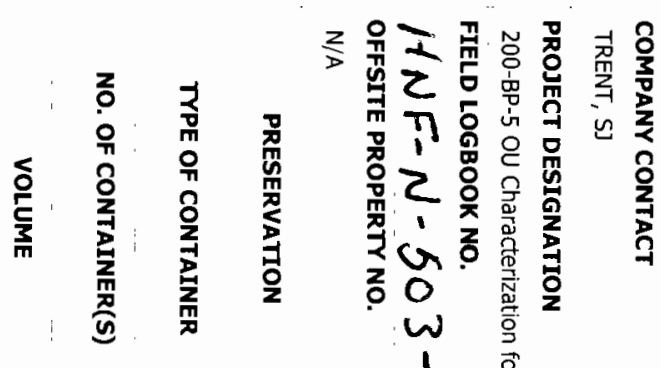

ㄴ.
总

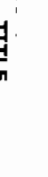

总暑 


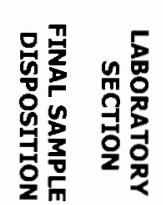

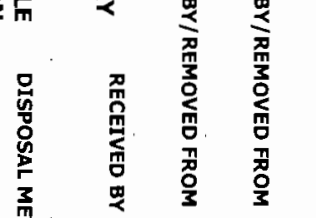

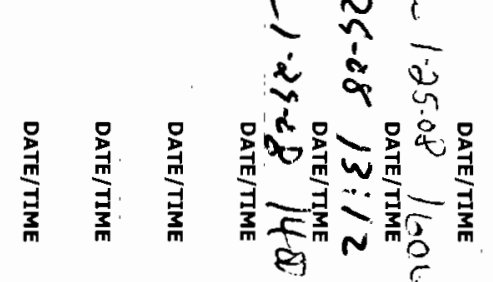
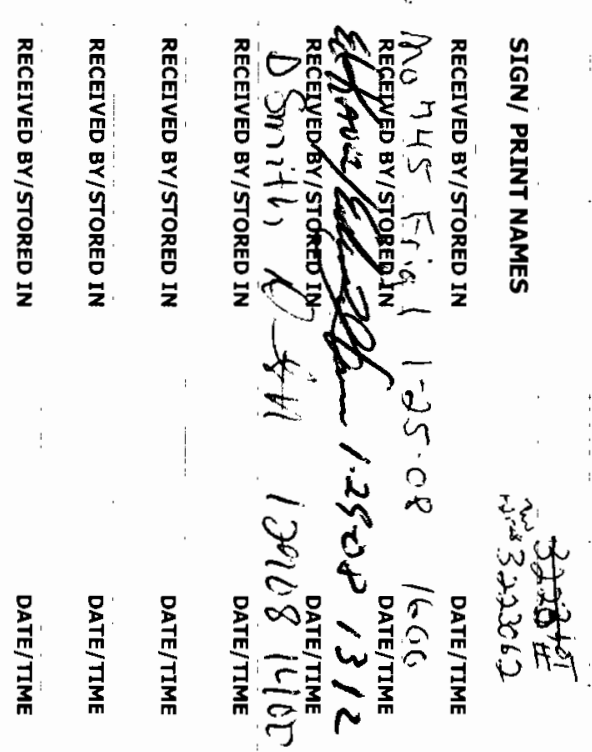

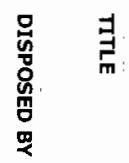

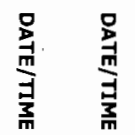
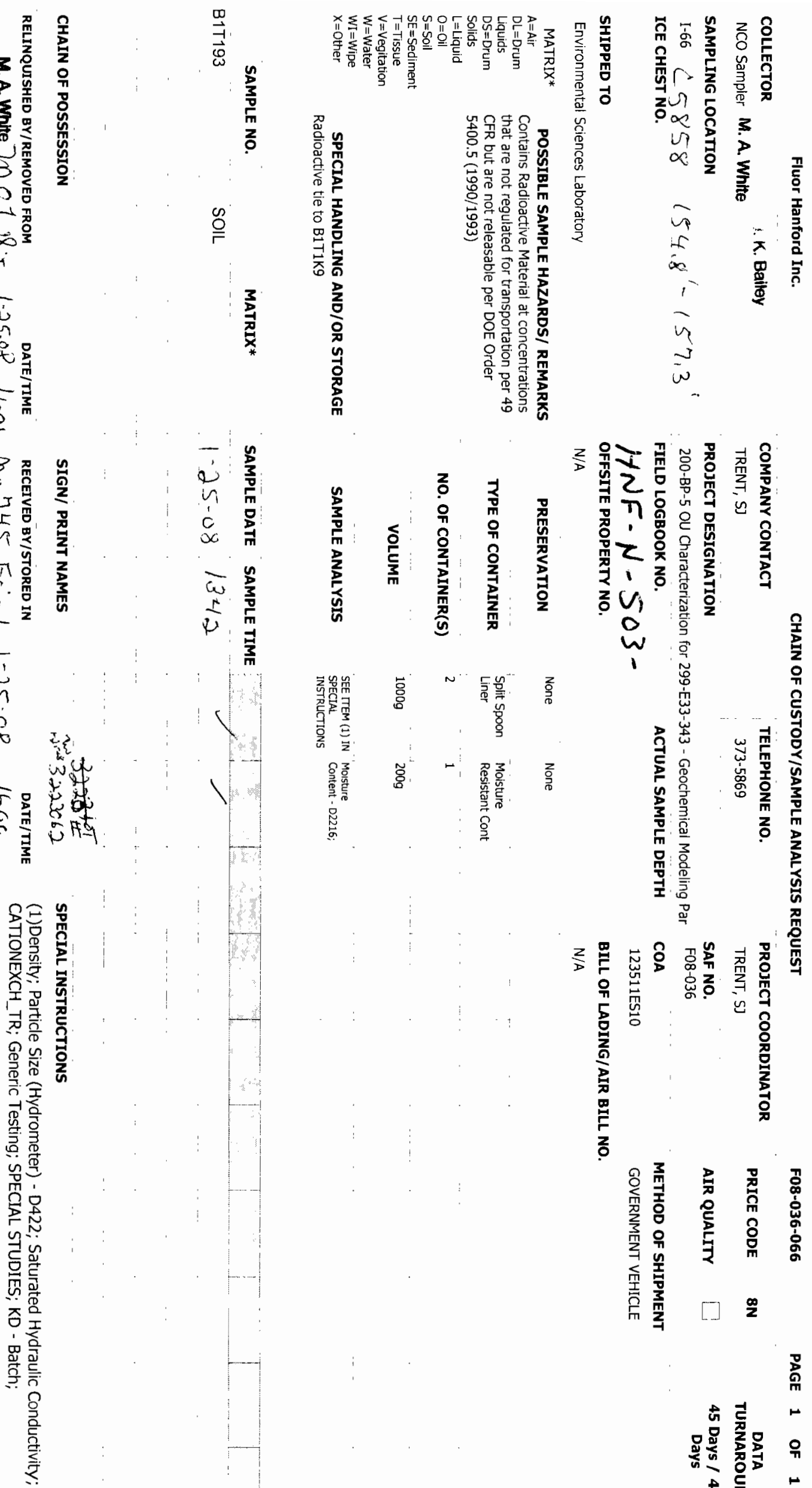

.

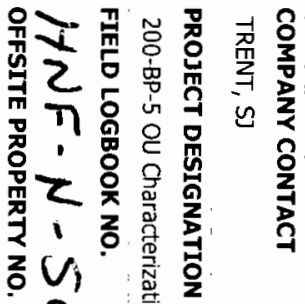

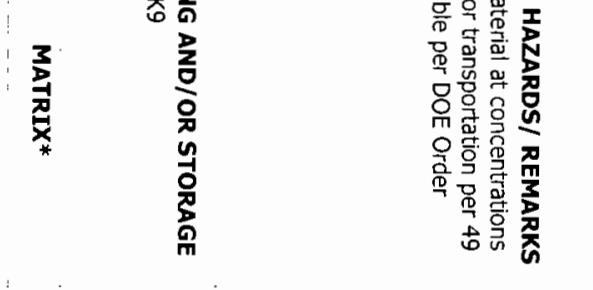

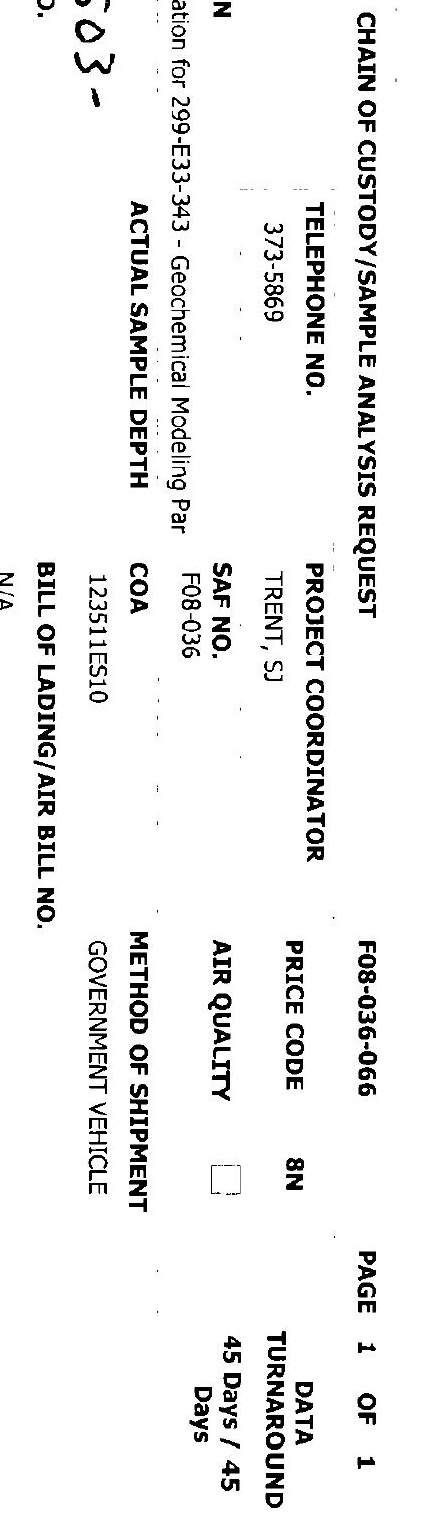

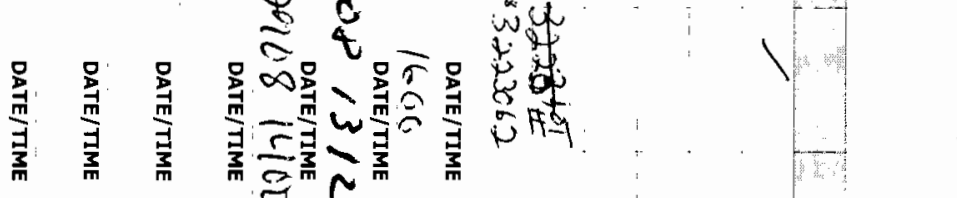




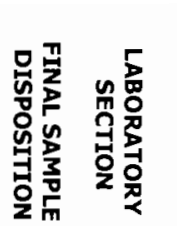
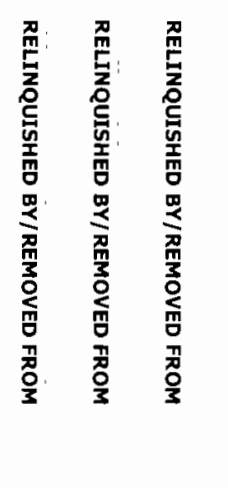

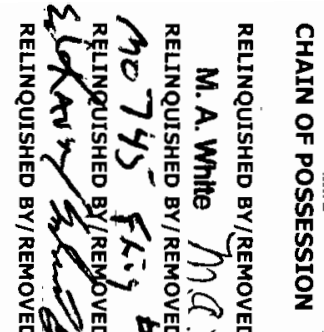

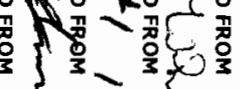

సै

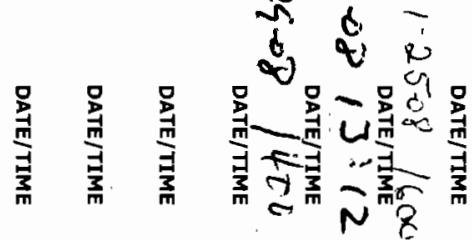

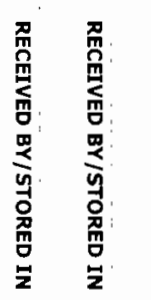

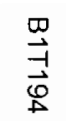

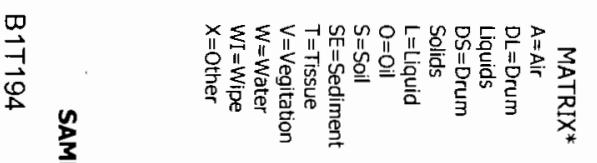

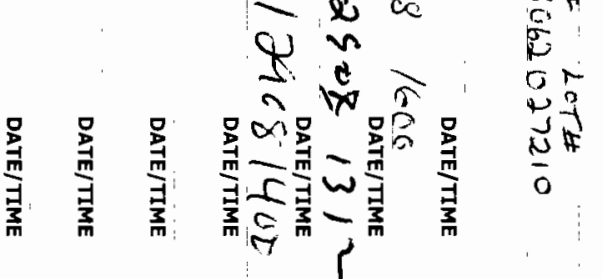

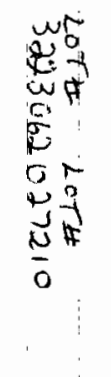

$\int \frac{1}{4} \quad$ 㝕

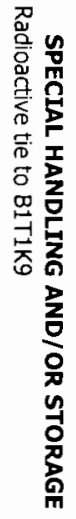

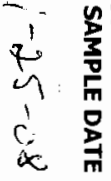

i $\frac{n}{v}$

Q 青

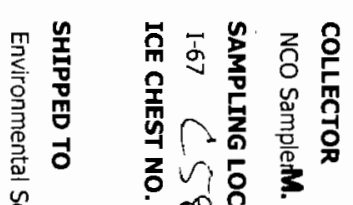

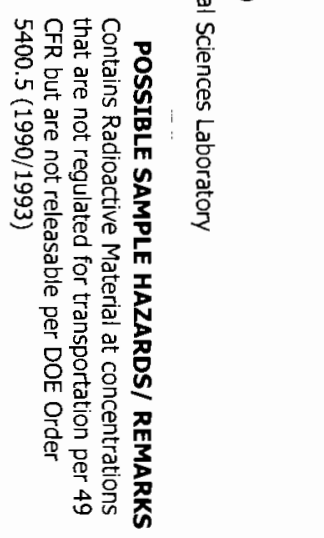

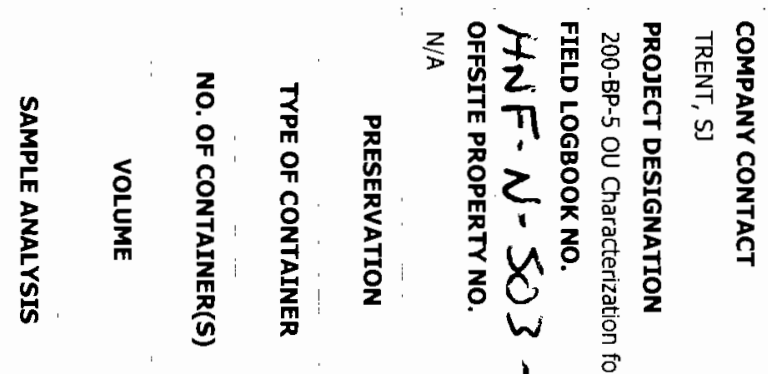

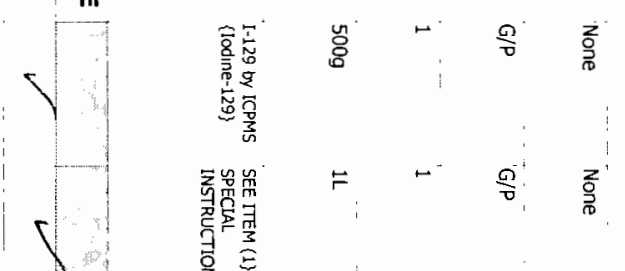

$\frac{2}{3}$ $\vec{\exists}$

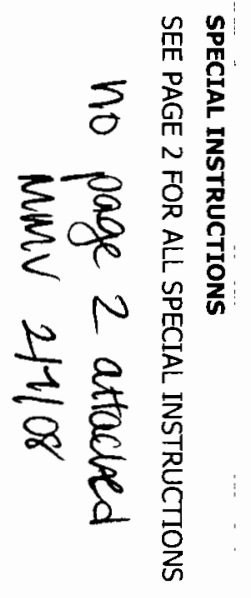




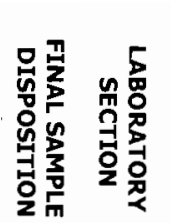

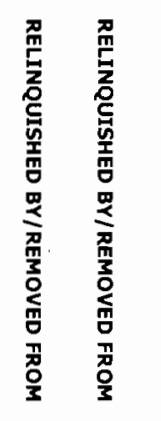

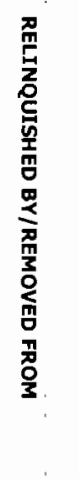

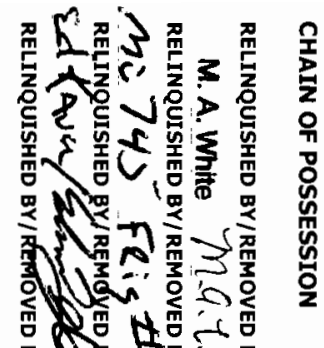

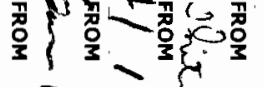

i i i

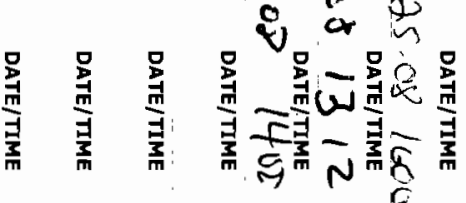

焉

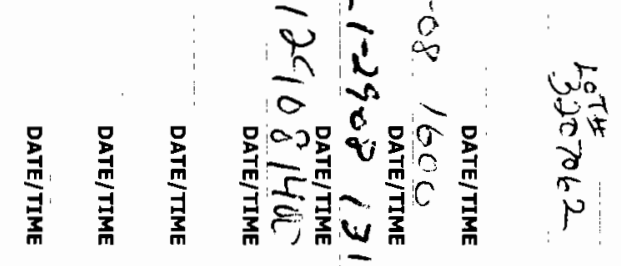

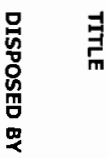

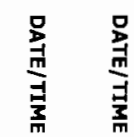
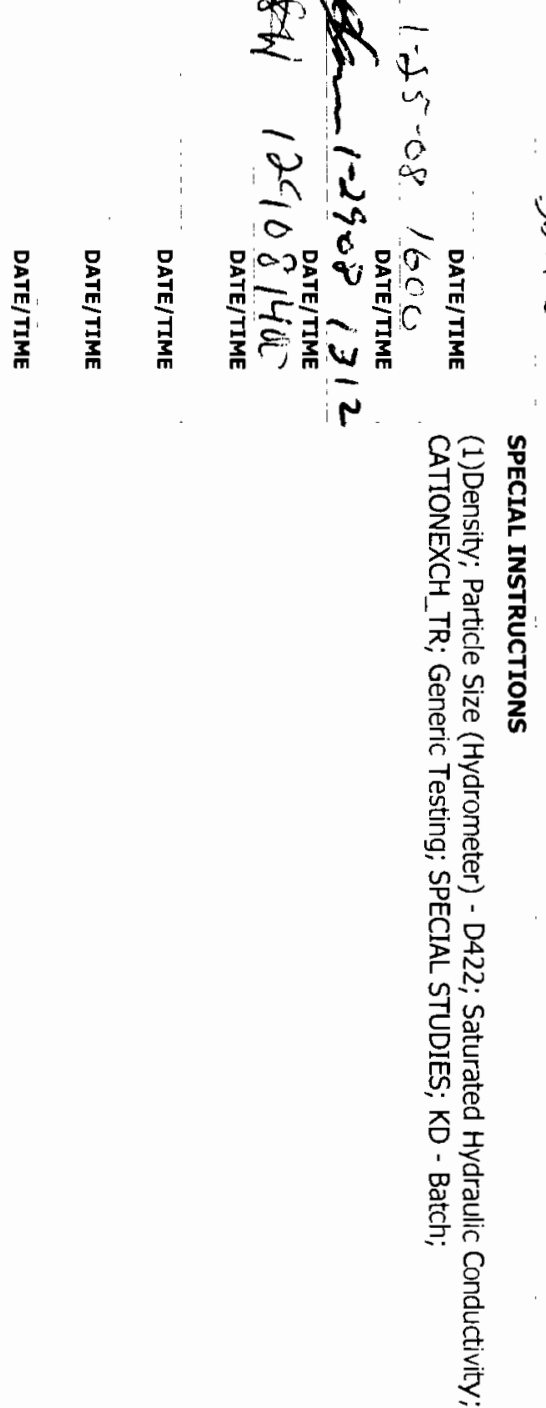

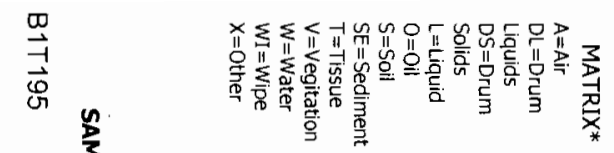

$\frac{1}{3}$
$\frac{\mathbf{z}}{\mathbf{m}}$
$\mathbf{0}$

$\stackrel{\mathscr{O}}{\rightleftharpoons}$

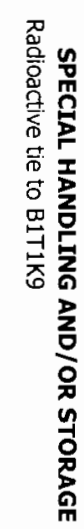

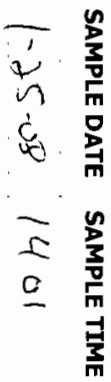

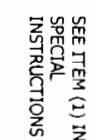

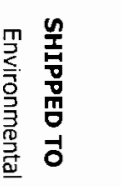

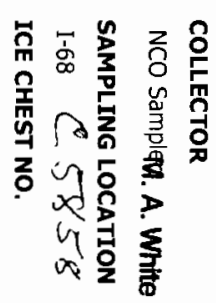

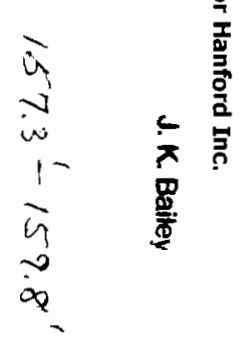

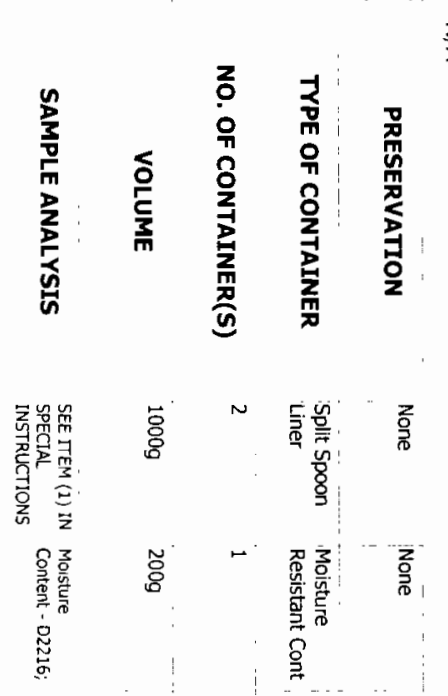

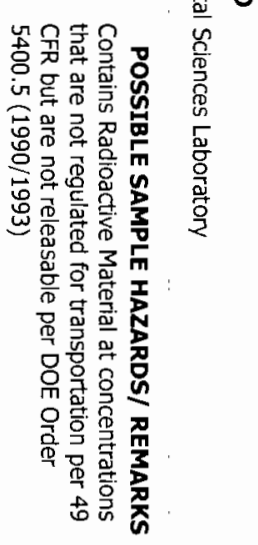

s

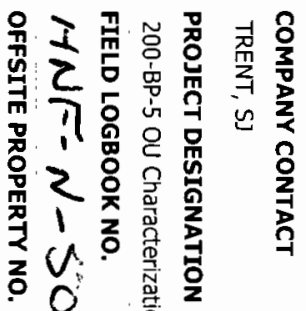

0
1
1

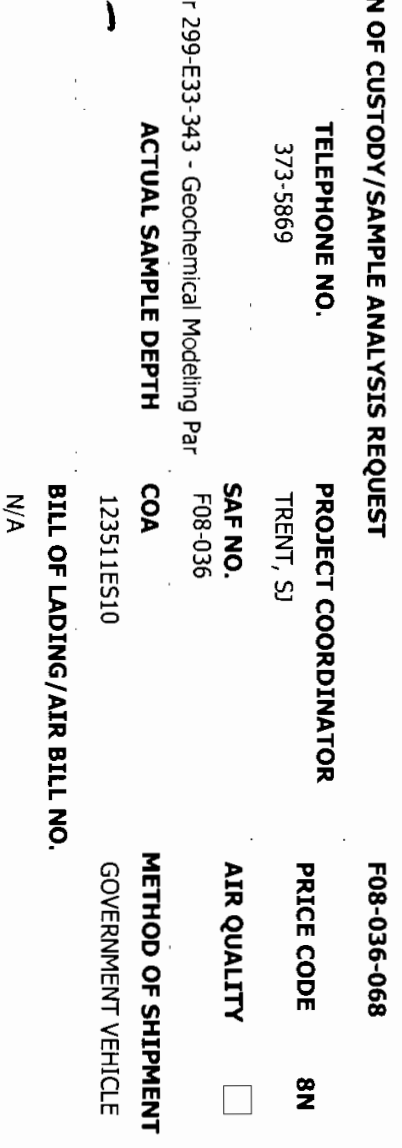

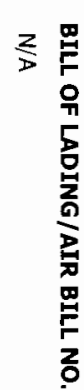

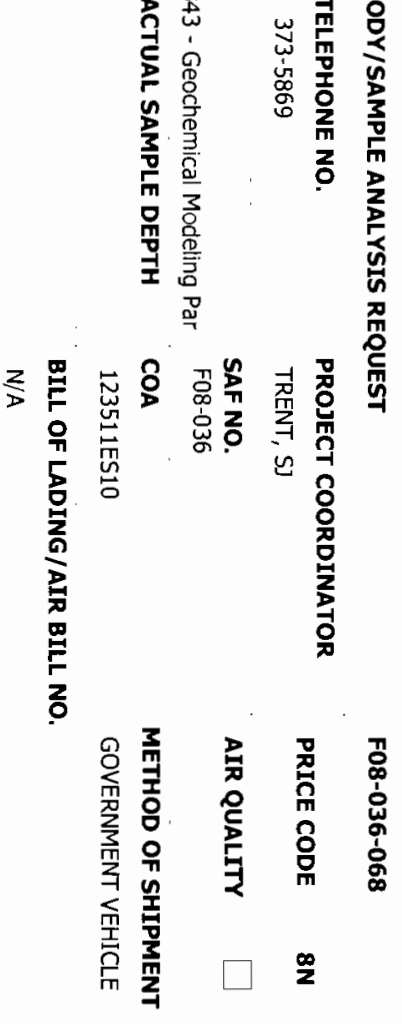

군

而

ำ

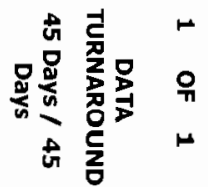


$\mathbb{1}$

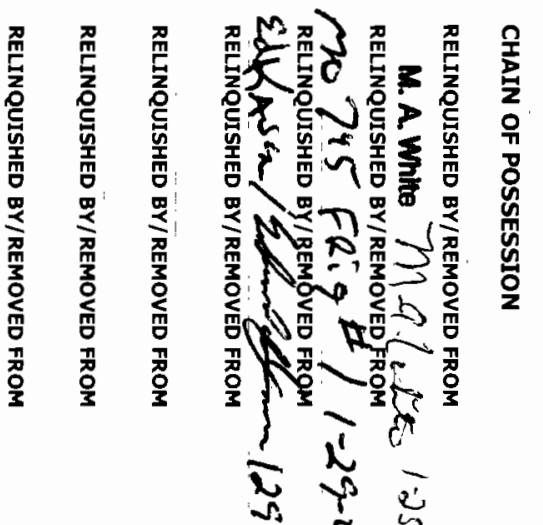

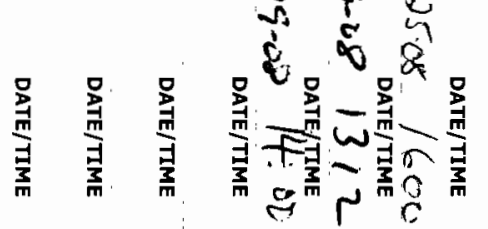
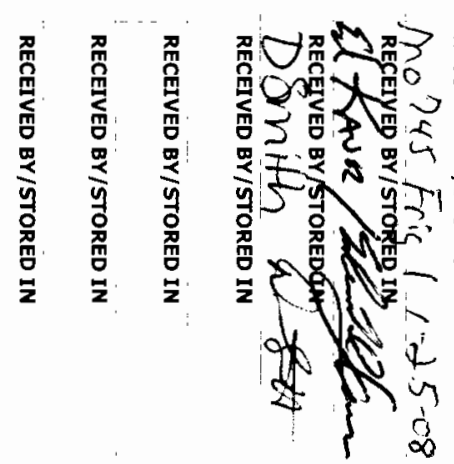

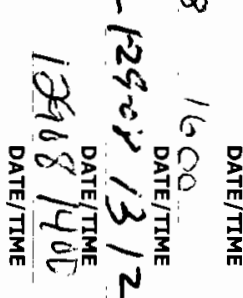

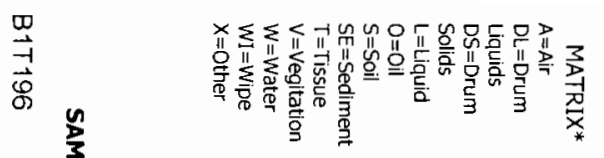

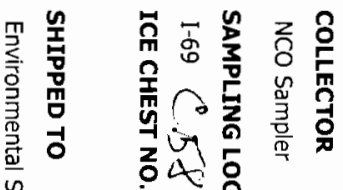

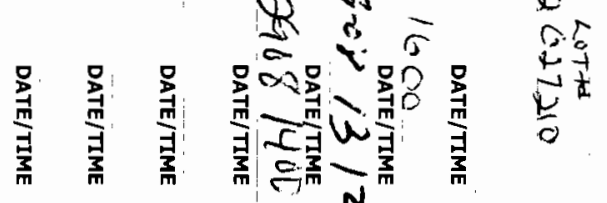

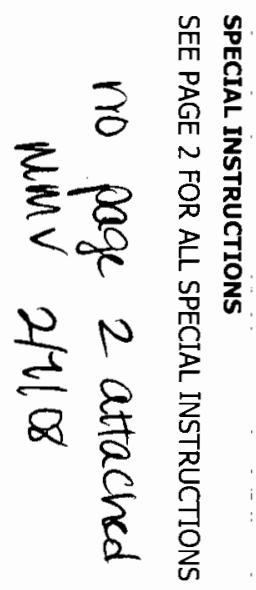

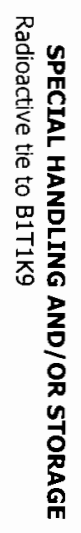

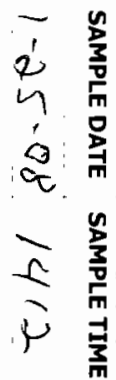

w5

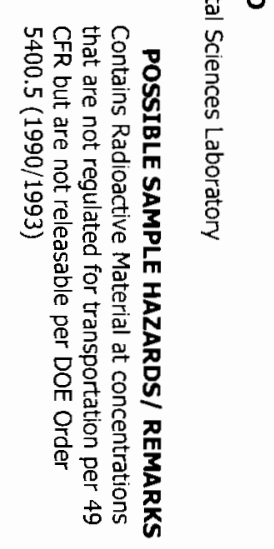

棺

$\stackrel{\infty}{\circ}$

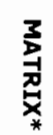

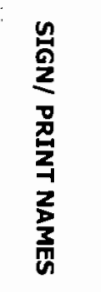

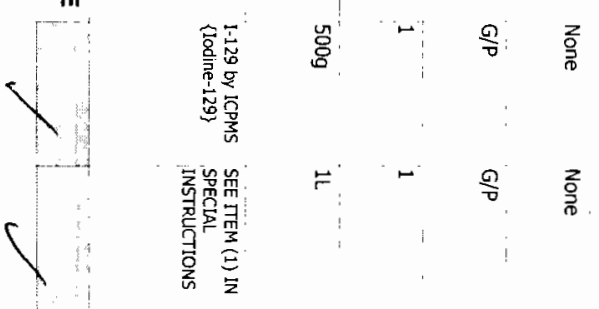

$\frac{2}{>}$
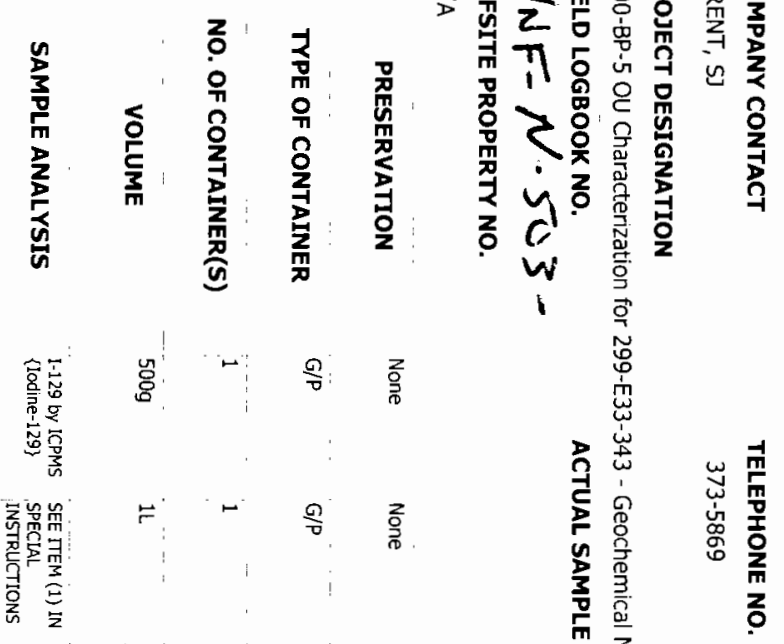

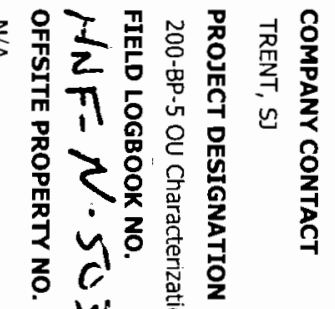

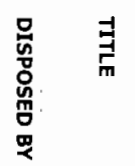

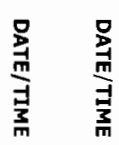
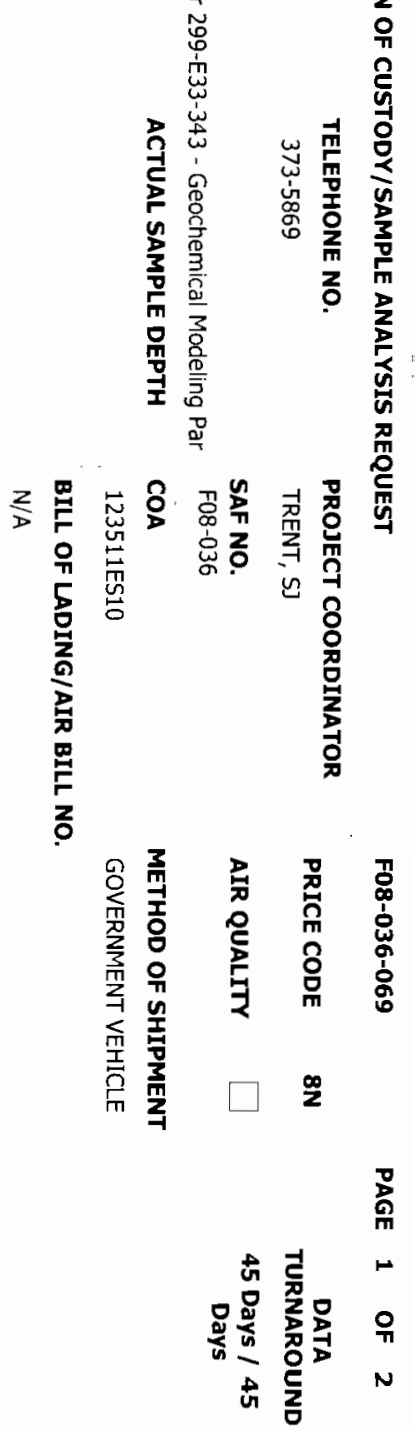


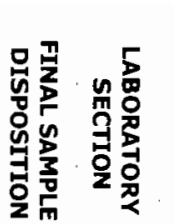
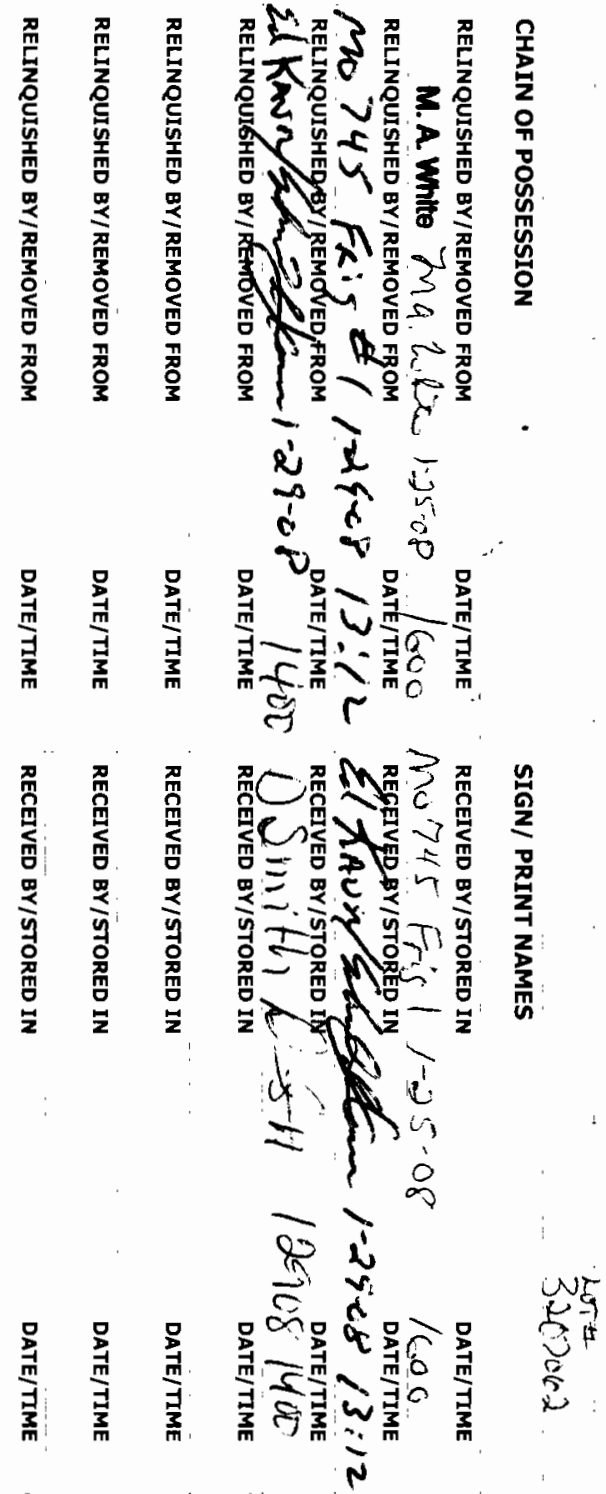

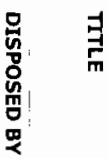

臀
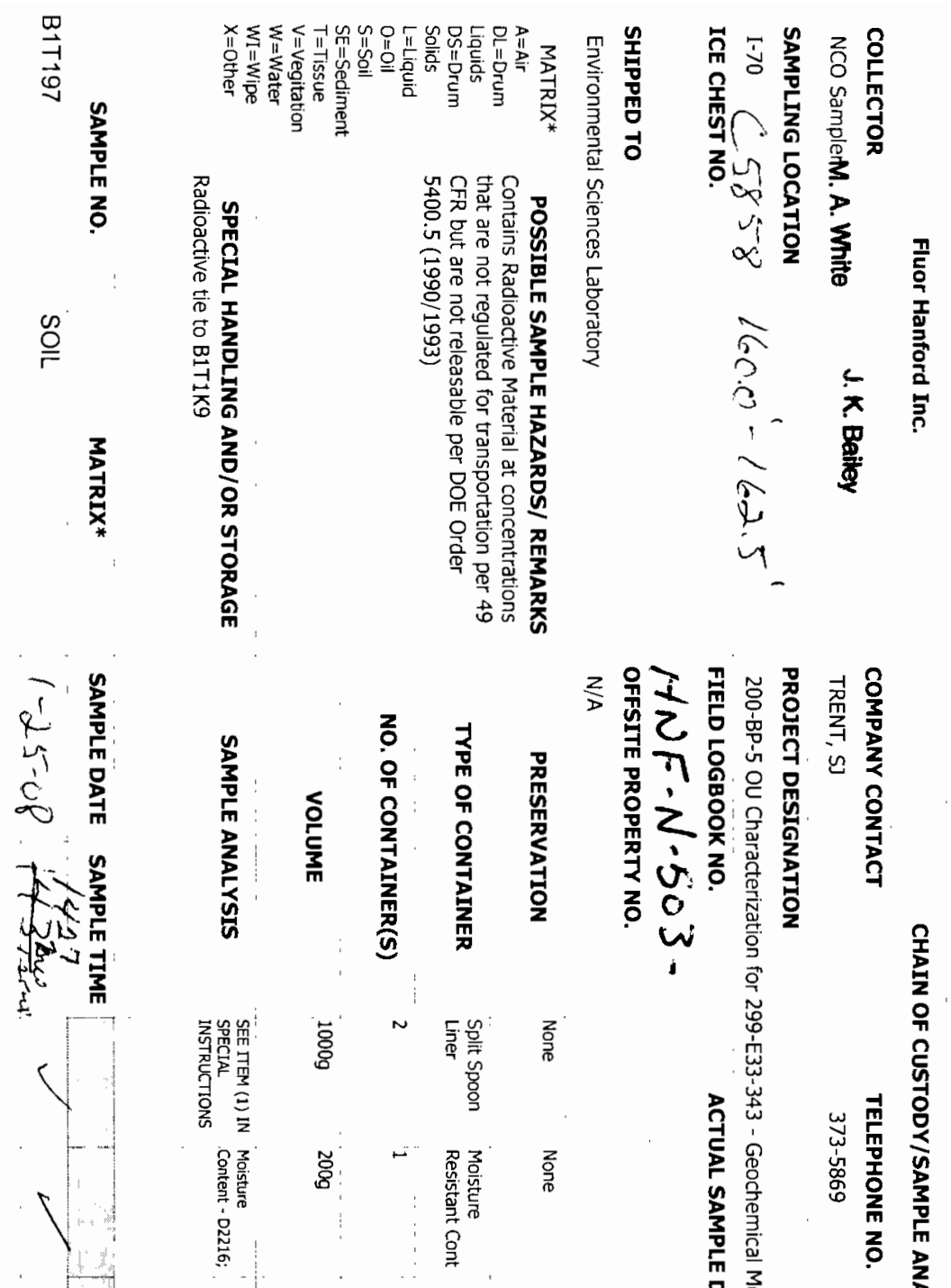

2
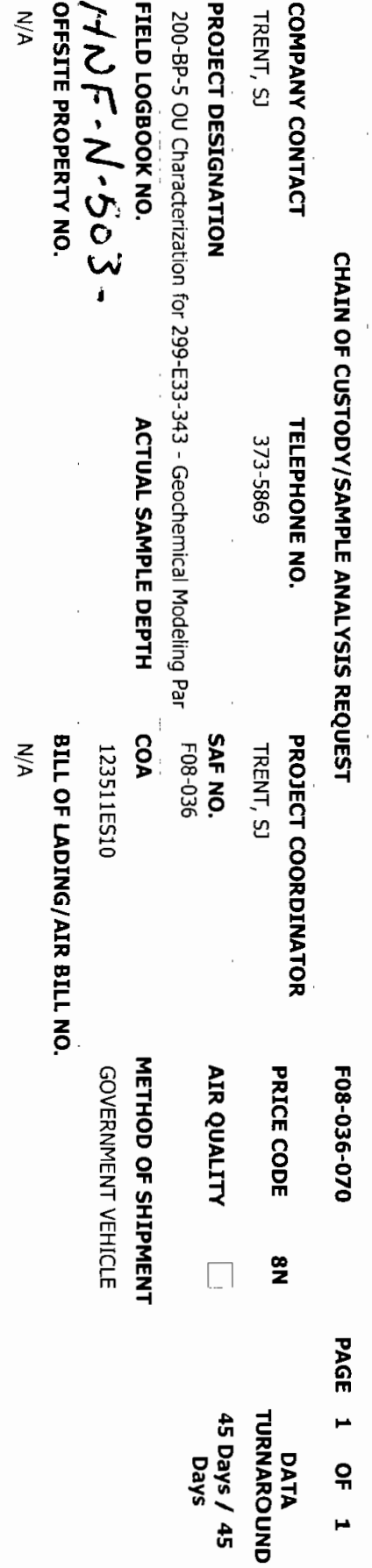


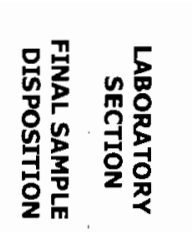
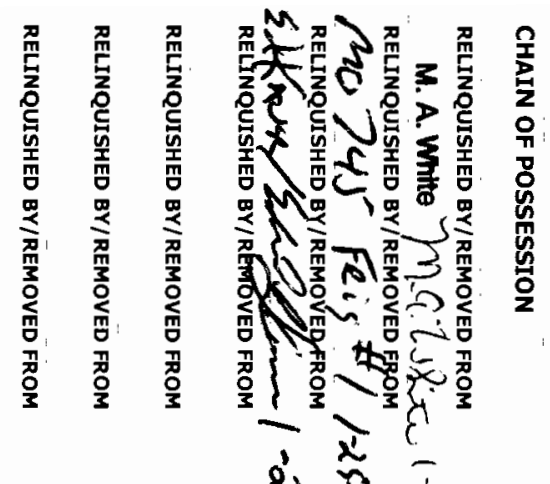

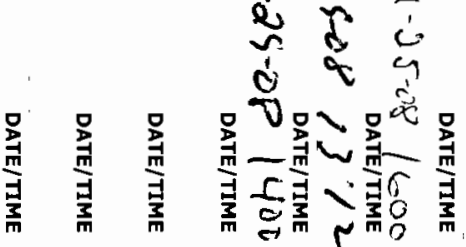
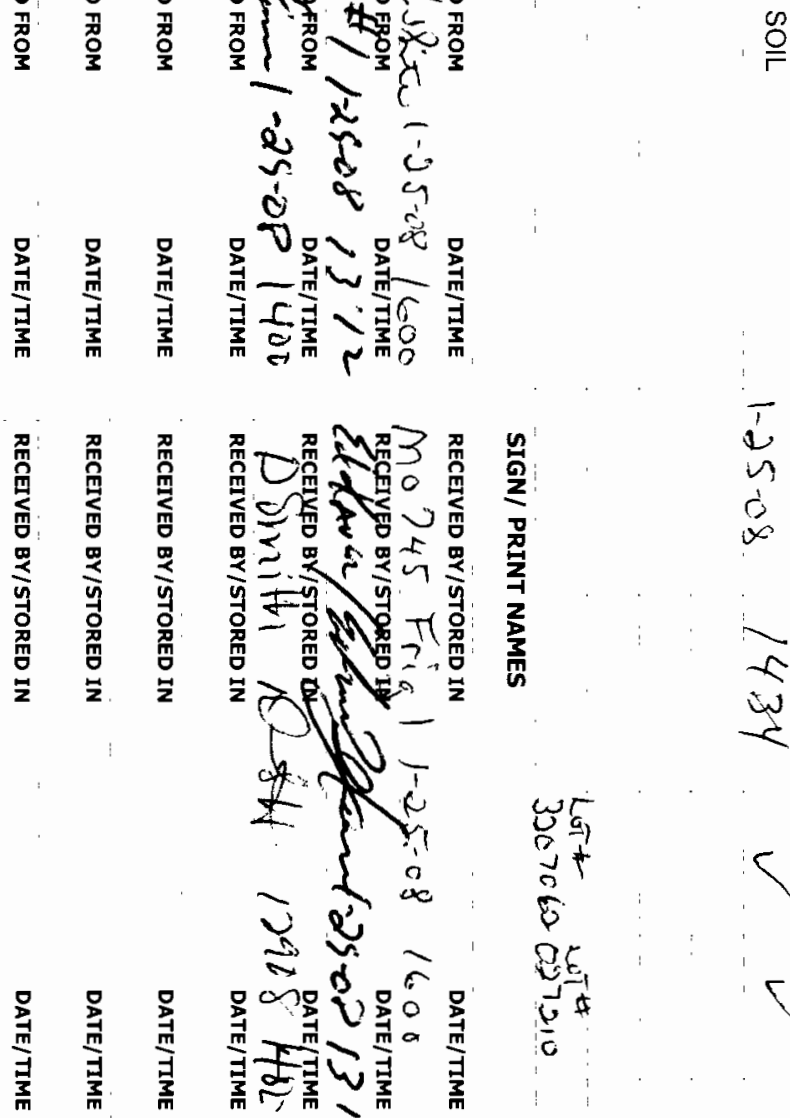

$r$

일

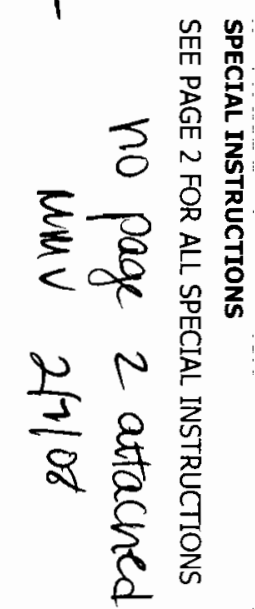

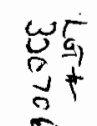

S

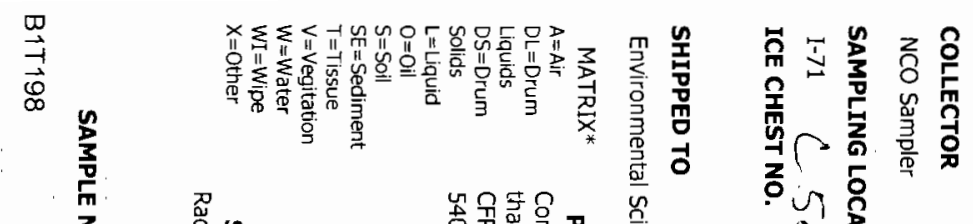

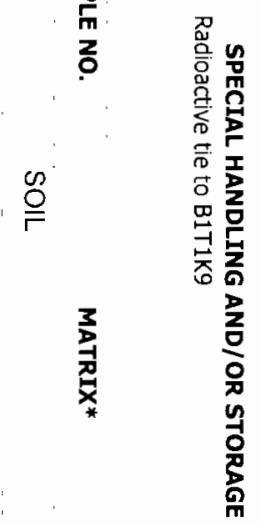

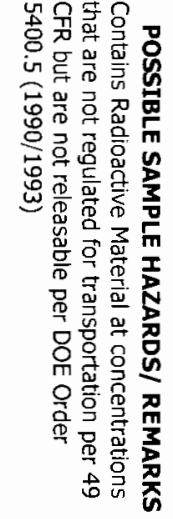

$\begin{array}{ll}\text { L } & \text { n: } \\ \sim & \frac{3}{3} \\ \text { d } & \frac{\pi}{7} \\ \infty & 0\end{array}$

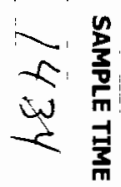

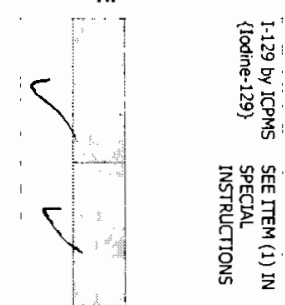

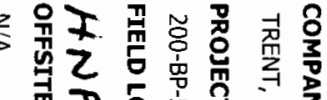

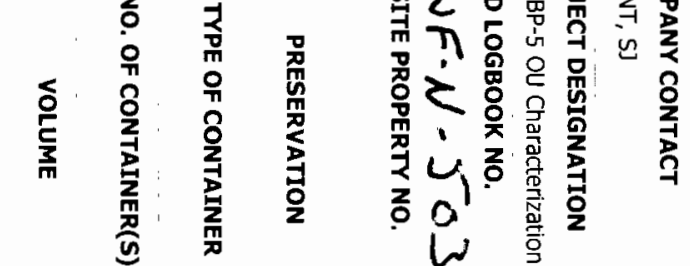

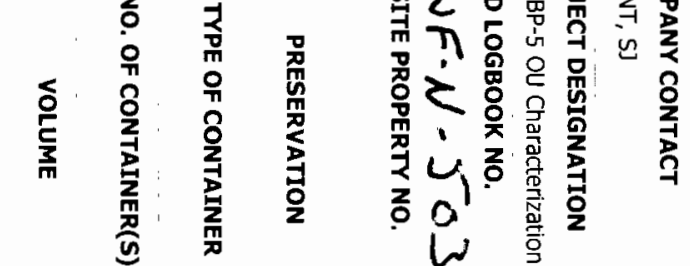

高 $-\frac{9}{8} \frac{2}{\frac{9}{\sigma}}$

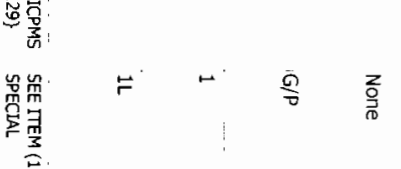

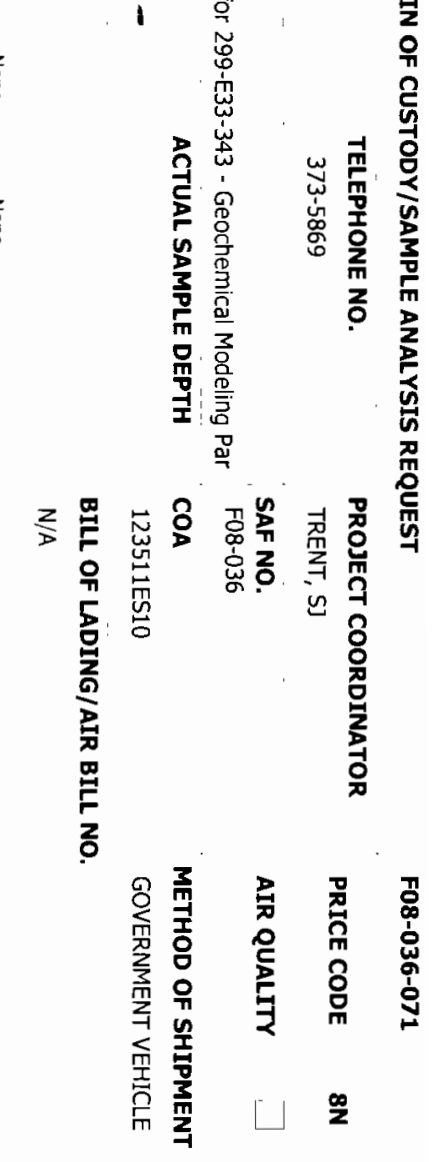

ว 
$\mid \|$
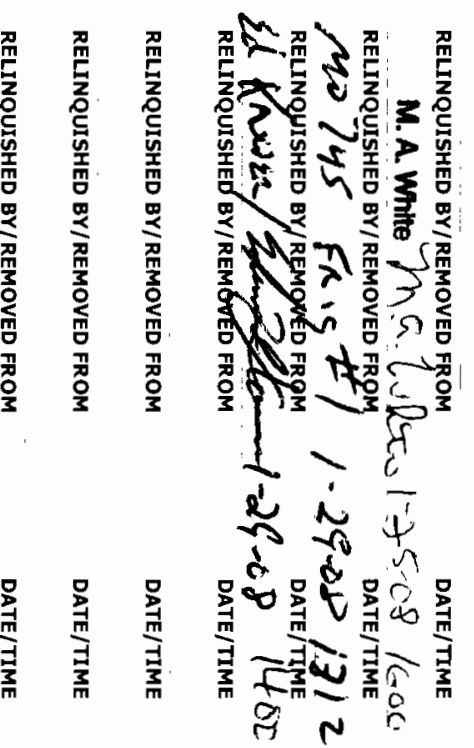

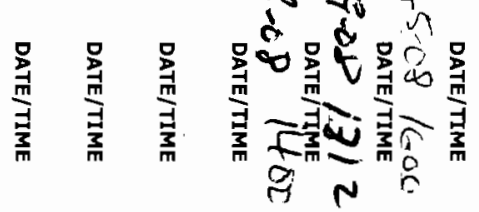

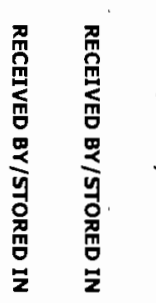

$\stackrel{\infty}{\vec{\exists}}$

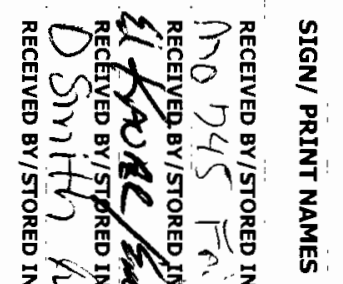

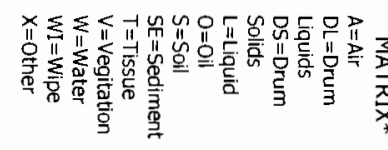

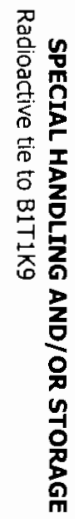

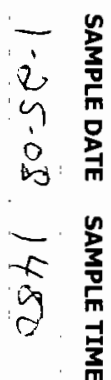

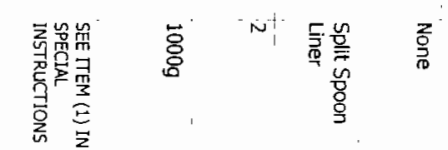

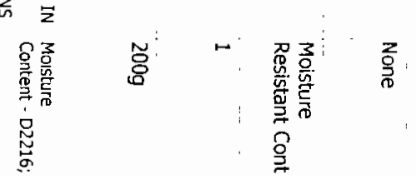

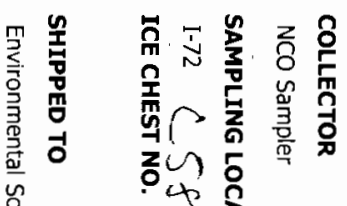
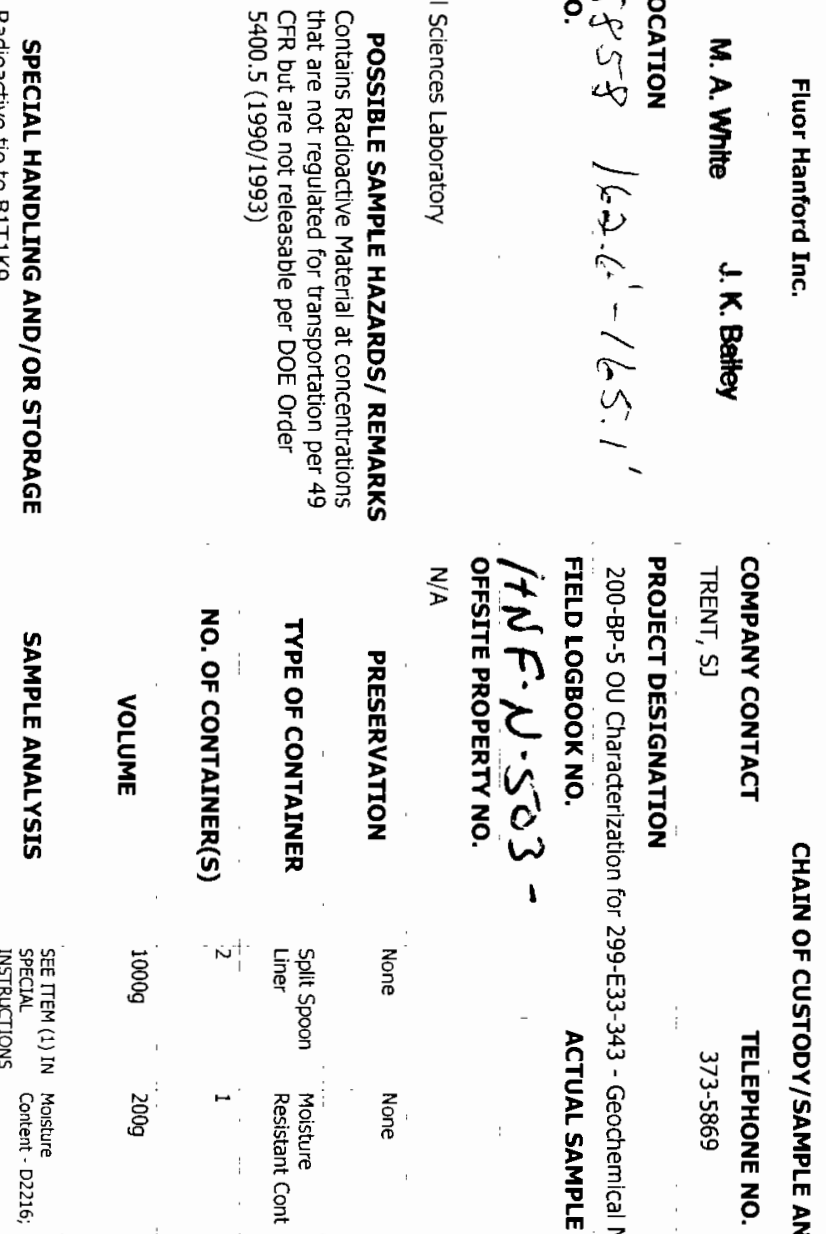

焉
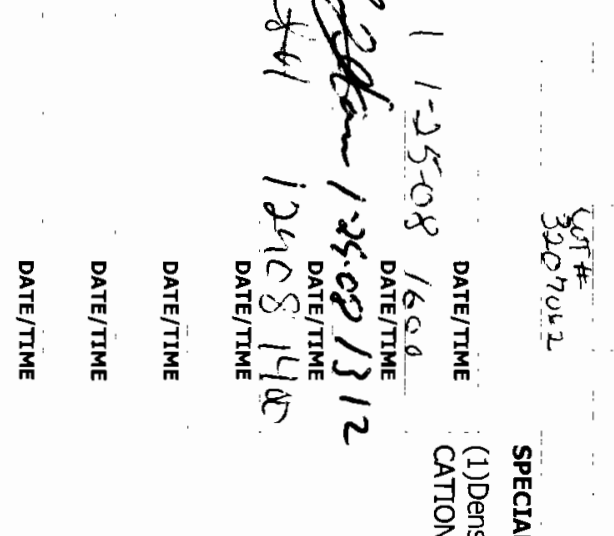

穿夏

旁?

고을

里

글

总高

实寻

ํํㄴ

足

窟管

증 목

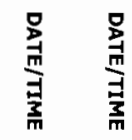



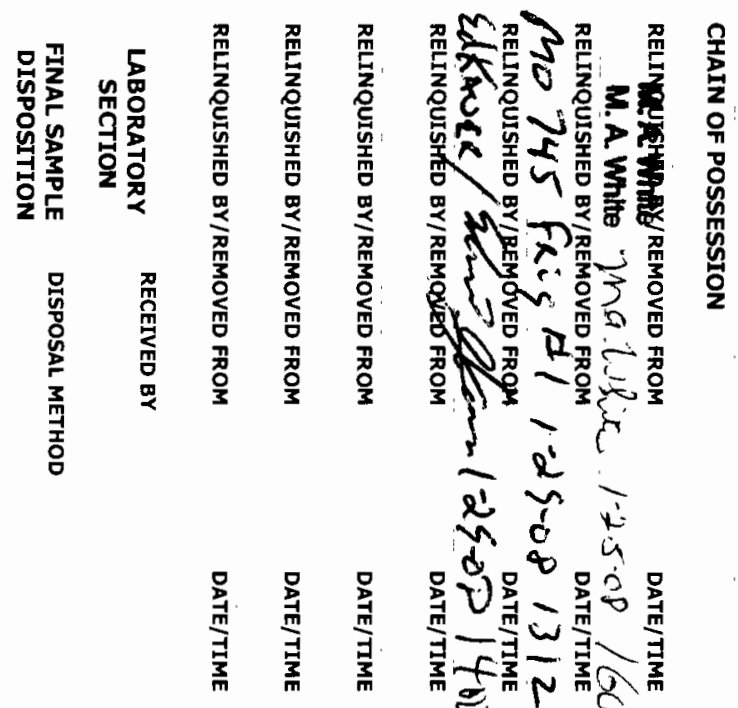

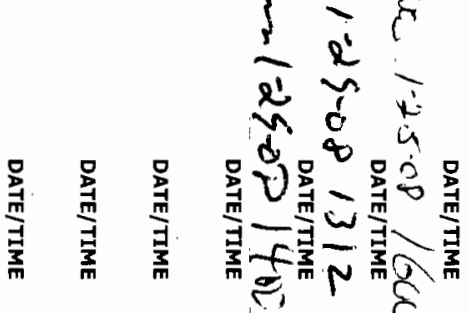

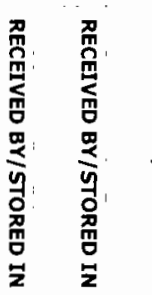

I

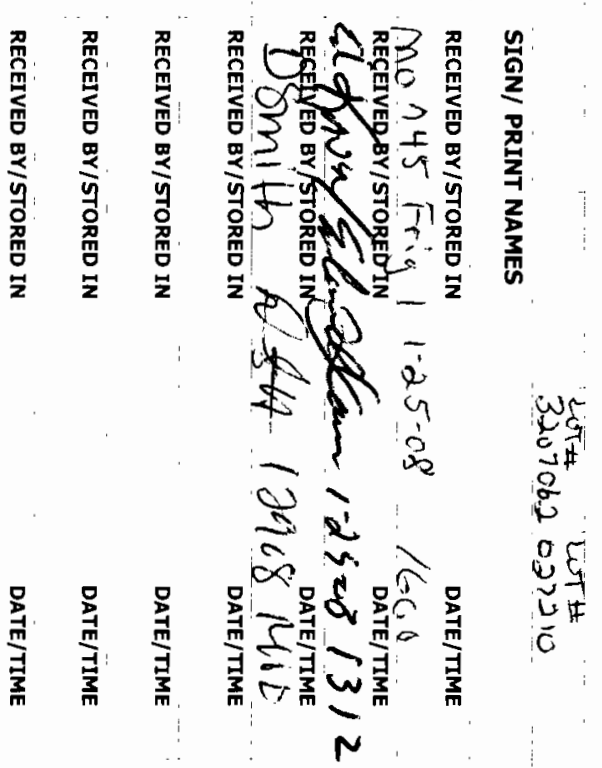

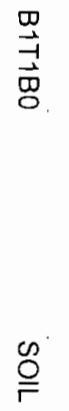

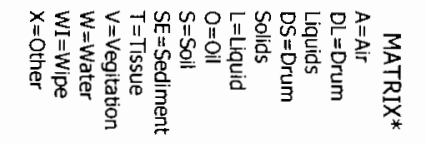

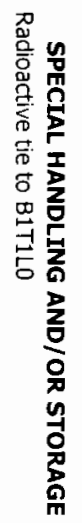

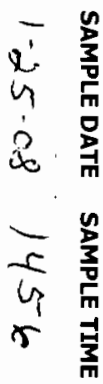

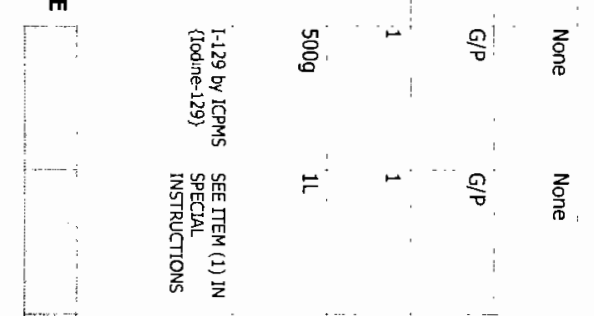

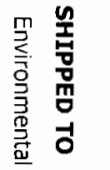

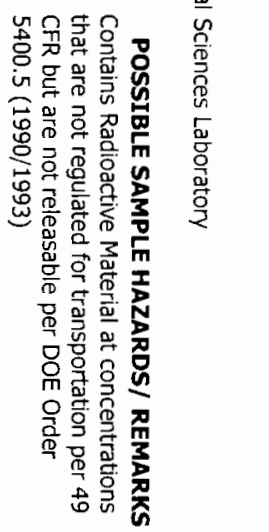

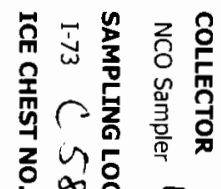

败蛋蛋

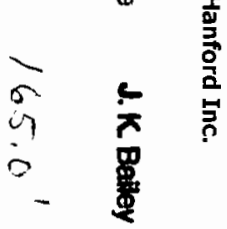

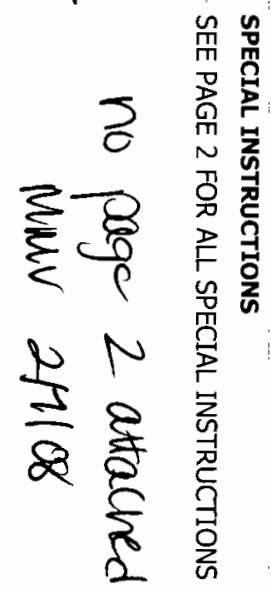

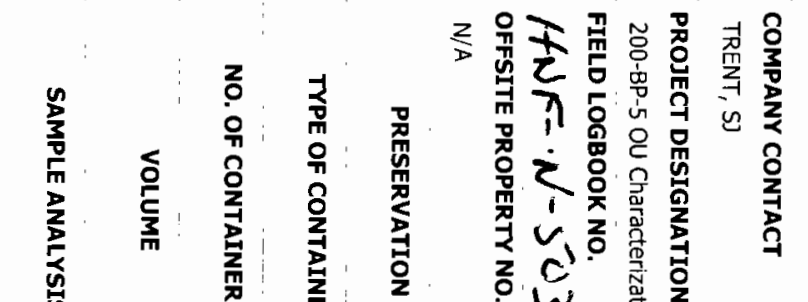

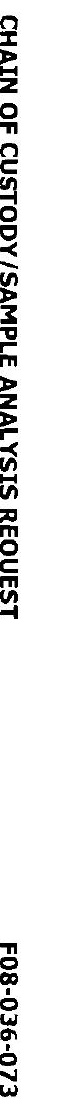

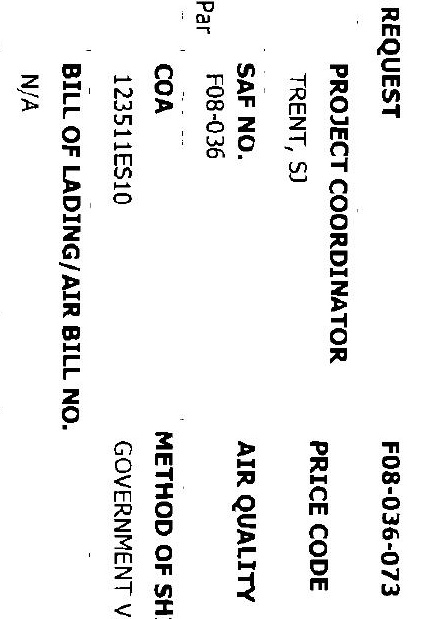

$\stackrel{\infty}{2}$

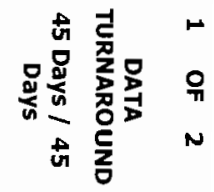



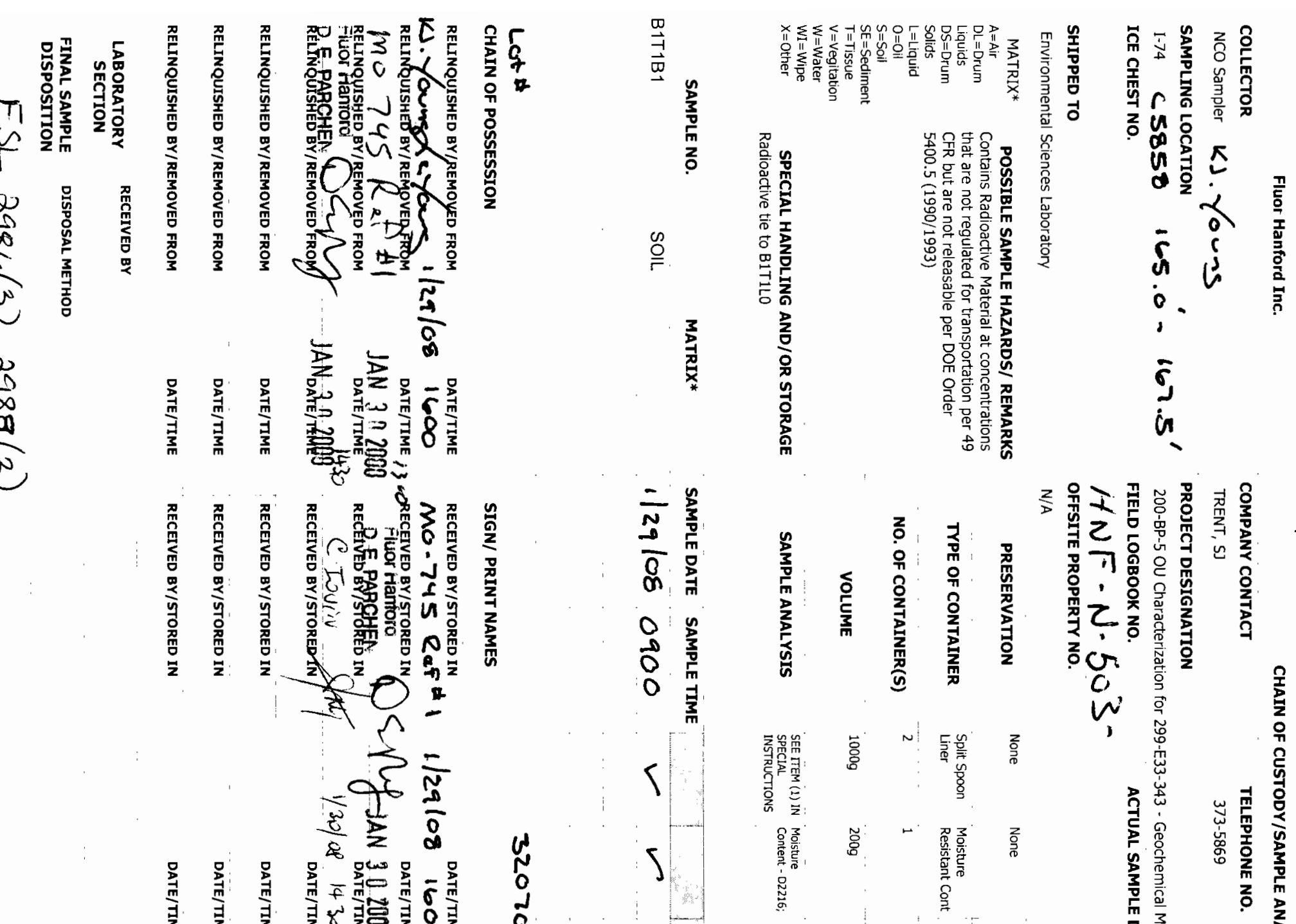

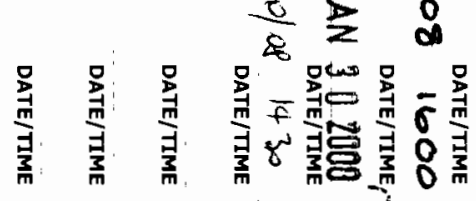

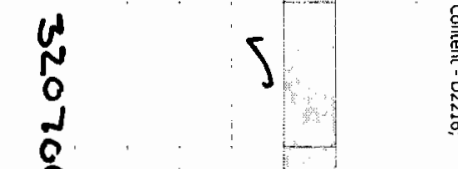

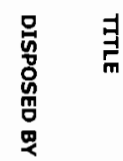
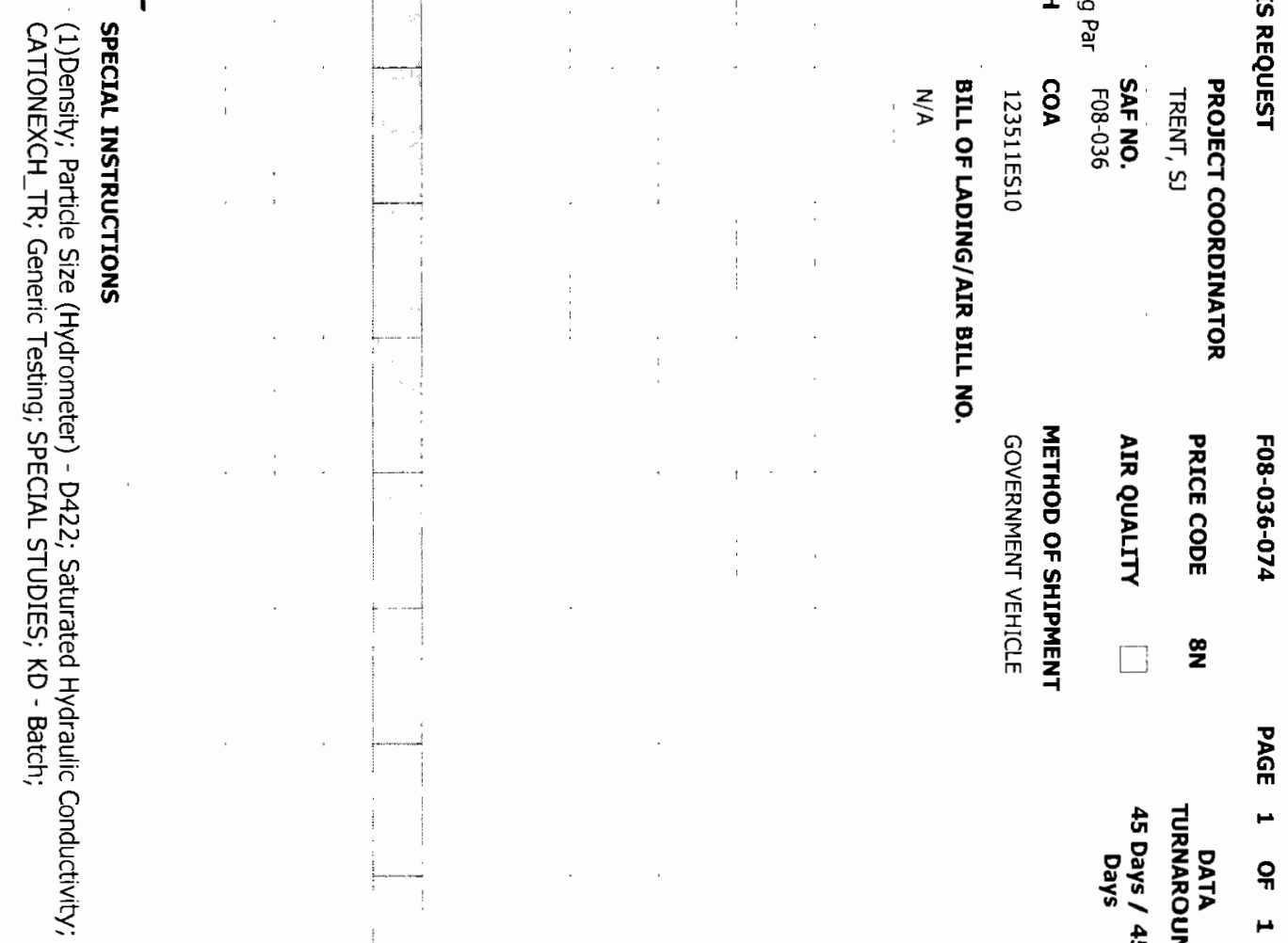

$\begin{array}{ll}\frac{9}{2} & \frac{D}{2} \\ \frac{7}{m} & \frac{7}{3} \\ \frac{3}{3} & \frac{7}{3} \\ \frac{3}{m}\end{array}$ 


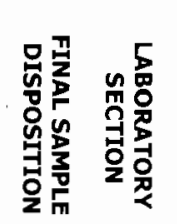

$\|$

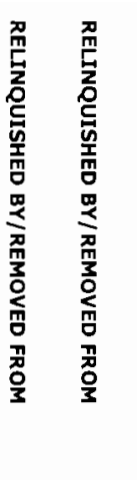

II

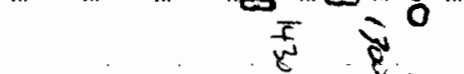

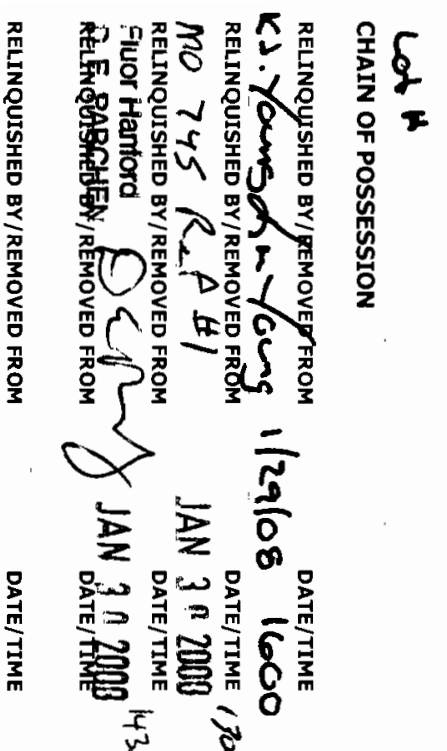

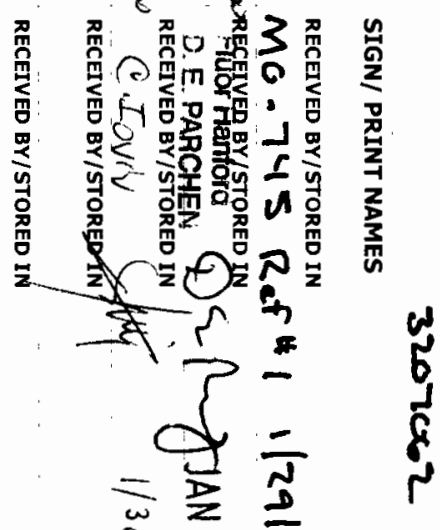

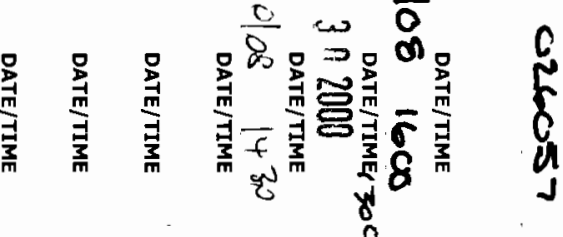

量

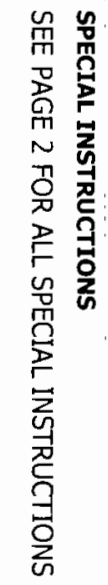

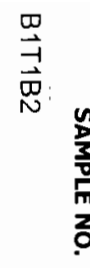

$\stackrel{8}{\circ}$

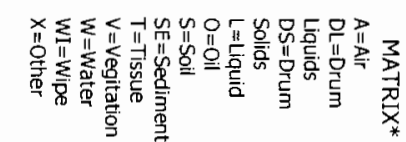

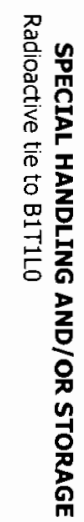

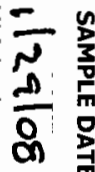

$\overbrace{\substack{0 \\ \frac{1}{3}}}^{\frac{n}{3}}$

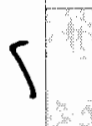

$\Gamma$

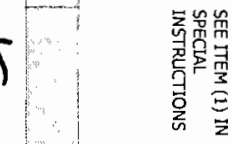

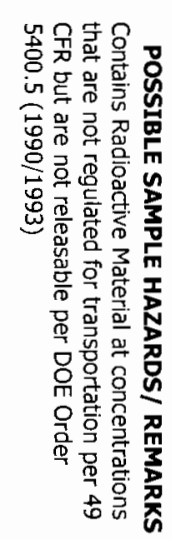
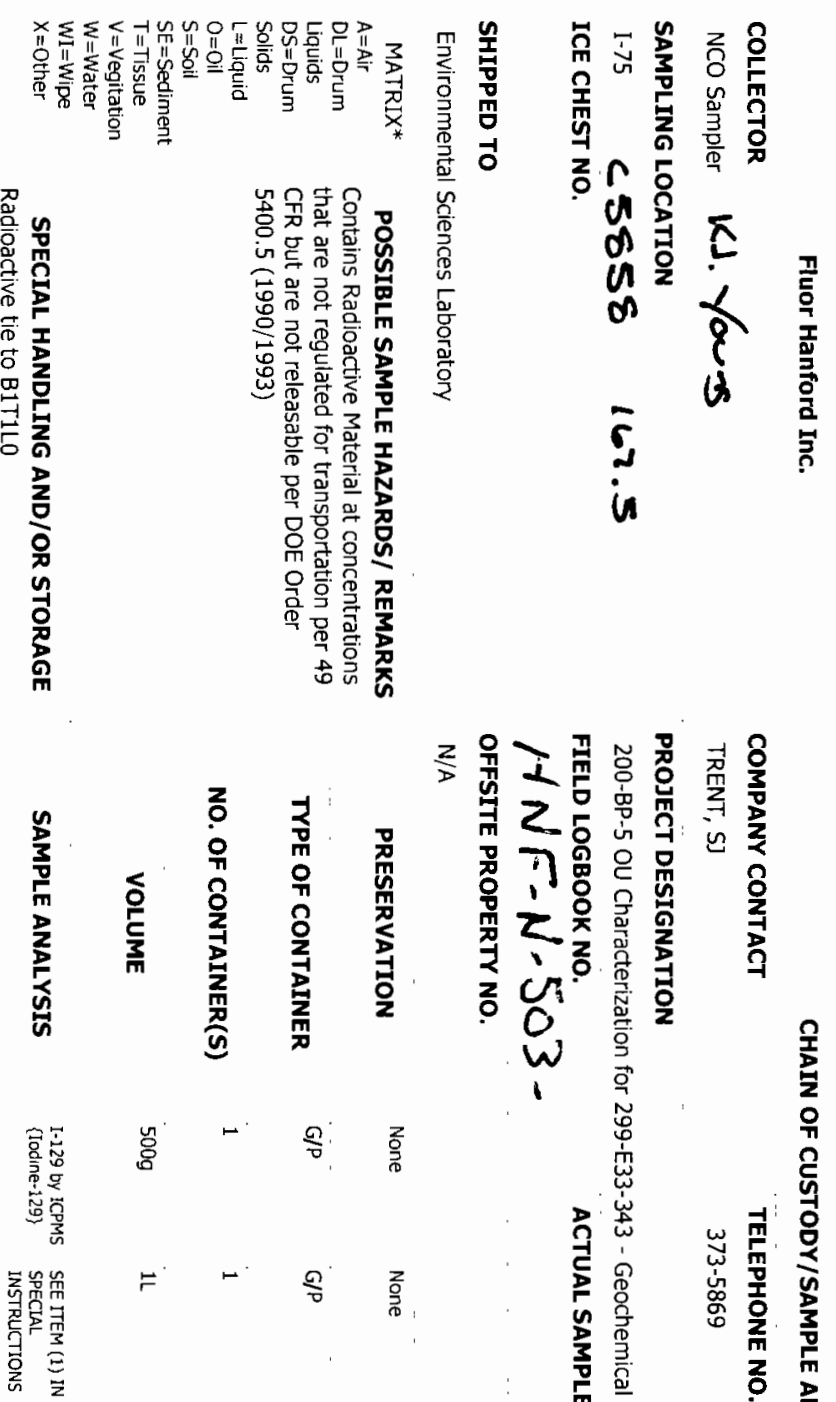

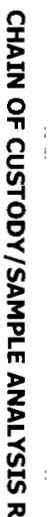
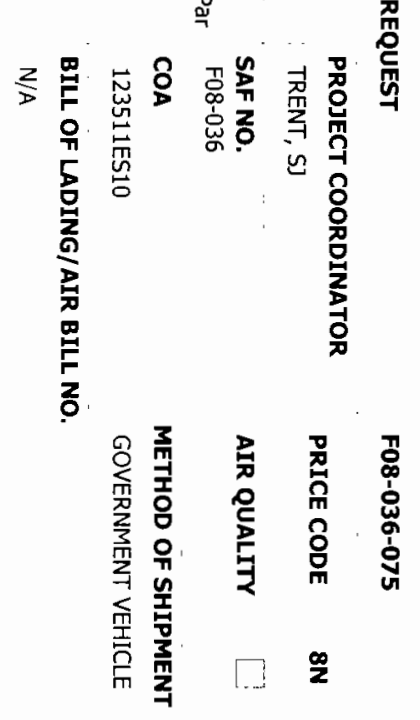

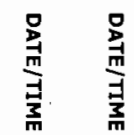

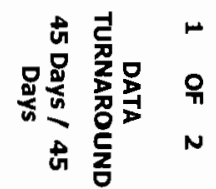




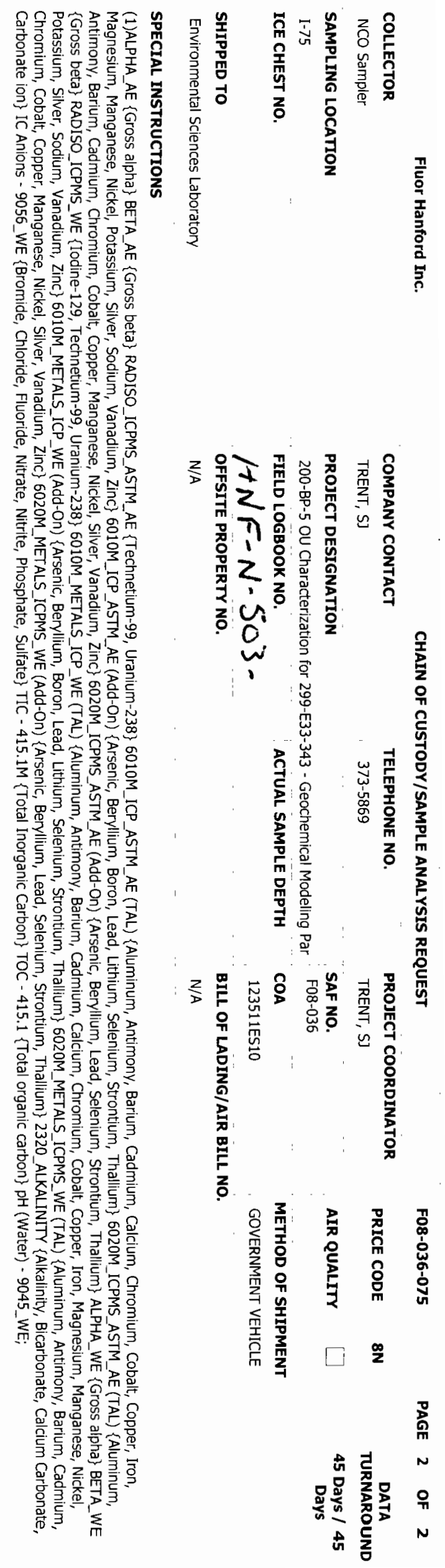




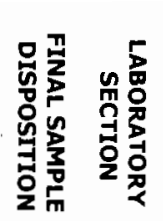

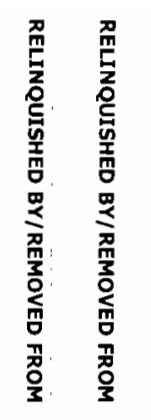

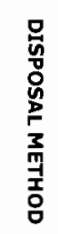

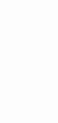

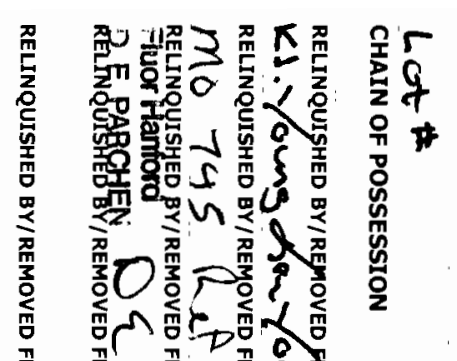

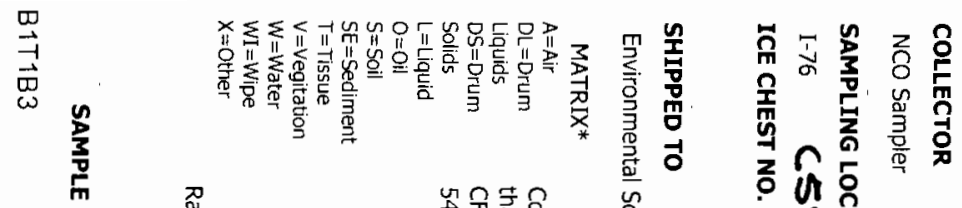

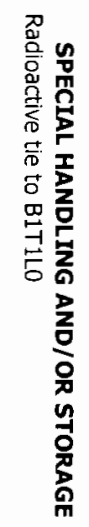
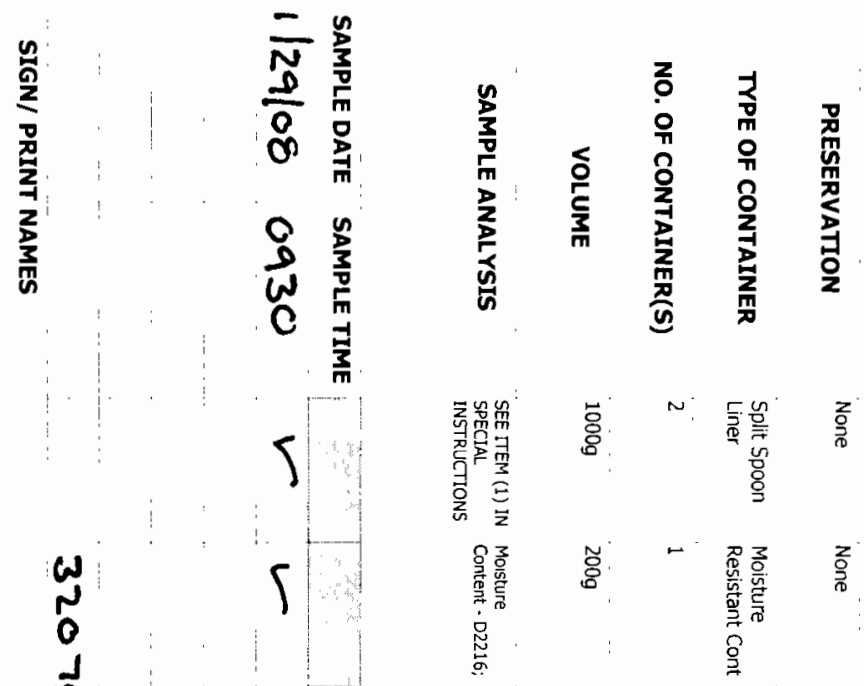

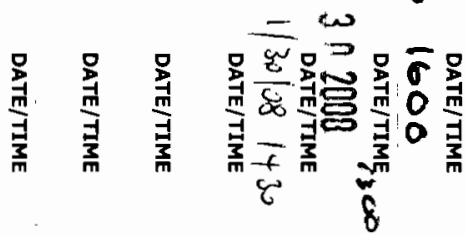

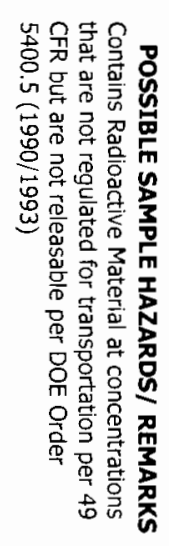

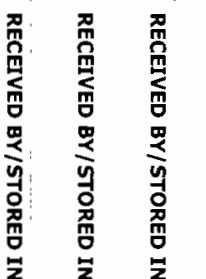

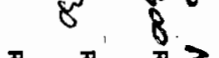

昜 寻

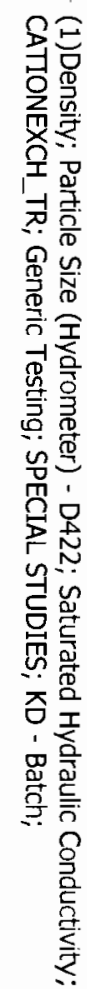

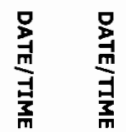

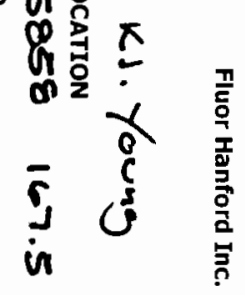

$\bar{c}$

$\dot{0}$

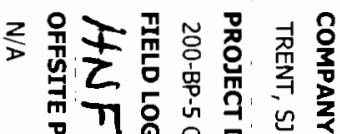

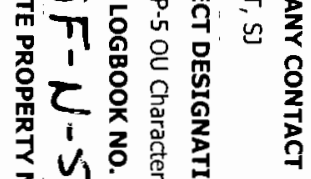
zo

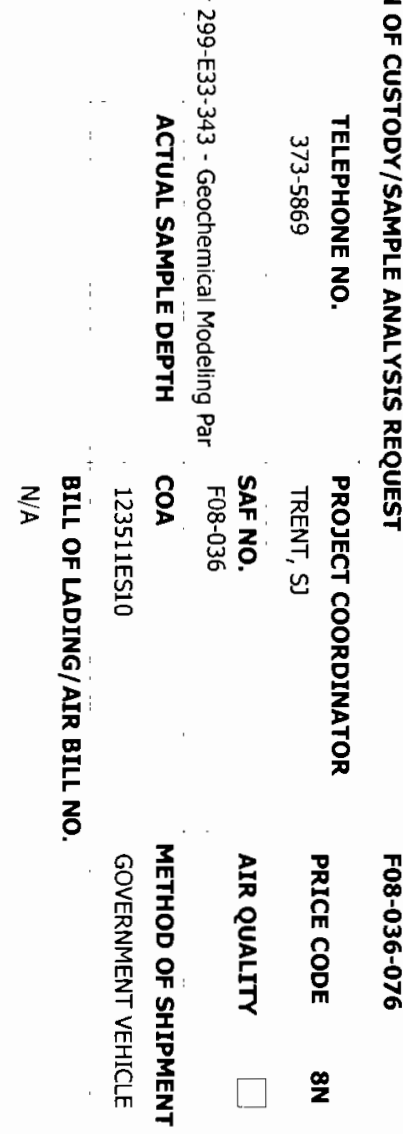




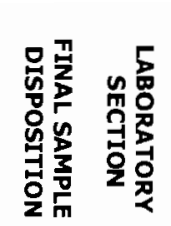

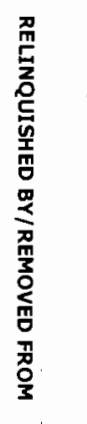

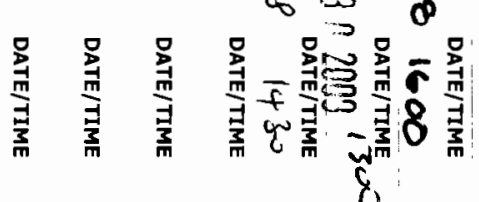

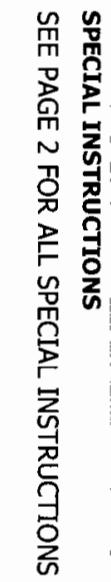

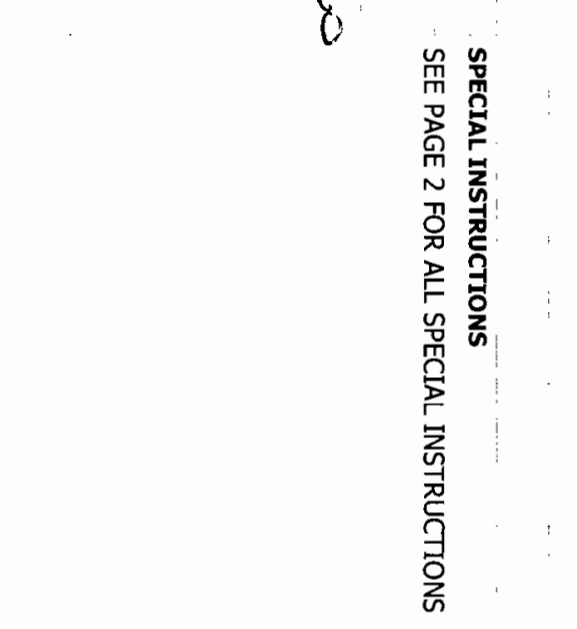

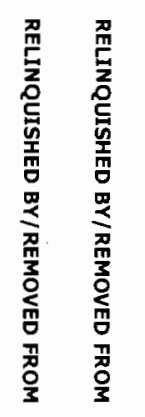

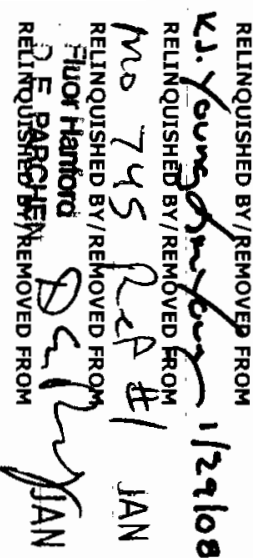

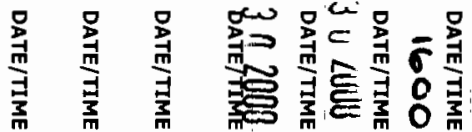

क्ष $\overline{\tilde{\varepsilon}}$

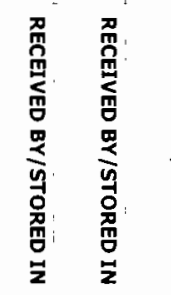

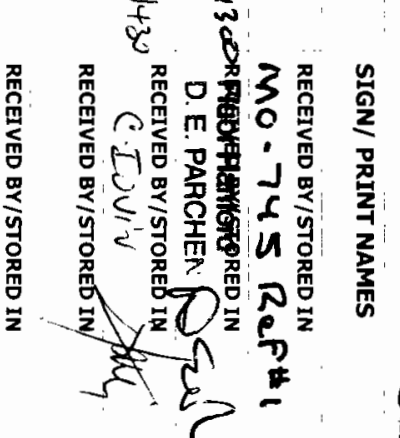

$\frac{\bar{z}}{\frac{\delta}{2}} \sum_{\infty}^{\frac{1}{2}}$

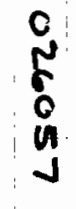

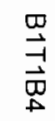

$\stackrel{\mathscr{O}}{\digamma}$

$\frac{1}{3}$
$\frac{5}{3}$
0
0

$\frac{0}{8} \frac{n}{3}$

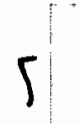

量

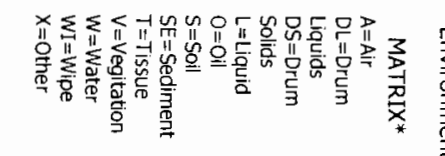

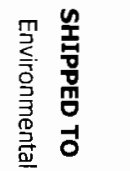

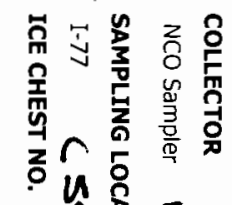

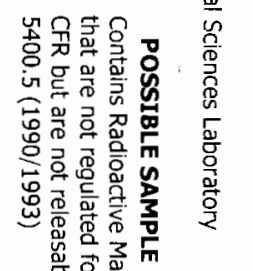

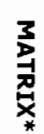

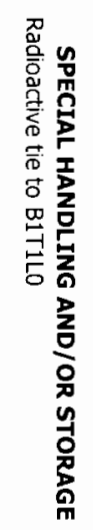

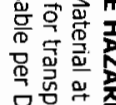

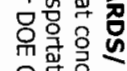

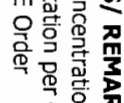

古总突

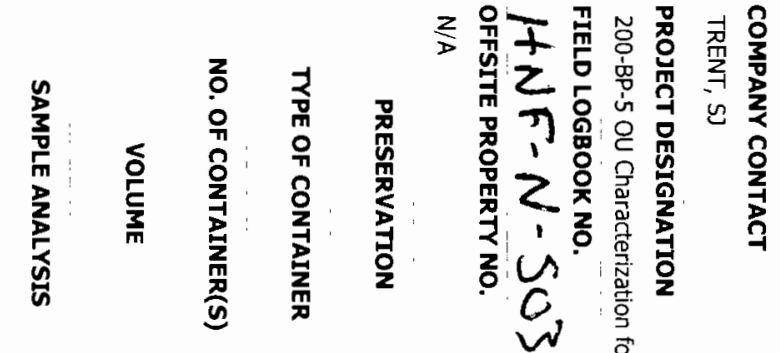

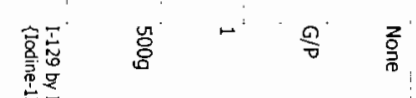

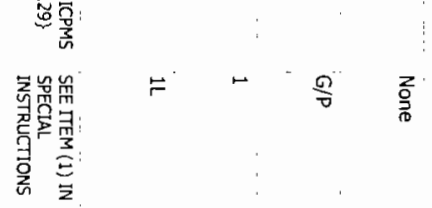

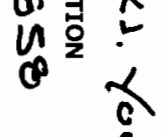

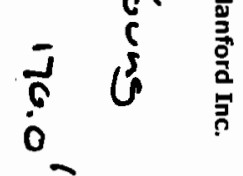



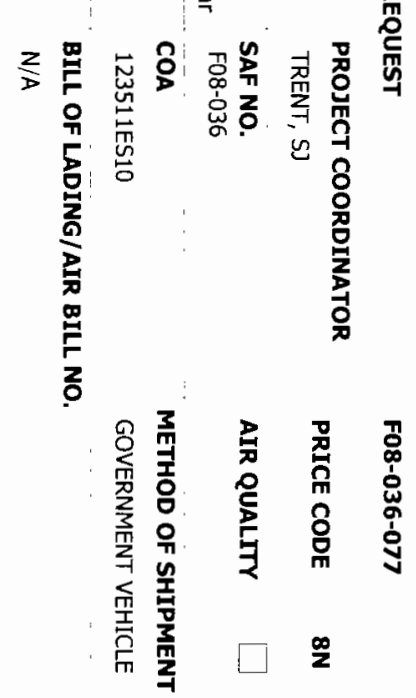

旁

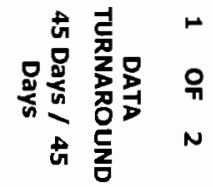



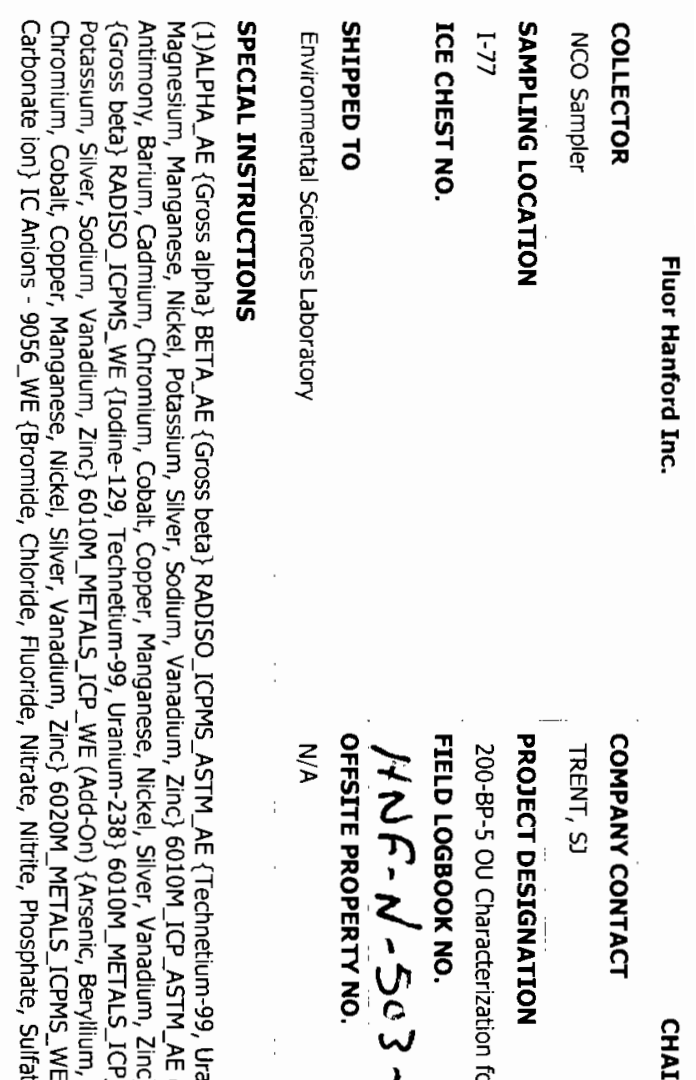

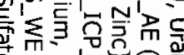

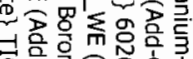

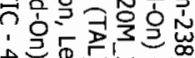

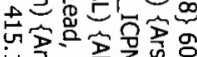

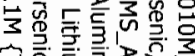

品思害当罗

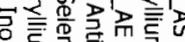

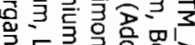

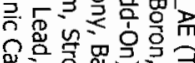

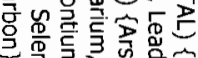

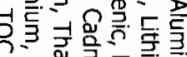

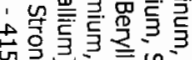

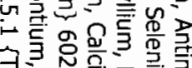

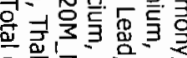

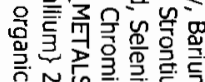

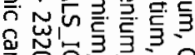

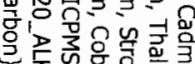

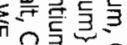

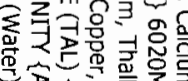

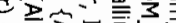

唂

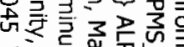

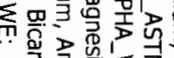

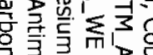

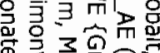

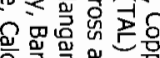

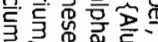

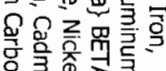

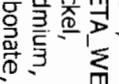

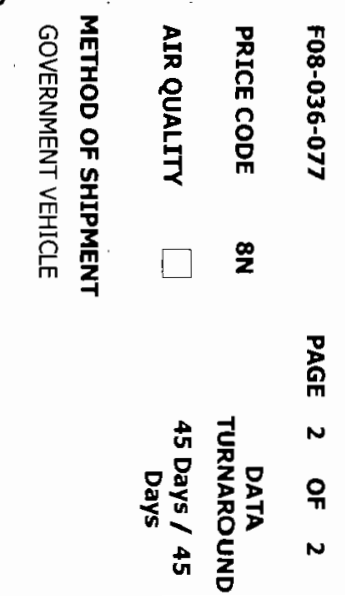




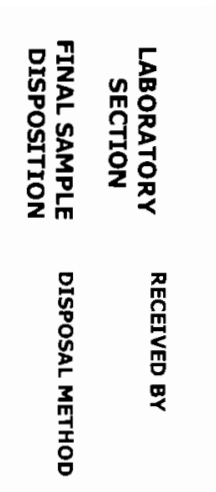

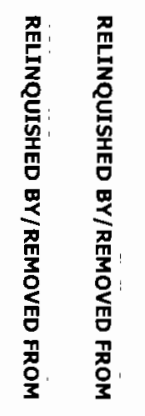

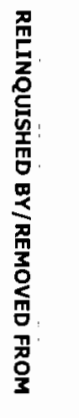

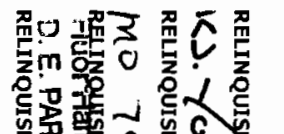

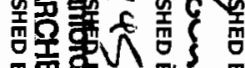

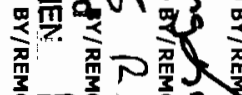

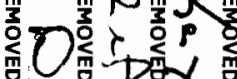

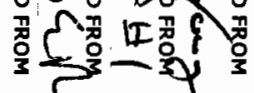

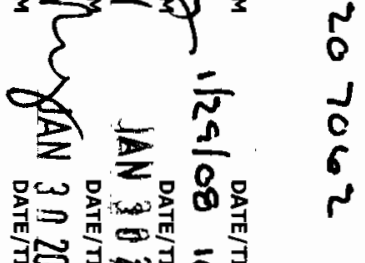

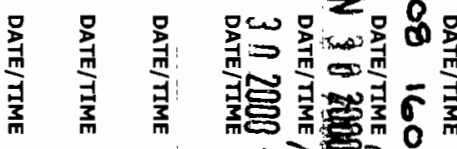

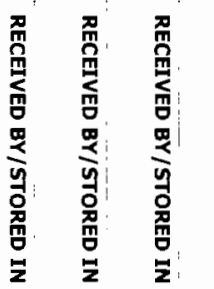

政造 8

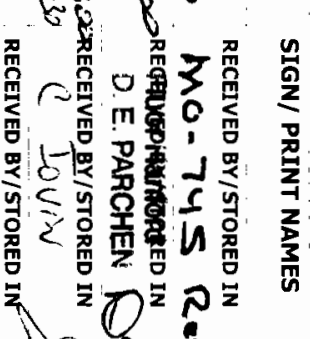

L $\pi$ \%

है $5=$

$=\frac{\pi}{2} \frac{1}{2}$

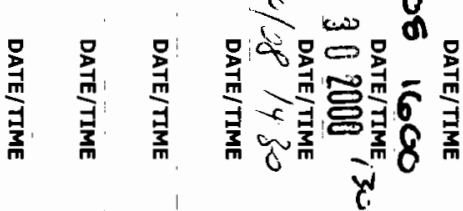

总

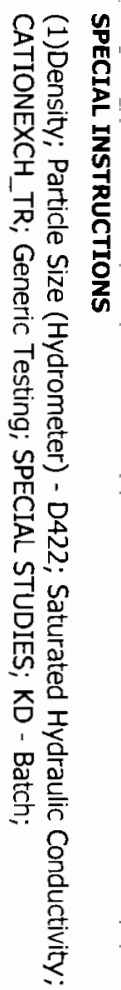

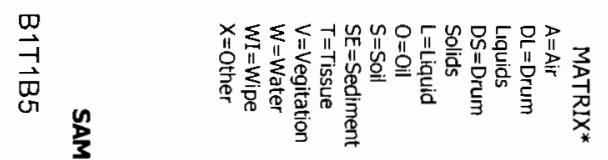

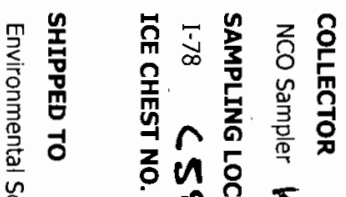
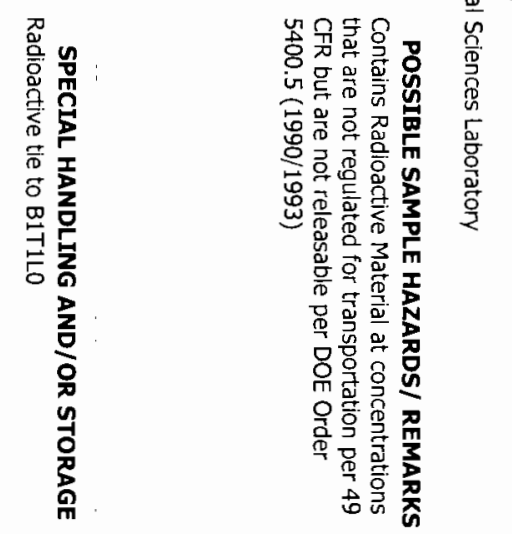

of $\pi$

$\stackrel{\mathscr{O}}{\stackrel{\circ}{ }}$

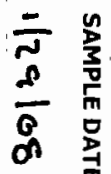

$=\frac{n}{\sqrt{3}}$

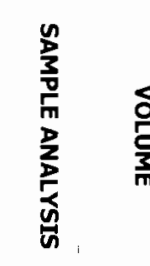

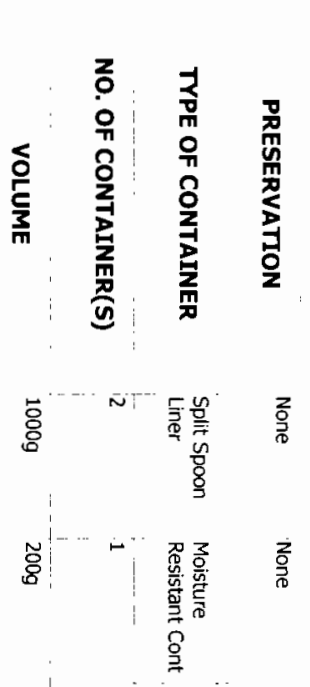

$\frac{2}{>}$

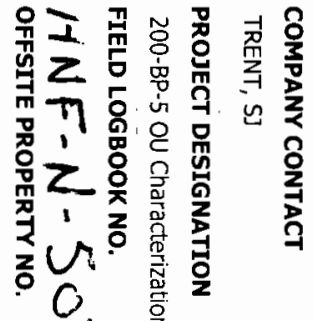

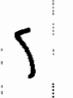

章策

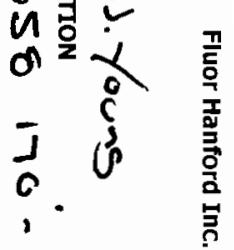

3

a 


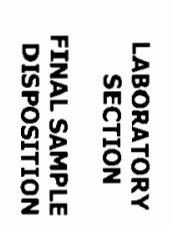

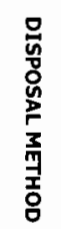

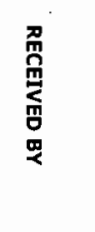

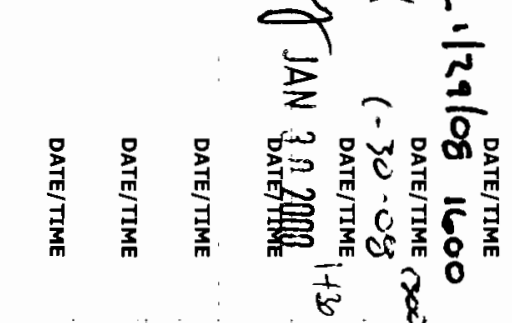
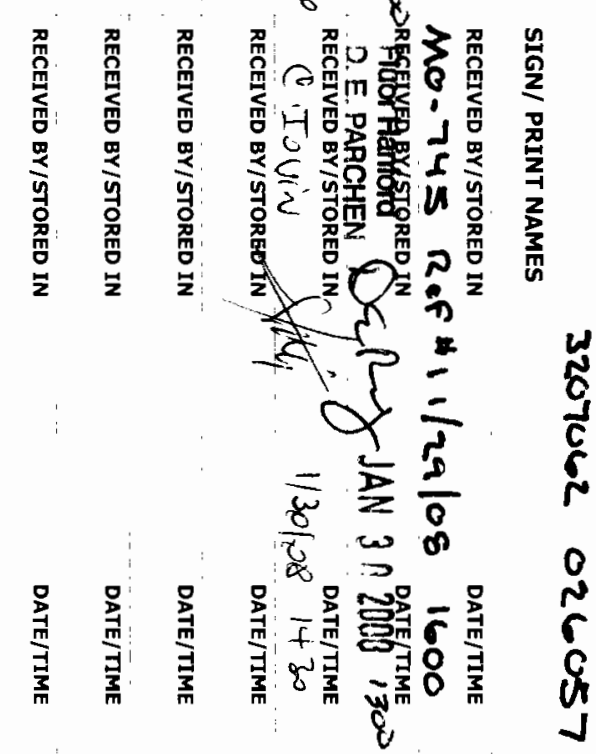

量

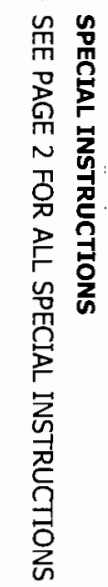

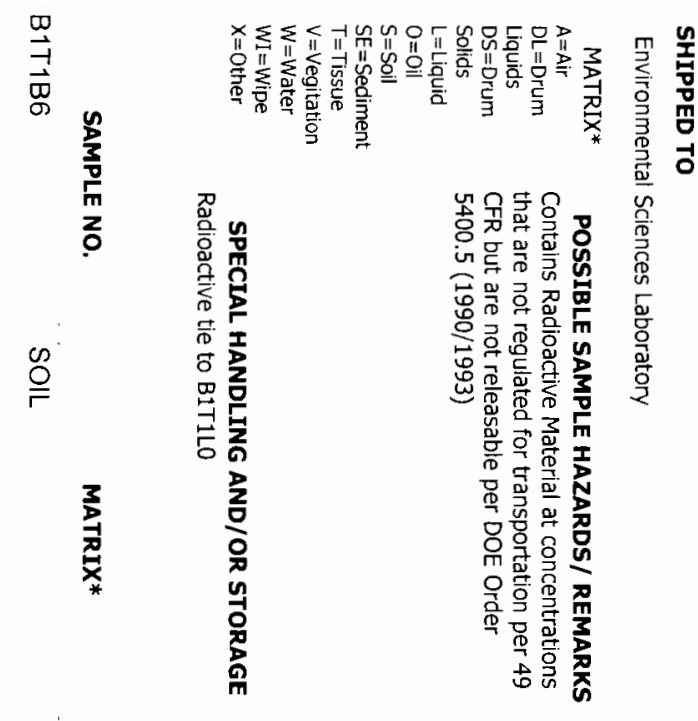

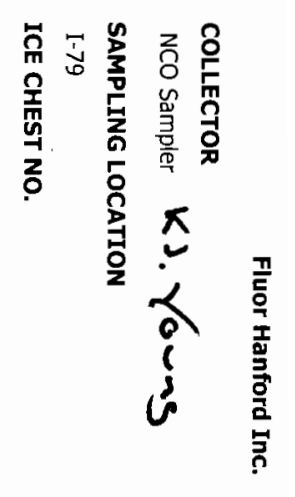

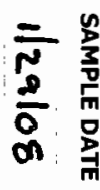

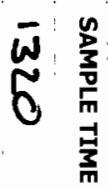

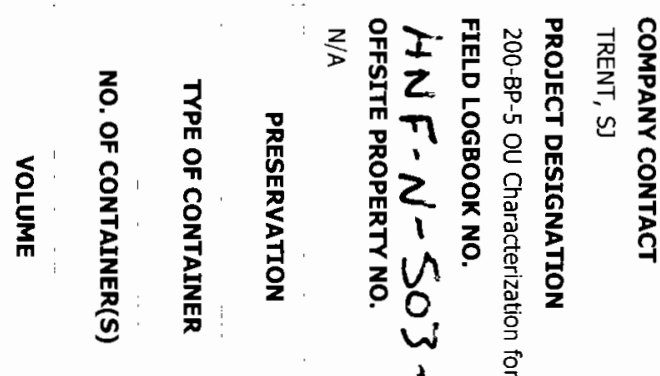

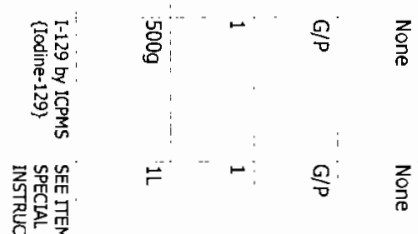

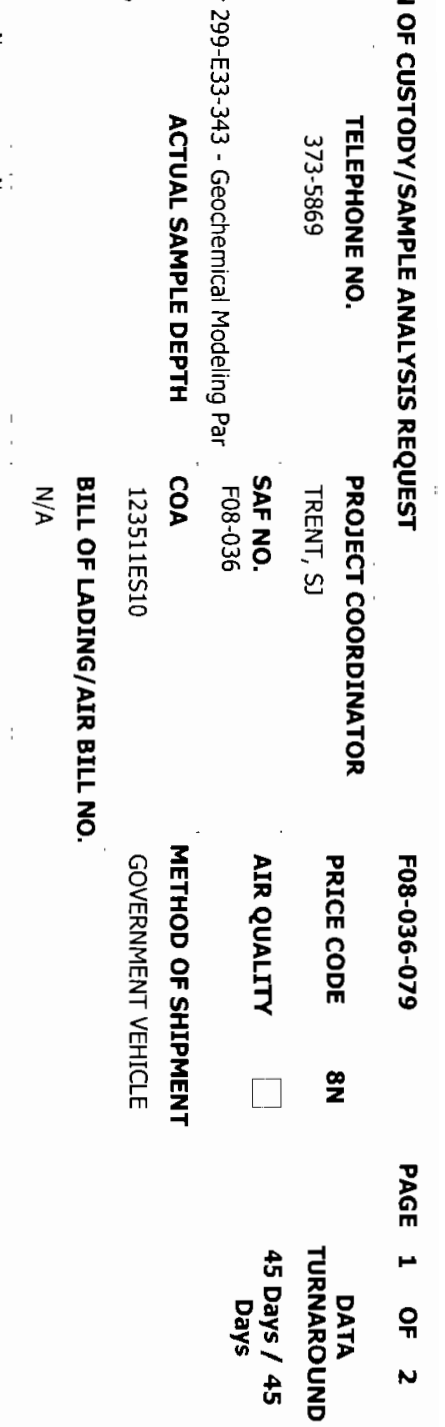




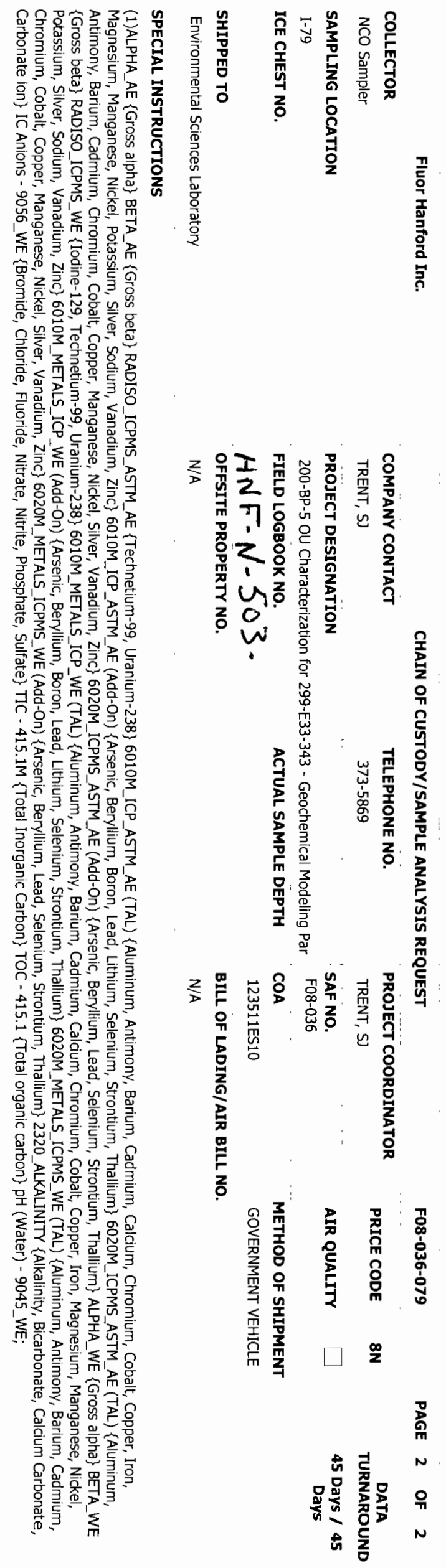




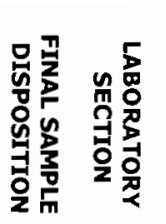

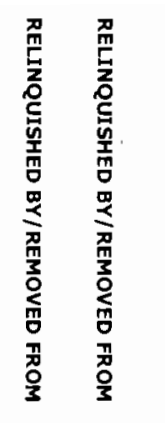

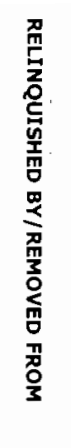

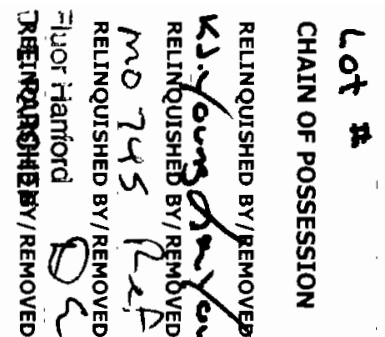

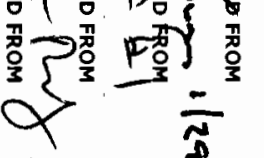

$=\overline{8}$

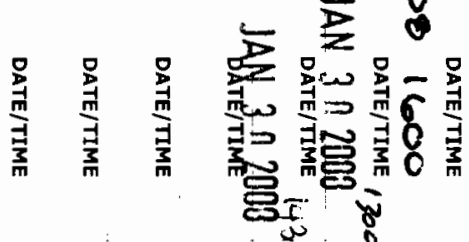

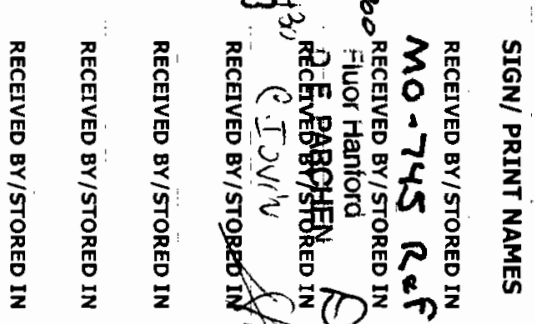

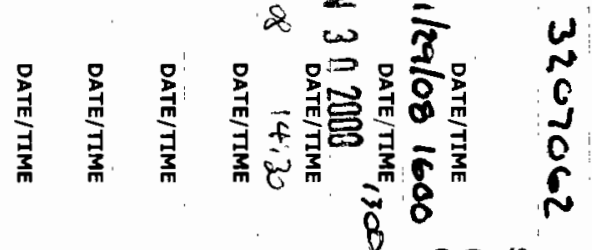

品 者

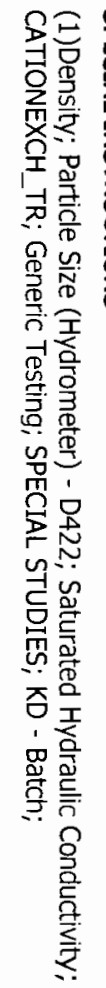

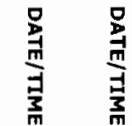

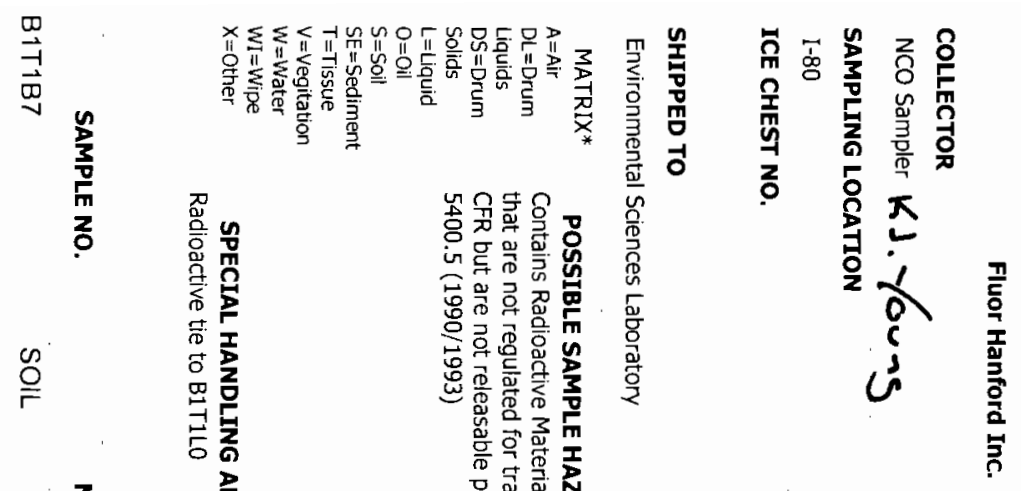

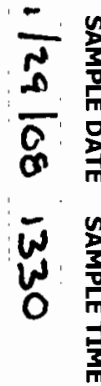

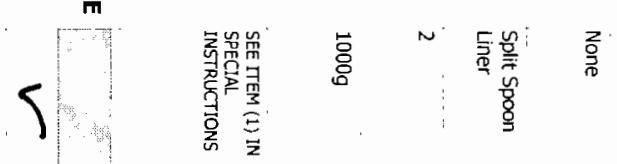

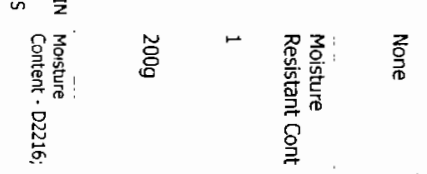

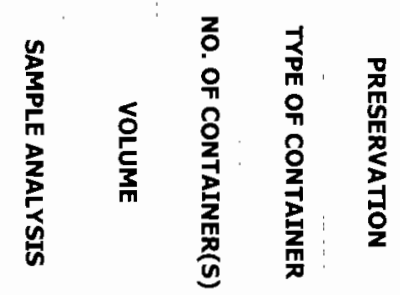

$2 ㅇ$

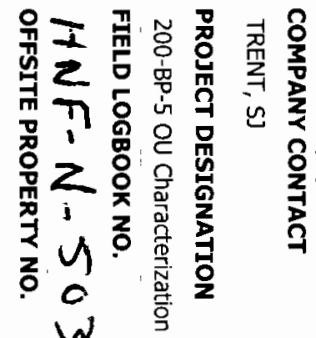

吕施

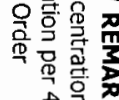

迹

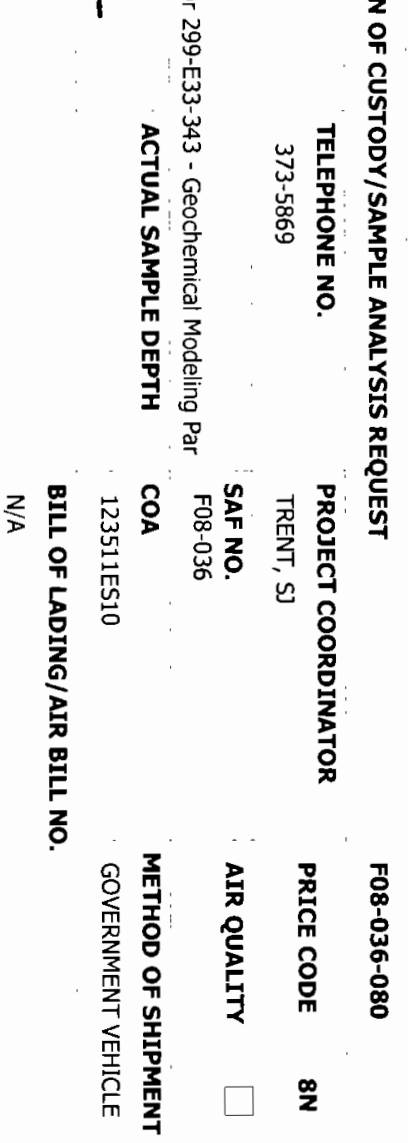




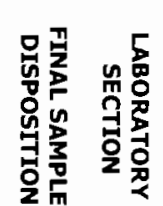

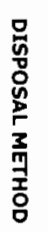

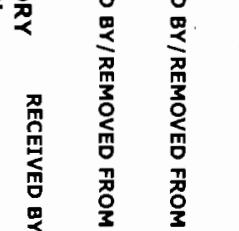

年

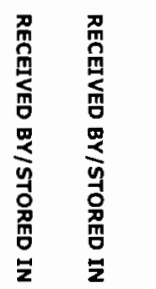

$$
\text { 备 }
$$
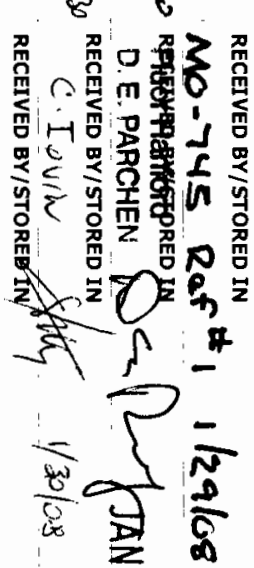

总总
率早

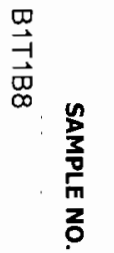

$\stackrel{\mathscr{O}}{\circ}$

$\underset{\sharp}{\stackrel{3}{3}}$

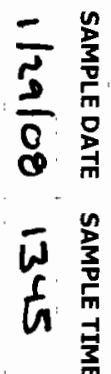

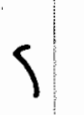

声

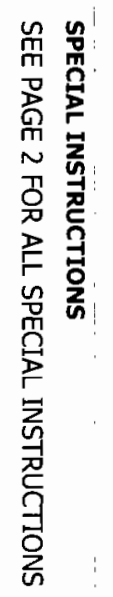

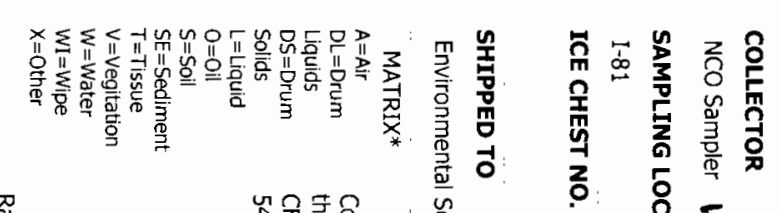

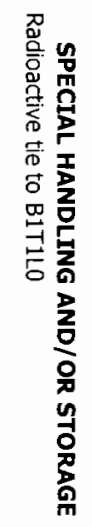

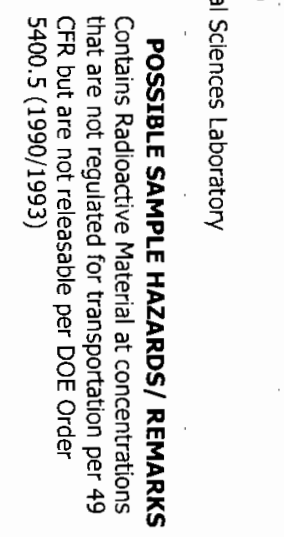

文

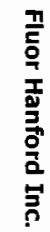
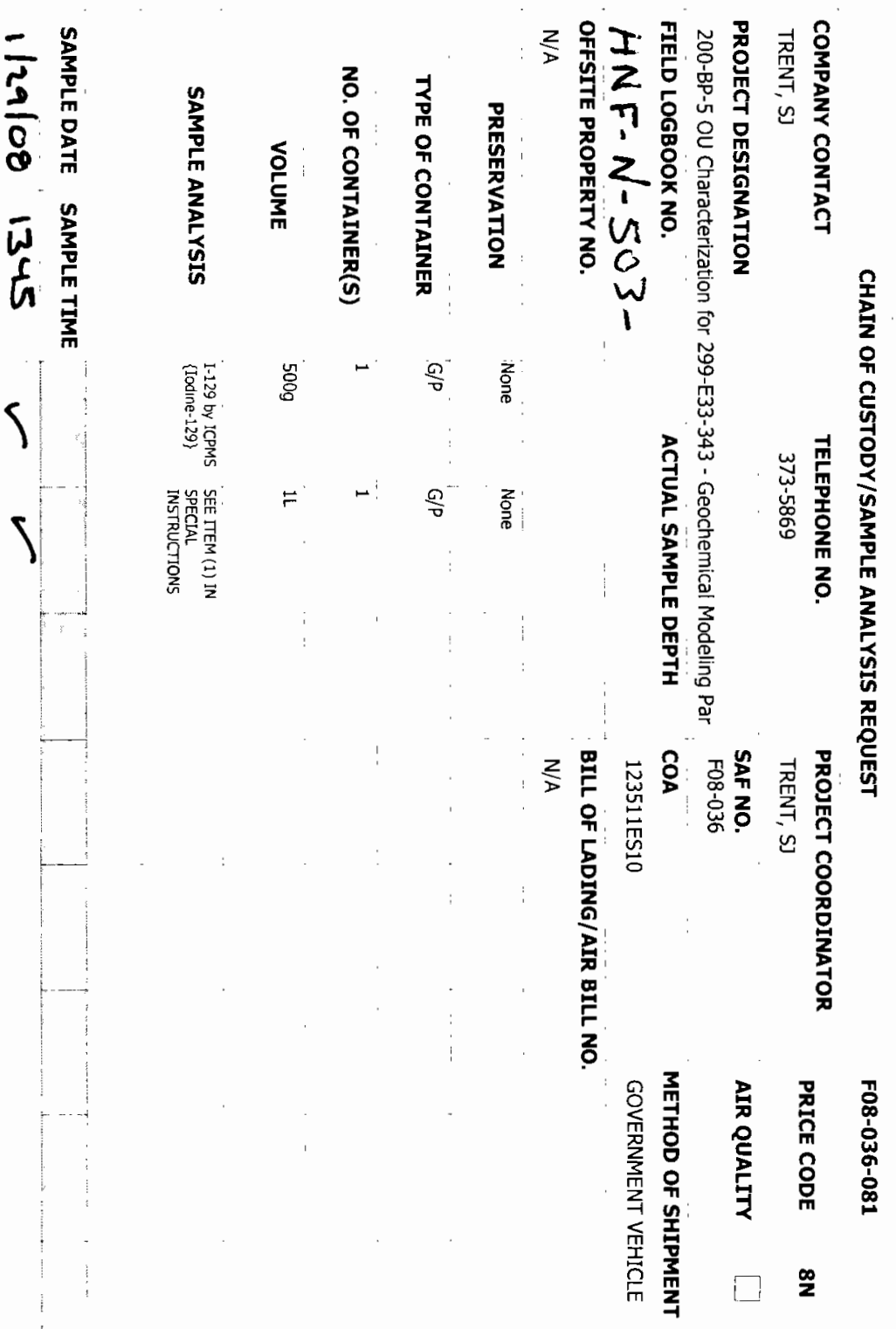

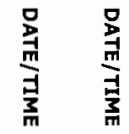

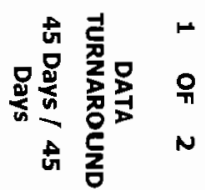




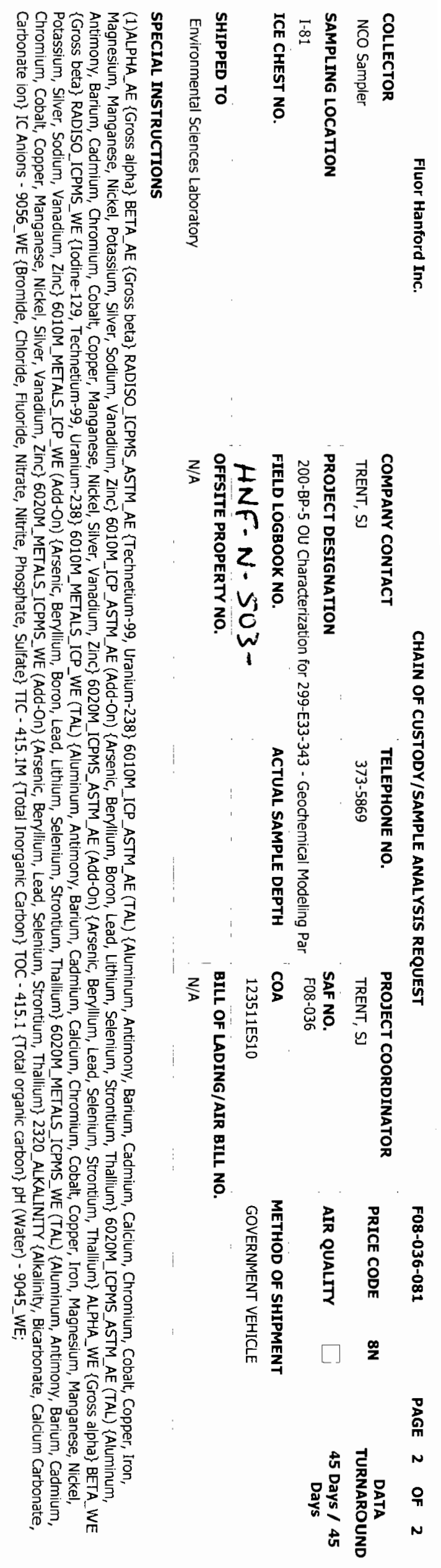




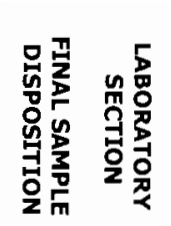
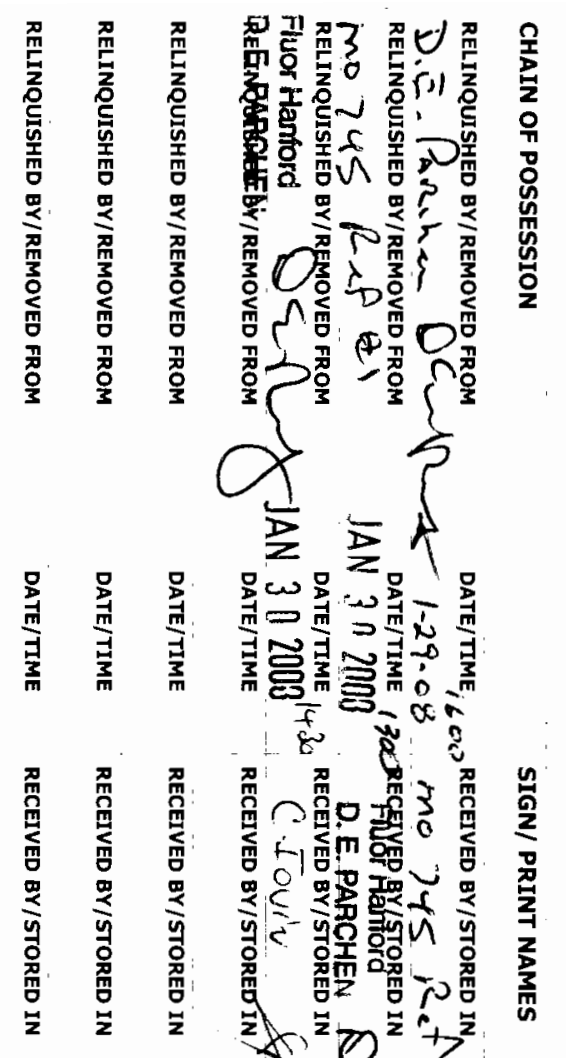

星

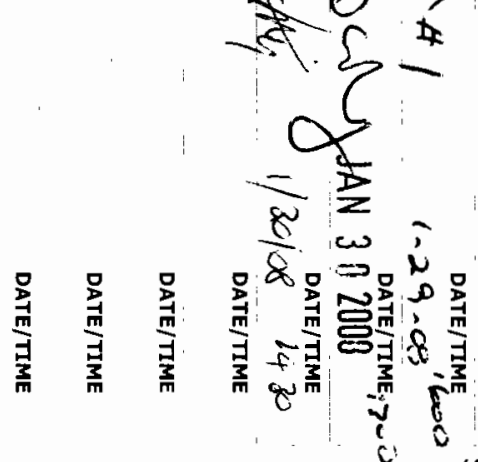

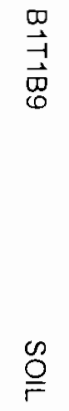

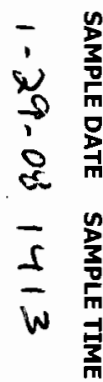

$w$
$w$
0
$x$
$x$

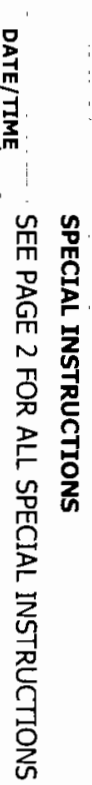

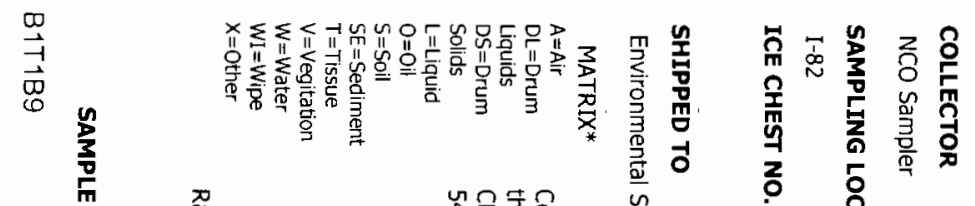

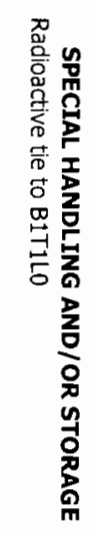

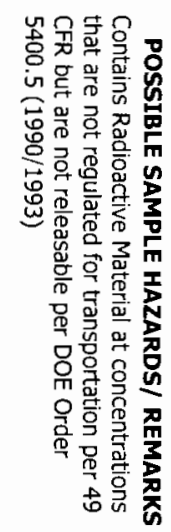

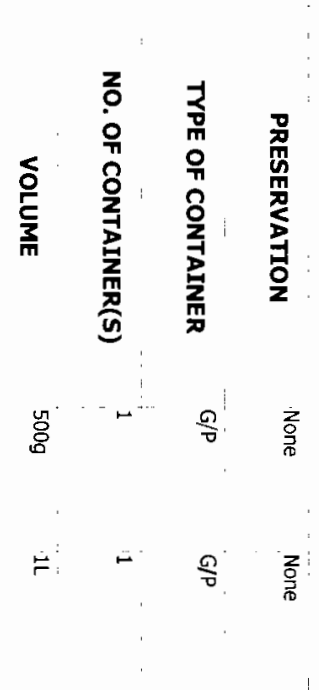

욕

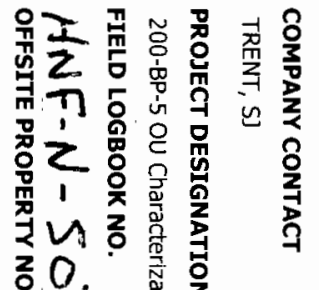

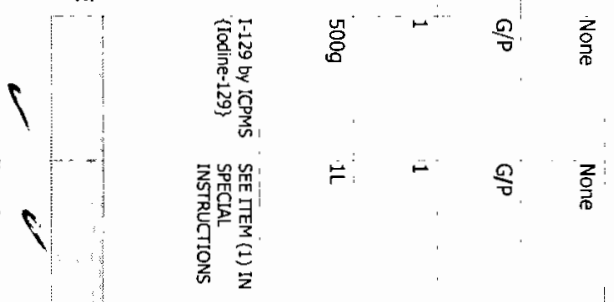<smiles>C1CCCC1</smiles><smiles>C1CC1</smiles>

z

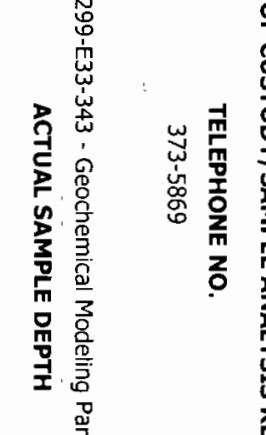

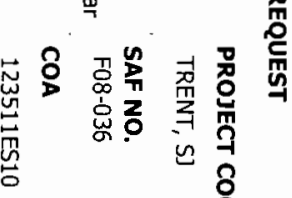

总

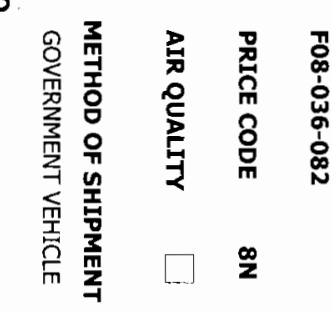

号

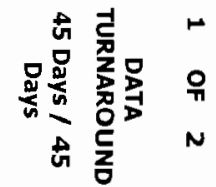




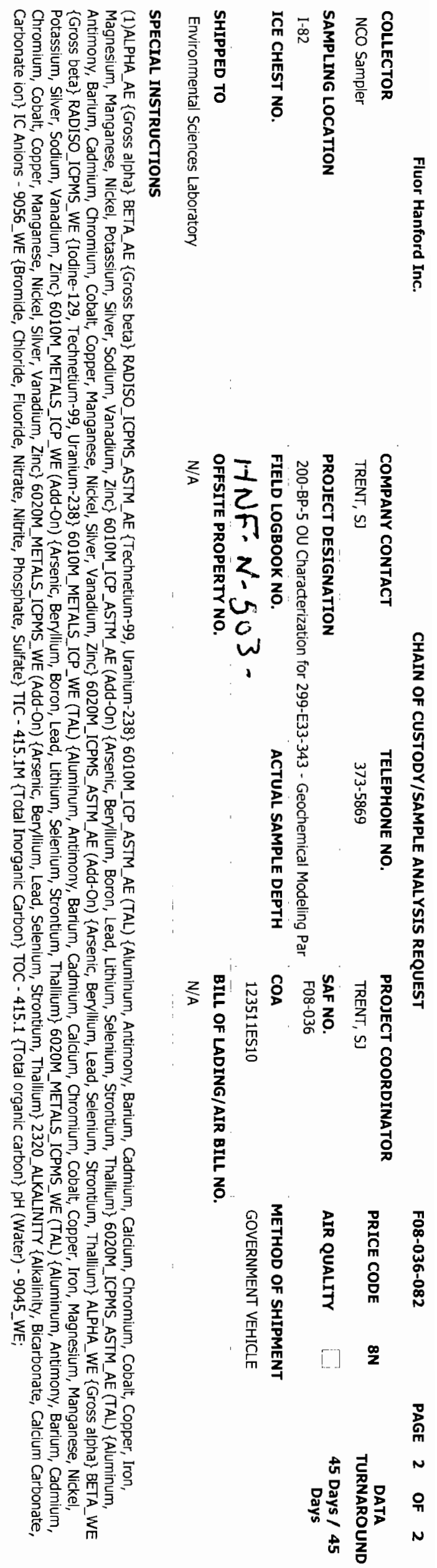




\section{$\|$}
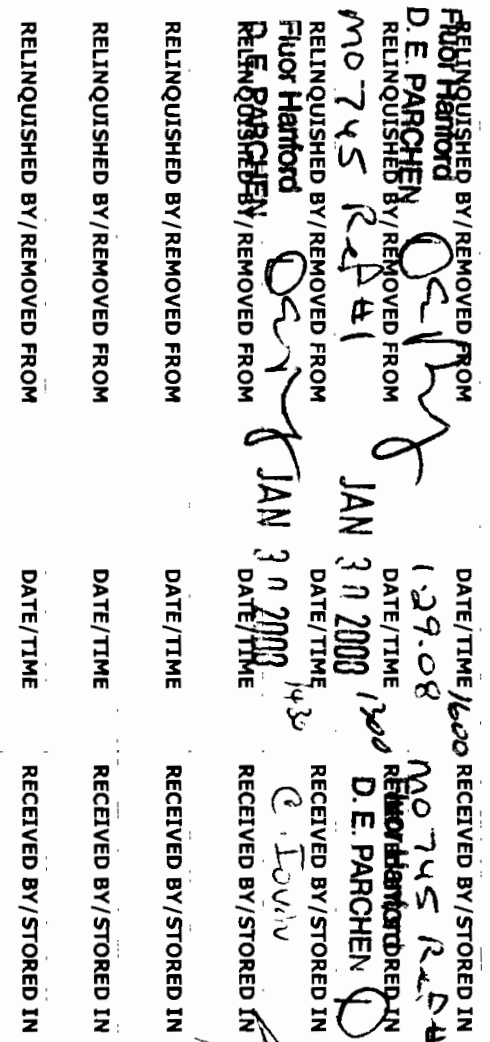

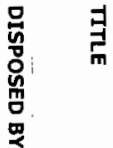

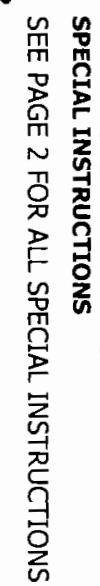

\section{. 每}

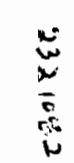

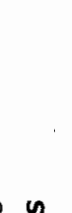

侐

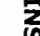
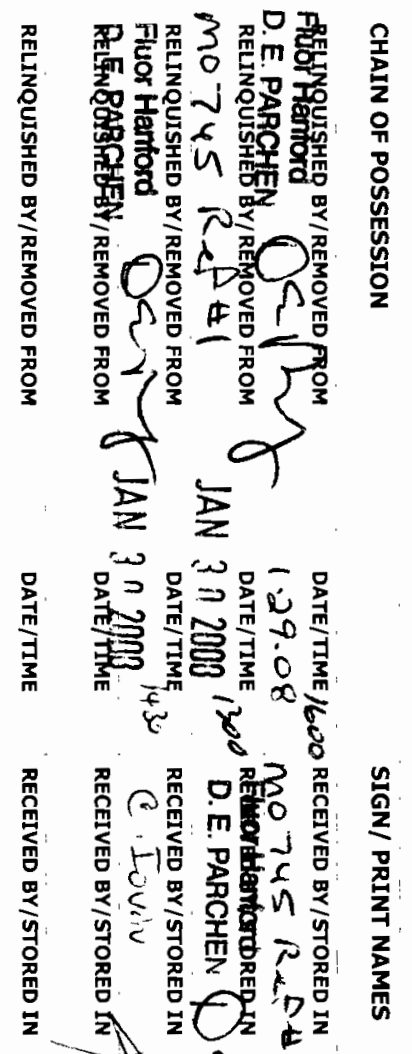

$\stackrel{\infty}{\vec{\exists}}$

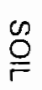

妾

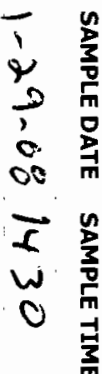

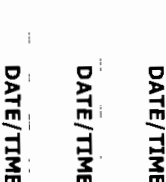<smiles>[C+]1CCCC1</smiles>

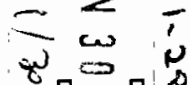

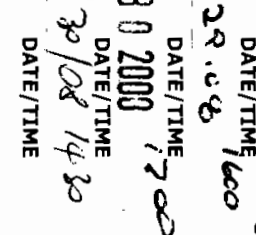




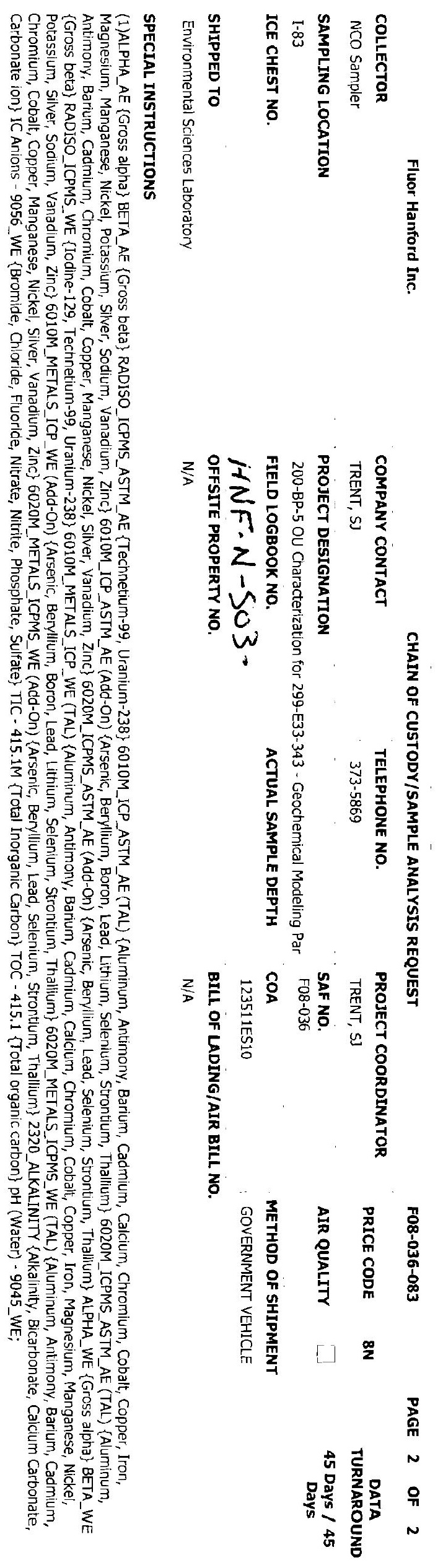




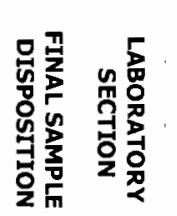
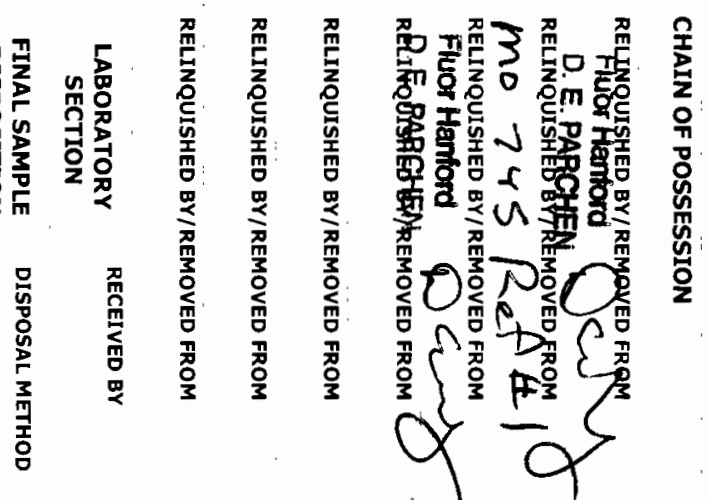

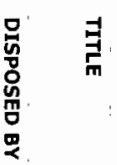
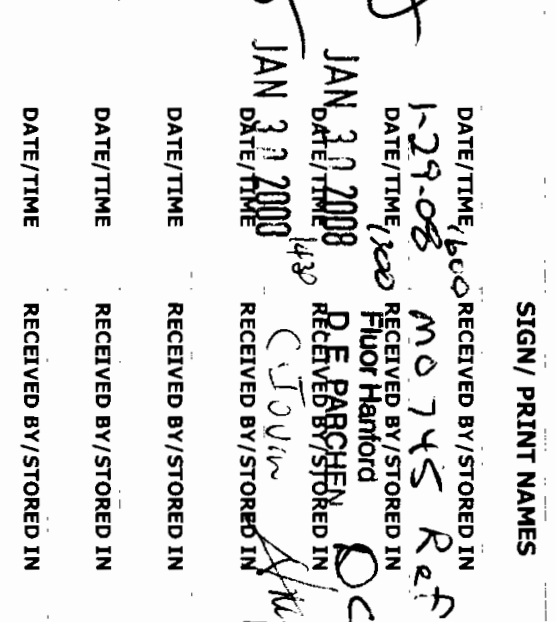

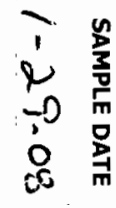

4

$\infty \quad$

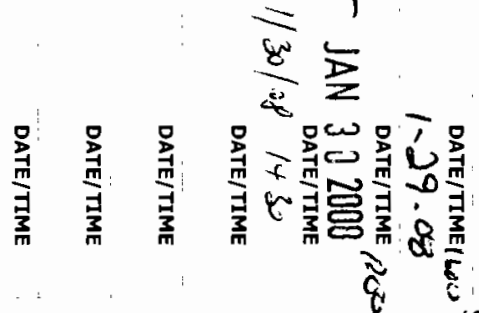

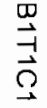

$\stackrel{\infty}{\rightleftharpoons}$

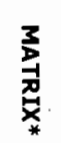

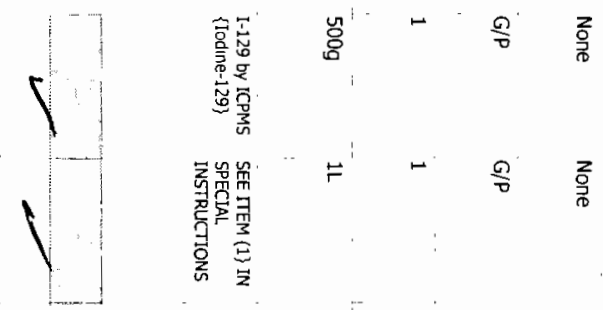

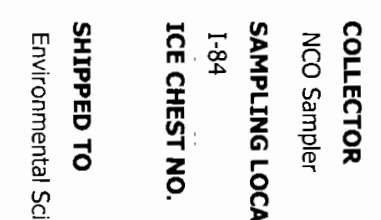

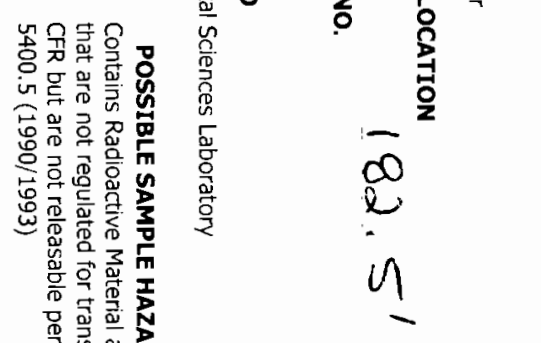

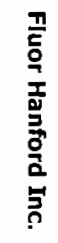
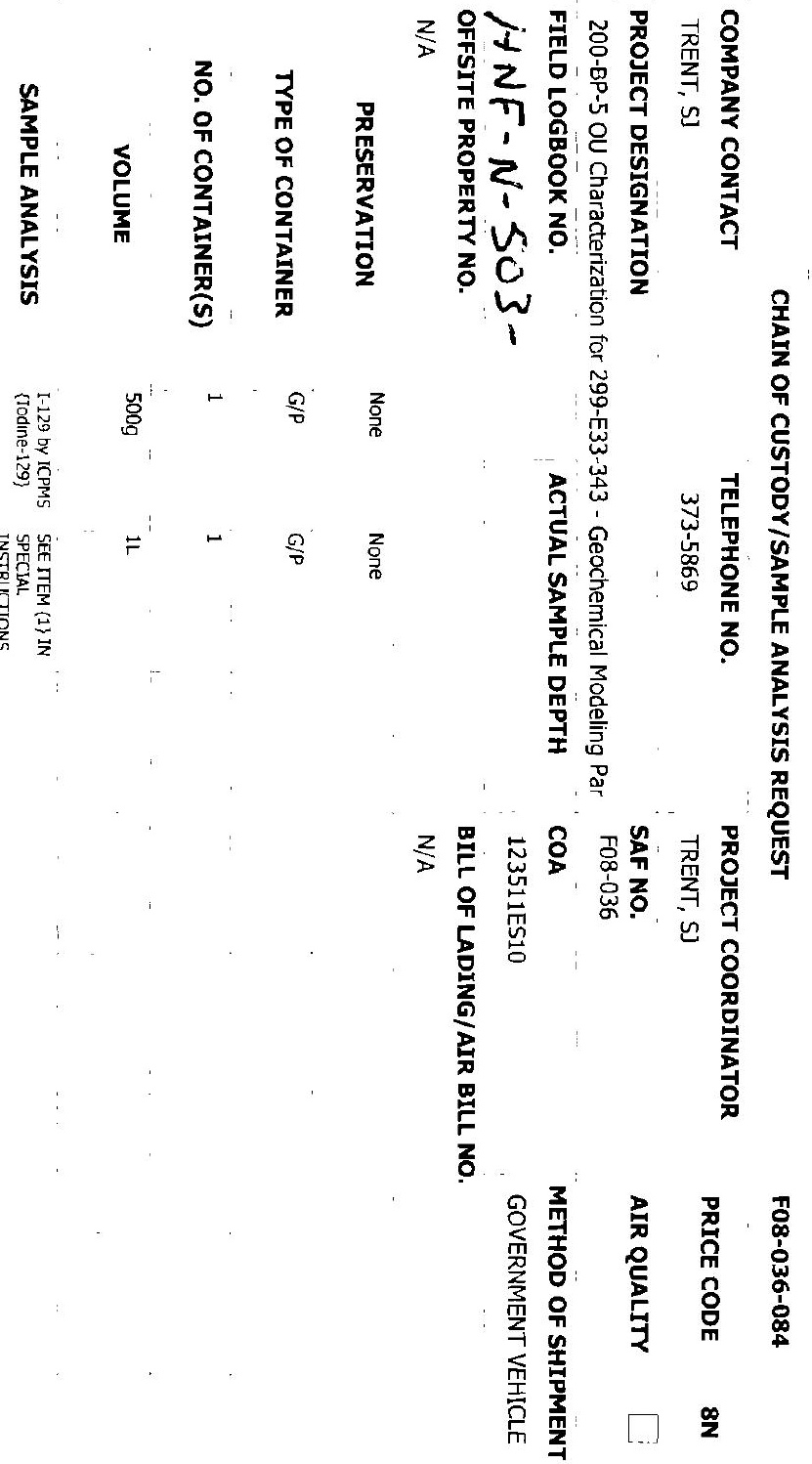

尊

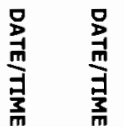




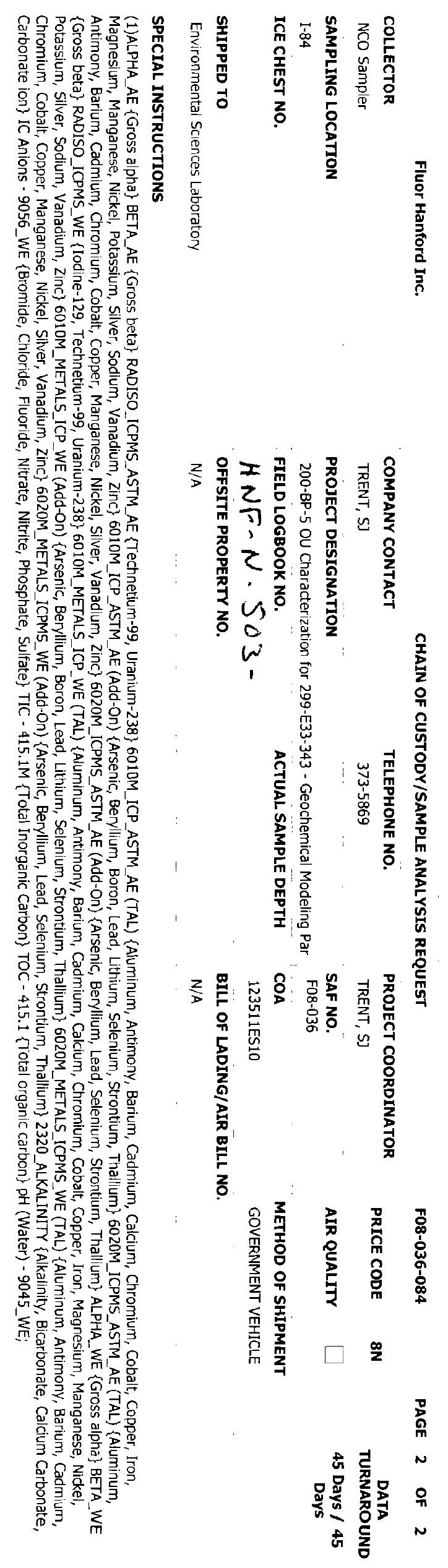




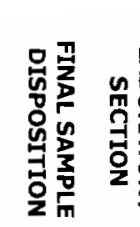

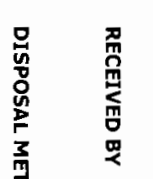

$\stackrel{w}{8}$

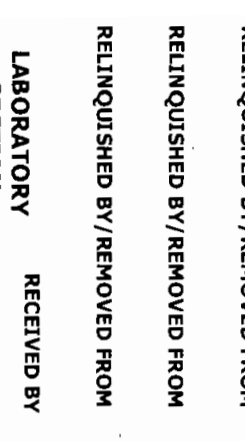

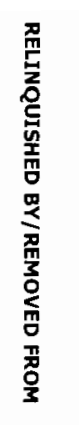

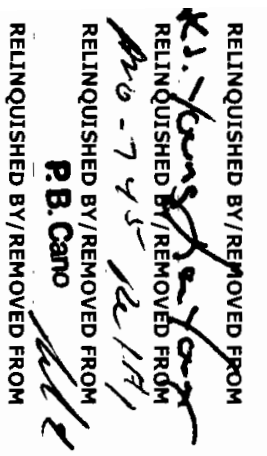

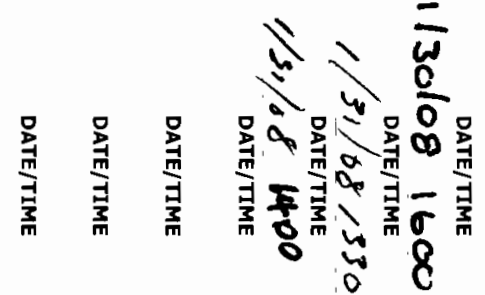

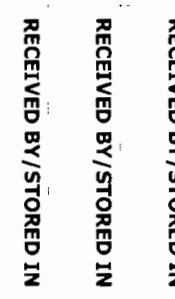

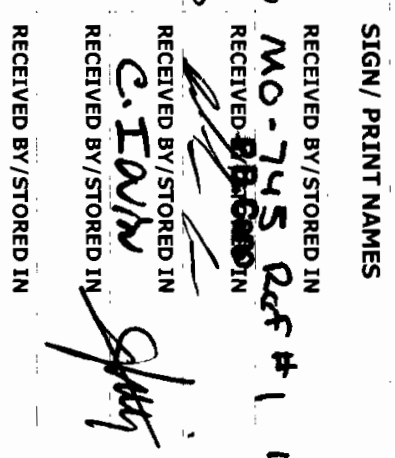

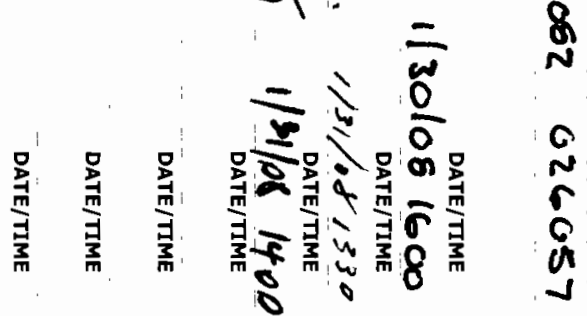
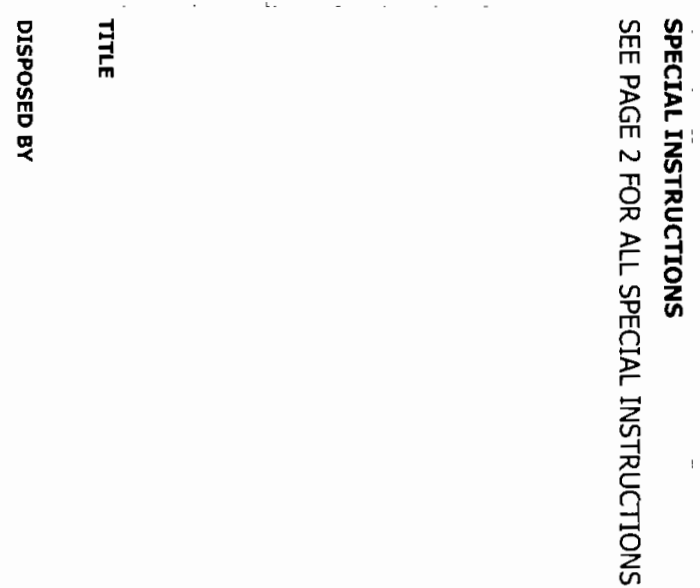

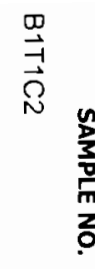

$\stackrel{8}{ }$

紊

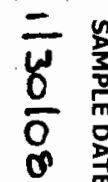

$\begin{array}{ll}0 & n \\ 0 & \frac{3}{3} \\ w & \frac{3}{0} \\ 0 & \frac{1}{3} \\ & \frac{1}{n}\end{array}$

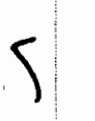

1

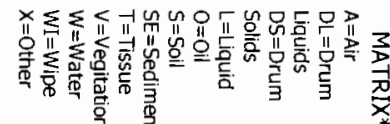

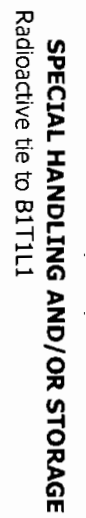

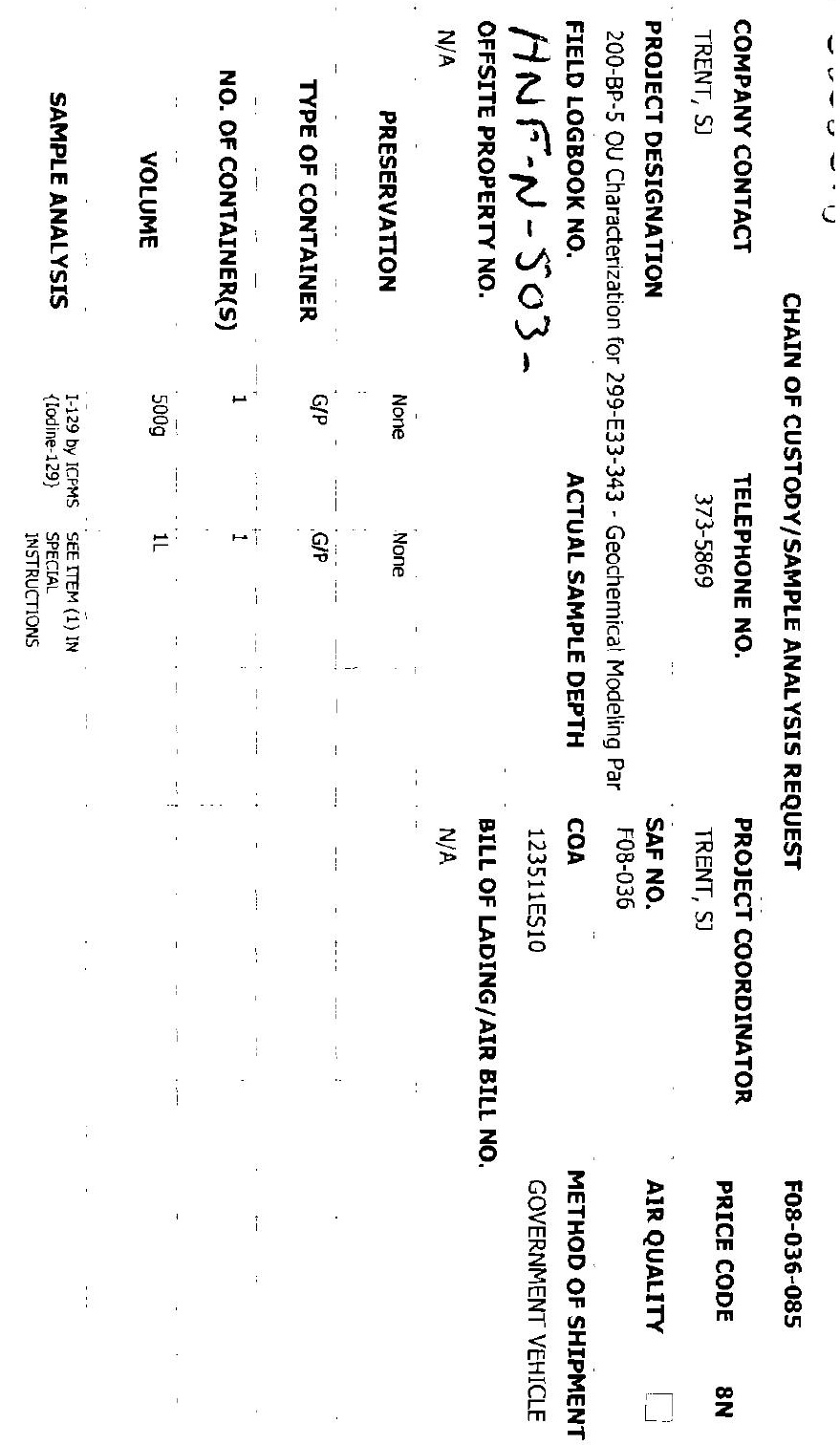

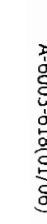

尊

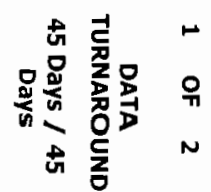




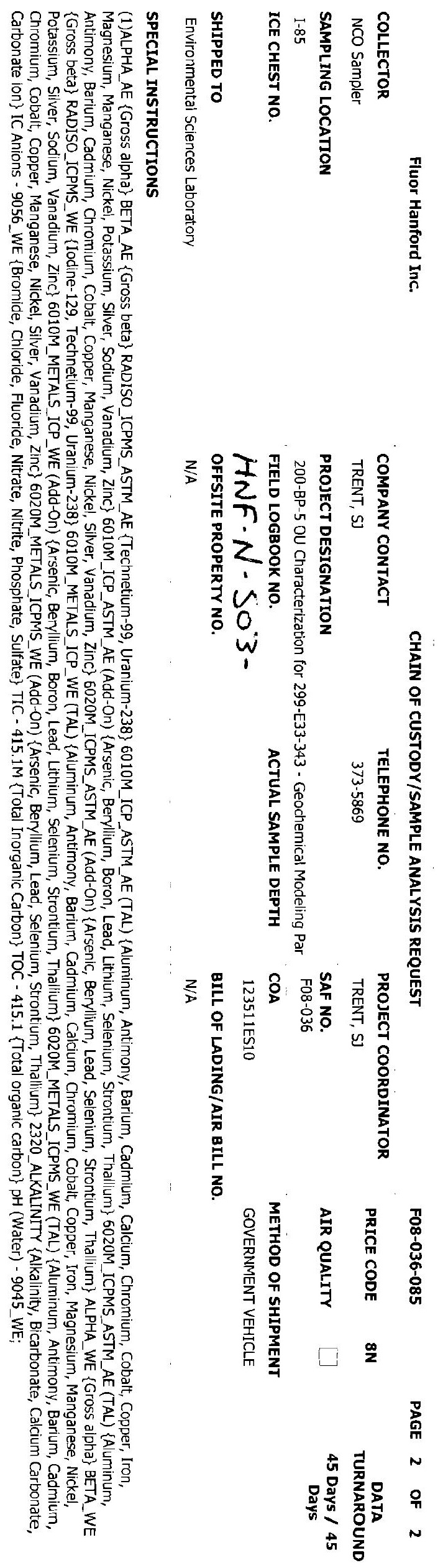




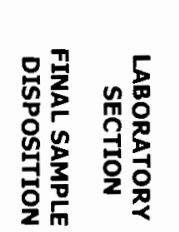
产

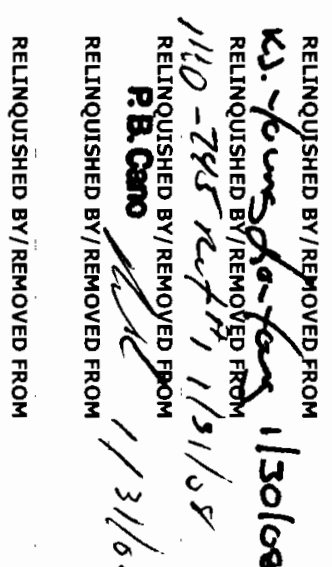

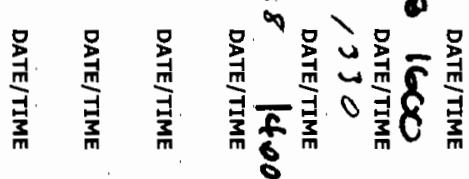

旁

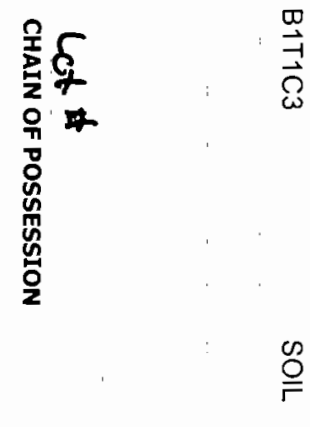

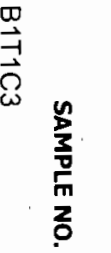

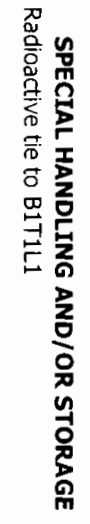

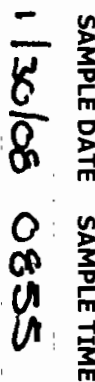

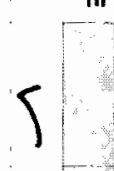

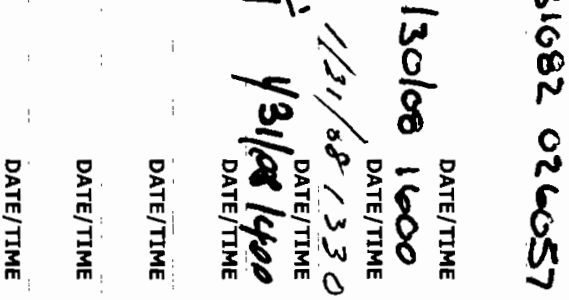

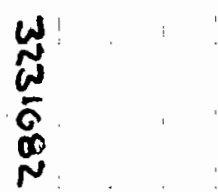

I

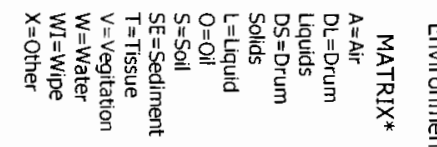

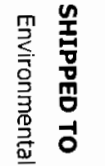

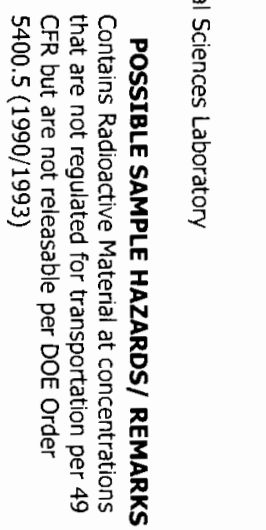

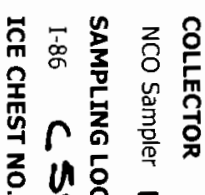

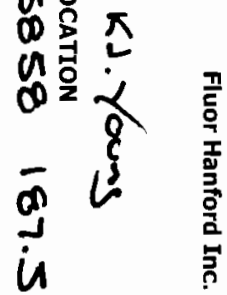
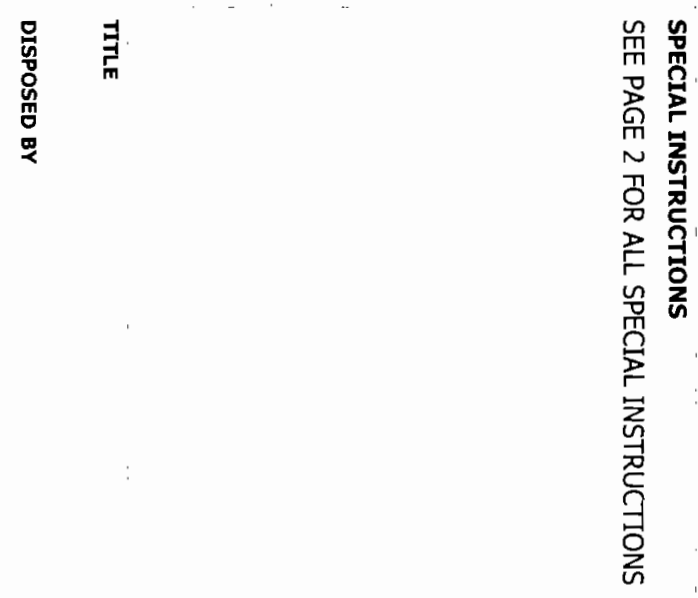

\section{章}

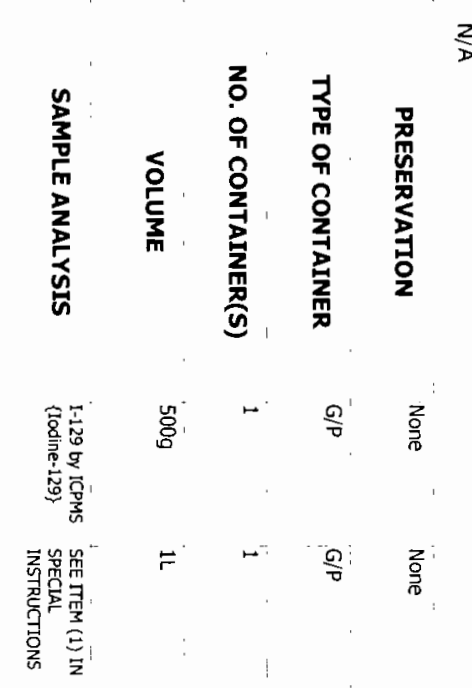

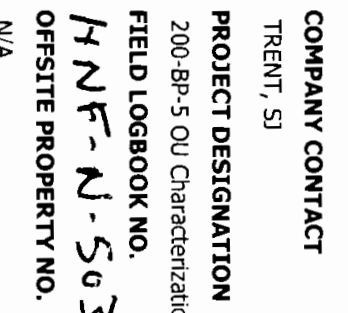

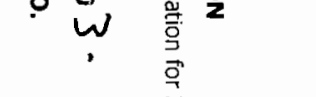




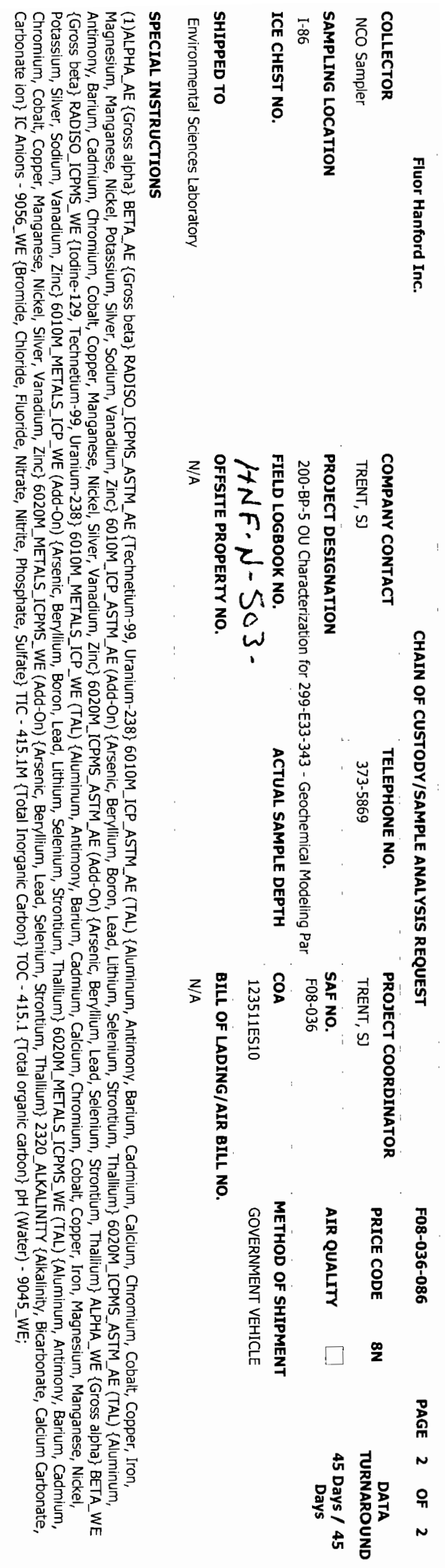



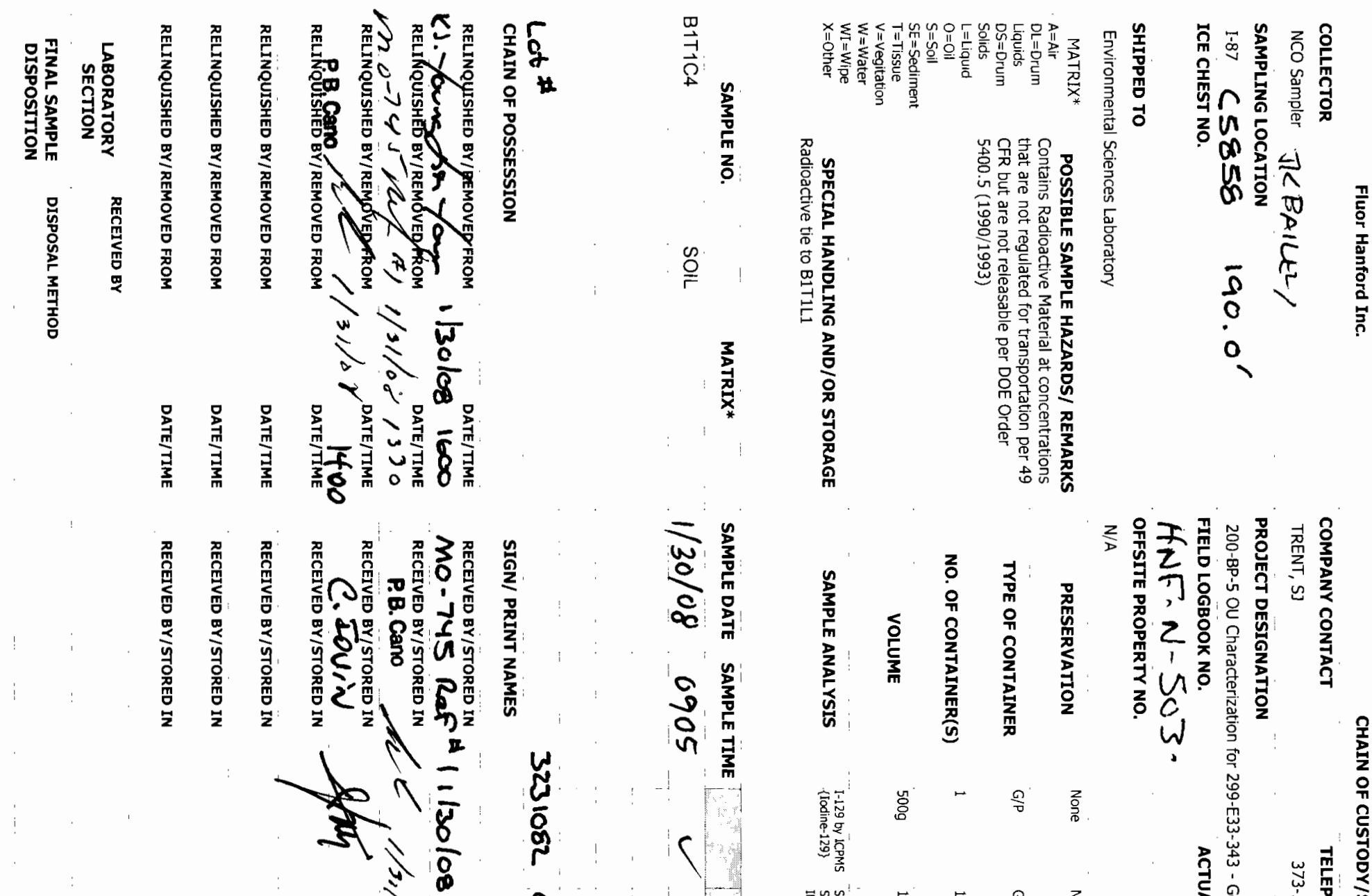

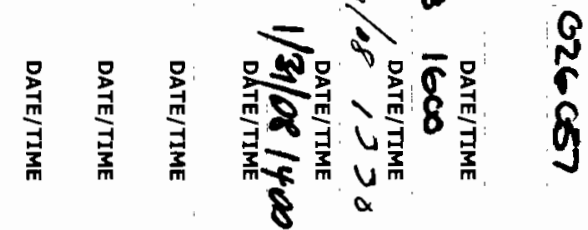

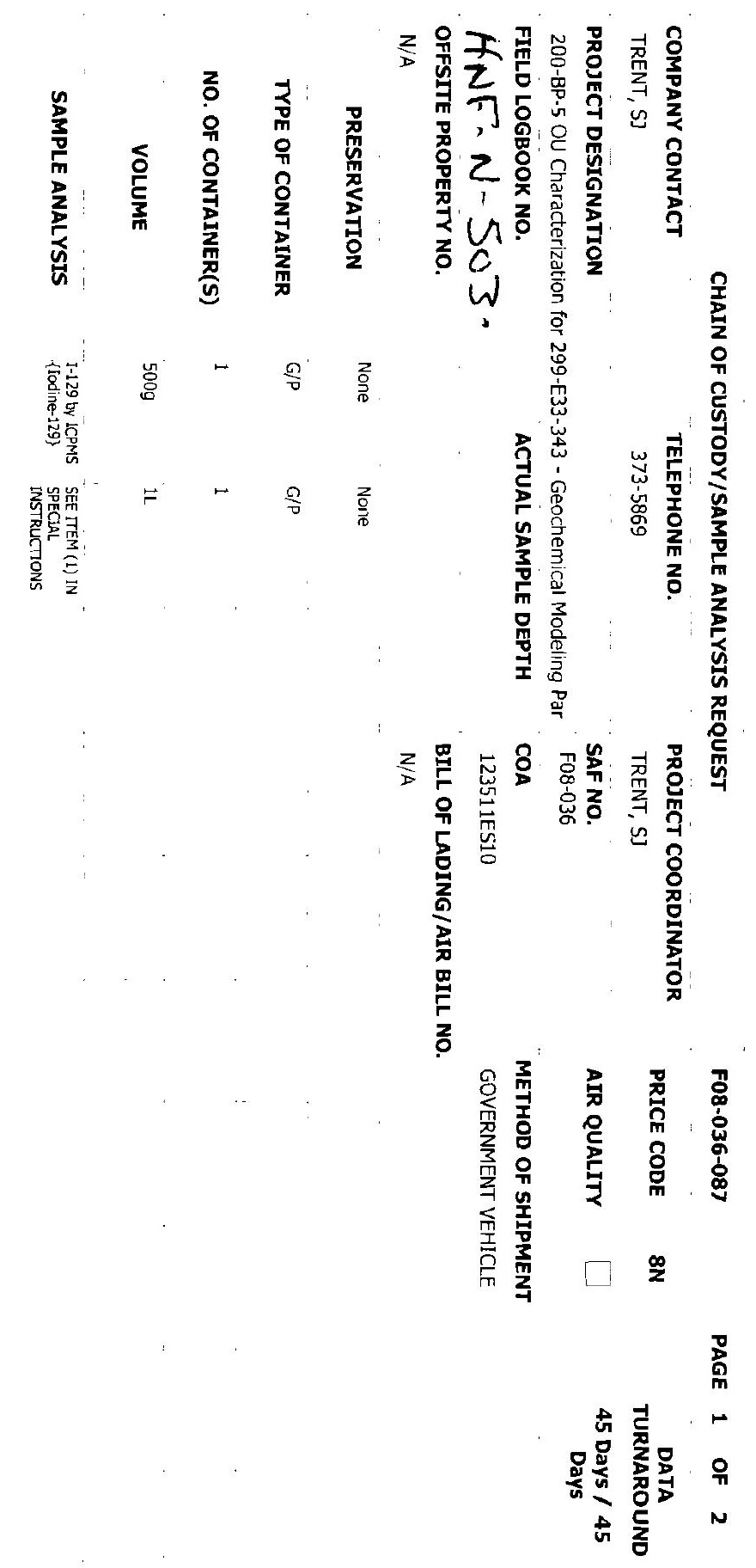




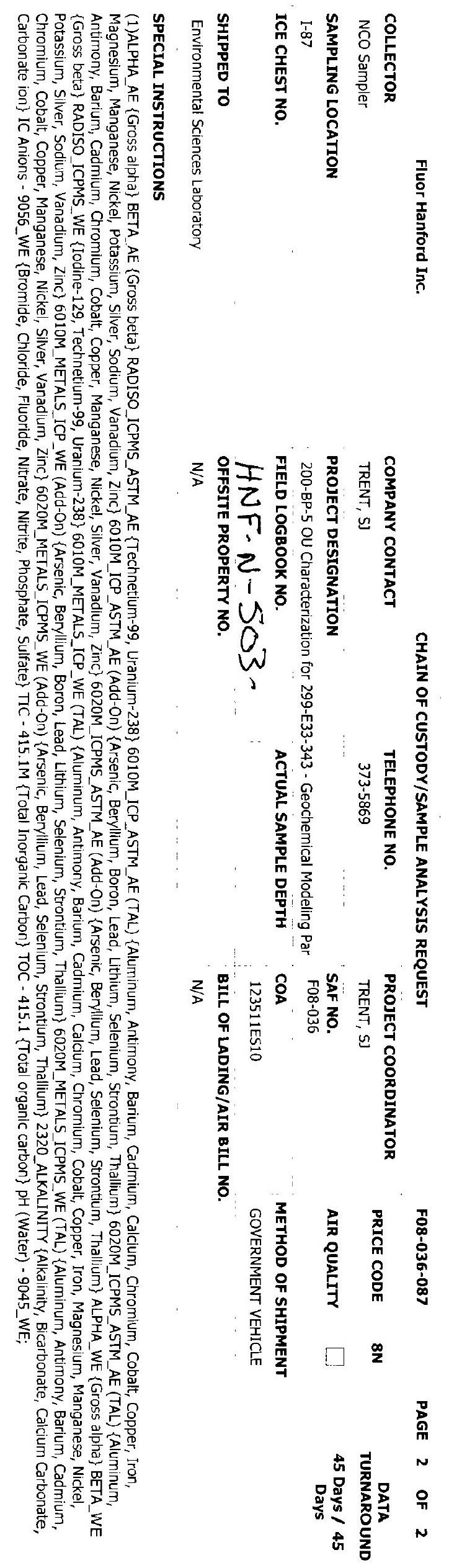




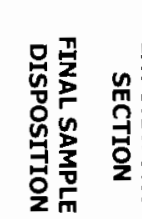

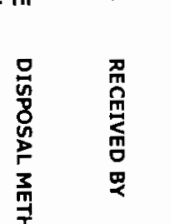

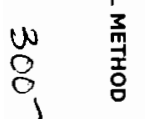
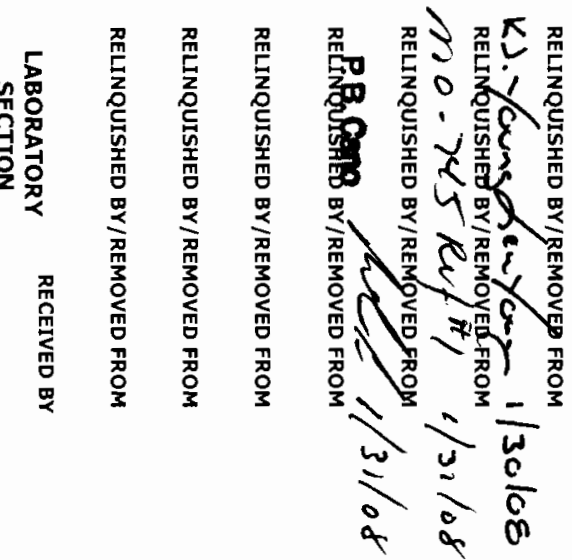

9
$\frac{1}{2}$
2
0
0

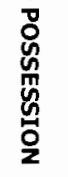

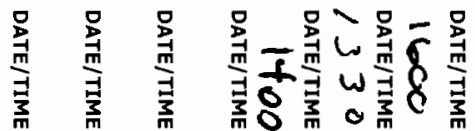

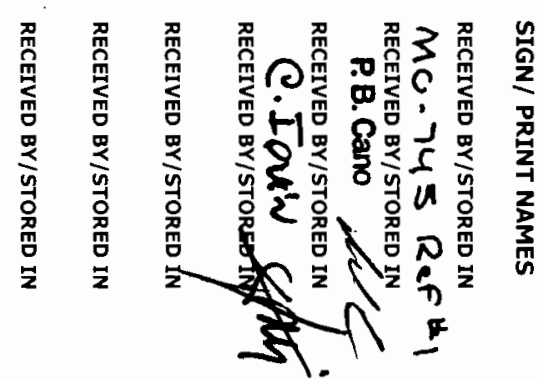

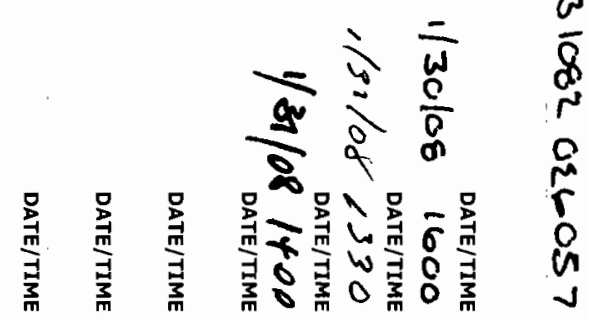
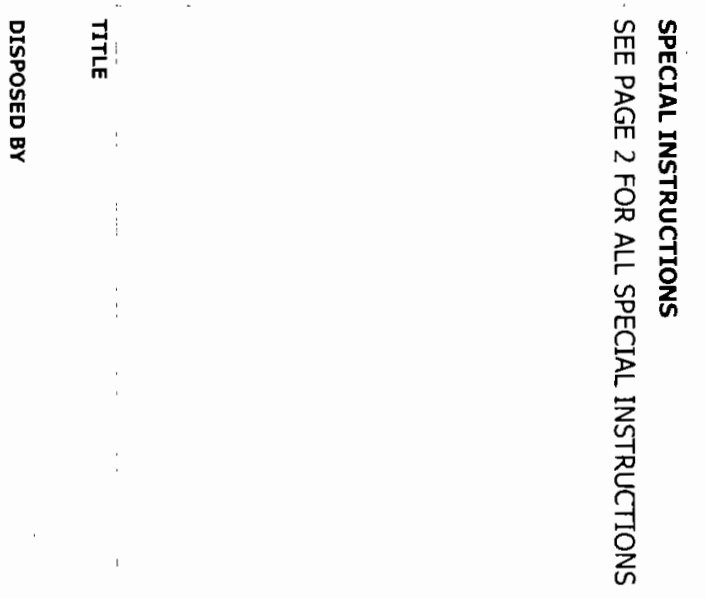

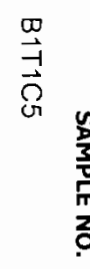

$\stackrel{\varrho}{\circ}$

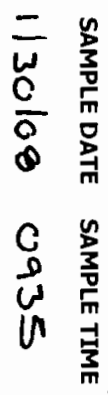

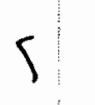

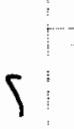

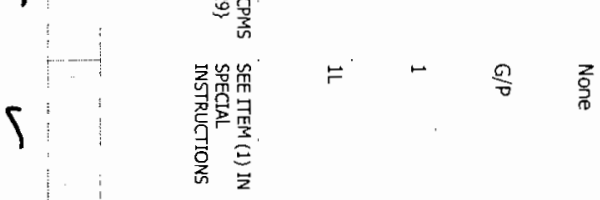

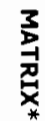

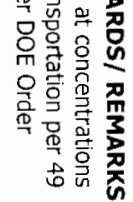

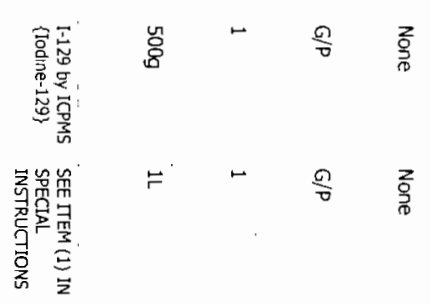

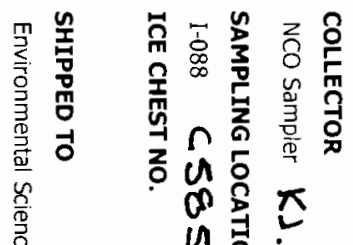

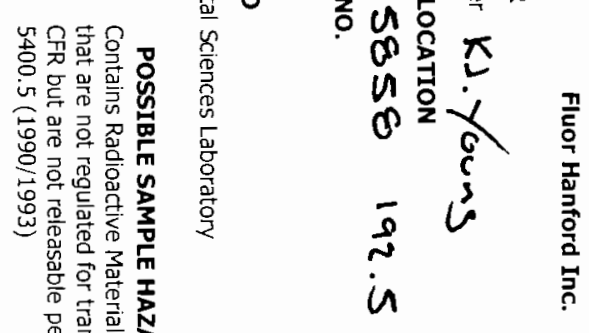

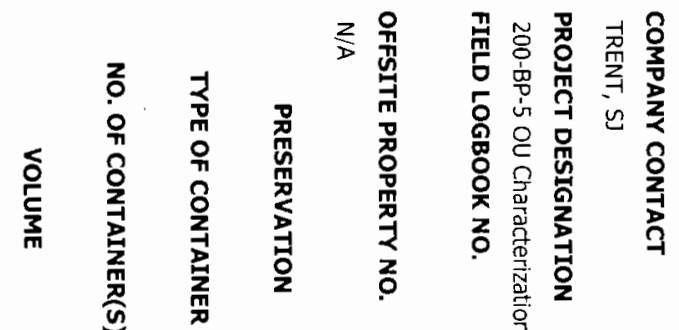

\section{$\frac{9}{2}$}

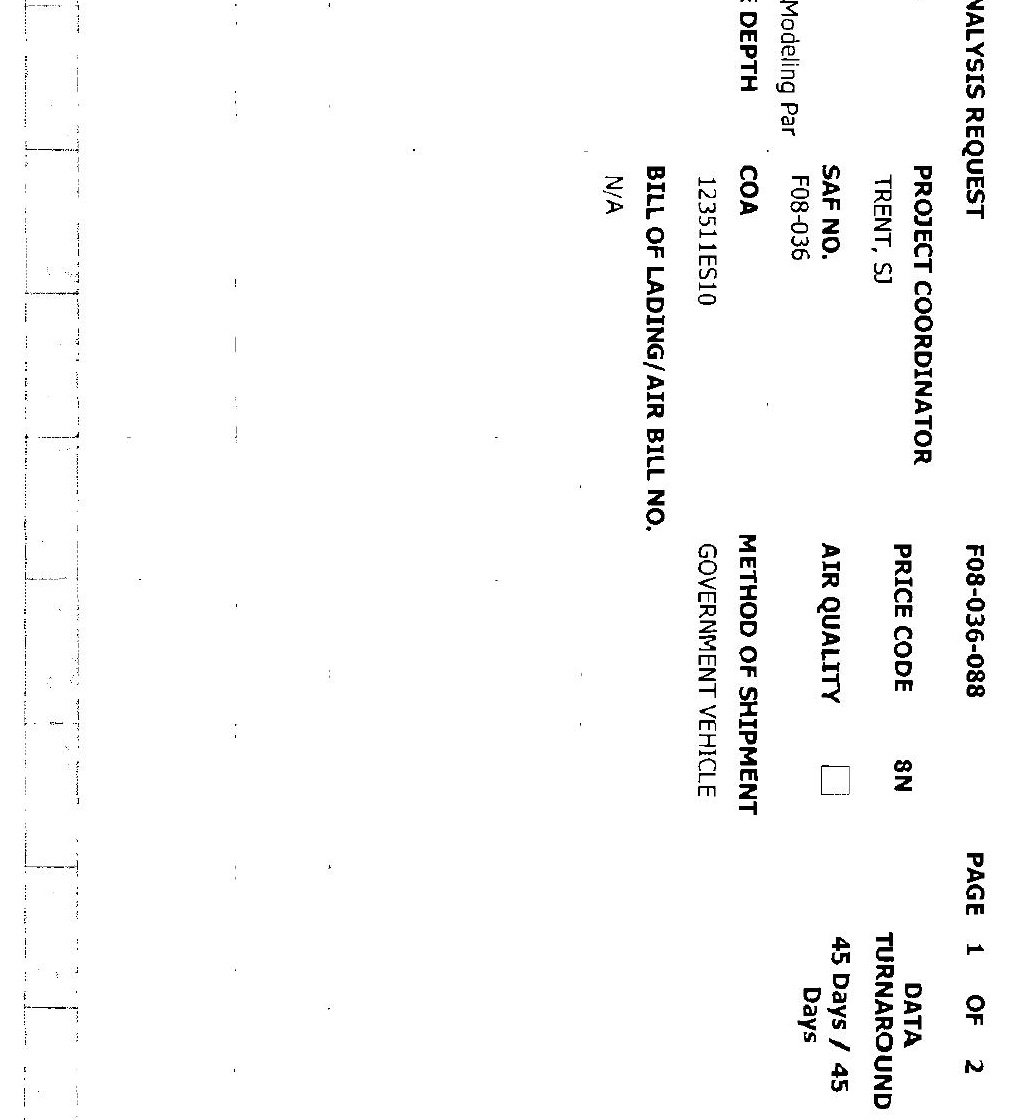




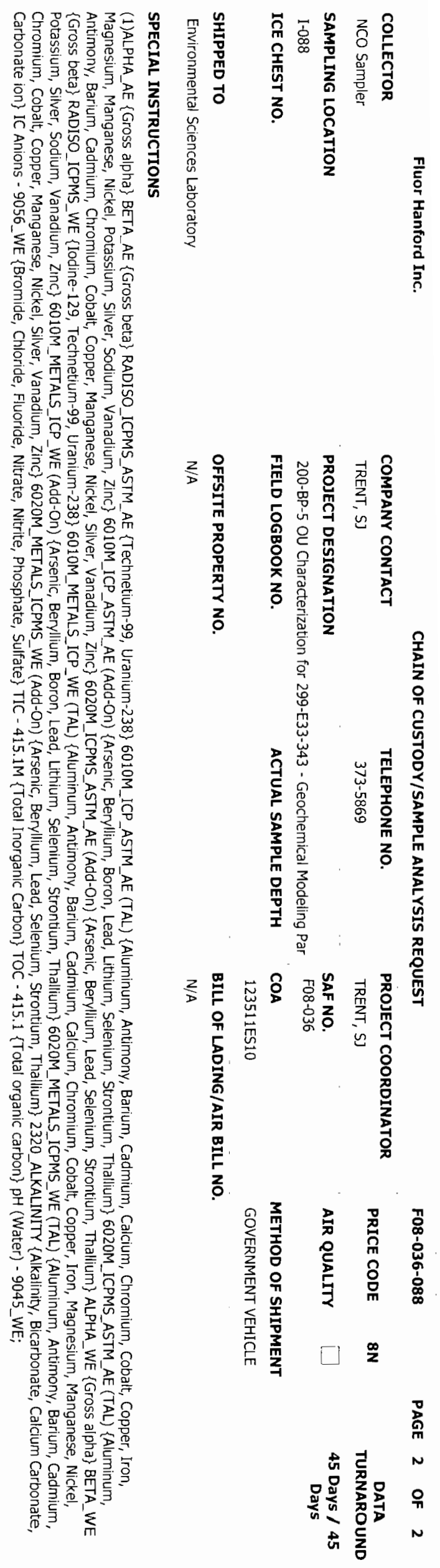



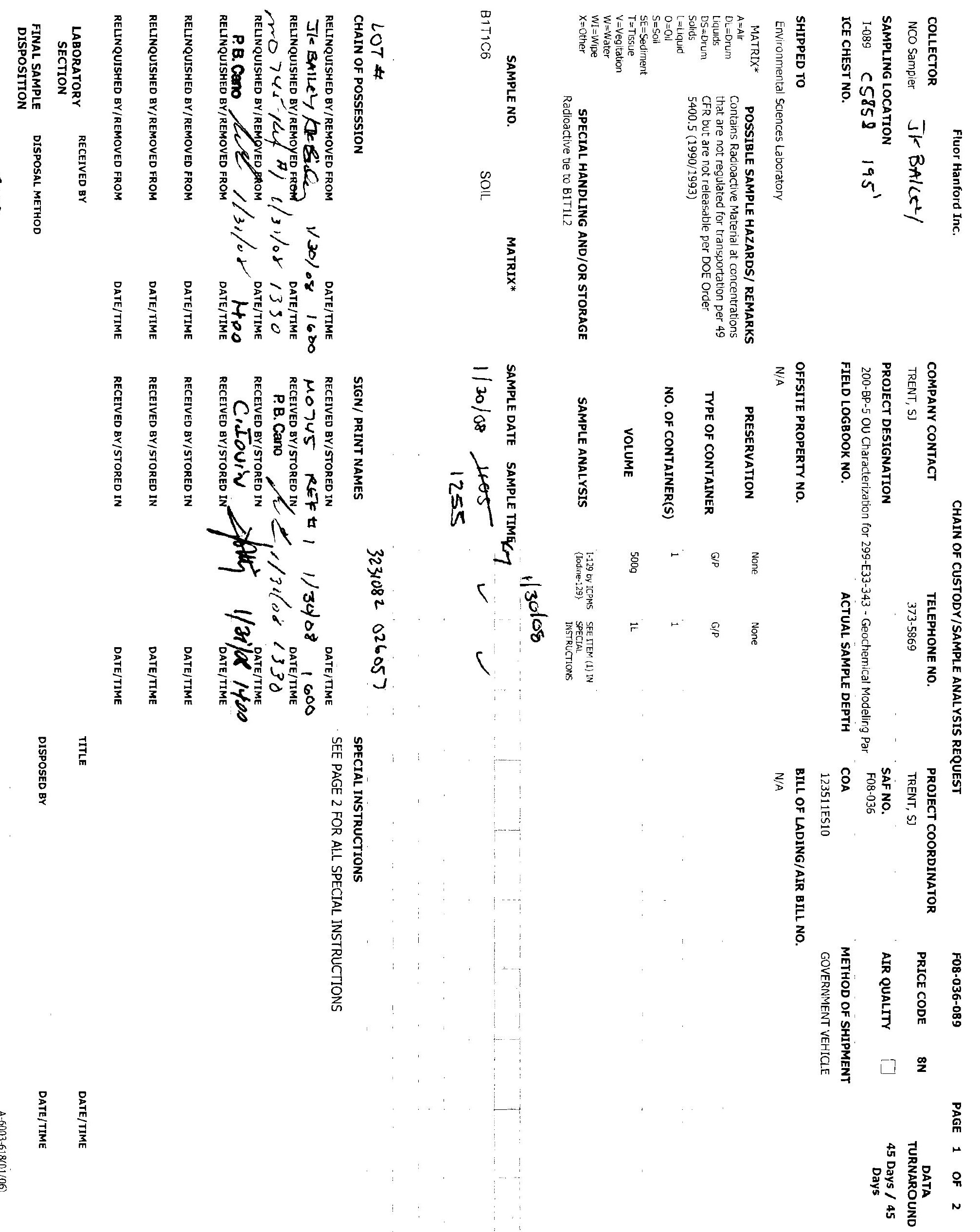


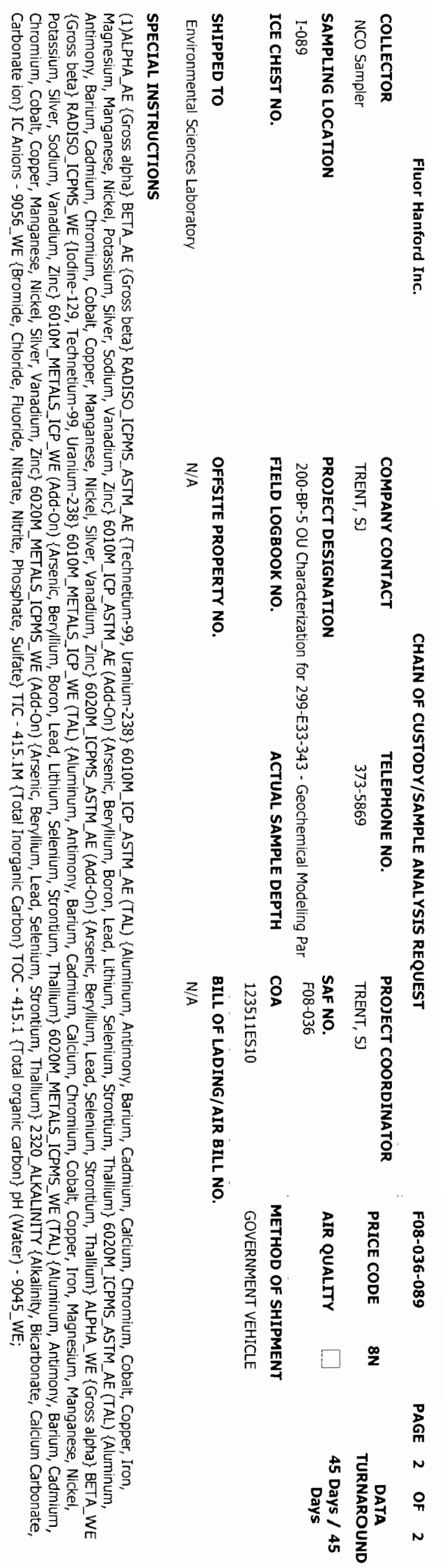


$\|$
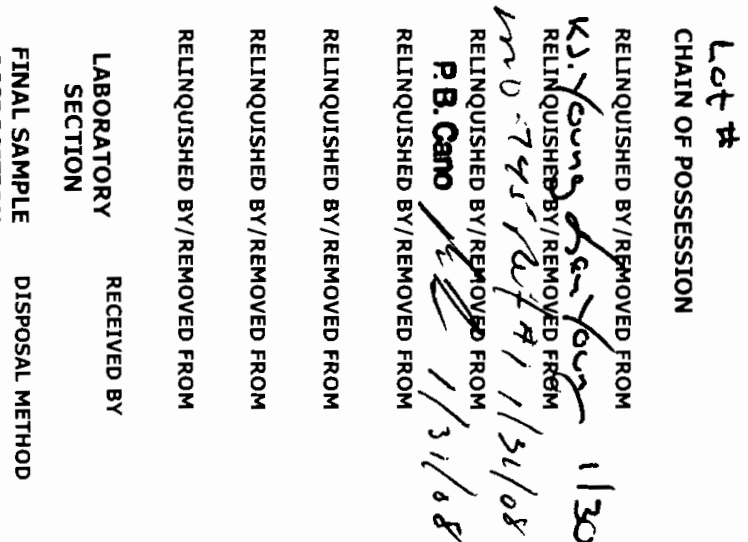

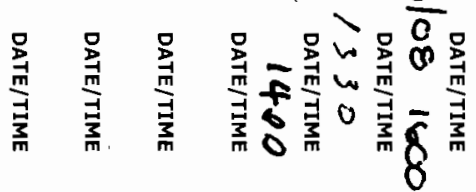
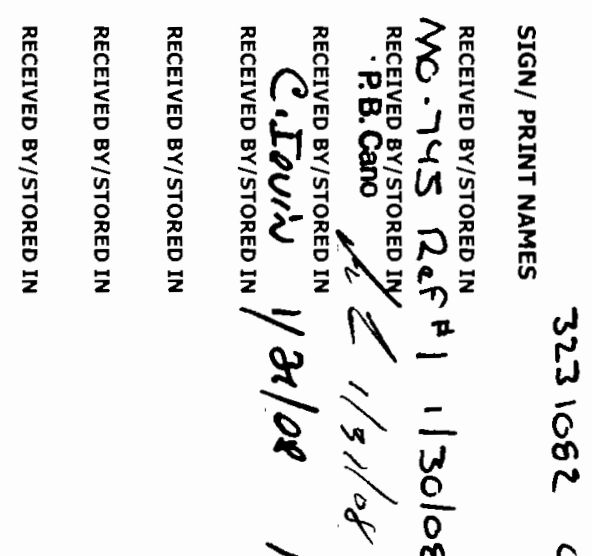

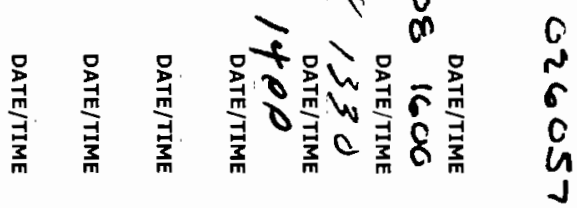

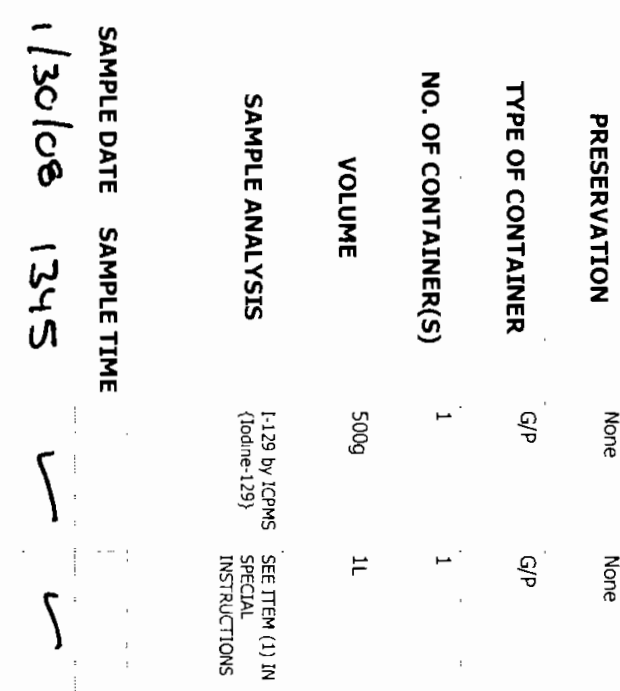

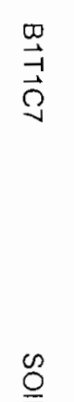

$\underset{\sharp}{\stackrel{3}{7}}$
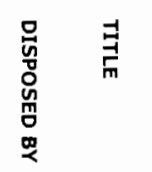

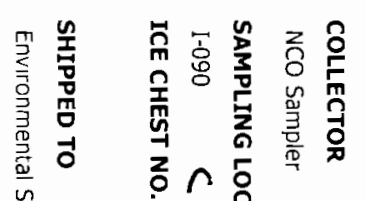

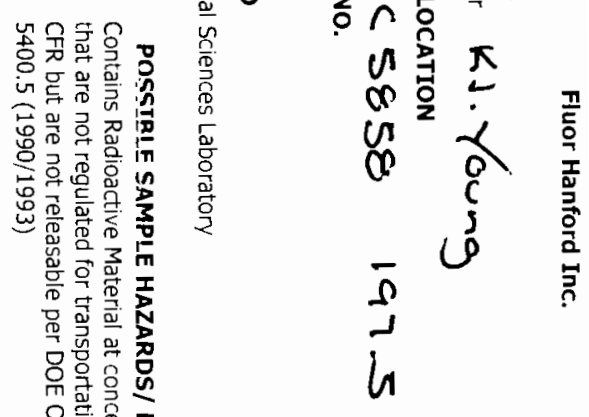

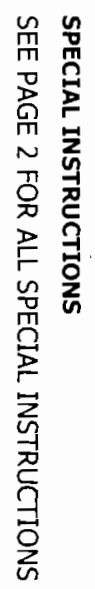

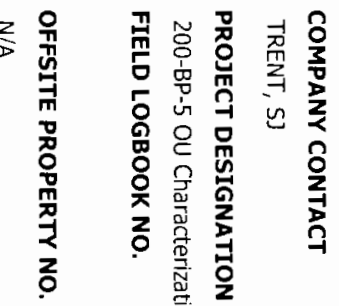

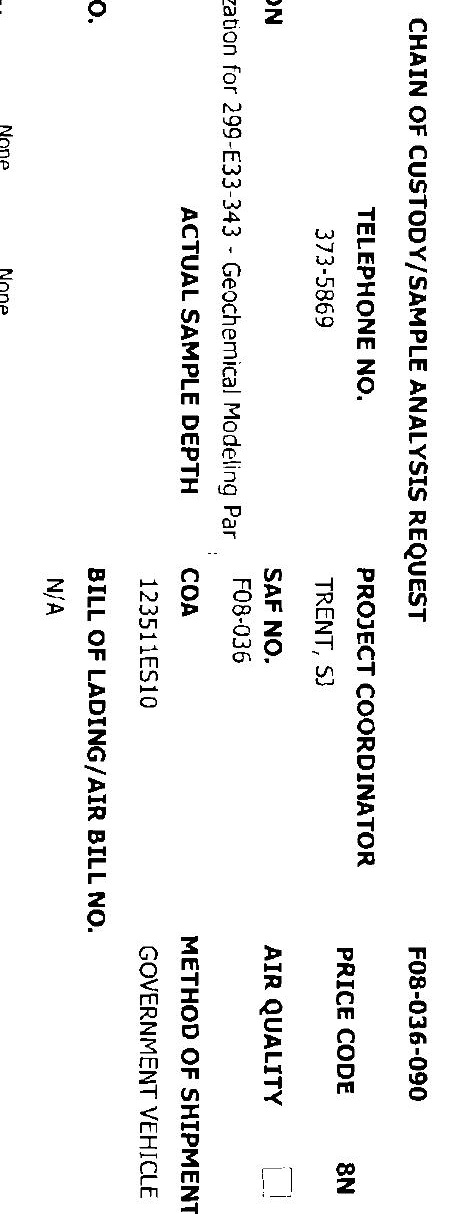

$\stackrel{\infty}{2}$

品

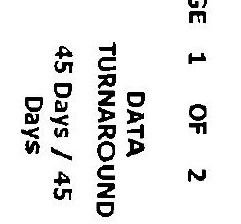




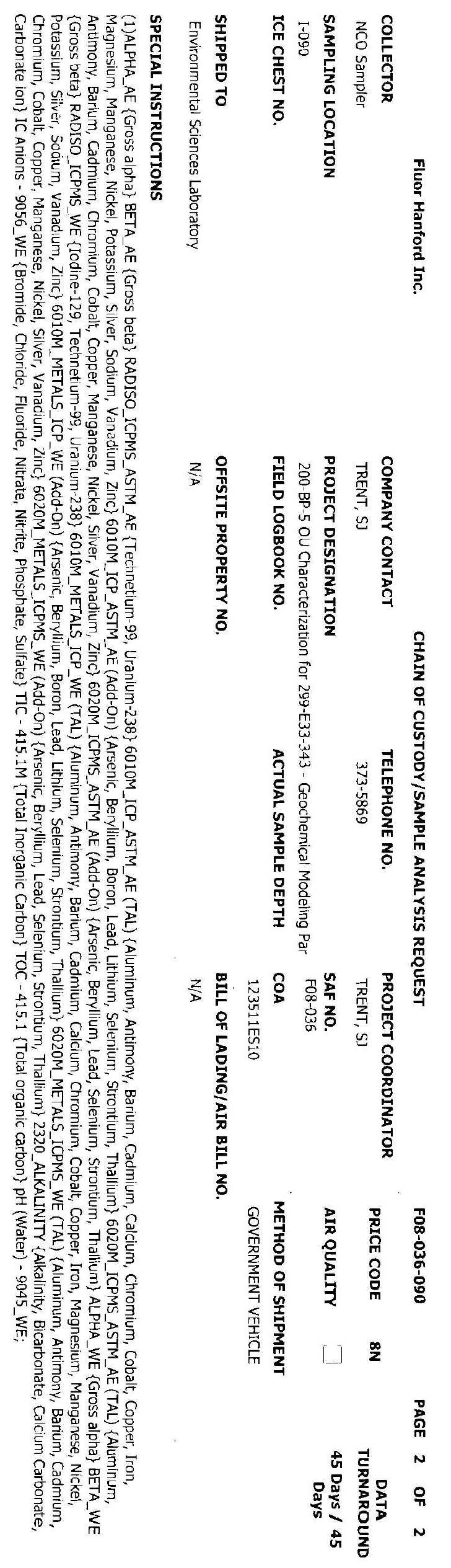




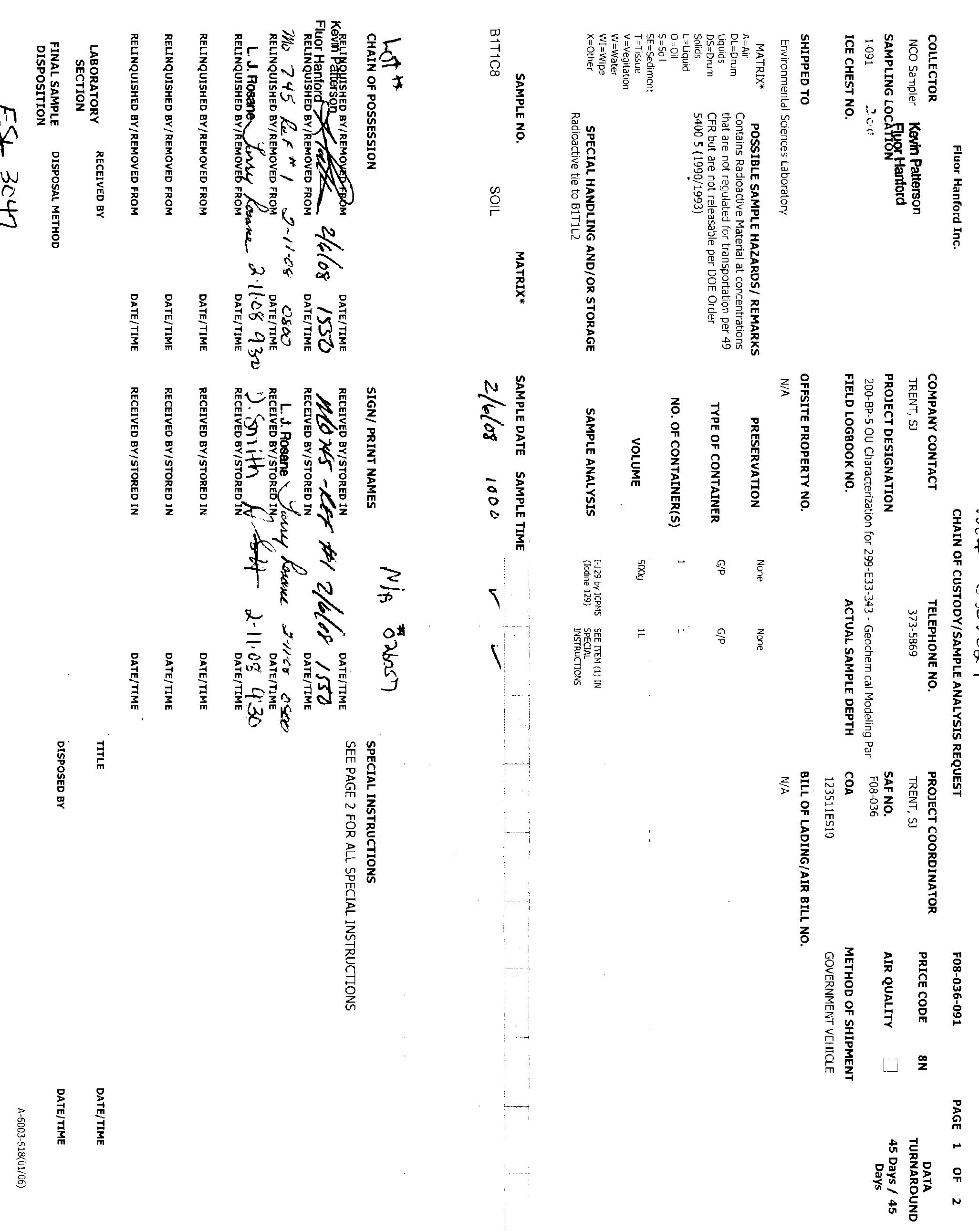




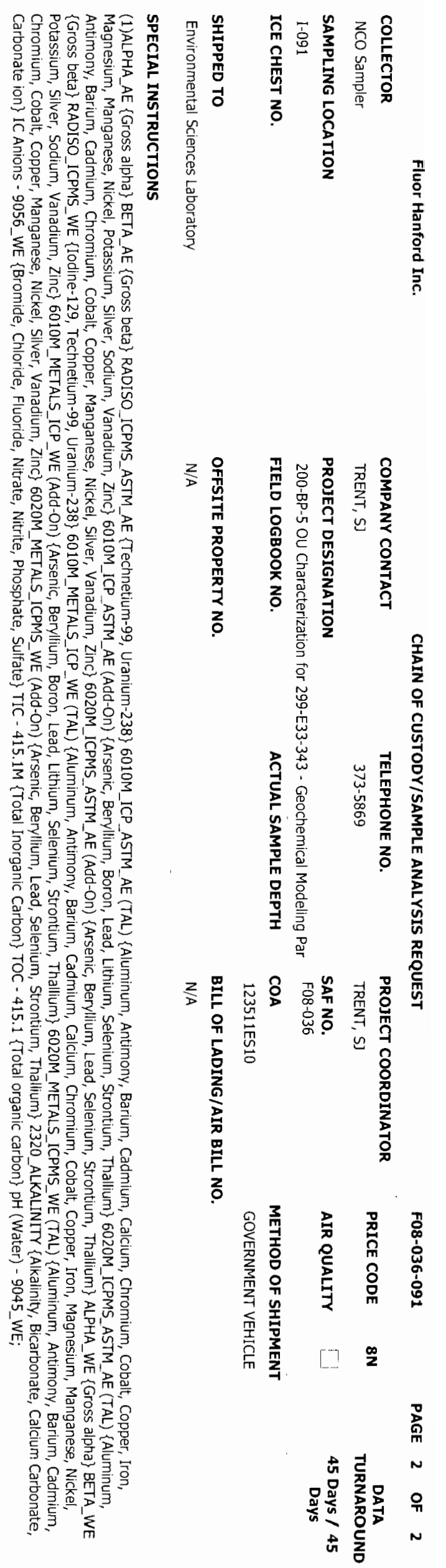




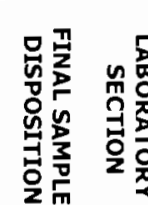

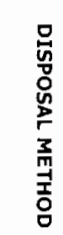

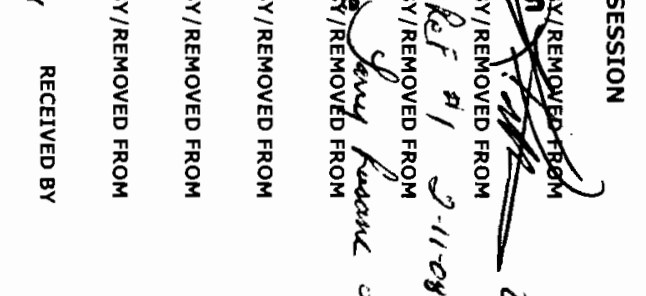

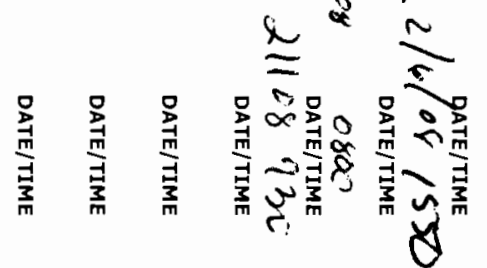
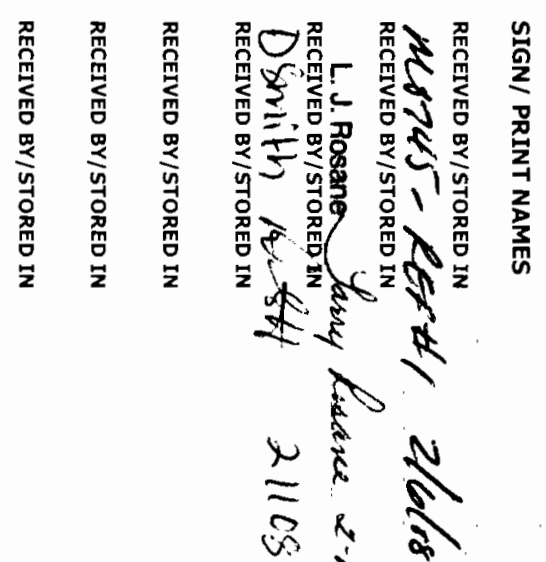

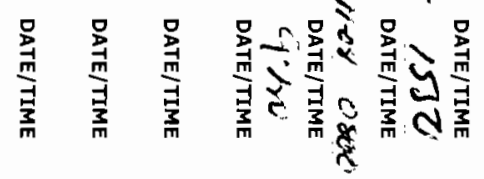

$\stackrel{\text { D }}{\overrightarrow{7}}$

$\stackrel{\infty}{\stackrel{0}{r}}$

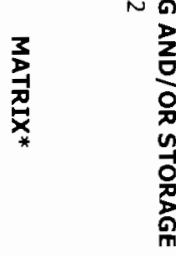

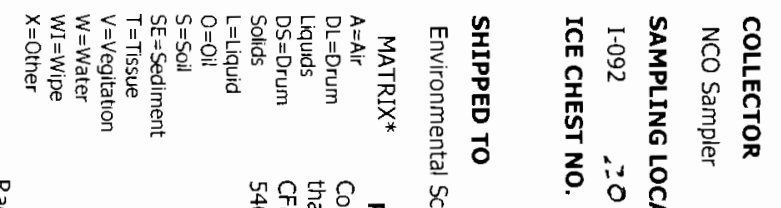

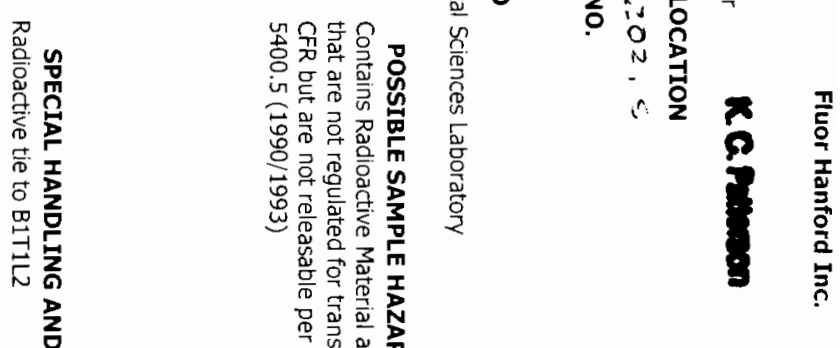

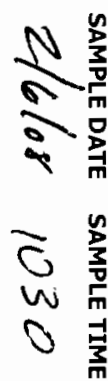

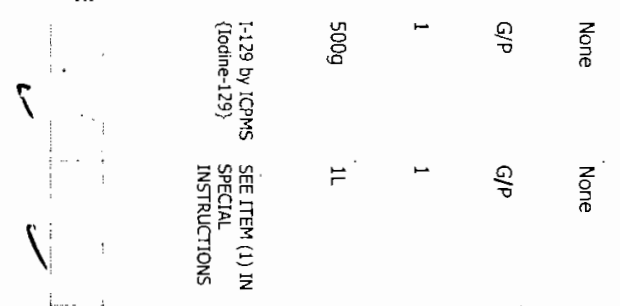

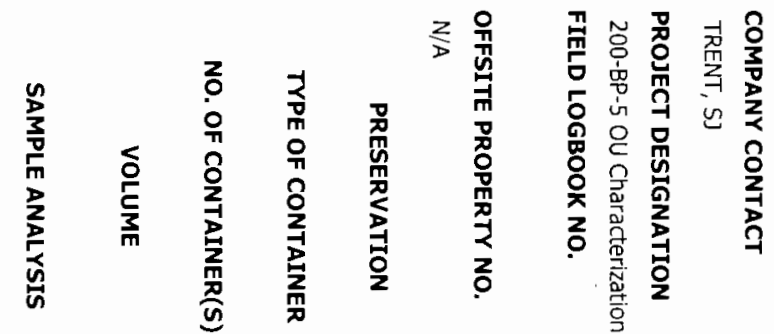

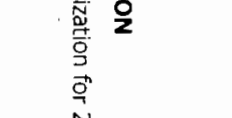

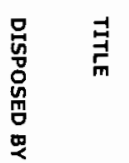

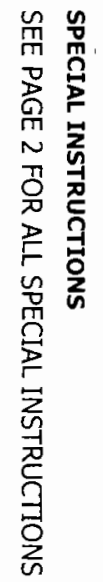

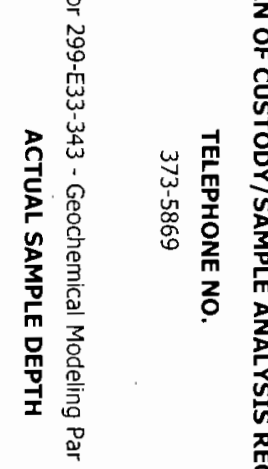

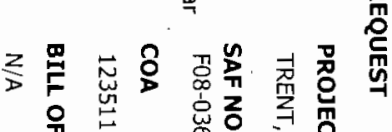

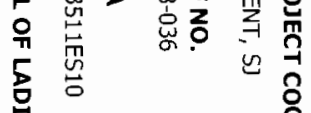

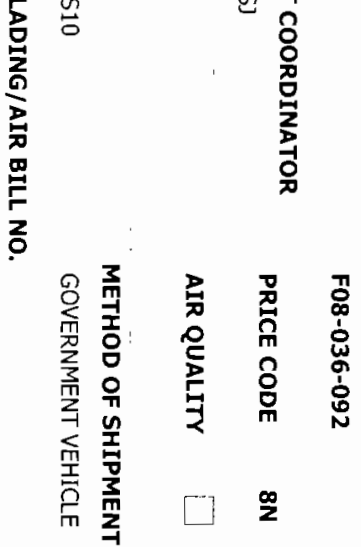

晃

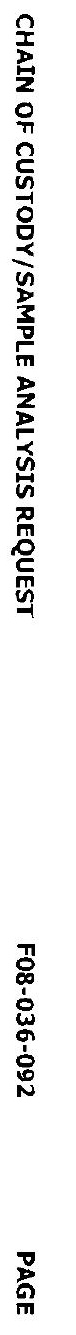

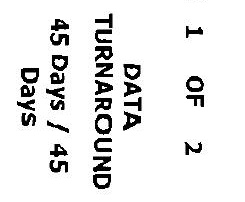




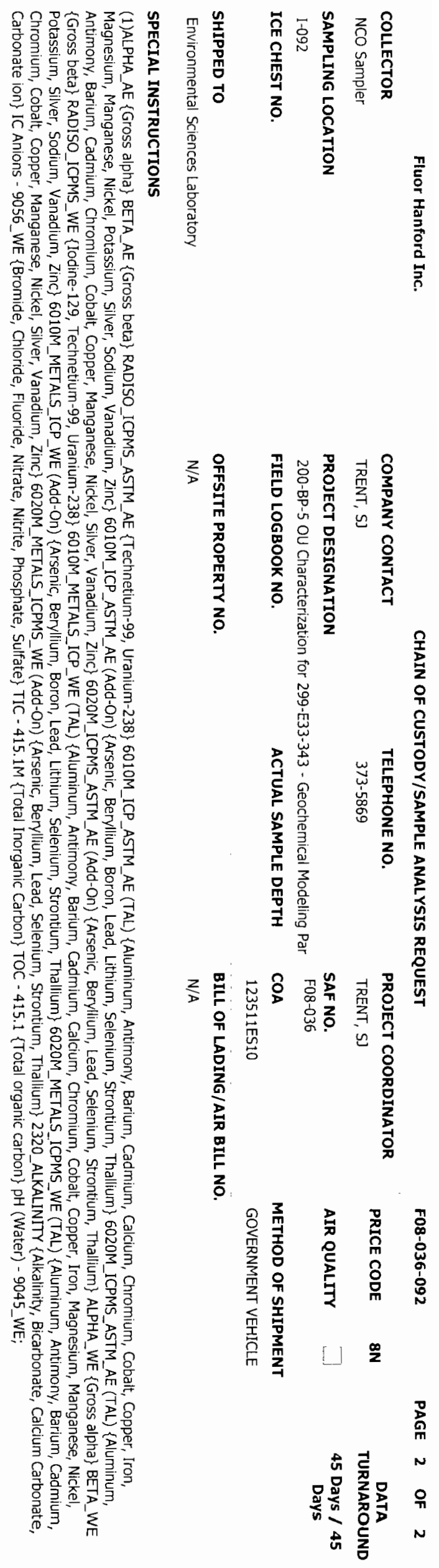




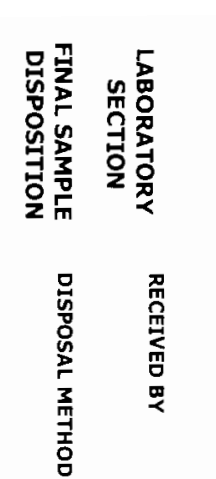
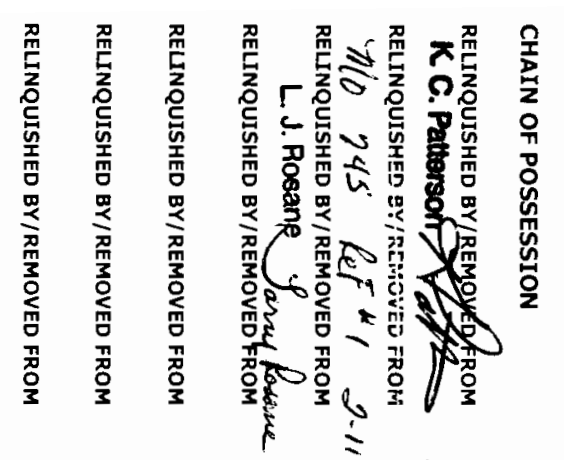

美
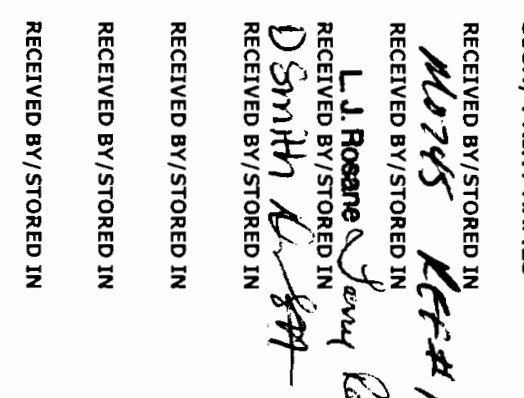

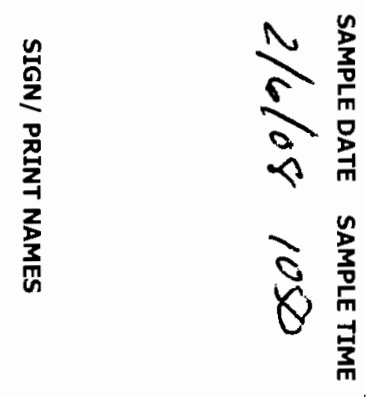

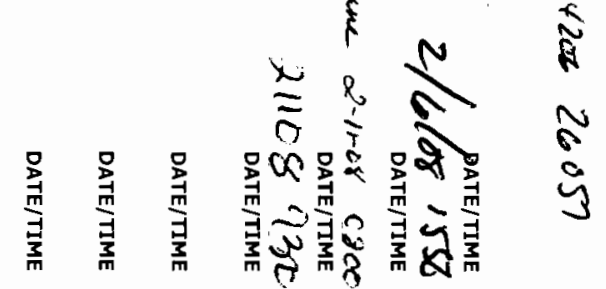

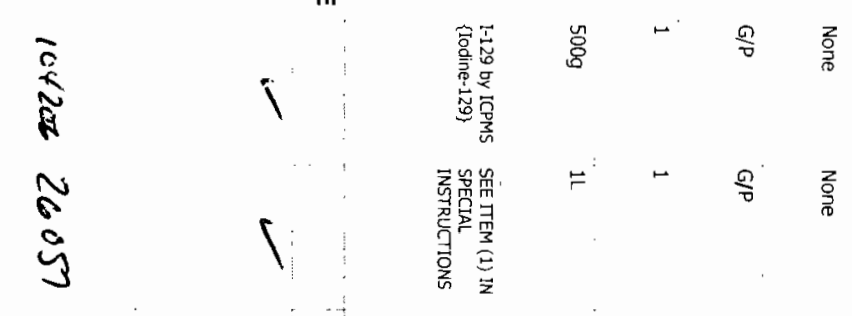

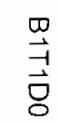

$\stackrel{0}{\circ}$

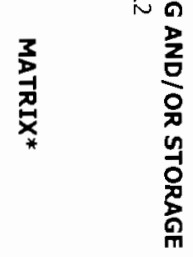

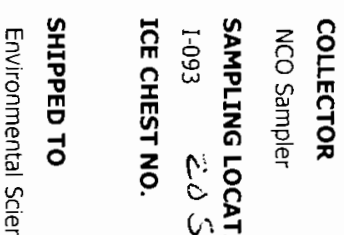

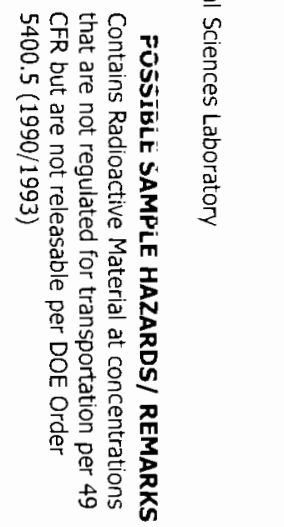

녕

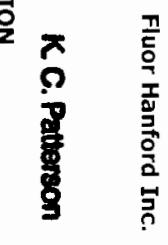

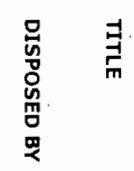

品总

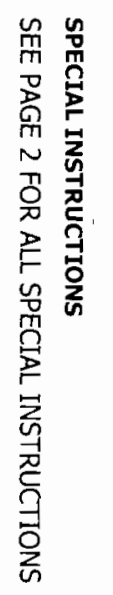

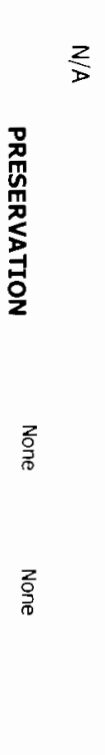




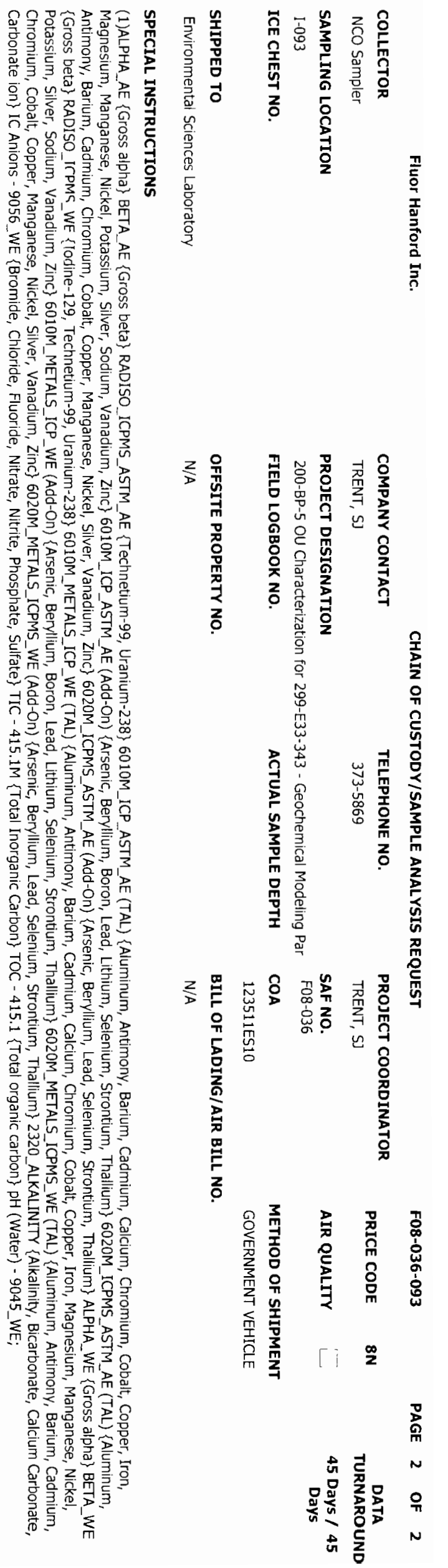




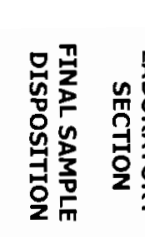

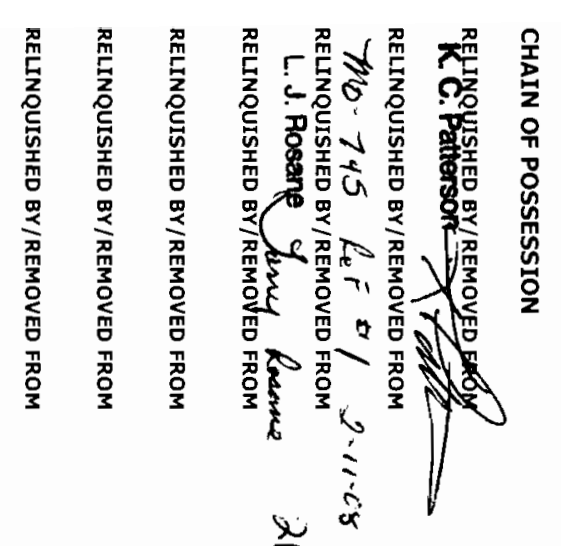

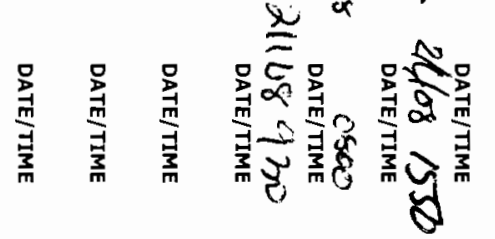
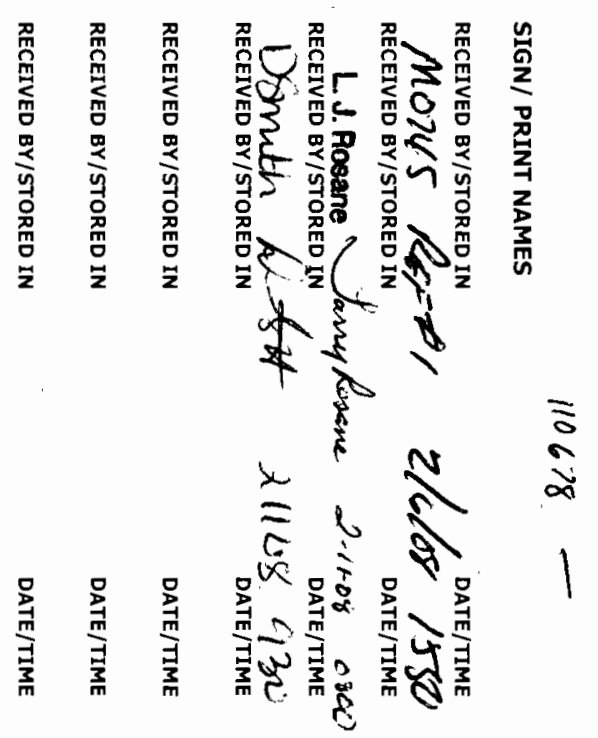

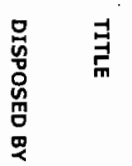

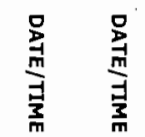

$\stackrel{\square}{\vec{\exists}}$

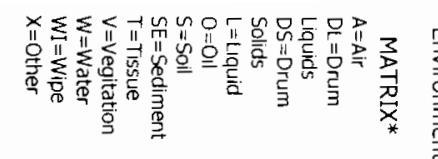

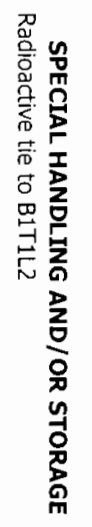

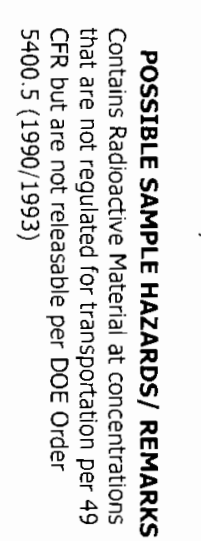

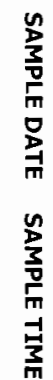

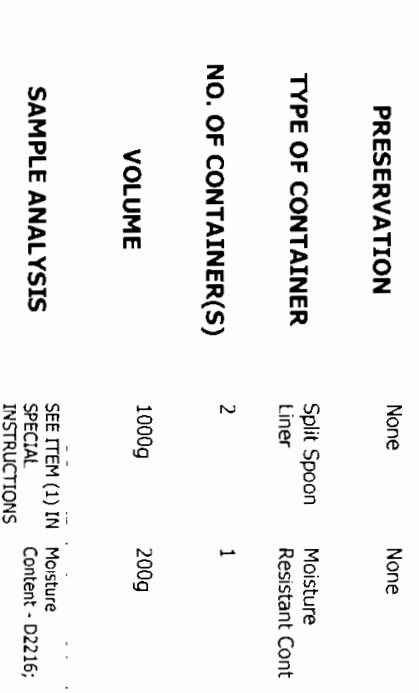

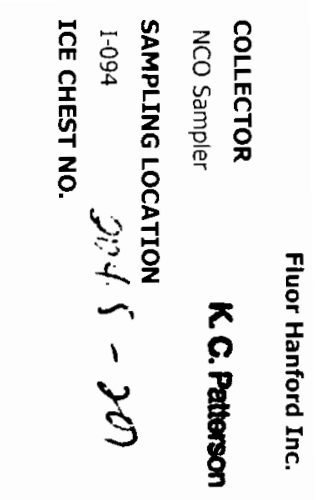

$z$

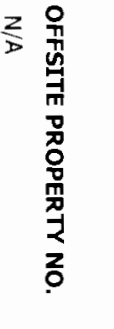

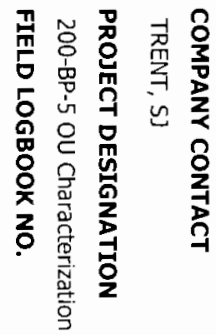

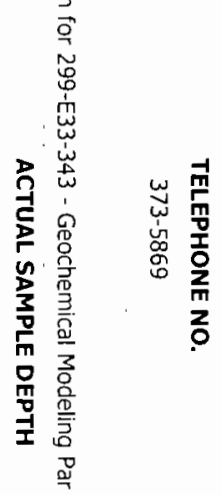

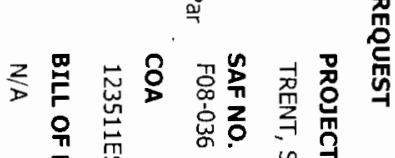

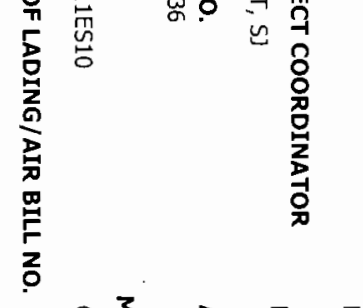

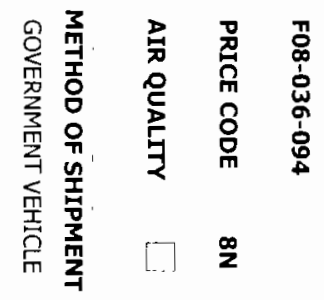

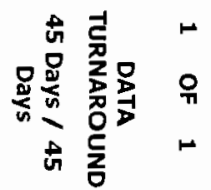




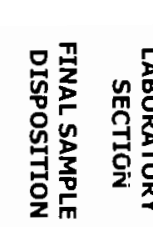

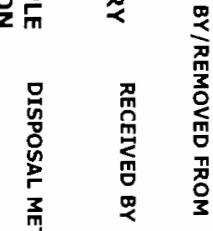

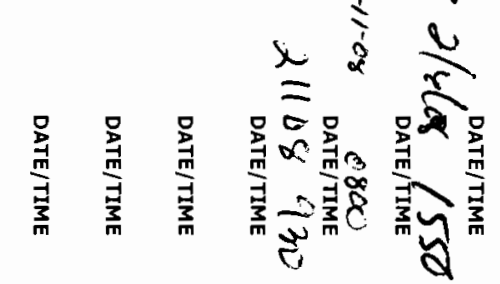

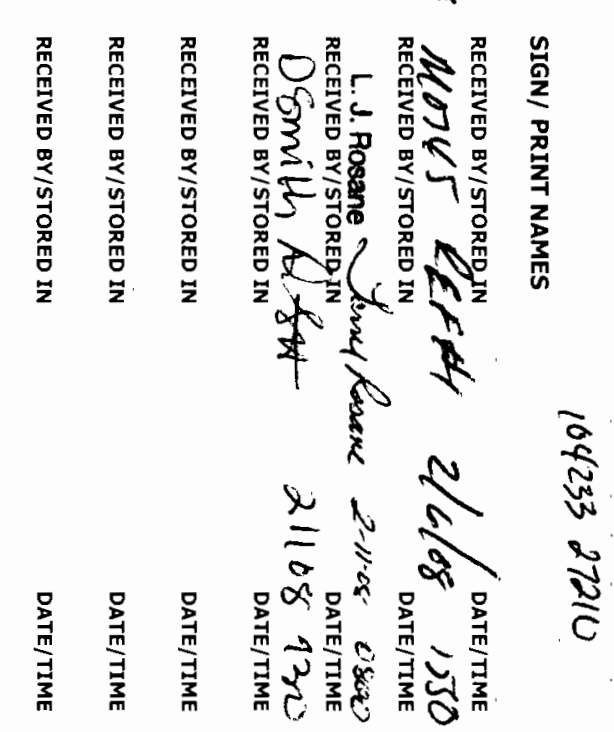

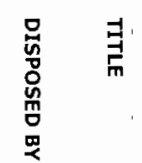

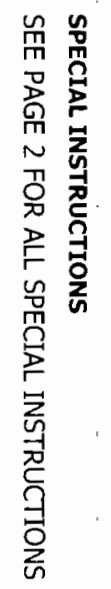

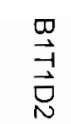

$\stackrel{\infty}{\rightleftharpoons}$

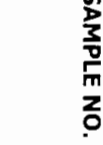

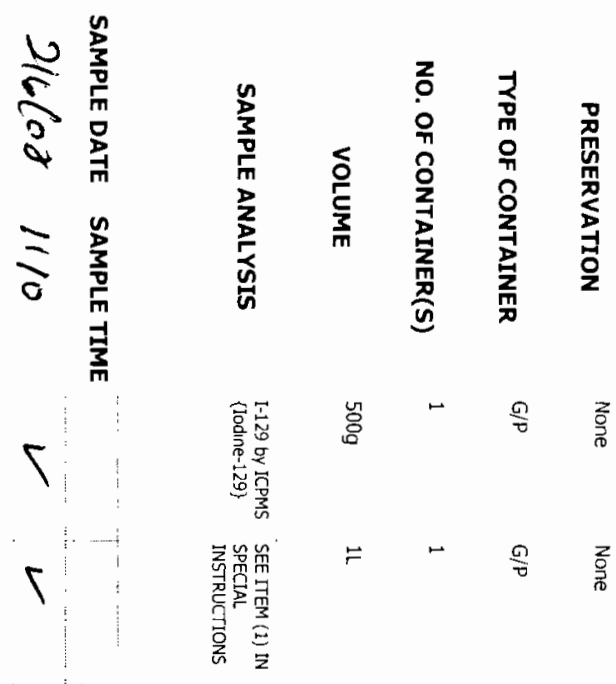

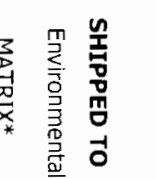

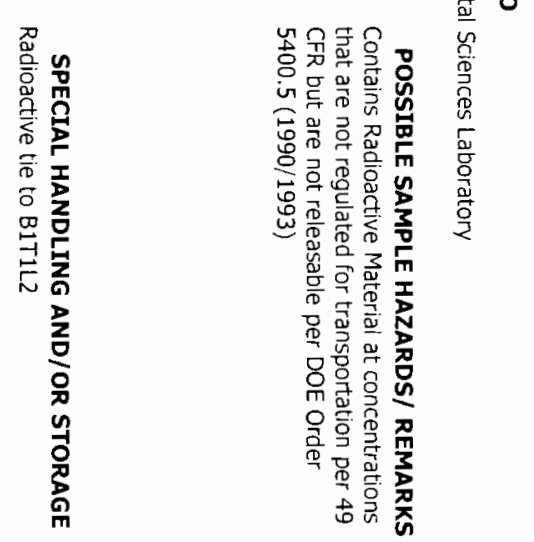

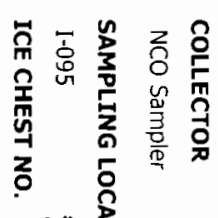

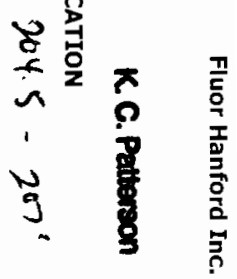

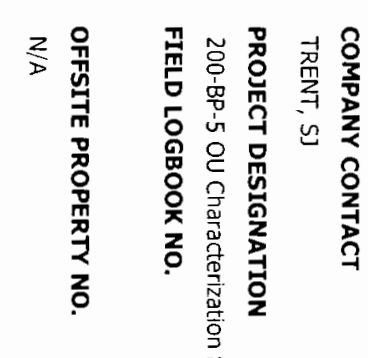<smiles>CCC</smiles>

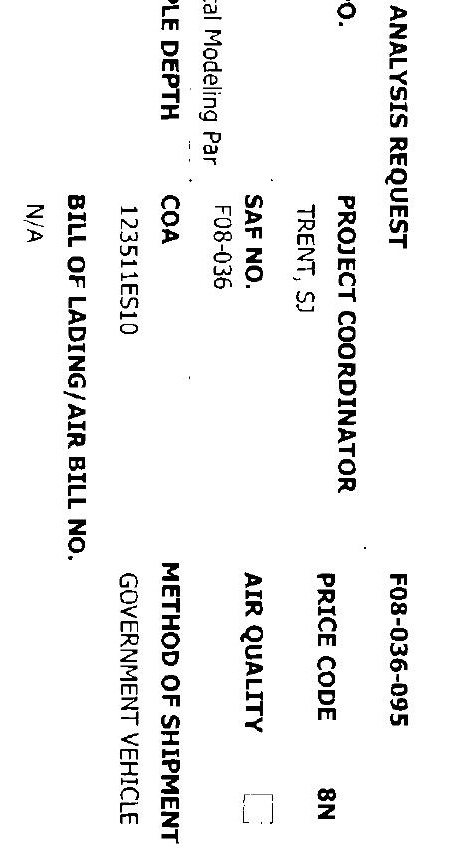

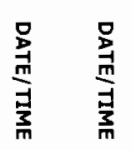

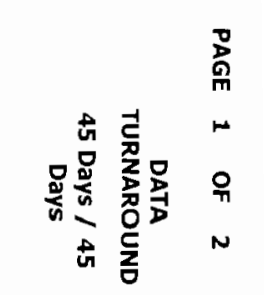




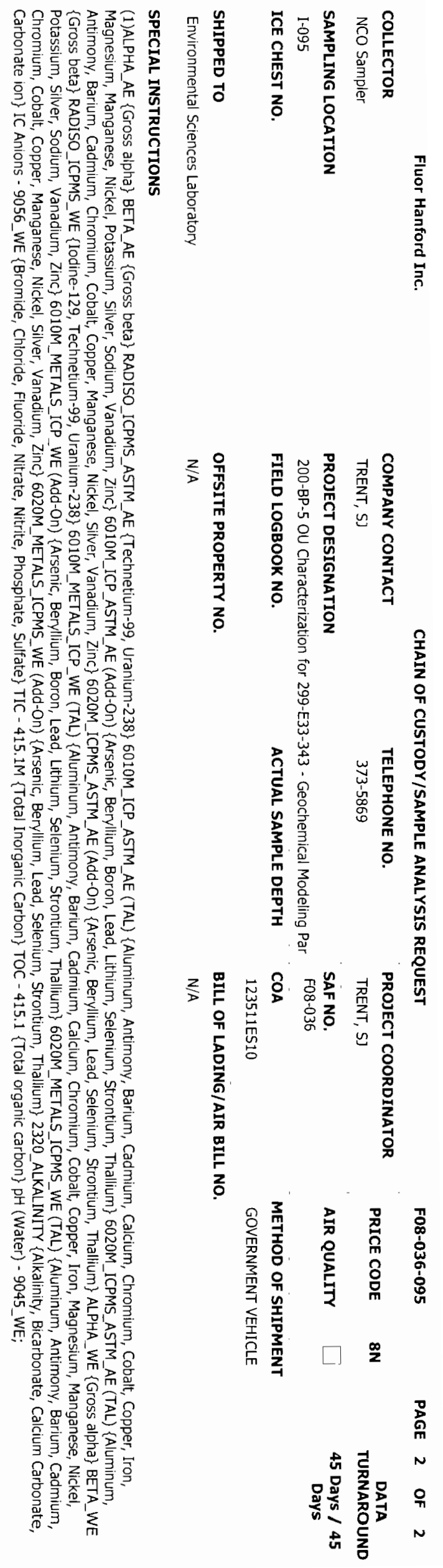




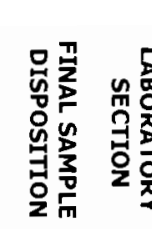
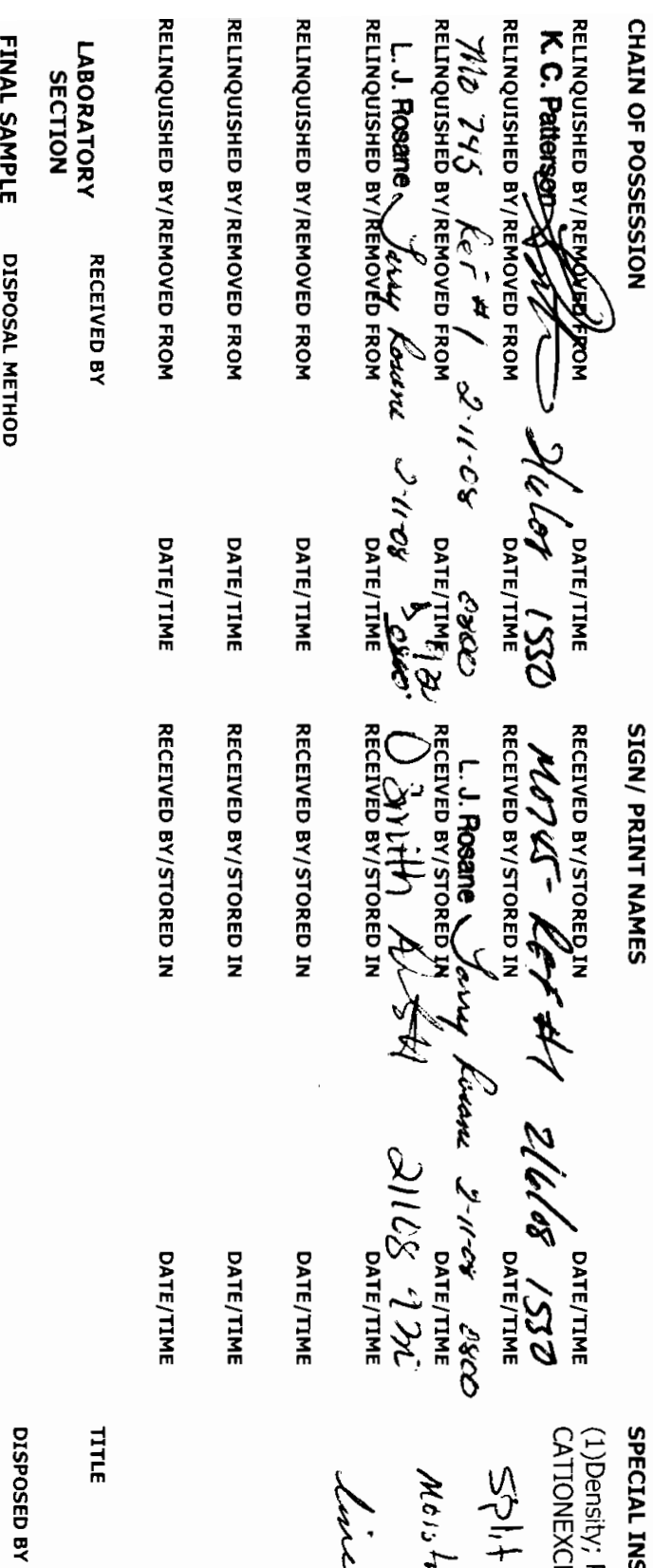

录

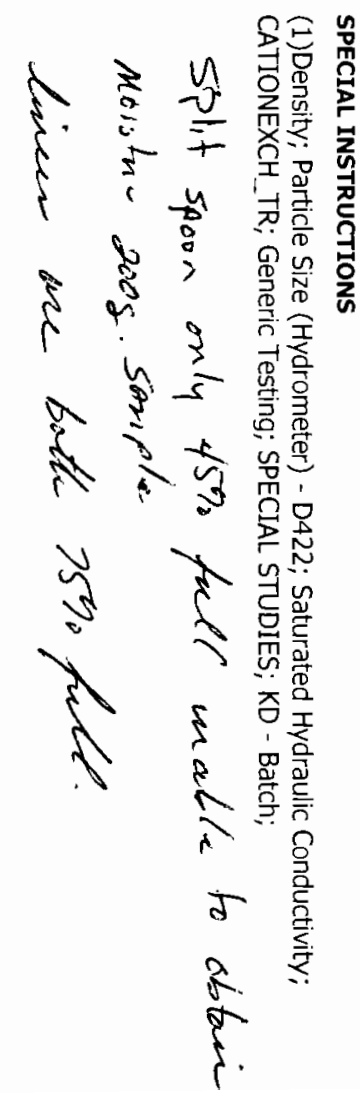

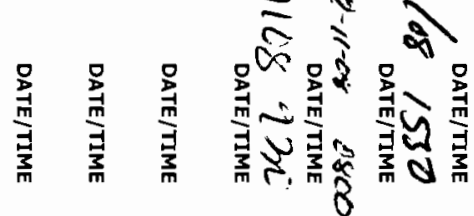

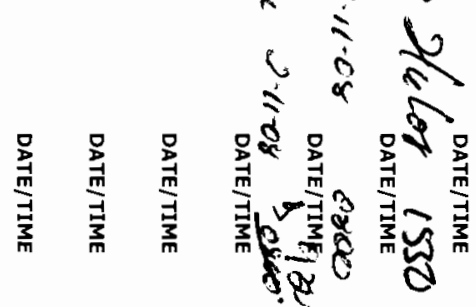

$\stackrel{\text { D }}{\overrightarrow{1}}$

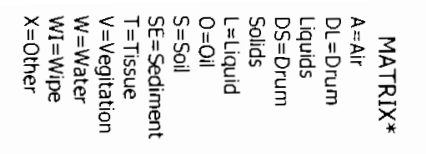

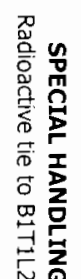

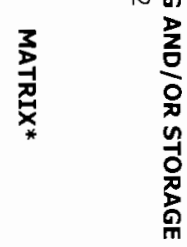

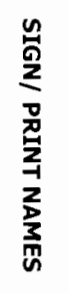
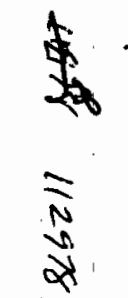

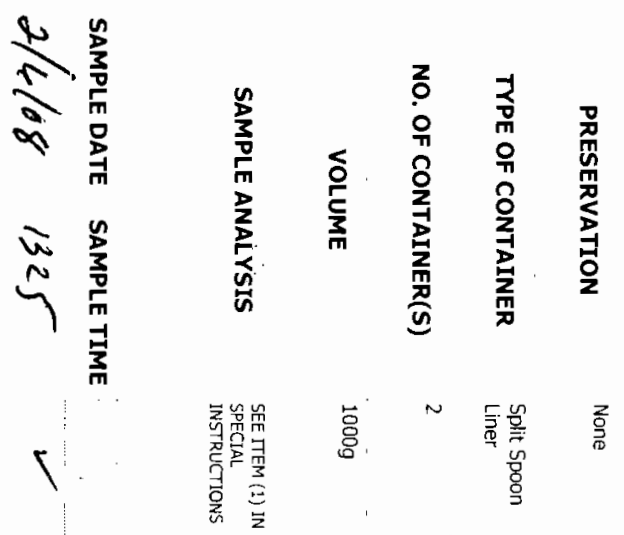
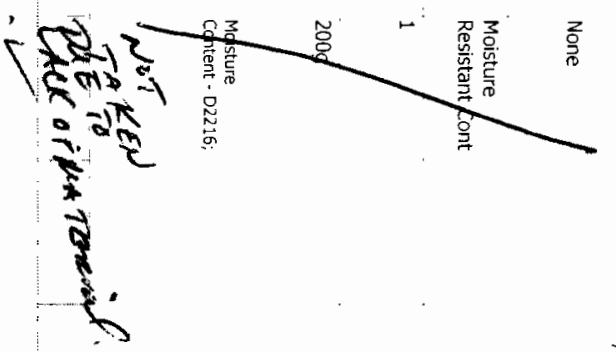

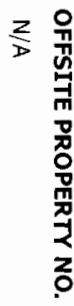

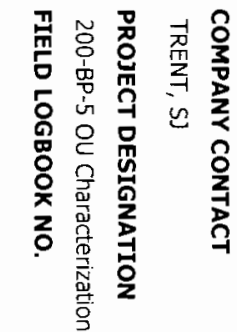

高

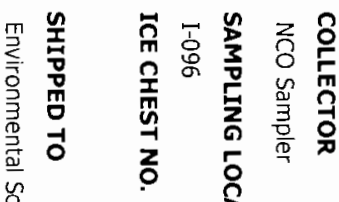

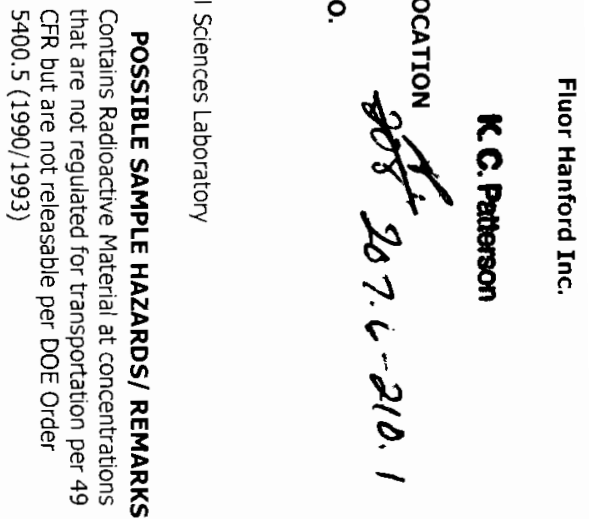

호

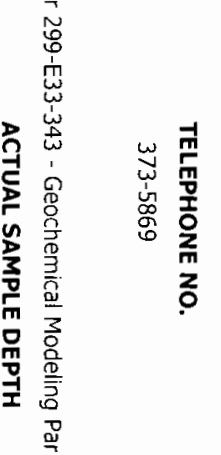

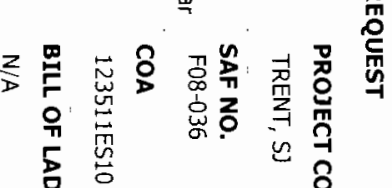

总

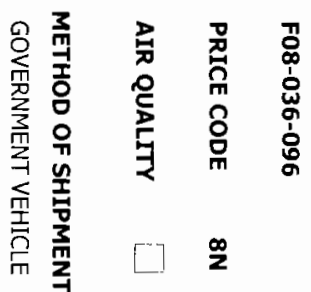

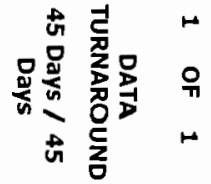




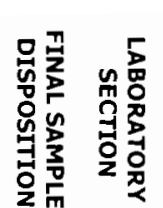

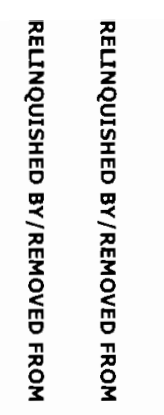

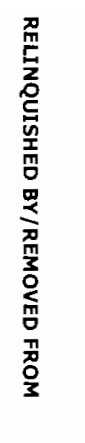

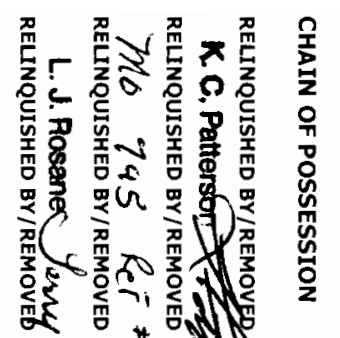

读

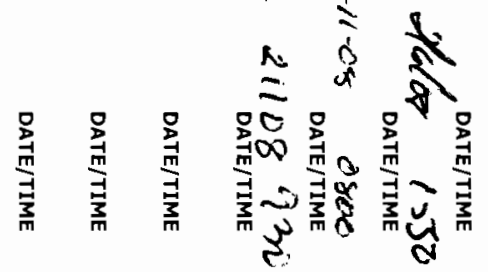
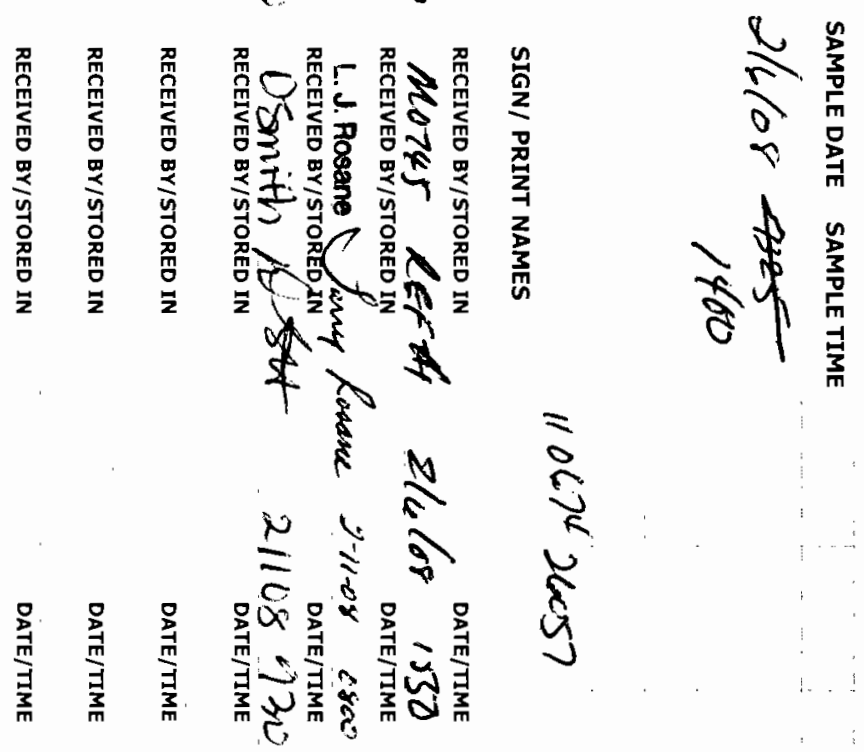

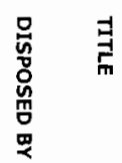

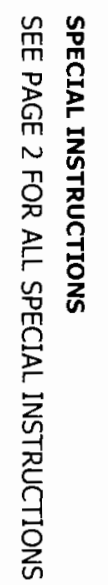

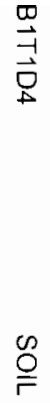

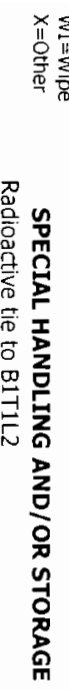

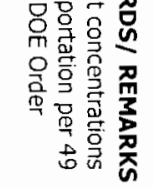
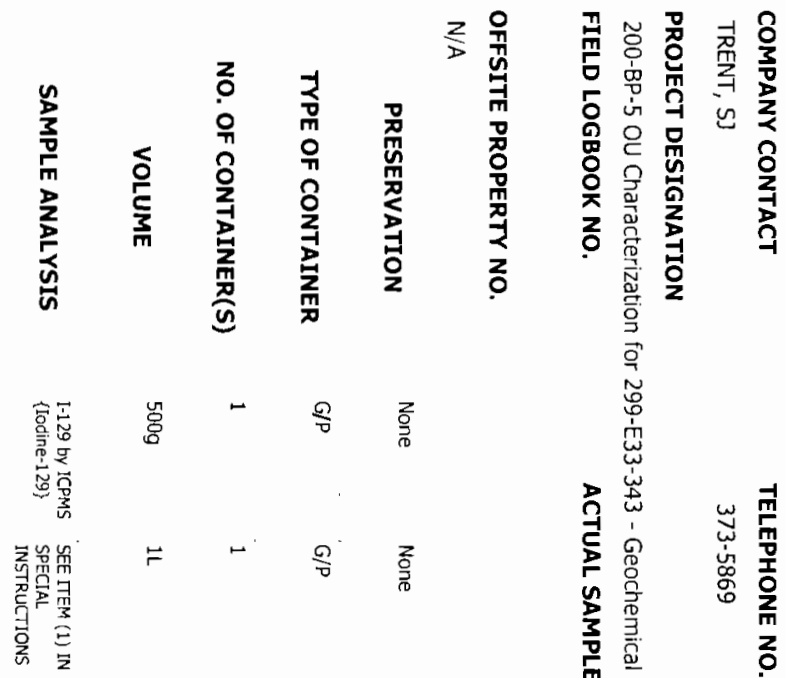

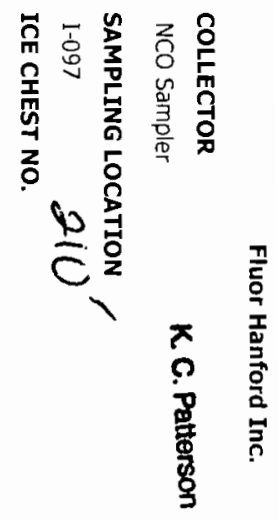

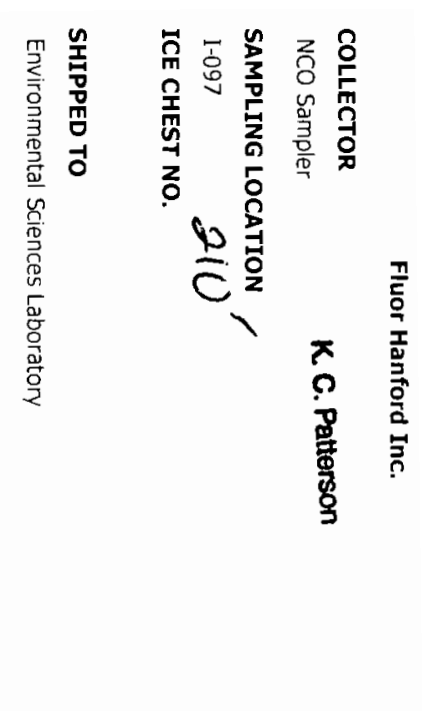

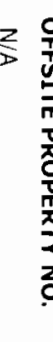

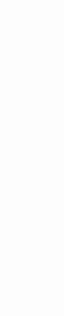




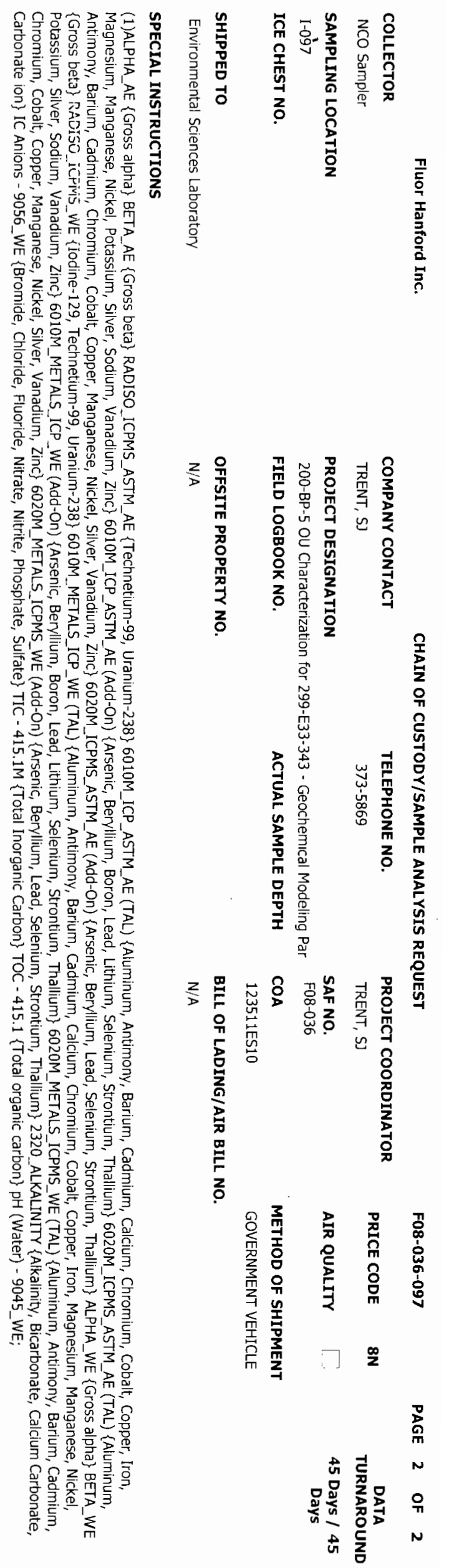




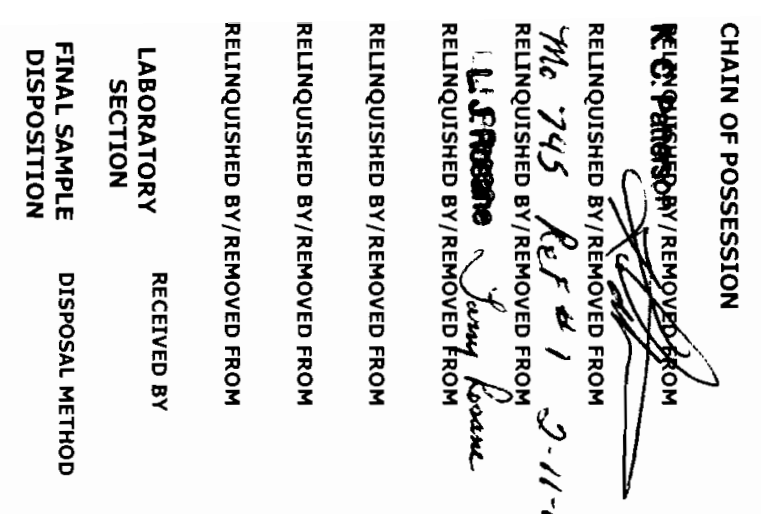

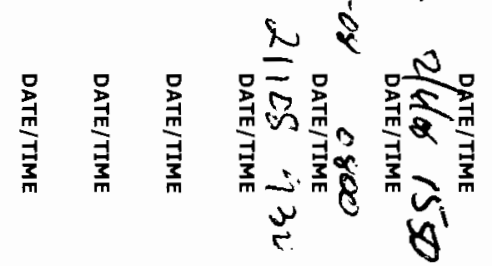

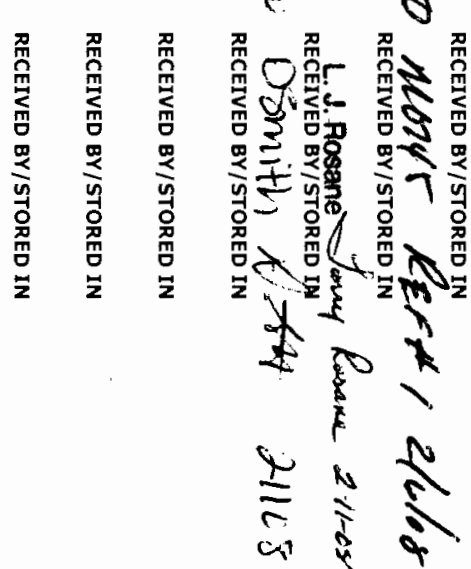

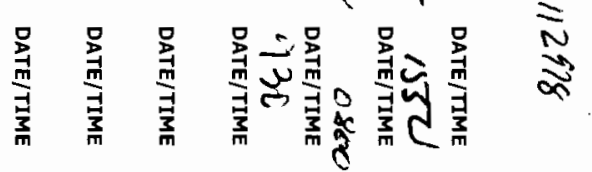

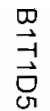

$\stackrel{\infty}{\ominus}$

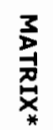

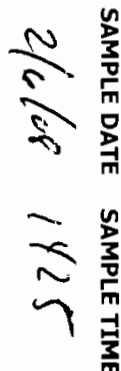

$\leqslant$

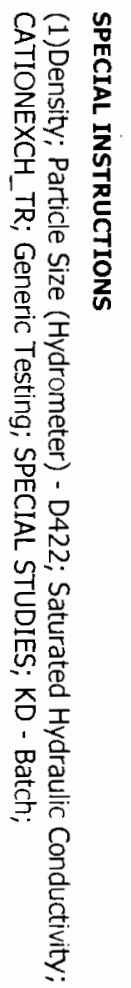

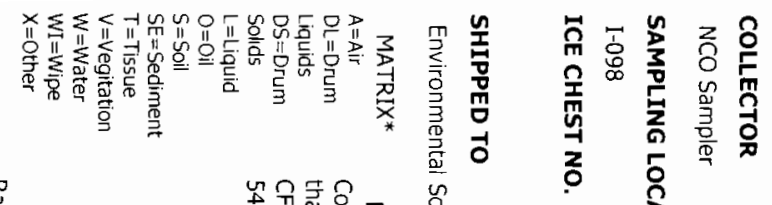

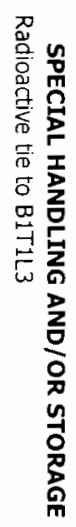

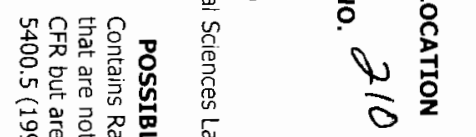

간

8 零

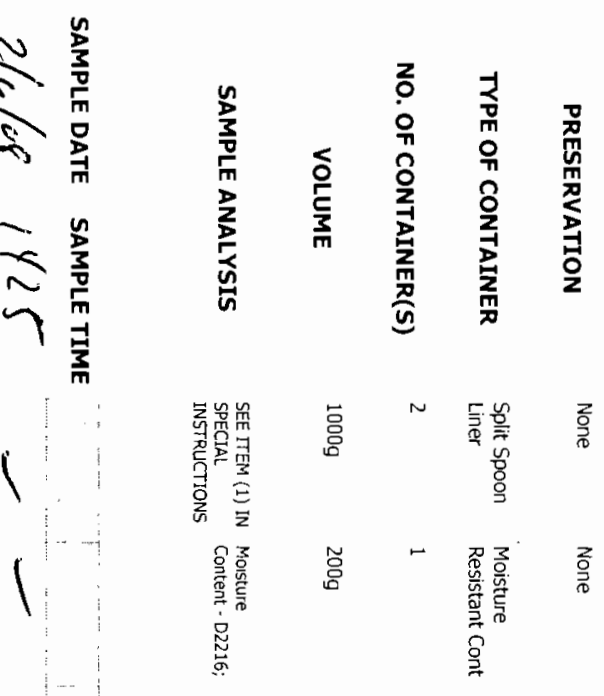

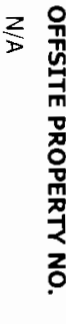

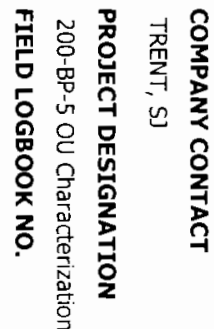

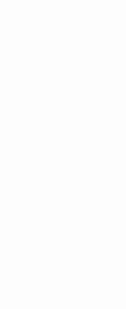

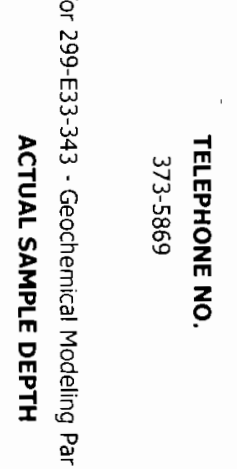

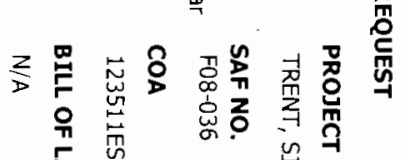

울

홈

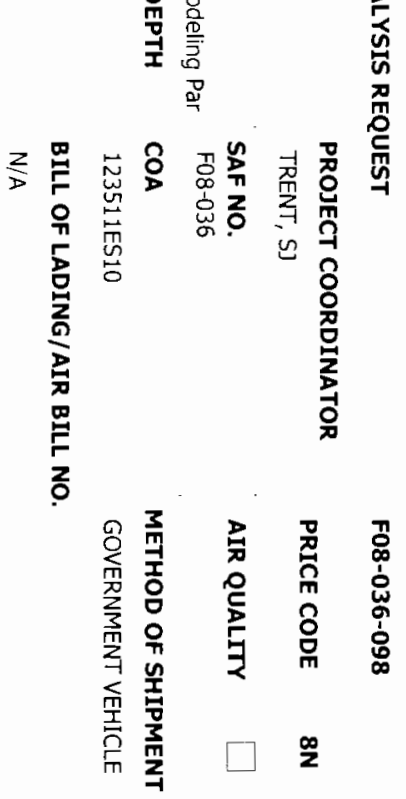

品品

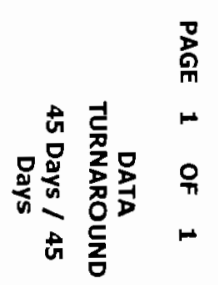


$\|$

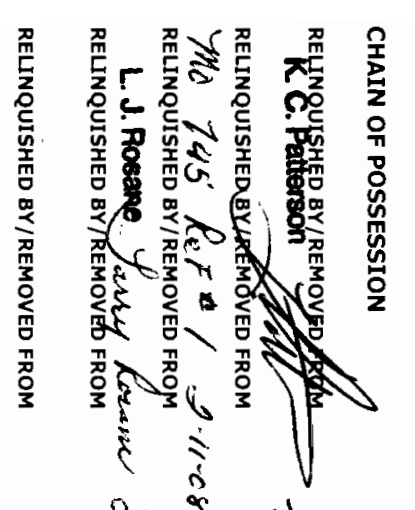

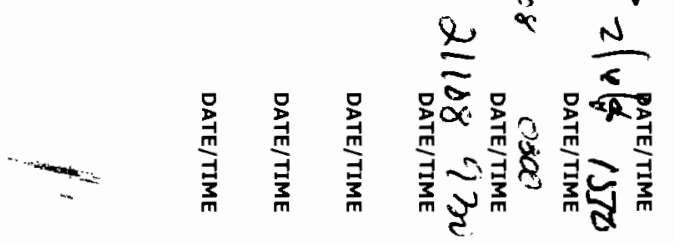
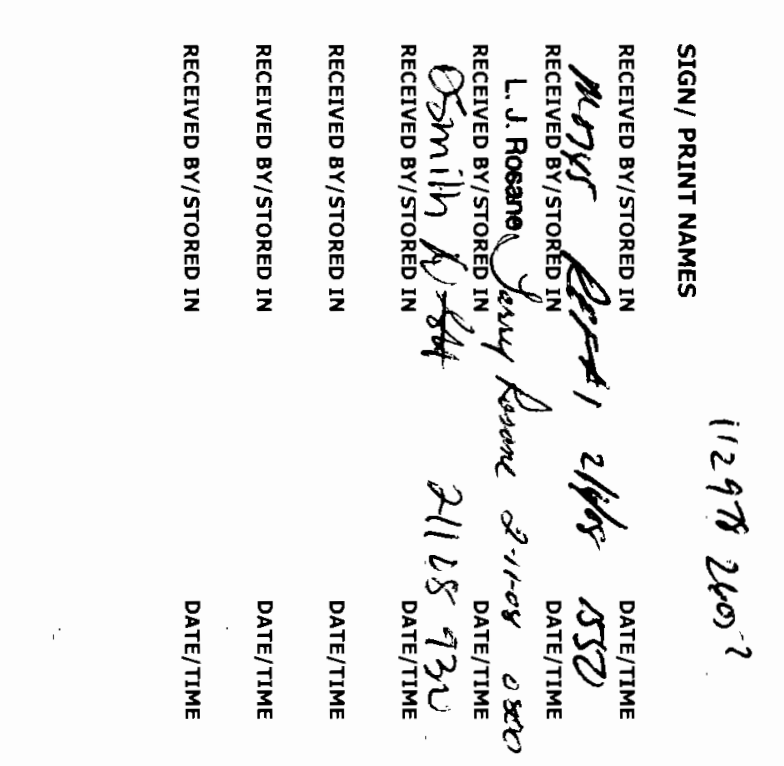

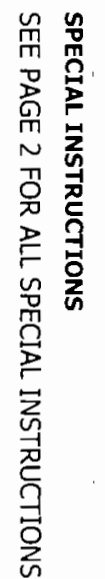

$\underset{ \pm}{二}$
$\overrightarrow{0}$
$\stackrel{\sigma}{\vec{\sigma}}$

$\stackrel{\infty}{\circ}$

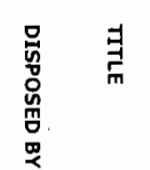

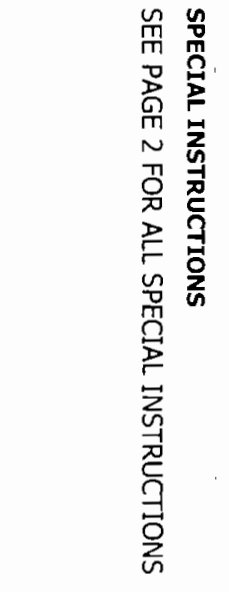

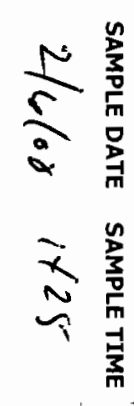

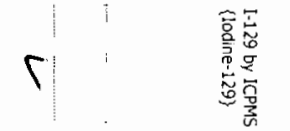

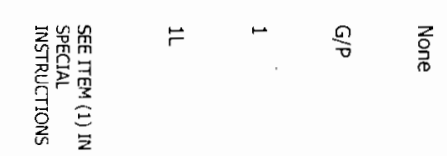

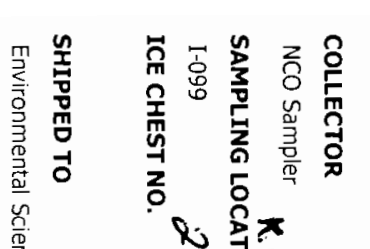

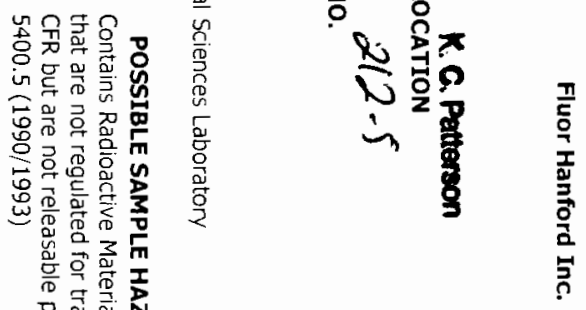

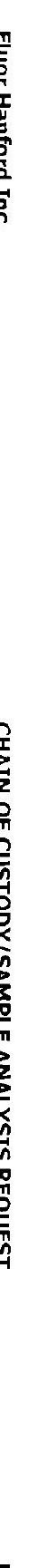

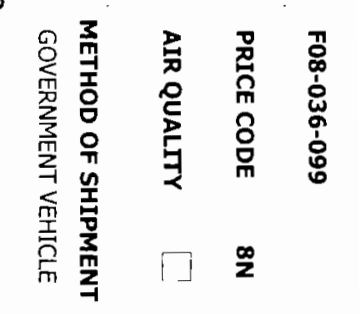

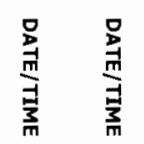

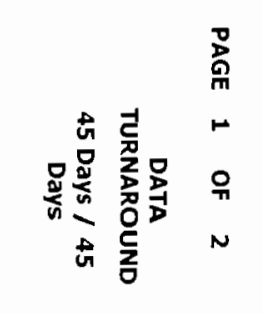




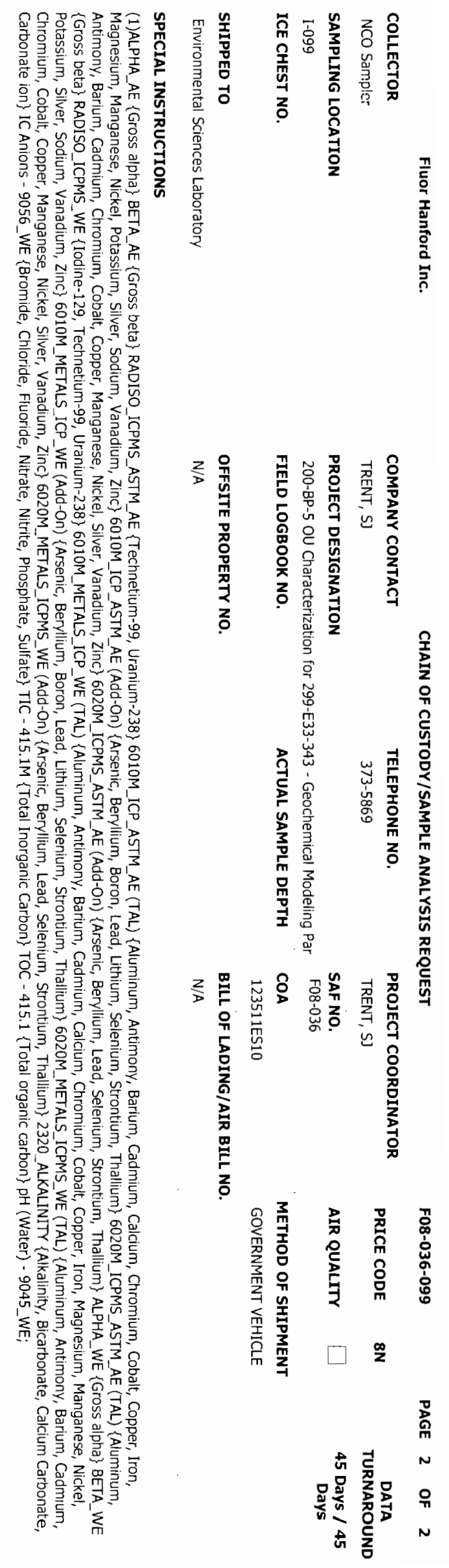




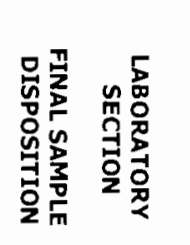

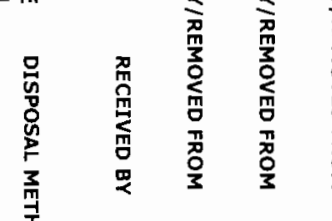

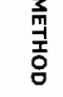
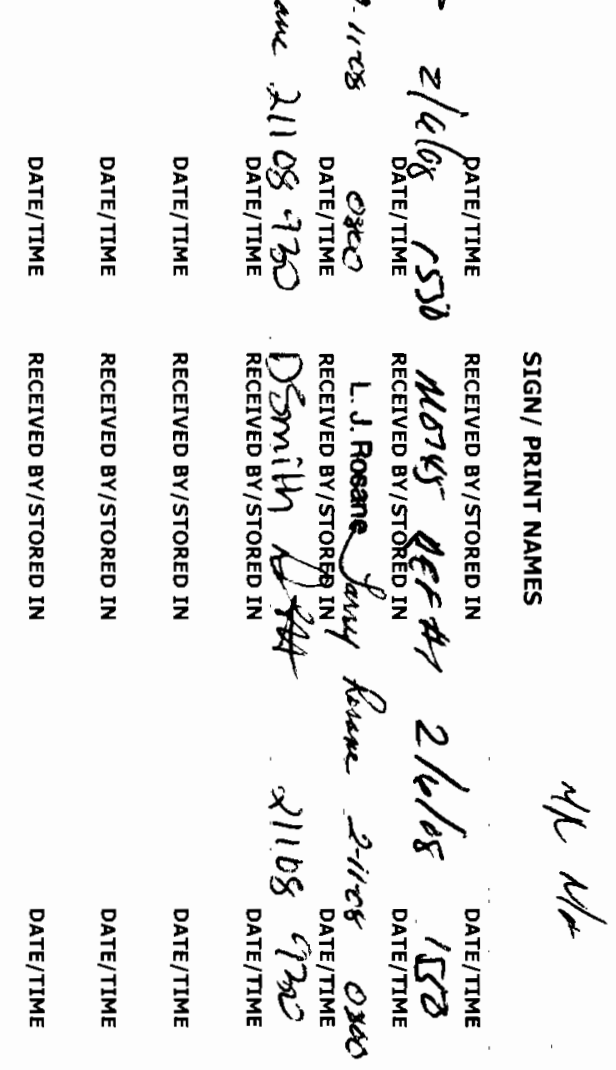

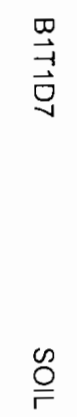

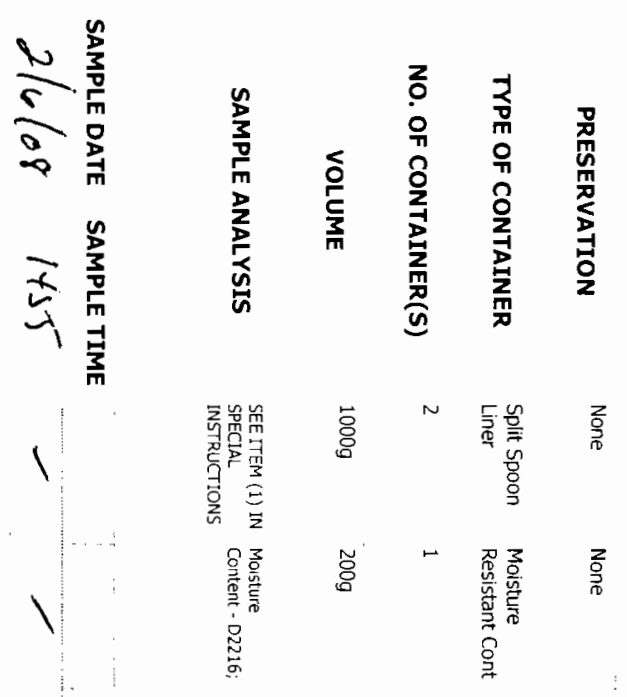

윰음

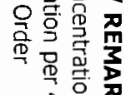

量

章

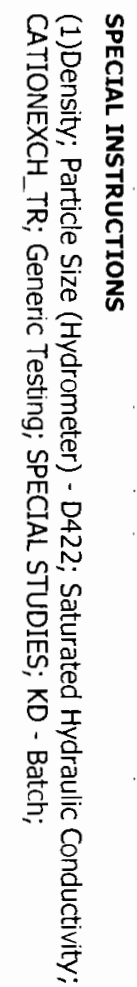

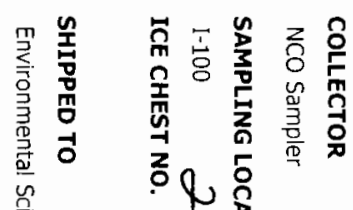

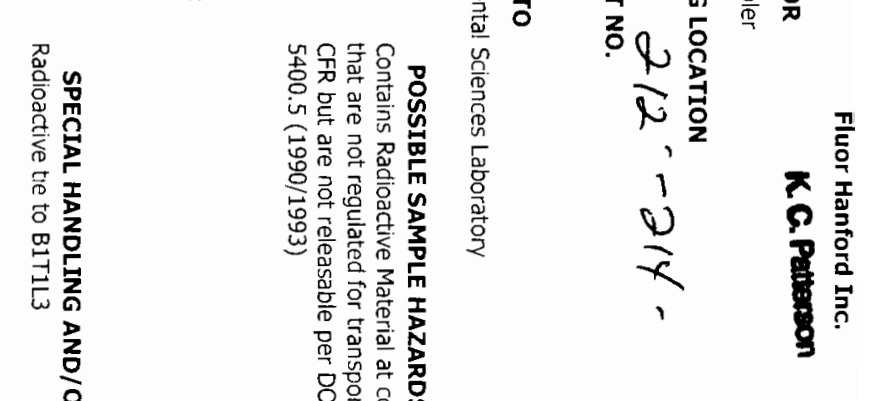

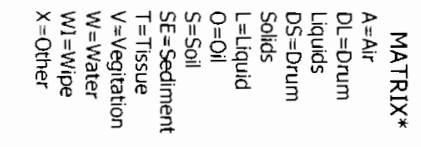

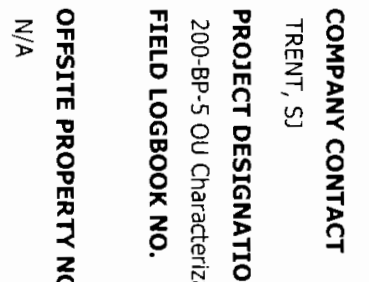$$
\text { 골 }
$$

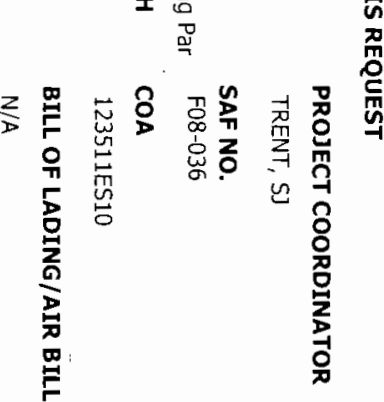

${ }^{\frac{2}{\overline{9}}}{ }^{2}$

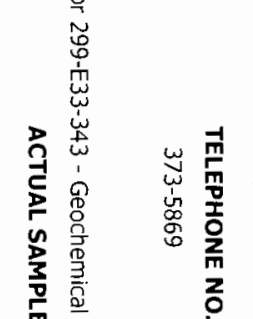

而

员

올

증
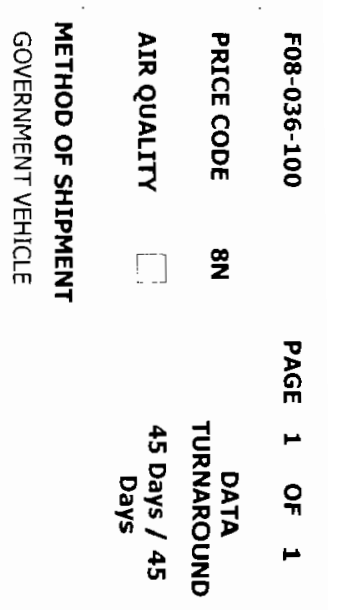
En

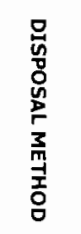

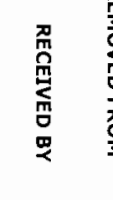

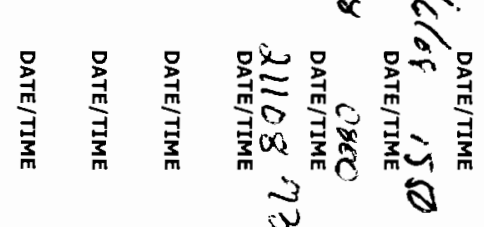

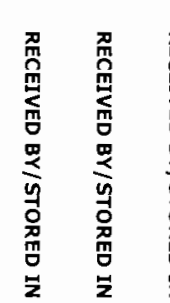

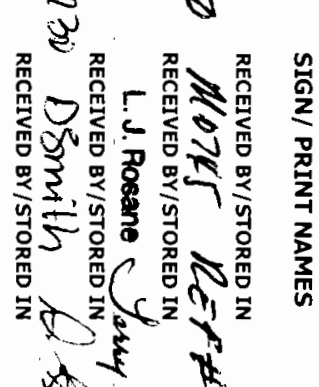

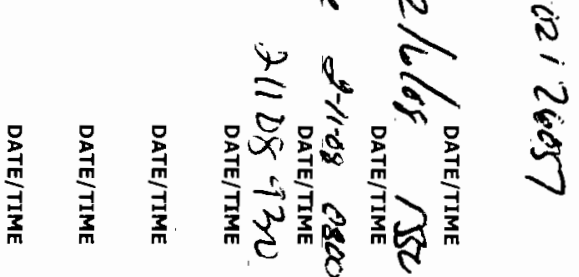

莍

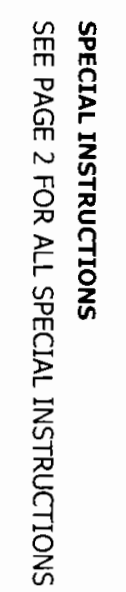

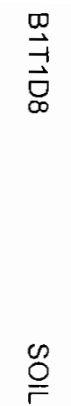

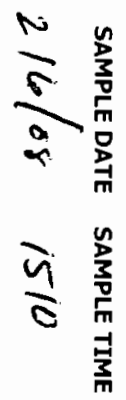

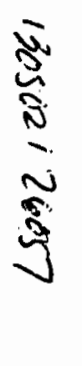

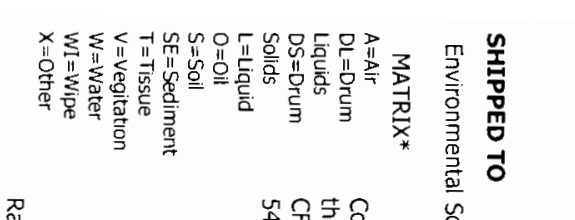

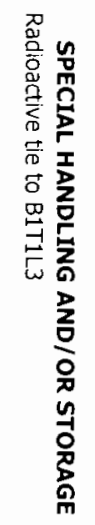

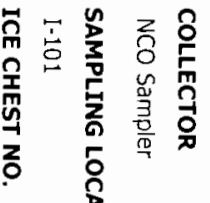

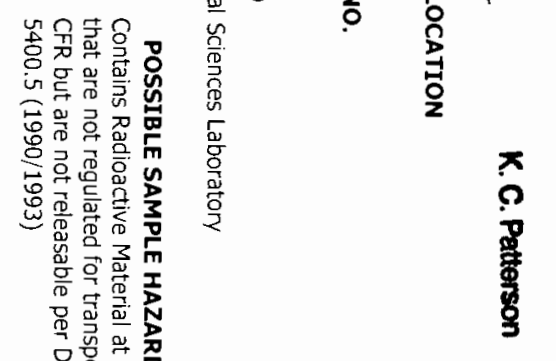

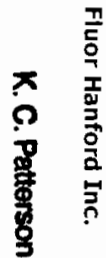

앙응

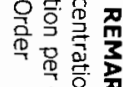

战离

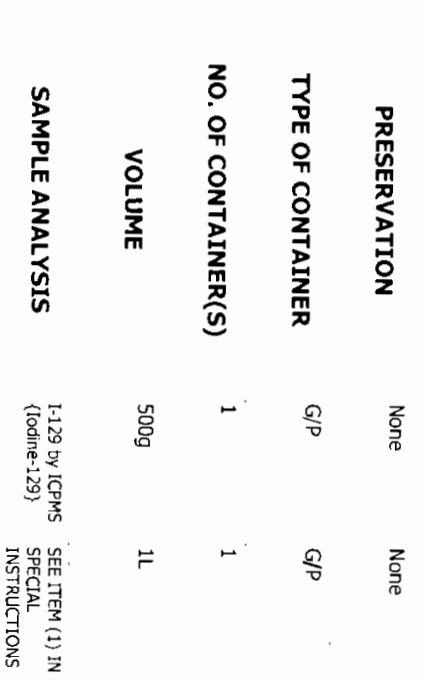

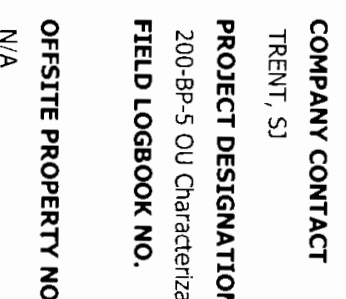

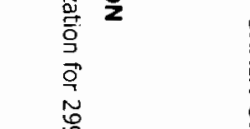

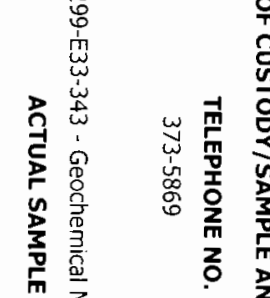

밈

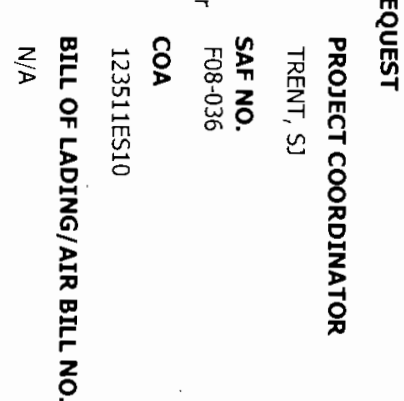

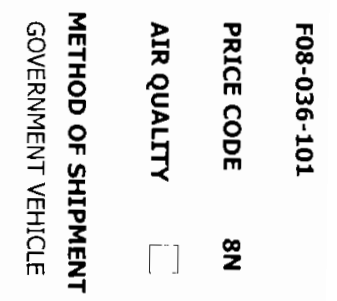




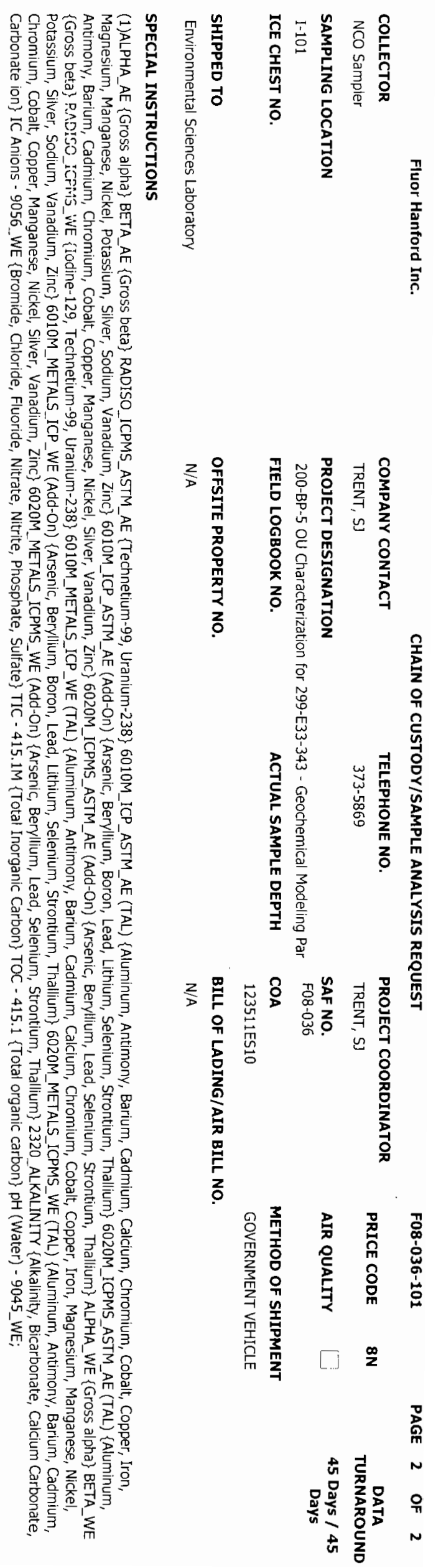




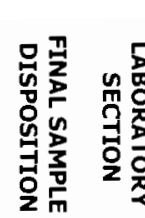

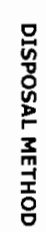

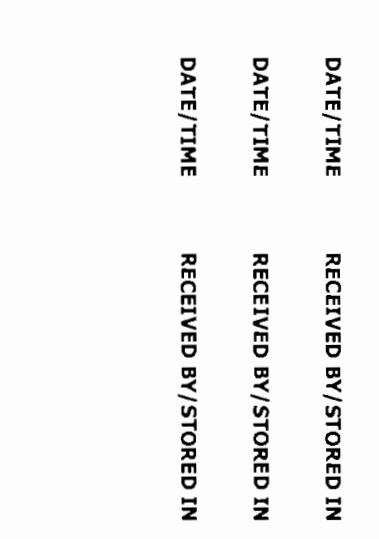

(1) 8

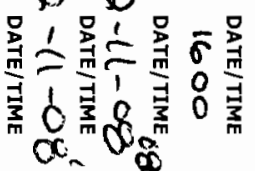

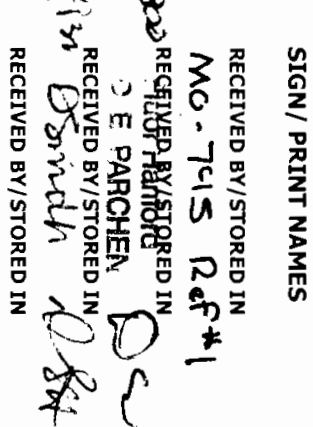

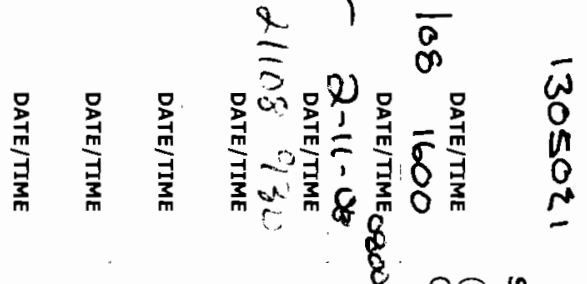

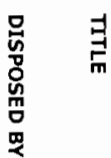

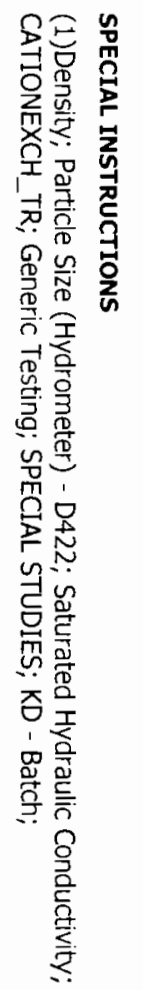

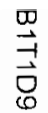

?

$\stackrel{\varrho}{ }$

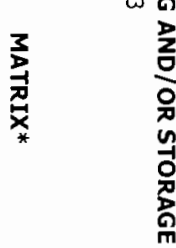

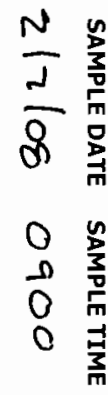

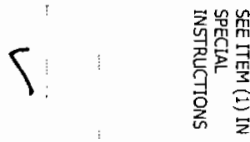

s

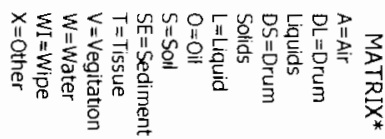

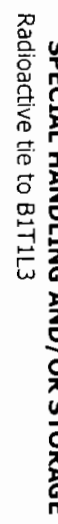

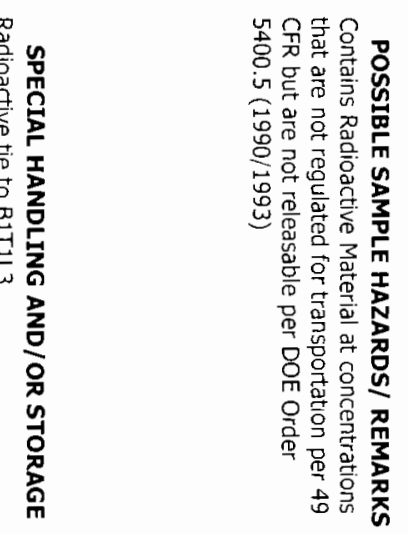

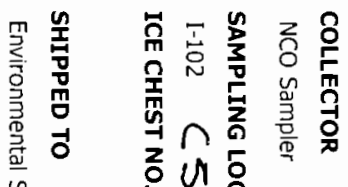

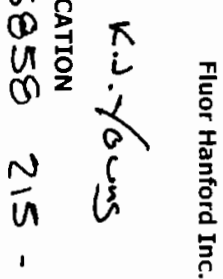

$\frac{1}{N}$

in

졸

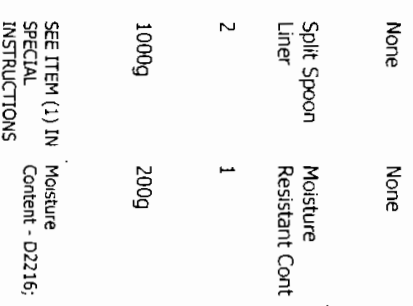

$z$

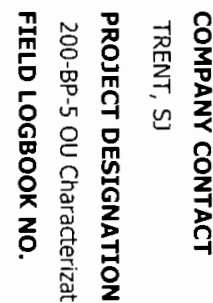

产 2

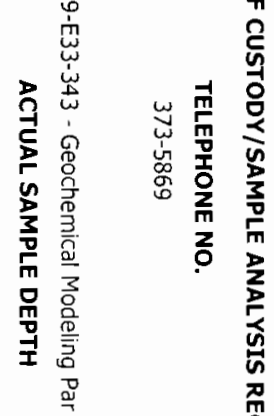

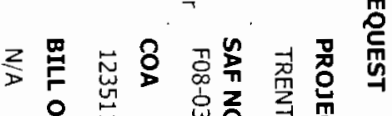

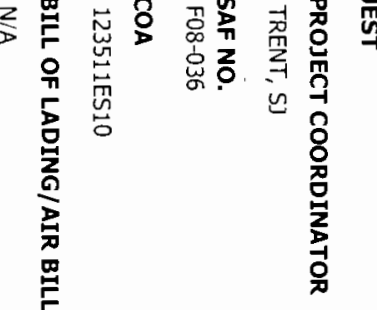

z

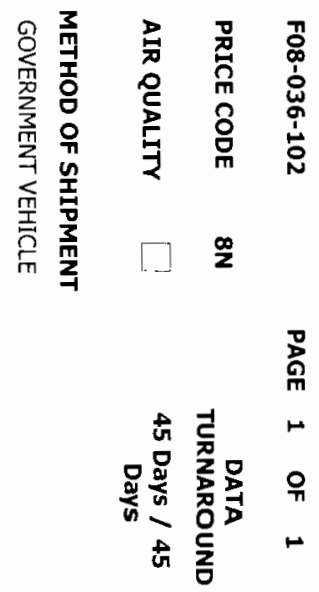




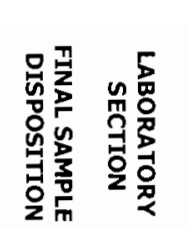

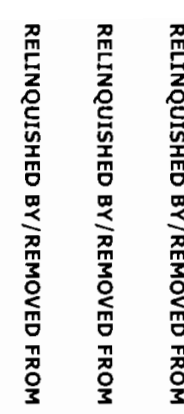

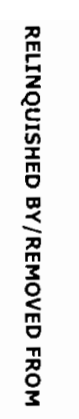

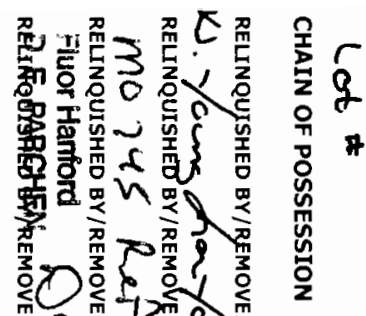

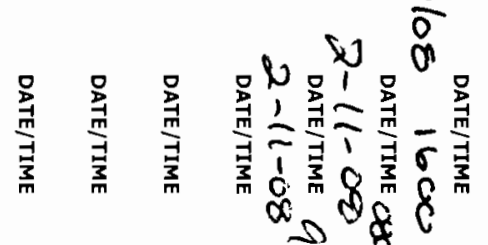

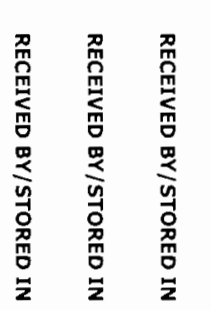

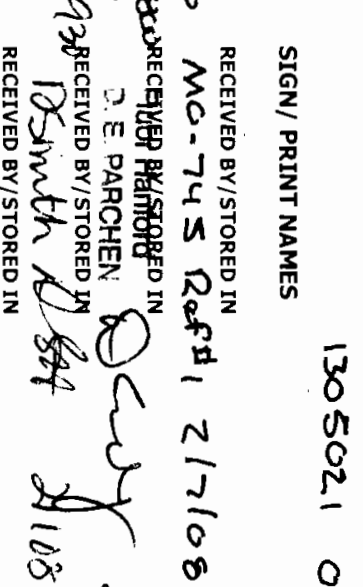

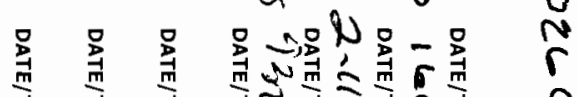

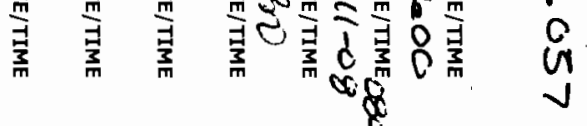

$\underset{n}{\exists}$

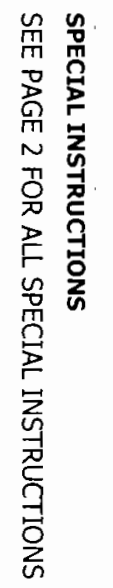

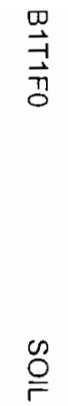

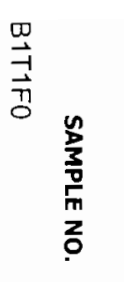

$\stackrel{\infty}{\circ}$

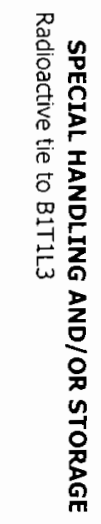

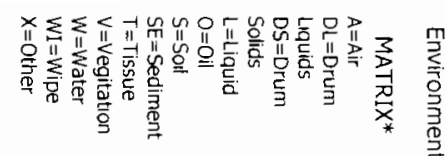

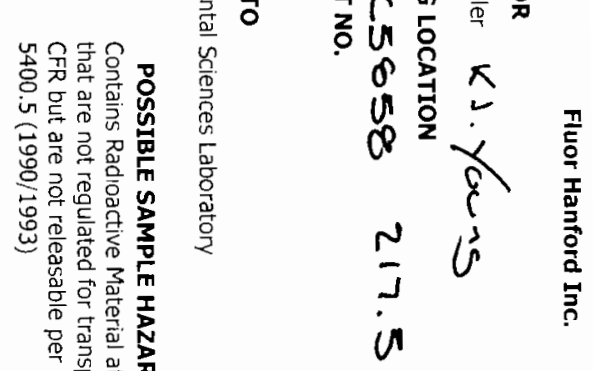

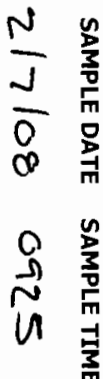

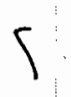

S

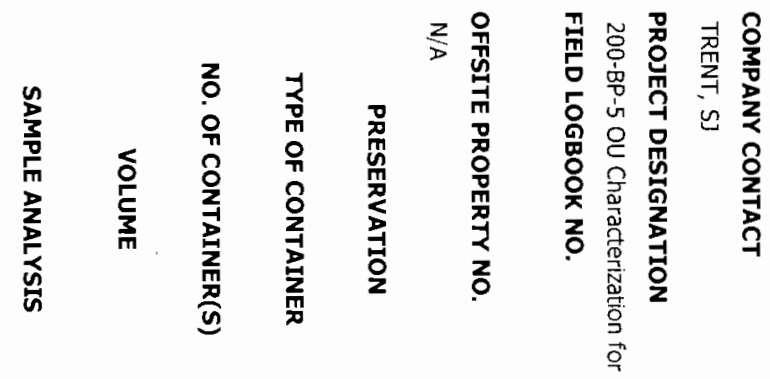

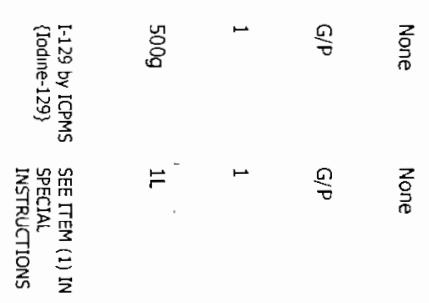

온

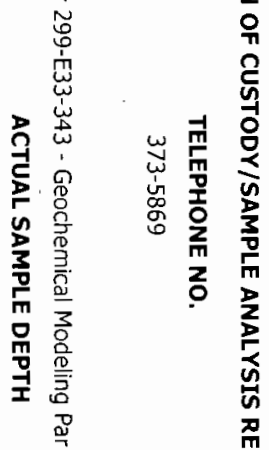

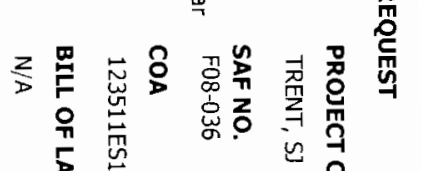

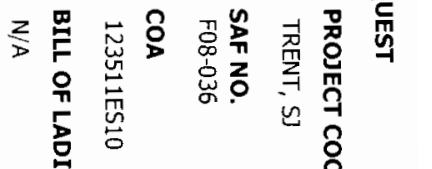

(2)

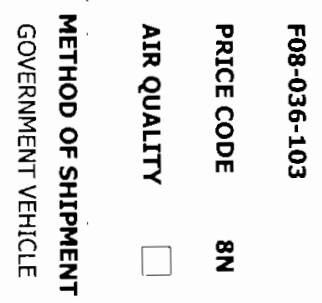

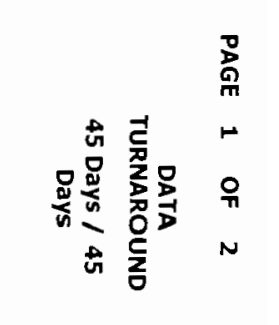




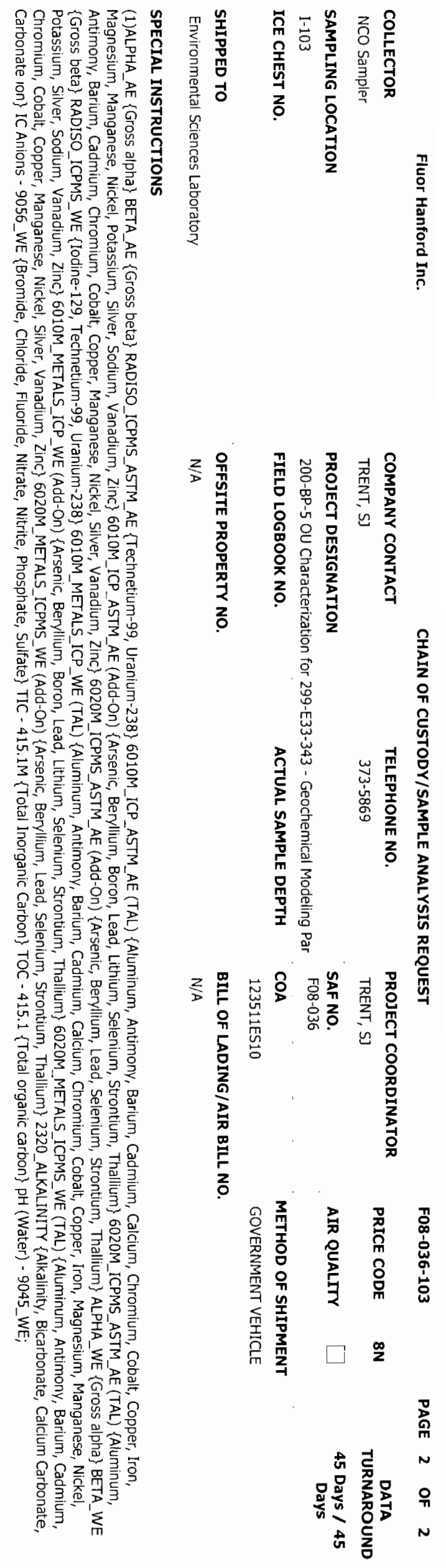



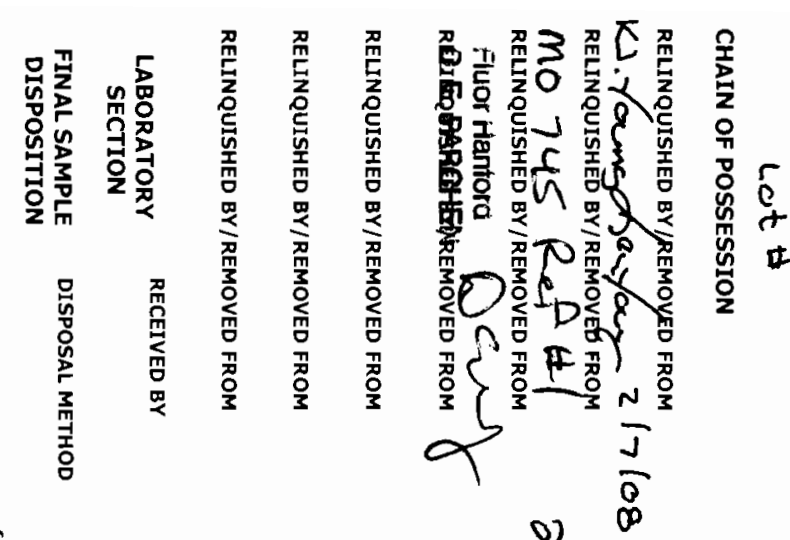

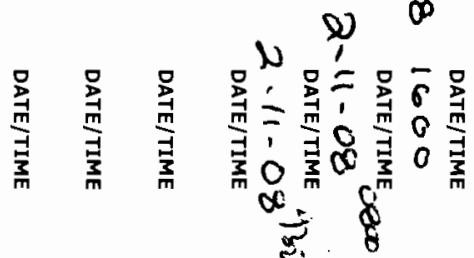
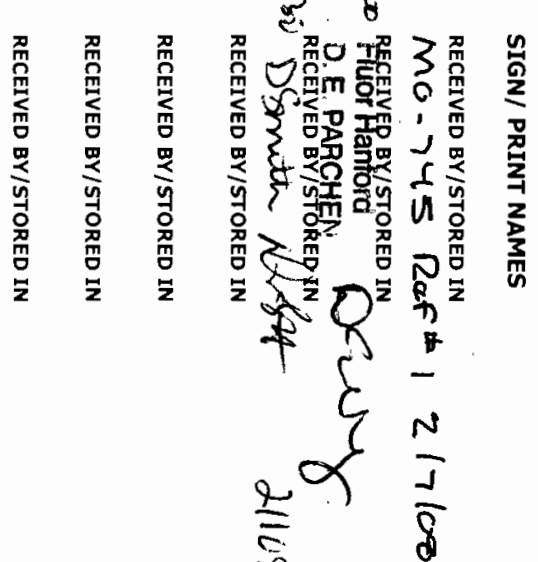

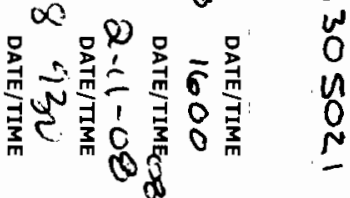

品

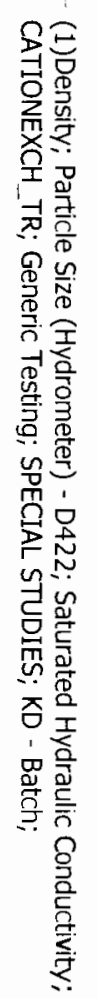

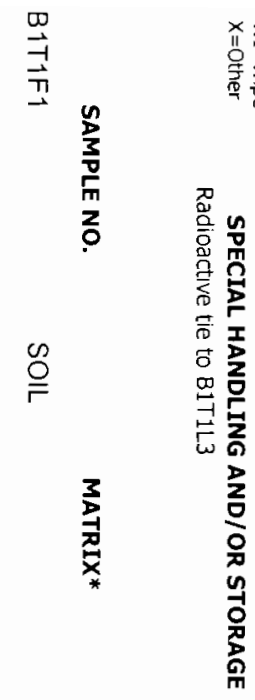

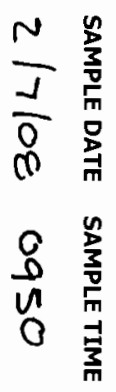

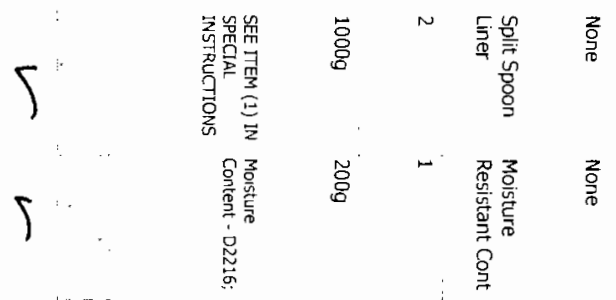

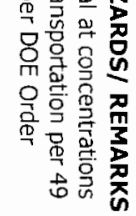

함

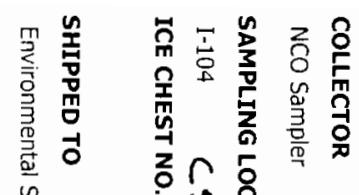

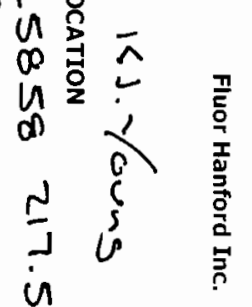

I

N

0 .
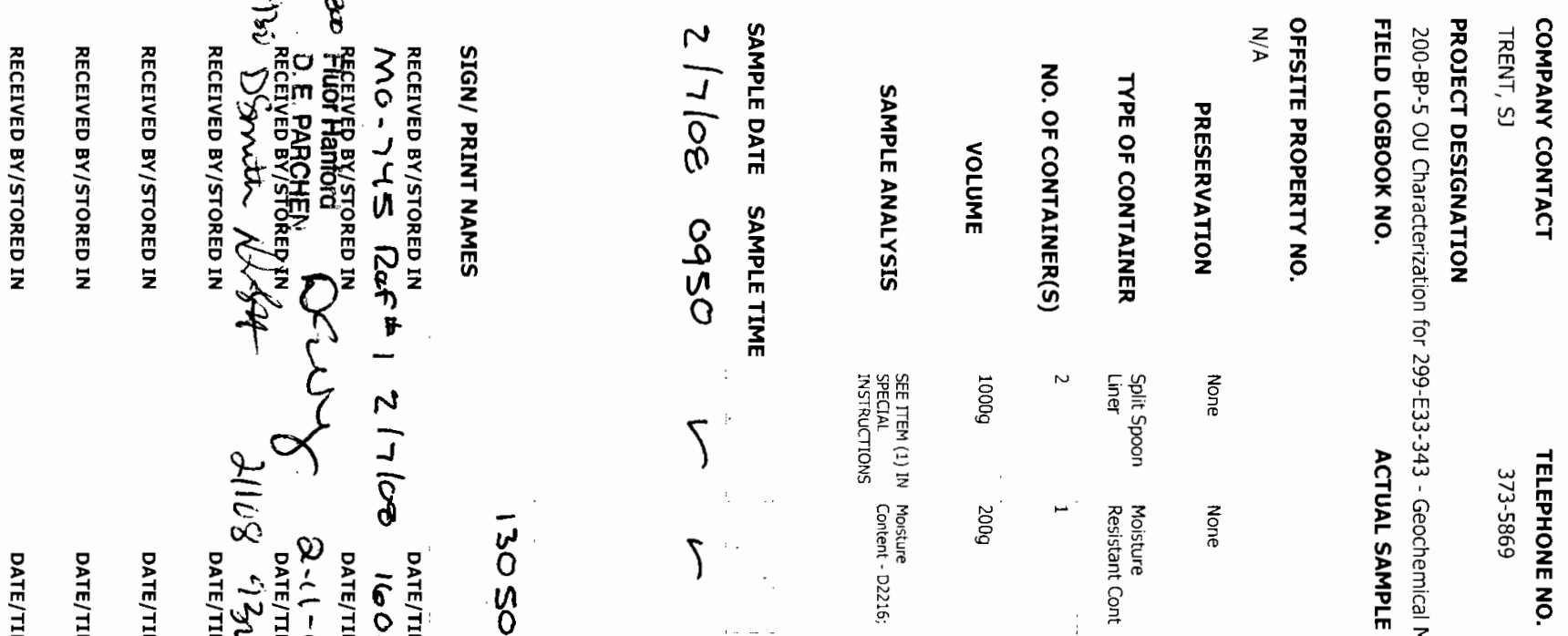

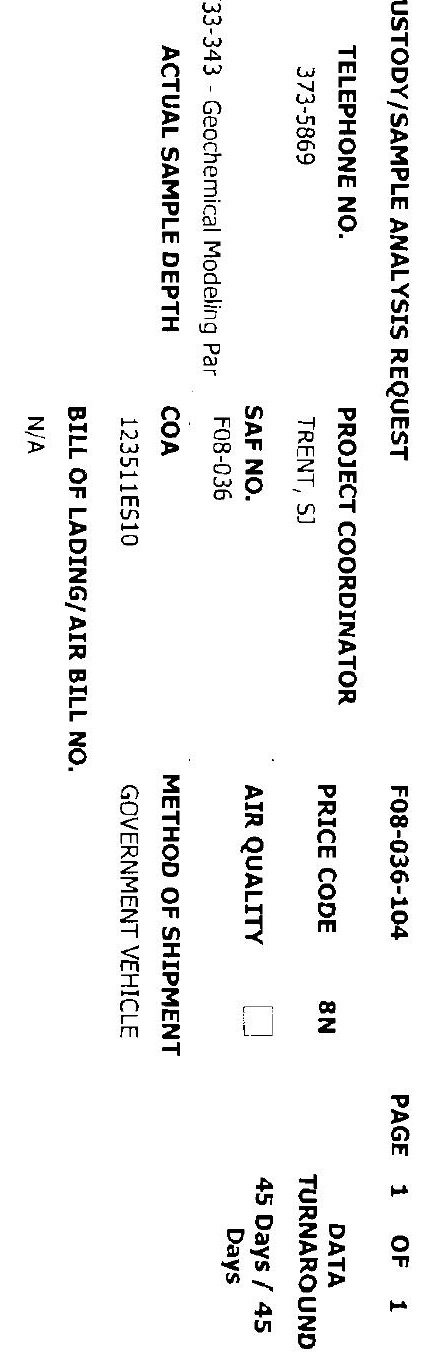



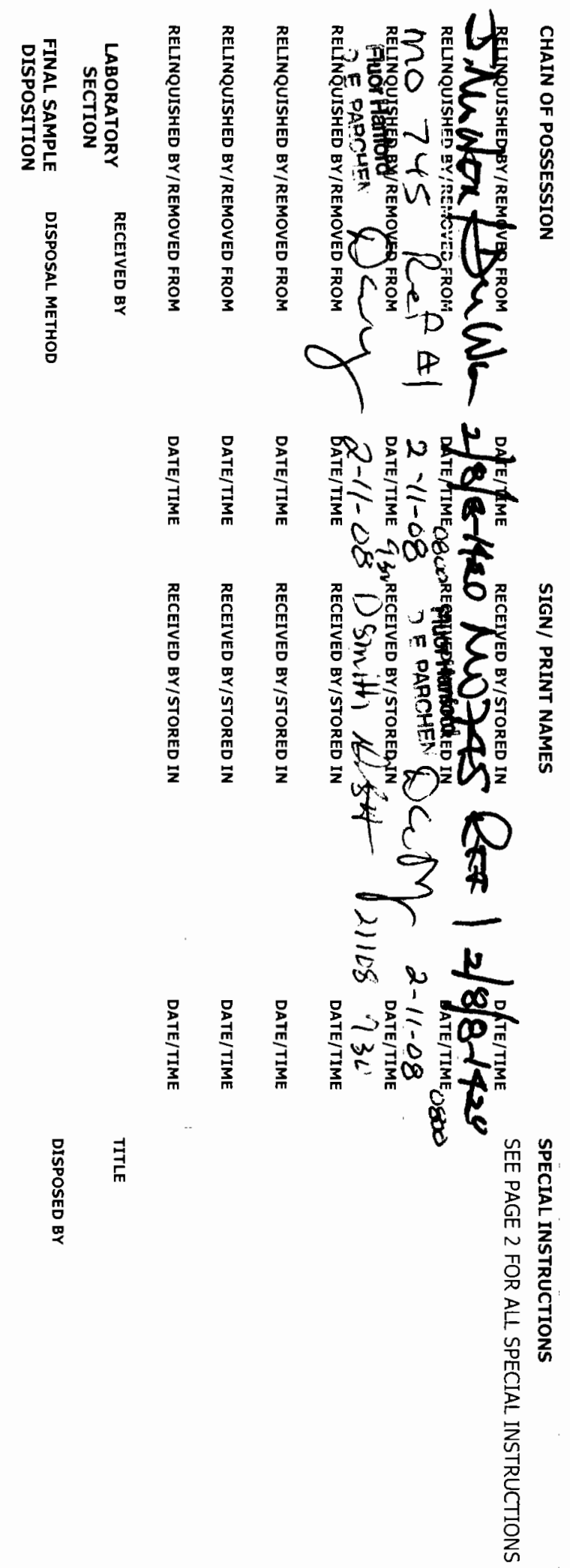
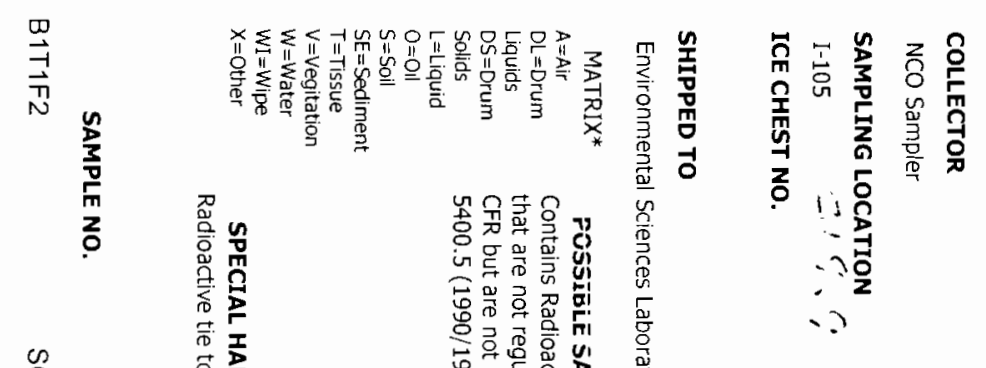

$\stackrel{\infty}{\circ}$

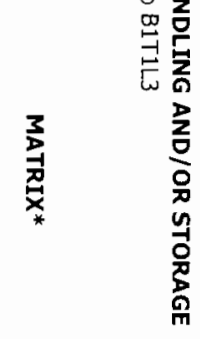

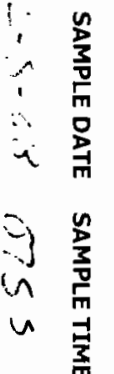
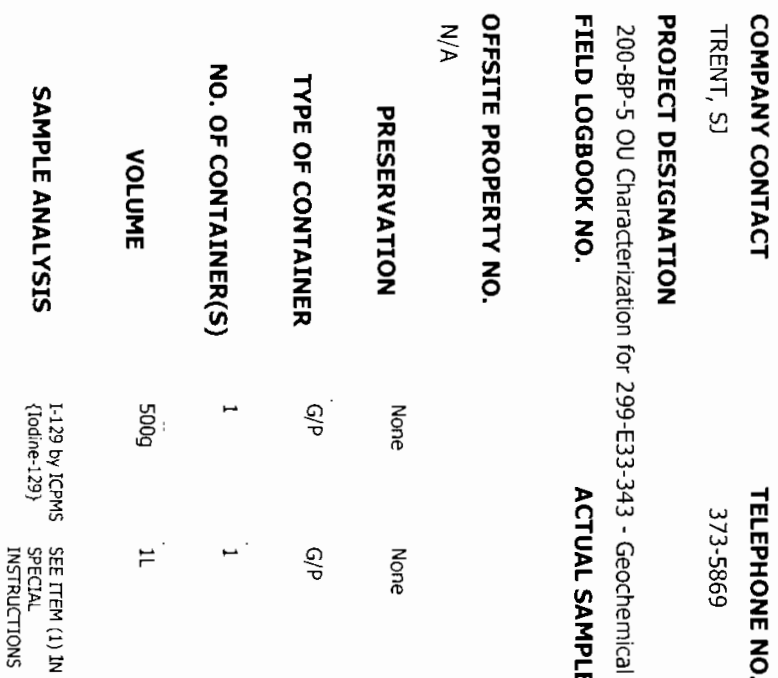

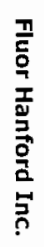

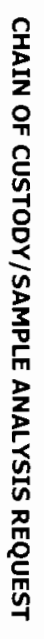

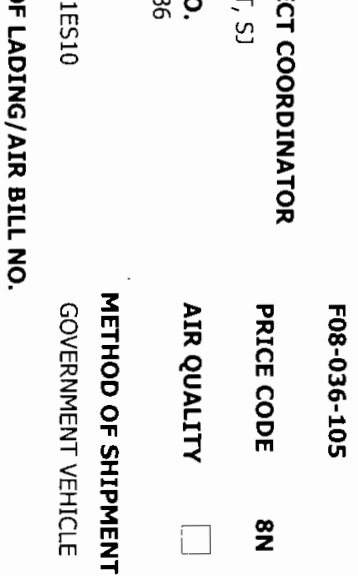

荈品

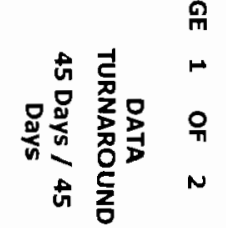




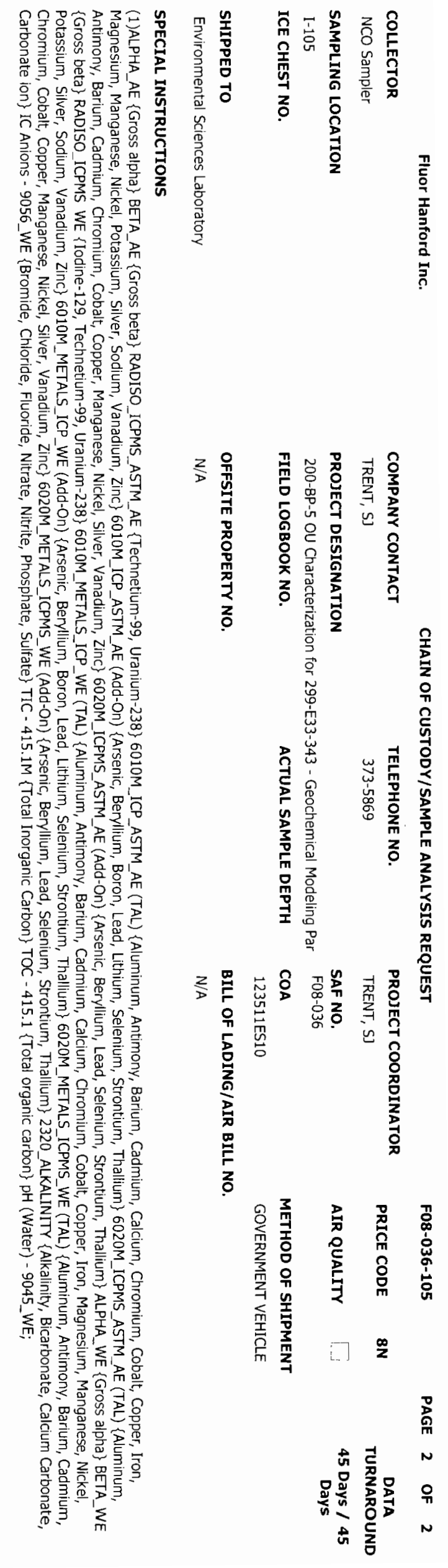




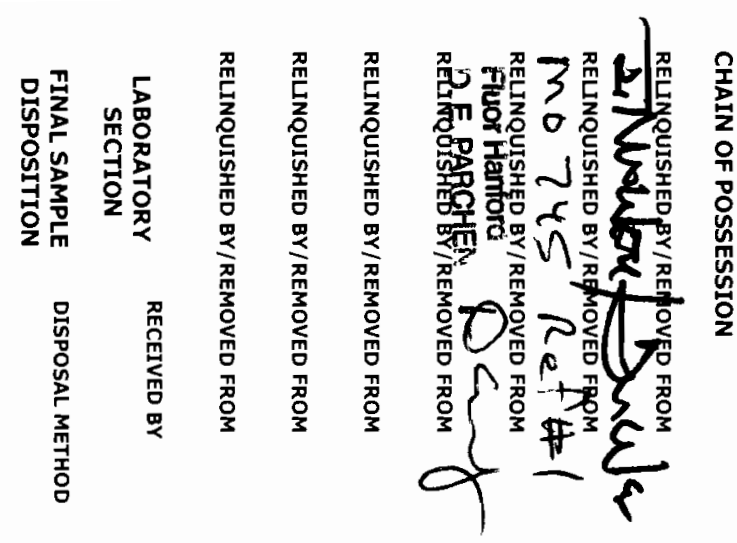

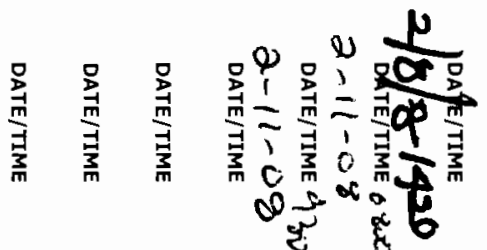
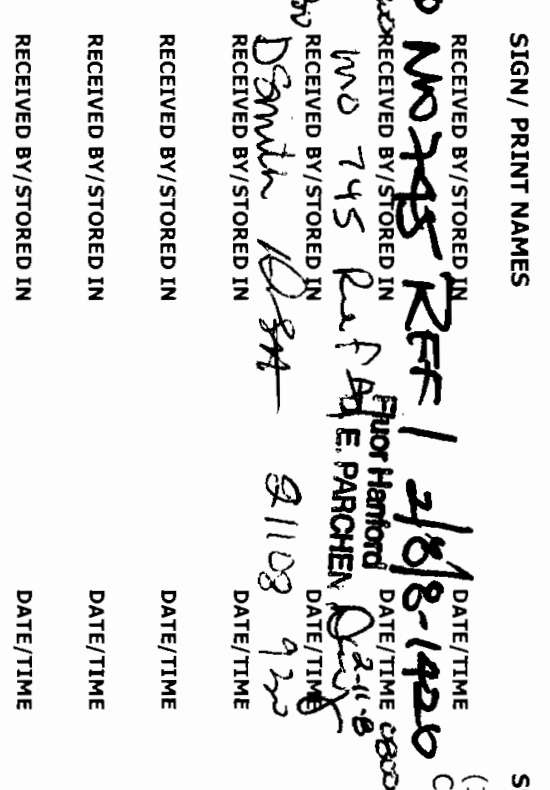

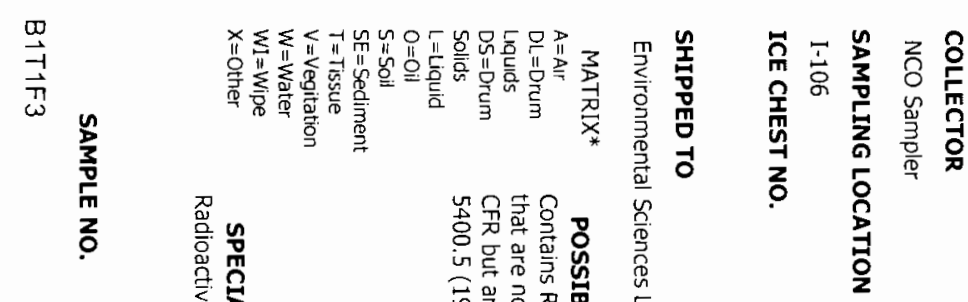

$\stackrel{\infty}{ }$

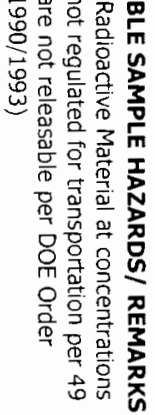

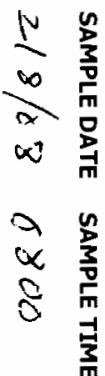
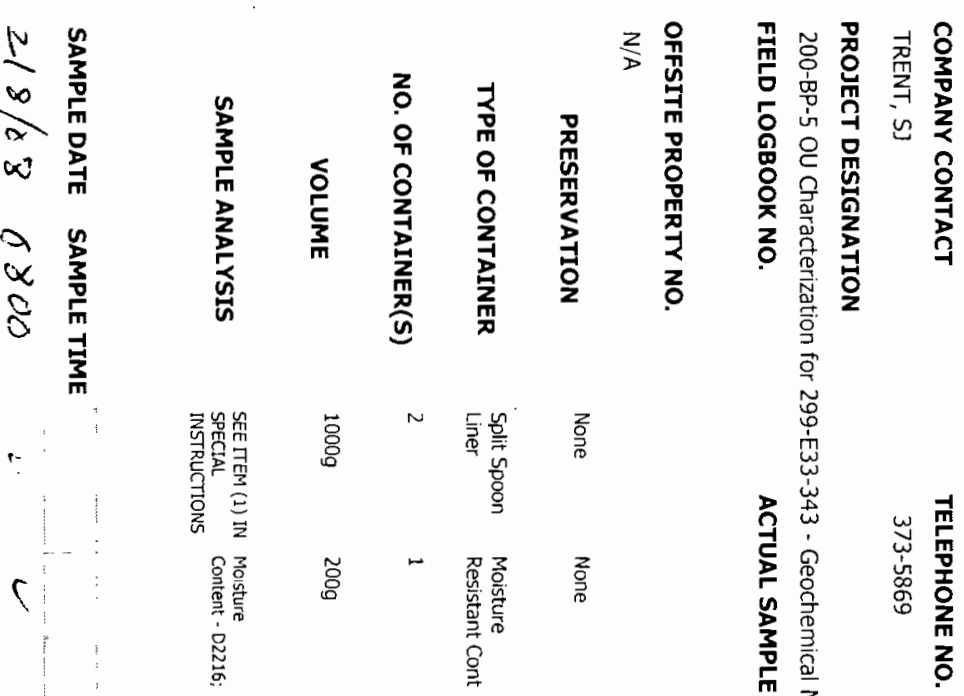

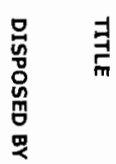

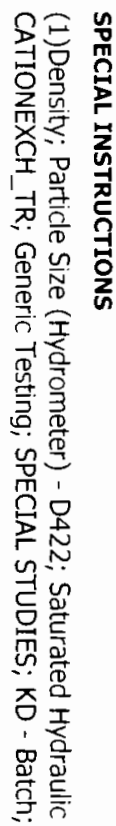

品暑

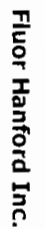

,

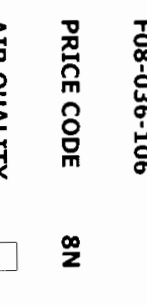

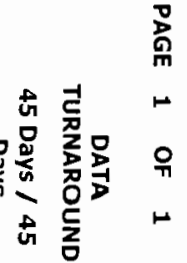




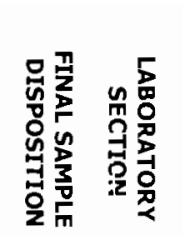
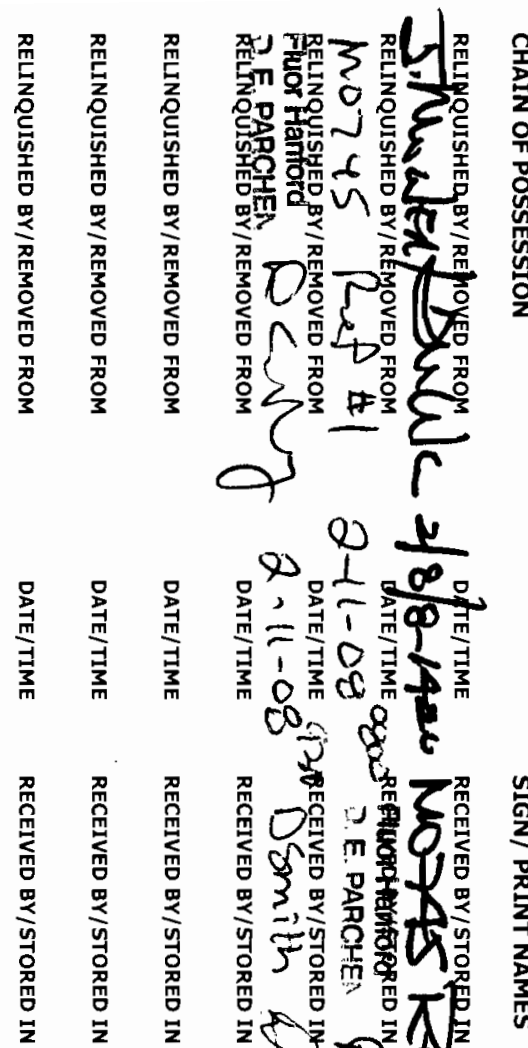

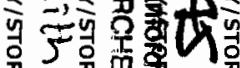

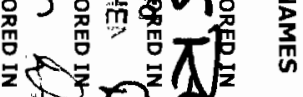

号总

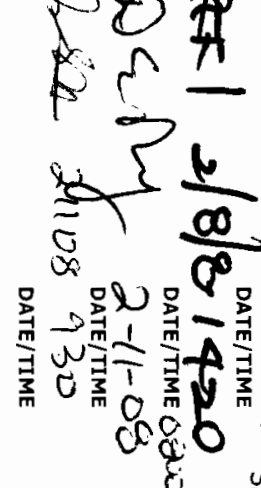

$\stackrel{\square}{\vec{\exists}}$

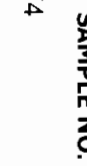

$\stackrel{0}{\stackrel{0}{ }}$

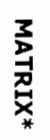

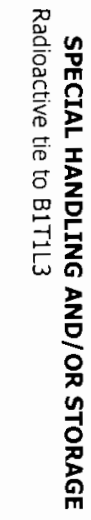

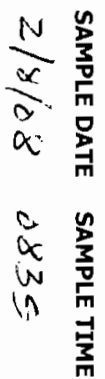

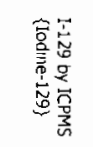

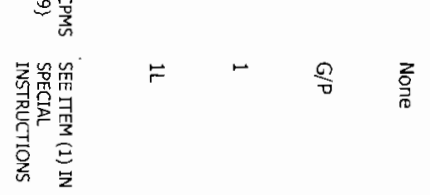

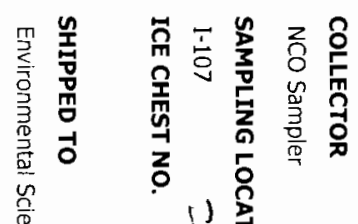

$N$ 을

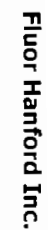

前言 党

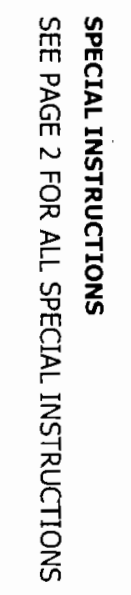

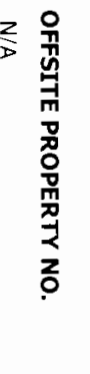

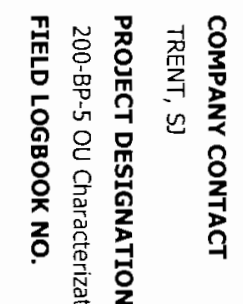

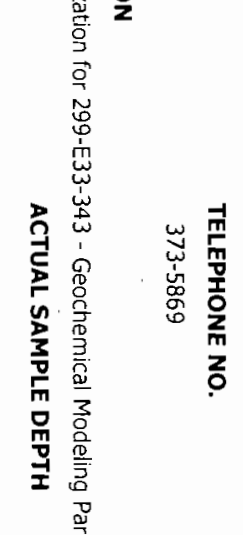

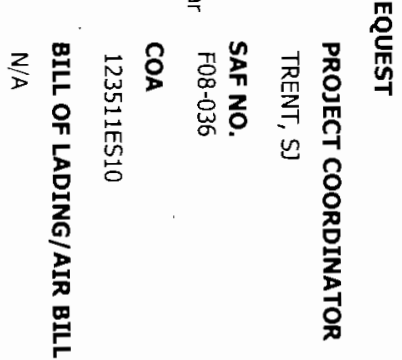

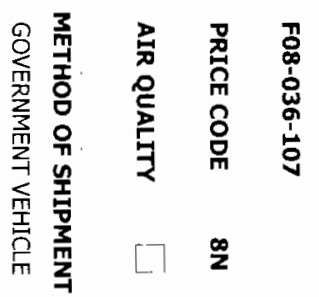

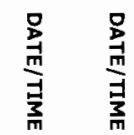

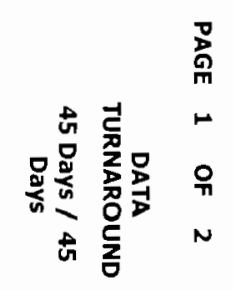




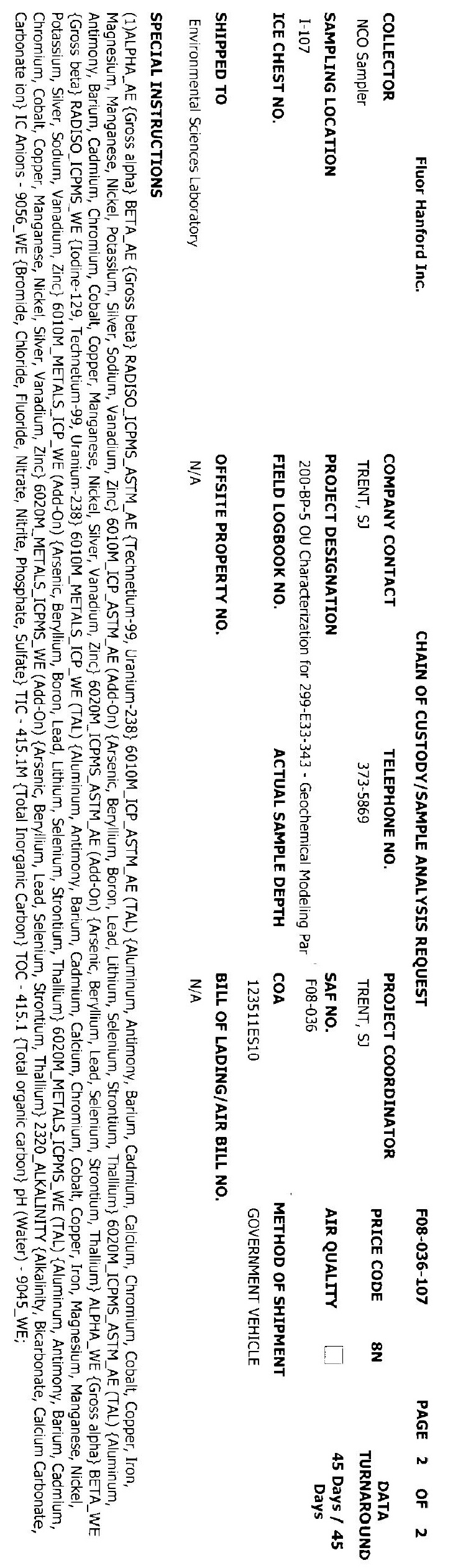



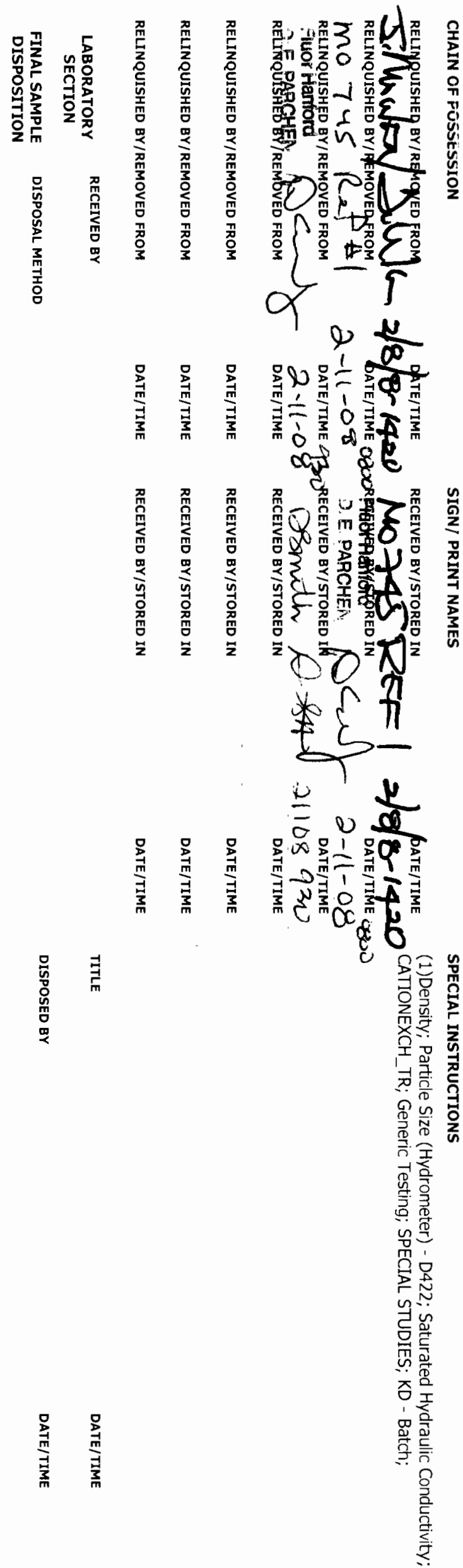

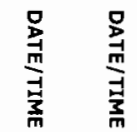

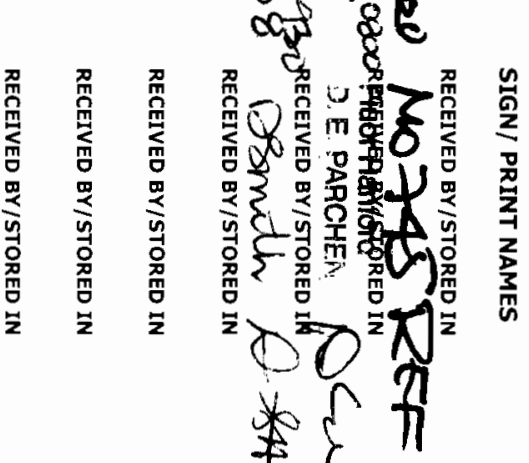

罢

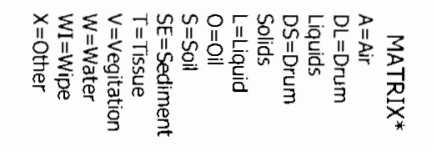

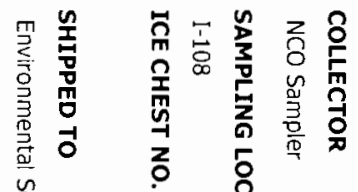

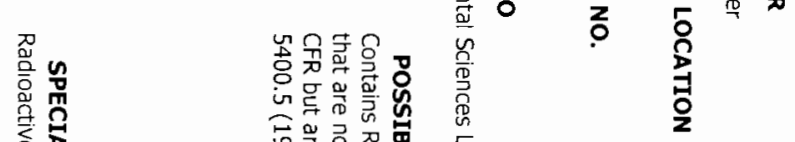

$\stackrel{\infty}{ }$

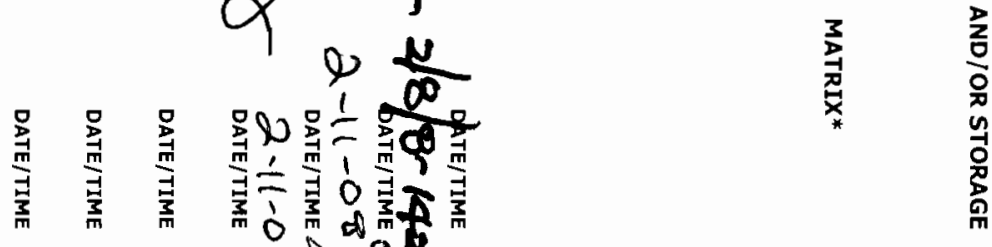

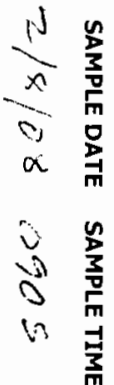

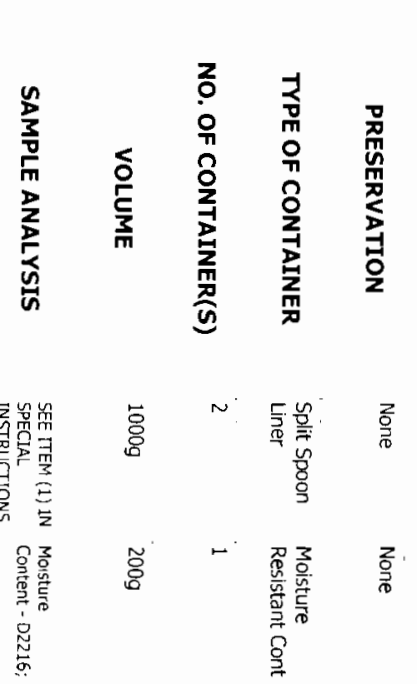

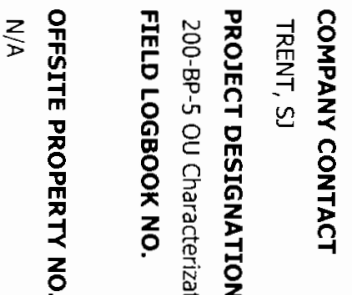

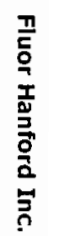

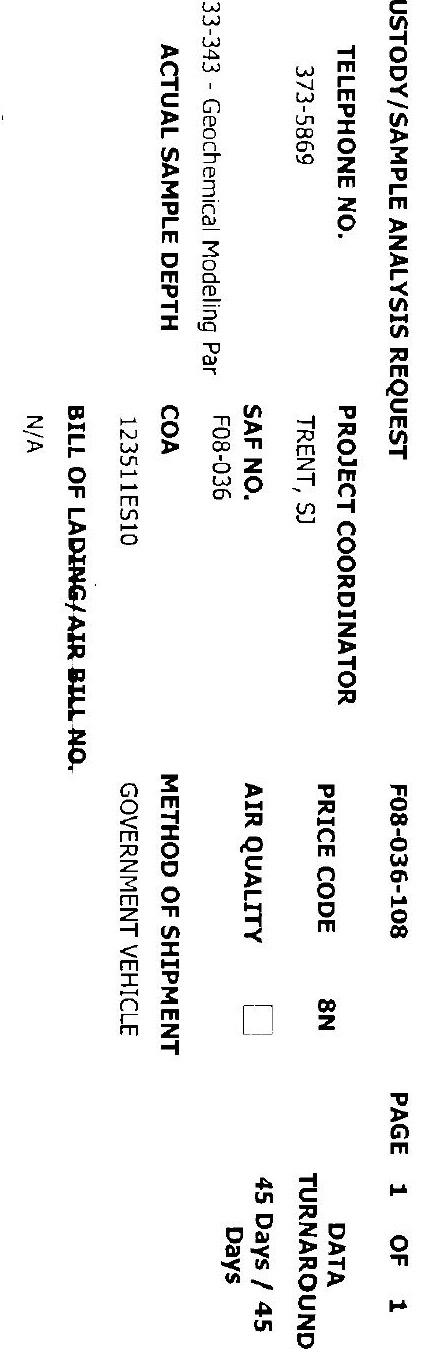




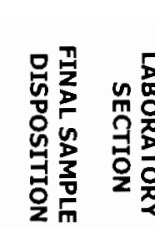
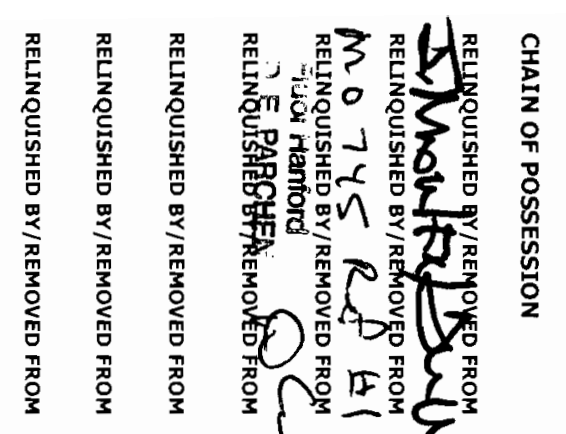

$\sum_{5}^{n}$

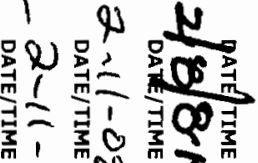

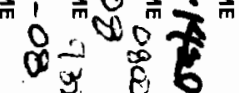

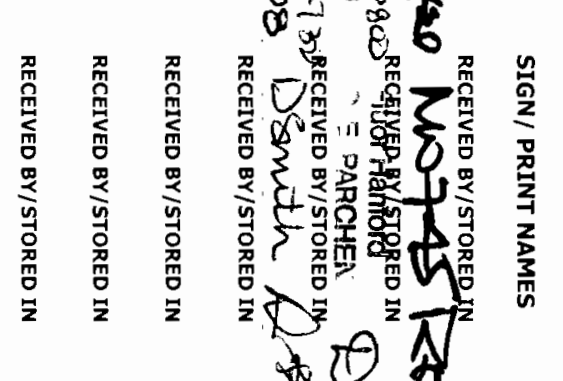

春

管

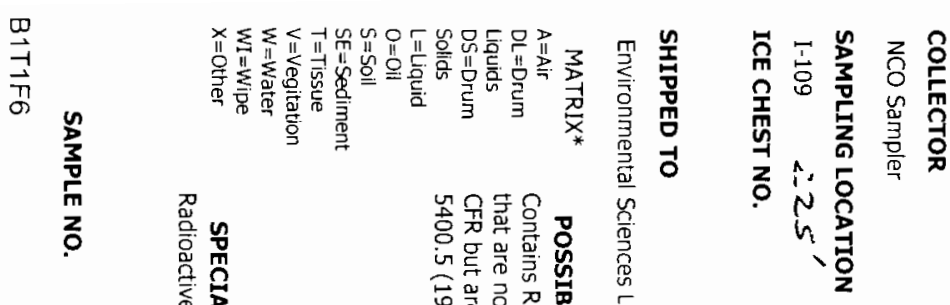

$\stackrel{\leftrightarrow}{\circ}$

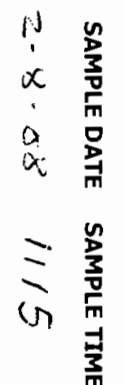

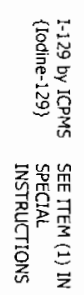
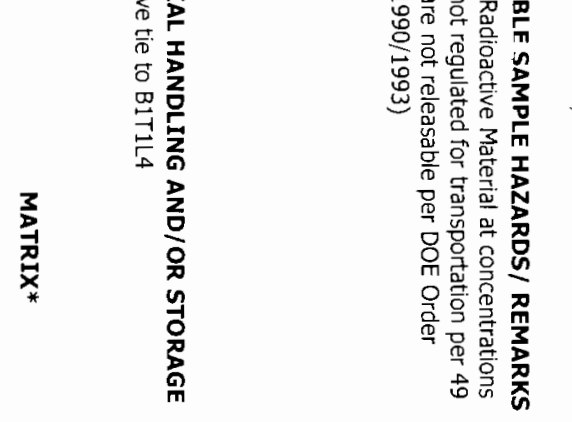

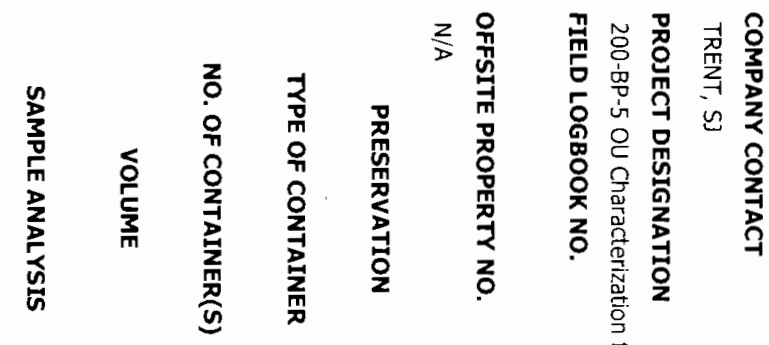

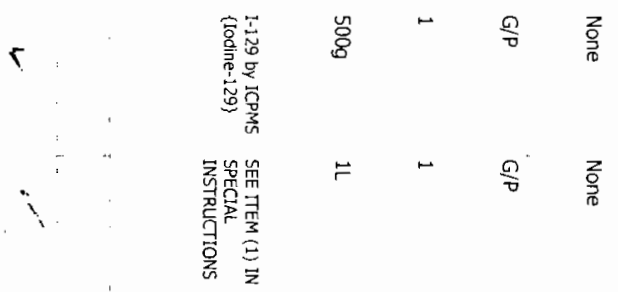<smiles>CCC</smiles>

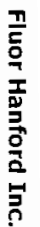

$\frac{9}{3}$

监 誉

N

辛

잉 곯

$\stackrel{9}{F}$

讨

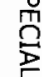

离

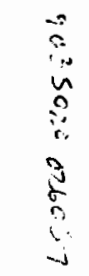

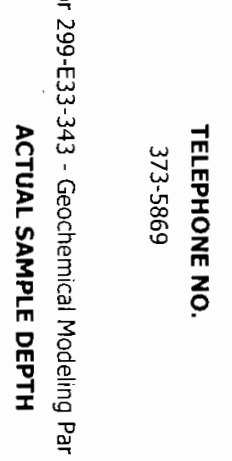

列

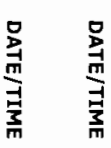

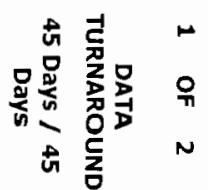




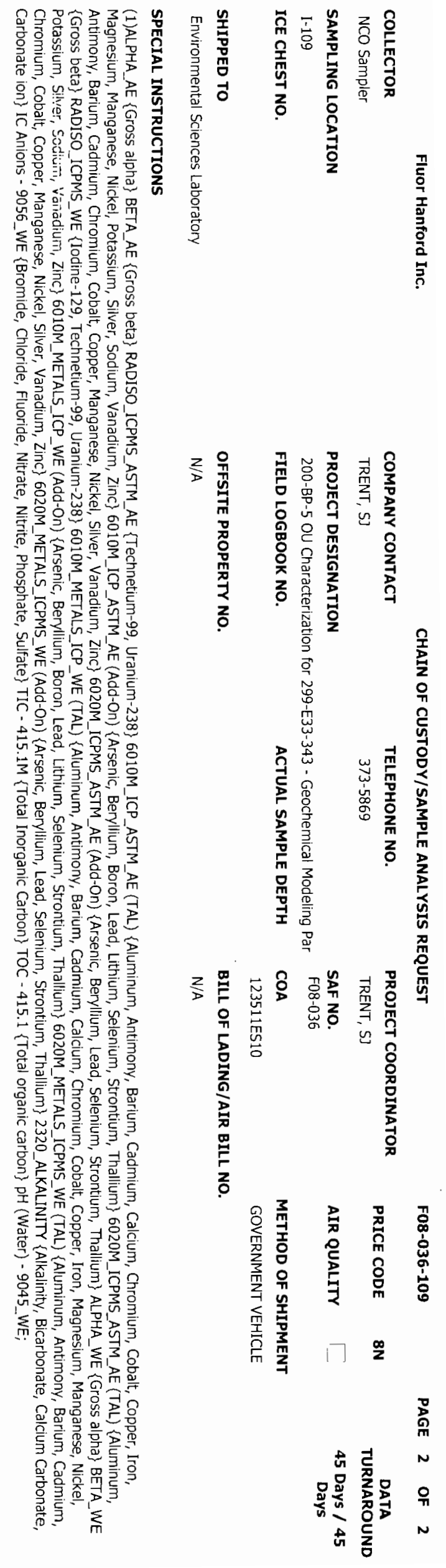




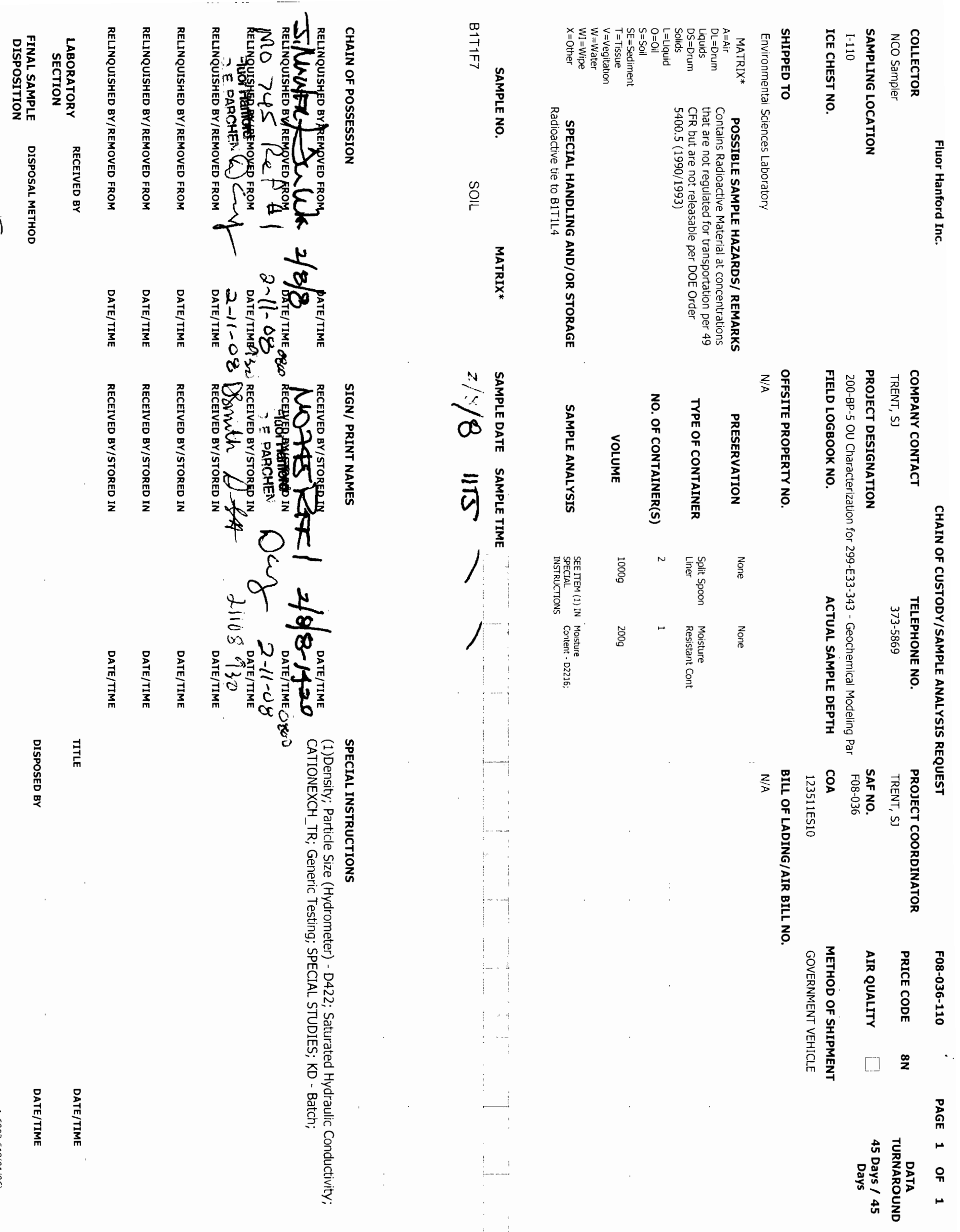




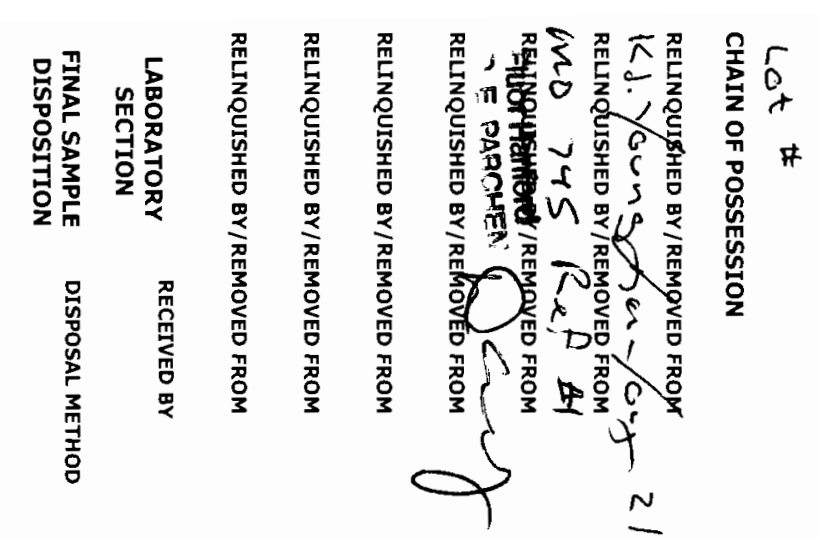

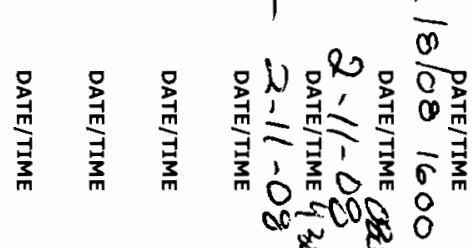

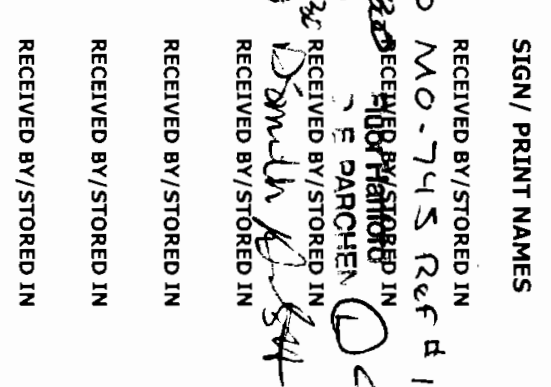

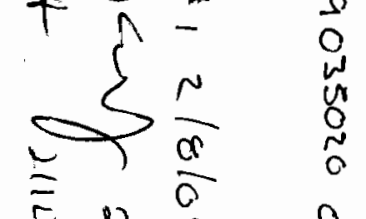

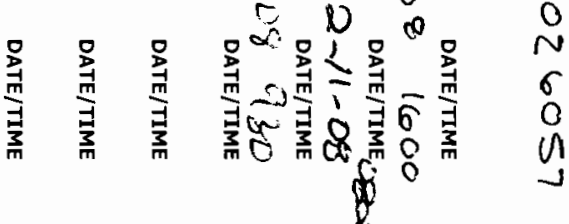

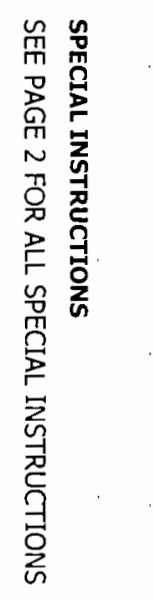

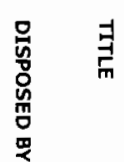

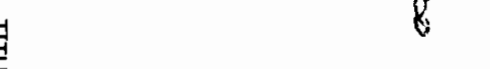

$\underset{\infty}{\stackrel{\square}{\overrightarrow{1}}}$

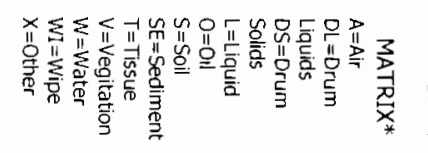

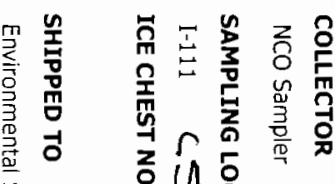

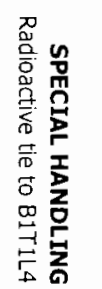

$\underset{\substack{x\\}}{3}$

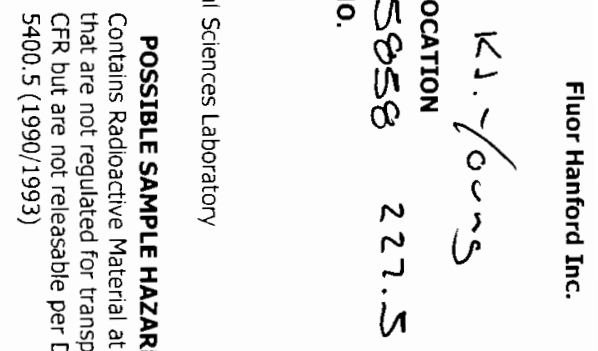

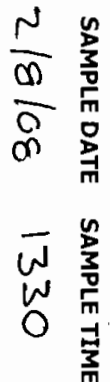

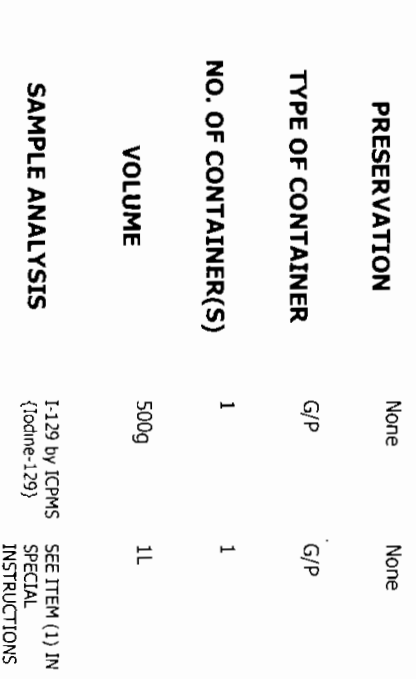

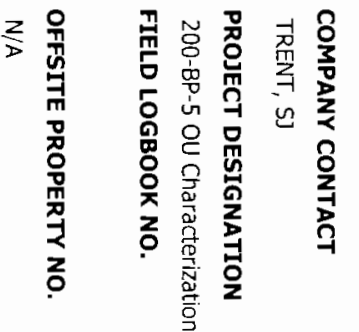

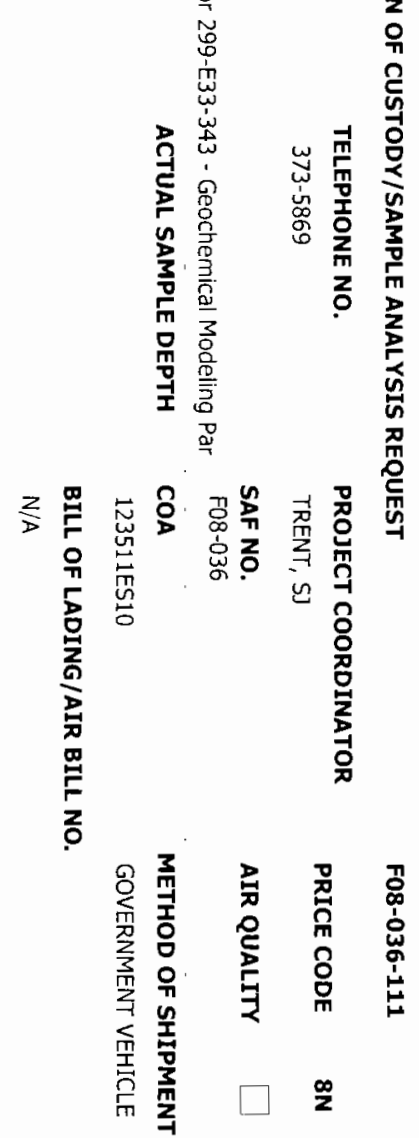

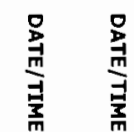




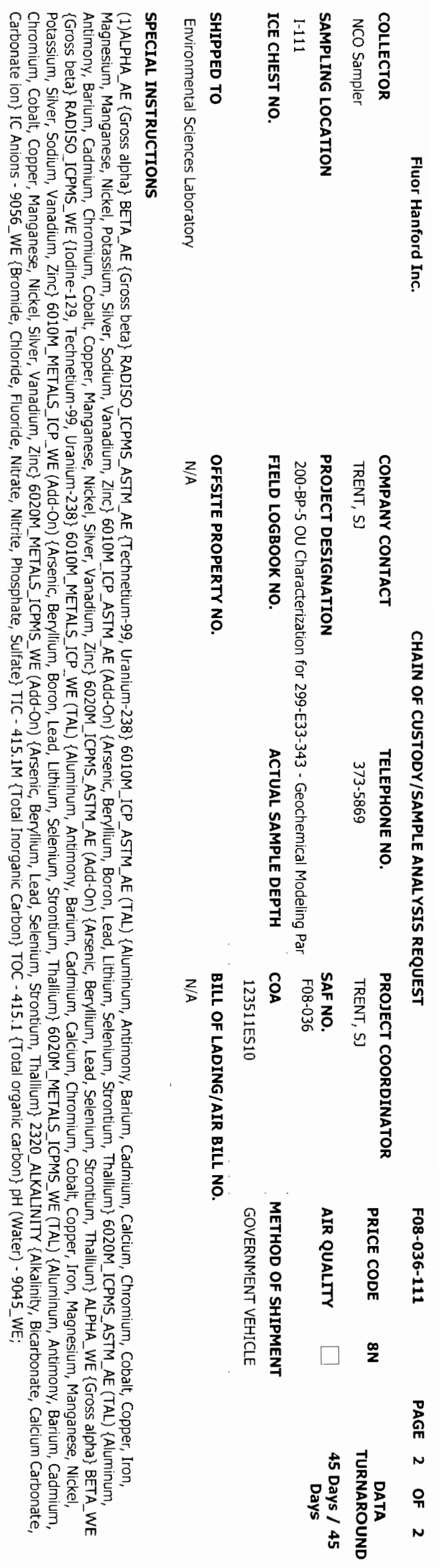



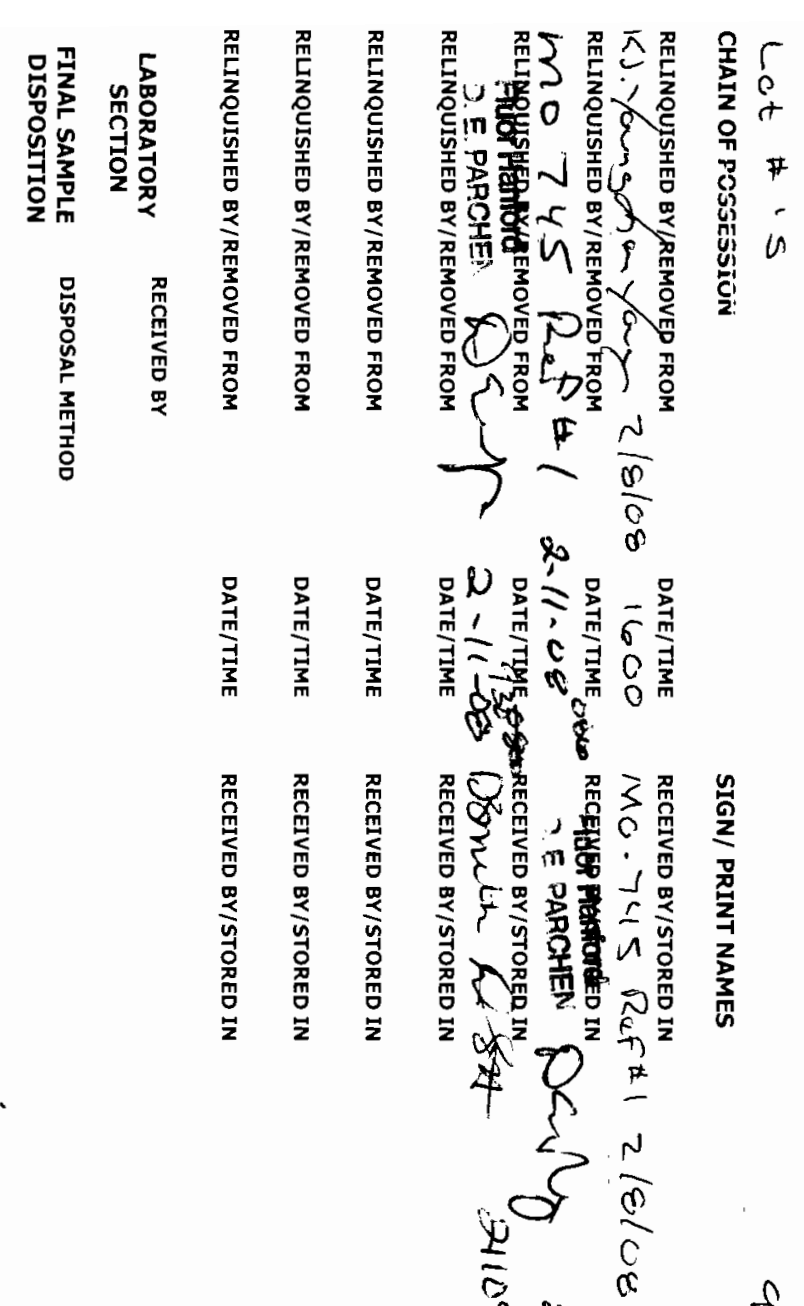

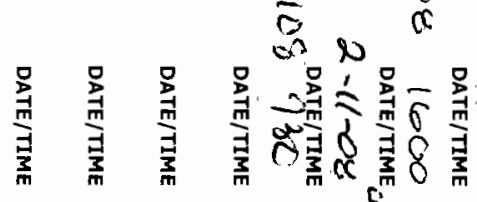<smiles></smiles>

亲

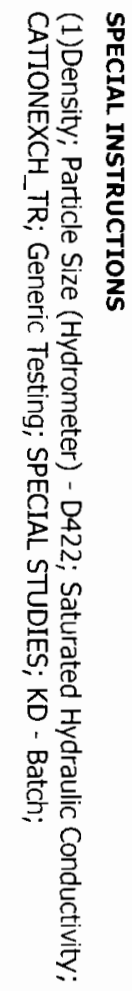

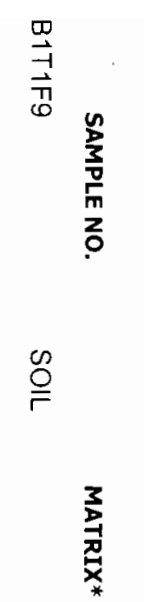

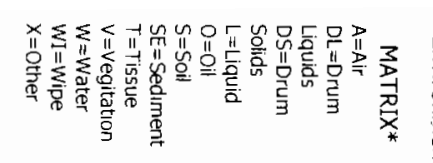

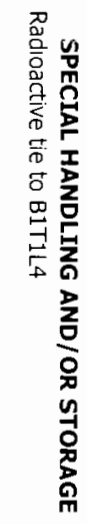

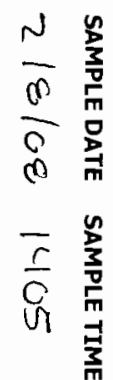

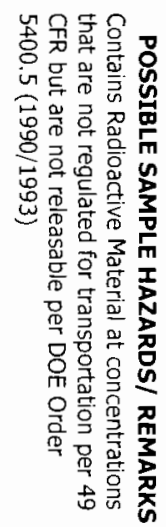

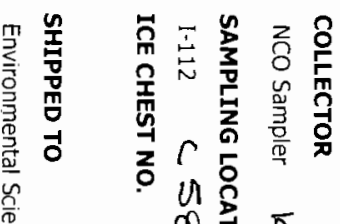

的㐘

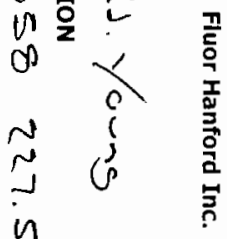

$\stackrel{0}{\omega}$

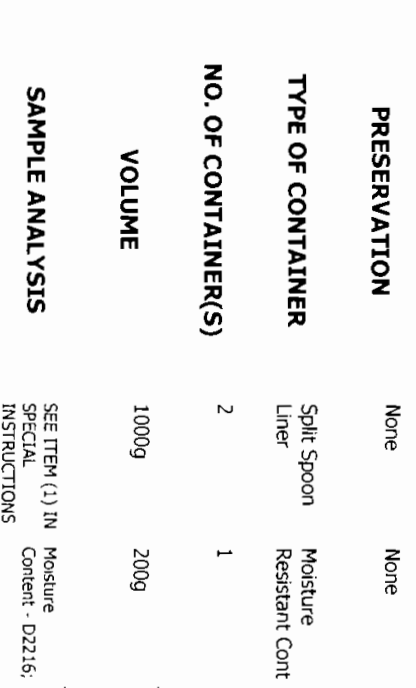

$\frac{2}{>}$

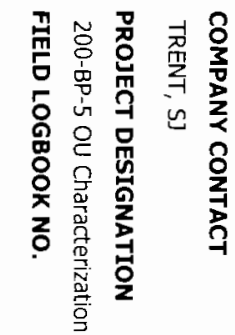
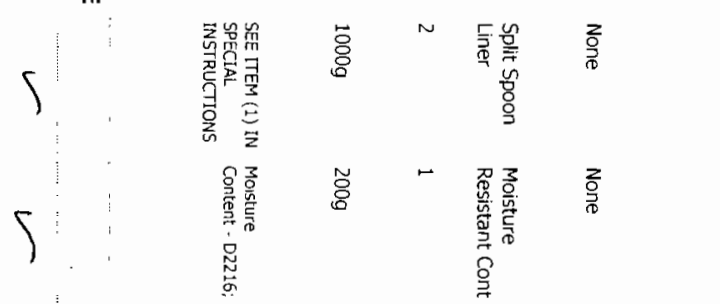

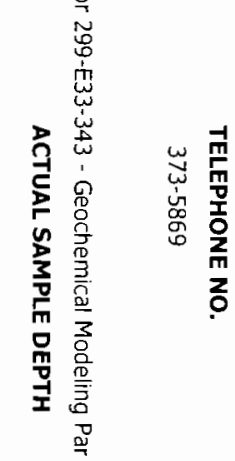

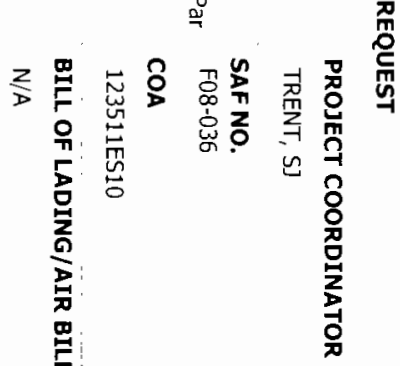

긍.

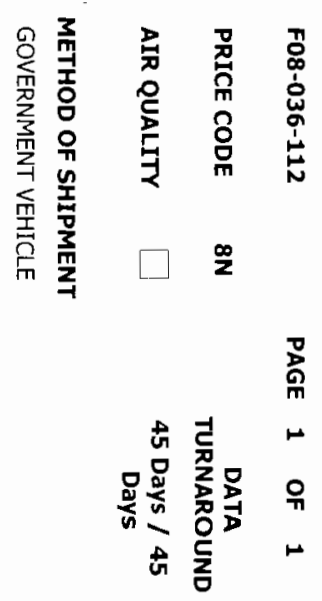




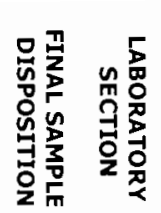

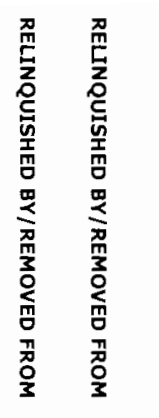

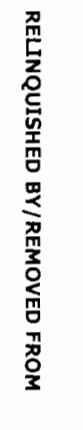

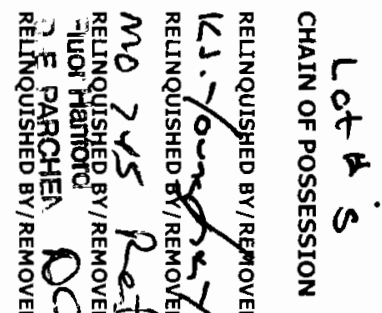

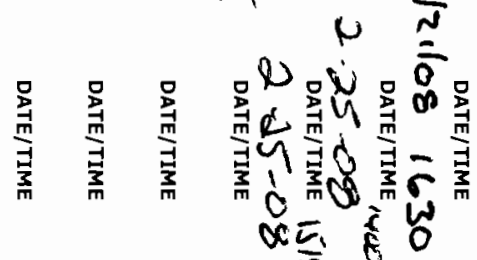

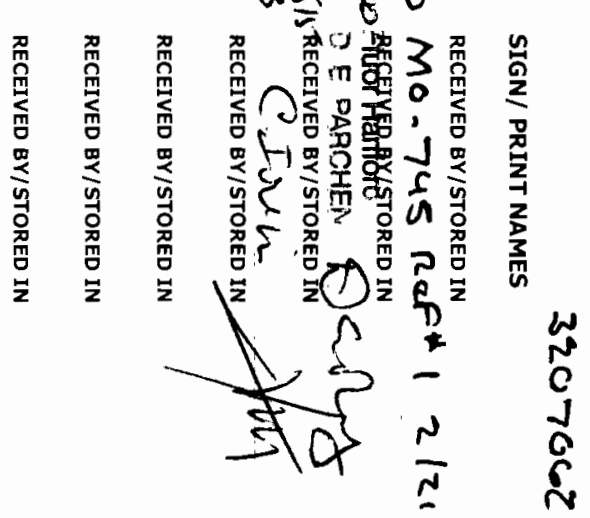

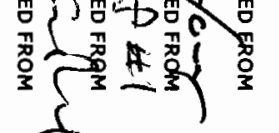<smiles>CCCCC(C)C</smiles>

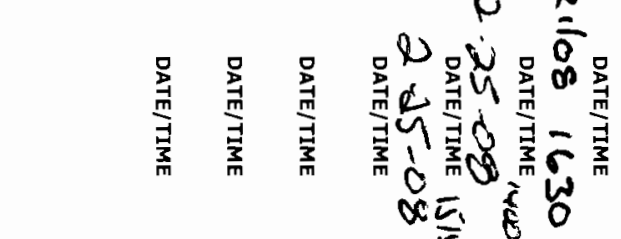

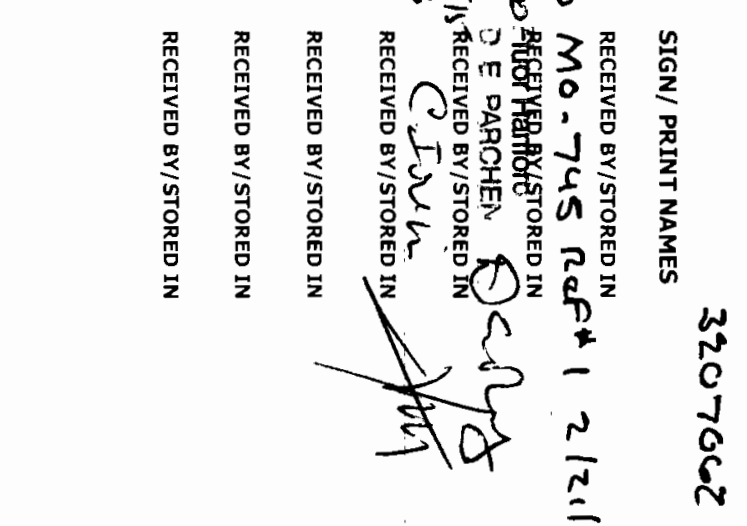

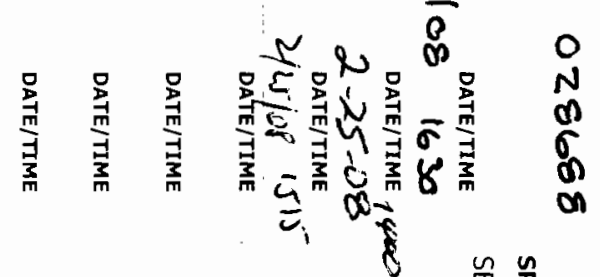

\section{요욜}

.

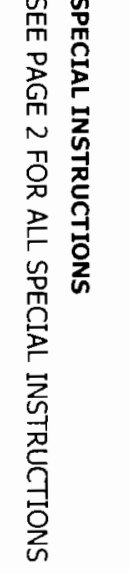

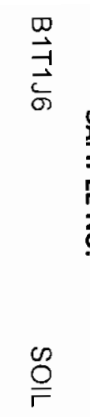

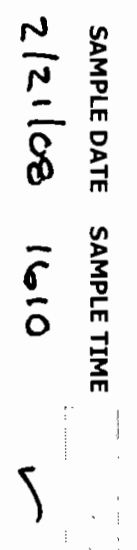

1

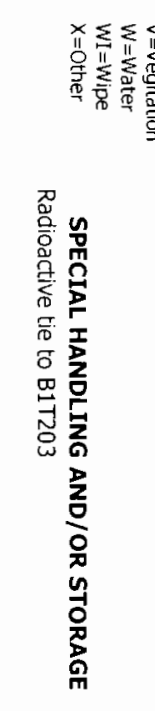

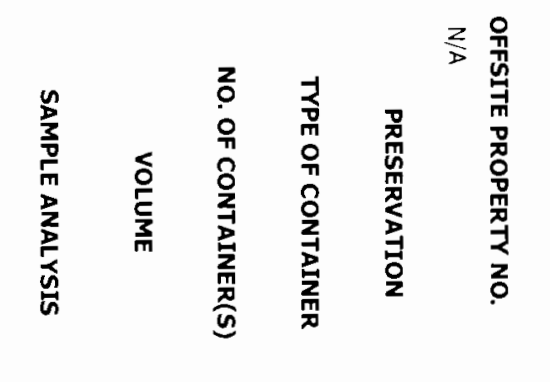
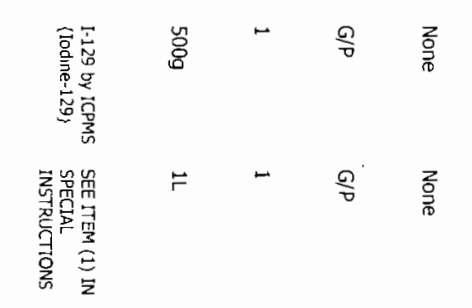

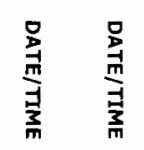

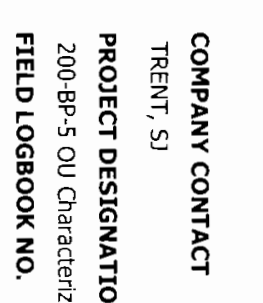

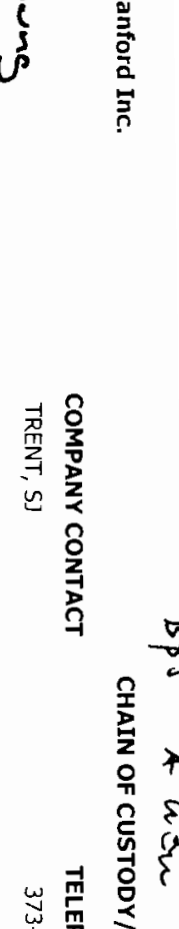




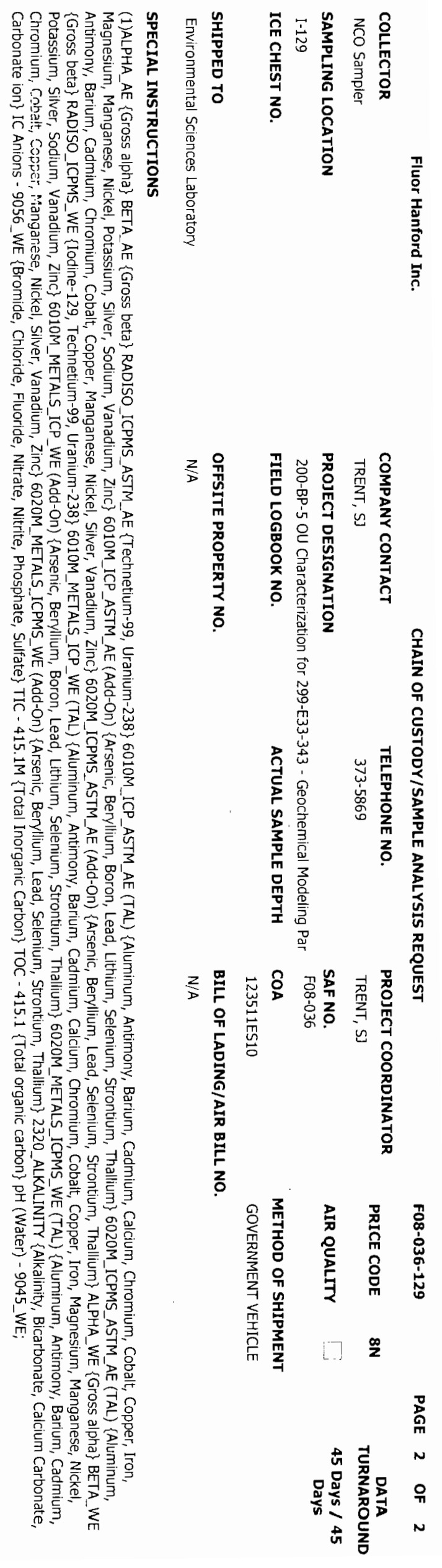




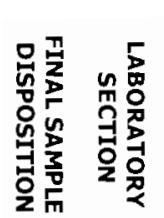
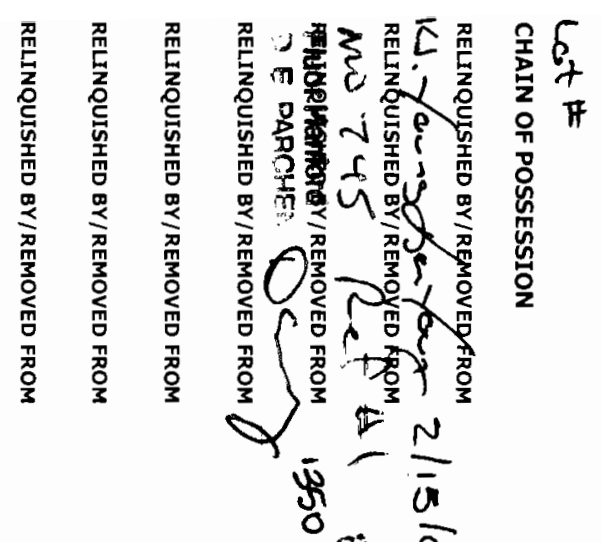

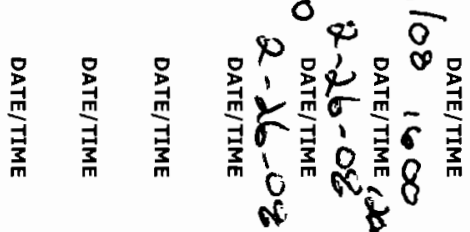
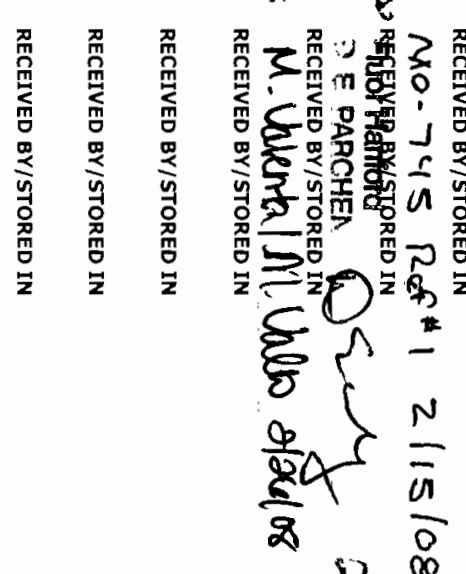

兴兴

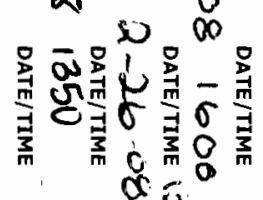
象

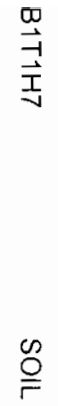

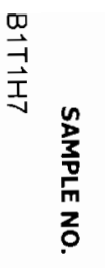

$\stackrel{\mathscr{\rho}}{\rightleftharpoons}$

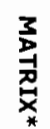

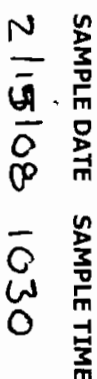

$<$

i

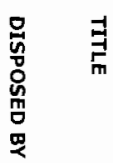

$\frac{3}{m}$

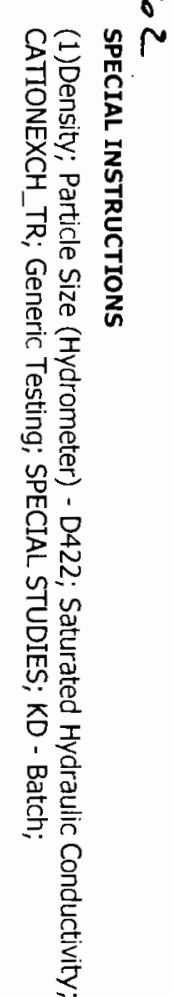

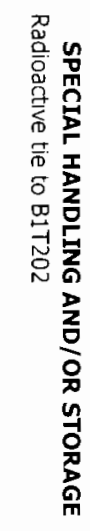

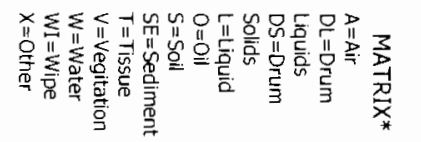

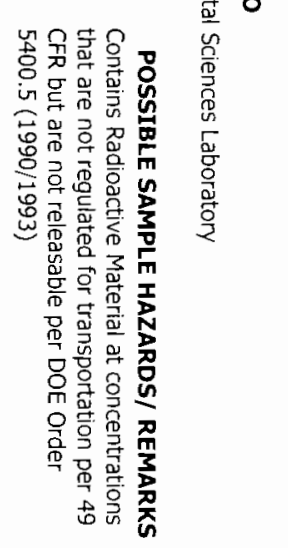

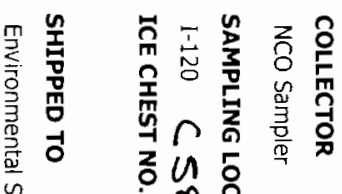

的豆

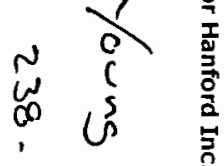

$\frac{1}{1}$

0

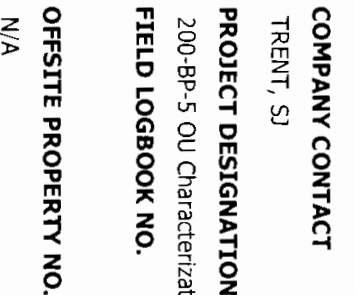

爱

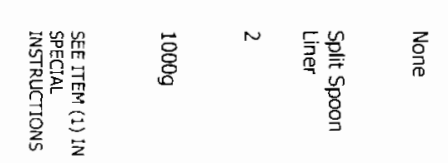

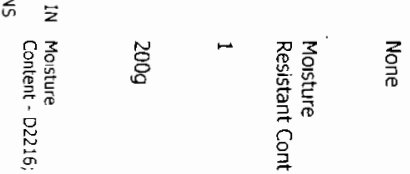

旁量 


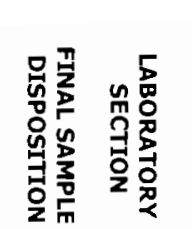
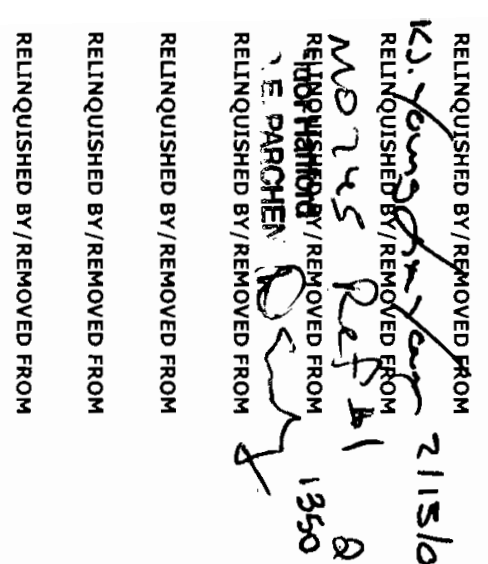

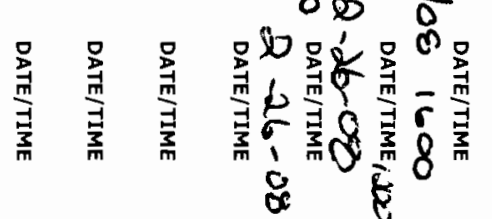

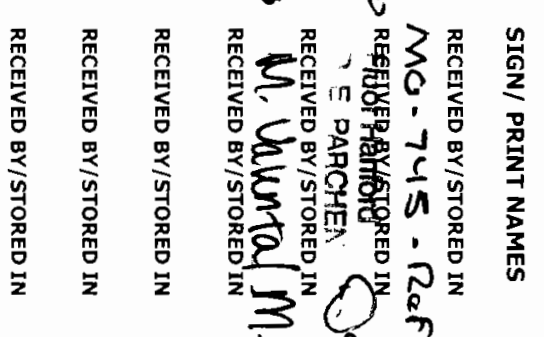

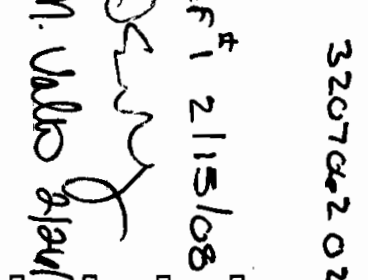

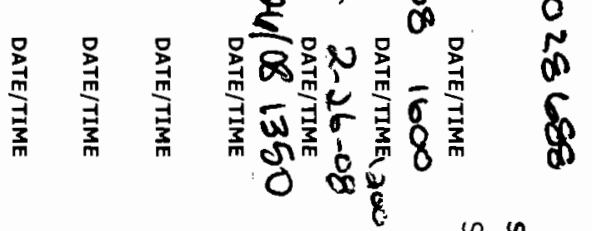

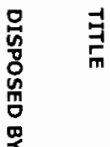

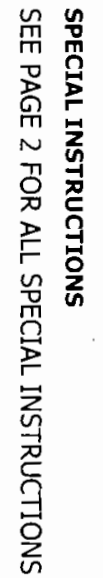

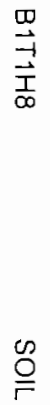

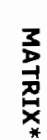

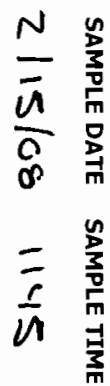

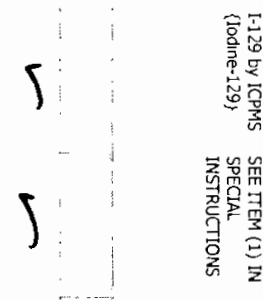

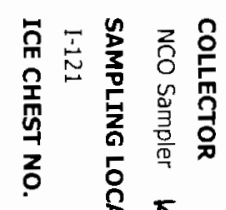

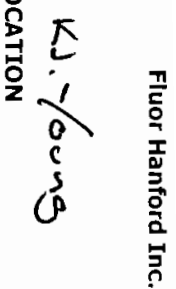

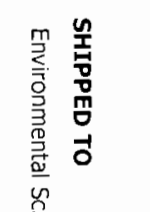

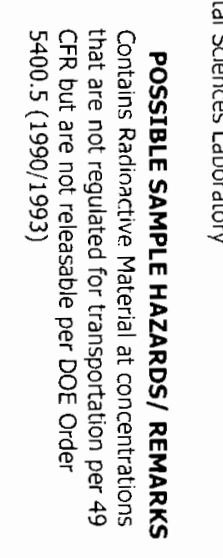

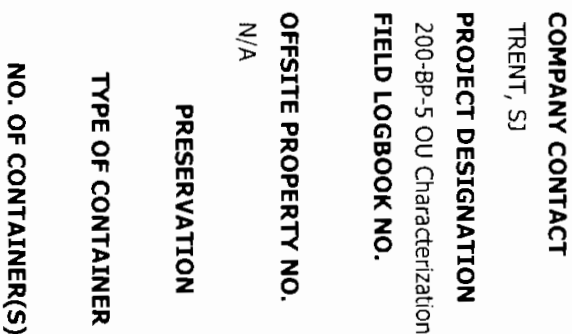

용 $\rightarrow \frac{1}{\partial}$

$\mapsto \quad+\frac{g}{\frac{9}{0}}$

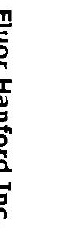

愛

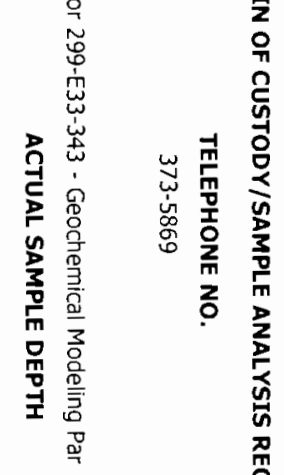

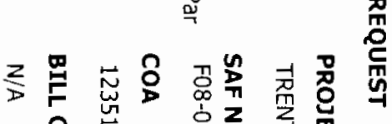

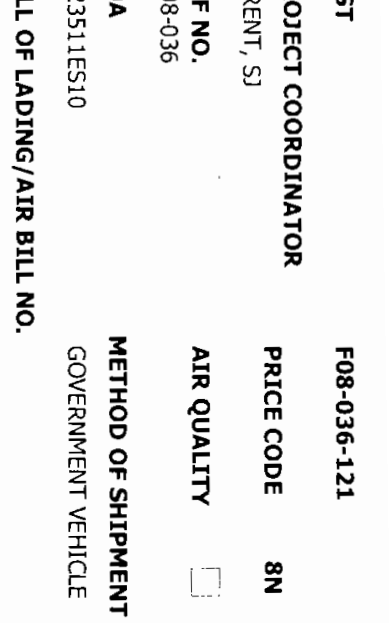

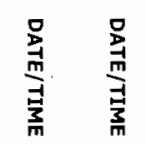

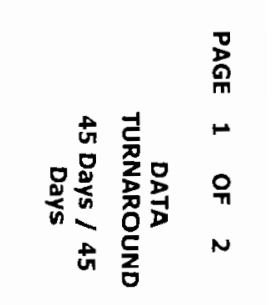




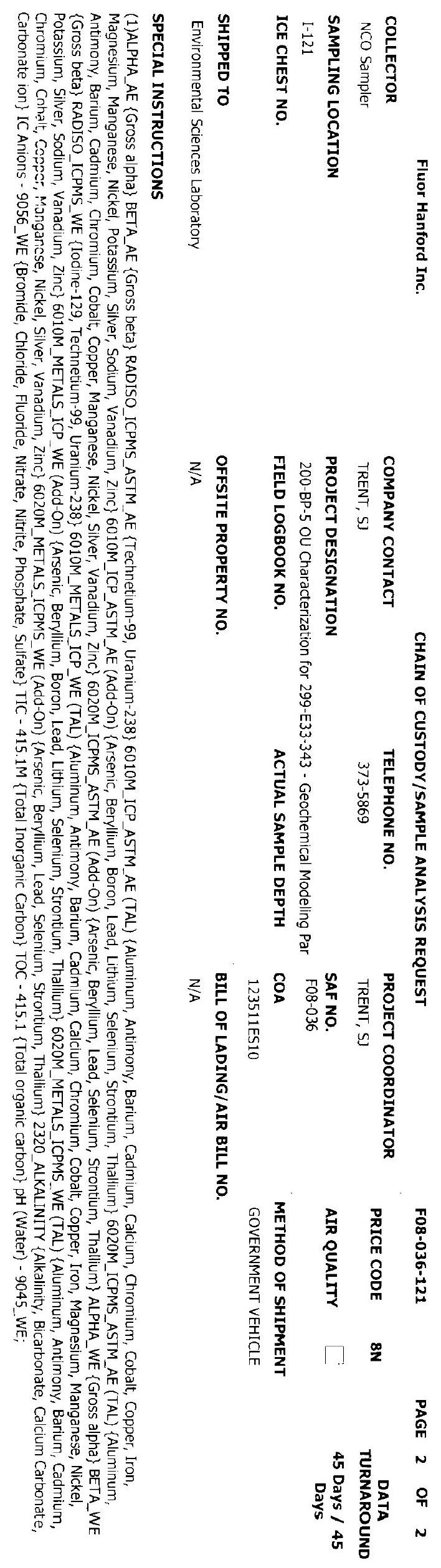



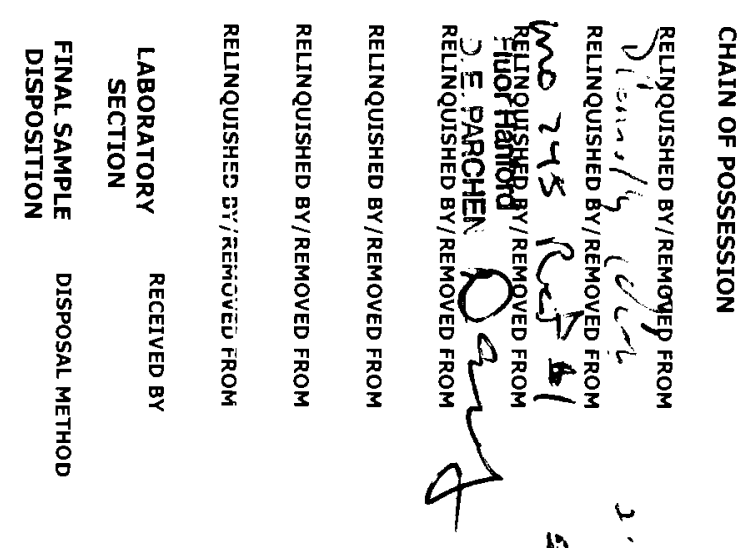

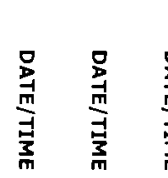

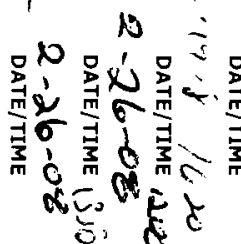

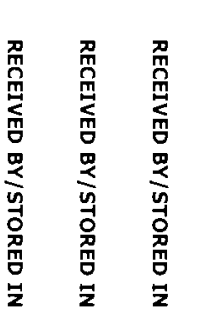

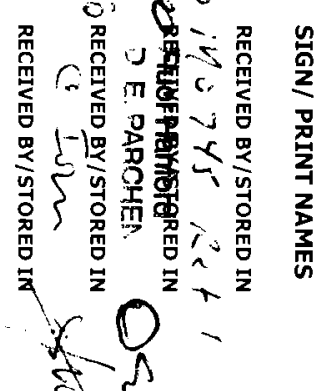

号 青

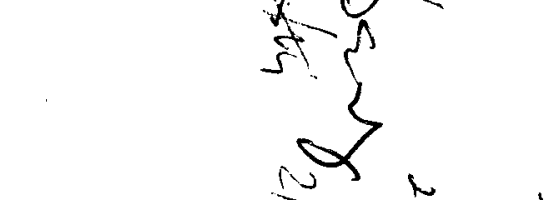

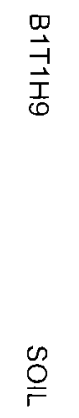

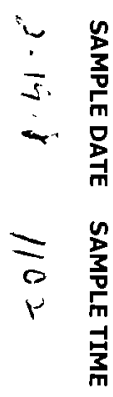

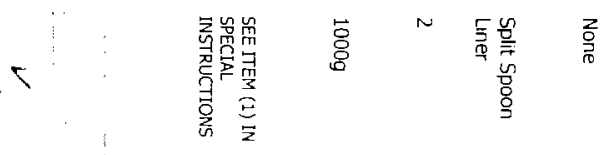

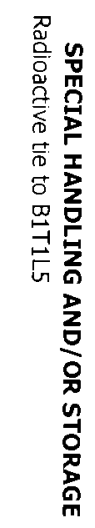

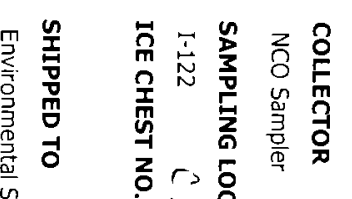
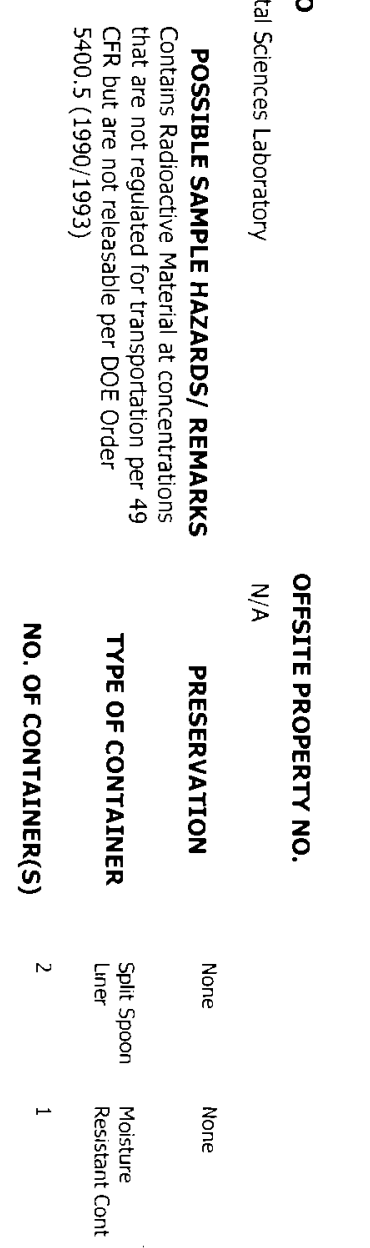

공

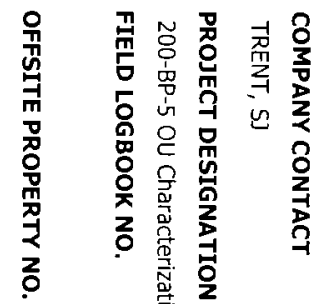

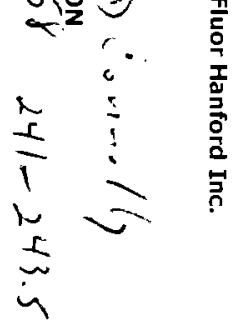

众量

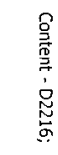

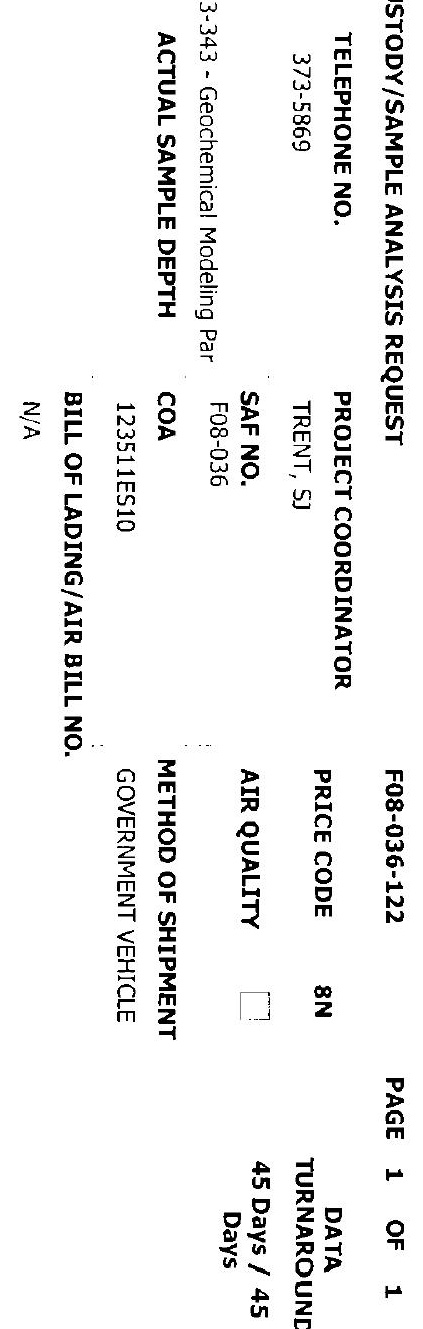




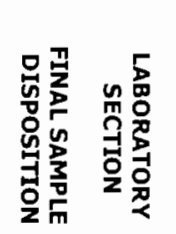

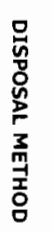

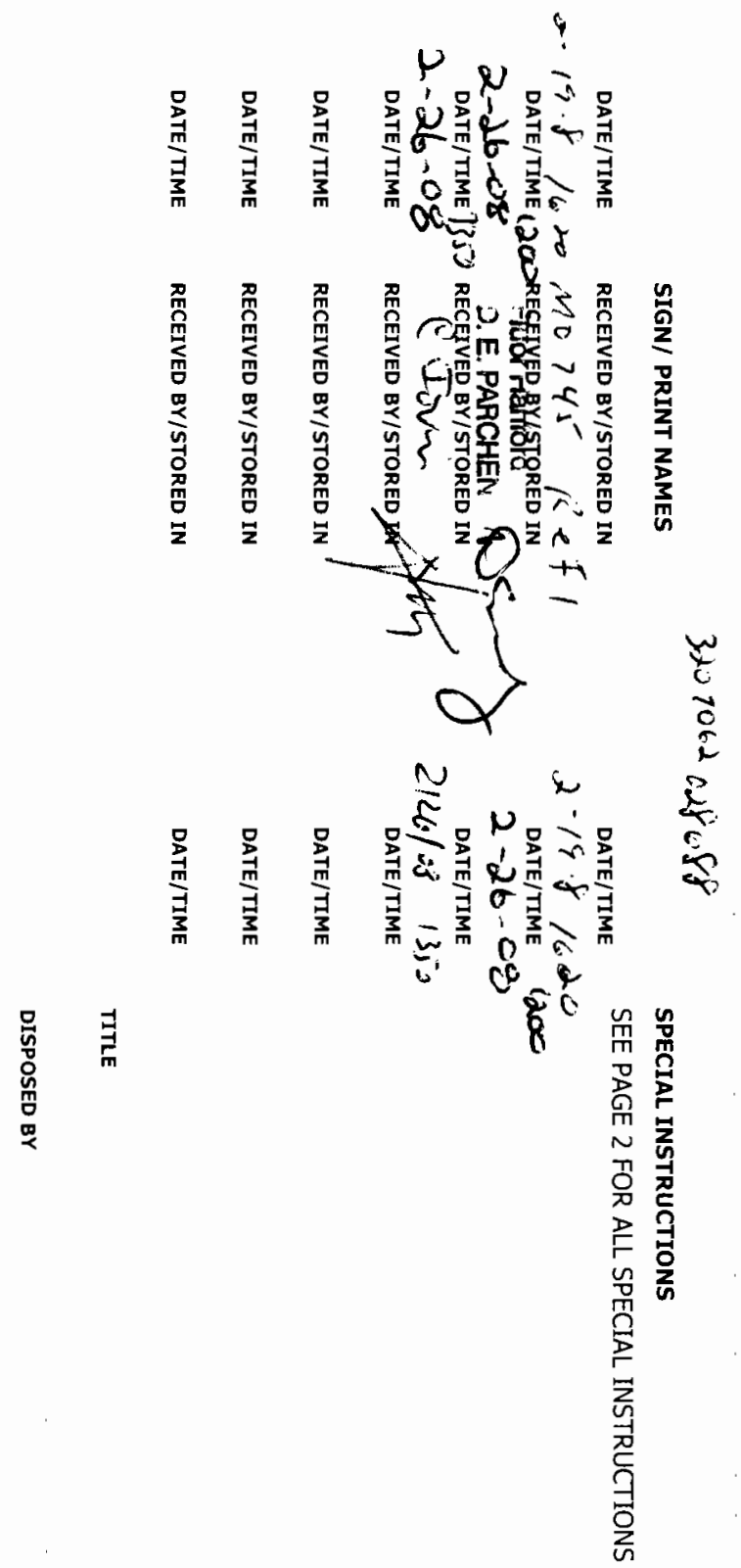

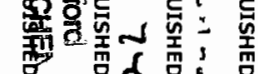

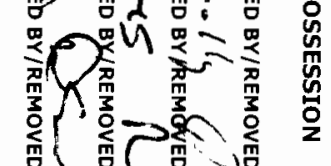
$\begin{array}{lll}\pi & \pi \\ 0 & \frac{\pi}{3} \\ 3 & 3\end{array}$

1

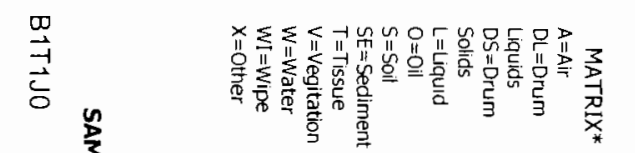

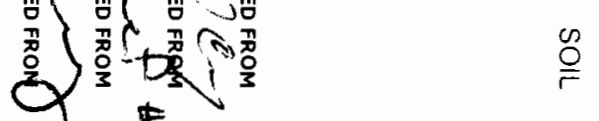

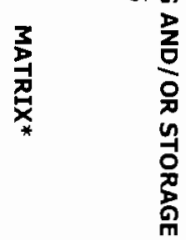

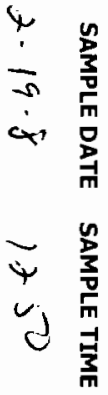
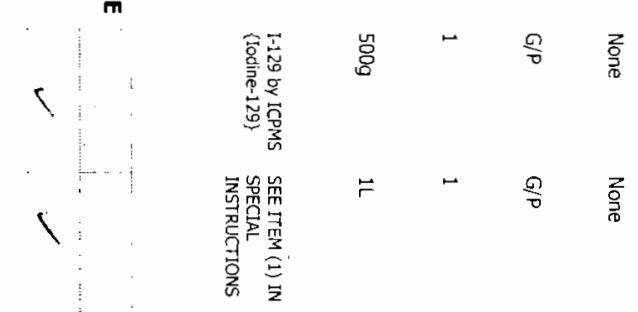

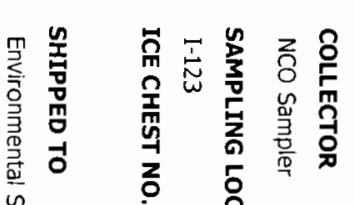

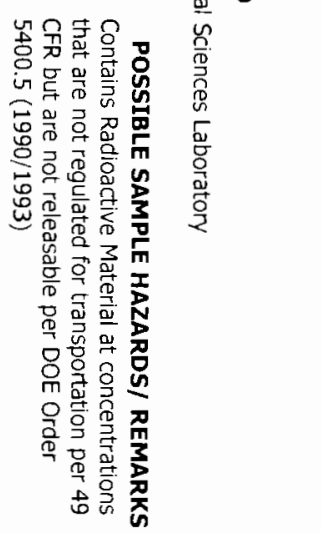

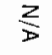

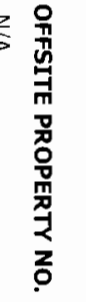

0

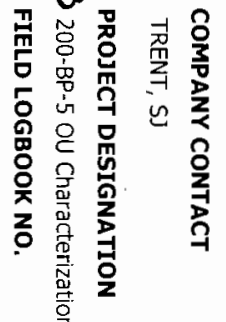

芯<smiles>C1CCC1</smiles>

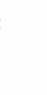

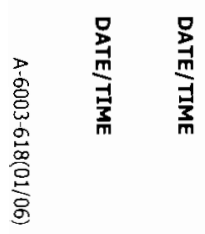




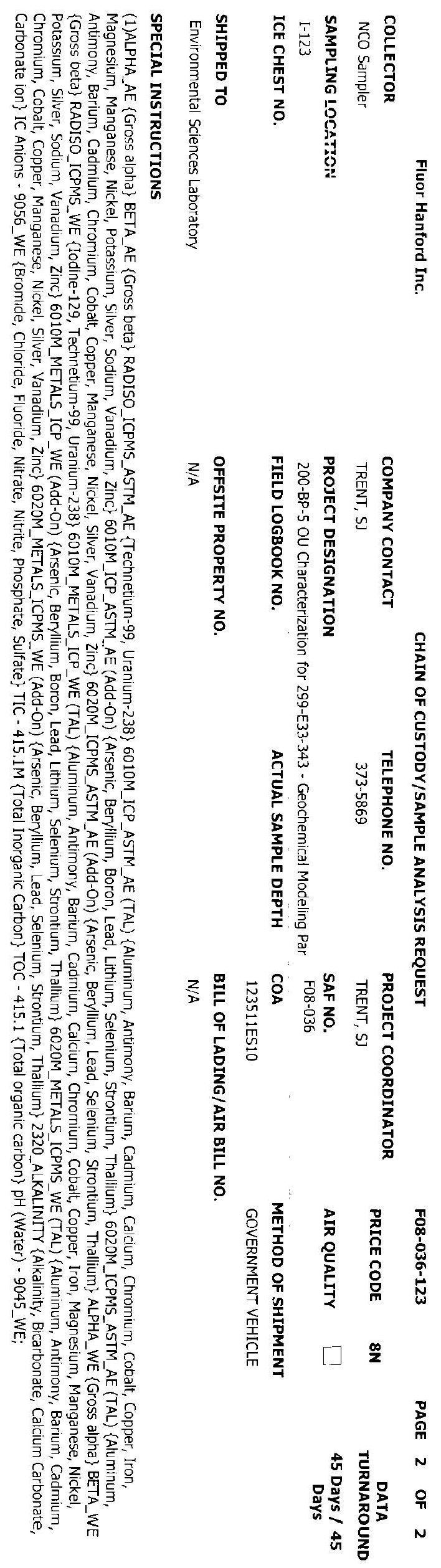




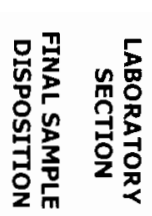
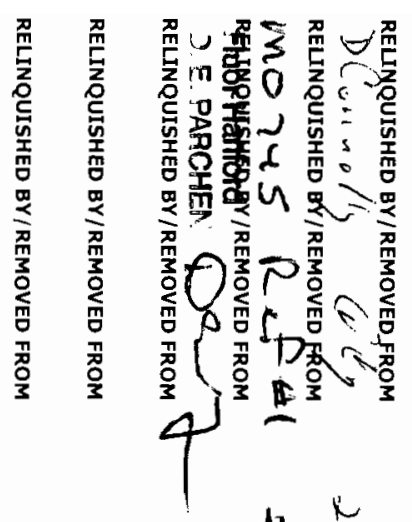

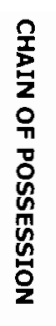

8
5
8
0
2
2
9
8

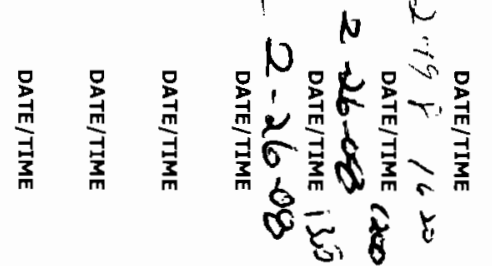

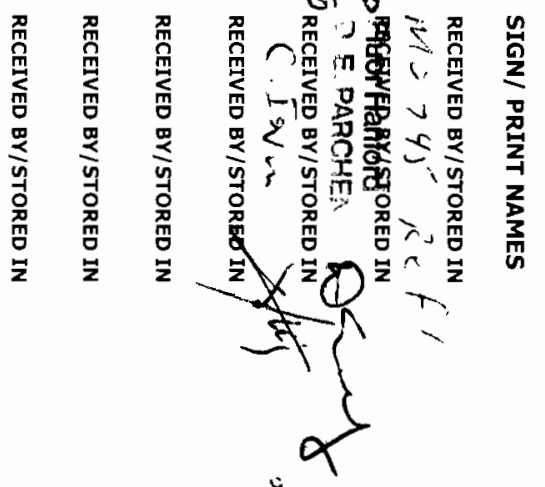

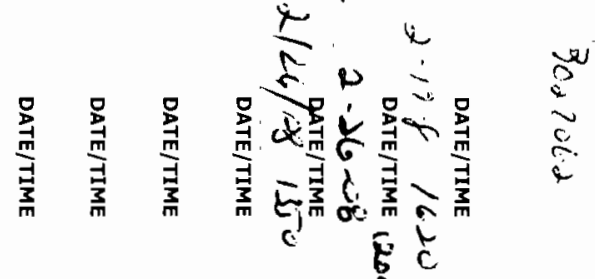

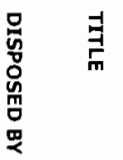

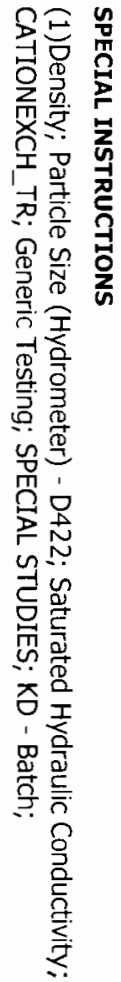

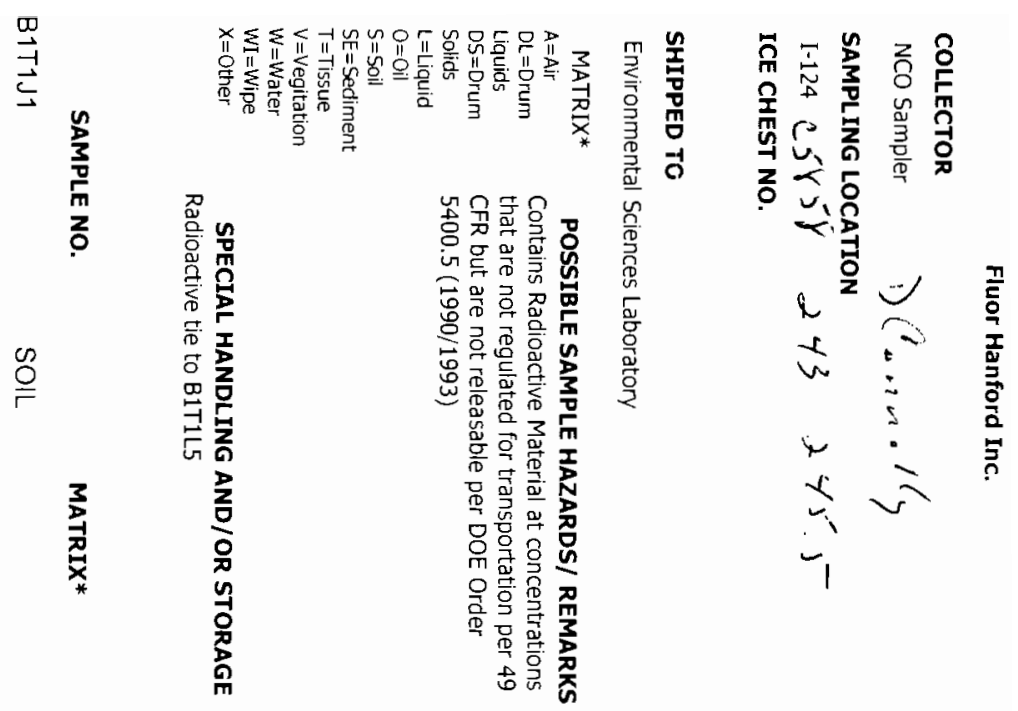

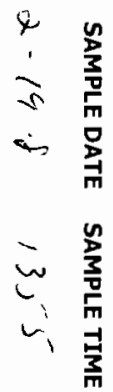

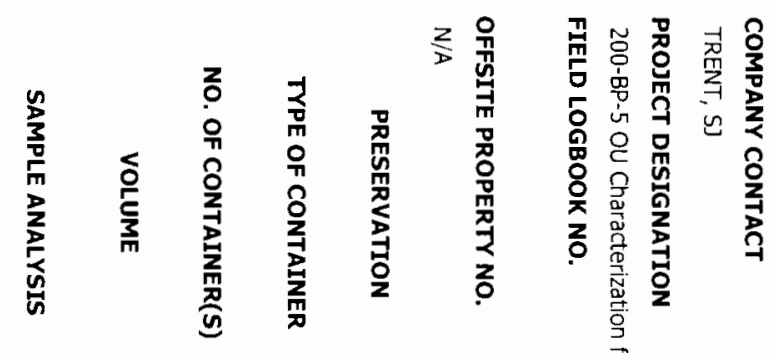

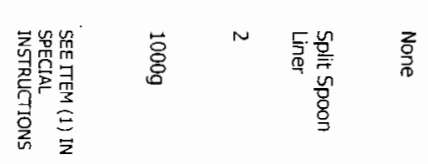
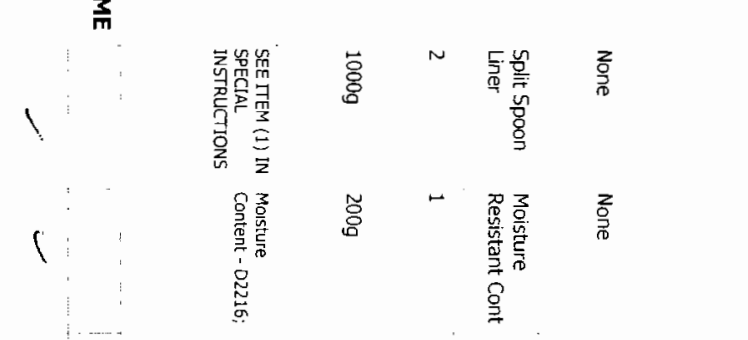


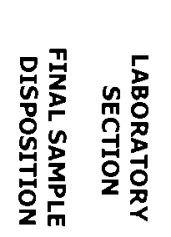

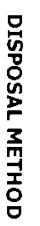

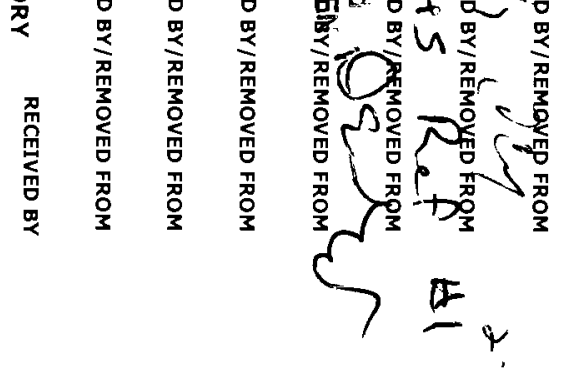

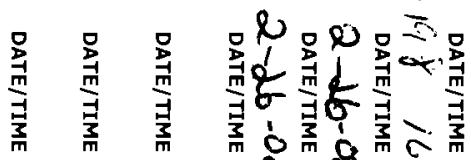

"रे कह है

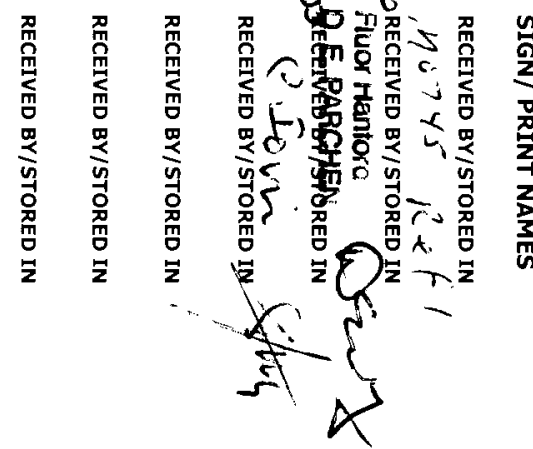

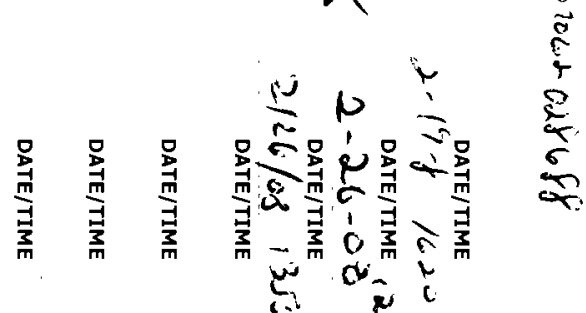

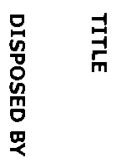

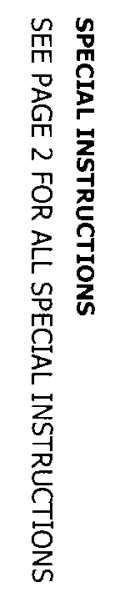

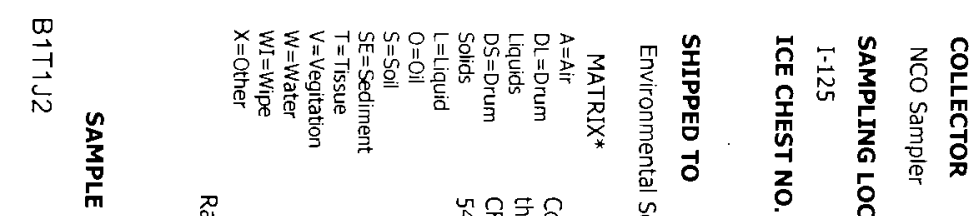

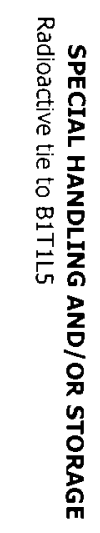

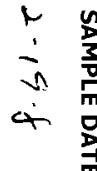

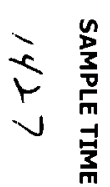

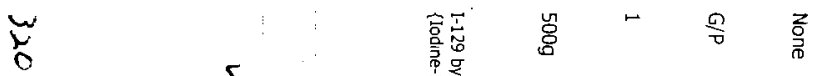

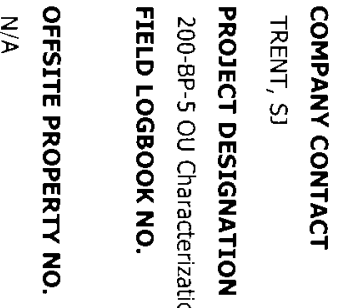

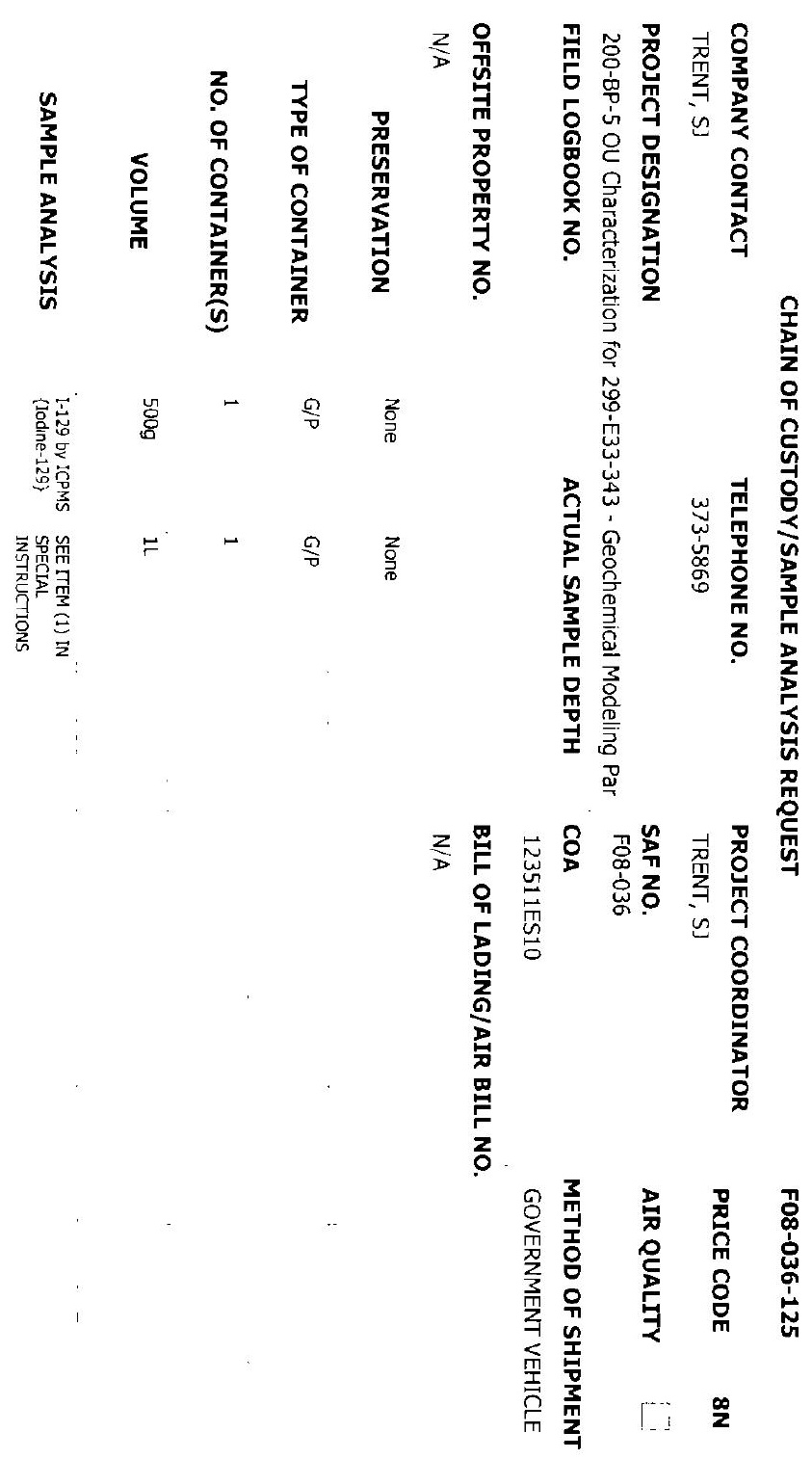

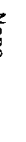

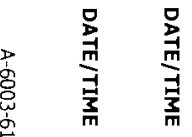




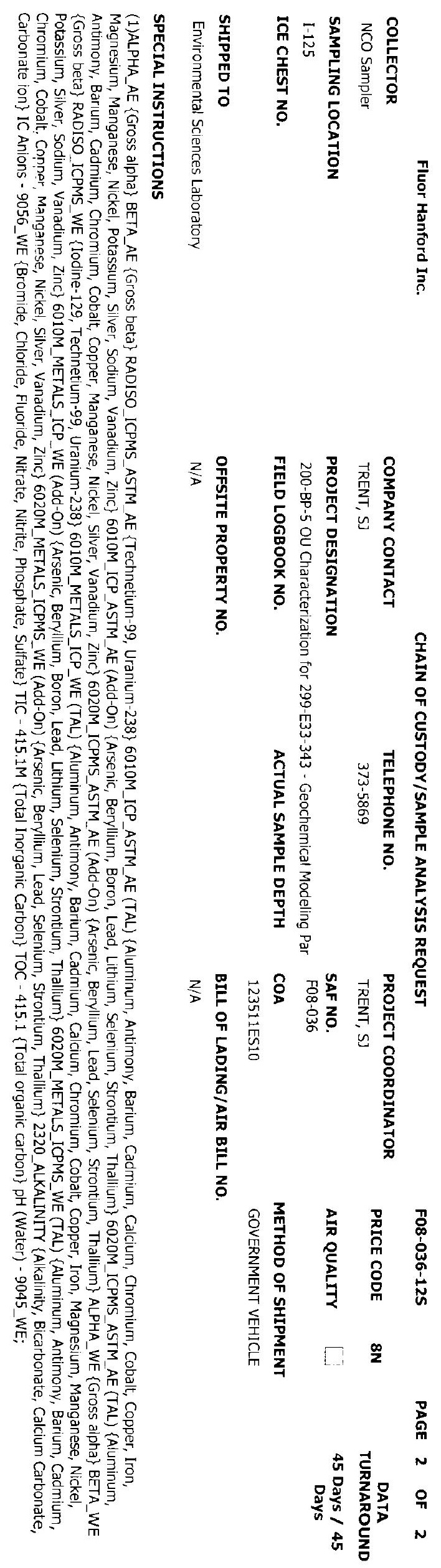


$\|$

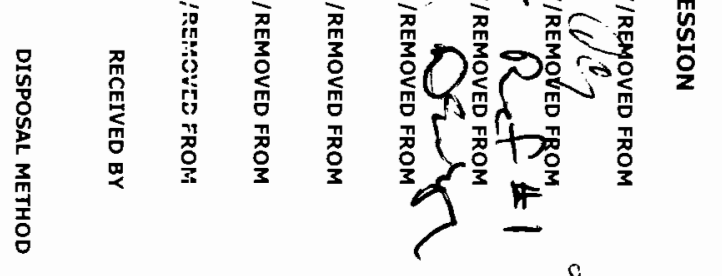

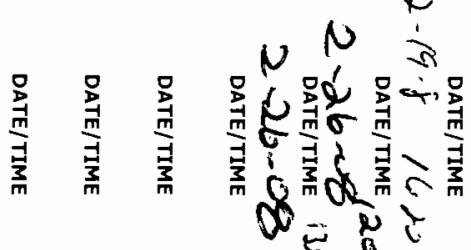

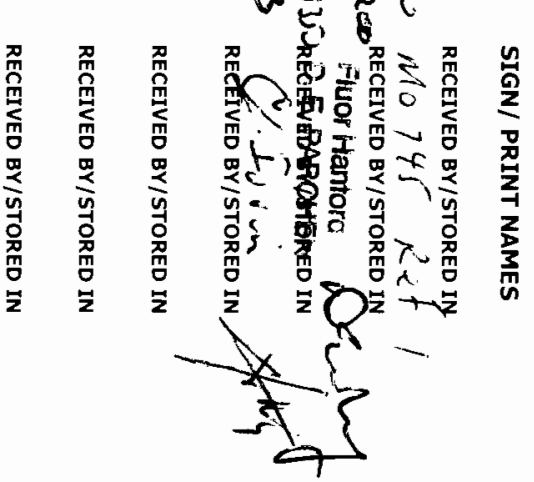

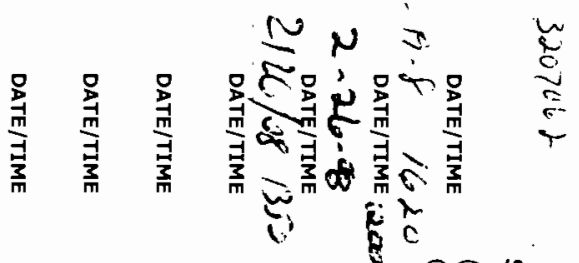

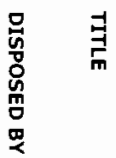
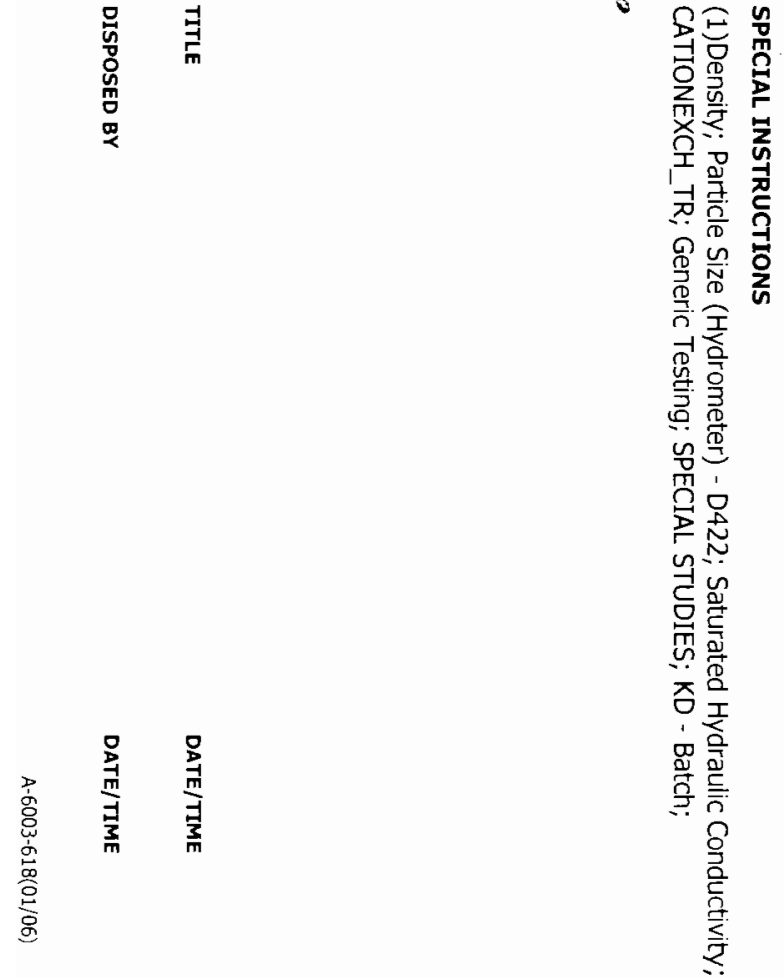

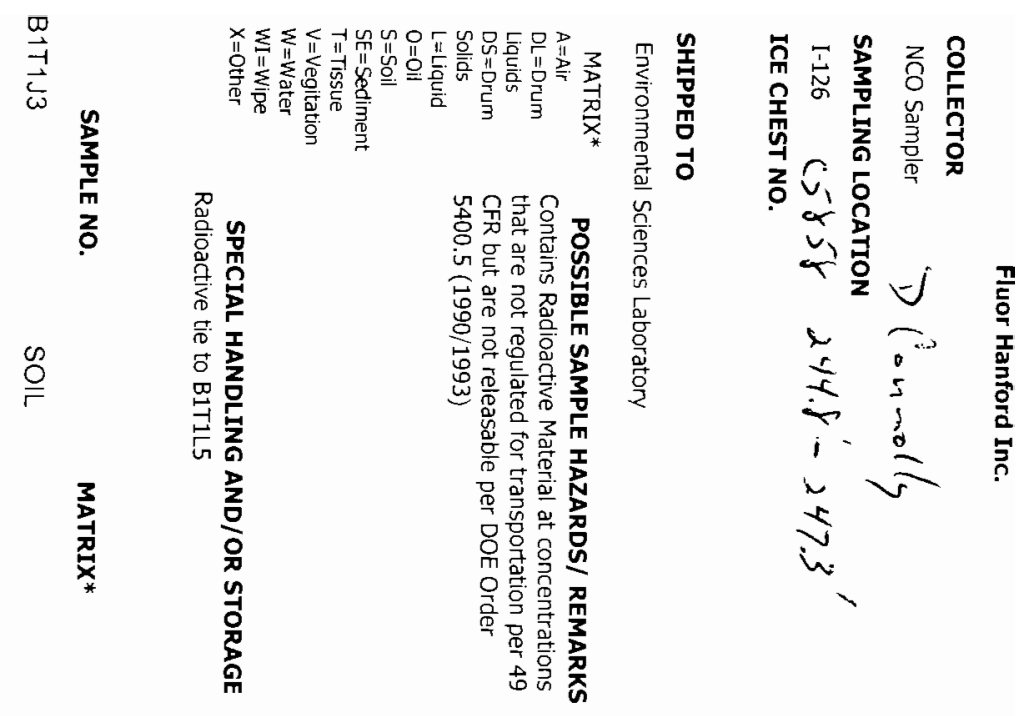

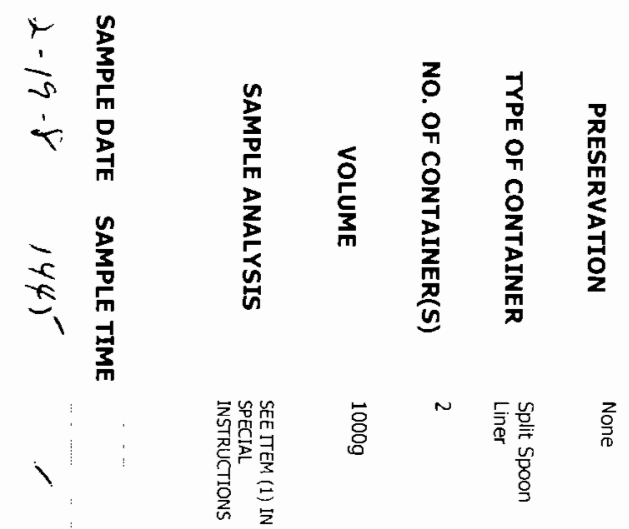

z 윻

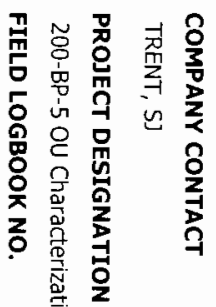

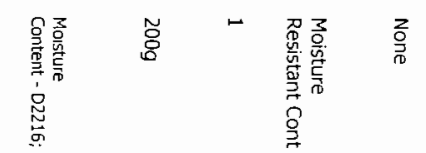

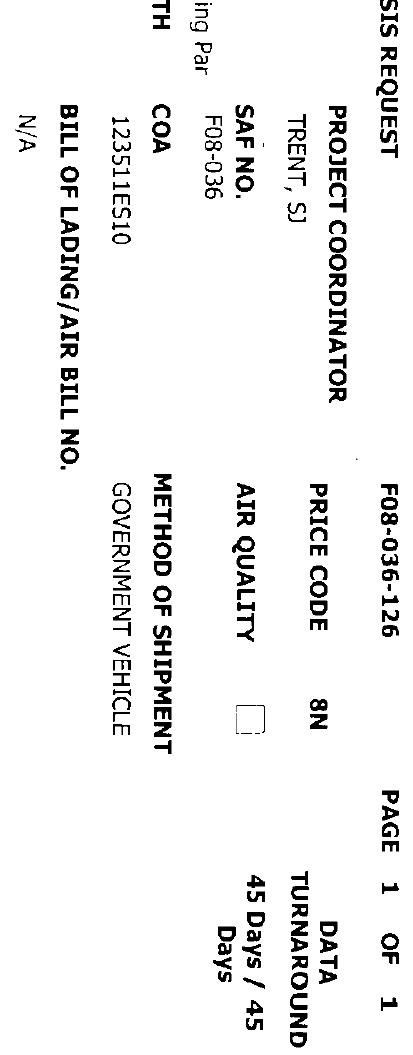



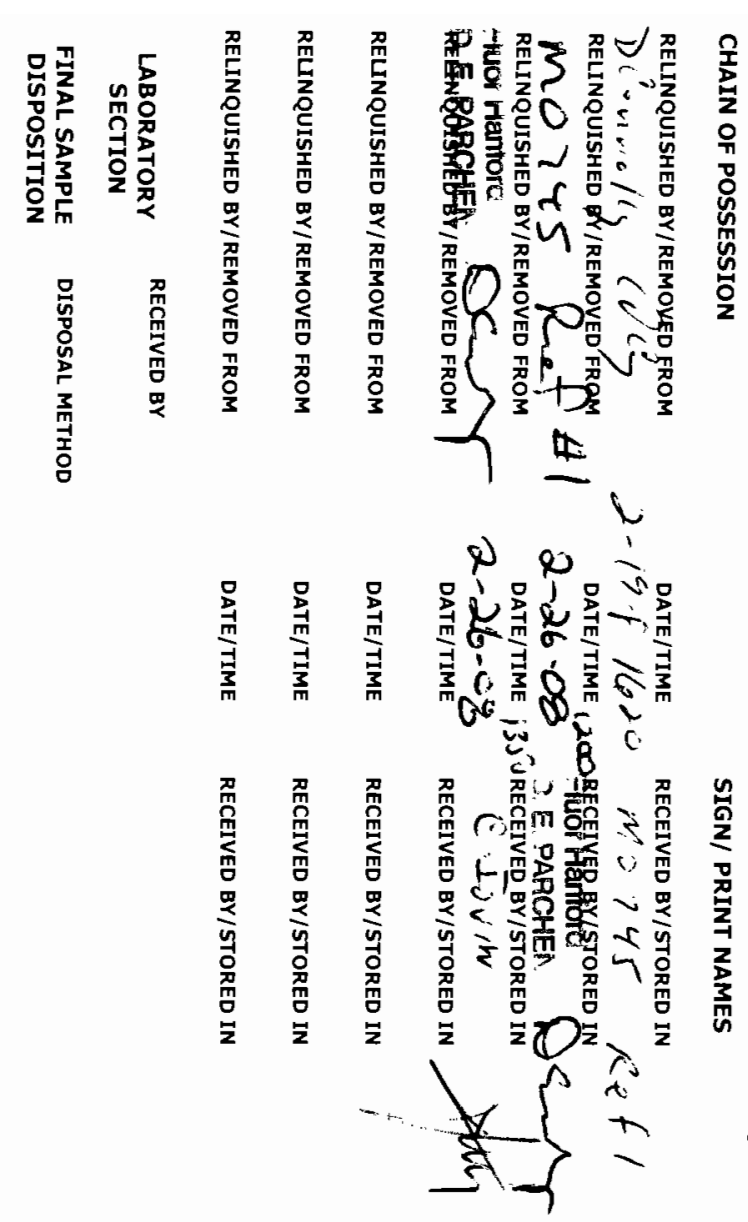

要
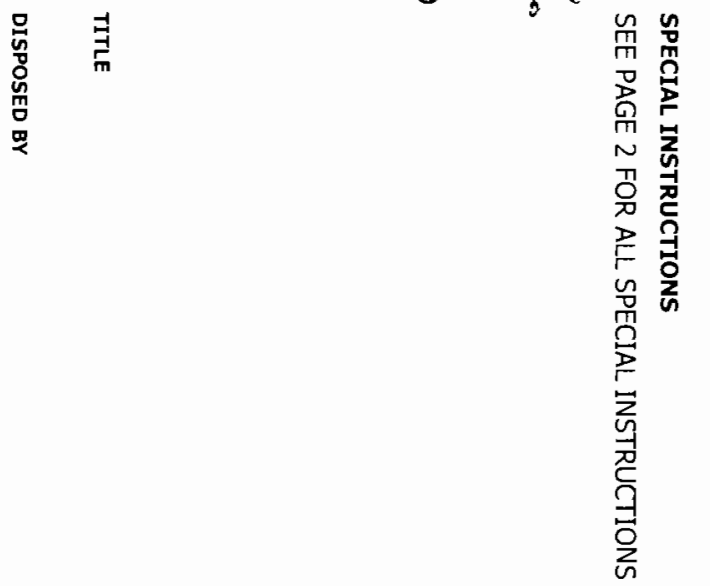

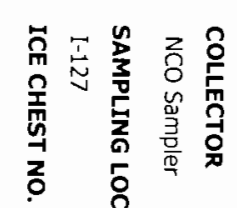

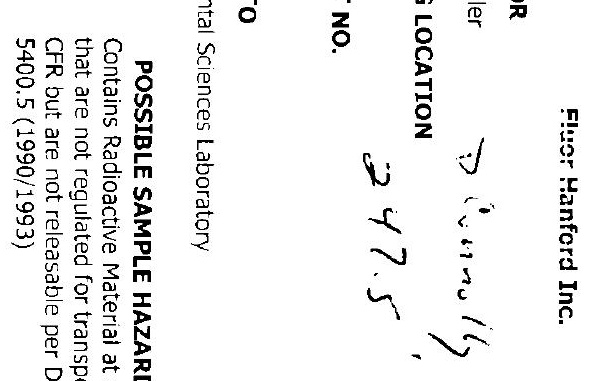

ㅁํㅇ응 즘

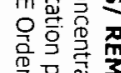

总害 变

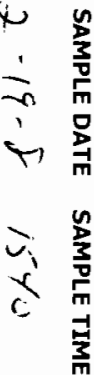

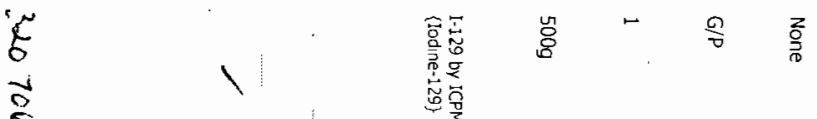

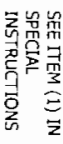

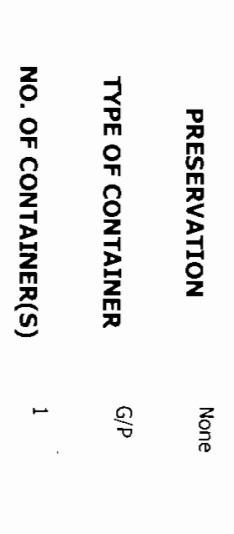

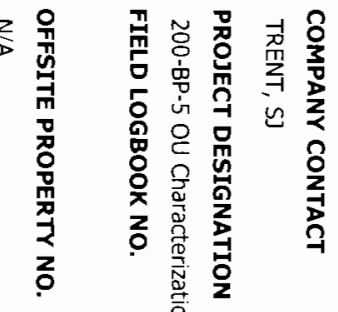

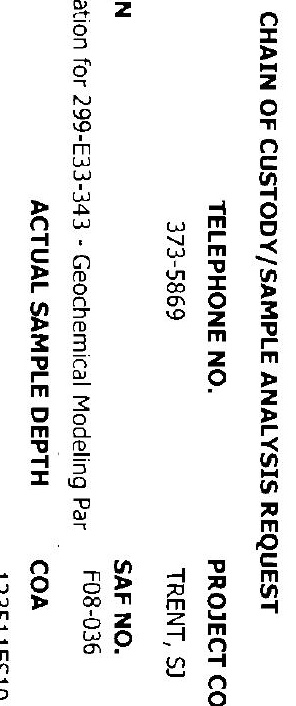

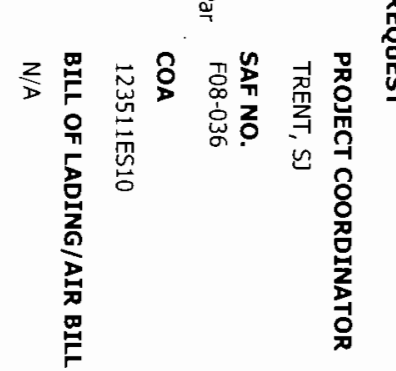

总

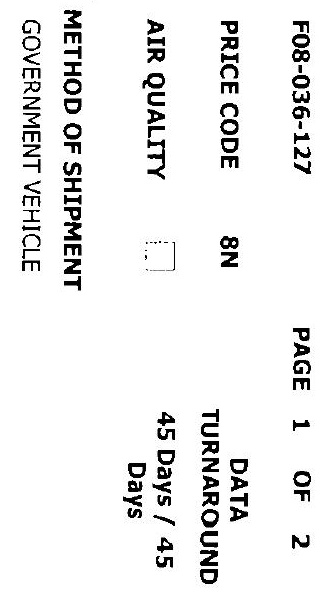




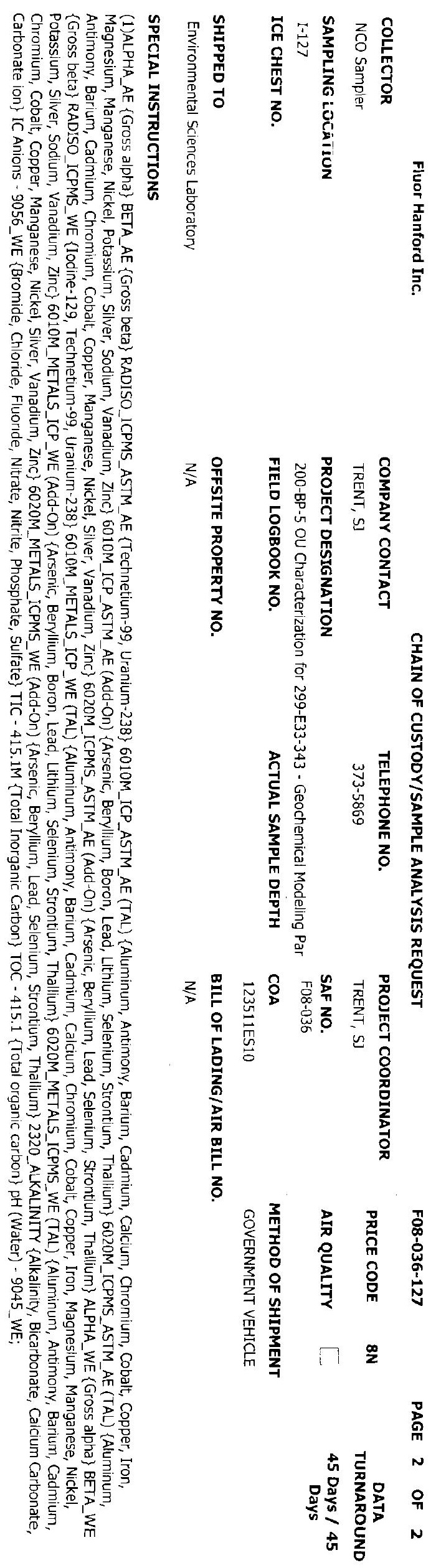




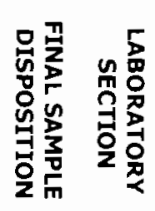
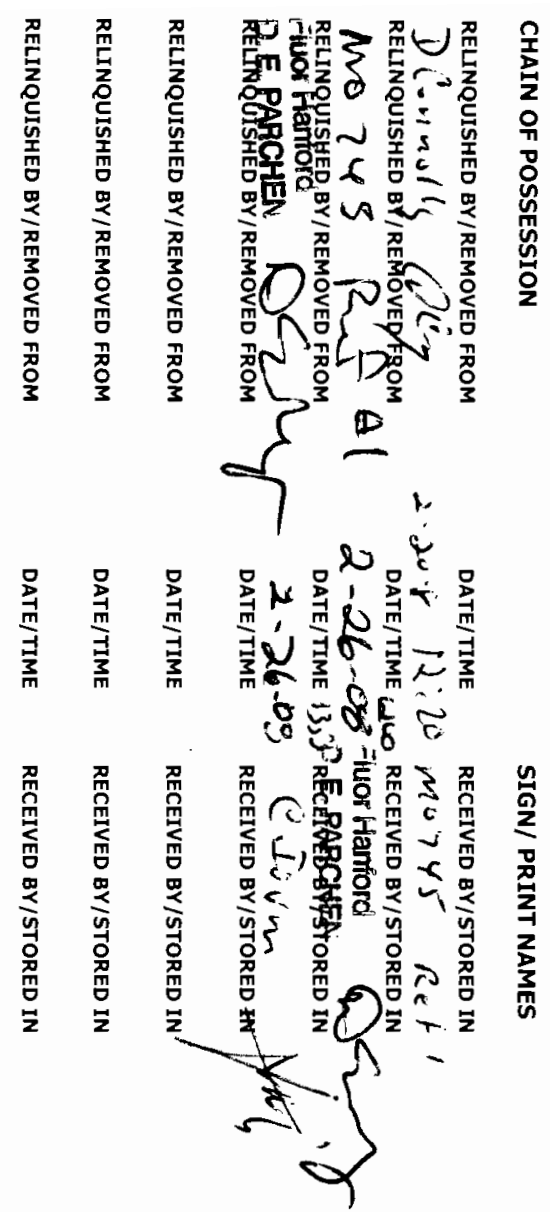

蒠

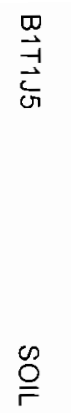

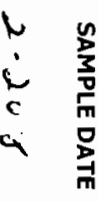

$\begin{array}{ll}\text { c. } & \frac{n}{3} \\ v & \frac{m}{0} \\ & \frac{1}{3} \\ \frac{1}{3} & \end{array}$

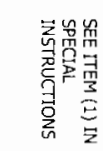

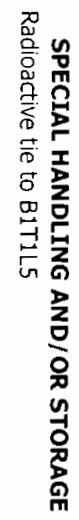

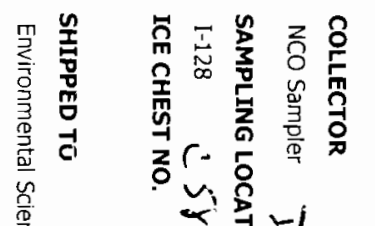

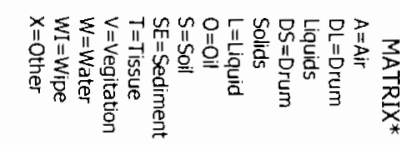

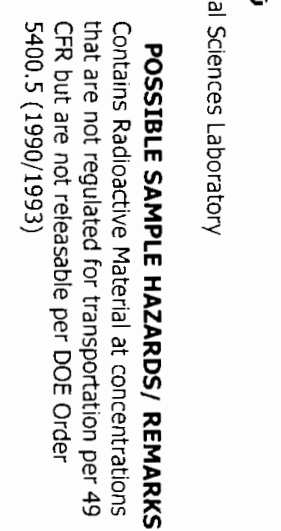

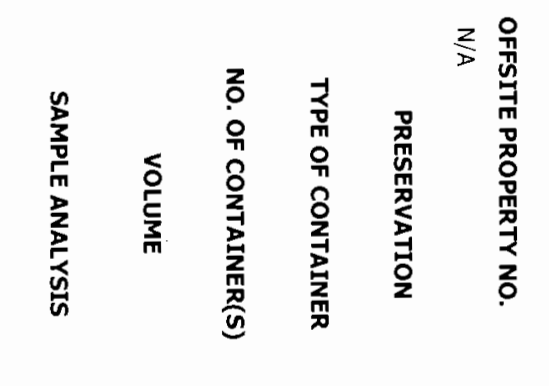

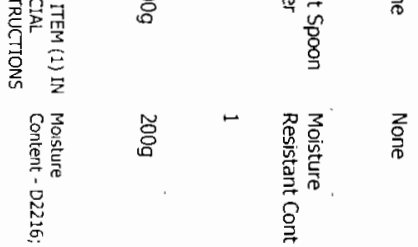

品暑量

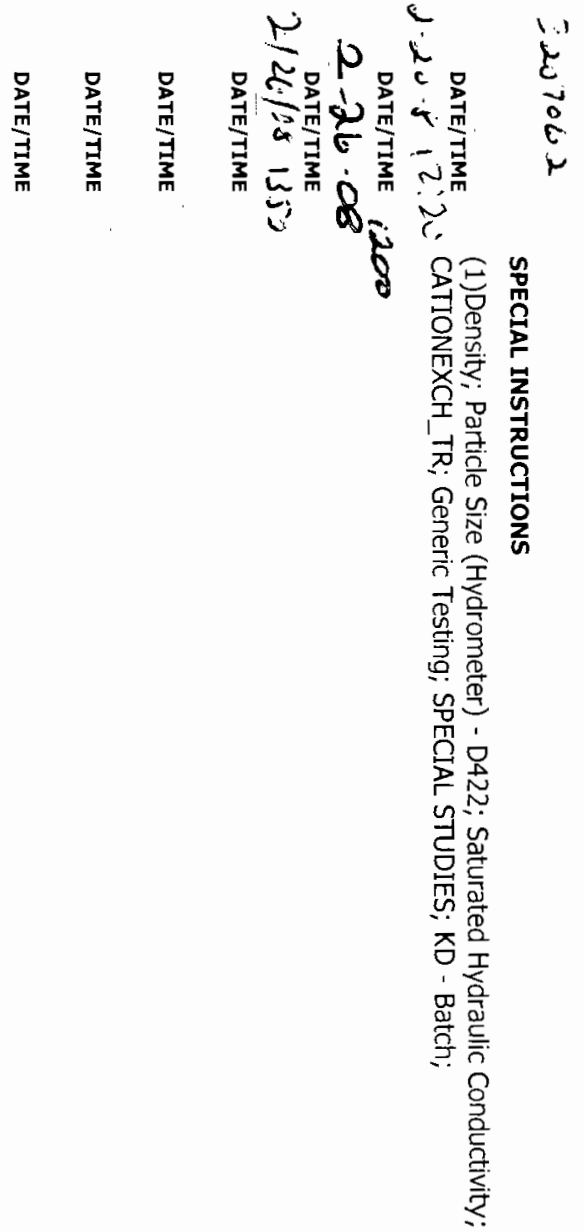




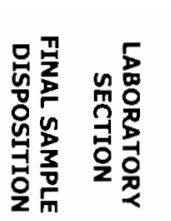
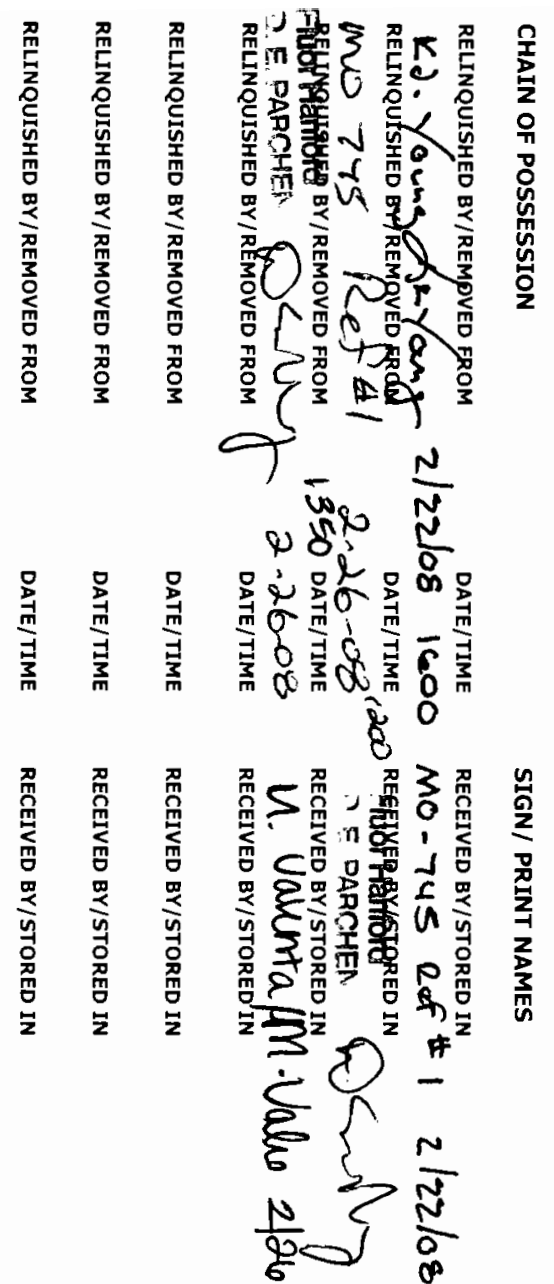

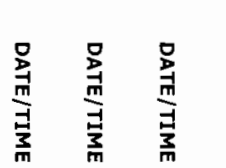

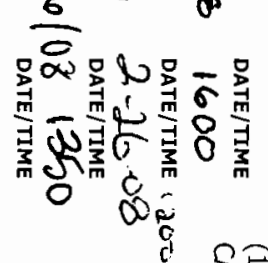

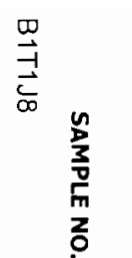

$\stackrel{p}{\risingdotseq}$

$\underset{*}{\stackrel{3}{\operatorname{d}}}$

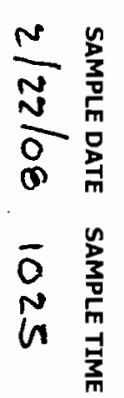
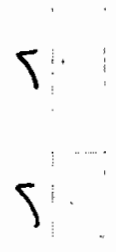

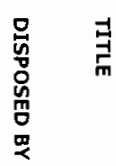

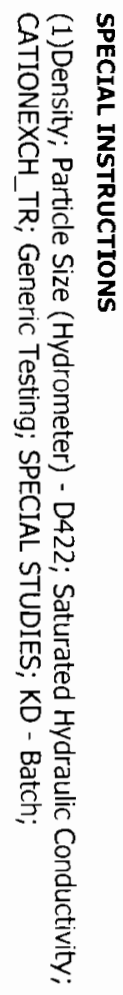

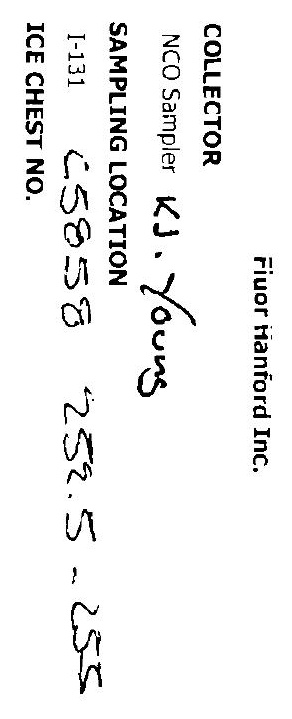

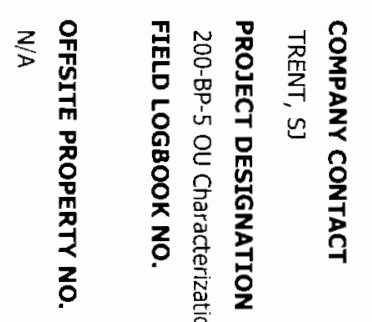

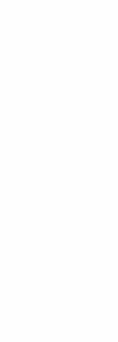

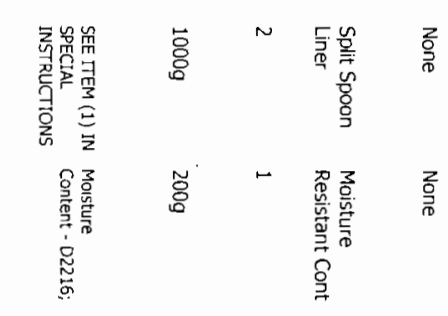

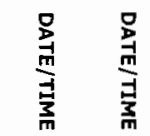

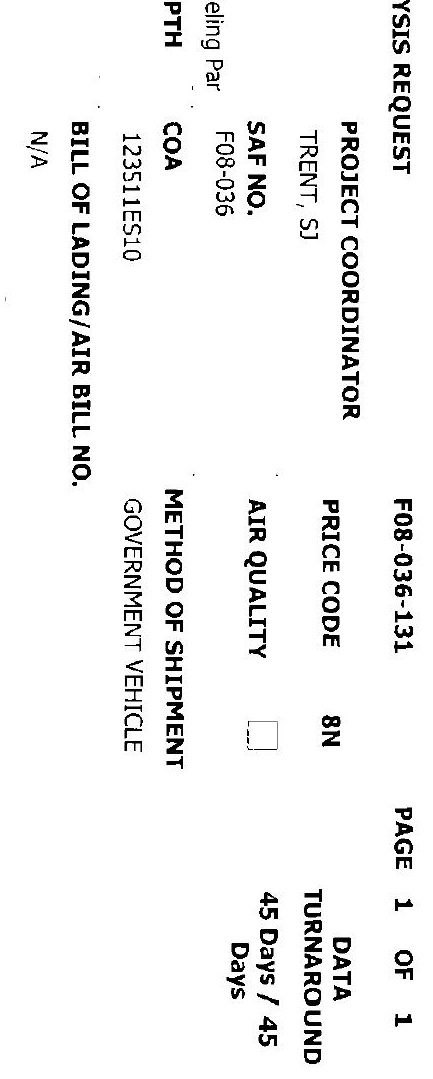




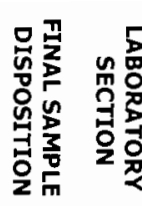

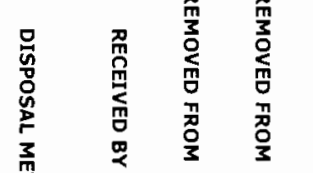

$\frac{3}{3}$

寻

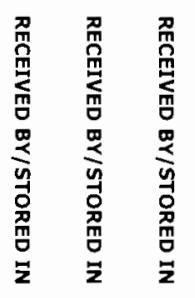

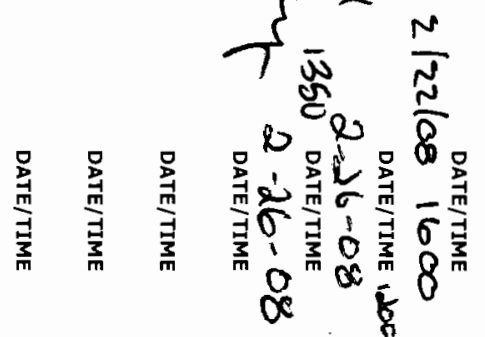
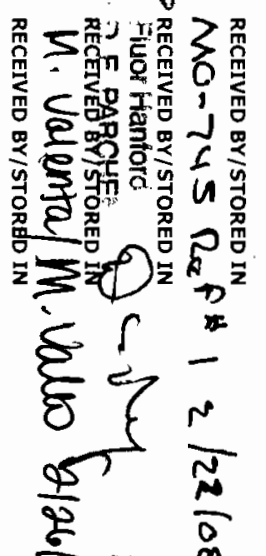

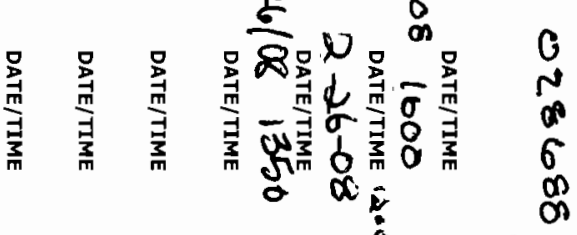

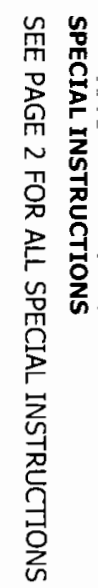

$\stackrel{\varpi}{\exists}$

$\stackrel{\varrho}{\circ}$

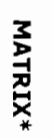

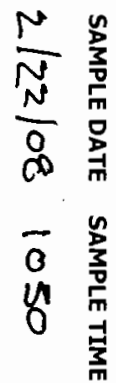

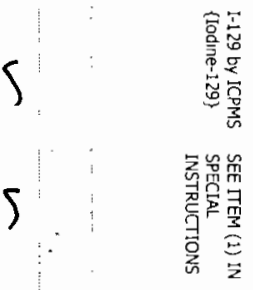

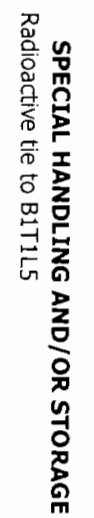

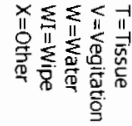

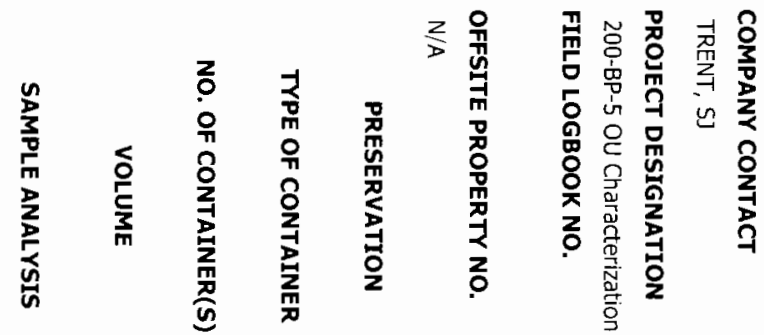

峞 $-\frac{9}{8} \frac{2}{\frac{5}{\sigma}}$

$+\quad-\frac{1}{2} \frac{\overline{0}}{\frac{1}{2}}$

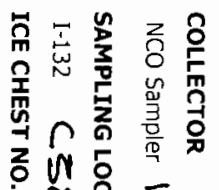

on $\overline{1}$ 弯

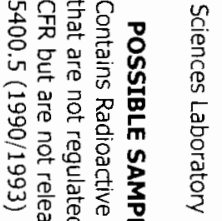

岁

亭焉

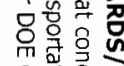

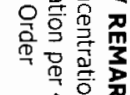

क高

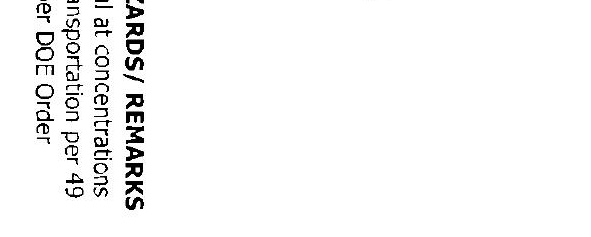

是

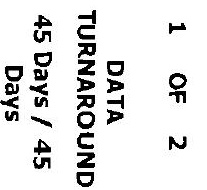




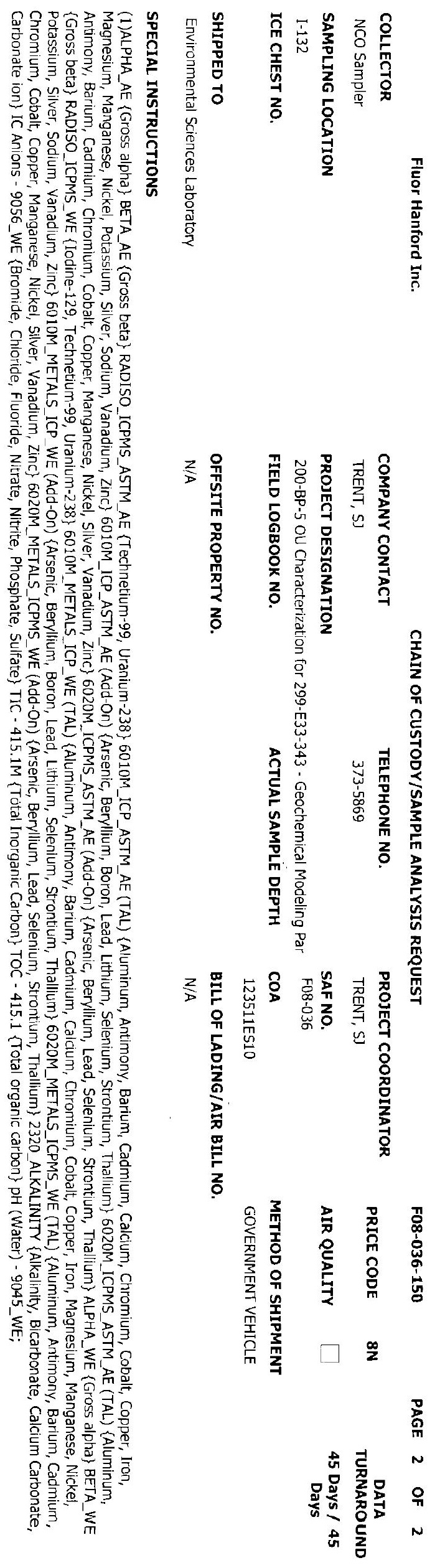




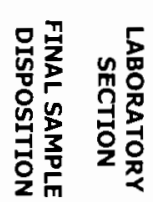

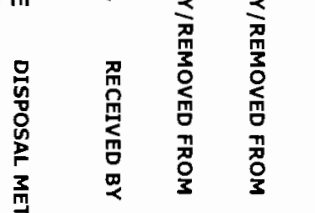

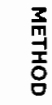

录

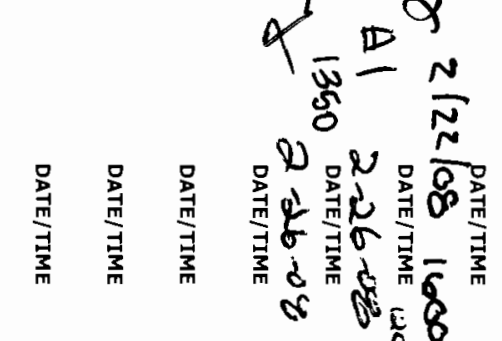
$\frac{n}{5} \quad \frac{N}{d}$

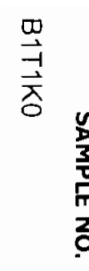

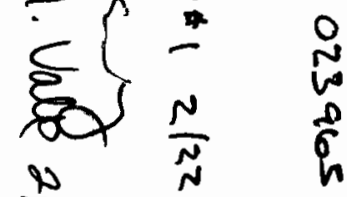

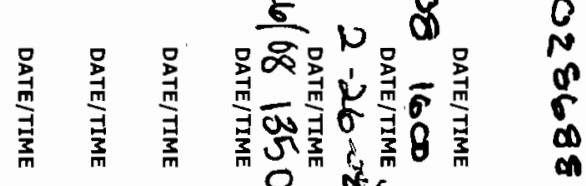

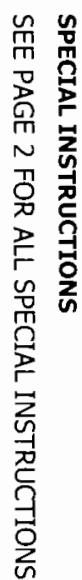

$\stackrel{\rho}{\circ}$

$\underset{3}{3}$

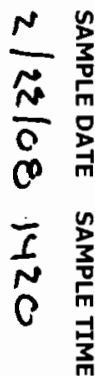

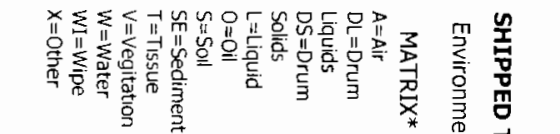

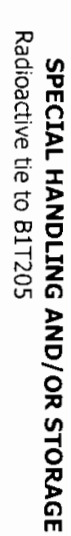

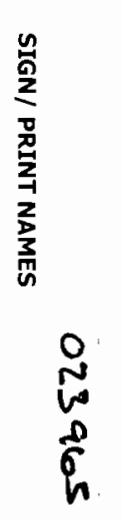

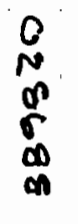

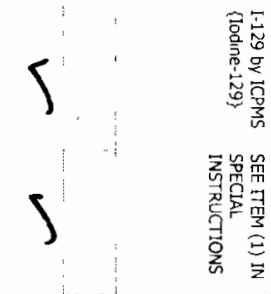

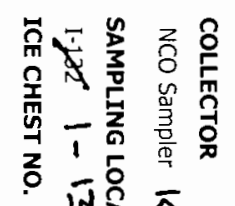

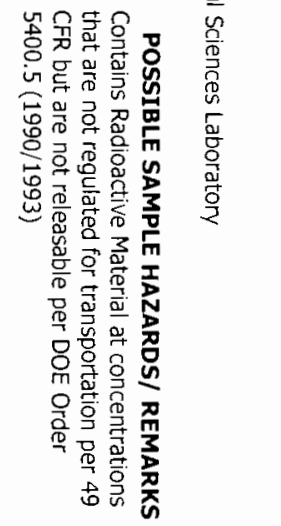

z위

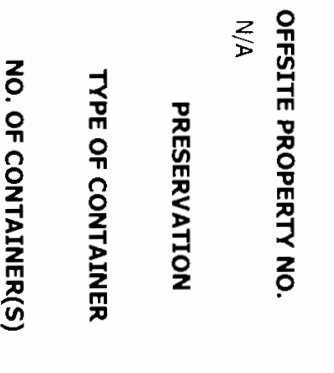

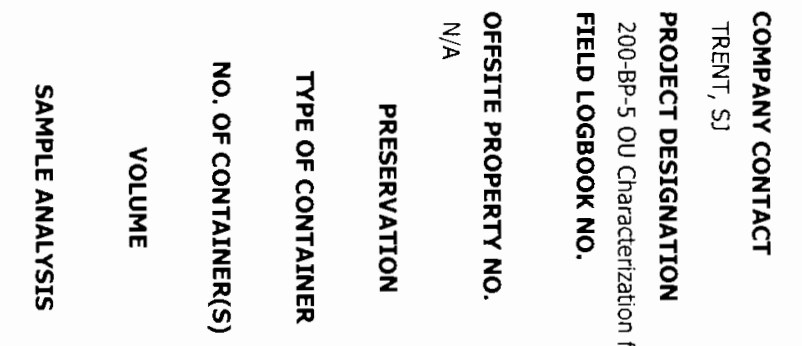

녕 $+\frac{0}{8}$ 홍 


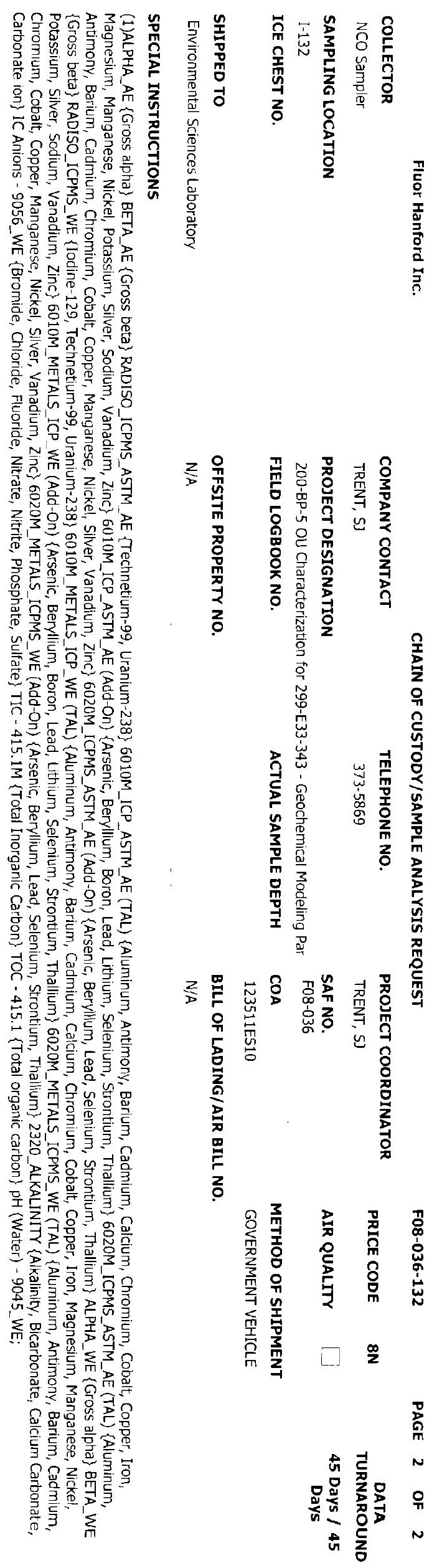




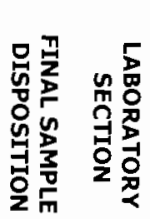

$\frac{\pi}{5}$

W

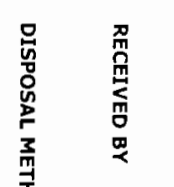

F

p

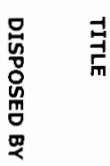

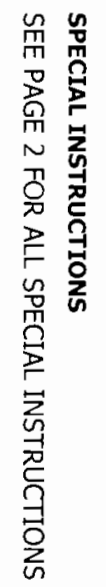

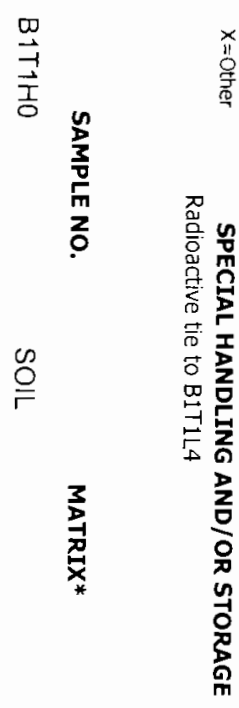

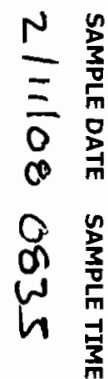

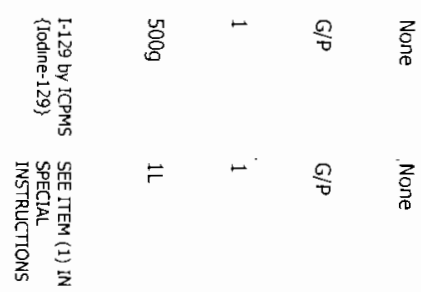

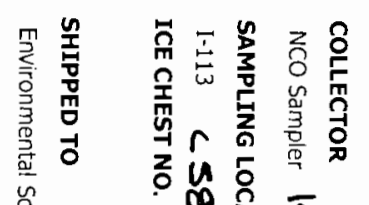

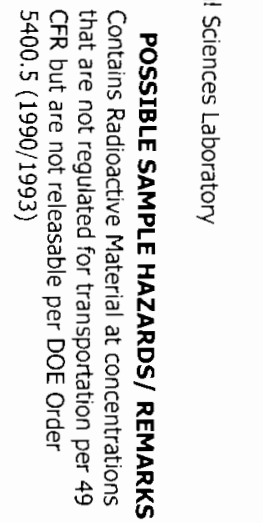

舫

$\infty$ ऽ)

त) is

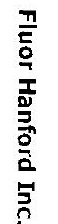

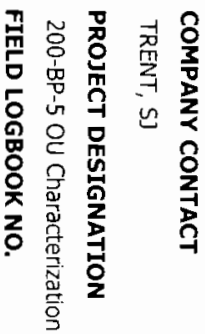

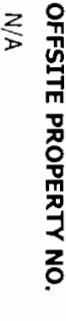

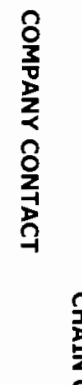

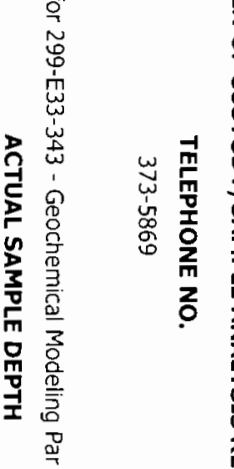

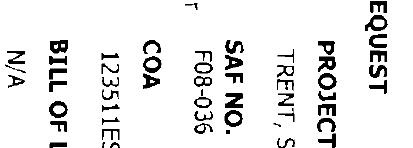

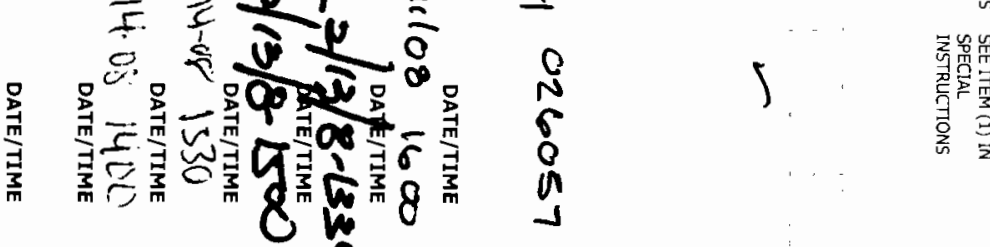

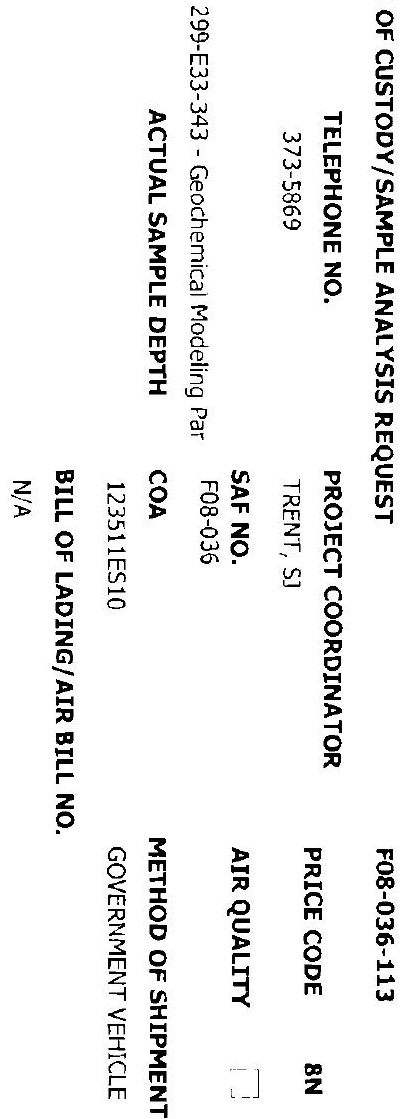

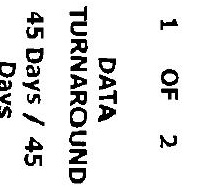




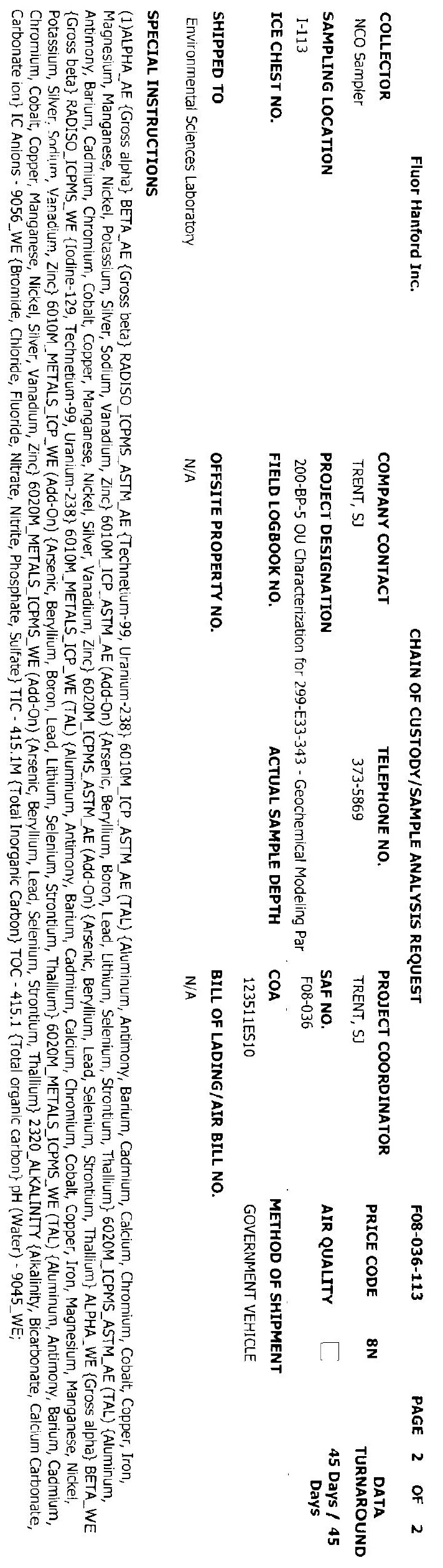




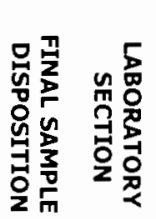

$w$

$\frac{w}{w}$

$\frac{w}{f}$

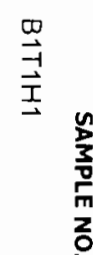

$\stackrel{\infty}{\circ}$

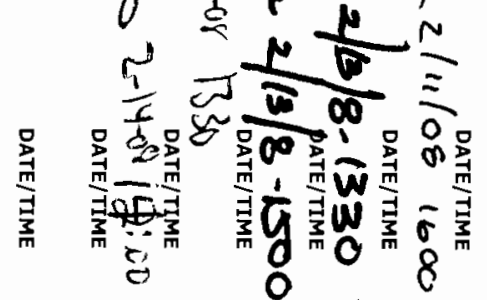

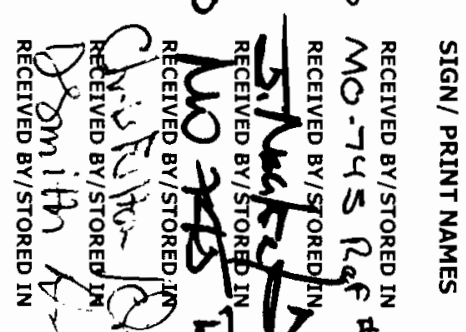

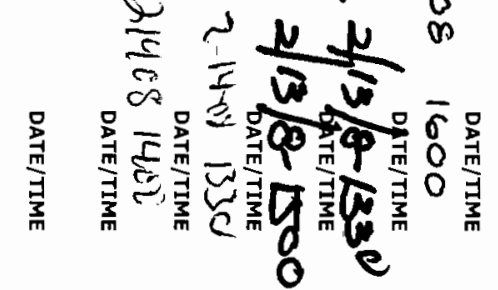

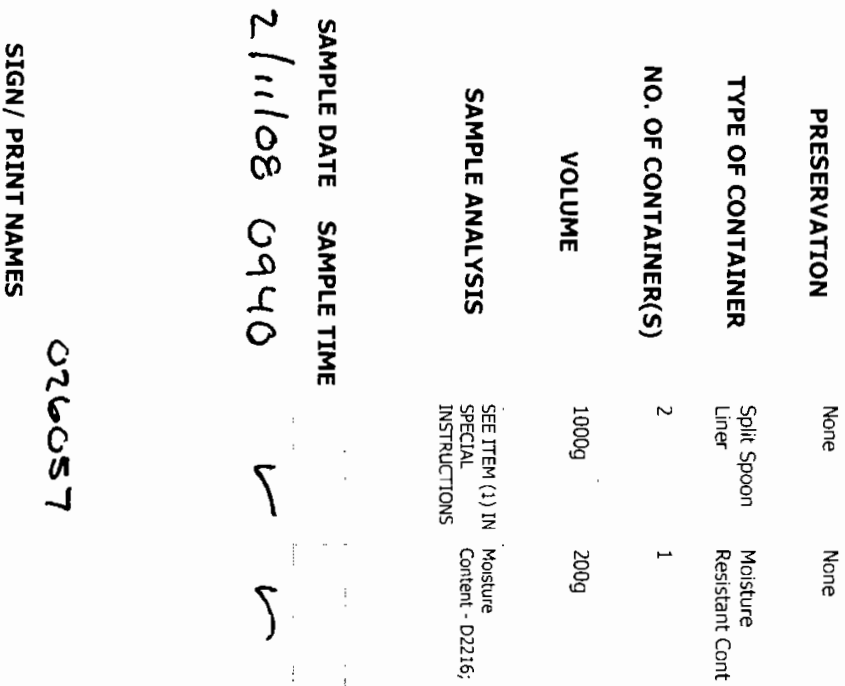

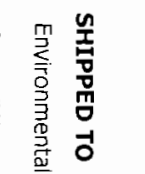

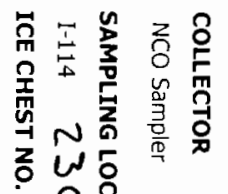

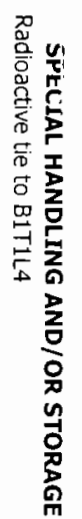

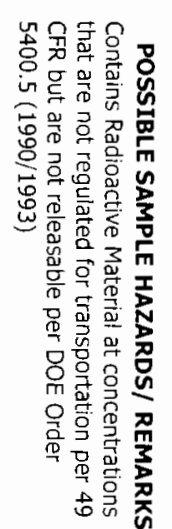

.

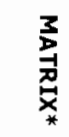

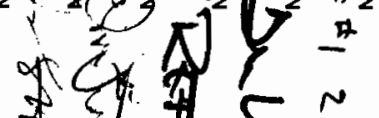

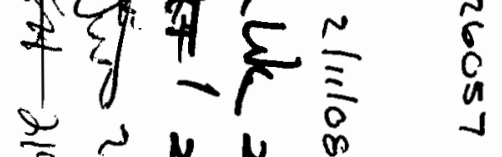

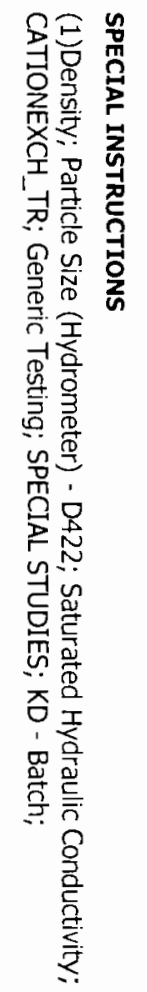

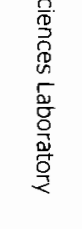

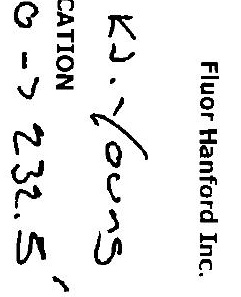

$\overbrace{n}^{n}$

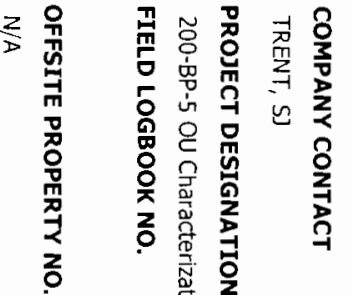

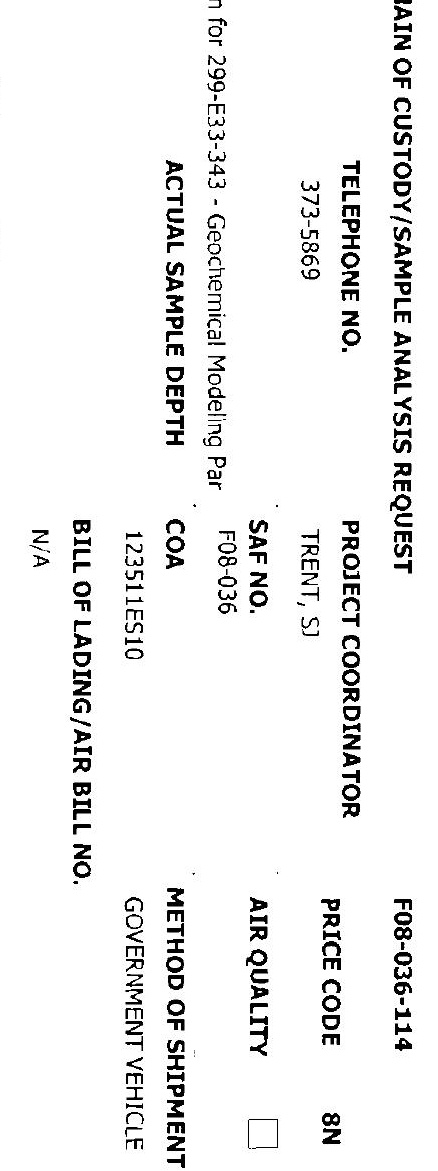

ลำ

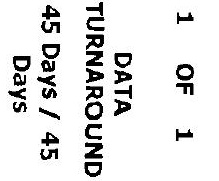




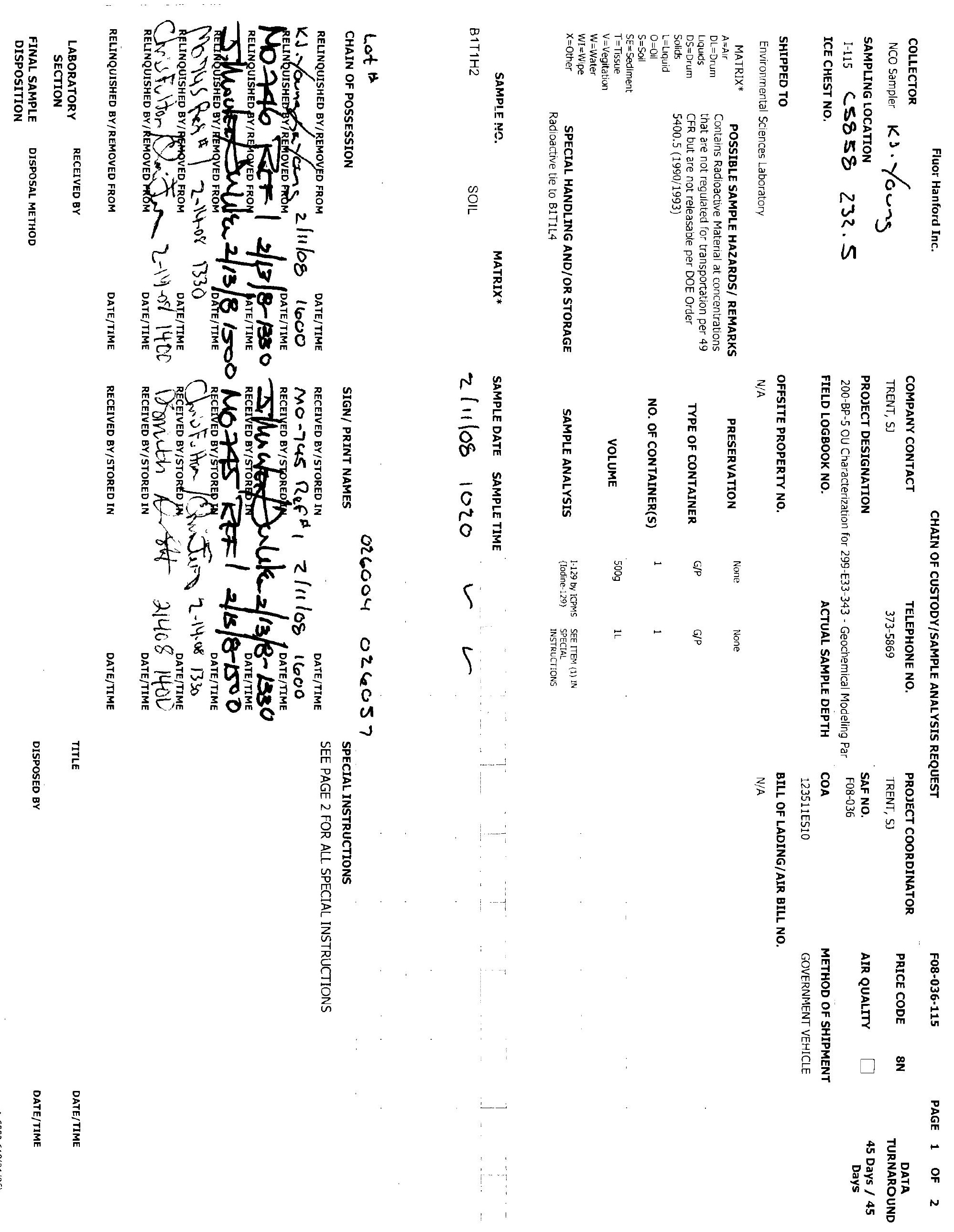




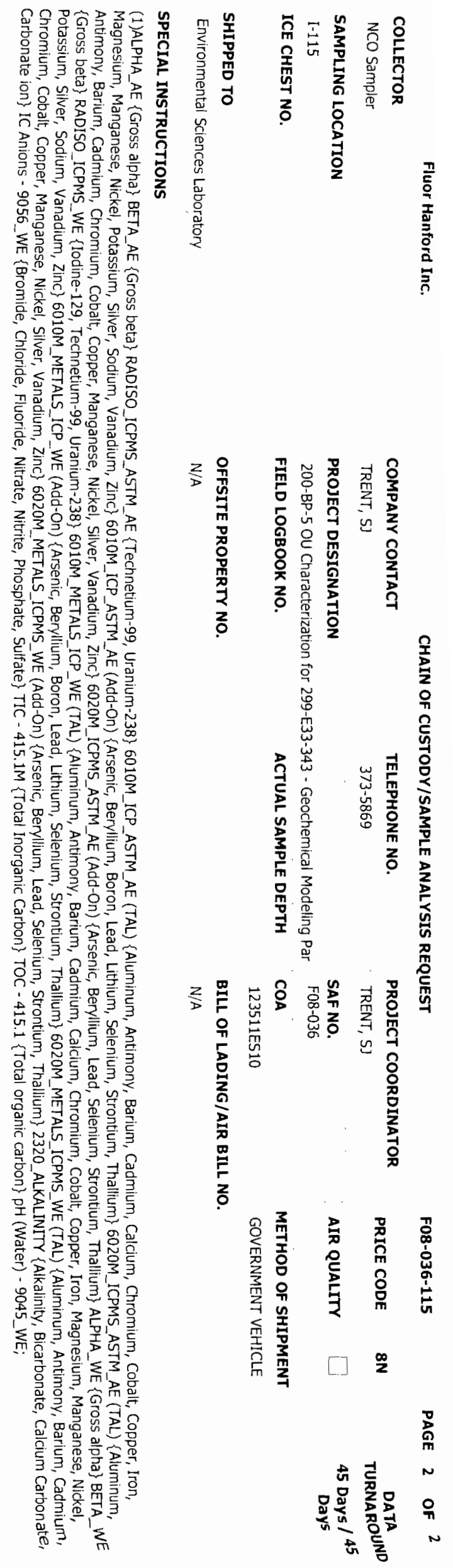




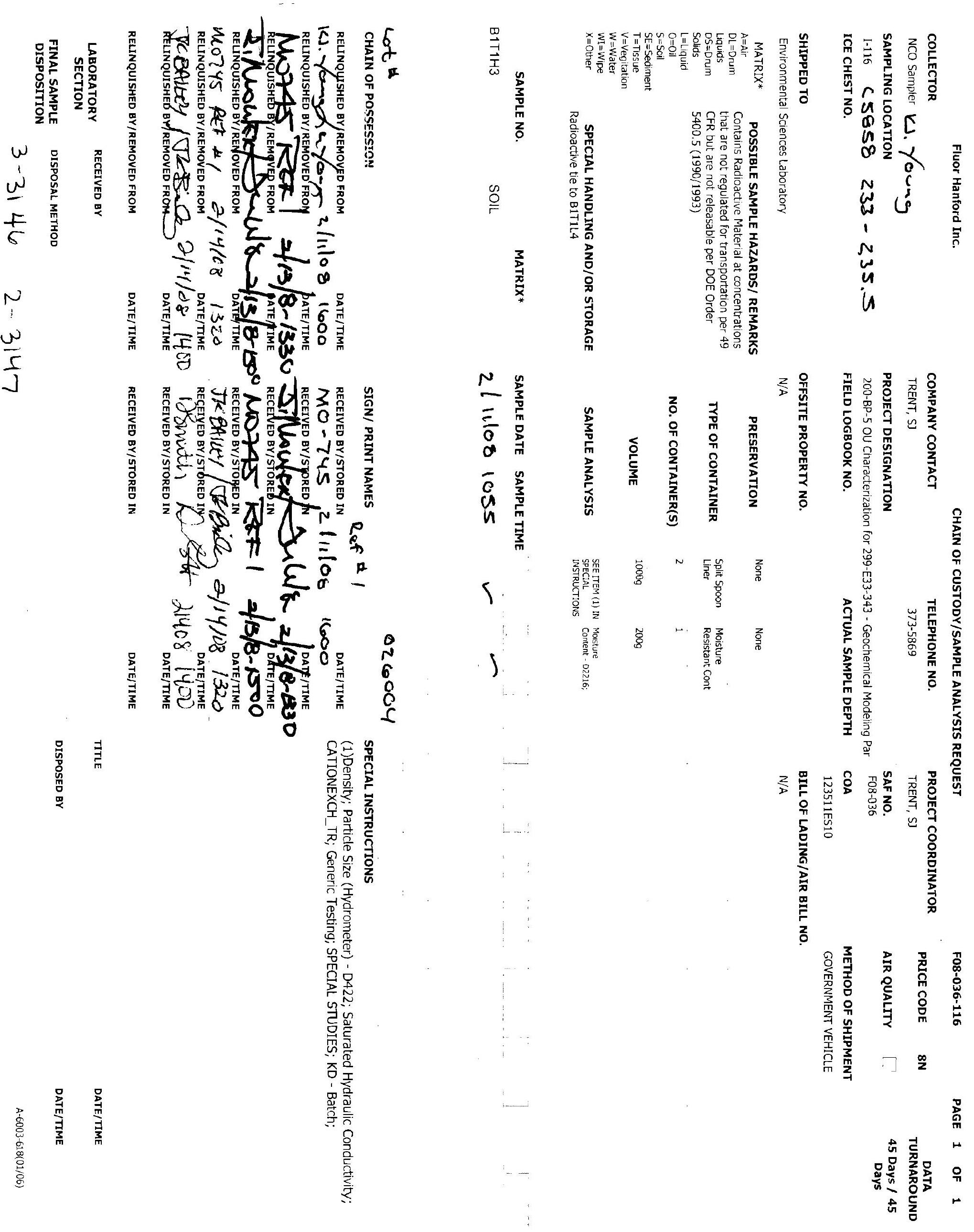




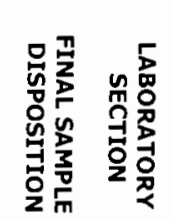

$w$

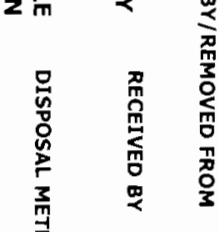

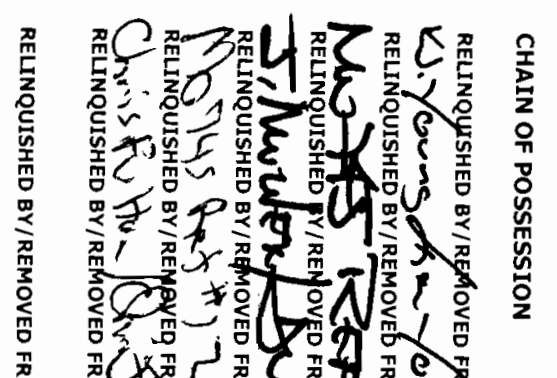

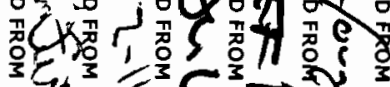

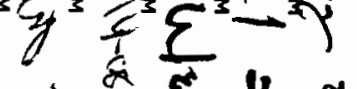

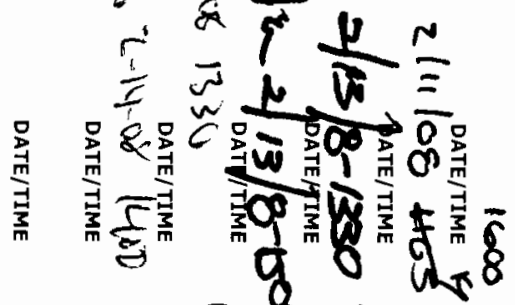

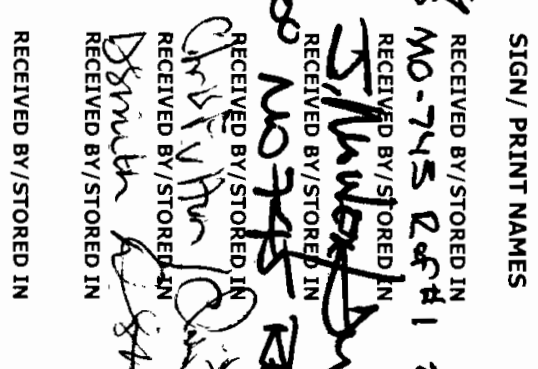

क

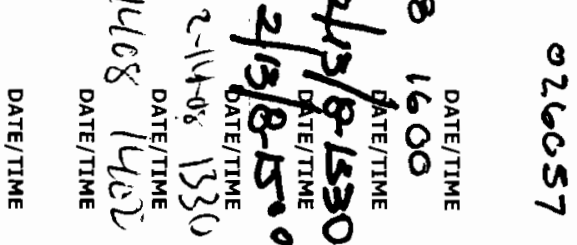

鿷

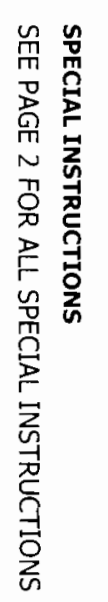

:
$\underset{\vec{I}}{\stackrel{0}{\vec{I}}}$

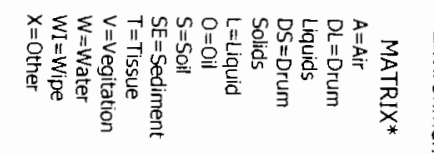

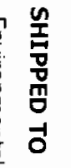

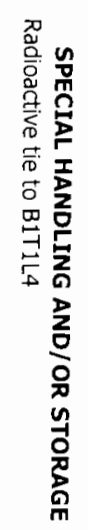

$\begin{array}{ll}N & \\ = & \frac{3}{3} \\ = & \frac{3}{\pi} \\ 0 & 0 \\ 0 & \frac{1}{7}\end{array}$

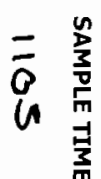

S
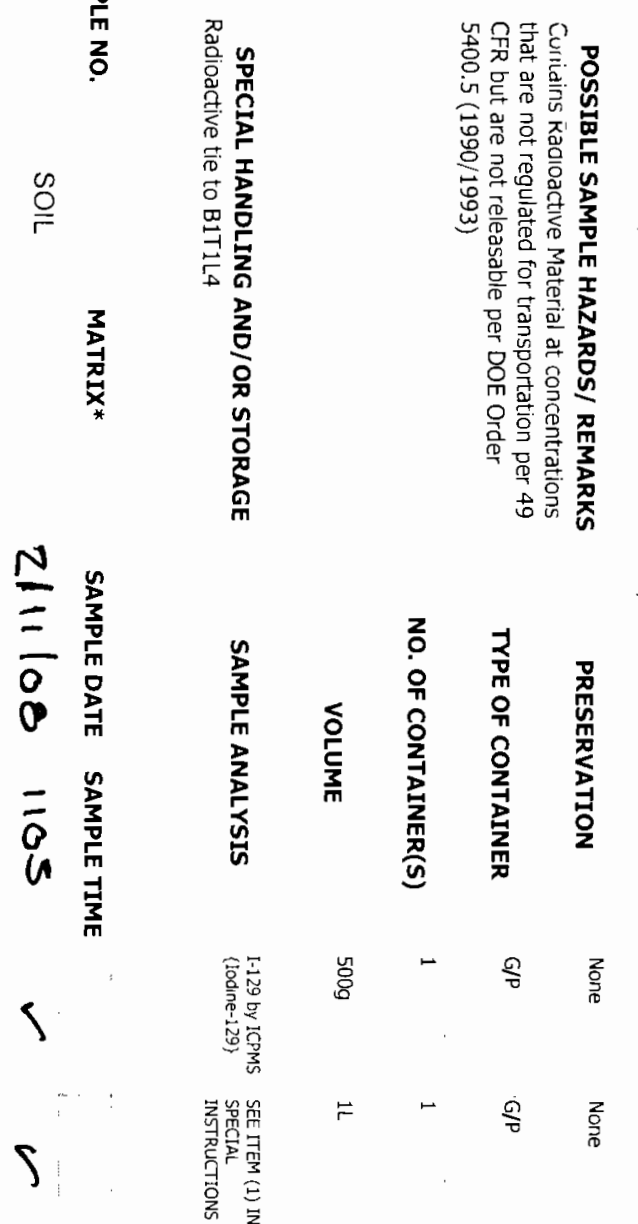

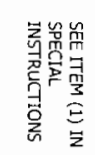

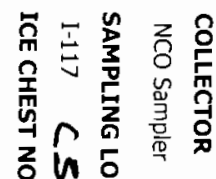

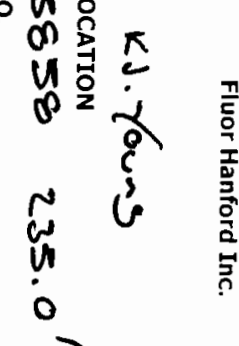

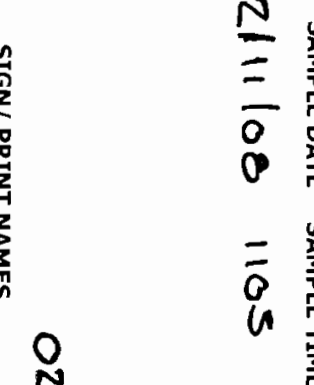

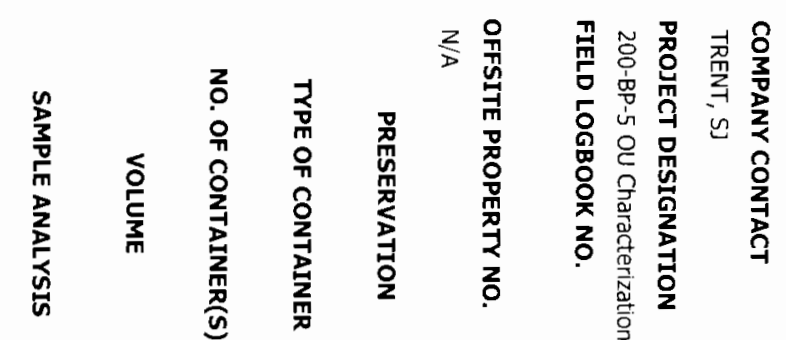
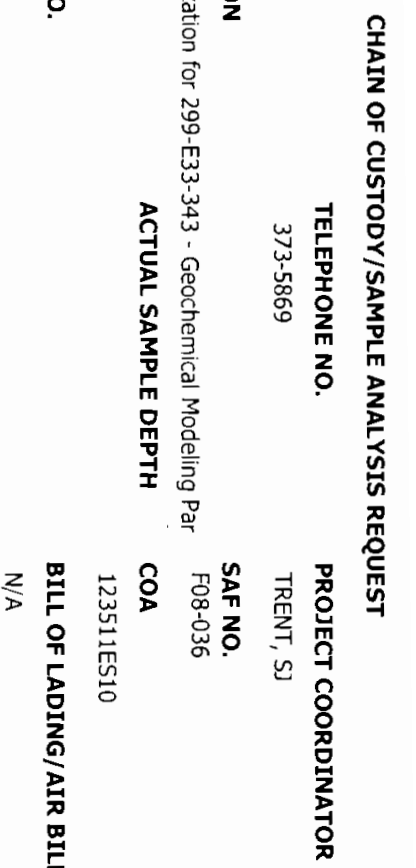

z

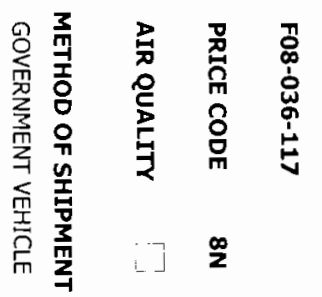

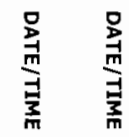

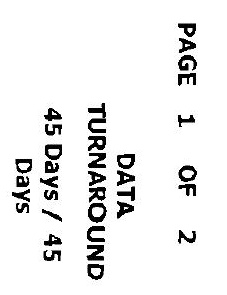




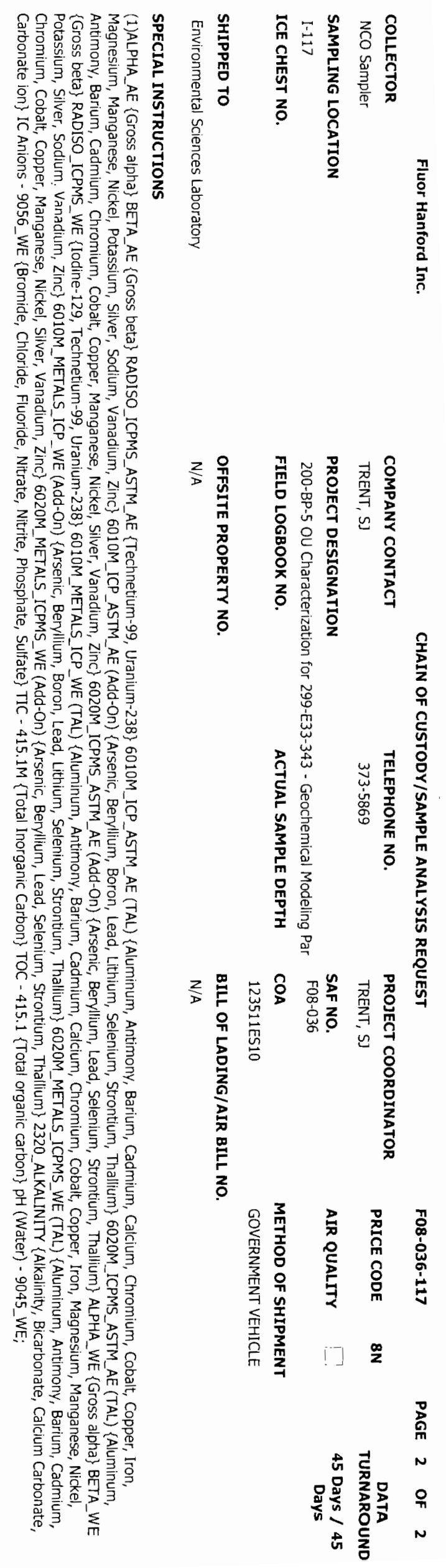




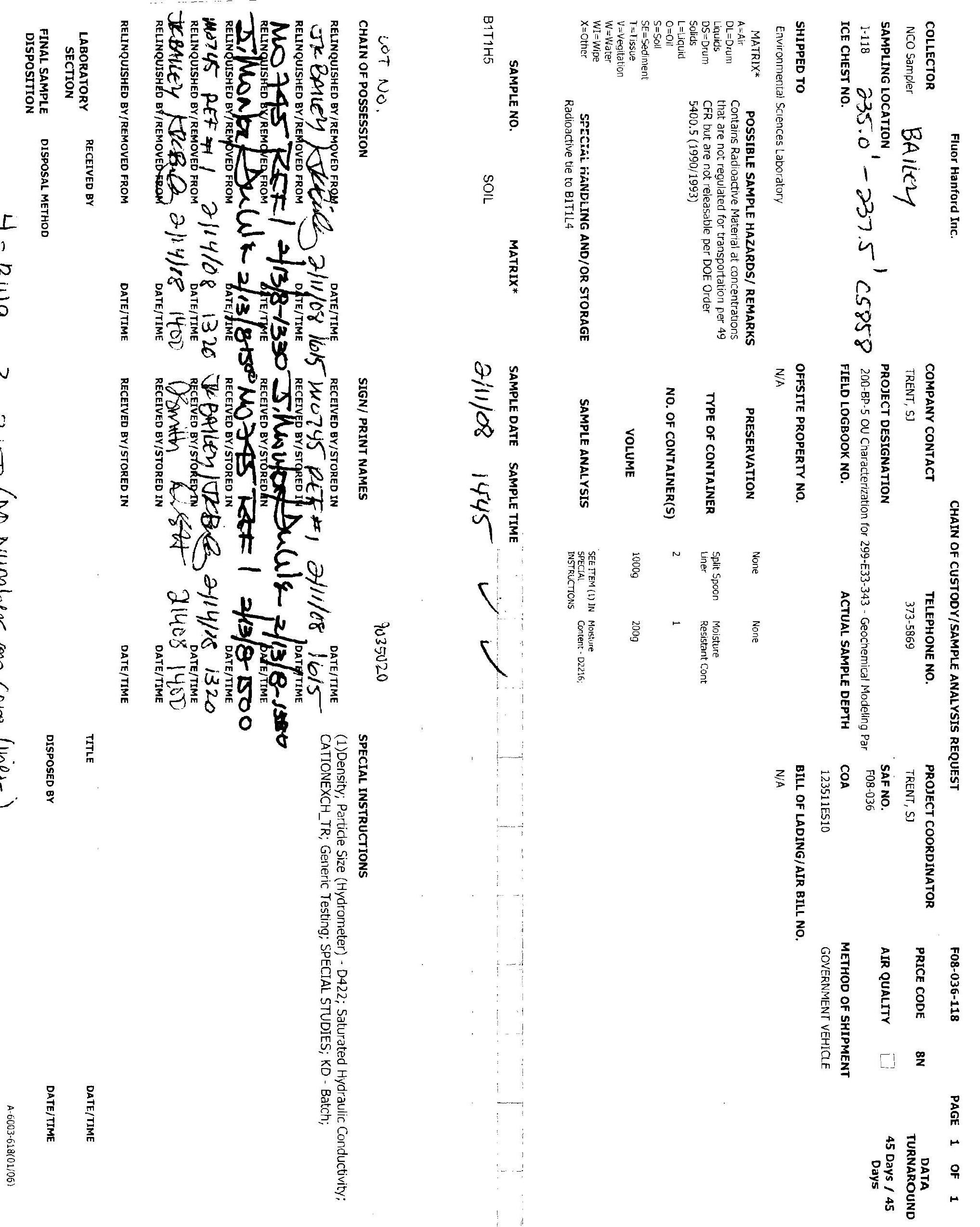




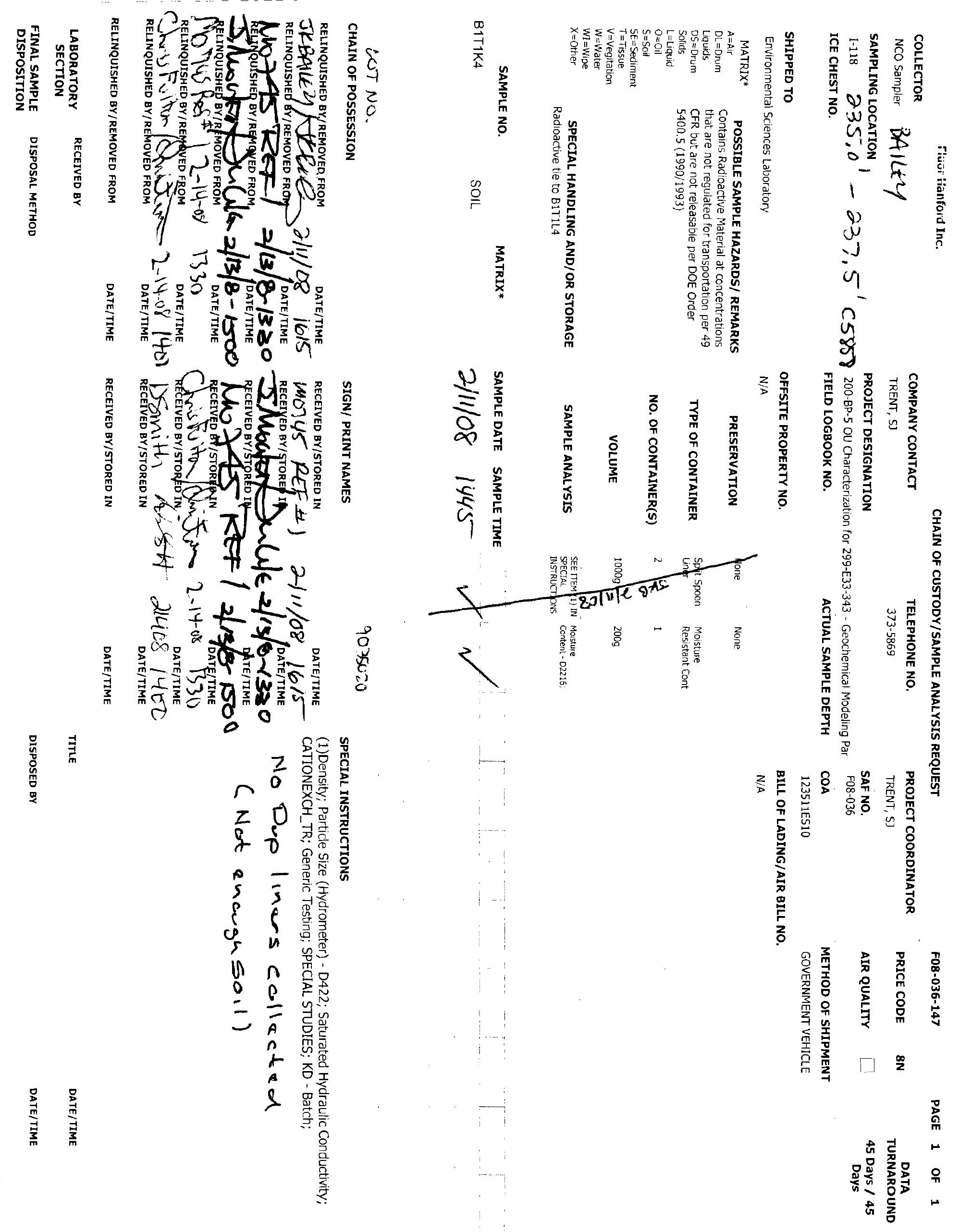




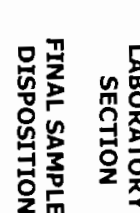
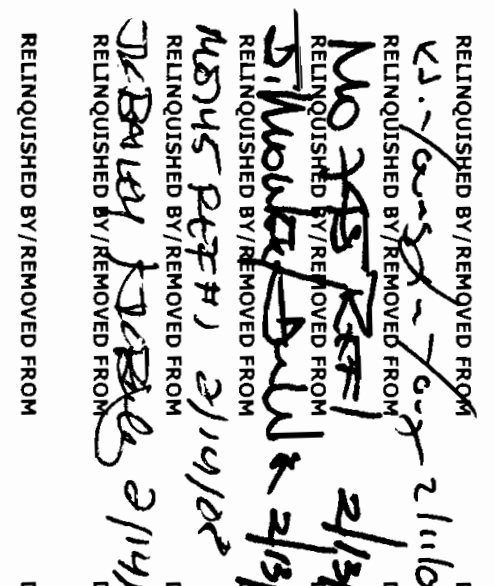

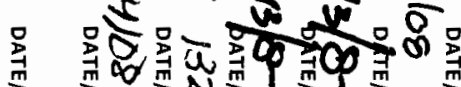

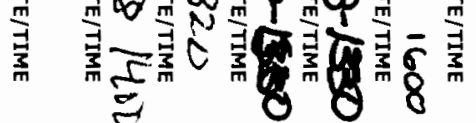

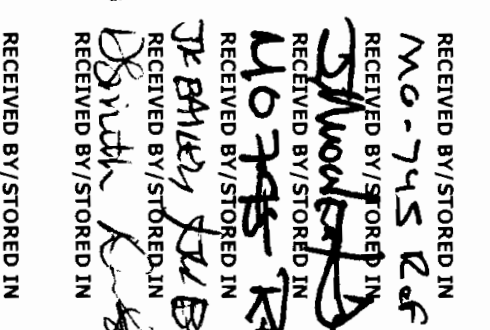

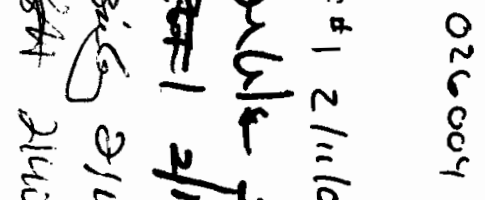

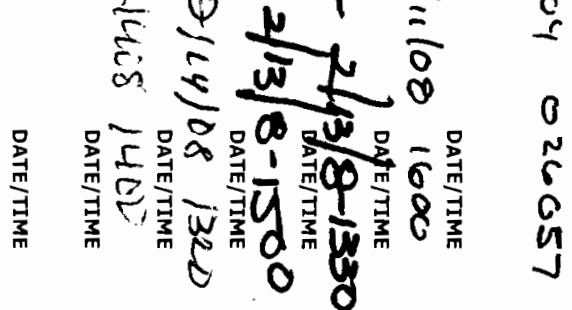

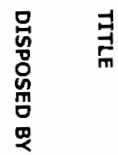

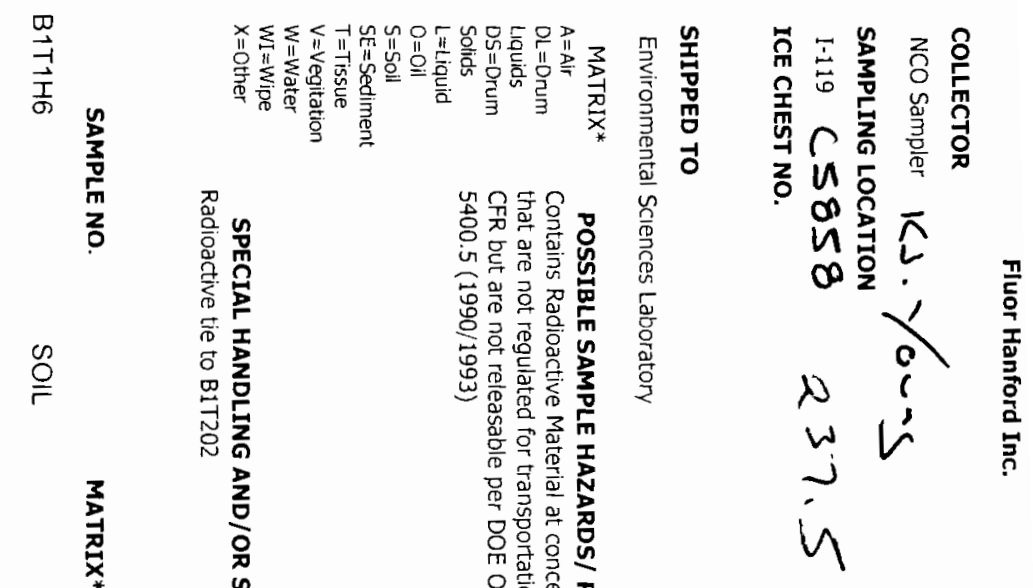

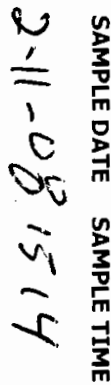

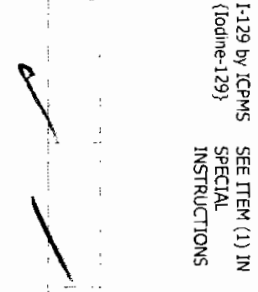

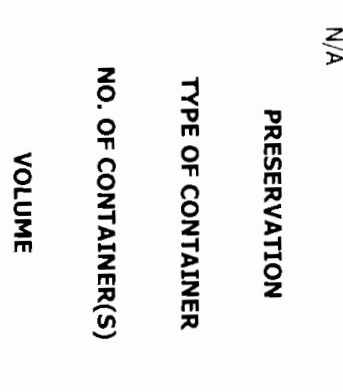

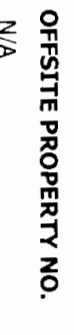

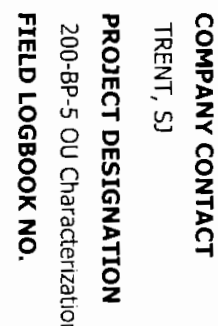

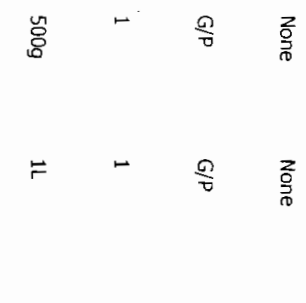

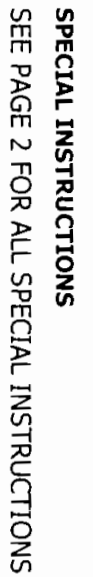

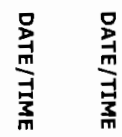




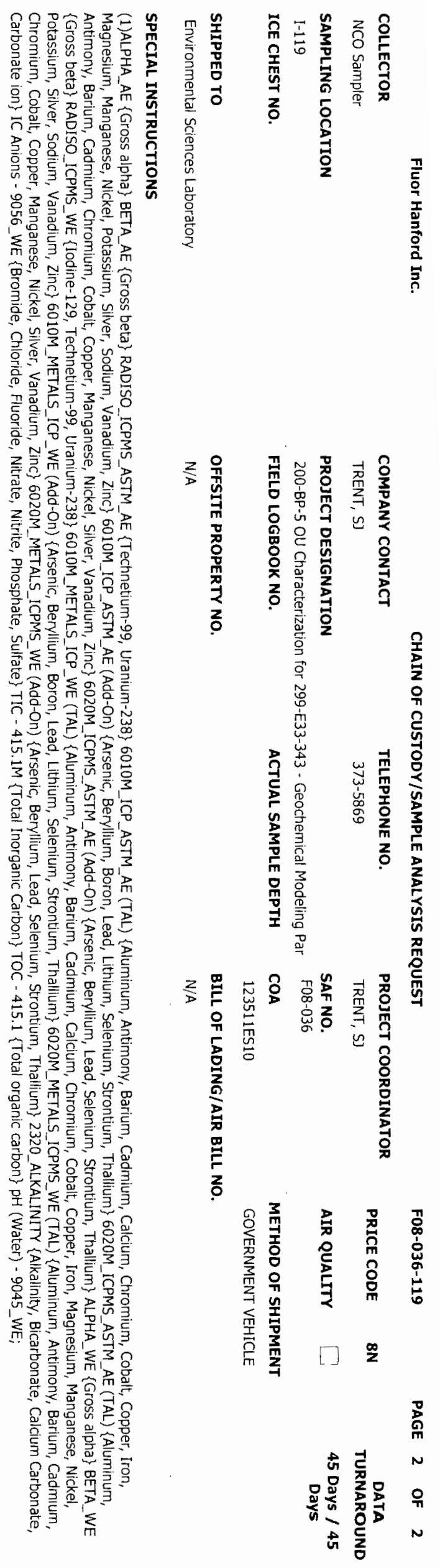

